DOE/CH-9601

Distribution Category:

General, Miscellaneous, and

Progress Reports (UC-600)

\title{
DEVELOPMENT OF THE VITRIFICATION COMPOSITIONAL ENVELOPE TO SUPPORT COMPLEX-WIDE APPLICATION OF MAWS TECHNOLOGY
}

Contract No. 32662402

\author{
Edited by \\ James J. Mazer \\ Argonne National Laboratory \\ Contributors \\ Isabelle S. Muller, Hao Gan, Andrew C. Buechele, \\ Shan-Tao Lai, and Ian L. Pegg \\ Vitreous State Laboratory \\ The Catholic University of America \\ Washington, D.C. \\ and \\ GTS Duratek, Inc. \\ Columbia, Maryland \\ DISTRABUTION OF THIS DOCUMENT IS UALLRITED \\ September 1996 \\ Prepared for

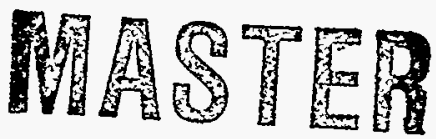 \\ Office of Waste Management \\ U.S. Department of Energy \\ 1000 Independence Avenue \\ Washington, DC 20585-0002 \\ Environmental Programs Group \\ Technical Team \\ Chicago Field Office \\ U.S. Department of Energy \\ 9800 S. Cass Avenue \\ Argonne, IL 60439 \\ Waste Management Department-Nuclear Waste Management Section \\ Chemical Technology Division, Argonne National Laboratory \\ 9700 S. Cass Avenue, Argonne, IL 60439 \\ under Prime Contract W-31-109-Eng-38 to the U.S. Department of Energy
}


GTS Duratek

The Catholic University of America
MAWS Compositional Envelope

Phase I Final Report

\section{ACKNOWLEDGMENTS}




\section{Table of Contents}

List of Tables $\ldots \ldots \ldots \ldots \ldots \ldots \ldots \ldots \ldots \ldots \ldots \ldots \ldots \ldots \ldots \ldots \ldots \ldots \ldots$ vi

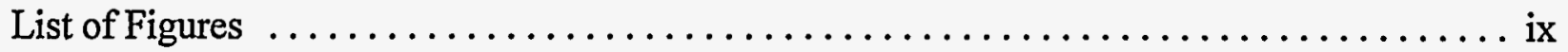

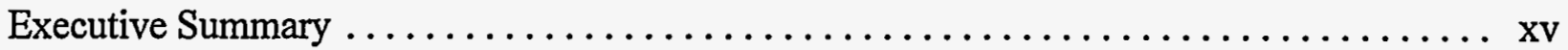

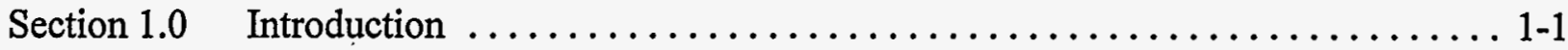

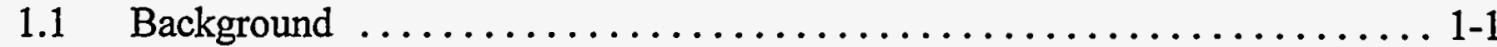

1.2 The MAWS Vitrification Compositional Envelope $\ldots \ldots \ldots \ldots \ldots \ldots \ldots \ldots$

1.3 Methodology and Approach ......................... 1-4

1.3.1 Waste Stream Characterization ..................... 1-5

1.3.2 Glass Formulation Development ................. 1-6

1.4 Facilities, Equipment, and Experimental Methods $\ldots \ldots \ldots \ldots \ldots \ldots \ldots \ldots$

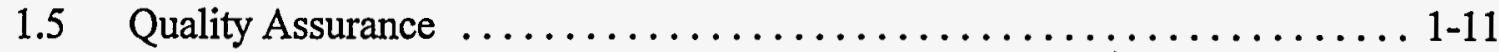

Section 2.0 MAWS Vitrification of Hanford Tank Wastes and Contaminated Soils . . . . . 2-1

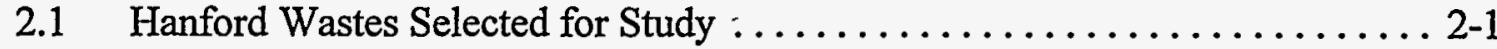

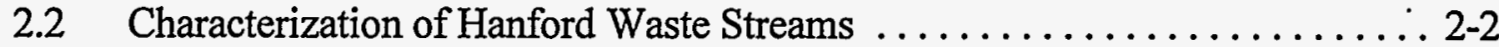

2.2.1 Non-Radioactive Soil . . . . . . . . . . . . . . . . . . . . 2-2

2.2.2 Radioactive Soil ........................... 2-2

2.2.2.1 Chemical Analysis and Physical Characterization $\ldots \ldots \ldots .2-2$

2.2.3 Hanford Tank Waste Surrogate . . . . . . . . . . . . . . 2-4

2.3 Hanford Vitrification Testing ......................... 2-5

2.3.1 Melts to Maximize Tank Waste Loading . . . . . . . . . . . . 2-5

2.3.2 Melts to Maximize Soil Loading . . . . . . . . . . . . . 2-7

2.3.2.1 Surrogate Melts ...................... 2-7

2.3.2.2 Melts Using Radioactive Soil $\ldots \ldots \ldots \ldots \ldots \ldots \ldots \ldots$. . . . . . .

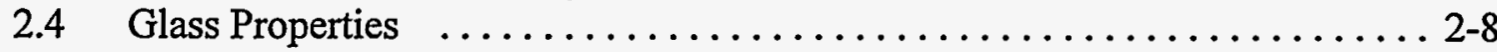

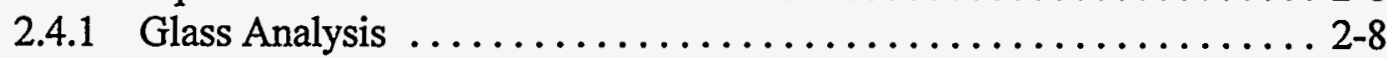

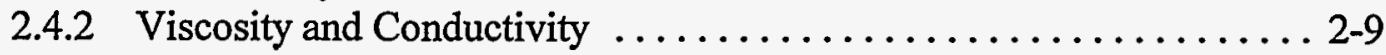

2.4.3 Glass Leach Testing - TCLP ... . . . . . . . . . . . . . . . . . . . 2-11

2.4 .4 Glass Leach Testing - PCT ...................... 2-11

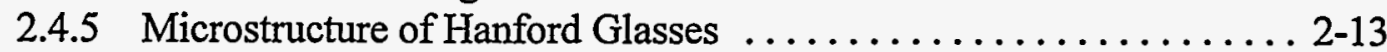

2.5 Summary and Conclusions ........................ 2-15

Section 3.0 MAWS Vitrification of Idaho Waste Streams: Contaminated Asbestos,

Eutectic Salt, WERF Fly Ash, and Soil .................... 3-1

3.1 Idaho Wastes Selected for Study ....................... $3-1$

3.2 Characterization of Idaho Waste Streams $\ldots \ldots \ldots \ldots \ldots \ldots \ldots \ldots . \ldots \ldots$ 
3.2.1 Idaho Radioactive Asbestos-Containing Material (ACM) . . . . . . . 3-2 3.2.1.1 Chemical Analysis and Physical Characterization ........ 3-2

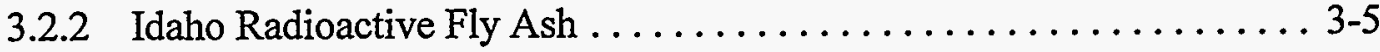

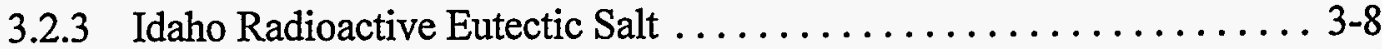

3.2.4 Idaho Non-Radioactive Soil . . . . . . . . . . . . . . . . . . . 3-10

3.2.5 Summary of Waste Stream Characterization Results ......... 3-11

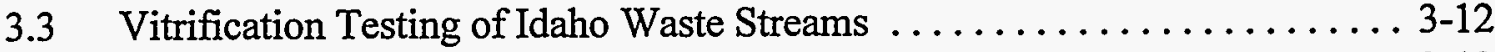

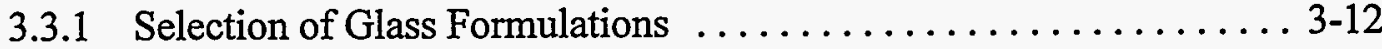

3.3.2 Glass Characterization . . . . . . . . . . . . . . . . . 3-14

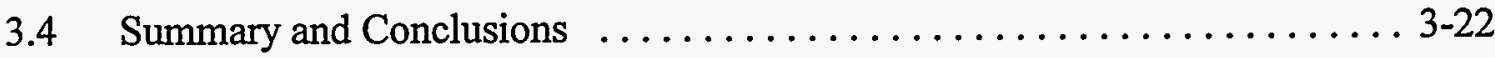

Section 4.0 MAWS Vitrification of Oak Ridge K-1407 Pond Waste, West End

Treatment Facility Sludge, TSCA Ash, and Soil . . . . . . . . . . . . . 4-1

4.1 Oak Ridge Wastes Selected for Study . . . . . . . . . . . . . . . . 4-1

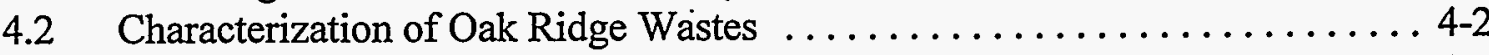

4.2.1 Oak Ridge K-1407 Pond Waste . . . . . . . . . . . . . . . 4-2

4.2 .2 TSCA Bottom Ash . . . . . . . . . . . . . . . . . . . 4-2

4.2 .3 Oak Ridge Soil ......................... 4-2

4.2.4 Oak Ridge West End Treatment Facility Sludge . . . . . . . . . . . 4-4

4.2.5 Summary of Waste Stream Characterization Results .......... 4-5

4.3 Oak Ridge Vitrification Testing . . . . . . . . . . . . . . . . . 4-6

4.3.1 Melts to Maximize $\mathrm{K}-1407$ Pond Waste Loading . . . . . . . . . . . . 4-6

4.3.2 Melts to Maximize WETF Sludge or TSCA Bottom Ash . . . . . . . 4 4-7

4.3 .3 Glass Testing . . . . . . . . . . . . . . . . . . . . . . . .

4.3.4 Summary and Conclusions ................. 4-12

Section 5.0 Development of Compositional Envelope Database and

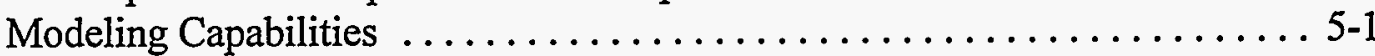

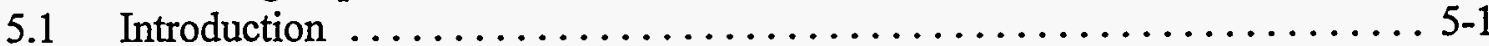

5.2 Glass Composition-Property Database $\ldots \ldots \ldots \ldots \ldots \ldots \ldots \ldots \ldots \ldots \ldots$. $\ldots \ldots \ldots$

5.2.1 Database Management System .................. 5-1

5.2.2 Glass Composition-Property Database: Contents and Structure . . . . . 5-2

5.2.3 Database User Interface: Client Application Development . . . . . . . . 5-2

5.3 Glass Composition-Property Model Development Results . . . . . . . . . 5-4

5.3.1 Sample Distribution in Compositional Space .............. 5-4

5.3.1.1 Sample Distribution of Viscosity Table . . . . . . . . . . 5-4

5.3.1.2 Sample Distribution for Conductivity Table . . . . . . . . 5-8

5.3.1.3 Sample Distribution for Hanford, Oak Ridge, and Idaho

Glasses ........................ 5-8

5.3.1.4 Sample Distribution for 7-day PCT Table . . . . . . . . . 5-8

5.3.2 Property-Composition Models and Optimization Algorithm . . . . . . 5-9 
5.3.2.1 Property-Composition Models for Viscosity, Conductivity, and 7-day PCT Data ...................... 5-9

5.3.2.2 Weighted Least Squares Regression ............. 5-12

5.3.2.3 Variable Selection and Model Performance Evaluation .... . 5-13

5.3.3 Modeling Results and Performance Evaluation ............. 5-16

5.3.3.1 Conductivity-Composition Model ................ 5-16

5.3.3.2 Viscosity Compositional Model ................. 5-19

5.3.3.3 7-Day PCT Compositional Models ............... 5-21

5.4 Example Model Results for Hanford, Idaho, and Oak Ridge Glasses . . . . . . 5-22

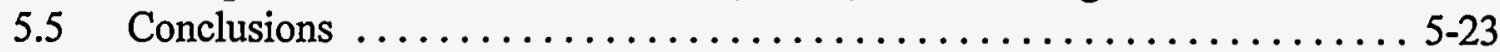

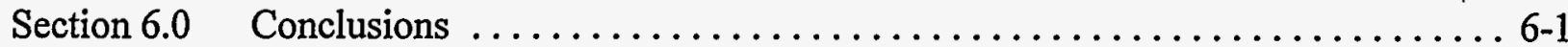

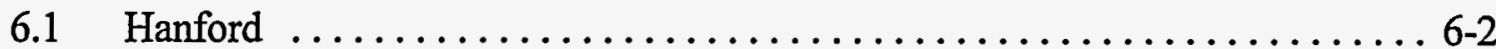

$6.2 \quad$ Idaho $\ldots \ldots \ldots \ldots \ldots \ldots \ldots \ldots \ldots \ldots \ldots \ldots \ldots \ldots \ldots \ldots, 6,3$

6.3 Oak Ridge $\ldots \ldots \ldots \ldots \ldots \ldots \ldots \ldots \ldots \ldots \ldots \ldots \ldots \ldots, 6,4$

6.4 Volume Reductions $\ldots \ldots \ldots \ldots \ldots \ldots \ldots \ldots \ldots \ldots \ldots \ldots \ldots, 6,5$

6.5 Database and Glass Property Modeling $\ldots \ldots \ldots \ldots \ldots \ldots \ldots \ldots \ldots, 6,7$

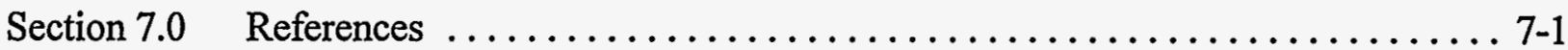


GTS Duratek

The Catholic University of America

\section{List of Tables}

Table 1.1 Summary of Crucible Melts Prepared and Testing Performed $\ldots \ldots \ldots \ldots$ 1-12

Table 2.1 Summary of DCP Analysis of the non Radioactive Soil Received

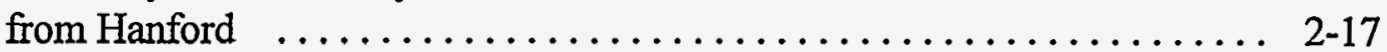

Table 2.2 Summary of Results of Analysis of Hanford Radioactive Soil. ........ 2-18

Table 2.3 Results of ICP-MS Analysis of Hanford Radioactive Soil

Samples (ppm) .............................. 2-19

Table 2.4 Results of Physical Characterization of Hanford Soils ............ 2-20

Table 2.5 Results of Total Carbon (TC) Content Analyses of Hanford Soils ...... . 2-21

Table 2.6 Results of Total Organic Carbon (TOC) Analyses of

Hanford Soils .............................. 2-22

Table 2.7 Results of Gamma Counting Analyses of Hanford Soil

Samples (in $\mathrm{nCi} / \mathrm{g}$ ) ................................ 2-23

Table 2.8a Nominal Low-Level Tank Feed Stream (Total Weight in Kg)

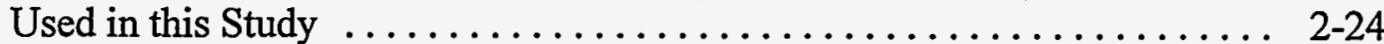

Table 2.8b Composition of the Low-Level Waste Simulant Expressed

on an Oxide Basis ............................. 2-25

Table 2.9 Summary of Glass Compositions of Hanford Surrogate

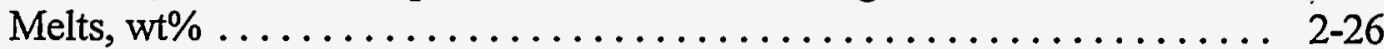

Table 2.10 Summary of Glass Composition Produced from Hanford Soil and

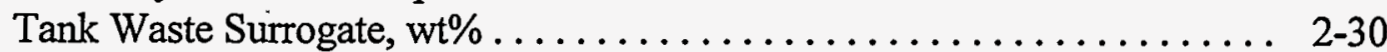

Table 2.11 Summary of Glass Composition Produced from Hanford Soil and

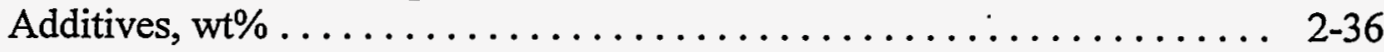

Table 2.12a Summary of Composition and Properties of Surrogate Soil Melts ...... 2-38

Table 2.12b Viscosity and Conductivity of Glass Samples Prepared from

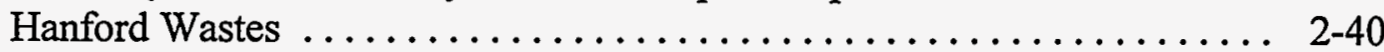

Table 2.13a Results from ICPMS Analysis of Glass Dissolutions ............ 2-43

Table 2.13b Sodium and Technetium Retention in Crucible Melts from Tank

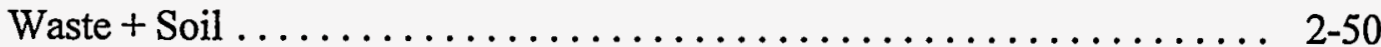

Table 2.14 Concentration of RCRA Metals (ppm) in TCLP Leachates from

Hanford Glasses ............................. 2-51

Table 2.15 Concentrations of Tc-99 Determined by ICPMS in TCLP Leachates

from Hanford Glasses. ......................... 2-52

Table 2.16 Summary of Results from PCT Leach Tests of Hanford Glasses ....... 2-53

Table 2.17 Percentage of Original Glass Dissolved, Calculated from the Sodium Concentration in the PCT Leachate $\ldots \ldots \ldots \ldots \ldots \ldots .2$. 2-59

Table 2.18 Crystallization Behavior in $\mathrm{Na}_{2} \mathrm{O}$ Series of Hanford Surrogate

Glasses ....................................... 2-60 
Table 2.19 Cristobalite-Type Phase Composition in $\mathrm{Na}_{2} \mathrm{O}$ Series of Hanford

Surrogate Glasses ............................ 2-61

Table 2.20 Pyroxene Composition in $\mathrm{Na}_{2} \mathrm{O}$ Series of Hanford Surrogate Glasses .... 2-62

Table 2.21 Pyroxene Composition in $\mathrm{CaO}$ Series of Hanford Surrogate Glasses ..... 2-63

Table 2.22 List of Hanford Glasses with Complete Viscosity, Conductivity,

TCLP, and PCT Data (i.e., Glasses in the Core Data Set of 40

Glasses) and Representative Data for those Properties ............ 2-64

Table 3.1 Summary of Idaho Waste Physical Characterization $\ldots \ldots \ldots \ldots \ldots$. 3-25

Table 3.2 Summary of the Asbestos Containing Material Analyses (wt\%) ........ 3-26

Table 3.3 Summary of Analysis on Idaho Fly Ash $\ldots \ldots \ldots \ldots \ldots \ldots \ldots \ldots . . \ldots \ldots .27$

Table 3.4 Summary of Gamma Spectroscopy on Idaho Wastes in $\mathrm{Ci} / \mathrm{g} \ldots \ldots \ldots$ 3-28

Table 3.5 Summary of Waste Composition as Oxides Used in Glass

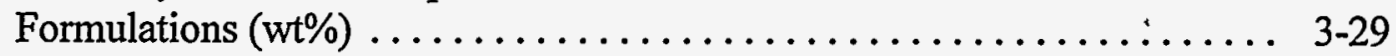

Table 3.6 Summary of Compositions of Glass Made from Idaho

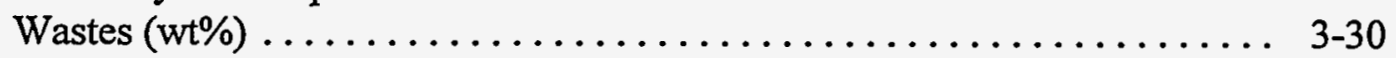

Table 3.7 Summary of Viscosity and Conductivity of Idaho Glasses $\ldots \ldots \ldots \ldots \ldots$ 3-35

Table 3.8 Summary of SEM Observation on Idaho Reduced Glasses . . . . . . . . . . . 3-37

Table 3.9 Summary of Analysis of PCT Leachates of Idaho Glasses ........... 3-38

Table 3.10a Results of TCLP Leaching Tests on Untreated Idaho Wastes (ppm) ..... 3-41

Table 3.10 Summary of TCLP Test Results on Idaho Glasses (ppm) ............ 3-41

Table 3.11 List of Idaho Glasses with Complete Viscosity, Conductivity,

TCLP, and PCT Data (i.e., Glasses in the Core Data Set of 40

Glasses) and Representative Data for those Properties ........... 3-42

Table $4.1 \quad$ Oak Ridge Surrogates Waste Formulations .................. 4-14

Table 4.2 Summary of Oak Ridge Waste Physical Characterization ........... 4-15

Table 4.3 Composition of Wastes Used in Oak Ridge Vitrification (wt\%) ........ 4-16

Table 4.4 Summary of Oak Ridge Glass Compositions (wt\%) . . . . . . . . . . . 4-17

Table 4.5 ICPMS Analysis of Glass Made from Spiked Oak Ridge K-1407

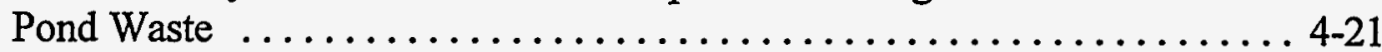

Table 4.6 Viscosity and Conductivity of Oak Ridge Glasses $\ldots \ldots \ldots \ldots \ldots \ldots \ldots$ 4-23

Table 4.7 Summary of PCT Results for Oak Ridge Glasses $\ldots \ldots \ldots \ldots \ldots \ldots \ldots$ 4-25

Table 4.8 Summary of TCLP Results for Oak Ridge Glasses and Waste (ppm) . . . . 4-28

Table 4.9 List of Oak Ridge Glasses with Complete Viscosity, Conductivity,

TCLP, and PCT Data (i.e., Glasses in the Core Data Set of 40

Glasses) and Representative Data for those Properties ............ 4-29

Table 5.1 Table Contents of Glass Composition-Property Database (GCPD). . . . . . 5 5-24

Table $5.2 \quad$ Oracle7 Client Applications. . . . . . . . . . . . . . . . . . . . . . 5 25 
Table 5.3 Custom Client Applications for Glass-Composition-Property Database. .................................. 5-26

Table 5.4 Results of Conductivity Models by Weighted and Unweighted Least-Squares Regressions. ........................ 5-27

Table 5.5 Results of Conductivity Model of Hanford Glasses by Unweighted Least-Squares Regression. ........................ 5-30

Table 5.6 Results of Viscosity Models by Weighted and Unweighted LeastSquares Regressions. ........................... 5-31

Table $5.7 \quad$ Viscosity Model by Unweighted Least-Squares Regression. . . . . . . . . 5-32

Table 5.8 Viscosity Model of Hanford Glasses by Unweighted Least-Squares Regression. ................................ 5-33

Table 5.9 Results of Modeling 7-Day PCT of $\mathrm{Si}, \mathrm{B}, \mathrm{Na}, \mathrm{Li}$, and $\mathrm{pH}$ by Unweighted Least Squares Regression. ................ 5-34

Table 5.10 7-Day PCT Model Coefficients for $\mathrm{Si}, \mathrm{B}, \mathrm{Na}, \mathrm{Li}$, and $\mathrm{pH} . \ldots \ldots \ldots \ldots .5$. 35

Table 5.11 Prediction of 7-Day PCT of $\mathrm{Si}, \mathrm{B}, \mathrm{Na}, \mathrm{Li}$, and $\mathrm{pH} . \ldots \ldots \ldots \ldots \ldots \ldots . \ldots .36$

Table 5.12 Division of 40 Glasses into Three Groups; Each Group was Sequentially Removed from the Database to Examine the Predictive Capabilities of the Models for "Unknown" Glasses . . . . . . . 5-38

Table 6.1a List of Hanford Glasses with Complete Viscosity, Conductivity, TCLP, and PCT Data (i.e., Glasses in the Core Data Set of 40 Glasses) and Representative Data for Those Properties ................. 6-9

Table 6.1b List of Idaho Glasses with Complete Viscosity, Conductivity, TCLP, and PCT Data (i.e., Glasses in the Core Data Set of 40 Glasses) and Representative Data for Those Properties ................ 6-10

Table 6.1c List of Oak Ridge Glasses with Complete Viscosity, Conductivity, TCLP, and PCT Data (i.e., Glasses in the Core Data Set of 40 Glasses) and Representative Data for Those Properties ................ 6-11

Table 6.2a Volume Reduction Calculated for Hanford Waste Streams $\ldots . \ldots \ldots \ldots .6$ 6-12 Table $6.2 \mathrm{~b}$ Volume Reduction Calculated for Idaho Waste Streams ............ 6-13 Table 6.2c Volume Reduction Calculated for Oak Ridge WETF Sludge, Pond Waste, TSCA Ash and Soil ............................. 6-14 


\section{List of Figures}

Figure 2.1 Particle Size Distribution in Hanford Soils. . . . . . . . . . . . . . 2 65

Figure 2.2

Figure 2.3

Figure 2.4

Gamma Spectrum of Hanford Soil. ....................... 2-66

Relative Volume of Glass Produced vs. Waste Loading. . . . . . . . . . 2-67

Viscosity of Hanford Tank Waste Glasses. Variation with Temperature and Sodium Content. ............................. 2-68

Figure 2.5 Hanford Soil - Example of Use of Glass Property Data to Determine Processing Temperature for a Given Set of Process Constraints. . . . . . . . 2-69

Figure 2.6 A Comparison of Results of the TCLP Leach Test for Tc-99 with the DOE 5400.5 Drinking Water Limit. ................... 2-70

Figure 2.7 Comparison of the Effect of Various Additives on TCLP Leaching of Calcium. ................................. 2-71

Figure 2.8 Comparison of PCT Release of Hanford Glasses with the SRL-EA Standard Glass. ............................... 2-72

Figure 2.9 Dependence of Percentage of Glass Dissolved in 7-day PCT Test on Glass Sodium Content. ........................... 2-73

Figure 2.10 PCT Data for Hanford Glasses. Silicon Concentrations vs. Time Compared to SRL-EA Glass. ........................ 2-74

Figure 2.11 PCT Data for Hanford Glasses. Silicon Concentrations vs. Time Compared to SRL-EA Glass. . . . . . . . . . . . . . . . . . . . . . .

Figure 2.12 PCT Data of Hanford Glasses. Sodium Concentrations vs. Time Compared to SRL-EA Glass. ....................... 2-76

Figure 2.13 PCT Data of Hanford Glasses. Sodium Concentrations vs. Time Compared to SRL-EA Glass. . . . . . . . . . . . . . . . . . . . . .

Figure 2.14 Results from 7-day PCT Tests as a Function of Sodium Loading ........ 2-78

Figure 2.15 Normalized PCT Leachate Concentrations after 7 days for Hanford Glasses and the SRL-EA Glass. .................... 2-79

Figure 2.16 Dehydrated, Polished Surface of HAN29E Showing Extreme Sensitivity of this Glass to Water. . . . . . . . .

Figure 2.17 Augite Crystals in HAN2 Heat Treated 18 Hours at $1100^{\circ} \mathrm{C} \ldots \ldots \ldots \ldots .2-81$

Figure 2.18 XRD Pattern from Crystals in Figure 2.17. ................. 2-81

Figure 2.19 Cristobalite-Type Phase in HAN9, Heat Treated 2 Hours at $900^{\circ} \mathrm{C} . \ldots \ldots .2-82$ Figure $2.20 \mathrm{XRD}$ Pattern from Crystals in Figure 2.19. . . . . . . . . . . . . . . 2-82

Figure 2.21 Phase III in HAN8 Heat Treated for 2 Hours at $900^{\circ} \mathrm{C} . \ldots \ldots \ldots \ldots . .2-83$

Figure 2.22 Phase IV in HAN9, Heat Treated 2 Hours at $900^{\circ} \mathrm{C} . \ldots \ldots \ldots \ldots \ldots . .2-83$

Figure 2.23 Crystallization Behavior in the CaO Series of Glasses Heat Treated at $1100^{\circ} \mathrm{C}$ for 24 Hours. ............................... 2-84

Figure 2.24 Augite Crystals in HAN13 Heat Treated for 24 Hours at $1100^{\circ} \mathrm{C} . \ldots \ldots \ldots$ 2-85 
Figure 2.25 Augite (Bright Polygonal) and Wollastonite (Grey Streaks)

Crystals in HAN15, Heat Treated for 24 Hours at $1100^{\circ} \mathrm{C}$. . . . . . . . . 2-85

Figure 2.26

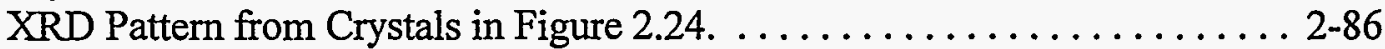

Figure 2.27

XRD Pattern from Crystals in Figure $2.25 . \ldots \ldots \ldots \ldots \ldots \ldots \ldots \ldots \ldots \ldots \ldots .286$

Figure 3.1 Type I Fiber in Idaho ACM. $\ldots \ldots \ldots \ldots \ldots \ldots \ldots \ldots \ldots \ldots \ldots \ldots \ldots, 43$

Figure 3.2

Figure 3.3a

Figure $3.3 \mathrm{~b}$

Figure 3.3c

Figure 3.4

Figure 3.5

Figure 3.6

Figure 3.7

Figure 3.8

Figure 3.9

Figure 3.10

Figure 3.11

Figure 3.12

Figure 3.13

Figure 3.14

Figure 3.15

Figure 3.16

Figure 3.17

Figure 3.18

Figure 3.19

Figure 3.20

Figure 3.21

Figure 3.22

Figure 3.23

Figure 3.24

Figure 3.25

Figure 3.26

Figure 3.27

Type II Fiber in Idaho ACM.

$3-43$

Results of Gamma Spectroscopy on ID1 (Asbestos). ............. 3-44

Results of Gamma Spectroscopy on ID3 (Flyash). .............. 3-44

Results of Gamma Spectroscopy of ID2 (Eutectic Salt). . .......... 3-44

Results of Particle Size Analysis of Idaho Wastes. . . . . . . . . . . 3-45

EDS Spectrum of Idaho Flyash (ID3), Average $\ldots \ldots \ldots \ldots \ldots \ldots \ldots . . \ldots \ldots$

EDS Spectrum of Idaho Flyash (ID3), Particle \#1 . . . . . . . . . . 3-46

EDS Spectrum of Idaho Flyash (ID3), Particle \#2 . . . . . . . . . . 3-47

EDS Spectrum of Idaho Flyash (ID3), Particle \#3. . . . . . . . . . . 3-47

EDS Spectrum of Idaho Flyash (ID3), Particle \#4. . . . . . . . . . . . 3-48

Crystals Formed after Heating Idaho Flyash at $1150^{\circ} \mathrm{C} . \ldots \ldots \ldots \ldots .3-49$

EDS Spectrum of Crystals from Center in Figure 3.10. .......... 3-49

EDS Spectrum of Long Blade-Shaped Crystals from Figure 3.10 . . . . . 3-50

EDS Spectrum of Small Polyhedral Crystals from Figure 3.10 . . . . . . 3-50

EDS Spectrum of Small Acicular Crystals from Figure $3.10 \ldots \ldots \ldots \ldots .3-51$

EDS Spectrum of Crystals at Edge of Crucible in Figure 3.10 . . . . . 3-51

EDS Spectrum of Crushed Borax Glass (IDG9), Average. . . . . . . . . . 3-52

EDS Spectrum of Crushed Borax Glass (IDG9), Particle \#1. . . . . . . 3-52

EDS Spectrum of Crushed Borax Glass (IDG9), Particle \#2. . . . . . . 3-53

EDS Spectrum of Crushed Borax Glass (IDG9), Particle \#3. . . . . . . . 3-53

EDS Spectrum of Crushed Borax Glass (IDG9), Particle \#4. . . . . . . . 3-54

Viscosity of Idaho Asbestos and Eutectic Salt Glasses. ........... 3-55

Crystallization in IDG14A Heat Treated at $1100^{\circ} \mathrm{C}$ for $20 \mathrm{hrs} . \ldots \ldots \ldots .3-56$

EDS Spectrum of Crystal in IDG10 Glass. ................. 3-57

EDS Spectrum of $5 \mu \mathrm{m}$ dia. Nodule in IDG10 Glass. . . . . . . . . . 3-57

EDS Spectrum of $15 \mu \mathrm{m}$ dia. Nodule in IDG10 Glass. . . . . . . . . . 3-58

EDS Spectrum of Region Containing Many Spherical Voids

in IDG10 Glass. . ................................. 3-58

Figure 3.28

EDS Spectrum from High-Backscatter-Intensity Region IDG10 Glass. . . . . 3-59

Figure 3.29

EDS Spectrum from Low-Backscatter-Intensity Region IDG10 Glass. . . . . 3-59

EDS Spectrum of $0.5 \mu \mathrm{m}$ dia. Nodule in IDG10 Glass. ............ 3-60

EDS Spectrum of $0.2 \mu \mathrm{m}$ dia. Nodule in IDG10 Glass. ........... 3-60

$\begin{array}{ll}\text { Figure 3.30 } & \text { EDS Spectrum of } 0.2 \mu \mathrm{m} \text { dia. Nodule in } \text { Sulfide Nodule Among Smaller Uranium Oxide Crystals }\end{array}$

in IDG14 Glass. . . . . . . . . . . . . . . . . . . . . . . . . . . . . 3-61 
Figure 3.32 Microstructure of Large Sediment Nodule from IDG15

Glass Elemental Distribution is Highly Varied. ............... 3-62

Figure 3.33 Microstructure of Crystalline Region in IDG15 Glass. . . . . . . . . . . 3-62

Figure 3.34 Mössbauer Spectrum of Idaho Glasses. ................... 3-63

Figure 3.35 EDS Spectrum of Sulfate Inclusion in IDG13B Glass. . . . . . . . . . . 3-64

Figure 3.36 Results from PCT Testing of Idaho Glasses. Sodium Concentration in

Leachate Normalized to that in the Glass. .................. 3-65

Figure 3.37 Results from PCT Testing of Idaho Glasses. Silicon Concentration in

Leachate Normalized to that in the Glass. .................. 3-66

Figure 3.38 Sodium PCT Leach Rate of Idaho Glasses Over One Year of Testing. . . . . . 3-67

Figure 3.39 Silicon PCT Leach Rate of Idaho Glasses Over One Year of Testing. . . . . . . 3-68

Figure 3.40 Summary of TCLP Performance of Idaho Glasses. ............. 3-69

Figure 3.41 Uranium Leaching in TCLP Testing of Idaho Glasses Compared to the Drinking Water Standard in DOE Order 5400.5 . . . . . . . . 3-70

Figure 4.1 Results from Particle Size Analysis of the Oak Ridge Soil .......... 4-30

Figure 4.2 Results from Gamma Spectrometry of WETF Sludge. .............. 4-31

Figure 4.3 Blocky Ni-Bearing Spinel Crystals in One of the Few Glassy Regions

of ORNL1. ................................. 4-32

Figure 4.4 Structure Found in the Majority of ORNL1 $\ldots \ldots \ldots \ldots \ldots \ldots \ldots \ldots .4-32$

Figure 4.5

Figure 4.6

Massive Dendritic Spinel Growth in ORNL2 ................ 4-33

Region in ORNL3 Showing Liquid-Liquid Phase Separation and Small

Crystals of a Variety of Phases. ........................ 4-34

Figure 4.7 Very Fine Scale Liquid-Liquid Phase Separation in ORNL3.......... 4-34

Figure 4.8 A Cluster of High Cr Spinel Crystals Surrounded by Some Dendritic

Growth of Ni-Fe Spinel. ........................... 4-35

Figure 4.9 The Wide Range of Spatial Scale Typical of Liquid-Liquid Phase

Separation in ORNL3 is Evident in this Micrograph. ............. 4-35

Figure 4.10 Viscosity of Some Oak Ridge Pond Waste Glasses. . . . . . . . . . . . 4-36

Figure 4.11 Viscosity of the WETF and TSCA Waste Glasses as a Function

of Temperature. ............................. 4-37

Figure 4.12 PCT Leaching of Oak Ridge Waste Glasses Over One Year. . . . . . . . . . 4-38

Figure 4.13 PCT Leaching of Oak Ridge Waste Glasses Over One Year. . . . . . . . . . . 4-39

Figure 4.14 PCT Leaching of Oak Ridge Waste Glasses Over One Year.. . . . . . . . . . . 4-40

Figure 4.15 PCT Leaching of Oak Ridge Waste Glasses Over One Year. . . . . . . . . . 4 4-41

Figure 4.16 PCT Leaching of Oak Ridge Waste Glasses Over One Year. . . . . . . . . 4 4-42

Figure 4.17 PCT Leaching of Oak Ridge Waste Glasses Over One Year. . . . . . . . . . . . 4-43

Figure 4.18 TCLP Performance of Selected Oak Ridge Pond Waste Glasses for Nickel and Uranium. . ............................... 4-44

Figure 4.19 TCLP Performance of the WETF and TSCA Waste Glasses for.Lead. . . . . 4-45 
Figure 5.1 The Oracle7 Client/Server Database Management System. SPX and TCP/IP are Communication Protocols. ................... 5-39

Figure 5.2 Table Structure of the Glass Composition-Property Database. . . . . . . . . 5-40

Figure 5.3 Example of Glass Entry Form: A List of Samples from the Hanford Project. ............................ 5-41

Figure 5.4 Example of Glass Entry Form: Displaying Glass Data for Selected

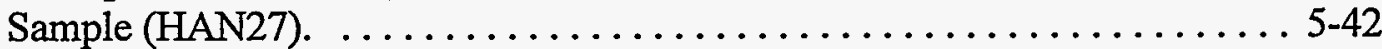

Figure $5.5 \quad$ Example of Glass Modeling Tool. $\ldots \ldots \ldots \ldots \ldots \ldots \ldots \ldots \ldots \ldots .43$

Figure 5.6 Data Frequency Distribution along $\mathrm{SiO}_{2}, \mathrm{Al}_{2} \mathrm{O}_{3}, \mathrm{~B}_{2} \mathrm{O}_{3}$ and $\mathrm{Na}_{2} \mathrm{O}$ wt $\% \ldots . .5-44$

Figure 5.7 Box and Whisker Plot of Glass Compositions in Viscosity Table. . ...... 5-46

Figure 5.8 Sample Distribution in Reduced Compositional Space of NBOT versus M3T. ................................ 5-47

Figure 5.9 Data Frequency Distribution in Reduced Compositional Space of NBOT versus M3T . ............................ 5-48

Figure 5.10 Data Frequency Distribution on M3T Axis (from viscosity table). $\ldots \ldots \ldots$ 5-48

Figure 5.11 Sample Distribution in Reduced Compositional Space of NBOT versus M3T ............................... 5-49

Figure 5.12 Sample Distribution in Reduced Compositional Space of NBOT versus M1M12 ............................. 5-50

Figure 5.13 Sample Distribution in Reduced Compositional Space of NBOT versus M3T ............................... 5-51

Figure 5.14 Scatter Plot Matrix of 11 of the Most Abundant Elements in the Viscosity Table. .................................. 5-52

Figure 5.15 Scatter Plots of 11 of the Most Abundant Elements versus their Products with $1 / \mathrm{T}$ (from viscosity table). .................... 5-53

Figure 5.16 Standard Deviations of 16 of the Most Abundant Elements and their Products with $1 / \mathrm{T}$ (data from viscosity table). . .............. 5-54

Figure 5.17 Weight is a Function of Euclidean Distance between Two Sample Points in Multi-Dimensional Compositional Space ................ 5-54

Figure 5.18 Frequency Distributions of Raw Conductivity Observations along Weight Axis. ................................ 5-55

Figure 5.19 Frequency Distributions of Weighted Conductivity Observations along Weight Axis. ................................ 5

Figure 5.20 Conductivities Predicted by Models of the Weighted and Unweighted Least-Squares Regressions. ........................ 5-57

Figure 5.21 Normalized Residuals (residual/observed) of Conductivities Predicted by Models of Weighted and Unweighted Least-Squares Regression. . . . . . . 5 5-57

Figure 5.22 Standard Deviations of 13 Most Abundant Oxides (wt\%) of Hanford Glasses. ................................... 5-58

Figure 5.23 Goodness of Fit of Conductivity Model of Hanford Glasses. . . . . . . . . 5-58 
Figure 5.24 Predicted Conductivity is a Function of Incremental Changes of Glass Components (HAN7 glass as reference). . ................. 5-59

Figure 5.25 Conductivity Change with Incremental Variations in $\mathrm{Na}_{2} \mathrm{O}$ or $\mathrm{CaO}$ wt\% (HAN7 as reference glass).

Figure 5.26 Viscosities Predicted by Models of Weighted and Unweighted

Least-Squares Regression. . ....................... 5-60

Figure 5.27 Goodness of Fit of Viscosity Model by Unweighted Least-Squares

Regression. ................................ 5-60

Figure 5.28 Normalized Residuals (residual/observed) of Viscosity Models of

Weighted and Unweighted Least-Squares Regression. ............ 5-61

Figure 5.29 Goodness of Fit of Viscosity Model of Hanford Glasses by Unweighted Least-Squares Regression. .......................... 5-61

Figure 5.30 Viscosity Change with Incremental Variations in Glass

Composition (adding/removing one glass component in wt\%). . . . . . 5-62

Figure 5.31

Viscosity Change with Incremental Variations in $\mathrm{Na}_{2} \mathrm{O}$ or $\mathrm{CaO}$ wt\%

(HAN7 as reference glass). ........................ 5-62

Figure 5.32a Goodness of Fits for 7-Day PCT Models for Si. .............. 5-63

Figure 5.32b Goodness of Fits for 7-Day PCT Models for B. . . . . . . . . . . . . 5-63

Figure 5.32c Goodness of Fits for 7-Day PCT Models for Na. . . . . . . . . . . . . . 5 5 64

Figure 5.32d Goodness of Fits for 7-Day PCT Models for Li. . . . . . . . . . . . . 5-64

Figure 5.32e Goodness of Fits for 7-Day PCT Models for $\mathrm{pH} . \ldots \ldots \ldots \ldots \ldots \ldots \ldots$ 5-65

Figure 5.33a Glass Component Effects on 7-Day PCT Values. $\ldots \ldots \ldots \ldots \ldots \ldots \ldots$ 5-66

Figure 5.33b Glass Component Effects on 7-Day PCT Values. $\ldots \ldots \ldots \ldots \ldots \ldots \ldots$ 5-66

Figure 5.33c Glass Component Effects on 7-Day PCT Values. ............... 5-67

Figure 5.33d Glass Component Effects on 7-Day PCT Values. .............. 5-67

Figure 5.34 Normalized Residuals (residual/observed) of 7-Day PCT Models for

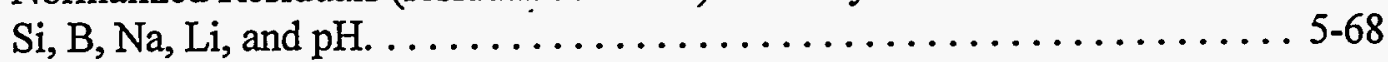

Figure 5.35a Correlation between Gibbs Free Energy of Hydration and the Normalized Release of Si from 7-day PCT. . ..................... 5-69

Figure 5.35b Correlation between Gibbs Free Energy of Hydration and the Normalized Release of B from 7-day PCT. . ..................... 5-69

Figure 5.35c Correlation between Gibbs Free Energy of Hydration and the Normalized Release of Na from 7-day PCT. . ..................... 5-70

Figure 5.35d Correlation between Gibbs Free Energy of Hydration and the Normalized Release of $\mathrm{Li}$ from 7 -day PCT. ..................... 5-70

Figure 5.36a Predicted versus Measured Viscosity of Group 1 Glasses . . . . . . . . . 5-71

Figure 5.36b Predicted versus Measured Viscosity of Group 2 Glasses . . . . . . . . . . 5-71

Figure 5.36c Predicted versus Measured Viscosity of Group 3 Glasses . . . . . . . . . . . 5-72

Figure 5.37a Predicted versus Measured Sodium Concentrations for 7-day PCT . . . . . 5-73

Figure 5.37b Predicted versus Measured Sodium Concentrations for 7-day PCT ....... 5-73

Figure 5.37c Predicted versus Measured Sodium Concentrations for 7-day PCT . . . . . 5-74 
Figure $6.1 \quad$ Waste Percentages used in the Hanford Glasses. . . . . . . . . . . 6-15

Figure 6.2 Waste Percentages used in the Idaho Glasses. . . . . . . . . . . . . 6-16

Figure 6.3 Waste Percentages used in the Oak Ridge Glasses . . . . . . . . . . 6-17 


\section{EXECUTIVE SUMMARY}

This report presents the results from a study of the application of the Minimum Additive Waste Stabilization (MAWS) approach using vitrification as a treatment technology to a variety of waste streams across the DOE complex. This work has involved both experimental vitrification work using actual mixed wastes and surrogate waste streams from several DOE sites (Hanford, Idaho, and Oak Ridge) as well as the development of a computer-based, integrated glass propertycomposition database. The long-term objective is that this data base will assist glass formulation studies with single waste streams or combinations of waste streams subject to a variety of userimposed constraints including waste stream usage priorities, process related constraints (e.g., melt viscosity, electrical conductivity, etc.), and waste form performance related constraints (e.g., TCLP and PCT leaching results).

The following waste streams were investigated in this work; actual samples of these materials were used except where noted:

- $\quad$ Hanford LLW Tank Waste (Radioactive surrogate with Tc and U)

Major Cations: $\quad \mathrm{Na}, \mathrm{Al}, \mathrm{P}$

- Hanford Contaminated Soils

Major Cations: $\quad \mathrm{Si}, \mathrm{Al}, \mathrm{Fe}, \mathrm{Ca}$

- Idaho Radioactive Asbestos-Containing Material (ACM)

Major Cations: $\quad \mathrm{Si}, \mathrm{Ca}, \mathrm{Al}, \mathrm{Mg}, \mathrm{Fe}$

- Idaho WERF Fly Ash

Major Cations: $\quad \mathrm{Zn}, \mathrm{S}, \mathrm{Pb}, \mathrm{Na}, \mathrm{Si}$

- Idaho Eutectic Evaporator Salt

Major Cations: $\quad \mathrm{K}, \mathrm{Na}, \mathrm{U}, \mathrm{Li}$,

- Idaho Soil (non-radioactive site soil)

Major Cations: $\quad \mathrm{Si}, \mathrm{Ca}, \mathrm{Al}, \mathrm{Mg}, \mathrm{Fe}$

- $\quad$ Oak Ridge WETF Sludge

Major Cations: $\quad \mathrm{Ca}, \mathrm{Al}, \mathrm{Mg}$

- $\quad$ Oak Ridge K-1407 Pond Waste (surrogate with U and Tc)

Major Cations: $\quad \mathrm{Si}, \mathrm{Ca}, \mathrm{P}, \mathrm{Al}, \mathrm{K}$, 
- $\quad$ Oak Ridge TSCA Incinerator Ash (surrogate with U)

Major Cations: $\quad \mathrm{Ca}, \mathrm{P}, \mathrm{Al}, \mathrm{Fe}$

- $\quad$ Oak Ridge Soil (non-radioactive soil)

Major Cations: $\quad \mathrm{Si}, \mathrm{Al}, \mathrm{Fe}$

Extensive efforts were made to include actual samples of waste streams in this study, with the exception of the Hanford tank waste where this was not possible. Surrogates were substituted for all but one of the waste streams selected from the Oak Ridge site.

The experimental work conducted in this project resulted in detailed characterization data with respect to properties of importance for vitrification for several waste streams. A core data set consists of 40 glasses on which viscosity, electrical conductivity, and TCLP and PCT leachabilty measurements have been made; the other glasses were partially characterized with respect to these properties.

Overall, the results suggest that viable glass formulations can be developed for a wide range of combinations of the waste streams investigated with high waste loadings and, in many cases, no additions of chemical additives (i.e., 100\% waste loading). While the limited number of formulations investigated in this study would be unlikely to lead to fully optimized compositions (given the number of waste streams and possible combinations thereof), the results do show the general ranges of glass and melt properties that are achievable with minimal use of chemical additives. The melt properties span ranges that would make the formulations suitable for processing in a variety of vitrification technologies, including the lower-temperature systems. The waste forms produced showed good leach resistance using both the TCLP and the PCT procedures.

The waste streams selected for this study cover a wide range of chemical compositions; this suggests that waste streams not included in this study could be beneficially blended in similar fashions. The compositional diversity also makes the data of additional value with respect to the compositional envelope modeling objectives of this project in that a relatively wide range of glass composition space is spanned which should improve the range of applicability of the models that have been developed.

The compositions of the Hanford soils are typical of most soils; the soils are rich in glass formers (such as $\mathrm{SiO}_{2}$ and $\mathrm{Al}_{2} \mathrm{O}_{3}$ ) and contain small amounts of fluxes (such as $\mathrm{Na}_{2} \mathrm{O}, \mathrm{Li}_{2} \mathrm{O}$, etc.). Conversely, the tank waste is primarily a solution of sodium salts and is, therefore, poor in glass formers but rich in fluxes. Samples of contaminated Hanford soils were characterized and vitrified in combination with a radioactive simulant of the Hanford low-level tank waste. Two groups of glasses were produced; those targeted toward maximized tank waste loadings and those targeted toward maximized soil loadings. The data collected in this study show that, in both cases, these Hanford waste streams can be treated by vitrification to produce leach resistant waste forms. 
Glasses with high tank waste loadings (up to about $31 \mathrm{wt} \%$ tank waste oxides) can be made with good chemical durability by using site soil as the major ingredient. With small amounts (around $5 \mathrm{wt} \%$ ) of additives, such as $\mathrm{ZrO}_{2}$, good leach resistance can be achieved for a waste form that is composed of $95 \%$ waste (on an oxide basis). A comparison has been made which shows that for a poor durability glass composition, replacement of the site soil ingredient by $\mathrm{SiO}_{2}$ sand results in a glass of poorer durability. It is unknown if similar observations would be made for other, more durable glasses. Realistically achievable tank waste loadings with the use of soil appear to be as high as $31 \%$ on an oxide basis using the existing TCLP and PCT specifications. The melts that were produced from Hanford tank wastes had properties that would permit vitrification using a variety of technologies. However, given the fact that the tank waste is predominantly sodium (a "flux" which lowers the glass melting temperature range) and the concerns with respect to volatility (especially for technetium and cesium), a low-temperature process may be favored.

Other data obtained from this study suggest that treatment of contaminated Hanford soils alone may require higher temperature vitrification processes. Small amounts ( $<10 \mathrm{wt} \%)$ of additives would be required to vitrify the site soils using processes operating in the $1400-1500^{\circ} \mathrm{C}$ range.

The results from the chemical characterization of the Idaho waste streams selected for this study (ACM, salt, flyash, and soil) suggest that their compositions are amenable to blending and vitrification in a MAWS approach. Furthermore, from a compositional envelope perspective, these waste streams span a wide range of compositions both in terms of their bulk constituents and the hazardous and radioactive contaminants that are present. For example, the silica contents are $0.4 \%$ for the salt, $5.1 \%$ for the fly ash, $45 \%$ for the ACM and $65 \%$ for the soil; similarly, alumina ranges from $0.1 \%$ to $13 \%$, calcia from $0.1 \%$ to $25 \%$, lithia from $0.1 \%$ to $9 \%$ and soda from $1 \%$ to $35 \%$; uranium is present at $9 \%$ in the salt while the flyash contains six RCRA metals at levels of up to a few percent.

The Idaho waste streams exhibit bulk densities between 0.3 to $1.3 \mathrm{~g} / \mathrm{cm}^{3}$ (though particle densities are as high as $2.9 \mathrm{~g} / \mathrm{cm}^{3}$ ) and weight losses upon heating at $1150^{\circ} \mathrm{C}$ are as much as $64 \%$. These data indicate the potential for large volume reductions upon vitrification, if high waste loading can be achieved. Glasses formulated from blends of these waste streams with $100 \%$ waste loadings have been prepared and characterized.

The results obtained show that the four Idaho waste streams studied can be effectively blended and vitrified without additives as either binary or quaternary mixtures; other combinations than those investigated are possible, however. In the binary approach, mixtures of salt and ACM were studied. The glass formulations investigated, containing around $10 \mathrm{wt} \%$ salt and $90 \mathrm{wt} \% \mathrm{ACM}$, produced viable glass products in replicate melts despite the composition variability found in the ACM waste stream. A series of formulations was also investigated using all four waste streams.

The leach resistance of the waste forms produced are uniformly high on the basis of the PCT procedure. Many of the glasses produced met all of the TCLP release limits. The high concentration 
of lead in the fly ash was the primary factor for glasses that exceeded the TCLP release limits.

The most problematic (from a vitrification perspective) of the waste streams investigated in this study was the fly ash due to the high concentrations of carbon, sulfur, and heavy metals, many of which are relatively redox sensitive. The use of the fly ash waste was found to have significant impacts on the oxidation state of the glass melts produced due to the high carbon content of that material. The oxidation state variations are in turn coupled to the speciation of the redox sensitive species in the glass (primarily sulfur, carbon, and the heavy metals) and therefore lead to variations in both the physical and chemical properties of the product and the melt (e.g., the formation of insoluble sulfides, immiscible sulfate phases, and metallic phases, notably lead). There are kinetic effects that must be considered in this regard since the crucible melts that were studied were produced in a finite time under an oxidizing atmosphere but with reductants in the batch. Some caution should be exercised in using the crucible melt observations to predict what might be expected to occur in a full-scale process since factors such as residence time and oxygen availability are highly dependent on the specific vitrification process concerned. Thus, while selection and control of the oxidation state range is an important consideration for full scale processing of wastes such as the Idaho fly ash, the specific preferred values must be determined on the basis of the particular process characteristics and limitations. However, an important component of such a determination is, of course, a knowledge of the characteristics and properties of melts made from various waste stream proportions under various conditions.

One sample of actual waste (WETF sludge) was obtained from the Oak Ridge site and three other waste streams (Pond Waste, TSCA Ash, and soil) were simulated; however, radioactive and hazardous constituents of concern were added to the simulants. The WETF sludge is a relatively large volume waste stream and an example of a large family of wastes generated by the precipitation of heavy metals from various process wastes (in this case, a biodenitration process) with calcium. The TSCA ash is another example of a high-calcium waste, although otherwise of quite different composition and origin from the WETF sludge. After calcium, the next most abundant cation in the WETF sludge is aluminum that, as the oxide, constitutes about $20 \mathrm{wt} \%$. The soil is high in silica and represents an obvious source of silica that would otherwise be lacking; together these observations suggest that high waste loading glasses would be of the general silica-lime-alumina type. Both the TSCA ash and the Pond Waste contain significant amounts of phosphorus that can lead to phase separation in silicate melts. The primary contaminants in these wastes are uranium and nickel, which are present in significant amounts in both the Pond Waste and the WETF sludge. Barium, chromium and lead are present at the tenth of a percent level while arsenic, silver, and mercury are present at the tens of ppm level.

It was found that both the WETF sludge and the TSCA ash wastes can be blended with contaminated soil and minimal amounts of additives (0-20\%) to produce glasses with good leach resistance as well as acceptable melt characteristics for processing. The ternary systems investigated were the WETF/Pond Waste/Soil system and the TSCA ash/Pond Waste/Soil system. Both of these combinations can be used to produce glasses and melts with acceptable characteristics for 
vitrification; in addition, the glasses formed from these blends show a significant reduction in the measured TCLP leachate concentrations.

The results of this study of the potential of MAWS vitrification applied to Oak Ridge wastes suggest that viable glass formulations can be developed for a wide range of combinations of the waste streams investigated with high waste loadings (and in some cases 100\%). The leach resistance of the waste forms produced are uniformly high on the basis of the PCT procedure. All but one of the glasses produced leached considerably less than any of the RCRA TCLP release limits.

In summary, the use of vitrification appears to be both viable and beneficial for the treatment of the particular waste streams selected. It is likely that other waste streams could also be incorporated in a similar manner.

In addition to the experimental work, an integrated glass property composition database and modeling facility has been designed, a substantial portion of the development work has been completed, and evaluation tests of the system have been conducted. All of the data from the experimental studies outlined above are included in the system, together with data from other sources. We have focused on three properties in this development phase of the program: Melt viscosity, electrical conductivity, and PCT leaching. The database spans a wide range of glass compositions and the property models provide both a good representation of those data as well as reasonable predictions for the properties of glasses that were not included in the data set used to generate the models.

The diversity of mixed and low-level waste streams in the DOE complex necessitates a facility of the type developed here that is based firmly on data from vitrification studies conducted predominantly on a similarly diverse set of actual waste streams. The present integrated databasemodeling facility incorporates useful models, well explored algorithms to rapidly build new models under different conditions, and a carefully organized glass-composition-property database. 


\section{SECTION 1.0 INTRODUCTION}

This report presents the results from a study of the application of the Minimum Additive Waste Stabilization (MAWS) approach, using vitrification as a potential treatment technology, to treat a variety of waste streams across the DOE complex. This work has involved both experimental vitrification development work using actual mixed wastes from several DOE sites (Hanford, Idaho, and Oak Ridge) as well as the development of a computer-based, integrated glass propertycomposition database. The major objective of this project is to support the design and formulation of glass compositions using the MAWS approach. The longer term objective is that this modeling capability will allow glasses to be formulated from single waste streams or combinations of waste streams subject to a variety of user-imposed constraints including waste stream usage priorities, process related constraints (e.g., melt viscosity, electrical conductivity, etc.), and performance related constraints (e.g., TCLP and PCT leaching characteristics). This capability will be useful in guiding and expediting future glass formulation studies to support any future applications of vitrification technologies.

\subsection{Background}

Using the MAWS concept, waste streams are viewed as resources for the stabilization process. The chemical properties of these resources are fully exploited to minimize the need for the purchased additives usually required for stabilization, and both total system and lifecycle factors are considered to maximize the economic benefits of blending the optimum proportions of multiple waste streams in an integrated system composed of an appropriate combination of treatment technologies.

Vitrification was selected as the best available stabilization technology for use in the MAWS program for a number of reasons including: the well-developed technology base that exists from high-level waste vitrification programs and the commercial glass industry; the large volume reductions achievable; the destruction of organic contaminants by thermal processing; the ability of glass to chemically incorporate a wide range of hazardous and radioactive components; and production of a stable homogeneous wasteform that can be made highly resistant to aqueous corrosion.

The MAWS approach has been taken from the development phase through supporting laboratory studies to on-site demonstration of an integrated, multiple-technology system to vitrify 
ends of actual radioactive mixed waste streams at the Fernald Environmental Management Project site in Fernald, Ohio (Fu-1994a, Pegg-1994a, Pegg-1994b, Bowan-1994).

The key concepts that are applied in the MAWS approach are as follows (Fu-1994a, Pegg1994a, Pegg-1994b, Bowan-1994):

- Increased volume reduction translates into lower life-cycle treatment costs due to the generally high volume-based costs of disposal.

- The need for non-waste additives should be minimized since these materials must be purchased, processed, and disposed (since they form part of the final waste form) and consequently affect treatment costs.

- Waste streams are resources for the treatment process since, by blending available waste streams of different compositions, the requirements for additives can frequently be reduced considerably.

- In general, no single treatment technology will fulfill the diverse requirements that are typical of site remediation activities. Often, a combination of technologies may provide the best solution to a particular waste treatment problem. Additional synergisms are then possible by blending waste streams and integrating treatment subsystems.

- Focus on optimizing the performance of the complete treatment system, not the individual subsystems since, in general, the optimum system performance is not obtained by optimizing the performance of the components individually.

- $\quad$ Produce a highly leach resistant waste form that meets or exceeds treatment requirements (to the extent that these are defined) and offers the best possible prospects of long-term stability.

- The treatment system should be designed to minimize production of sidestream wastes.

The general MAWS approach could be manifested in a variety of system configurations and process flows, depending on the quantities and compositions of the waste streams that are available at a given site. 


\subsection{The MAWS Vitrification Compositional Envelope}

The general MAWS approach should be widely applicable across the DOE complex, especially since many types of compositionally diverse waste streams (e.g., contaminated soils, transite, asbestos, and fly ash) are present at most DOE sites. However, the bulk of the hazardous and radioactive constituents are frequently in ponds or tanks in the form of sludges whose compositions are unique. Application of the MAWS concept or more traditional single waste stream vitrification, to a new remediation problem usually requires characterization of the waste streams, a crucible glass melting study to identify the optimum formulations and waste blends, and largerscale testing to obtain necessary process data. There is a need to develop a database of such information as it is accumulated in site specific studies, such that the development time for each successive application of the treatment technology can be reduced. A key to meeting this need is the development of glass composition-property correlations that are supported by the database and are capable of providing the glass property predictions necessary to guide and expedite the glass formulation optimization process.

An important feature of the experimental vitrification studies conducted in the present work is that they were not process-specific and therefore the data generated support studies using a variety of vitrification process technologies (e:g., Joule-heated melting, cyclone melting, plasma melting, etc.); in particular, the data were collected over a wide temperature range in order to support a correspondingly wide range of processing technologies.

The data collected from this work were used to extend and enhance property-composition correlations that have been developed for both the MAWS program and for the high-level nuclear waste vitrification program. In areas where the uniqueness of the data made this impractical, new correlations were found to represent the data. Despite the tremendous efforts expended in the development of optimum formulations for high-level waste vitrification at sites such as West Valley, Savannah River, and Hanford, the composition range that has been explored in detail is actually quite small (all are mixed-alkali borosilicate glasses). Vitrification of low-level radioactive and mixed wastes must emphasize process economics and make use of composition regions, which currently remain relatively unexplored. The recent development studies, conducted in support of the MAWS demonstration at the Fernald site, are an example of this strategy; this work led to unusual but cost effective glass composition formulations (specifically, high-fluoride, low-silica, high-calcium, highmagnesium, low-alkali, low-boron). Predictions made on the basis of existing data bases (e.g., from high-level nuclear waste vitrification studies) would not have led to these formulations and, consequently, direct laboratory studies were essential. There is thus a clear need to expand the requisite broad compositional database on key glass properties and to represent those data in terms of quantitative correlations, in order to minimize the incremental development cost incurred per additional site waste stream that must be addressed. 


\subsection{Methodology and Approach}

The experimental portion of this work involved waste stream selection; waste stream characterization; glass formulation and melting using crucible melts; and glass characterization to obtain data on key process parameters and waste form performance parameters. The resulting data were analyzed to obtain an understanding of the relationships between glass composition and these key parameters. These relationships could then be represented in the form of models that can be used to guide the selection of formulations for treatment by vitrification. The specific objectives of this study can be summarized as follows:

(a) Conduct screening/scoping characterization of up to six waste streams;

(b) Conduct detailed characterization of three waste streams;

(c) Conduct glass formulation testing involving at least 40 crucible melts;

(d) Conduct detailed glass characterization tests on each of the melts produced in terms of parameters affecting processability and leach resistance;

(e) Develop property-composition correlations based on these data.

Several melt and glass product properties are of importance with respect to waste treatment by vitrification, as discussed further in Section 1.3.2. Melt viscosity, as a function of temperature, is an important factor in determining processing temperature since a high viscosity results in slow throughput rates and very low viscosity melts are often more corrosive. A similar compromise is necessary with the electrical conductivity of the melt for acceptable processing by joule-heated melting; high conductivity melts lead to electrode current density limitations whereas low conductivity melts increase the conduction through the melter refractories. Phase stability and glass microstructural data are necessary to determine the lower limit of processing temperatures beyond which melt crystallization occurs, since this could clog some melters. Leach resistance is an important performance criterion since the role of the glass waste form is to immobilize the hazardous and radioactive constituents and minimize their release into the environment. Two leach tests were used in this study: The Environmental Protection Agency Toxicity Characteristic Leaching Procedure (EPA TCLP) (US EPA-1990) and the Savannah River Product Consistency Test (PCT) (Jantzen1989). The EPA TCLP is an 18 hour test in an acetate-buffered leachant ( $\mathrm{pH} \mathrm{5)}$ at $22^{\circ} \mathrm{C}$ for release of hazardous components to the leaching solution. The PCT test is a seven-day test in deionized water at $90^{\circ} \mathrm{C}$ using $75-150 \mu \mathrm{m}$ glass powder. In addition, data from the PCT test permit direct comparison of the glass leaching behavior with that of high-level nuclear waste glasses. Finally, there is waste loading, since increased waste loading decreases overall treatment costs.

The forty glasses produced in this work were characterized with respect to these properties; a list of the glasses provided in Table 1.1. Other glasses were produced and partially characterized. A summary of these data are presented in the Appendix. Some of the appendix formulations 
produced inhomogeneous melts or highly phase separated products, or both. These materials were not subjected to the complete characterization testing. The durability testing was reserved primarily for glasses that would be preferred on the basis of visual observations, other characterization data (e.g. melt viscosity), or waste loadings. In addition, PCT tests were conducted only on glasses that contained the relevant radioactive and hazardous constituents. The data are organized and presented in separate Sections by site of origin of the wastes, and, in each case, the core data set is highlighted

\subsubsection{Waste Stream Characterization}

The starting point in evaluating the suitability of vitrification as a treatment method for a particular waste stream is characterization of the waste material. The characterization data are also essential in the development of glass formulations for the particular problem. The primary data requirements are for chemical composition, with particular emphasis on the major components. It is often the case that detailed data on contaminant concentrations have been collected for regulatory purposes, but only very gross information is available on the bulk constituents; it is the latter data that are most important for vitrification development since precise information on trace constituents is of little benefit for that purpose.

In a broad sense, waste streams can be classified on a spectrum of "silica-rich" (or more generally "glass-former rich") to "flux-rich", with these extremes having opposite effects on melting temperature and melt viscosity. The characteristics of the waste then determine the types and amounts of additives that are required in order to meet the design objectives. Generally, flux-rich wastes form glasses with poor leach resistance or crystallization. Similarly, glass-former rich wastes will only form glasses at high temperatures and typically require the addition of fluxing agents to reduce the processing temperature.

Waste stream compositional information for glass development purposes should include data for all major components (greater than about the $1 \mathrm{wt} \%$ level on a dry basis). This should include anions as well as cations. Total carbon content is useful for assessing potential redox effects, as is the content of metallic components. Thermogravimetric measurements (weight loss versus temperature) and particle size analyses also provide useful data from a process perspective. Since it is ultimately necessary to determine the fate of all of the contaminant species of concern, the concentrations of these species in the waste must be determined but are not critical in the glass design process.

While there are many classes of glass forming systems, including chalcogenides, heavy metal fluorides, and oxynitrides, only two - silicates and phosphates - have found significant applications in waste treatment. However, silicates are by far the most commonly used due mainly to the ubiquity, 
and low cost, of silicon-bearing minerals in nature. Silicate glasses can be formulated to incorporate a wide range of concentrations of the majority of the elements of the periodic table.

\subsubsection{Glass Formulation Development}

The development of viable glass formulations needs to consider a number of factors including the following:

The process should operate as economically as possible, for obvious reasons. Some of the major factors that influence the overall economics are the following:

(i) Waste loading, or conversely stated, minimum addition of non-waste additives in order to produce the stabilized waste form. Additives affect the overall economics from several perspectives: the cost of the additives; the cost of processing; and the cost of disposing the additives.

(ii) Phase Stability. If crystalline phases are formed during vitrification, sedimentation can occur which may ultimately lead to clogging in some melter designs as those materials accumulate. The liquidus temperature - the highest temperature at which crystals will form from the melt - is an important processing parameter and is strongly composition dependent. Feed formulations may need to be selected such that the liquidus temperature remains below the processing temperature for all reasonable variations in feed composition for some vitrification technologies.

(iii) Melt Viscosity. The melt viscosity is strongly dependent on the melt composition and temperature and may strongly affect a particular vitrification technology.

(iv) Electrical Conductivity. The electrical conductivity of the melt is an important factor affecting processability in vitrification systems that rely on electrical conduction through the melt in order to produce power dissipation into the melt by the joule heating effect. The conductivity determines such parameters as electrode sizes and spacing and power supply specifications. Again, the electrical conductivity of the melt is strongly dependent on its composition and temperature. Therefore, this dependence must be quantified in order to select target feed formulations for a particular system design and in order to determine acceptable ranges of variation in feed composition.

(v) Leach Resistance. Generally, the most important requirement from a product performance perspective is that the waste form be resistant to leaching of the contaminants of concern, since a major objective of the remediation process is to effectively isolate those contaminants and prevent their release into the environment. As with other properties, glass leach resistance is strongly 
dependent on composition. The observed rate of reaction of glass with water is highly dependent on factors including the temperature, the composition of the leachant (e.g., pure water, a natural groundwater, a pH-buffered solution, etc.) the time elapsed since the start of the reaction (initial rates are typically greater than later rates), and the ratio of the surface area of the glass to the volume of leachant. To minimize the possible effects of these variable, standardized test are used to evaluate leach resistance

Phase stability can also impact the leach resistance of the resulting waste form due to the formation of secondary phases as the molten glass cools. The leach resistance of a homogeneous glass can be easily controlled by careful formulation of the glass composition. However, if secondary phases are formed to any significant extent, the problem becomes more complicated since the secondary phases could be more soluble than the glass or may sequester constituents from the host glass that could degrade its leach resistance. The types and amounts of secondary phases and the waste form microstructure are all factors that can influence the leach resistance.

It is important to appreciate the dependence of the leachability measurement on the test conditions. For example, if leachant $\mathrm{pH}$ is considered, the TCLP is conducted under slightly acidic conditions and show good acidic leach resistance. This may be of little relevance in terms of actual performance if the disposal conditions are likely to be alkaline. Unfortunately, disposal conditions are often unknown or poorly defined during the waste form development phase. We have, therefore, used both the TCLP and the PCT, since these evaluate leachability under two different sets of conditions.

(vi) Compressive Strength. The compressive strength of the waste form is important for land disposal due to the possibility of subsidence. If subsidence should occur, it provides a means for water collection and pooling, rather than run-off, which would tend to increase contaminant release. Glass-based waste forms have extremely high compressive strengths and this is rarely a constraining factor for these materials.

(vii) Volume reduction. While volume reduction was considered as an economic factor above, it should also be included as a performance consideration since there is an incentive to minimize the size and number of the disposal facilities that are required to store the treated waste.

The role of glass formulation development is then to arrive at the optimum compromise between these competing factors by variation of the target glass composition. It should, however, be emphasized that the selection of a particular process or set of operating conditions should not be divorced from the glass formulation development and vice versa. 


\subsection{Facilities, Equipment, and Experimental Methods}

The major operations included analysis and characterization of the waste streams; glass melting; standard leach tests on the vitrified products; analysis of the vitrified products, and leachates; and product characterization. Standard glass characterization techniques include viscosity, conductivity, Direct Current Plasma (DCP) analysis, and microstructure determination using Scanning Electron Microscopy - Energy Dispersive X-Ray Spectroscopy (SEM-EDS). A short description of the techniques used is given below. Each of these techniques is described in detail in VSL technical procedures (CUA VSL 1992).

Specific Gravity: The specific gravity of wastes was determined using a pycnometer and the procedure specified in American Society for Testing and Materials (ASTM) D854-83. At least two measurements were made for each sample and the expected uncertainty in the specific gravity measurement is, as described in ASTM D854-83; within a standard deviation of $0.021 \mathrm{~g} \mathrm{~cm}^{-3}$.

Carbon Content: The carbon content of wastes was measured using a Dohrmann DC-80 Carbon Analyzer fitted with a 183 Boat Sampling Module (BSM). This system is capable of measuring the total carbon (TC), total organic carbon (TOC), and the inorganic carbon (IC) contents of wastewater, slurries, sludges, and solid samples. Each liquid sample is measured at least five times and the measurements typically agree to within $\pm 10 \%$. We estimate the error of measuring the carbon content of solids to be also within $\pm 10 \%$. These errors are purely analytical, however, and do not include sampling errors that can be quite significant for some waste streams.

Sieve Analysis: The distributions of particle sizes in wastes were determined using ASTM procedure D422-63. The major sources of error for these measurements are: accuracy of sieve size, particles left on the sieve which should have passed through, and weighing errors. We estimate the accumulation of these errors to be about $\pm 10 \%$.

Weight Loss: The weight loss data were obtained by heating representative samples of wastes as follows: $110^{\circ} \mathrm{C}$ for 18 hours; $450^{\circ} \mathrm{C}$ for 4 hours; $850^{\circ} \mathrm{C}$ for 4 hours; and $1150^{\circ} \mathrm{C}$ for 4 hours. Programmable Deltech furnaces (DT-28-12 and DT-28-06) were used for the weight loss measurements and consequently the time and heating rates could be easily controlled. The major source of error in our weight loss measurements is due to errors in weighing, which we estimate to be $\pm 5 \%$. Uncertainties from sample-to-sample variations (sampling errors) are estimated to be $\pm 15 \%$.

Gamma Spectroscopy: A Canberra Gamma Spectroscopy system coupled to a personal computer was used to obtain gamma spectroscopy measurements. Quantitative measurements were made by first effecting a total dissolution of the sample (typically in $\mathrm{HF} / \mathrm{HNO}_{3}$ ) and making measurements on the 
solution; this procedure simplified the calibration since a liquid standard could be used in the same geometry.

Glass Melting: Crucible melts were made with blends of wastes and additives; the ingredients were manually mixed by shaking in a plastic container and transferred to a crucible (platinum-gold or clay). The crucible was then placed in a furnace at the selected melt temperature (typically 1100 to $1450^{\circ} \mathrm{C}$ ), and the blend was typically melted for one hour; the melts were then stirred using a motordriven Pt-Rh stirrer during the last half hour of the melt time. In instances, in which the melt was judged to be too viscous or incompletely melted, the temperature was increased, typically for a further period of thirty minutes.

Heat Treatments: Approximately two-gram samples of glass were heat treated for specified times and temperatures. Unless otherwise stated, each sample was premelted at a temperature slightly above the original melt temperature for one hour before the heat treatment in order to dissolve any pre-existing crystals or crystal nuclei that may have been present in the as-melted glass.

Viscosity Measurement: The viscosity was calculated from measurements of the torque and rotation speed of a calibrated spindle attached to a Brookfield viscometer (Napolitano-1965). Measurements were made over a range of temperatures, usually between $1000^{\circ} \mathrm{C}$ to $1450^{\circ} \mathrm{C}$, and the data interpolated to standard temperatures. The equipment was calibrated using standard oils of known viscosity and checked using a National Institute of Standards and Technology (NIST) standard reference glass (SRM711). The precision and accuracy of the viscosity measurements are estimated to be within $\pm 10 \%$.

Conductivity Measurement: The electrical conductivity of the glass was determined by measuring the resistance of the glass melt as a function of frequency using a calibrated platinum electrode probe (Tickle-1967). The results were extrapolated to zero frequency to obtain the DC conductivity. These measurements were taken over a temperature range of typically $1000-1450^{\circ} \mathrm{C}$, and the data interpolated to standard temperatures. The equipment was calibrated using salt solutions of known concentrations. Estimated uncertainties in the conductivity measurements are $\pm 5 \%$.

Glass Redox State: The redox states of glass samples were determined using Mössbauer spectroscopy to determine the ratio of $\mathrm{Fe}^{2+}$ to $\mathrm{Fe}^{3+}$ in the glass using a Ranger Mössbauer spectrometer with a cobalt source (Mysen-1989, Yaschenko-1994). The estimated uncertainty in the redox ratio measurements is $\pm 5 \%$.

TCLP Test: The TCLP leach test was used to determine the leach resistance of the glasses that were produced by measuring the leachate concentrations obtained after 18 hours at $22^{\circ} \mathrm{C}$ in a sodium acetate buffer solution (US EPA-1990). The leachate concentrations were measured by both DCP 
and Inductively Coupled Plasma Mass Spectrometry (ICPMS). The overall uncertainty is estimated to be $\pm 20 \%$.

PCT Test: The PCT test evaluates the relative chemical durability of glasses by measuring the concentrations of the chemical species released from crushed glass $(75-150 \mu \mathrm{m})$ to the test solution (deionized water in this case) at $90^{\circ} \mathrm{C}$ (Jantzen-1989). All tests were conducted in triplicate with a standard glass (West Valley Reference 5) included in each test set. The overall uncertainty in the test results is estimated to be $\pm 15 \%$. The leachates were sampled after $7,28,56,120,240$, and 360 days. At each sampling date, $4.0 \mathrm{ml}$ of the leachate was removed for analysis from each test vessel and was replaced by $4.0 \mathrm{ml}$ of deionized water (Feng-1990, Feng-1994).

Scanning Electron Microscopy/Energy Dispersive Spectroscopy (SEM/EDS): Scanning electron microscopy was used to characterize the microstructure of the glasses and permit analysis of the glassy and crystalline phases using energy dispersive $x$-ray spectrometry. This equipment permits determination of both the volume fractions and compositions of crystalline phases in both as-melted and heat treated glasses. The percentage of crystals is estimated by examining several regions of a $\sim 1 \mathrm{~cm}^{2}$ sample, and is accurate to about $\pm 20 \%$. The identity of major crystalline phases was also confirmed by $\mathrm{X}$-ray diffraction measurements.

Microwave Dissolution of Sludges, Soils, and Glasses: Various combinations of acids (typically $\mathrm{HF} / \mathrm{HNO}_{3}$ ) are used to dissolve waste samples and glasses, depending on the composition of the sample. The samples are dissolved completely by heating (typically $100 \mathrm{mg}$ in $200 \mathrm{ml}$ of solution) then in sealed pressure vessels in a microwave oven (US EPA-1986).

Solution Analyses: Direct Current Plasma Emission Spectroscopy (DCP-ES), Inductively Coupled Plasma Mass Spectrometry (ICP-MS), ion chromatography, and ion selective electrode techniques were used for solution analyses. The overall uncertainty associated with DCP-ES analysis is $\pm 5 \%$, that for ion chromatography and ion selective electrode analyses is $\pm 10 \%$, and for ICP-MS, the uncertainty is estimated to be $\pm 30 \%$ for semi-quantitative analysis and $\pm 15 \%$ for quantitative analysis.

Fluoride Analysis: The fluoride content was determined by a modified Environmental Protection Agency (EPA) SW846 procedure that involves microwave dissolution of the glass in a two-step process using $\mathrm{NaOH}$ then aqua regia. Once the fluoride is in solution, a fluoride electrode is used to determine its concentration. The experimental reproducibility of our fluoride analysis is within $\pm 15 \%$. 


\subsection{Quality Assurance}

This work was conducted under a Quality Assurance (QA) program in order to ensure the reliability, verifiability, and traceability of data obtained in the laboratory. The program is based upon the applicable quality assurance program requirements for nuclear facilities outlined in American Nuclear Standards Institute/American Society of Mechanical Engineers (ANSI/ASME) NQA-1. A full-time Quality Assurance Officer (QAO) supervised the implementation of the QA program throughout the duration of the project. Technical procedure requirements were reviewed and several new Standard Operating Procedures (SOP) were developed as necessary to complete the work. Regular internal surveillances are conducted by the VSL QAO, and external audits are conducted by other Department of Energy (DOE) and commercial project sponsors. Data obtained at each stage of the project were reviewed by the Laboratory Managers, the Project Manager, and the Principal Investigator for accuracy and reproducibility. Periodic validation by the QAO and statistical quality controls were in place to ensure the reliability of the data and the results. 
Table 1.1.

Summary of Crucible Melts Prepared and Testing Performed. (indicated by check marks)

\begin{tabular}{|c|c|c|c|c|c|c|c|}
\hline Glass & Name & $\begin{array}{c}\text { Waste } \\
\text { Loading \% }\end{array}$ & Waste Types & Viscosity & Conductivity & TCLP & PCT \\
\hline 1 & HAN22B & $80 \%$ & Rad. Soil (HA2) & $\checkmark$ & $\checkmark$ & $\checkmark$ & $\checkmark$ \\
\hline 2 & HAN24 & $100 \%$ & Rad. Soil+Tank Waste & $\checkmark$ & $\checkmark$ & $\checkmark$ & $\checkmark$ \\
\hline 3 & HAN25 & $95 \%$ & Rad. Soil+Tank Waste & $\checkmark$ & $\checkmark$ & $\checkmark$ & $\checkmark$ \\
\hline 4 & HAN26 & $95 \%$ & Rad. Soil+Tank Waste & $\checkmark$ & $\checkmark$ & $\checkmark$ & $\checkmark$ \\
\hline 5 & HAN27 & $95 \%$ & Rad. Soil+Tank Waste & $\checkmark$ & $\checkmark$ & $\checkmark$ & $\checkmark$ \\
\hline 6 & HAN28 & $95 \%$ & Rad. Soil+Tank Waste & $\checkmark$ & $\checkmark$ & $\checkmark$ & $\checkmark$ \\
\hline 7 & HAN28A & $95 \%$ & Rad. Soil+Tank Waste & $\checkmark$ & $\checkmark$ & $\checkmark$ & $\checkmark$ \\
\hline 8 & HAN29A & $95 \%$ & Rad. Soil+Tank Waste & $\checkmark$ & $\checkmark$ & $\checkmark$ & $\checkmark$ \\
\hline 9 & HAN29B1 & $95 \%$ & Rad. Soil+Tank Waste & $\checkmark$ & $\checkmark$ & $\checkmark$ & $\checkmark$ \\
\hline 10 & HAN29E & $86 \%$ & Rad. Soil+Tank Waste & $\checkmark$ & $\checkmark$ & $\checkmark$ & $\checkmark$ \\
\hline 11 & HAN29F & $95 \%$ & Rad. Soil+Tank Waste & $\checkmark$ & $\checkmark$ & $\checkmark$ & $\checkmark$ \\
\hline 12 & HAN30A & $95 \%$ & Rad. SoiI+Tank Waste & $\checkmark$ & $\checkmark$ & $\checkmark$ & $\checkmark$ \\
\hline 13 & HAN33 & $85 \%$ & Rad. Soil HA2 & $\checkmark$ & $\checkmark$ & $\checkmark$ & $\checkmark$ \\
\hline 14 & HAN34 & $85 \%$ & Rad. Soil HA2 & $\checkmark$ & $\checkmark$ & $\checkmark$ & $\checkmark$ \\
\hline 15 & HAN35 & $90 \%$ & Rad. Soil HA2 & $\checkmark$ & $\checkmark$ & $\checkmark$ & $\checkmark$ \\
\hline 16 & HAN36 & $95 \%$ & Rad. Soil+Tank Waste & $\checkmark$ & $\checkmark$ & $\checkmark$ & $\checkmark$ \\
\hline 17 & HAN37 & $95 \%$ & Rad. Soil+Tank Waste & $\checkmark$ & $\checkmark$ & $\checkmark$ & $\checkmark$ \\
\hline 18 & HAN38 & $95 \%$ & Rad. Soil+Tank Waste & $\checkmark$ & $\checkmark$ & $\checkmark$ & $\checkmark$ \\
\hline 19 & HAN39 & $32 \%$ & Tank Waste+additives & $\checkmark$ & $\checkmark$ & $\checkmark$ & $\checkmark$ \\
\hline 20 & IDG7A & $100 \%$ & Rad. Salt/ACM & $\checkmark$ & $\checkmark$ & $\checkmark$ & $\checkmark$ \\
\hline 21 & DG7B & $100 \%$ & RAD. Salt/ACM & $\checkmark$ & $\checkmark$ & $\checkmark$ & $\checkmark$ \\
\hline 22 & IDG7C & $100 \%$ & Rad. Salt/ACM & $\checkmark$ & $\checkmark$ & $\checkmark$ & $\checkmark$ \\
\hline
\end{tabular}




\begin{tabular}{|c|c|c|c|c|c|c|c|}
\hline Glass & Name & $\begin{array}{c}\text { Waste } \\
\text { Loading \% } \\
\end{array}$ & Waste Types & Viscosity & Conductivity & TCLP & PCT \\
\hline 23 & IDG8A & $100 \%$ & Rad. Salt/ACM & $\checkmark$ & $\checkmark$ & $\checkmark$ & $\checkmark$ \\
\hline 24 & IDG8C & $100 \%$ & Rad. Salt/ACM & $\checkmark$ & $\checkmark$ & $\checkmark$ & $\checkmark$ \\
\hline 25 & IDG12C & $100 \%$ & $\begin{array}{l}\text { Rad. Ash/Salt/ } \\
\text { ACM/Soil }\end{array}$ & $\checkmark$ & $\checkmark$ & $\checkmark$ & $\checkmark$ \\
\hline 26 & IDG13A & $100 \%$ & $\begin{array}{l}\text { Rad. Ash/Salt/ } \\
\text { ACM/Soil }\end{array}$ & $\checkmark$ & $\checkmark$ & $\checkmark$ & $\checkmark$ \\
\hline 27 & IDG13B & $100 \%$ & $\begin{array}{l}\text { Rad. Ash/Salt/ } \\
\text { ACM/Soil }\end{array}$ & $\checkmark$ & $\checkmark$ & $\checkmark$ & $\checkmark$ \\
\hline 28 & IDG13C & $100 \%$ & $\begin{array}{l}\text { Rad. Ash/Salt/ } \\
\text { ACM/Soil }\end{array}$ & $\checkmark$ & $\checkmark$ & $\checkmark$ & $\checkmark$ \\
\hline 29 & IDG14A & $100 \%$ & $\begin{array}{l}\text { Rad. Ash/Salt/ } \\
\text { ACM/Soil }\end{array}$ & $\checkmark$ & $\checkmark$ & $\checkmark$ & $\checkmark$ \\
\hline 30 & IDG14B & $100 \%$ & $\begin{array}{l}\text { Rad. Ash/Salt/ } \\
\text { ACM/Soil }\end{array}$ & $\checkmark$ & $\checkmark$ & $\checkmark$ & $\checkmark$ \\
\hline 31 & IDG15A & $100 \%$ & $\begin{array}{l}\text { Rad. Ash/Salt/ } \\
\text { ACM/Soil } \\
\end{array}$ & $\checkmark$ & $\checkmark$ & $\checkmark$ & $\checkmark$ \\
\hline 32 & ORNL3 & $82.7 \%$ & Pond Waste & $\checkmark$ & $\checkmark$ & $\checkmark$ & $\checkmark$ \\
\hline 33 & ORNL9A & $75 \%$ & Pond Waste & $\checkmark$ & $\checkmark$ & $\checkmark$ & $\checkmark$ \\
\hline 34 & ORNL10 & $65 \%$ & Pond Waste & $\checkmark$ & $\checkmark$ & $\checkmark$ & $\checkmark$ \\
\hline 35 & ORNL11 & $100 \%$ & WETF/Soil & $\checkmark$ & $\checkmark$ & $\checkmark$ & $\checkmark$ \\
\hline 36 & ORNL12 & $100 \%$ & WETF/Pond Waste/Soil & $\checkmark$ & $\checkmark$ & $\checkmark$ & $\checkmark$ \\
\hline 37 & ORNL13 & $80 \%$ & WETF/Soil & $\checkmark$ & $\checkmark$ & $\checkmark$ & $\checkmark$ \\
\hline 38 & ORNL14 & $100 \%$ & TSCA Ash/Soil & $\checkmark$ & $\checkmark$ & $\checkmark$ & $\checkmark$ \\
\hline 39 & ORNL15 & $100 \%$ & $\begin{array}{l}\text { TSCA Ash/Pond } \\
\text { Waste/Soil } \\
\end{array}$ & $\checkmark$ & $\checkmark$ & $\checkmark$ & $\checkmark$ \\
\hline 40 & ORNL16 & $80 \%$ & TSCA Ash/Soil & $\checkmark$ & $\checkmark$ & $\checkmark$ & $\checkmark$ \\
\hline
\end{tabular}




\section{Appendix}

Summary of Crucible Melts Prepared and Testing Performed.

(indicated by check marks)

\begin{tabular}{|c|c|c|c|c|c|c|c|}
\hline Glass & Name & $\begin{array}{c}\text { Waste } \\
\text { Loading \% }\end{array}$ & Waste Types & Viscosity & Conductivity & TCLP & $\overline{\mathrm{PCT}}$ \\
\hline 1 & HAN1 & $100 \%$ & Soil Surrogate & $\checkmark$ & G & $\mathrm{A}$ & A \\
\hline 2 & HAN2 & $95 \%$ & Soil Surrogate & $\checkmark$ & $\checkmark$ & $\mathrm{A}$ & A \\
\hline 3 & HAN3 & $91 \%$ & Soil Surrogate & $\checkmark$ & $\checkmark$ & A & A \\
\hline 4 & HAN4 & $87 \%$ & Soil Surrogate & $\checkmark$ & $\checkmark$ & A & A \\
\hline 5 & HAN5 & $83 \%$ & Soil Surrogate & $\checkmark$ & $\checkmark$ & A & A \\
\hline 6 & HAN6 & $80 \%$ & Soil Surrogate & $\checkmark$ & $\checkmark$ & A & A \\
\hline 7 & HAN7 & $77 \%$ & Soil Surrogate & $\checkmark$ & $\checkmark$ & $\mathrm{A}$ & A \\
\hline 8 & HAN8 & $74 \%$ & Soil Surrogate & $\checkmark$ & $\checkmark$ & $\mathrm{A}$ & $\mathrm{A}$ \\
\hline 9 & HAN9 & $71 \%$ & Soil Surrogate & $\checkmark$ & $\checkmark$ & $\mathrm{A}$ & A \\
\hline 10 & HAN10 & $69 \%$ & Soil Surrogate & $\checkmark$ & $\checkmark$ & $\mathrm{A}$ & A \\
\hline 11 & HAN11 & $66 \%$ & Soil Surrogate & $\checkmark$ & $\checkmark$ & $\mathrm{A}$ & $\mathrm{A}$ \\
\hline 12 & HAN12 & $95 \%$ & Soil Surrogate & $\checkmark$ & $\checkmark$ & $\mathrm{A}$ & $\mathrm{A}$ \\
\hline 13 & HAN13 & $91 \%$ & Soil Surrogate & $\checkmark$ & $\checkmark$ & $\mathrm{A}$ & $\mathrm{A}$ \\
\hline 14 & HAN14 & $87 \%$ & Soil Surrogate & $\checkmark$ & $\checkmark$ & A & $\mathrm{A}$ \\
\hline 15 & HAN15 & $83 \%$ & Soil Surrogate & $\checkmark$ & $\checkmark$ & $\mathrm{A}$ & $\mathrm{A}$ \\
\hline 16 & HAN16 & $80 \%$ & Soil Surrogate & $\checkmark$ & $\checkmark$ & $\mathrm{A}$ & $\mathrm{A}$ \\
\hline 17 & HAN17 & $83 \%$ & Soil Surrogate & $\checkmark$ & $\checkmark$ & $\mathrm{A}$ & $\mathrm{A}$ \\
\hline 18 & HAN18 & $83 \%$ & Soil Surrogate & $\checkmark$ & $\checkmark$ & $\mathrm{A}$ & $\mathrm{A}$ \\
\hline 19 & HAN19 & $83 \%$ & Soil Surrogate & $\checkmark$ & $\checkmark$ & A & A \\
\hline 20 & HAN20 & $80 \%$ & Non-Rad. Soil (HA1) & $\checkmark$ & $\checkmark$ & A & $\mathrm{A}$ \\
\hline 21 & HAN28B & $95 \%$ & Rad. Soil+Tank Waste & B & B & $\checkmark$ & $\mathrm{B}$ \\
\hline 22 & HAN29 & $95 \%$ & Rad. Soil+Tank Waste & $\checkmark$ & $\checkmark$ & B & B \\
\hline
\end{tabular}




\begin{tabular}{|c|c|c|c|c|c|c|c|}
\hline Glass & Name & $\begin{array}{c}\text { Waste } \\
\text { Loading \% } \\
\end{array}$ & Waste Types & Viscosity & Conductivity & TCLP & PCT \\
\hline 23 & HAN29B & $95 \%$ & Rad. Soil+Tank Waste & B & B & $\checkmark$ & B \\
\hline 24 & HAN29B2 & $95 \%$ & Rad. Soil+Tank Waste & B & B & $\checkmark$ & B \\
\hline 25 & HAND29 & $95 \%$ & Rad. Soil+Tank Waste & $\checkmark$ & $\checkmark$ & C & $\mathrm{C}$ \\
\hline 26 & HAN29G & $86 \%$ & Rad. Soil+Tank Waste & $\mathrm{C}$ & C & $\mathrm{C}$ & $\mathrm{C}$ \\
\hline 27 & HAN30B & $95 \%$ & Rad. Soil+Tank Waste & B & B & $\checkmark$ & B \\
\hline 28 & HAN30C & $95 \%$ & Rad. Soil+Tank Waste & B & B & B & B \\
\hline 29 & HAN32 & $90 \%$ & Rad. Soil HA2 & $\checkmark$ & $\checkmark$ & $\mathrm{H}$ & $\mathrm{H}$ \\
\hline 30 & IDG4 & $100 \%$ & ID2+surrogate ACM & A & A & A & A \\
\hline 31 & IDG5 & $100 \%$ & $\mathrm{ID} 2$ +surrogate $\mathrm{ACM}$ & A & A & A & A \\
\hline 32 & IDG6 & $100 \%$ & $\mathrm{ID} 2$ +surrogate $\mathrm{ACM}$ & A & A & A & A \\
\hline 33 & IDG8B & $100 \%$ & Rad. Salt/ACM & $\checkmark$ & $\checkmark$ & $\checkmark$ & G \\
\hline 34 & IDG10 & $100 \%$ & Rad. Salt/ACM & $\mathrm{D}$ & $\mathrm{D}$ & $\mathrm{D}$ & $\mathrm{D}$ \\
\hline 35 & IDG11 & $100 \%$ & $\begin{array}{l}\text { Rad. Ash/Salt/ } \\
\text { ACM/Soil. }\end{array}$ & $\mathrm{D}$ & D & $\mathrm{D}$ & $\mathrm{D}$ \\
\hline 36 & DG12A & $100 \%$ & $\begin{array}{l}\text { Rad. Ash/Salt/ } \\
\text { ACM/Soil }\end{array}$ & F & $\mathrm{F}$ & $\mathrm{F}$ & F \\
\hline 37 & IDG12B & $100 \%$ & $\begin{array}{l}\text { Rad. Ash/Salt/ } \\
\text { ACM/Soil }\end{array}$ & F & $\mathrm{F}$ & F & F \\
\hline 38 & IDG14C & $100 \%$ & $\begin{array}{l}\text { Rad. Ash/Salt/ } \\
\text { ACM/Soil }\end{array}$ & F & $\mathrm{F}$ & F & F \\
\hline 39 & IDG15B & $100 \%$ & $\begin{array}{l}\text { Rad. Ash/Salt/ } \\
\text { ACM/Soil }\end{array}$ & $\checkmark$ & $\checkmark$ & $\checkmark$ & F \\
\hline 40 & IDG15C & $100 \%$ & $\begin{array}{l}\text { Rad. Ash/Salt/ } \\
\text { ACM/Soil }\end{array}$ & $\checkmark$ & $\checkmark$ & $\checkmark$ & F \\
\hline 41 & ORNL1 & $100 \%$ & Pond Waste & $\mathrm{E}$ & $E$ & $\checkmark$ & A \\
\hline 42 & ORNL2 & $91.4 \%$ & Pond Waste & $\mathrm{E}$ & $\mathrm{E}$ & $\checkmark$ & A \\
\hline 43 & ORNL4 & $85 \%$ & Pond Waste/Soil & D & D & $\mathrm{D}$ & $\mathrm{D}$ \\
\hline 44 & ORNL5 & $95 \%$ & Pond Waste/Soil & $\mathrm{D}$ & D & D & $\mathrm{D}$ \\
\hline 45 & ORNL6 & $75 \%$ & Pond Waste/Soil & A & A & A & $\mathrm{A}$ \\
\hline
\end{tabular}




\begin{tabular}{|l|l|l|l|l|l|l|l||}
\hline Glass & \multicolumn{1}{|c|}{ Name } & $\begin{array}{c}\text { Waste } \\
\text { Loading \% }\end{array}$ & \multicolumn{1}{|c|}{ Waste Types } & Viscosity & Conductivity & TCLP & PCT \\
\hline \hline 46 & ORNL7 & $75 \%$ & Pond Waste/Soil & $\checkmark$ & $\checkmark$ & $\checkmark$ & A \\
\hline 47 & ORNL8 & $100 \%$ & Pond Waste & $\checkmark$ & $\checkmark$ & $\checkmark$ & H \\
\hline
\end{tabular}

\section{NOTES:}

$A=$ Surrogate glasses, therefore not leach tested.

$\mathrm{B}=$ Glasses prepared to examine sodium/technetium retention; limited further testing.

$\mathrm{C}=$ Testing of similar glasses $\left(\mathrm{Na}_{2} \mathrm{O}>40 \mathrm{wt} \%\right)$ showed very poor durability.

$\mathrm{D}=$ Glasses which were visibly multi-phase on pouring.

$\mathrm{E}=$ The metals in these glasses attacked the platinum equipment. The measurement lacked accuracy and the cost of repair of the equipment is prohibitive.

$\mathrm{F}=\quad$ The Idaho glasses using the radioactive ACM were intentionally melted in triplicate in order to examine waste composition variability; not all glasses were leach tested.

$\mathrm{G}=$ Glass extremely viscous even above $1400^{\circ} \mathrm{C}$.

$\mathrm{H}=$ Good leach resistance expected on the basis of similar compositions, therefore other melts took priority for leach testing.

Waste Stream Abbreviations:

Tank Waste $=$ Hanford LLW Tank Waste (Radioactive surrogate with Tc and U)

Soil = Hanford Contaminated Soils (for HAN glasses)

$\mathrm{ACM}=$ Idaho Radioactive Asbestos-Containing Material

Ash = Idaho WERF Fly Ash (for IDG glasses)

Salt $=$ Idaho Eutectic Evaporator Salt

Soil = Idaho Soil (non-radioactive site soil) (for IDG glasses)

WETF = Oak Ridge WETF Sludge

Pond Waste = Oak Ridge K-1407 Pond Waste (surrogate with U and Tc)

TSCA Ash = Oak Ridge TSCA Incinerator Ash (surrogate with U)

Soil = Oak Ridge Soil (non-radioactive soil) (for ORNL glasses) 


\section{SECTION 2.0 \\ MAWS VITRIFICATION OF HANFORD TANK WASTES AND CONTAMINATED SOILS}

\subsection{Hanford Wastes Selected for Study}

Two Hanford waste streams were, selected for this study: the tank wastes and the contaminated soils. Treatment alternatives for the tank wastes at the Hanford site are presently under review (Boomer-1993). The process selected will most likely involve separation of the wastes into high-level and low-level streams followed by vitrification of each stream separately.

The tank waste is a high-volume waste stream for which vitrification has been selected as the preferred treatment technology. Since the major cation constituent of this waste is sodium (Boomer1993), the waste behaves as a "flux" from a vitrification perspective. This leads to a wide range of potential blending scenarios using the MAWS approach since waste streams that are rich in glass formers are relatively common (the most obvious one at the Hanford site being contaminated soils). This section presents the results obtained from a study of the application of MAWS vitrification to the combination of the Hanford low-level tank waste and contaminated soil waste streams.

Defense wastes stored at the Hanford site are currently contained in double shell tanks and older single shell tanks in the form of liquids, slurries, sludges, and salt cakes. The projected pretreatment process will separate the wastes into a high-level waste component and a low-level waste component. The low-level waste component will be Class $\mathrm{C}$ or lower in activity and will have an estimated volume of about $230,000 \mathrm{~m}^{3}$ (61 million gallons); this stream will consist mostly of sodium nitrate and nitrite in an alkaline liquid slurry form. The high sodium content of this waste and the fact that glass leach resistance generally decreases with increasing sodium content means that waste loadings are not expected to exceed about 20-30 wt $\%$ on an oxide basis. Thus, the bulk of the waste form would be composed of non-waste additives. In the present study, we have investigated the benefits of substituting those additives by other wastes in a MAWS approach. Specifically, we have investigated the use of contaminated site soils to provide the necessary glass-forming constituents and, in turn, provide a viable treatment alternative for the soils at little additional cost.

From a MAWS perspective, the low-level stream from the Hanford tanks is a valuable commodity since it is composed predominantly of sodium. Sodium is an effective fluxing agent in glass making, which is often purchased as an additive. The large volumes of contaminated soils at 
Hanford (about 22 million $\mathrm{yd}^{3}$ in the 100 and 300 areas alone) will likely require volume reduction by soil washing and, since the contaminant-enriched stream that is produced will require subsequent stabilization, the MAWS concept has considerable potential at Hanford. The low-level sodium stream from the tank-waste separations process could be combined with soil-washing concentrates to produce a vitrifiable blend that could be melted to form a volume-reduced, glass waste form. This section summarizes the results obtained from a vitrification formulation study to evaluate such an approach for these Hanford waste streams.

\subsection{Characterization of Hanford Waste Streams}

\subsubsection{Non-Radioactive Soil}

Samples of contaminated soils were requested from the Hanford site for this study. Preliminary tests were performed on a sample of non-radioactive site soils. Direct current plasma (DCP) analysis of this soil sample (HA1) was performed to obtain compositional information for vitrification scoping melts. Table 2.1 summarizes the results of $\mathrm{DCP}$ analyses of a triplicate sampling and triplicate microwave dissolutions of each sample. The consistency between the three analyses and dissolutions is good, indicating that the soil is homogeneous. The composition of the nonradioactive soil sample is in general agreement with the typical compositional range provided to us by Hanford personnel (Ludowise-1993) with some exceptions; alumina and silica fall on the high side of that range and calcium, iron, potassium, magnesium, and sodium are at the low end of the range.

\subsubsection{Radioactive Soil}

The radioactive soil sample was received from Hanford in three containers (for shipping convenience), although it was described as a single sample. A screening test using a Geiger counter showed a significant difference between the three containers, however. The samples were given separate identification labels (HA2, HA3, and HA4) and separate analyses were performed on each.

\subsubsection{Chemical Analysis and Physical Characterization}

Separate subsamples of each of the three soil samples were dried at $1150^{\circ} \mathrm{C}$ and at $450^{\circ} \mathrm{C}$ to remove volatile constituents. Triplicate subsamples of each of the dried samples were subjected to acid dissolution using a microwave digestion technique, and the resulting solutions were analyzed by DCP emission spectroscopy. The results are summarized in Table 2.2. The triplicate dissolutions showed good reproducibility. There is generally good agreement between these results and the 
composition range provided by Hanford personnel with the exceptions of $\mathrm{Fe}_{2} \mathrm{O}_{3}$, which is about half of the expected value, and $\mathrm{SiO}_{2}$, which is slightly higher than expected.

Contaminants in the soil were characterized using ICPMS analyses; the soil samples were dried at $450^{\circ} \mathrm{C}$ and then dissolved in a $\mathrm{HF} / \mathrm{HNO}_{3}$ solution by microwave. The Uranium concentration was approximately $4 \mathrm{ppm}$ for soil samples $\mathrm{HA} 2$ and $\mathrm{HA} 4$, while $\mathrm{HA} 3$ continued twice that amount. Of the other contaminants detected, $\mathrm{Cu}, \mathrm{Zn}, \mathrm{Pb}, \mathrm{Ni}$, and $\mathrm{Mn}$ were found at approximately equal concentrations in all three soils, as shown in Table 2.3. (Note that the value for each element ("Avg (ppb) X 2000") reflects the 2000-fold dilution factor involved in the dissolution of the solid sample.)

\section{Thermogravimetry}

The results of our thermogravimetric analysis (weight loss versus temperature) of each of the three samples are presented in Table 2.4. The soil has a low moisture content (below 6.5\%) and the total volatile losses (volatilization and combustion of organics, decomposition of carbonates, hydroxides, etc.) are only about $8 \%$; little sample-to-sample variation was found.

\section{Specific Gravity}

The specific gravity of the soil samples, both as-received as well as after drying at $110^{\circ} \mathrm{C}$ for 18 hours, was measured according to ASTM procedure D854-83; the results are summarized in Table 2.4 .

\section{Particle Size distribution}

The particle size distribution was measured, according to the ASTM procedure D422-63, after preparation of the soil, as described in ASTM D421-85. The results are summarized in Table 2.4 and Figure 2.1. Note that the soil was sieved at Hanford and the fraction below $2 \mathrm{~mm}$ was shipped. Almost $70 \%$ of the soil falls within the $0.25 \mathrm{~mm}$ to $2 \mathrm{~mm}$ range. 


\section{Total Carbon Analysis (TC) and Total Organic Carbon (TOC)}

The TC was measured by direct high-temperature combustion $\left(800^{\circ} \mathrm{C}\right)$ followed by infrared detection of the resulting carbon dioxide, while TOC was measured using the same technique, but after the soil was acidified and sparged to remove inorganic carbon by decomposition of carbonates to carbon dioxide. The difference between the TC to TOC values reflects, therefore, the amount of carbon due to carbonates in the soil (versus organic material only, given by TOC). Both TC and TOC measurements are summarized in Tables 2.5 and 2.6, respectively. As discussed previously, these results are consistent with the low weight loss between $450^{\circ} \mathrm{C}$ and $850^{\circ} \mathrm{C}$. The soil (at least the fraction below $2 \mathrm{~mm}$ studied here) contains very little organic material; the amount present as carbonate is also very minor; the total carbon content is about $1 \mathrm{wt} \%$ in all three samples with about 0.1 to $0.4 \%$ due to carbonates.

\section{Gamma Counting of Sieved Fractions}

Gamma counting spectroscopy was performed on liquid samples obtained by total dissolution of $1 \mathrm{~g}$ of each soil sample. The solutions were contained in a $500 \mathrm{ml}$ Marinelli beaker to achieve the required calibrated geometry. The results are presented in Table 2.7; a typical spectrum is shown in Figure 2.2.

The activity of $\mathrm{HA} 2$ is much higher than that of the other two samples. A further observation is the enrichment of activity in the finer fractions; this result is of practical importance since it provides the basis of a possible simple means of volume reduction through concentration of the contaminants by sieving. ${ }^{137} \mathrm{Cs}$ is the isotope that is responsible for the majority of the gamma activity. ${ }^{57} \mathrm{Co}$ was found at a level close to the detection limit in the finer soil fractions and ${ }^{234} \mathrm{Th}$ was detected at slightly above background.

\subsubsection{Hanford Tank Waste Surrogate}

The nominal composition of the low-level waste feed stream from the Hanford tanks that was used in this study is given in Table 2.8a as the total mass of each component in both the liquid and the solid phases. A recipe for preparing the surrogate tank waste (Double Shell Slurry Feed, DSSF), which was used in the grout waste form development program at Hanford (Voogd-1993), was used to guide the selection of the specific chemical compounds and the preparation method. The third column in Table 2.8a lists the source of each constituent in the surrogate formulation. Table $2.8 \mathrm{~b}$ lists the composition of the surrogate LLW tank waste on an oxide basis. The surrogate formulation consists of the following components: 
- A solid mix of $\mathrm{NaOH}+\mathrm{NaNO}_{3}$, and $\mathrm{BiOCl}$

- An aqueous solution of most of the remaining components made up by mixing three solutions

- A spike solution containing the RCRA metals

- A spike solution containing selected radionuclides

The preparation of the surrogate in this way has been successfully tested on a small scale to ensure that there is no precipitation from the aqueous phase that would cause problems in obtaining representative subsamples for crucible melts. Enough liquid fraction was prepared in order to provide for at least five crucible melts, while the solid components and radioactive components $(\mathrm{U}, \mathrm{Tc}$, and non-radioactive $\mathrm{Cs}$, $\mathrm{Sr}$, and I) were added individually to each crucible melt batch.

\subsection{Hanford Vitrification Testing}

Our composition study for Hanford wastes was directed along two paths with the following separate objectives:

(A) Maximize waste loading with respect to the low-level tank waste stream but make full use of contaminated site soil in order to minimize the use of additives.

(B) Maximize the waste loading with respect to site soil and minimize the use of additives.

\subsubsection{Melts to Maximize Tank Waste Loading}

Table 2.10 lists the compositions of the glasses prepared from Hanford radioactive soil combined with the LLW tank waste simulant (which includes heavy metals and several radionuclides or their corresponding non-radioactive isotopes). The composition of each glass is summarized in terms of the soil content, the total $\mathrm{Na}_{2} \mathrm{O}$ content, the $\mathrm{Na}_{2} \mathrm{O}$ content contributed by the tank waste component, and the additives. This format is preferred over stating the oxide waste loading with respect to the tank waste since, as discussed below, significant amounts of sodium were frequently lost in the glass melting process. However, all of the glasses were subjected to direct chemical analysis and the actual sodium content was used in assessing property-composition relationships.

Since the tank waste yields only about $14.3 \%$ oxides (about $83 \%$ of which is $\mathrm{Na}_{2} \mathrm{O}$ ) on a mass basis (due to volatile losses from water, nitrates, hydroxides, etc., based on information obtained from Hanford), large volume reductions can be obtained upon vitrification, particularly if high waste 
loadings can be achieved. Using a $20 \%$ oxide waste loading with respect to tank waste as a baseline, Figure 2.3 shows the reduction in the total volume of glass that will be produced as a consequence of increased waste loading; the volume could be reduced by one-third relative to this baseline by increasing the waste loading from $20 \%$ to $30 \%$. Due to the high sodium content of the tank waste, it is likely that the upper limit of waste loading will be determined by the glass leach resistance. The results presented below provide an indication of the effects of tank waste loading on glass leach resistance as determined by both the TCLP and the PCT.

Several observations were made during the melting of glasses with higher tank waste loadings that differed somewhat from those at lower waste loadings. In all cases, the liquid tank waste surrogate was mixed with the soil directly in the clay crucible used for melting. As the tank waste fraction increased from $31 \%$ to $40 \%$ (on an oxide basis), an effervescence became more noticeable presumably due to a chemical reaction of the soil with the very basic tank solution. The mixture became increasingly frothy and the temperature increased to as high as $40^{\circ} \mathrm{C}$. While the lower waste loading glasses (HAN24 to HAN28) produced a visually homogeneous glass product after melting at $1200^{\circ} \mathrm{C}$, the HAN29 and HAN30 glasses, although less viscous and easier to pour, produced a glass that was very foamy and that fractured easily. We subsequently followed the melting step at $1200^{\circ} \mathrm{C}$ by a brief higher temperature fining step of about 5 minutes at $1400^{\circ} \mathrm{C}$; this procedure produced a product that was visually homogeneous. Interactions of the corrosive tank waste material and the clay crucible were also detected from the DCP analyses of the resultant glasses and revealed a small but systematic increase of the silica level and a concurrent decrease in the sodium level. We believe that the observed loss of sodium was due to a combination of soluble components in the waste penetrating the crucible and to evaporation of sodium-containing species upon melting. The former effect was avoided in later melt preparations (HAN29F and HAN36 to HAN39) by melting them in platinum-gold crucibles. Sodium loss due to evaporation was reduced by minimizing the melt temperature and time to $1200^{\circ} \mathrm{C}$ and 70 minutes, respectively. The efforts to increase sodium retention in the glass were successful; glass HAN29F was prepared with $42.7 \%$ $\mathrm{Na}_{2} \mathrm{O}$ and the fraction of $\mathrm{Na}_{2} \mathrm{O}$ retained could be increased to as much as $95 \%$ when the target level was about $29 \% \mathrm{Na}_{2} \mathrm{O}$.

The glass formulations in this series made use of the site soil to provide the necessary glass forming components. We found that the leach resistance, and therefore the tank waste loading, could be further increased by including small amounts (about $5 \mathrm{wt} \%$ ) of additives, as discussed below. A single glass melt (HAN39) was made to demonstrate the difference of using site soil in a MAWS approach over an alternative such as using $\mathrm{SiO}_{2}$ sand. This poorly durable glass composition (see Section 2.4.4) may not be representative of the response of all glasses to $\mathrm{SiO}_{2}$ substitutions, but is intended to indicate how one composition responds at this substitution. 


\subsubsection{Melts to Maximize Soil Loading}

Even if the LLW tank wastes would not be available as a source of flux material for treatment of soils or soil-wash concentrates, vitrification may still be considered as a viable treatment alternative. In this case, a major objective would be to formulate glasses with the lowest possible amount of additives. This strategy was also investigated in this study. A series of scoping melts were prepared using a soil surrogate.

\subsubsection{Surrogate Melts}

A series of glass melts (presented in Table 2.9) were prepared using chemicals instead of actual soil to investigate additive requirements for vitrification in this soil composition range. As with any surrogate, some differences from the actual material are inevitable; in particular, trace constituents and organics (which may affect glass oxidation state) were not incorporated. However, samples of actual Hanford soil contained low concenrations of organics ( $<1 \mathrm{wt} \%$, see Table 2.6$)$ and, furthermore, glasses were also made from the actual soils, as discussed below. The composition of the surrogate soil was based on an average soil composition obtained from the Hanford site, shown in Table 2.2 (column headed "Average Hanford"). The compositions and properties of these glasses (HAN1 to HAN19) are listed in Table 2.12a.

\subsubsection{Melts Using Radioactive Soil}

The radioactive soil HA2 was used for all subsequent tests, with the single exception of HAN20 which used the non-radioactive HA1 soil. HAN23 was a test melt of the radioactive soil alone; even at $1450^{\circ} \mathrm{C}$, this melt was so viscous that it could not be poured from the crucible; consequently, no further analyses were performed on this glass. HAN22 was a melt made using the radioactive soil with $25 \%$ added $\mathrm{Na}_{2} \mathrm{O}$, which corresponds to the surrogate melt HAN6. As described above and as shown in Table 2.2, the radioactive soil sample used for this work was richer in silicon and depleted in iron relative to the soil composition used to prepare the surrogate melts. Thus, we would expect HAN22 and HAN23 to be more viscous than their respective surrogate melts, HAN6 and HAN1, as was found experimentally. HAN22 produced a glass with acceptable viscosity and conductivity for processing below $1500^{\circ} \mathrm{C}$.

Other glasses were melted from the same radioactive soil sample (HA2) with small amounts of additives based upon the results from the surrogate study. Glasses HAN31 to HAN34 were melted with the compositions shown in Table 2.11; HAN31 could not be poured due to its high viscosity. Melt HAN35 used $\mathrm{CaF}_{2}$ as an additive in place of $\mathrm{CaO}$ since $\mathrm{CaF}_{2}$ is more effective in reducing the melt viscosity. 


\subsection{Glass Properties}

\subsubsection{Glass Analysis}

DCP analyses of $\mathrm{HF} / \mathrm{HNO}_{3}$ dissolutions of all of the radioactive glasses are included in Tables 2.10 and 2.11. These dissolutions were done in duplicate or triplicate, and the results showed good consistency with each other. However, the target sodium level was consistently higher than the value measured (for example, $24 \%$ measured level for a target of $29 \% \mathrm{Na}_{2} \mathrm{O}$ in $\mathrm{HAN} 25$ ). The same trend was found for other alkali and alkali-earths, although to a lesser extent since their target concentrations were not as high. The rest of the components, and especially silica, were consequently enriched due to both sodium evaporation and crucible corrosion. The effect of crucible corrosion was avoided for the subsequent glasses (HAN35 - HAN39) by replacing the fireclay crucible with a platinum crucible. The sodium loss in these glasses was reduced, indicating that some of the sodium loss in the earlier melts was probably due to penetration of the LLW simulant into the crucible.

ICPMS analyses of the glass dissolution samples are presented in Table 2.13a; the analytes include ${ }^{99} \mathrm{Tc}$. This element is known to exhibit some volatility in the glass melting process and comparison of the batched and analyzed concentrations permits an evaluation of this effect. The analyzed concentrations in the dissolution sample $(0.1 \mathrm{~g}$ of glass in $200 \mathrm{ml})$ as well as the original concentration in the glass are included in Table 2.13a. The batched concentration of technetium was $10 \mathrm{ppm}$ compared to the measured concentrations in the glasses of between about 0.5 and $3 \mathrm{ppm}$. Table $2.13 \mathrm{~b}$ presents a summary of the correlation between heating time and melt temperature and the retention levels of both ${ }^{99} \mathrm{Tc}$ and sodium. A substantial fraction of the technetium was lost from all of the melts. However, care should be exercised in interpreting these results from a processing perspective since the extent of volatilization depends on the characteristics of the particular process considered.

As discussed above, analyses of the glasses also showed that the loss of sodium during melting was significant, as shown in Table $2.13 \mathrm{~b}$. Similar caveats to those expressed for technetium with respect to extrapolating these results to actual process conditions are appropriate; the extent of volatilization will be highly dependent upon the specific process. In the present study, the variation of the glass properties with sodium content was of interest, and it was therefore necessary to prepare glasses with a range of sodium contents. While practical difficulties arose due to sodium loss, these are essentially a detail since the final glass composition was determined by chemical analysis, and, consequently, the resulting data on the glass properties are no less valuable.

The highest sodium retention value was reached for HAN36, which was melted in a platinum crucible with the melt time reduced to 70 minutes. In the case of glasses HAN29 and HAN30, which 
had increased target sodium levels, the retention decreased to between 60 and $70 \%$ (the target was $35.8 \% \mathrm{Na}_{2} \mathrm{O}$ while the measured did not exceed 26.3\%). In melts HAN36 through HAN39, the total treatment time was decreased to two hours ( 1 hour drying at $250^{\circ} \mathrm{C}$ and 70 minutes at $1200^{\circ} \mathrm{C}$ ). The analyses of these samples show that the retention can be increased to as much as $95 \%$. The glass prepared with the most soda contains $42.7 \%$ sodium oxide based on the analyzed composition, but the highest retention was reached for a lower target of $29 \%$.

Three methods were tested to bring the glasses closer to the targeted contents of $\mathrm{Na}_{2} \mathrm{O}$ :

1) Minimizing the drying and melting time.

2) Compensating for sodium loss by adding an excess to the original formulation. For example, since the sodium oxide level was measured in HAN29 at $25 \mathrm{wt} \%$ for a targeted value of $35 \mathrm{wt} \%$, an extra $10 \mathrm{wt} \%$ is added to the formulation in the next melt.

3) Replacement of the use of fireclay crucibles by platinum-gold.

Approach (2) led to a number of experimental difficulties, however, (intense boiling of the formulation) and the test had to be repeated. In the most successful attempts, glasses with more than $43 \mathrm{wt} \%$ sodium oxide were made by combinations of these methods. However, glasses containing such high levels of $\mathrm{Na}_{2} \mathrm{O}$ often showed obvious signs of reaction with the humidity in the air when left on the bench for a few hours. Such samples were discarded and only glasses HAN29E and HAN29F (sodium oxide at 33.2 and $42.7 \mathrm{wt} \%$, respectively) were characterized further, as discussed below.

ICPMS analyses of the other contaminants after glass dissolution indicated that they were generally retained in the glass. The glasses were prepared with the nominal concentrations of bismuth, lead, and uranium provided by the tank waste, and with cesium, strontium, iodine, and technetium concentrations of $100 \mathrm{ppm}, 100 \mathrm{ppm}, 100 \mathrm{ppm}$, and $10 \mathrm{ppm}$, respectively. Table $2.13 \mathrm{a}$ summarizes the ICPMS analyses of glasses HAN29F, HAN30A, and HAN30B, when $0.1 \mathrm{~g}$ of glass were dissolved in $200 \mathrm{ml}$ of solution and the corresponding concentrations in the original glass. Note that for some analytes, the glass contained more than the amount provided by the tank waste due to the contribution from the soil.

\subsubsection{Viscosity and Conductivity}

Data on melt viscosity and electrical conductivity, both key processing parameters, are summarized in Table 2.12. There is an inevitable loss of sodium during the conductivity and viscosity measurements due to the high temperatures that are required; this causes the viscosity data 
to be slightly higher and the conductivity data to be slightly lower than expected. The procedures for these measurements were therefore modified to minimize the time required to make the measurements in an attempt to decrease the amount of sodium that evaporates. To evaluate the extent of this effect, some glasses were reanalyzed after the conductivity and viscosity measurements were completed. The sodium loss during the measurement was decreased to less than $3 \%$ using this technique. The amount of sodium retained in the glass will significantly affect the melt viscosity; this is evident in the results for five glasses prepared from the formulation HAN29, presented in Figure 2.4. The formulation is $55.1 \%$ soil, $5 \%$ zirconia, and a targeted tank waste of $39.9 \%$, but the sodium level varied between $25.3 \%$ and $42.4 \%$; the higher values were reached by compensating for the evaporation by adding sodium in the preparation. As shown in the figure, the data follow the expected trend according to their respective analyzed sodium contents; the higher the retention, the lower the viscosity.

It is interesting to compare the results for HAN36 with those for HAN39, in which site soil was replaced by $\mathrm{SiO}_{2}$ (both included $5 \mathrm{wt} \%$ added $\mathrm{Al}_{2} \mathrm{O}_{3}$ ). This comparison shows that the site soil produces a melt with significantly lower viscosity for the same tank waste loading. This is probably due to the additional fluxes that are provided by the soil (the alkali and alkaline earths and, to a lesser extent, iron). The glass prepared with soil also shows better leach resistance in the PCT and TCLP (Sections 2.4.3 and 2.4.4).

The electrical conductivity of the melt is of considerable importance with a joule-heated melter technology. The results in Table $2.12 \mathrm{~b}$ show that these glasses have high electrical conductivities due to their high sodium contents, since sodium ions are the primary current carriers in these glasses. High-level waste melters have typically used an upper limit of $0.55 \mathrm{~S} / \mathrm{cm}$, which would restrict the tank waste loading if applied here. However, melts of considerably higher conductivities have been processed at Catholic University (Hojaji-1995).

The systematic composition variations, made in the series of surrogate glasses, permit a simple illustration of the interaction of the composition dependence of glass properties and the constraints imposed by any given vitrification process in determining the waste loadings that are achievable and the acceptable range of operating temperatures. For the purpose of this illustration, we will consider a process that requires a melt viscosity of below 100 Poise and an electrical conductivity of below $1 \mathrm{~S} / \mathrm{cm}$ at the processing temperature. Based on data from the surrogate melts, we can determine the temperature at which each of these constraints is encountered as a function of the amount of added sodium oxide, as shown in Figure 2.5. Increasing the sodium oxide content reduces both the 100 Poise temperature and the $1 \mathrm{~S} / \mathrm{cm}$ temperature, but at different rates. The region between these two curves represents the combinations of sodium oxide contents (or conversely, waste loadings) and processing temperatures that will satisfy these constraints. Thus, for vitrification of the soils, higher waste loading and larger operating ranges are possible for higher processing 
temperatures. Other factors, such as leach resistance, liquidus temperature, volatilization of contaminants, and melter lifetime, must also be considered in such an analysis. Furthermore, this represents the effect of only one composition variation (i.e., with respect to sodium oxide), while many others are possible, some of which may lead to more acceptable solutions. Reliable correlations that represent the relationship between glass properties and glass composition can be useful in this respect; this subject is addressed in Section 5.

\subsubsection{Glass Leach Testing - TCLP}

The TCLP was performed on most of the radioactive Hanford glasses. The results for the RCRA metals are shown in Table 2.14 and show that all of the glasses pass. In particular, ${ }^{99} \mathrm{Tc}$, which is often the limiting radionuclide in performance assessment scenarios, shows low levels (Table 2.15): Between 0.26 and $1.2 \mathrm{ppb}$ which is below the drinking water level given in DOE 5400.5 of $5.9 \mathrm{ppb}$ (Figure 2.6). Note that the technetium analyses of the TCLP leachates show excellent reproducibility and the spikes were reliably recovered down to the lowest concentration attempted (2 ppt). Since calcium is one of the major glass constituents present at a comparable concentration in all of the glasses (2.5 to 3\%), it can be used for intercomparison of the effects of the various additives tested. Figure 2.7 shows how the addition of components such as alumina, silica, and zirconia can improve the leach resistance of the glass matrix, as monitored by the amount of calcium in the leachate.

In the course of developing methods to improve the retention of sodium during glass preparation, samples were taken at various stages of the melting/fining process. For example, HAN29B1 was melted at $1200^{\circ} \mathrm{C}$ for 70 minutes and HAN29B2 was melted in the same way but with an additional 30 minutes at $1300^{\circ} \mathrm{C}$. The results of the DCP analysis indicate that HAN29B2 contained $2 \%$ less sodium oxide; that glass was found to leach four timies less uranium in the TCLP than did HAN29B1 (Table 2.14).

\subsubsection{Glass Leach Testing - PCT}

The Product Consistency Test (PCT), was also used to evaluate the leach resistance of Hanford glasses. The results obtained after the nominal 7-day leaching period are compared with those for the Savannah River Environmental Assessment Glass (SRL-EA) in Table 2.16. (Note: Each glass was tested in triplicate with two blanks and triplicates of a West Valley Reference glass as an internal standard. Values obtained from previous tests were used for the SRL-EA glass; these agree well with results from other laboratories (Pegg-1994c).) Leachate concentrations normalized to the glass composition (expressed in grams of element leached per liter of solution) and leach rate (in 
grams per square meter of glass exposed per day over the 7-day test duration) are also shown. The boron leach rate, which could be calculated only for HAN26, is 20 times lower than that of SRL-EA. Sodium leach rates are also below the SRL-EA value by factors of two to fifteen. Silicon release shows similar trends. These results are summarized in Figure 2.8.

In general, the PCT performance is closely related to the sodium content of the glasses. As an illustration of this, the sodium concentrations in the leachates were converted to the corresponding minimum amount of glass that must have dissolved to reach the analyzed leachate concentration of sodium; the results are shown in Table 2.17 where the glasses have been ordered according to their respective sodium contents. These results for the 7-day data are also plotted in Figure 2.9. While the general trend of increasing glass dissolution with increasing sodium content is evident, it is also apparent that PCT durable glasses with high sodium contents can be made, certainly in comparison to the SRL-EA glass. It is interesting to note the drastic difference between HAN39 and the other glasses with similar sodium contents. The former used a mixture of $\mathrm{SiO}_{2}$ and $\mathrm{Al}_{2} \mathrm{O}_{3}$ as the source of glass formers, while the latter glasses used Hanford soil with small amounts of additives. These results demonstrate the wide range of variation that is possible even at fixed sodium content.

The long-term PCT data (up to 1 year) for both silicon (Figures 2.10 and 2.11) and sodium (Figures 2.12 and 2.13) show some interesting effects of small changes (a few wt\%) in the glass composition. The leach rates of these glasses appear to stabilize after the 7-day sampling. However, after a period of time, which depends on the glass composition, the leach rates are observed to show dramatic increases. This increase occurs earliest for the glass without additives and progressively later for additives of $\mathrm{SiO}_{2}, \mathrm{~B}_{2} \mathrm{O}_{3}, \mathrm{Al}_{2} \mathrm{O}_{3}$, and $\mathrm{ZrO}_{2}$ (for which no increase was observed over the time frame of the measurements). This phenomenon, of a sudden increase in the leach rate, has been observed previously for certain glasses in PCT testing (Adel-Hadadi-1988) and also by other researchers (Patyn-1990). There is reason to believe that the general behavior is related to the onset of secondary phase formation, which can remove species from solution and thereby maintain a nonzero reaction affinity (Bourcier-1990, Patyn-1990). It appears that the slightly lower waste loading coupled with the addition of small amounts of other additives $\left(5 \% \mathrm{ZrO}_{2}, \mathrm{Al}_{2} \mathrm{O}_{3}\right.$, or $\left.\mathrm{B}_{2} \mathrm{O}_{3}\right)$ has a stabilizing effect on the long-term behavior and suppresses, or at least delays, the rise in the leach rate. This too is in agreement with previous findings for West Valley glasses (Adel-Hadadi-1988, Feng-1989, Muller-1992, Xing-1994a, Xing-1994b).

In Figure 2.14, the analyzed sodium oxide content is included as a label above each bar graph for each glass. As both Figures 2.14 and 2.15 show, when soil is used as the source of glass formers (HAN29F, HAN36, HAN37, HAN38), all but the glass with the highest sodium content (corresponding to about $40 \%$ tank waste) are better than or comparable to SRL-EA glass on the 7day PCT. If, however, pure silica is used in place of the soil, the glass (HAN39) performs poorly (note that although the tank waste loading is nominally $31 \%$, due to the sodium content of the soil, 
this glass actually contains less sodium than glasses HAN36-38). The target glass composition is one of the least durable glasses. It is unclear if this observation persists for other Hanford glass compositions. Comparison of the compositions shows that the soil glasses have higher concentrations of $\mathrm{Al}_{2} \mathrm{O}_{3}, \mathrm{CaO}, \mathrm{Fe}_{2} \mathrm{O}_{3}, \mathrm{~K}_{2} \mathrm{O}, \mathrm{MgO}$, and $\mathrm{TiO}_{2}$. These increases would be expected (with the possible exception of $\mathrm{K}_{2} \mathrm{O}$ ) to improve the leach resistance; as examples, it is well known that soda-lime-silicates and calcium aluminosilicate glasses are more leach resistant than the corresponding sodium silicate glasses (Paul-1990). Thus, the composition of the soil happens to be closer to the optimum combination of additives (from a leach resistance perspective) than is pure silica for this particular glass composition. It is possible that there is some effect of the oxidation state, although this seems unlikely since the Hanford soils contain low concentrations $(<1 \mathrm{wt} \%)$ of organic materials (see Table 2.6), and all of the glasses were melted in air, which from previous experience produces oxidized glasses in the presence of low concentrations of organic materials. The difference is therefore most likely a result of the oxide composition of the soil.

\subsubsection{Microstructure of Hanford Glasses}

Scanning electron microscopy coupled with energy dispersive $\mathrm{x}$-ray spectroscopy (SEMEDS) was used to examine the as-melted glasses, HAN24, HAN25, HAN26, and HAN28. Small amounts of undissolved material were found in all four, although there was very little in HAN24 (no additive) and in $\mathrm{HAN} 26\left(5 \% \mathrm{~B}_{2} \mathrm{O}_{3}\right.$ additive).

SEM analyses of as-melted HAN28 found areas of undissolved $\mathrm{ZrO}_{2}$. Three were found in the prepared surface and were about $20-30 \mu \mathrm{m}$ in diameter. Grains of zirconia were surrounded by irregular regions of a zirconia-silica glass phase. There was also a region in this sample, about 50 x $200 \mu \mathrm{m}$, that exhibited a different hardness than the surrounding area as indicated by the relief caused by the polishing operation. The composition was similar to the surrounding glass, but slightly elevated in $\mathrm{Si}$ and $\mathrm{Al}$, and possibly in $\mathrm{Na}$ and $\mathrm{Zr}$, suggesting that the material was crystalline. These results suggest that the $5 \mathrm{wt} \%$ addition of $\mathrm{ZrO}_{2}$, which was effective in improving the leach resistance, may not be completely soluble in this melt, although the effect could be entirely kinetic given the relatively short melt duration.

As-melted HAN24 showed only a small anomalous region, $\sim 25 \times 50 \mu \mathrm{m}$, that was slightly enriched in $\mathrm{Fe}$ relative to the surrounding glass and had a smooth boundary without the faceting characteristic of a crystalline material.

All four glasses were heat treated for two hours at $1050^{\circ} \mathrm{C}$, after a 1 hour pre-melt at $1200^{\circ} \mathrm{C}$, in order to determine a bound on the liquidus temperature. The only feature visible by SEM on the polished surfaces of the heat treated glasses was in HAN25 where a region was found containing 
undissolved alumina; the quantity of this material $(<0.1 \mathrm{vol} \%)$ is inconsequential for many processing technologies. The region was about $50 \mu \mathrm{m}$ diameter and was subdivided into thin platelet-shaped crystals of alumina, high-alumina glass and possibly crystalline alumina-silica regions. The lack of any significant devitrification, even in this rather short heat treatment, suggests that the liquidus temperature should not be a processing concern for these glasses.

During preparation of a sample of HAN29E (42.7 wt\% $\mathrm{Na}_{2} \mathrm{O}$ ) for microstructural examination by SEM, the sample apparently absorbed water due to the high $\mathrm{Na}_{2} \mathrm{O}$ content. After polishing and carbon coating under vacuum, the loss of absorbed water caused severe cracking of the surface as shown in Figure 2.16. This glass also produced high leachate concentrations under PCT conditions (Table 2.16).

Several of the surrogate glasses were examined to determine the phase stability and dependence of liquidus temperature on composition for these formulations. Samples of these glasses were heat treated and examined in the SEM-EDS to tentatively identify and quantify any crystal phases present. Two series of glasses were studied; one in which successive increments were made to the sodium content of the base soil composition, and the other in which increments were made to the calcium content (Table 2.11a). A summary of results for the sodium series is presented in Table 2.18.

Crystallization of augite $\left((\mathrm{Ca}, \mathrm{Na})(\mathrm{Mg}, \mathrm{Fe}, \mathrm{Al}, \mathrm{Ti})(\mathrm{Si}, \mathrm{Al})_{2} \mathrm{O}_{6}\right)$ on the order of $10-15$ vol\% was found (Figure 2.17) in glasses containing less than about $12 \mathrm{wt} \%$ soda which were heat treated for 18 hours at $1100^{\circ} \mathrm{C}$. The crystallization of augite reduced the $\mathrm{MgO}$ content of the remaining glass (initially about $3 \mathrm{wt} \%$ ) by about $50 \%$. The particular augite composition observed was rich in calcium, magnesium, and silicon and the average composition in the two glasses in which it appeared are shown in Table 2.19. The identity of this phase was supported by $\mathrm{x}$-ray diffraction data (Figure 2.18). At $15 \mathrm{wt} \% \mathrm{Na}_{2} \mathrm{O}$ and above, no augite crystallized in samples that were heat treated for 24 hours at $1100^{\circ} \mathrm{C}$.

At $\mathrm{Na}_{2} \mathrm{O}$ levels of about $25 \mathrm{wt} \%$ or greater and lower heat treatment temperatures $\left(900^{\circ} \mathrm{C}\right.$ for 2 hours), an aluminosilicate (identity uncertain but having a diffraction pattern similar to $0.33\left(\mathrm{Na}_{2} \mathrm{O}\right) \mathrm{NaAlSiO}_{4}$ and a cristobalite-like structure) crystallizes with large-scale dendritic morphology (Figures 2.19 and 2.20). The quantity of this phase appears to increase with soda content of the glass; its composition varies with that of the parent glass and with conditions during crystallization (see Table 2.20). Note that the liquidus of this phase increases with increasing soda content. In some composition ranges, this phase exhibits considerable sensitivity to water. In fact, the two additional "phases" listed in Table 2.18 (III and IV) may simply be the remains of a water soluble phase that dissolved during polishing; close examination of the microstructure of polished specimens suggested such dissolution (Figures 2.21 and 2.22). The aluminosilicate phase contains 
magnesium and depletes the remaining glass of $\mathrm{Mg}$ but not as severely as does augite. The high soda ( $25 \mathrm{wt} \% \mathrm{Na}_{2} \mathrm{O}$ and above) glasses are also noticeably more subject to beam damage in the SEM, probably because of reduction and loss of $\mathrm{Na}$, or possibly because they have absorbed water and are dehydrated by the beam.

The crystallization behavior in the calcium series is summarized in Figure 2.23. Only augite formed (Figure 2.24) until the $\mathrm{CaO}$ content reached about $20 \mathrm{wt} \%$ at which point wollastonite $\left(\mathrm{CaSiO}_{3}\right)$ also formed (Figure 2.25). The identities of both phases were confirmed by x-ray diffraction (Figures 2.26 and 2.27). Increasing the calcia content did not significantly alter the volume fraction of augite (10-15 vol\%), but did shift the composition of the augite to higher $\mathrm{Ca}, \mathrm{Mg}$, and $\mathrm{Si}$ and lower $\mathrm{Al}, \mathrm{Fe}$, and $\mathrm{Na}$ (see Table 2.21). Once wollastonite crystallized, the silicon, magnesium, and calcium contents of the augite decreased with compensating increases in iron, sodium, and aluminum (see Table 2.21).

\subsection{Summary and Conclusions}

Samples of contaminated Hanford soils have been characterized and vitrified in combination with a simulant of the Hanford low-level tank waste. The resulting glasses have been subjected to characterization with respect to melt viscosity and electrical conductivity, microstructure, and TCLP and PCT leach resistance. Two groups of glasses were produced; those targeted toward maximized tank waste loadings and those targeted toward maximized soil loadings.

The data collected in this study show that glasses with high waste loadings (up to about 31 wt\% tank waste oxides) can be made with good chemical durability by using site soil as the major ingredient. With small amounts (around $5 \mathrm{wt} \%$ ) of additives, such as $\mathrm{ZrO}_{2}$, good leach resistance can be achieved for a waste form that is composed of $95 \%$ waste (on an oxide basis). A single comparison has been made which shows that replacement of the site soil ingredient by $\mathrm{SiO}_{2}$ sand results in a glass of poorer durability. Realistically achievable tank waste loadings with the use of soil appear to be as high as $31 \%$ on an oxide basis using the presently existing TCLP and PCT specifications.

Other data obtained from this study show that the treatment of contaminated soils alone may require higher temperature vitrification processes. Very small amounts $(<10 \mathrm{wt} \%)$ of additives would be required to vitrify the site soils using processes operating in the $1400-1500^{\circ} \mathrm{C}$ range.

The Hanford glasses that have been subjected to viscosity, conductivity, TCLP, and PCT testing (i.e., those glasses in the core data set of 40 glasses, as discussed in Section 1.3) are listed in Table 2.22. For convenience, Table 2.22 also lists representative data for each characteristic, 
consisting of the viscosity and conductivity values at $1250^{\circ} \mathrm{C}$, the uranium concentrations in the TCLP leachates, and the sodium concentration in the PCT leachates after seven days. 
Table 2.1

Summary of DCP Analysis of the non Radioactive Soil Received from Hanford

\begin{tabular}{|c|c|c|c|c|c|}
\hline \multirow[b]{2}{*}{$\begin{array}{c}\text { Oxide } \\
\text { wt\% }\end{array}$} & \multirow{2}{*}{$\begin{array}{l}\text { Average } \\
\text { on HA1* }\end{array}$} & \multirow{2}{*}{ Std. Dev. } & \multicolumn{3}{|c|}{ Range for Hanford Site Data ${ }^{(1)}$} \\
\hline & & & Min. & Max. & $\begin{array}{l}\text { Average } \\
\text { Hanford }\end{array}$ \\
\hline $\mathrm{Al}_{2} \mathrm{O}_{3}$ & 14.45 & 0.22 & 12.50 & 14.40 & 13.45 \\
\hline $\mathrm{B}_{2} \mathrm{O}_{3}$ & 0.20 & 0.13 & & & \\
\hline $\mathrm{BaO}$ & 0.09 & 0.01 & & & \\
\hline $\mathrm{CaO}$ & 4.85 & 0.07 & 5.37 & 6.81 & 6.09 \\
\hline $\mathrm{Fe}_{2} \mathrm{O}_{3}$ & 7.52 & 0.14 & 8.16 & 11.50 & 9.83 \\
\hline $\mathrm{K}_{2} \mathrm{O}$ & 1.99 & 0.06 & 1.43 & 2.45 & 1.94 \\
\hline $\mathrm{MgO}$ & 2.04 & 0.03 & 1.41 & 3.40 & 2.41 \\
\hline $\mathrm{MnO}_{2}$ & 0.20 & 0.01 & & & \\
\hline $\mathrm{Na}_{2} \mathrm{O}$ & 2.66 & 0.05 & 2.69 & 3.23 & 2.96 \\
\hline $\mathrm{P}_{2} \mathrm{O}_{5}$ & 0.46 & 0.02 & & & \\
\hline $\mathrm{SiO}_{2}$ & 63.99 & 0.46 & 58.00 & 64.40 & 61.20 \\
\hline $\mathrm{SrO}$ & 0.05 & 0.00 & & & \\
\hline $\mathrm{TiO}_{2}$ & 1.40 & 0.04 & & & \\
\hline $\mathrm{U}_{3} \mathrm{O}_{8}$ & 0.05 & 0.01 & & & \\
\hline Total & 100.00 & & & & 97.88 \\
\hline
\end{tabular}

This average is calculated from triplicate samplings of the soil container and triplicate microwave dissolution of each sample (i.e., a total of nine analyses).

(1) (Ludowise-1993). 
Table 2.2

Summary of Results of Analysis of Hanford Radioactive Soil

\begin{tabular}{||c|c|c|c|c|c|c||}
\hline $\begin{array}{c}\text { Oxide } \\
\text { wt\% }\end{array}$ & $\begin{array}{c}\text { Average } \\
\text { on HA2 }\end{array}$ & $\begin{array}{c}\text { Average } \\
\text { on HA3 }\end{array}$ & $\begin{array}{c}\text { Average } \\
\text { on HA4 }\end{array}$ & $\begin{array}{c}\text { Average } \\
\text { Soil }\end{array}$ & $\begin{array}{c}\text { Maximum } \\
\text { Std. Dev.* }\end{array}$ & $\begin{array}{c}\text { Average } \\
\text { Hanford }\end{array}$ \\
\hline $\mathrm{Al}_{2} \mathrm{O}_{3}$ & 12.76 & 12.31 & 12.95 & 12.67 & 0.56 & 13.45 \\
\hline $\mathrm{BaO}$ & 0.12 & 0.11 & 0.11 & 0.11 & 0.00 & \\
\hline $\mathrm{CaO}$ & 4.80 & 3.64 & 3.65 & 4.03 & 0.16 & 6.09 \\
\hline $\mathrm{Fe}_{2} \mathrm{O}_{3}$ & 4.29 & 3.83 & 3.67 & 3.93 & 0.16 & 9.83 \\
\hline $\mathrm{K}_{2} \mathrm{O}$ & 2.41 & 2.31 & 2.30 & 2.34 & 0.12 & 1.94 \\
\hline $\mathrm{MgO}^{*}$ & 2.80 & 1.74 & 0.96 & 1.83 & 0.68 & 2.41 \\
\hline $\mathrm{MnO}_{2}$ & 0.30 & 0.15 & 0.14 & 0.20 & 0.01 & \\
\hline $\mathrm{Na}_{2} \mathrm{O}$ & 4.36 & 3.37 & 3.29 & 3.67 & 0.18 & 2.96 \\
\hline $\mathrm{P}_{2} \mathrm{O}_{5}$ & 0.48 & 0.30 & 0.27 & 0.35 & 0.02 & \\
\hline $\mathrm{SiO}_{2}$ & 64.75 & 70.95 & 71.81 & 69.17 & 1.33 & 61.20 \\
\hline $\mathrm{SrO}$ & 0.05 & 0.05 & 0.05 & 0.05 & 0.00 & \\
\hline $\mathrm{TiO}_{2}$ & 0.58 & 0.59 & 0.60 & 0.59 & 0.02 & \\
\hline $\mathrm{U}_{3} \mathrm{O}_{8}$ & 0.13 & 0.04 & 0.03 & 0.07 & 0.01 & \\
\hline $\mathrm{Sum}$ & 97.83 & 99.39 & 99.83 & 99.02 & & 97.88 \\
\hline
\end{tabular}

"This is the composition (after renormalization to 100\%) used to calculate the target compositions of the glasses. "Each of these three samples was submitted for DCP analysis after triplicate microwave dissolution of each of two samples, one dried at $450^{\circ} \mathrm{C}$ and the other dried at $1150^{\circ} \mathrm{C}$. The standard deviation is calculated from all 18 analyses. 
Table 2.3

Results of ICP-MS Analysis of Hanford Radioactive Soil Samples (ppm)

\begin{tabular}{|l|c|c|c||}
\hline & HA2D450H & HA3D450H & HA4D50H \\
\hline $\mathrm{U}-238$ & 4.4 & 8.8 & 4.3 \\
\hline $\mathrm{Cu}$ & 29 & 33 & 32 \\
\hline $\mathrm{Zn}$ & 37 & 59 & 51 \\
\hline $\mathrm{Pb}$ & 44 & 30 & 21 \\
\hline $\mathrm{Ni}$ & 304 & 351 & 323 \\
\hline $\mathrm{Mn}$ & 649 & 758 & 723 \\
\hline
\end{tabular}

The contents of $\mathrm{Ag}, \mathrm{Cd}, \mathrm{Ra}$, and $\mathrm{Pu}-239$ are each less than $1 \mathrm{ppb}$.

The contents of $\mathrm{Hg}$ and As are below $3.5 \mathrm{ppb}$.

The content of Se is below $10 \mathrm{ppb}$.

Note: $\quad \mathrm{Ni}-58$ has possible interference from $\mathrm{Fe}, \mathrm{Si}, \mathrm{CaO}, \mathrm{KOH}$ and the cones.

$\mathrm{Cu}-63$ has possible interference from $\mathrm{TiO}, \mathrm{ArNa}$ and $\mathrm{PO}_{2}$.

$\mathrm{Zn}-68$ has possible interference from $\mathrm{Ba}^{++}, \mathrm{Ce}^{++}$, and $\mathrm{SiF}$.

Therefore, the true contents of $\mathrm{Ni}, \mathrm{Cu}$ and $\mathrm{Zn}$ could be lower than their corresponding given values here. 
Table 2.4

Results of Physical Characterization of Hanford Soils

\begin{tabular}{|c|c|c|c|}
\hline \multicolumn{4}{|l|}{ Thermogravimetric Analysis (VSL Procedure) } \\
\hline & HA2 & HA3 & HA4 \\
\hline $\begin{array}{r}\text { Wt. Loss@ } 110^{\circ} \mathrm{C}, \% \\
@ 450^{\circ} \mathrm{C}, \% \\
@ 850^{\circ} \mathrm{C}, \% \\
@ 1150^{\circ} \mathrm{C}, \%\end{array}$ & $\begin{array}{l}4.3 \\
7.1 \\
7.4 \\
7.6\end{array}$ & $\begin{array}{l}6.1 \\
7.7 \\
7.9 \\
8.1\end{array}$ & $\begin{array}{l}6.5 \\
7.5 \\
8.0 \\
8.2\end{array}$ \\
\hline \multicolumn{4}{|l|}{ Specific Gravity (ASTM D854-83), $\mathrm{g} / \mathrm{cm}^{3}$} \\
\hline & HA2 & HA3 & HA4 \\
\hline $\begin{array}{r}\text { As Received } \\
\text { Dried @ } 110^{\circ} \mathrm{C}\end{array}$ & $\begin{array}{l}2.39 \\
2.52\end{array}$ & $\begin{array}{l}2.44 \\
2.64\end{array}$ & $\begin{array}{l}2.45 \\
2.67\end{array}$ \\
\hline \multicolumn{4}{|l|}{ Particle Size Distribution } \\
\hline Coarse material, size of particles $>2 \mathrm{~mm}$ & $0.65 \%$ & $0.44 \%$ & $0.30 \%$ \\
\hline $0.425 \mathrm{~mm}<$ size of particles $<2 \mathrm{~mm}$ & $29.97 \%$ & $34.23 \%$ & $30.08 \%$ \\
\hline $0.25 \mathrm{~mm}<$ size of particles $<0.425 \mathrm{~mm}$ & $40.37 \%$ & $31.47 \%$ & $37.41 \%$ \\
\hline $0.15 \mathrm{~mm}<$ size of particles $<0.25 \mathrm{~mm}$ & $10.55 \%$ & $10.94 \%$ & $11.99 \%$ \\
\hline $0.075 \mathrm{~mm}<$ size of particles $<0.15 \mathrm{~mm}$ & $7.46 \%$ & $11.04 \%$ & $11.25 \%$ \\
\hline size of particles $<0.075 \mathrm{~mm}$ & $11.00 \%$ & $8.54 \%$ & $8.97 \%$ \\
\hline TOTAL & $100.00 \%$ & $100.00 \%$ & $100.00 \%$ \\
\hline
\end{tabular}


Table 2.5

Results of Total Carbon (TC) Content Analyses of Hanford Soils

\begin{tabular}{|c|c|c|c|c|}
\hline & & HA2 & HA3 & HA4 \\
\hline (1) & Mass taken for TC analysis, $\mathrm{g}$ & 1.03 & 1.08 & 0.52 \\
\hline (2) & DI (deionized) water added, $\mathrm{g}$ & 5.00 & 10.00 & 2.00 \\
\hline (3) & Dilution factor, $(1)+(2) /(1)$ & 5.87 & 10.26 & 4.86 \\
\hline \multicolumn{5}{|c|}{ Measurements of TC } \\
\hline (4) & Sample \#1, ppm & 1770 & 975.7 & 1983 \\
\hline (5) & Sample \#2, ppm & 1917 & 940 & 1799 \\
\hline (6) & Sample \#3, ppm & 1683 & 879.9 & 2061 \\
\hline$(7)$ & Sample \#4, ppm & 1837 & 1072 & 2074 \\
\hline (8) & Sample \#5, ppm & 1706 & 876.6 & 2115 \\
\hline (9) & Sample \#6, ppm & 1728 & 929.1 & 1667 \\
\hline$(10)$ & Sample \#7, ppm & & 863.9 & 1870 \\
\hline (11) & Sample \#8, ppm & & & 2198 \\
\hline (12) & Average value (for solution), ppm & 1773.5 & 933.9 & 1970.9 \\
\hline (13) & Standard deviation (for solution), ppm & 88.83 & 73.01 & 178.61 \\
\hline (14) & Relative standard deviation & $5.01 \%$ & $7.82 \%$ & $9.06 \%$ \\
\hline \multicolumn{5}{|c|}{ Results } \\
\hline$(15)$ & Average value (for soil), ppm & 10416 & 9581 & 9580 \\
\hline$(16)$ & Standard deviation (for soil), ppm & 522 & 749 & 868 \\
\hline (17) & Relative standard deviation & $5.01 \%$ & $7.82 \%$ & $9.06 \%$ \\
\hline
\end{tabular}


Table 2.6

Results of Total Organic Carbon (TOC) Analyses of Hanford Soils

\begin{tabular}{|c|c|c|c|c|}
\hline & & HA2 & HA3 & HA4 \\
\hline (1) & Mass taken for TOC analysis, $g$ & 0.33 & 1.53 & 2.50 \\
\hline (2) & DI (deionized) water added, $\mathrm{g}$ & 2.00 & 4.00 & 5.00 \\
\hline (3) & Dilution factor, $(1)+(2) /(1)$ & 7.02 & 3.62 & 3.00 \\
\hline \multicolumn{5}{|c|}{ Measurements of TOC } \\
\hline (4) & Sample \#1, ppm & 862.2 & 2478 & 2014 \\
\hline (5) & Sample \#2, ppm & 893.7 & 2341 & 1794 \\
\hline (6) & Sample \#3, ppm & 864.5 & 2271 & 1917 \\
\hline (7) & Sample \#4, ppm & 902.6 & 2363 & 1917 \\
\hline (8) & Sample \#5, ppm & 844.4 & 2465 & 1999 \\
\hline (9) & Sample \#6, ppm & 825.6 & 2523 & 2015 \\
\hline$(10)$ & Sample \#7, ppm & 896 & 2389 & 1994 \\
\hline (11) & Sample \#8, ppm & & 2245 & 2149 \\
\hline (12) & Average value (for solution), ppm & 869.9 & 2384.4 & 1996.1 \\
\hline (13) & Standard deviation (for solution), ppm & 28.92 & 99.37 & 106.52 \\
\hline (14) & Relative standard deviation & $3.32 \%$ & $4.17 \%$ & $5.34 \%$ \\
\hline \multicolumn{5}{|c|}{ Results } \\
\hline (15) & Average value (for soil), ppm & 6110 & 8630 & 5982 \\
\hline (16) & Standard deviation (for soil), ppm & 203 & 360 & 319 \\
\hline (17) & Relative standard deviation & $3.32 \%$ & $4.17 \%$ & $5.34 \%$ \\
\hline
\end{tabular}

Page 2-22 
Table 2.7

Results of Gamma Counting Analyses of Hanford Soil Samples (in nCi/g)

\begin{tabular}{|c|c|c|c|}
\hline Soil I.D. & HA2 & . $\mathrm{HA3}$ & HA4 \\
\hline $\begin{array}{l}75 \mu \mathrm{m} \text { to } \\
150 \mu \mathrm{m}\end{array}$ & $\begin{array}{l}\text { Cs }-137=3.15 \\
\text { Co- }-57=0.02\end{array}$ & $\begin{array}{l}\text { Cs- } 137=0.77 \\
\text { Co- } 57=0.003\end{array}$ & $\begin{array}{l}\text { Cs }-137=0.76 \\
\text { Co- } 57=0.004\end{array}$ \\
\hline $\begin{array}{l}150 \mu \mathrm{m} \text { to } \\
250 \mu \mathrm{m}\end{array}$ & $\begin{array}{l}\text { Cs }-137=2.3 \\
\text { Co- } 57=0.01\end{array}$ & $\begin{array}{c}\text { Cs- } 137=0.39 \\
\text { Co- } 57<\text { LLD }=0.002\end{array}$ & $\begin{array}{c}\text { Cs-137 }=0.46 \\
\text { Co- } 57<\text { LLD }=0.002\end{array}$ \\
\hline $\begin{array}{l}250 \mu \mathrm{m} \text { to } \\
425 \mu \mathrm{m}\end{array}$ & $\begin{array}{c}\text { Cs- } 137=1.22 \\
\text { Co-57 }<\text { LLD }=0.002\end{array}$ & $\begin{array}{c}\text { Cs- } 137=0.33 \\
\text { C0-57 }<\text { LLD }=0.02\end{array}$ & $\begin{array}{c}\text { Cs- } 137=0.36 \\
\text { Cd- } 57<\text { LLD }=0.002\end{array}$ \\
\hline $\begin{array}{c}425 \mu \mathrm{m} \text { to } \\
2 \mathrm{~mm}\end{array}$ & $\begin{array}{c}\text { Cs-137 = } 1.01 \\
\text { Co-57 }<\text { LLD }=0.002\end{array}$ & $\begin{array}{c}\text { Cs-137 }=0.4 \\
\text { Co-57 }<\text { LLD }=0.002\end{array}$ & $\begin{array}{c}\text { Cs-137 }=0.43 \\
\text { Cd- } 57<\text { LLD }=0.002\end{array}$ \\
\hline
\end{tabular}


Table 2.8a

Nominal Low-Level Tank Feed Stream (Total Weight in Kg) Used in this Study ${ }^{(1)}$

\begin{tabular}{|c|c|c|c|}
\hline & Liquid & Solid & Source used in surrogate \\
\hline 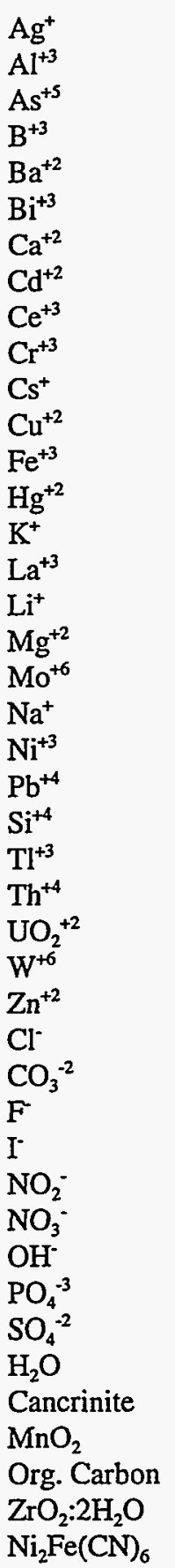 & $\begin{array}{r}3901720.00 \\
\\
14.0 \\
299.0 \\
64100.0 \\
10800.0 \\
2190.0 \\
2340.0 \\
116000.0 \\
8.3 \\
209.0 \\
7370.0 \\
1160.0 \\
1400000.0 \\
278.0 \\
4.3 \\
524.0 \\
2760.0 \\
71700000.0 \\
\\
2610.0 \\
11000.0 \\
\\
132.0 \\
85100.0 \\
3270.0 \\
1930.0 \\
484000.0 \\
1410000.0 \\
1100000.0 \\
219.0 \\
10400000.0 \\
114000000.0 \\
19200000.0 \\
8260000.0 \\
1880000.0 \\
547000000.0 \\
\\
21400.0 \\
772000.0 \\
23400.0\end{array}$ & $\begin{array}{r}0.6 \\
1720.0 \\
2.1 \\
9.8 \\
4.8 \\
1300.0 \\
905.0 \\
14.9 \\
1550.0 \\
211.0 \\
0.8 \\
3.5 \\
4730.0 \\
0.1 \\
91.4 \\
207.0 \\
0.0 \\
20.8 \\
33.6 \\
4360.0 \\
40.4 \\
3.4 \\
81.4 \\
5.6 \\
85.7 \\
9540.0 \\
\\
8.1 \\
13.9 \\
1100.0 \\
370.0 \\
370.0 \\
401.0 \\
6720.0 \\
11300.0 \\
1290.0 \\
256.0 \\
\\
14900.0 \\
1240.0 \\
\end{array}$ & 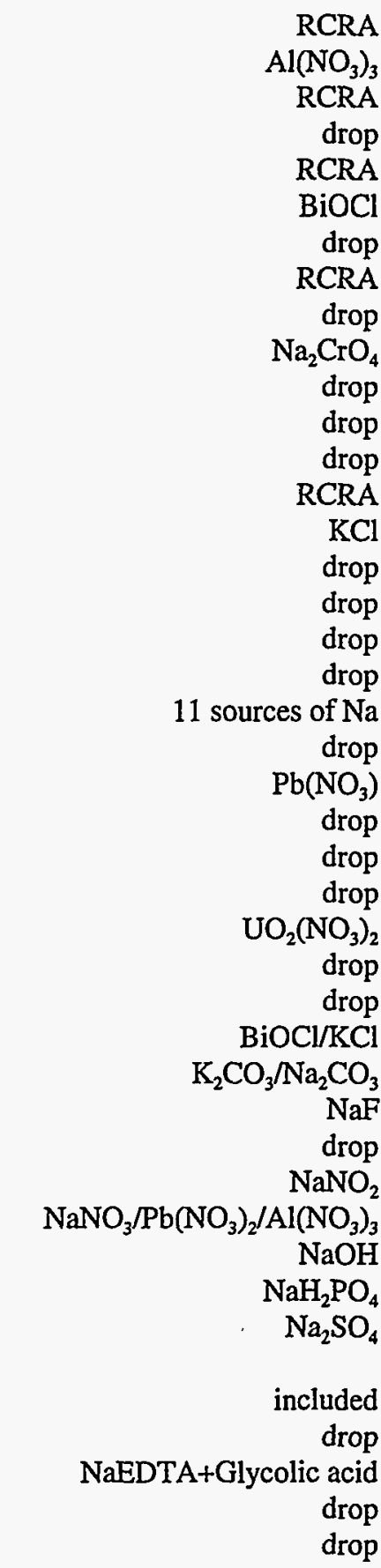 \\
\hline Sum (kg) & 781864838 & 66161 & \\
\hline
\end{tabular}

(1) (Boomer-1993)

RCRA $=$ RCRA element spike solution. 
Table 2.8b

- Composition of the Low-Level Waste Simulant Expressed on an Oxide Basis (calculated from (Boomer-1993))

\begin{tabular}{||l|c||}
\hline \multicolumn{1}{|c|}{ Oxides } & $w t \%$ \\
\hline $\mathrm{Al}_{2} \mathrm{O}_{3}$ & 6.39 \\
$\mathrm{BiO}$ & 0.06 \\
$\mathrm{Cr}_{2} \mathrm{O}_{3}$ & 0.15 \\
$\mathrm{~K}_{2} \mathrm{O}$ & 1.47 \\
$\mathrm{Na}_{2} \mathrm{O}$ & 83.63 \\
$\mathrm{PbO}$ & 0.0004 \\
$\mathrm{U}_{3} \mathrm{O}_{8}$ & 0.25 \\
$\mathrm{Cl}-$ & 0.42 \\
$\mathrm{~F}-$ & 0.95 \\
$\mathrm{P}_{2} \mathrm{O}_{5}$ & 5.34 \\
$\mathrm{SO}{ }_{3}$ & 1.36 \\
\hline & ppm \\
\hline $\mathrm{U}-238$ & 800 \\
$\mathrm{Tc}-99$ & $\sim 30^{*}$ \\
$\mathrm{Cs}$ (nat.) & $\sim 300^{*}$ \\
$\mathrm{Sr}$ (nat.) & $\sim 300^{*}$ \\
$\mathrm{I}$ (nat.) & $\sim 300^{*}$ \\
\hline
\end{tabular}

"These were adjusted to nominal levels of $10 \mathrm{ppm}, 100 \mathrm{ppm}, 100 \mathrm{ppm}$, and $100 \mathrm{ppm}$, respectively, in the resultant glasses. 
Table 2.9

Summary of Glass Compositions of Hanford Surrogate Melts, wt\%

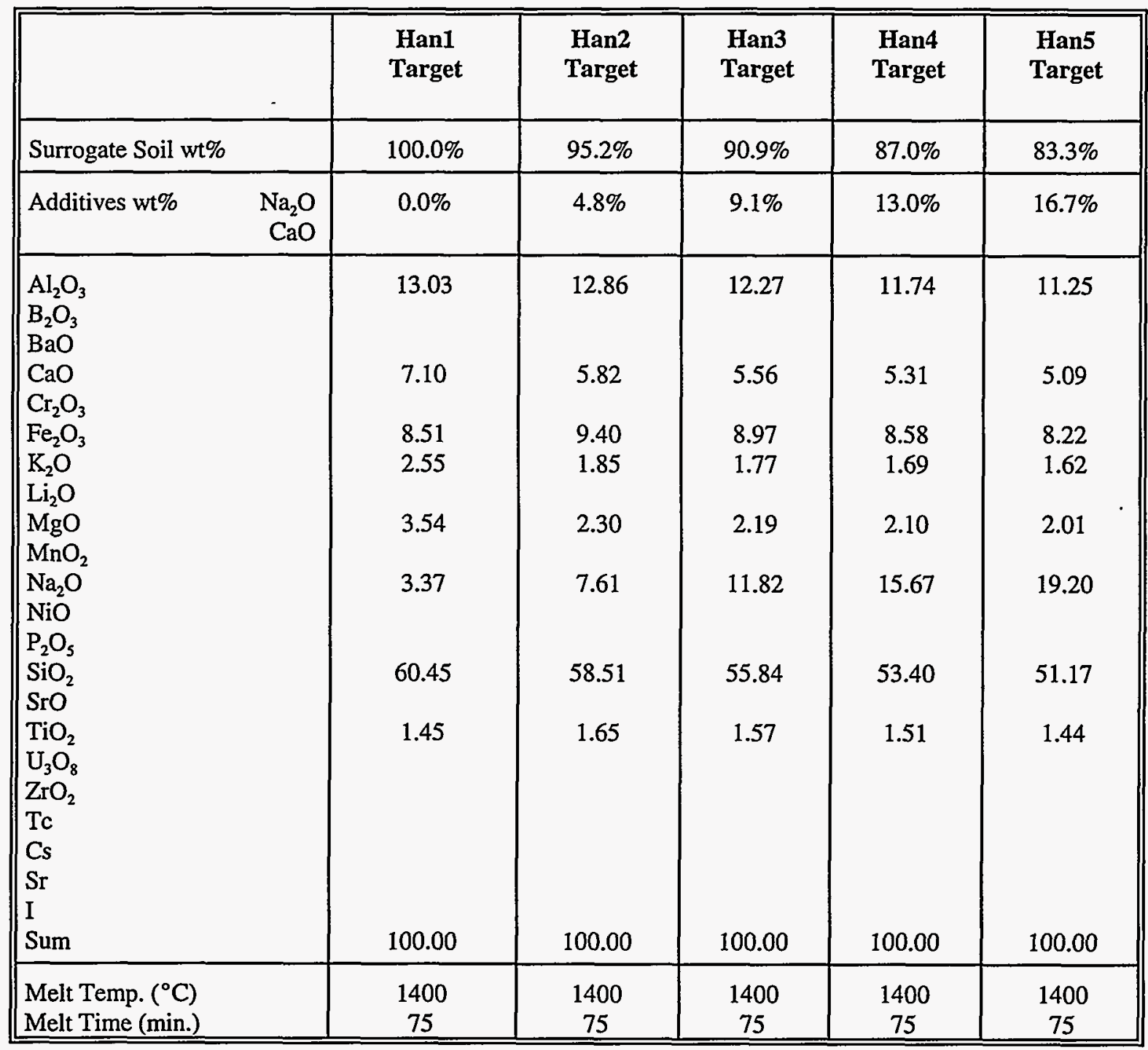




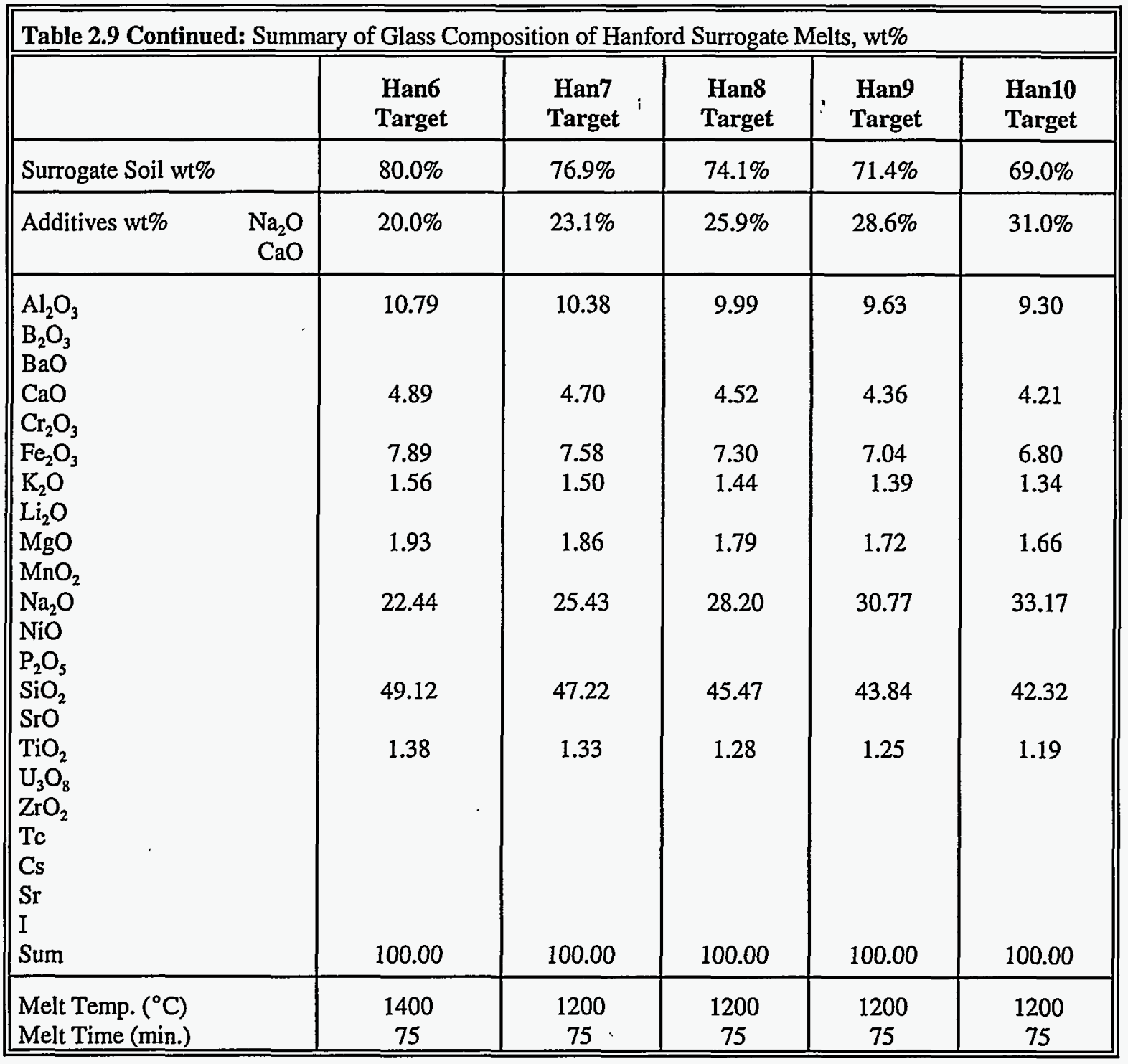




\begin{tabular}{|c|c|c|c|c|c|}
\hline & $\begin{array}{l}\text { Han11 } \\
\text { Target }\end{array}$ & $\begin{array}{l}\text { Han12 } \\
\text { Target }\end{array}$ & $\begin{array}{l}\text { Han13 } \\
\text { Target }\end{array}$ & $\begin{array}{l}\text { Han14 } \\
\text { Target }\end{array}$ & $\begin{array}{l}\text { Han15. } \\
\text { Target }\end{array}$ \\
\hline Surrogate Soil wt\% & $66.7 \%$ & $95.2 \%$ & $90.9 \%$ & $87.0 \%$ & $83.3 \%$ \\
\hline 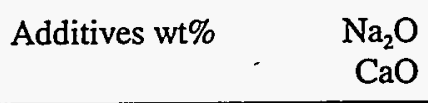 & $\begin{array}{l}33.3 \% \\
0.0 \% \\
\end{array}$ & $\begin{array}{l}0.0 \% \\
4.8 \%\end{array}$ & $\begin{array}{l}0.0 \% \\
9.1 \%\end{array}$ & $\begin{array}{c}0.0 \% \\
13.0 \%\end{array}$ & $\begin{array}{c}0.0 \% \\
16.7 \% \\
\end{array}$ \\
\hline $\begin{array}{l}\mathrm{Al}_{2} \mathrm{O}_{3} \\
\mathrm{~B}_{2} \mathrm{O}_{3} \\
\mathrm{BaO} \\
\mathrm{CaO} \\
\mathrm{Cr}_{2} \mathrm{O}_{3} \\
\mathrm{Fe}_{2} \mathrm{O}_{3} \\
\mathrm{~K}_{2} \mathrm{O} \\
\mathrm{Li}_{2} \mathrm{O} \\
\mathrm{MgO} \\
\mathrm{MnO}_{2} \\
\mathrm{Na}_{2} \mathrm{O} \\
\mathrm{NiO} \\
\mathrm{P}_{2} \mathrm{O}_{5} \\
\mathrm{SiO} \\
\mathrm{SrO} \\
\mathrm{TiO}_{2} \\
\mathrm{U}_{3} \mathrm{O}_{8} \\
\mathrm{ZrO} \\
\mathrm{Tc} \\
\mathrm{Cs} \\
\mathrm{Sr} \\
\mathrm{I} \\
\mathrm{Sum}\end{array}$ & $\begin{array}{l}8.99 \\
\\
4.07 \\
6.57 \\
1.30 \\
1.61 \\
35.40 \\
40.91 \\
1.15\end{array}$ & $\begin{array}{l}12.86 \\
10.60 \\
\\
9.40 \\
1.85 \\
\\
2.30 \\
2.83 \\
\\
58.51 \\
1.65 \\
\end{array}$ & $\begin{array}{l}12.27 \\
14.68 \\
8.97 \\
1.77 \\
2.19 \\
2.70 \\
\\
55.84 \\
1.57\end{array}$ & $\begin{array}{l}11.74 \\
\\
18.40 \\
\\
8.58 \\
1.69 \\
2.10 \\
2.58 \\
\\
53.40 \\
1.51\end{array}$ & $\begin{array}{l}11.25 \\
21.81 \\
\\
8.22 \\
1.62 \\
\\
2.01 \\
2.47 \\
\\
51.17 \\
1.44 \\
\end{array}$ \\
\hline $\begin{array}{l}\text { Melt Temp. }\left({ }^{\circ} \mathrm{C}\right) \\
\text { Melt Time (min.) }\end{array}$ & $\begin{array}{c}1200 \\
75 \\
\end{array}$ & $\begin{array}{c}1200 \\
75 \\
\end{array}$ & $\begin{array}{c}1200 \\
75 \\
\end{array}$ & $\begin{array}{c}1200 \\
75 \\
\end{array}$ & $\begin{array}{c}1200 \\
75 \\
\end{array}$ \\
\hline
\end{tabular}




\begin{tabular}{|c|c|c|c|c|c|}
\hline & $\begin{array}{l}\text { Han16 } \\
\text { Target }\end{array}$ & $\begin{array}{l}\text { Han17 } \\
\text { Target }\end{array}$ & $\begin{array}{l}\text { Han18 } \\
\text { Target }\end{array}$ & $\begin{array}{l}\text { Han19 } \\
\text { Target }\end{array}$ & $\begin{array}{c}\text { Han20* } \\
\text { Target }\end{array}$ \\
\hline Surrogate Soil wt\% & $80.0 \%$ & $83.3 \%$ & $83.3 \%$ & $83.3 \%$ & $80.0 \%$ \\
\hline $\begin{array}{lr}\text { Additives wt } \% & \mathrm{Na}_{2} \mathrm{O} \\
\mathrm{CaO}\end{array}$ & $\begin{array}{c}0.0 \% \\
20.0 \%\end{array}$ & $\begin{array}{l}8.3 \% \\
8.3 \%\end{array}$ & $\begin{array}{l}4.2 \% \\
12.5 \%\end{array}$ & $\begin{array}{l}12.5 \% \\
4.2 \%\end{array}$ & $20.0 \%$ \\
\hline $\begin{array}{l}\mathrm{Al}_{2} \mathrm{O}_{3} \\
\mathrm{~B}_{2} \mathrm{O}_{3} \\
\mathrm{BaO} \\
\mathrm{CaO} \\
\mathrm{Cr}_{2} \mathrm{O}_{3} \\
\mathrm{Fe}_{2} \mathrm{O}_{3} \\
\mathrm{~K}_{2} \mathrm{O} \\
\mathrm{Li}_{2} \mathrm{O} \\
\mathrm{MgO} \\
\mathrm{MnO}_{2} \\
\mathrm{Na}_{2} \mathrm{O} \\
\mathrm{NiO} \\
\mathrm{P}_{2} \mathrm{O}_{5} \\
\mathrm{SiO} \\
\mathrm{SrO}_{2} \\
\mathrm{TiO}_{2} \\
\mathrm{U}_{3} \mathrm{O}_{8} \\
\mathrm{ZrO} \\
\mathrm{Tc}_{2} \\
\mathrm{Cs} \\
\mathrm{Sr} \\
\mathrm{I} \\
\mathrm{Sum}\end{array}$ & $\begin{array}{l}10.79 \\
24.95 \\
7.89 \\
1.56 \\
1.93 \\
2.38 \\
49.12 \\
1.38\end{array}$ & $\begin{array}{l}11.25 \\
13.45 \\
8.22 \\
1.62 \\
2.01 \\
10.84 \\
51.17 \\
1.44\end{array}$ & $\begin{array}{l}11.25 \\
\\
17.63 \\
\\
8.22 \\
1.62 \\
\\
2.01 \\
6.66 \\
\\
51.17 \\
1.44 \\
\end{array}$ & $\begin{array}{l}11.25 \\
\\
9.27 \\
\\
8.22 \\
1.62 \\
\\
2.01 \\
15.02 \\
\\
51.17 \\
1.44 \\
\end{array}$ & $\begin{array}{l}11.66 \\
3.91 \\
\\
6.07 \\
1.60 \\
1.65 \\
22.32 \\
51.65 \\
1.13 \\
\\
100.00\end{array}$ \\
\hline $\begin{array}{l}\text { Melt Temp. }\left({ }^{\circ} \mathrm{C}\right) \\
\text { Melt Time (min.) }\end{array}$ & $\begin{array}{c}1200 \\
75\end{array}$ & $\begin{array}{l}1200 \\
75\end{array}$ & $\begin{array}{l}1200 \\
75\end{array}$ & $\begin{array}{c}1200 \\
75 \\
\end{array}$ & $\begin{array}{c}1200 \\
75 \\
\end{array}$ \\
\hline
\end{tabular}

*This glass was prepared using the non radioactive soil HA1 and the HAN6 formulation. 
Table 2.10

Summary of Glass Composition Produced from Hanford Soil and Tank Waste Surrogate, wt\%

\begin{tabular}{|c|c|c|c|c|c|c|}
\hline & \multicolumn{2}{|c|}{ HAN24 } & \multicolumn{2}{|c|}{ HAN25 } & \multicolumn{2}{|c|}{ HAN26 } \\
\hline & Target & Analyzed & Target & Analyzed & Target & Analyzed \\
\hline $\begin{array}{l}\text { Soil wt\% } \\
\text { Total } \mathrm{Na}_{2} \mathrm{O} \text { wt } \% \\
\text { Tank Waste } \mathrm{Na}_{2} \mathrm{O} \% \\
\text { Additives } \mathrm{wt} \% \\
\end{array}$ & $\begin{array}{r}67.0 \% \\
30.1 \% \\
27.6 \% \\
\text { None } \\
\end{array}$ & $\begin{array}{r}67.0 \% \\
24.9 \% \\
22.4 \% \\
\text { None } \\
\end{array}$ & $\begin{array}{r}63.7 \% \\
28.6 \% \\
26.3 \% \\
5 \% \mathrm{Al}_{2} \mathrm{O}_{3} \\
\end{array}$ & $\begin{array}{r}63.7 \% \\
23.8 \% \\
21.3 \% \\
5 \% \mathrm{Al}_{2} \mathrm{O}_{3} \\
\end{array}$ & $\begin{array}{r}63.7 \% \\
28.6 \% \\
26.3 \% \\
5 \% \mathrm{~B}_{2} \mathrm{O}_{3} \\
\end{array}$ & $\begin{array}{r}63.7 \% \\
24.8 \% \\
22.5 \% \\
5 \% \mathrm{~B}_{2} \mathrm{O}_{3} \\
\end{array}$ \\
\hline & & & & & & \\
\hline $\mathrm{Al}_{2} \mathrm{O}_{3}$ & 10.69 & 10.98 & 15.15 & 15.44 & 10.15 & 10.68 \\
\hline $\mathrm{B}_{2} \mathrm{O}_{3}$ & 0.00 & 0.09 & 0.00 & 0.08 & 5.00 & 4.83 \\
\hline $\mathrm{BaO}$ & 0.08 & 0.09 & 0.02 & 0.09 & 0.02 & 0.10 \\
\hline $\mathrm{CaO}$ & 2.73 & 2.35 & 2.59 & 2.21 & 2.59 & 3.25 \\
\hline $\mathrm{Cr}_{2} \mathrm{O}_{3}$ & 0.05 & 0.29 & 0.05 & 0.19 & 0.05 & 0.07 \\
\hline $\mathrm{Fe}_{2} \mathrm{O}_{3}$ & 2.66 & 3.60 & 2.53 & 3.29 & 2.53 & 3.09 \\
\hline $\mathrm{K}_{2} \mathrm{O}$ & 2.07 & 1.92 & 1.96 & 1.89 & 1.96 & 1.91 \\
\hline $\mathrm{Li}_{2} \mathrm{O}$ & 0.00 & 0.05 & 0.00 & 0.06 & 0.00 & 0.04 \\
\hline $\mathrm{MgO}$ & 1.24 & 1.32 & 1.18 & 1.24 & 1.18 & 1.72 \\
\hline $\mathrm{MnO}_{2}$ & 0.13 & 0.09 & 0.13 & 0.09 & 0.13 & 0.08 \\
\hline $\mathrm{Na}_{2} \mathrm{O}$ & 30.13 & 24.88 & 28.62 & 23.83 & 28.62 & 24.85 \\
\hline $\mathrm{NiO}$ & 0.00 & 0.09 & 0.00 & 0.13 & 0.00 & 0.02 \\
\hline $\mathrm{P}_{2} \mathrm{O}_{5}$ & 2.00 & 1.76 & 1.90 & 1.67 & 1.90 & 1.51 \\
\hline $\mathrm{SiO}_{2}$ & 46.80 & 51.72 & 44.46 & 49.06 & 44.46 & 47.25 \\
\hline $\mathrm{SrO}^{2}$ & 0.03 & 0.06 & 0.03 & 0.06 & 0.03 & 0.05 \\
\hline $\mathrm{TiO}_{2}$ & 0.40 & 0.56 & 0.38 & 0.56 & 0.38 & 0.50 \\
\hline $\mathrm{U}_{3} \mathrm{O}_{8}$ & 0.0715 & 0.10 & 0.0679 & 0.09 & 0.0679 & $* .03$ \\
\hline $\mathrm{ZrO}_{2}$ & 0.00 & 0.09 & 0.00 & 0.03 & 0.00 & 0.02 \\
\hline $\mathrm{Cl}$ & 0.14 & & 0.13 & & 0.13 & \\
\hline $\mathrm{F}$ & 0.32 & & 0.30 & & 0.31 & \\
\hline $\mathrm{Tc}$ & 0.001 & 0.00025 & 0.001 & 0.00033 & 0.001 & 0.00033 \\
\hline Cs & 0.01 & & 0.01 & & 0.01 & \\
\hline $\mathrm{Sr}$ & 0.01 & & 0.01 & & 0.01 & \\
\hline & 0.01 & & 0.01 & & 0.01 & \\
\hline SUM & 100.00 & 100.00 & 100.00 & 100.00 & 100.00 & 100.00 \\
\hline Melt Temp., ${ }^{\circ} \mathrm{C}$ & & 1200 & & 1200 & & 1200 \\
\hline Melt Time, min. & & 75 & & 75 & & 70 \\
\hline
\end{tabular}


Table 2.10 Continued: Summary of Glass Composition Produced from Hanford Soil and Tank Waste Surrogate, wt\%

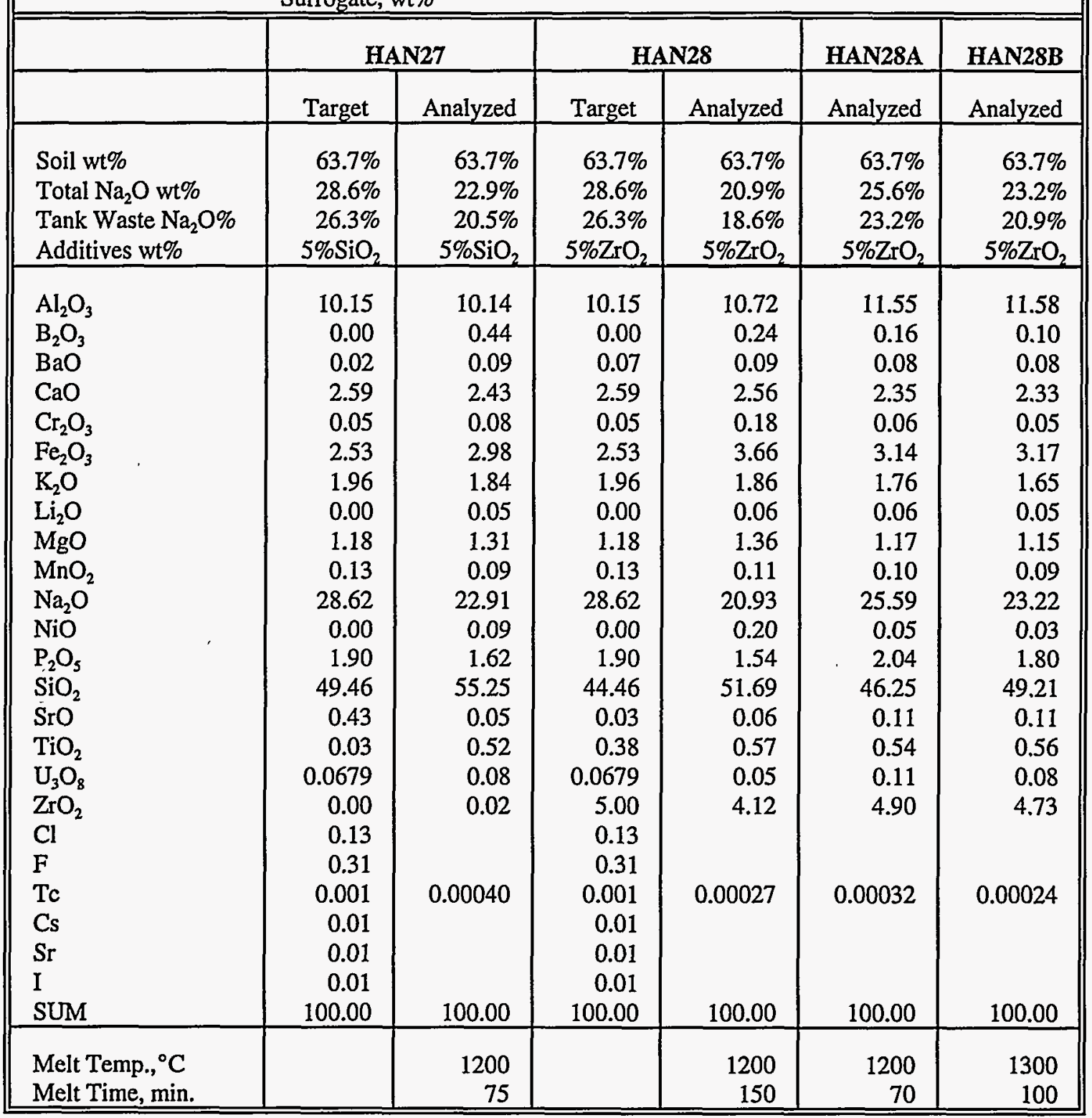




\begin{tabular}{|c|c|c|c|c|c|c|}
\hline & & $\sqrt{29}$ & HAN29A & HAN29B & HAN29B1 & HAN29B2 \\
\hline & Target & Analyzed & Analyzed & Analyzed & Analyzed & Analyzed \\
\hline $\begin{array}{l}\text { Soil wt\% } \\
\text { Total } \mathrm{Na}_{2} \mathrm{O} \text { wt\% } \\
\text { Tank Waste } \mathrm{Na}_{2} \mathrm{O} \% \\
\text { Additives wt\% }\end{array}$ & $\begin{array}{r}55.1 \% \\
35.5 \% \\
33.4 \% \\
5 \% \mathrm{ZrO}_{2} \\
\end{array}$ & $\begin{array}{r}55.1 \% \\
25.3 \% \\
23.2 \% \\
5 \% \mathrm{ZrO}_{2} \\
\end{array}$ & $\begin{array}{r}55.1 \% \\
28.8 \% \\
26.8 \% \\
5 \% \mathrm{ZrO}_{2} \\
\end{array}$ & $\begin{array}{r}55.1 \% \\
27.4 \% \\
25.4 \% \\
5 \% \mathrm{ZrO}_{2} \\
\end{array}$ & $\begin{array}{r}55.1 \% \\
29.3 \% \\
27.3 \% \\
5 \% \mathrm{ZrO}_{2} \\
\end{array}$ & $\begin{array}{r}55.1 \% \\
27.0 \% \\
25.0 \% \\
5 \% \mathrm{ZrO}_{2} \\
\end{array}$ \\
\hline $\begin{array}{l}\mathrm{Al}_{2} \mathrm{O}_{3} \\
\mathrm{~B}_{2} \mathrm{O}_{3} \\
\mathrm{BaO} \\
\mathrm{CaO} \\
\mathrm{Cr}_{2} \mathrm{O}_{3} \\
\mathrm{Fe}_{2} \mathrm{O}_{3} \\
\mathrm{~K}_{2} \mathrm{O} \\
\mathrm{Li}_{2} \mathrm{O} \\
\mathrm{MgO} \\
\mathrm{MnO}_{2} \\
\mathrm{Na}_{2} \mathrm{O} \\
\mathrm{NiO} \\
\mathrm{P}_{2} \mathrm{O}_{5} \\
\mathrm{SiO} \\
\mathrm{SrO} \\
\mathrm{TiO}_{2} \\
\mathrm{U}_{3} \mathrm{O}_{8} \\
\mathrm{ZrO} \\
\mathrm{Cl} \\
\mathrm{F} \\
\mathrm{Tc} \\
\mathrm{Cs} \\
\mathrm{Sr} \\
\mathrm{I} \\
\mathrm{SUM} \\
\end{array}$ & $\begin{array}{r}9.60 \\
0.00 \\
0.06 \\
2.24 \\
0.06 \\
2.19 \\
1.89 \\
0.00 \\
1.02 \\
0.11 \\
35.47 \\
0.00 \\
2.33 \\
38.49 \\
0.03 \\
0.33 \\
0.0690 \\
5.00 \\
0.17 \\
0.39 \\
0.001 \\
0.01 \\
0.01 \\
0.01 \\
100.00 \\
\end{array}$ & $\begin{array}{r}12.58 \\
0.14 \\
0.09 \\
2.68 \\
0.03 \\
3.02 \\
1.71 \\
0.06 \\
1.20 \\
0.11 \\
25.26 \\
0.01 \\
1.81 \\
45.94 \\
0.05 \\
0.55 \\
0.06 \\
4.68\end{array}$ & $\begin{array}{r}10.90 \\
0.10 \\
0.07 \\
2.11 \\
0.05 \\
2.81 \\
1.67 \\
0.05 \\
1.03 \\
0.07 \\
28.80 \\
0.02 \\
2.26 \\
44.35 \\
0.05 \\
0.50 \\
0.07 \\
5.10\end{array}$ & $\begin{array}{r}11.44 \\
0.08 \\
0.07 \\
1.89 \\
0.05 \\
2.60 \\
1.45 \\
0.05 \\
0.93 \\
0.07 \\
27.45 \\
0.01 \\
2.13 \\
46.50 \\
0.05 \\
0.51 \\
0.06 \\
4.69\end{array}$ & $\begin{array}{r}10.90 \\
0.13 \\
0.08 \\
2.29 \\
0.02 \\
3.13 \\
1.53 \\
0.04 \\
1.16 \\
0.09 \\
29.32 \\
0.03 \\
2.16 \\
48.43 \\
0.06 \\
0.54 \\
0.08 \\
0.02\end{array}$ & $\begin{array}{r}12.16 \\
0.07 \\
0.07 \\
2.15 \\
0.02 \\
2.88 \\
1.30 \\
0.04 \\
1.07 \\
0.09 \\
27.04 \\
0.03 \\
2.04 \\
50.33 \\
0.05 \\
0.58 \\
0.06 \\
0.02\end{array}$ \\
\hline $\begin{array}{l}\text { Melt Temp., }{ }^{\circ} \mathrm{C} \\
\text { Melt Time, min. }\end{array}$ & & $\begin{array}{r}1200 \\
120 \\
\end{array}$ & $\begin{array}{r}1200 \\
70 \\
\end{array}$ & $\begin{array}{r}1400 \\
100 \\
\end{array}$ & $\begin{array}{r}1200 \\
70 \\
\end{array}$ & $\begin{array}{r}1300 \\
100 \\
\end{array}$ \\
\hline
\end{tabular}




\begin{tabular}{|c|c|c|c|c|c|c|}
\hline & HAN29E & HAN29F & HAN30 & HAN30A & HAN30B & HAN30C \\
\hline & Analyzed & Analyzed & Target & Analyzed & Analyzed & Analyzed \\
\hline $\begin{array}{l}\text { Soil wt\% } \\
\text { Total } \mathrm{Na}_{2} \mathrm{O} \text { wt\% } \\
\text { Tank Waste } \mathrm{Na}_{2} \mathrm{O} \% \\
\text { Additives wt\% }\end{array}$ & $\begin{array}{r}50.1 \% \\
42.7 \% \\
40.7 \% \\
5 \% \mathrm{ZrO}_{2}+1 \\
0 \% \mathrm{Na}_{2} \mathrm{O} \\
\end{array}$ & $\begin{array}{r}55.1 \% \\
33.2 \% \\
31.2 \% \\
5 \% \mathrm{ZrO}_{2}\end{array}$ & $\begin{array}{r}55.1 \% \\
35.5 \% \\
33.4 \% \\
5 \% \mathrm{Al}_{2} \mathrm{O}_{3}\end{array}$ & $\begin{array}{r}55.1 \% \\
22.1 \% \\
20.1 \% \\
5 \% \mathrm{Al}_{2} \mathrm{O}_{3}\end{array}$ & $\begin{array}{r}55.1 \% \\
28.1 \% \\
26.1 \% \\
5 \% \mathrm{Al}_{2} \mathrm{O}_{3}\end{array}$ & $\begin{array}{r}55.1 \% \\
25.5 \% \\
26.1 \% \\
5 \% \mathrm{Al}_{2} \mathrm{O}_{3}\end{array}$ \\
\hline $\begin{array}{l}\mathrm{Al}_{2} \mathrm{O}_{3} \\
\mathrm{~B}_{2} \mathrm{O}_{3} \\
\mathrm{BaO} \\
\mathrm{CaO} \\
\mathrm{Cr}_{2} \mathrm{O}_{3} \\
\mathrm{Fe}_{2} \mathrm{O}_{3} \\
\mathrm{~K}_{2} \mathrm{O} \\
\mathrm{Li}_{2} \mathrm{O} \\
\mathrm{MgO} \\
\mathrm{MnO}_{2} \\
\mathrm{Na}_{2} \mathrm{O} \\
\mathrm{NiO} \\
\mathrm{P}_{2} \mathrm{O}_{5} \\
\mathrm{SiO}_{2} \\
\mathrm{SrO} \\
\mathrm{TiO}_{2} \\
\mathrm{U}_{3} \mathrm{O}_{8} \\
\mathrm{ZrO}_{2} \\
\mathrm{Cl} \\
\mathrm{F} \\
\mathrm{Tc} \\
\mathrm{Cs} \\
\mathrm{Sr} \\
\mathrm{I} \\
\mathrm{SUM}\end{array}$ & $\begin{array}{c}9.36 \\
0.03 \\
0.07 \\
2.11 \\
0.06 \\
2.50 \\
1.82 \\
0.06 \\
0.97 \\
0.09 \\
42.74 \\
0.01 \\
2.07 \\
34.00 \\
0.03 \\
0.40 \\
0.04 \\
3.63 \\
- \\
- \\
0 \\
- \\
- \\
- \\
100.00\end{array}$ & $\begin{array}{c}9.84 \\
0.27 \\
0.09 \\
2.58 \\
0.08 \\
2.77 \\
1.67 \\
0.07 \\
1.24 \\
0.11 \\
33.20 \\
0.02 \\
2.31 \\
40.36 \\
0.06 \\
0.45 \\
0.09 \\
4.80 \\
100.00 \\
- \\
0.00021 \\
- \\
- \\
- \\
100.00\end{array}$ & $\begin{array}{r}14.60 \\
0.00 \\
0.06 \\
2.24 \\
0.06 \\
2.19 \\
1.89 \\
0.00 \\
1.02 \\
0.11 \\
35.47 \\
0.00 \\
2.33 \\
38.49 \\
0.03 \\
0.33 \\
0.0690 \\
0.00 \\
0.17 \\
0.39 \\
0.001 \\
0.01 \\
0.01 \\
0.01 \\
100.00\end{array}$ & $\begin{array}{r}18.27 \\
0.13 \\
0.05 \\
1.91 \\
0.00 \\
1.77 \\
1.29 \\
0.03 \\
0.88 \\
0.05 \\
22.05 \\
0.00 \\
1.63 \\
51.19 \\
0.04 \\
0.64 \\
0.05 \\
0.02 \\
- \\
- \\
0.00022 \\
- \\
- \\
- \\
100.00\end{array}$ & $\begin{array}{r}15.16 \\
0.25 \\
0.07 \\
2.12 \\
0.04 \\
2.59 \\
1.57 \\
0.07 \\
1.08 \\
0.07 \\
28.11 \\
0.03 \\
2.62 \\
45.47 \\
0.05 \\
0.49 \\
0.20 \\
0.02 \\
- \\
- \\
0.00025 \\
- \\
- \\
- \\
100.00\end{array}$ & $\begin{array}{r}17.12 \\
0.88 \\
0.10 \\
2.61 \\
0.04 \\
2.68 \\
1.37 \\
0.07 \\
1.35 \\
0.10 \\
25.51 \\
0.02 \\
2.30 \\
45.04 \\
0.05 \\
0.55 \\
0.11 \\
0.10 \\
- \\
- \\
\text { NA } \\
- \\
- \\
- \\
100.00\end{array}$ \\
\hline $\begin{array}{l}\text { Melt Temp., }{ }^{\circ} \mathrm{C} \\
\text { Melt time, min. }\end{array}$ & $\begin{array}{r}1200 \\
60 \\
\end{array}$ & & & $\begin{array}{r}1400 \\
90 \\
\end{array}$ & $\begin{array}{r}1200 \\
100 \\
\end{array}$ & $\begin{array}{r}1400 \\
190 \\
\end{array}$ \\
\hline
\end{tabular}




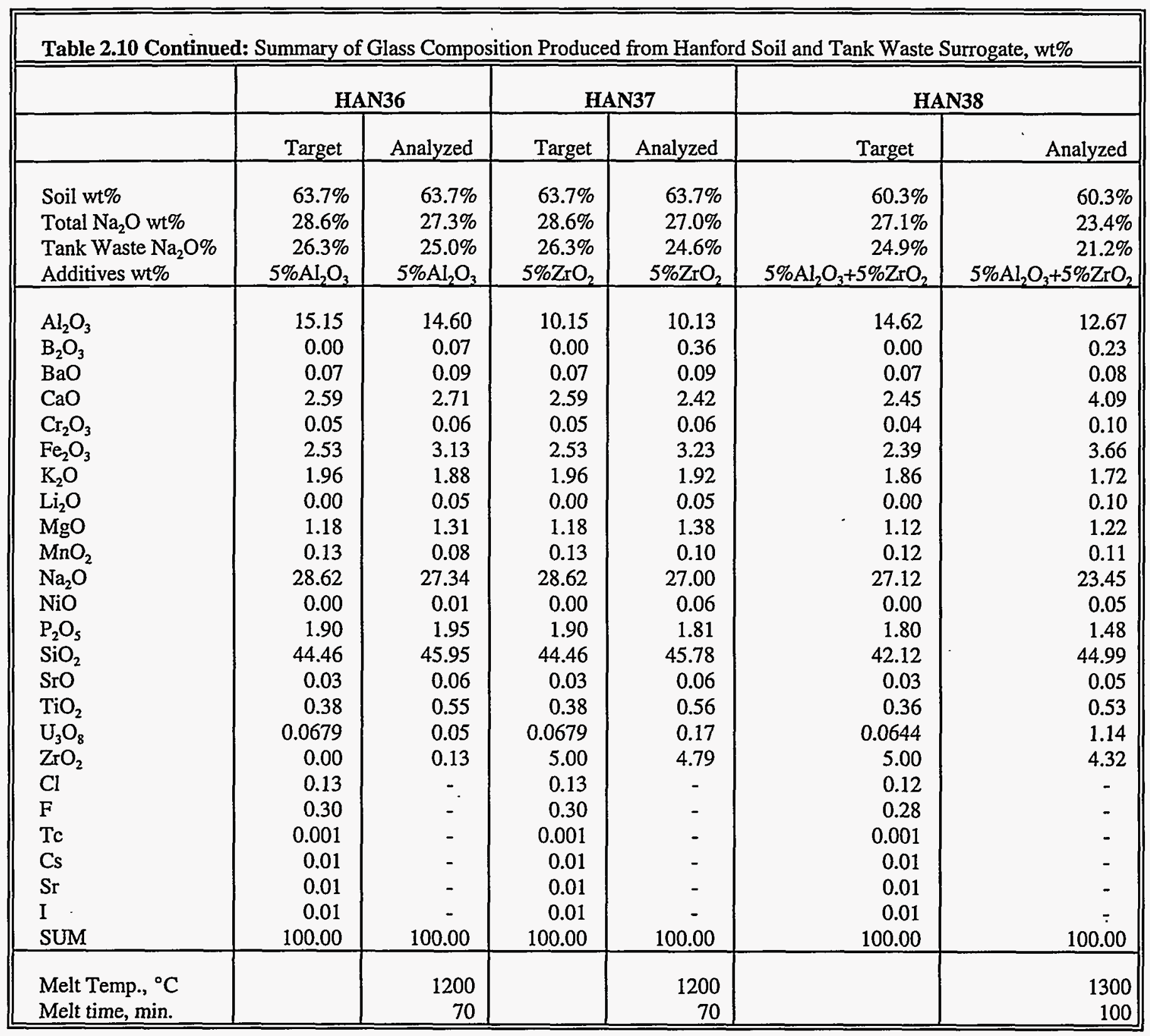




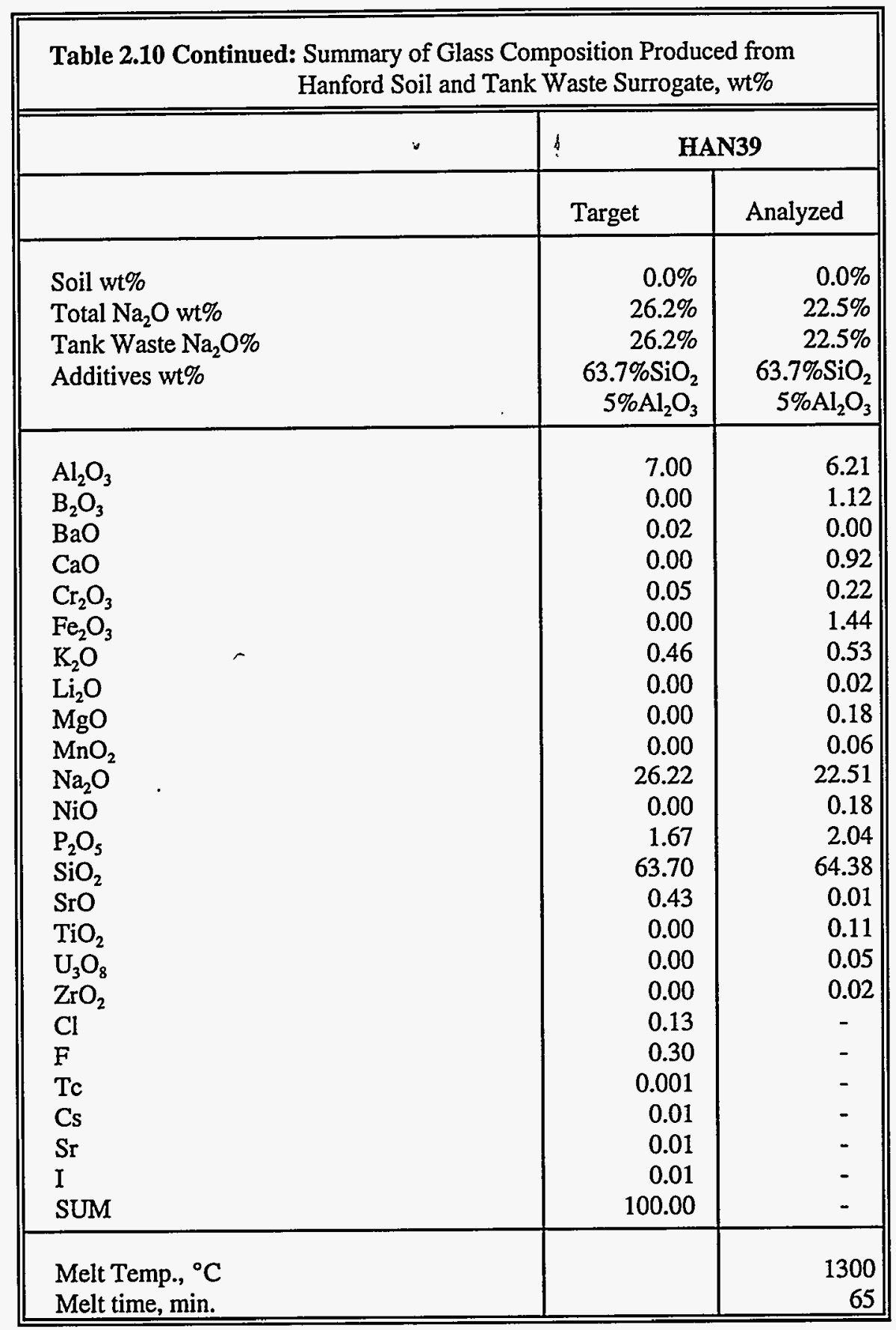


Table 2.11

Summary of Glass Composition Produced from Hanford Soil and Additives, wt\%

\begin{tabular}{|c|c|c|c|c|c|c|}
\hline & HA2 & \multicolumn{2}{|c|}{ HAN22B } & HAN31 & \multicolumn{2}{|c|}{ HAN32 } \\
\hline & Soil & Target & Analyzed & Target & Target & Analyzed \\
\hline $\begin{array}{l}\text { Soil wt\% } \\
\text { Additives wt\% }\end{array}$ & & \multicolumn{2}{|c|}{$\begin{array}{c}80 \% \\
20 \% \mathrm{Na}_{2} \mathrm{O} \\
\end{array}$} & $\begin{array}{c}95 \% \\
5 \% \mathrm{CaO} \\
\end{array}$ & \multicolumn{2}{|c|}{$\begin{array}{c}90 \% \\
10 \% \mathrm{CaO} \\
\end{array}$} \\
\hline $\mathrm{Al}_{2} \mathrm{O}_{3}$ & 12.80 & 10.24 & 10.92 & 12.39 & 11.74 & 11.22 \\
\hline $\mathrm{B}_{2} \mathrm{O}_{3}$ & 0.00 & 0.00 & 1.14 & 0.00 & 0.00 & 0.52 \\
\hline $\mathrm{BaO}$ & 0.11 & 0.09 & 0.11 & 0.12 & 0.11 & 0.10 \\
\hline $\mathrm{CaO}$ & 4.07 & 3.26 & 3.79 & 9.66 & 14.42 & 12.92 \\
\hline $\mathrm{Cr}_{2} \mathrm{O}_{3}$ & 0.00 & 0.00 & 0.03 & 0.00 & 0.00 & 0.01 \\
\hline $\mathrm{Fe}_{2} \mathrm{O}_{3}$ & 3.97 & 3.18 & 2.17 & 4.17 & 3.95 & 4.07 \\
\hline $\mathrm{K}_{2} \mathrm{O}$ & 2.36 & 1.89 & 1.88 & 2.34 & 2.22 & 1.93 \\
\hline $\mathrm{Li}_{2} \mathrm{O}$ & 0.00 & 0.00 & 0.07 & 0.00 & 0.00 & 0.07 \\
\hline $\mathrm{MgO}$ & 1.85 & 1.48 & 1.84 & 2.72 & 2.58 & 1.64 \\
\hline $\mathrm{MnO}_{2}$ & 0.20 & 0.16 & 0.14 & 0.29 & 0.28 & 0.11 \\
\hline $\mathrm{Na}_{2} \mathrm{O}$ & 3.71 & 22.97 & 22.56 & 4.23 & 4.01 & 3.05 \\
\hline $\mathrm{NiO}$ & 0.00 & 0.00 & 0.02 & 0.00 & 0.00 & 0.02 \\
\hline $\mathrm{P}_{2} \mathrm{O}_{5}$ & 0.35 & 0.28 & 0.26 & 0.47 & 0.44 & 0.36 \\
\hline $\mathrm{SiO}_{2}$ & 69.86 & 55.89 & 54.28 & 62.88 & 59.57 & 63.12 \\
\hline $\mathrm{SrO}$ & 0.00 & 0.00 & 0.05 & 0.05 & 0.05 & 0.05 \\
\hline $\mathrm{TiO}_{2}$ & 0.05 & 0.04 & 0.59 & 0.56 & 0.53 & 0.69 \\
\hline $\mathrm{U}_{3} \mathrm{O}_{8}$ & 0.60 & 0.48 & 0.08 & 0.13 & 0.12 & 0.05 \\
\hline $\mathrm{ZrO}_{2}$ & 0.07 & 0.05 & 0.01 & 0.00 & 0.00 & 0.07 \\
\hline $\mathrm{Cl}$ & 0.00 & 0.00 & - & - & - & - \\
\hline $\mathrm{F}$ & 0.00 & 0.00 & - & - & - & - \\
\hline SUM & 100.00 & 100.00 & 100.00 & 100.00 & 100.00 & 100.00 \\
\hline $\begin{array}{l}\text { Melt Temp }{ }^{\circ} \mathrm{C} \\
\text { Melt time, } \min \end{array}$ & & & $\begin{array}{r}1200 \\
90\end{array}$ & $\begin{array}{r}1400 \\
90\end{array}$ & & $\begin{array}{r}1400 \\
90\end{array}$ \\
\hline
\end{tabular}




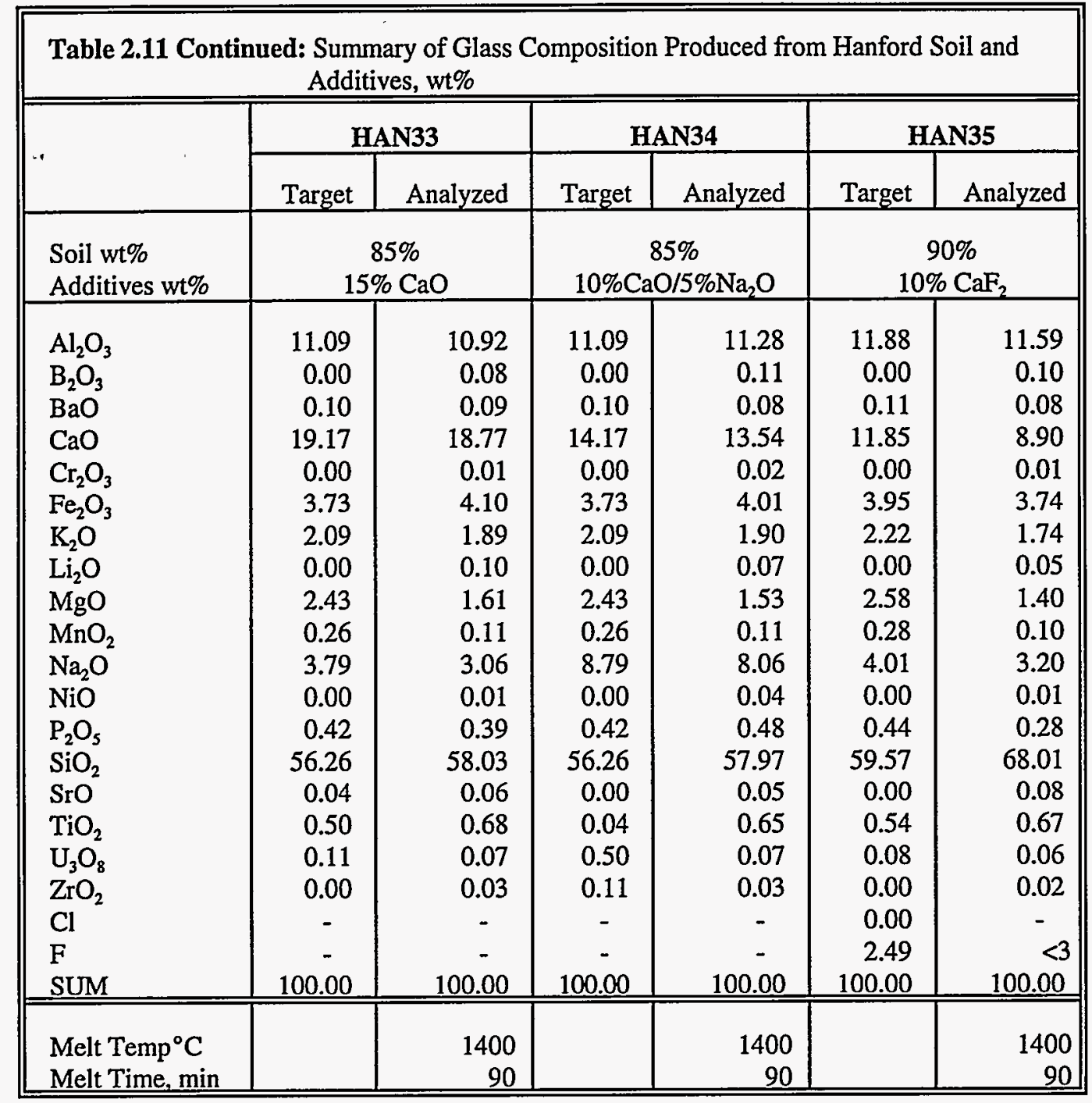


Table 2.12a

Summary of Composition and Properties of Surrogate Soil Melts

\begin{tabular}{|c|c|c|c|c|c|c|c|c|c|c|c|}
\hline Sample ID\# & $\begin{array}{c}\text { Han } \\
1\end{array}$ & Han2 & Han3 & Han4 & Han5 & Han6 & Han7 & Han8 & Han 9 & Han10 & Han11 \\
\hline Surrogate Soil & & $95.2 \%$ & $90.9 \%$ & $87.0 \%$ & $83.3 \%$ & $80.0 \%$ & $76.9 \%$ & $74.1 \%$ & $71.4 \%$ & $69.0 \%$ & $66.7 \%$ \\
\hline $\mathrm{Na}, \mathrm{O}$ wt\% & & $4.80 \%$ & $9.10 \%$ & $13.00 \%$ & $16.70 \%$ & $20.00 \%$ & $23.10 \%$ & $25.90 \%$ & $28.60 \%$ & $31.00 \%$ & $33.30 \%$ \\
\hline \multicolumn{12}{|l|}{$\mathrm{CaO}$ wt\% } \\
\hline \multicolumn{12}{|c|}{ Viscosity in [Poise] versus Temp. in Celsius } \\
\hline Temperature $\left({ }^{\circ} \mathrm{C}\right)$ & \multicolumn{11}{|c|}{ Viscosity in (Poise) } \\
\hline $\begin{array}{l}1050 \\
1100 \\
1150 \\
1200 \\
1250 \\
1300 \\
1350 \\
1400 \\
1450 \\
1500 \\
\end{array}$ & $\begin{array}{c}- \\
- \\
- \\
- \\
- \\
- \\
499 \\
305 \\
192\end{array}$ & $\begin{array}{r}- \\
- \\
- \\
- \\
1347 \\
702 \\
420 \\
277\end{array}$ & $\begin{array}{r}- \\
- \\
- \\
- \\
1383 \\
768\end{array}$ & $\begin{array}{r}- \\
1318 \\
706 \\
399 \\
237 \\
146 \\
94 \\
62 \\
43 \\
30 \\
\end{array}$ & $\begin{array}{r}715 \\
426 \\
262 \\
167 \\
110 \\
74 \\
51 \\
36 \\
26 \\
19 \\
\end{array}$ & $\begin{array}{r}566 \\
316 \\
186 \\
114 \\
73 \\
49 \\
33 \\
23 \\
17\end{array}$ & $\begin{array}{r}380 \\
208 \\
124 \\
79 \\
54 \\
38 \\
28 \\
21 \\
16\end{array}$ & $\begin{array}{r}197 \\
122 \\
78 \\
52 \\
35 \\
25 \\
18 \\
13 \\
9\end{array}$ & $\begin{array}{r}142 \\
83 \\
53 \\
37 \\
27 \\
20 \\
16 \\
13 \\
11\end{array}$ & $\begin{array}{r}108 \\
64 \\
40 \\
26 \\
18 \\
12 \\
9 \\
7 \\
5\end{array}$ & $\begin{array}{r}85 \\
51 \\
32 \\
21 \\
14 \\
10 \\
7 \\
5 \\
4\end{array}$ \\
\hline \multicolumn{12}{|c|}{ Temperature $\left({ }^{\circ} \mathrm{C}\right)$ predicted for a viscosity of $100 \mathrm{P}$} \\
\hline & 1526 & 1581 & 1516 & 1343 & 1261 & 1215 & 1173 & 1122 & 1082 & 1057 & 1036 \\
\hline \multicolumn{12}{|c|}{ Measurement range $\left({ }^{\circ} \mathrm{C}\right)$} \\
\hline $\begin{array}{l}\text { Lower } \\
\text { Higher }\end{array}$ & $\begin{array}{l}1363 \\
1460 \\
\end{array}$ & $\begin{array}{l}1271 \\
1403 \\
\end{array}$ & $\begin{array}{l}1243 \\
1317 \\
\end{array}$ & $\begin{array}{l}1112 \\
1303 \\
\end{array}$ & $\begin{array}{l}1243 \\
1454 \\
\end{array}$ & $\begin{array}{l}1168 \\
1362 \\
\end{array}$ & $\begin{array}{l}1113 \\
1316 \\
\end{array}$ & $\begin{array}{l}1054 \\
1243 \\
\end{array}$ & $\begin{array}{l}1026 \\
1211 \\
\end{array}$ & $\begin{array}{l}1065 \\
1257 \\
\end{array}$ & $\begin{array}{l}1020 \\
1209 \\
\end{array}$ \\
\hline \multicolumn{12}{|c|}{ Conductivity in $[\mathrm{S} / \mathrm{cm}]$ versus Temp. in Celsius } \\
\hline Temperature $\left({ }^{\circ} \mathrm{C}\right)$ & \multicolumn{11}{|c|}{ Conductivity in (S/cm) } \\
\hline $\begin{array}{l}1000 \\
1050 \\
1100 \\
1150 \\
1200 \\
1250 \\
1300 \\
1350 \\
1400 \\
1450 \\
1500 \\
\end{array}$ & $\begin{array}{l}- \\
- \\
- \\
- \\
- \\
- \\
- \\
- \\
- \\
- \\
-\end{array}$ & $\begin{array}{c}- \\
- \\
- \\
- \\
- \\
0.1020 \\
116 \\
0.131 \\
0.147 \\
0.164\end{array}$ & $\begin{array}{c}- \\
- \\
- \\
- \\
- \\
0.13 \\
0.11 \\
0.09\end{array}$ & $\begin{array}{l}0.148 \\
0.185 \\
0.227 \\
0.274 \\
0.327 \\
0.386 \\
0.451\end{array}$ & $\begin{array}{c}- \\
- \\
- \\
0.485 \\
0.551 \\
0.620 \\
0.693 \\
0.769 \\
0.848 \\
0.930 \\
1.015\end{array}$ & $\begin{array}{l}0.401 \\
0.517 \\
0.653 \\
0.809 \\
0.985 \\
1.183 \\
1.402 \\
1.641 \\
1.901 \\
2.182 \\
2.482 \\
\end{array}$ & $\begin{array}{c}- \\
- \\
0.389 \\
0.831 \\
1.036 \\
1.151\end{array}$ & $\begin{array}{l}0.446 \\
0.677 \\
0.929 \\
1.189 \\
1.450\end{array}$ & $\begin{array}{l}0.790 \\
1.051 \\
1.276 \\
1.467 \\
1.630\end{array}$ & $\begin{array}{l}1.136 \\
1.293 \\
1.458 \\
1.630 \\
1.809\end{array}$ & $\begin{array}{l}1.416 \\
1.653 \\
1.854 \\
2.024 \\
2.169\end{array}$ \\
\hline \multicolumn{12}{|c|}{ Measurement range $\left({ }^{\circ} \mathrm{C}\right)$} \\
\hline $\begin{array}{l}\text { Lower } \\
\text { Higher }\end{array}$ & & $\begin{array}{l}1301 \\
1392\end{array}$ & $\begin{array}{l}1276 \\
1340\end{array}$ & $\begin{array}{l}1018 \\
1227\end{array}$ & $\begin{array}{l}1219 \\
1460\end{array}$ & $\begin{array}{l}1038 \\
1463\end{array}$ & $\begin{array}{l}1169 \\
1298\end{array}$ & $\begin{array}{l}1085 \\
1214 \\
\end{array}$ & $\begin{array}{l}1041 \\
1189 \\
\end{array}$ & $\begin{array}{r}992 \\
1192 \\
\end{array}$ & $\begin{array}{r}992 \\
1191 \\
\end{array}$ \\
\hline
\end{tabular}




\begin{tabular}{|c|c|c|c|c|c|c|c|c|c|}
\hline Sample ID\# & Han12 & Han 13 & Han14 & Han15 & Han16 & Han17 & Han18 & Han19 & Han20 \\
\hline Surrogate Soil & $95.2 \%$ & $90.9 \%$ & $87.0 \%$ & $83.3 \%$ & $80.0 \%$ & $83.3 \%$ & $83.3 \%$ & $83.3 \%$ & $80.0 \%$ \\
\hline $\mathrm{Na}, \mathrm{O}$ wt\% & & & & & & $8.3 \%$ & $4.2 \%$ & $12.5 \%$ & $20.0 \%$ \\
\hline $\mathrm{CaO}$ wt\% & $4.8 \%$ & $9.1 \%$ & $13.0 \%$ & $16.7 \%$ & $20.0 \%$ & $8.3 \%$ & $12.5 \%$ & $4.2 \%$ & \\
\hline \multicolumn{10}{|c|}{ Viscosity in [Poise] versus Temp. in Celsius } \\
\hline Temperature $\left({ }^{\circ} \mathrm{C}\right)$ & \multicolumn{9}{|c|}{ Viscosity in (Poise) } \\
\hline $\begin{array}{l}1050 \\
1100 \\
1150 \\
1200 \\
1250 \\
1300 \\
1350 \\
1400 \\
1450 \\
1500 \\
\end{array}$ & $\begin{array}{r}- \\
- \\
- \\
- \\
1441 \\
752 \\
427 \\
260 \\
167\end{array}$ & $\begin{array}{r}- \\
- \\
- \\
1178 \\
573 \\
301 \\
169 \\
101 \\
63\end{array}$ & $\begin{array}{r}- \\
- \\
- \\
404 \\
227 \\
132 \\
79 \\
49 \\
31\end{array}$ & $\begin{array}{l}- \\
- \\
- \\
278 \\
150 \\
84 \\
49 \\
29 \\
18\end{array}$ & $\begin{array}{r}- \\
- \\
- \\
225 \\
101 \\
52 \\
30 \\
19 \\
12\end{array}$ & $\begin{array}{l}- \\
- \\
- \\
- \\
132 \\
78 \\
49 \\
31 \\
21\end{array}$ & $\begin{array}{c}- \\
- \\
- \\
- \\
122 \\
72 \\
45 \\
30 \\
21 \\
15 \\
\end{array}$ & $\begin{array}{r}1097 \\
587 \\
332 \\
196 \\
121 \\
77 \\
51 \\
34 \\
24 \\
17 \\
\end{array}$ & $\begin{array}{r}614 \\
357 \\
216 \\
135 \\
87 \\
58 \\
39 \\
27 \\
20 \\
14 \\
\end{array}$ \\
\hline \multicolumn{10}{|c|}{ Temperature $\left({ }^{\circ} \mathrm{C}\right)$ predicted for a viscosity of $100 \mathrm{P}$} \\
\hline & 1516 & 1401 & 1327 & 1285 & 1251 & 1276 & 1268 & 1270 & 1234 \\
\hline \multicolumn{10}{|c|}{ Measurement range $\left({ }^{\circ} \mathrm{C}\right)$} \\
\hline $\begin{array}{l}\text { Lower } \\
\text { Higher }\end{array}$ & $\begin{array}{l}1288 \\
1361 \\
\end{array}$ & $\begin{array}{l}1259 \\
1361 \\
\end{array}$ & $\begin{array}{l}1293 \\
1392 \\
\end{array}$ & $\begin{array}{l}1284 \\
1333 \\
\end{array}$ & $\begin{array}{l}1240 \\
1313 \\
\end{array}$ & $\begin{array}{l}1294 \\
1390 \\
\end{array}$ & $\begin{array}{l}1297 \\
1515 \\
\end{array}$ & $\begin{array}{l}1047 \\
1465 \\
\end{array}$ & $\begin{array}{l}1189 \\
1237 \\
\end{array}$ \\
\hline \multicolumn{10}{|c|}{ Conductivity in [S/cm] versus Temp. in Celsius } \\
\hline Temperature $\left({ }^{\circ} \mathrm{C}\right)$ & \multicolumn{9}{|c|}{ Conductivity in (S/cm) } \\
\hline $\begin{array}{l}1000 \\
1050 \\
1100 \\
1150 \\
1200 \\
1250 \\
1300 \\
1350 \\
1400 \\
1450 \\
1500 \\
\end{array}$ & $\begin{array}{c}- \\
- \\
- \\
- \\
0.005 \\
0.017 \\
0.028 \\
0.036 \\
0.043\end{array}$ & $\begin{array}{c}- \\
- \\
- \\
- \\
- \\
- \\
0.019 \\
0.028 \\
0.034\end{array}$ & $\begin{array}{c}- \\
- \\
- \\
- \\
- \\
0.063 \\
0.078 \\
0.091 \\
0.102 \\
0.111\end{array}$ & $\begin{array}{c}- \\
- \\
- \\
- \\
- \\
0.025 \\
0.050 \\
0.083\end{array}$ & $\begin{array}{c}- \\
- \\
- \\
- \\
0.018 \\
0.031 \\
0.043 \\
0.053\end{array}$ & $\begin{array}{c}- \\
- \\
0.084 \\
0.101 \\
0.120 \\
0.141 \\
0.164\end{array}$ & $\begin{array}{c}- \\
- \\
- \\
0.036 \\
0.052 \\
0.073 \\
0.101 \\
0.137 \\
0.182 \\
0.239\end{array}$ & $\begin{array}{c}- \\
- \\
- \\
0.298 \\
0.341 \\
0.387 \\
0.436 \\
0.487 \\
0.540 \\
0.596 \\
0.654 \\
\end{array}$ & $\begin{array}{c}- \\
0.642 \\
0.719 \\
0.799 \\
0.881 \\
0.966\end{array}$ \\
\hline \multicolumn{10}{|c|}{ Measurement range $\left({ }^{\circ} \mathrm{C}\right)$} \\
\hline $\begin{array}{l}\text { Lower } \\
\text { Higher }\end{array}$ & $\begin{array}{l}1274 \\
1347 \\
\end{array}$ & $\begin{array}{l}1307 \\
1383 \\
\end{array}$ & $\begin{array}{l}1271 \\
1427 \\
\end{array}$ & $\begin{array}{l}1224 \\
1291 \\
\end{array}$ & $\begin{array}{l}1206 \\
1275 \\
\end{array}$ & $\begin{array}{l}1170 \\
1260 \\
\end{array}$ & $\begin{array}{r}1210 \\
1390 \\
\end{array}$ & $\begin{array}{l}1178 \\
1415 \\
\end{array}$ & $\begin{array}{r}1118 \\
1194 \\
\end{array}$ \\
\hline
\end{tabular}


Table 2.12b

Viscosity and Conductivity of Glass Samples Prepared from Hanford Wastes

\begin{tabular}{|c|c|c|c|c|c|c|c|}
\hline NAME & HAN22B & HAN24 & HAN25 & HAN26 & HAN27 & HAN28 & HAN28A \\
\hline $\begin{array}{l}\text { Soil wt\% } \\
\text { Total } \mathrm{Na}_{2} \mathrm{O} \\
\text { Tank } \mathrm{Na}_{2} \mathrm{O} \\
\text { Additives \% }\end{array}$ & $\begin{array}{r}80.0 \% \\
23.0 \% \\
0.0 \% \\
20 \% \mathrm{Na}_{2} \mathrm{O} \\
\end{array}$ & $\begin{array}{r}67.0 \% \\
24.9 \% \\
22.4 \% \\
\text { None } \\
\end{array}$ & $\begin{array}{r}67.0 \% \\
23.8 \% \\
21.3 \% \\
5 \% \mathrm{Al}_{2} \mathrm{O}_{3} \\
\end{array}$ & $\begin{array}{r}63.7 \% \\
24.8 \% \\
22.5 \% \\
5 \% \mathrm{~B}_{2} \mathrm{O}_{3} \\
\end{array}$ & $\begin{array}{r}63.7 \% \\
22.9 \% \\
20.5 \% \\
5 \% \mathrm{SiO}_{2} \\
\end{array}$ & $\begin{array}{r}63.7 \% \\
20.9 \% \\
18.6 \% \\
5 \% \mathrm{ZrO}_{2} \\
\end{array}$ & $\begin{array}{r}63.7 \% \\
25.6 \% \\
23.2 \% \\
5 \% \mathrm{ZrO}_{2} \\
\end{array}$ \\
\hline \multicolumn{8}{|c|}{ Viscosity in [Poise] versus Temperature in Celsius } \\
\hline Temperature $\left({ }^{\circ} \mathrm{C}\right)$ & \multicolumn{7}{|c|}{ Viscosity in (Poise) } \\
\hline $\begin{array}{l}1050 \\
1100 \\
1150 \\
1200 \\
1250 \\
1300 \\
1350 \\
1400 \\
1450 \\
1500 \\
\end{array}$ & $\begin{array}{r}1004 \\
547 \\
320 \\
198 \\
129 \\
87 \\
61 \\
44 \\
33 \\
25 \\
\end{array}$ & $\begin{array}{r}1187 \\
679 \\
404 \\
249 \\
158 \\
103 \\
70 \\
48 \\
34 \\
24 \\
\end{array}$ & $\begin{array}{r}2885 \\
1550 \\
870 \\
507 \\
307 \\
192 \\
123 \\
81 \\
55 \\
38 \\
\end{array}$ & $\begin{array}{r}473 \\
278 \\
171 \\
110 \\
74 \\
51 \\
36 \\
26 \\
20 \\
15 \\
\end{array}$ & $\begin{array}{r}1875 \\
1013 \\
580 \\
350 \\
220 \\
144 \\
97 \\
68 \\
48 \\
36 \\
\end{array}$ & $\begin{array}{r}4195 \\
2078 \\
1082 \\
588 \\
333 \\
196 \\
119 \\
74 \\
48 \\
31 \\
\end{array}$ & $\begin{array}{r}1138 \\
579 \\
319 \\
188 \\
117 \\
76\end{array}$ \\
\hline \multicolumn{8}{|c|}{ Measurement range $\left({ }^{\circ} \mathrm{C}\right)$} \\
\hline $\begin{array}{l}\text { Lower } \\
\text { Higher }\end{array}$ & $\begin{array}{l}1009 \\
1525 \\
\end{array}$ & $\begin{array}{l}1109 \\
1473 \\
\end{array}$ & $\begin{array}{l}1140 \\
1473 \\
\end{array}$ & $\begin{array}{l}1001 \\
1440 \\
\end{array}$ & $\begin{array}{l}1041 \\
1439 \\
\end{array}$ & $\begin{array}{r}1129 \\
1462 \\
\end{array}$ & $\begin{array}{l}1132 \\
1323 \\
\end{array}$ \\
\hline \multicolumn{8}{|c|}{ Conductivity in $[\mathrm{S} / \mathrm{cm}]$ versus Temperature in Celsius } \\
\hline Temperature $\left({ }^{\circ} \mathrm{C}\right)$ & \multicolumn{7}{|c|}{ Conductivity in [S/cm] } \\
\hline $\begin{array}{l}1000 \\
1050 \\
1100 \\
1150 \\
1200 \\
1250 \\
1300 \\
1350 \\
1400 \\
1450 \\
1500 \\
\end{array}$ & $\begin{array}{l}0.539 \\
0.625 \\
0.718 \\
0.816 \\
0.920 \\
1.029 \\
1.143 \\
1.261 \\
1.383 \\
1.509 \\
1.638 \\
\end{array}$ & $\begin{array}{l}0.428 \\
0.511 \\
0.603 \\
0.703 \\
0.810 \\
0.926 \\
1.049 \\
1.180 \\
1.318 \\
1.462 \\
1.612 \\
\end{array}$ & $\begin{array}{l}0.447 \\
0.532 \\
0.625 \\
0.727 \\
0.836 \\
0.953 \\
1.077 \\
1.209 \\
1.347 \\
1.491 \\
1.642 \\
\end{array}$ & $\begin{array}{l}0.551 \\
0.631 \\
0.716 \\
0.805 \\
0.899 \\
0.995 \\
1.096 \\
1.199 \\
1.304 \\
1.412 \\
1.523 \\
\end{array}$ & $\begin{array}{l}0.574 \\
0.663 \\
0.758 \\
0.859 \\
0.965 \\
1.075 \\
1.191 \\
1.310 \\
1.433 \\
1.559 \\
1.689 \\
\end{array}$ & $\begin{array}{l}0.328 \\
0.397 \\
0.474 \\
0.558 \\
0.651 \\
0.752 \\
0.860 \\
0.975 \\
1.098 \\
1.227 \\
1.363 \\
\end{array}$ & $\begin{array}{l}0.568 \\
0.644 \\
0.724 \\
0.807 \\
0.894 \\
0.985 \\
1.078 \\
1.175\end{array}$ \\
\hline Measurement range & & & & & & & \\
\hline $\begin{array}{l}\text { Lower } \\
\text { Higher }\end{array}$ & $\begin{array}{r}988 \\
1466 \\
\end{array}$ & $\begin{array}{r}952 \\
1450 \\
\end{array}$ & $\begin{array}{l}1149 \\
1484 \\
\end{array}$ & $\begin{array}{l}1087 \\
1462 \\
\end{array}$ & $\begin{array}{l}1146 \\
1474 \\
\end{array}$ & $\begin{array}{l}1161 \\
1442 \\
\end{array}$ & $\begin{array}{l}1187 \\
1468 \\
\end{array}$ \\
\hline
\end{tabular}




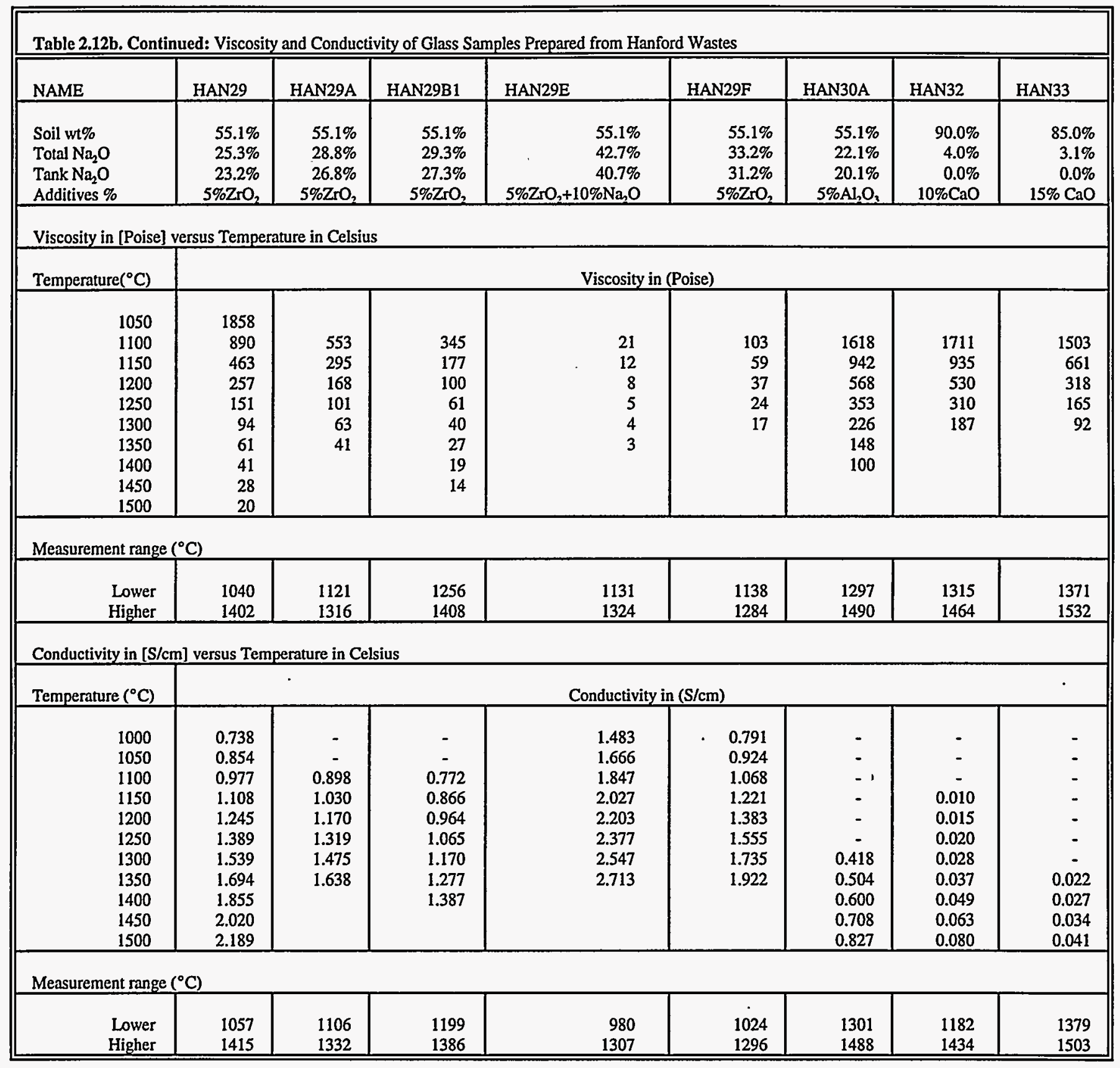




\begin{tabular}{|c|c|c|c|c|c|c|}
\hline NAME & HAN34 & HAN35 & HAN36 & HAN37 & HAN38 & HAN39 \\
\hline $\begin{array}{l}\text { Soil wt\% } \\
\text { Total } \mathrm{Na}_{2} \mathrm{O} \\
\text { Tank } \mathrm{Na}_{2} \mathrm{O} \\
\text { Additives \% }\end{array}$ & $\begin{array}{r}85.0 \% \\
8.1 \% \\
0.0 \% \\
10 \% \mathrm{CaO} / \\
5 \% \mathrm{Na}_{2} \mathrm{O} \\
\end{array}$ & $\begin{array}{r}90.0 \% \\
3.2 \% \\
0.0 \% \\
10 \% \mathrm{CaF}\end{array}$ & $\begin{array}{r}63.7 \% \\
27.3 \% \\
25.0 \% \\
5 \% \mathrm{Al}_{2} \mathrm{O}_{3}\end{array}$ & $\begin{array}{r}63.7 \% \\
27.0 \% \\
24.6 \% \\
5 \% \mathrm{ZrO}_{2}\end{array}$ & $\begin{array}{r}60.3 \% \\
23.4 \% \\
21.2 \% \\
5 \% \mathrm{Al}_{2} \mathrm{O}_{3} \\
+5 \% \mathrm{ZrO}_{2} \\
\end{array}$ & $\begin{array}{r}0.0 \% \\
22.5 \% \\
22.5 \% \\
63.7 \% \mathrm{SiO}_{2} \\
+5 \% \mathrm{Al}_{2} \mathrm{O}_{3} \\
\end{array}$ \\
\hline \multicolumn{7}{|c|}{ Viscosity in [Poise] versus Temperature in Celsius } \\
\hline Temperature $\left({ }^{\circ} \mathrm{C}\right)$ & \multicolumn{6}{|c|}{ Viscosity in (Poise) } \\
\hline $\begin{array}{l}1050 \\
1100 \\
1150 \\
1200 \\
1250 \\
1300 \\
1350 \\
1400 \\
1450 \\
1500 \\
\end{array}$ & $\begin{array}{r}1018 \\
549 \\
315 \\
191 \\
121 \\
80 \\
\end{array}$ & $\begin{array}{r}1652 \\
881 \\
507 \\
311 \\
201 \\
136 \\
\end{array}$ & $\begin{array}{r}719 \\
414 \\
250 \\
157 \\
102 \\
69\end{array}$ & $\begin{array}{r}481 \\
253 \\
145 \\
88 \\
57 \\
39\end{array}$ & $\begin{array}{r}1259 \\
632 \\
342 \\
197 \\
120 \\
77\end{array}$ & $\begin{array}{r}773 \\
471 \\
297 \\
192 \\
128 \\
88\end{array}$ \\
\hline \multicolumn{7}{|c|}{ Measurement range $\left({ }^{\circ} \mathrm{C}\right)$} \\
\hline $\begin{array}{l}\text { Lower } \\
\text { Higher }\end{array}$ & $\begin{array}{l}1275 \\
1459 \\
\end{array}$ & $\begin{array}{l}1268 \\
1464 \\
\end{array}$ & $\begin{array}{l}1144 \\
1313 \\
\end{array}$ & $\begin{array}{l}1139 \\
1326 \\
\end{array}$ & $\begin{array}{l}1146 \\
1315 \\
\end{array}$ & $\begin{array}{l}1171 \\
1365 \\
\end{array}$ \\
\hline \multicolumn{7}{|c|}{ Conductivity in [S/cm] versus Temperature in Celsius } \\
\hline Temperature $\left({ }^{\circ} \mathrm{C}\right)$ & \multicolumn{6}{|c|}{ Conductivity in $(\mathrm{S} / \mathrm{cm})$} \\
\hline $\begin{array}{l}1000 \\
1050 \\
1100 \\
1150 \\
1200 \\
1250 \\
1300 \\
1350 \\
1400 \\
1450 \\
1500 \\
\end{array}$ & $\begin{array}{c}- \\
- \\
- \\
- \\
- \\
0.073 \\
0.094 \\
0.120 \\
0.150 \\
0.185 \\
0.226 \\
\end{array}$ & $\begin{array}{c}- \\
- \\
- \\
- \\
- \\
0.025 \\
0.028 \\
0.033 \\
0.037 \\
0.041 \\
0.046 \\
\end{array}$ & $\begin{array}{c}- \\
- \\
0.628 \\
0.698 \\
0.771 \\
0.845 \\
0.922 \\
0.999\end{array}$ & $\begin{array}{c}- \\
- \\
0.631 \\
0.680 \\
0.730 \\
0.779 \\
0.828 \\
0.877 \\
0.926\end{array}$ & $\begin{array}{c}- \\
- \\
0.374 \\
0.442 \\
0.517 \\
0.598 \\
0.686 \\
0.780\end{array}$ & $\begin{array}{c}- \\
- \\
0.464 \\
0.522 \\
0.582 \\
0.645 \\
0.710 \\
0.776 \\
0.845\end{array}$ \\
\hline \multicolumn{7}{|c|}{ Measurement range $\left({ }^{\circ} \mathrm{C}\right)$} \\
\hline $\begin{array}{l}\text { Lower } \\
\text { Higher }\end{array}$ & $\begin{array}{l}1256 \\
1494\end{array}$ & $\begin{array}{l}1295 \\
1489\end{array}$ & $\begin{array}{l}1146 \\
1329\end{array}$ & $\begin{array}{l}1156 \\
1377\end{array}$ & $\begin{array}{l}1165 \\
1335\end{array}$ & $\begin{array}{l}1171 \\
1365\end{array}$ \\
\hline
\end{tabular}


Table 2.13a

Results from ICPMS Analysis of Glass Dissolutions

(The suffices M1; M2, etc. to the glass name refer to separate dissolutions)

\begin{tabular}{|c|c|c|c|c|c|c|c|c|}
\hline ELEM. & RUN-1 & RUN-2 & RUN-3 & RUN-4 & $\begin{array}{l}\text { AVG } \\
(\mathrm{ppb})\end{array}$ & $\begin{array}{l}\text { STD. } \\
\text { DEV. }\end{array}$ & $\begin{array}{l}\text { RSD } \\
(\%)\end{array}$ & $\begin{array}{l}\mathrm{OCS}^{*} \\
\text { (ppm) }\end{array}$ \\
\hline $\mathrm{Cr}$ & 206.9 & 220.1 & 224.5 & 212 & 215.9 & 6.9 & 3.2 & 426 \\
\hline $\mathrm{Ni}$ & & & & & $<30$ & & & $<60$ \\
\hline $\mathrm{Cu}$ & 89 & 102 & 65 & 54 & 77.2 & 18.9 & 24.5 & 152 \\
\hline $\mathrm{Sr}$ & 176 & 173 & 172 & 184 & 175.9 & 4.8 & 2.7 & 347 \\
\hline Cs & 34 & 33 & 35 & 33 & 33.6 & 0.6 & 1.6 & 66 \\
\hline $\mathrm{Ba}$ & 290 & 299 & 285 & 295 & 292.2 & 5.5 & 1.9 & 576 \\
\hline $\mathrm{Pb}$ & 40 & 42 & 38 & 37 & 39.3 & 2.0 & 5.0 & 78 \\
\hline $\mathrm{Bi}$ & 81 & 85 & 85 & 88 & 84.5 & 2.5 & 3.0 & 167 \\
\hline $\mathrm{U}-238$ & 219 & 222 & 212 & 210 & 215.8 & 5.0 & 2.3 & 426 \\
\hline \multicolumn{9}{|c|}{ SAMPLE NAME: HAN-29FM3 (Dissolved Solid $=0.10035 \mathrm{~g}$ ) } \\
\hline ELEM. & RUN-1 & RUN-2 & RUN-3 & RUN-4 & $\begin{array}{l}\text { AVG } \\
(\mathrm{ppb})\end{array}$ & $\begin{array}{l}\text { STD. } \\
\text { DEV. }\end{array}$ & $\begin{array}{l}\text { RSD } \\
(\%)\end{array}$ & $\begin{array}{c}\text { OCS } \\
\text { (ppm) }\end{array}$ \\
\hline $\mathrm{Cr}$ & 198 & 204 & 202 & 216 & 204.9 & 6.5 & 3.2 & 408 \\
\hline $\mathrm{Ni}$ & 41 & 24 & 23 & 28 & 29.0 & 7.3 & 25.2 & 58 \\
\hline $\mathrm{Cu}$ & 44 & 44 & 68 & 55 & 52.9 & 9.7 & 18.4 & 105 \\
\hline $\mathrm{Sr}$ & 159 & 160 & 171 & 166 & 164.1 & 4.8 & 2.9 & 327 \\
\hline Cs & 29 & 31 & 31 & 30 & 30.3 & 0.9 & 3.1 & 60 \\
\hline $\mathrm{Ba}$ & 282 & 291 & 289 & 283 & 286.4 & 3.9 & 1.4 & 571 \\
\hline $\mathrm{Pb}$ & 37 & 34 & 37 & 37 & 36.1 & 1.3 & 3.7 & 72 \\
\hline $\mathrm{Bi}$ & 82 & 85 & 87 & 86 & 84.9 & 2.0 & 2.3 & 169 \\
\hline U-238 & 205 & 216 & 206 & 210 & 209.2 & 4.4 & 2.1 & 417 \\
\hline
\end{tabular}

- OCS = Original concentration in solid (glass) 


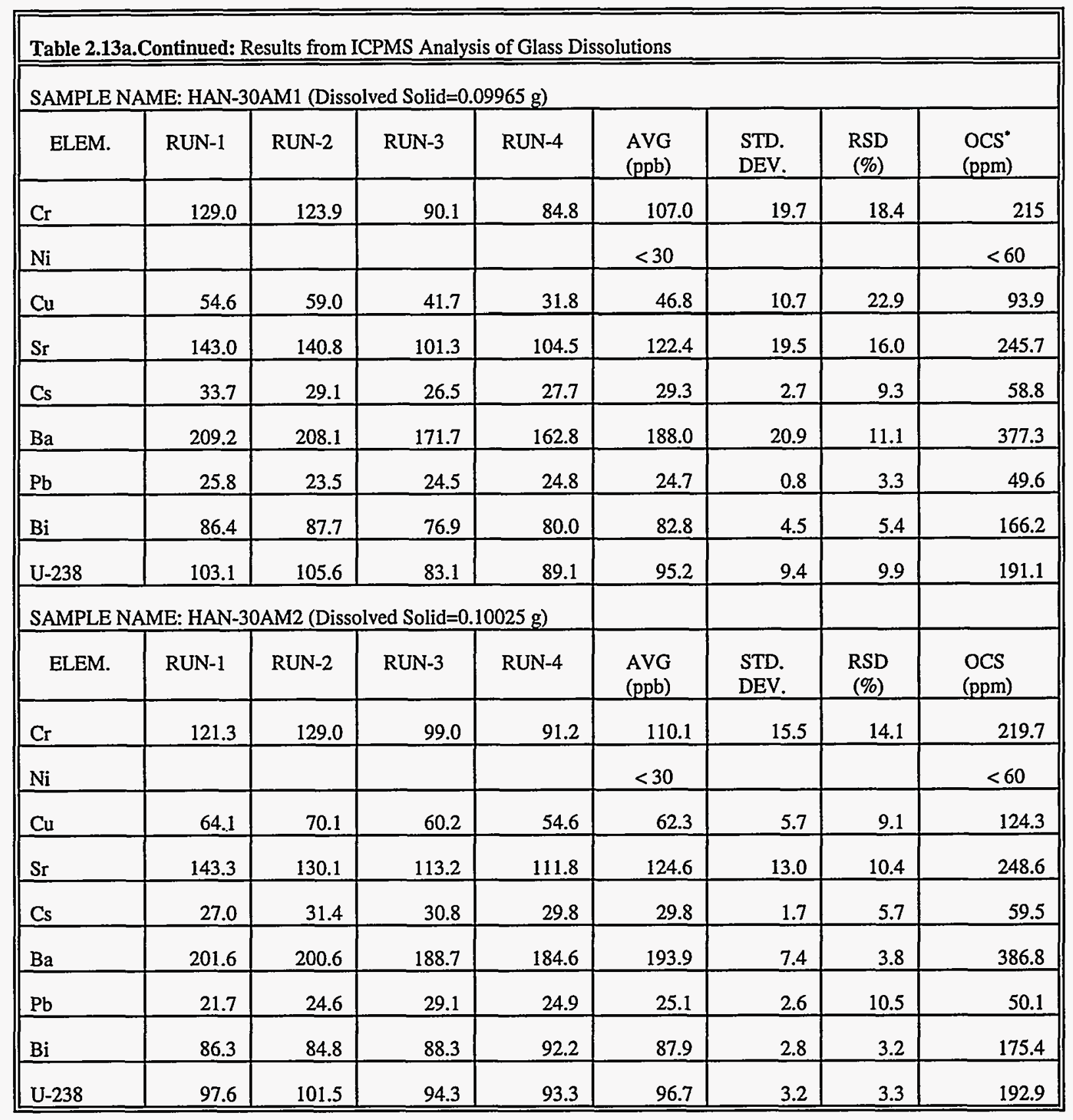

'OCS = Original concentration in solid (glass) 


\begin{tabular}{|c|c|c|c|c|c|c|c|c|}
\hline \multicolumn{9}{|c|}{ SAMPLE NAME: HAN-30AM3 (Dissolved Solid=0.10000 g) } \\
\hline ELEM. & RUN-1 & RUN-2 & RUN-3 & RUN-4 & $\begin{array}{l}\text { AVG } \\
(\mathrm{ppb})\end{array}$ & $\begin{array}{l}\text { STD. } \\
\text { DEV. }\end{array}$ & $\begin{array}{c}\text { RSD } \\
(\%) \\
\end{array}$ & $\begin{array}{l}\mathrm{OCS}^{*} \\
(\mathrm{ppm}) \\
\end{array}$ \\
\hline $\mathrm{Cr}$ & 142.7 & 131.3 & 99.8 & 99.0 & 118.2 & 19.2 & 16.3 & 236.4 \\
\hline $\mathrm{Ni}$ & & & & & $<30$ & & & $<60$ \\
\hline $\mathrm{Cu}$ & 63.5 & 56.0 & 33.2 & 31.6 & 46.1 & 13.9 & 30.3 & 92.2 \\
\hline $\mathrm{Sr}$ & 136.3 & 137.5 & 105.3 & 100.6 & 119.9 & 17.1 & 14.2 & 239.8 \\
\hline Cs & 31.8 & 31.6 & 30.0 & 30.5 & 31.0 & 0.7 & 2.4 & 62.0 \\
\hline $\mathrm{Ba}$ & 208.2 & 201.4 & 186.0 & 187.7 & 195.8 & 9.3 & 4.8 & 391.6 \\
\hline $\mathrm{Pb}$ & 22.6 & 22.2 & 26.2 & 25.0 & 24.0 & 1.7 & 6.9 & 48.0 \\
\hline $\mathrm{Bi}$ & 84.9 & 85.9 & 84.3 & 86.0 & 85.3 & 0.7 & 0.8 & 170.6 \\
\hline $\mathrm{U}-238$ & 101.1 & 103.6 & 93.2 & 90.9 & 97.2 & 5.3 & 5.4 & 194.4 \\
\hline \multicolumn{9}{|c|}{ SAMPLE NAME: HAN-30BM1 (Dissolved Solid=0.09927 g) } \\
\hline ELEM. & RUN-1 & RUN-2 & RUN-3 & RUN-4 & $\begin{array}{l}\text { AVG } \\
(\mathrm{ppb})\end{array}$ & $\begin{array}{l}\text { STD. } \\
\text { DEV. }\end{array}$ & $\begin{array}{c}\text { RSD } \\
(\%)\end{array}$ & $\begin{array}{l}\text { OCS } \\
(\mathrm{ppm})\end{array}$ \\
\hline $\mathrm{Cr}$ & 145 & 142 & 114 & 107 & 127.0 & 16.7 & 13.2 & 255.9 \\
\hline $\mathrm{Ni}$ & 99 & 107 & 98 & 83 & 96.7 & 9.0 & 9.3 & 194.8 \\
\hline $\mathrm{Cu}$ & 55 & 45 & 40 & 46 & 46.4 & 5.2 & 11.2 & 93.5 \\
\hline $\mathrm{Sr}$ & 176 & 179 & 160 & 160 & 168.8 & 8.7 & 5.1 & 340.1 \\
\hline Cs & 32 & 33 & 31 & 30 & 31.6 & 0.9 & 3.0 & 63.7 \\
\hline $\mathrm{Ba}$ & 265 & 259 & 267 & 256 & 261.6 & 4.5 & 1.7 & 527.0 \\
\hline $\mathrm{Pb}$ & - 26 & 26 & 30 & 30 & 27.9 & 2.1 & 7.4 & 56.2 \\
\hline $\mathrm{Bi}$ & 102 & 105 & 117 & 115 & 109.5 & 6.2 & 5.7 & 220.6 \\
\hline U-238 & 719 & 734 & 695 & 676 & 706.1 & 22.2 & 3.1 & $1,422.6$ \\
\hline
\end{tabular}

"OCS = Original concentration in solid (glass). 


\begin{tabular}{|c|c|c|c|c|c|c|c|c|}
\hline \multicolumn{9}{|c|}{ SAMPLE NAME: HAN-30BM2 (Dissolved Solid=0.10005 g) } \\
\hline ELEM. & RUN-1 & RUN-2 & RUN-3 & RUN-4 & $\begin{array}{l}\text { AVG } \\
(\mathrm{ppb})\end{array}$ & $\begin{array}{l}\text { STD. } \\
\text { DEV. }\end{array}$ & $\begin{array}{c}\text { RSD } \\
(\%)\end{array}$ & $\begin{array}{l}\mathrm{OCS}^{*} \\
\text { (ppm) }\end{array}$ \\
\hline $\mathrm{Cr}$ & 136.4 & 136.8 & 121.7 & 117.9 & 128.2 & 8.5 & 6.6 & 256.3 \\
\hline $\mathrm{Ni}$ & 95.5 & 96.7 & 87.3 & 81.1 & 90.2 & 6.4 & 7.0 & 180.3 \\
\hline $\mathrm{Cu}$ & 51.3 & 40.4 & 63.5 & 51.1 & 51.6 & 8.2 & 15.9 & 103.1 \\
\hline $\mathrm{Sr}$ & 173.4 & 181.4 & 178.0 & 162.2 & 173.8 & 7.2 & 4.2 & 347.4 \\
\hline Cs & 32.9 & 34.3 & 32.8 & 36.2 & 34.1 & 1.4 & 4.0 & 68.2 \\
\hline $\mathbf{B a}$ & 253.3 & 266.0 & 274.2 & 280.7 & 268.6 & 10.2 & 3.8 & 536.9 \\
\hline $\mathrm{Pb}$ & 19.8 & 24.7 & 29.4 & 29.5 & 25.9 & 4.0 & 15.5 & 51.8 \\
\hline $\mathrm{Bi}$ & 104.5 & 104.7 & 123.1 & 119.7 & 113.0 & 8.5 & 7.5 & 225.9 \\
\hline U-238 & 708.5 & 717.3 & 714.8 & 720.5 & 715.3 & 4.4 & 0.6 & 1430 \\
\hline \multicolumn{9}{|c|}{ SAMPLE NAME: HAN-30BM3 (Dissolved Solid=0.09935 g) } \\
\hline ELEM. & RUN-1 & RUN-2 & RUN-3 & RUN-4 & $\begin{array}{l}\text { AVG } \\
\text { (ppb) }\end{array}$ & $\begin{array}{l}\text { STD. } \\
\text { DEV. }\end{array}$ & $\begin{array}{c}\text { RSD } \\
(\%) \\
\end{array}$ & $\begin{array}{r}\text { OCS } \\
+\quad(\mathrm{ppm}) \\
\end{array}$ \\
\hline $\mathrm{Cr}$ & 150.3 & 127.7 & 119.1 & 113.4 & 127.6 & 14.0 & 11.0 & 256.9 \\
\hline $\mathrm{Ni}$ & 103.5 & 77.0 & 91.7 & 89.9 & 90.5 & 9.4 & 10.4 & 182.2 \\
\hline $\mathrm{Cu}$ & 59.1 & 61.9 & 50.7 & 54.1 & 56.5 & 4.3 & 7.7 & 113.7 \\
\hline $\mathrm{Sr}$ & 179.4 & 171.4 & 171.4 & 175.1 & 174.3 & 3.3 & 1.9 & 350.9 \\
\hline Cs & 30.0 & 29.3 & 35.4 & 32.8 & 31.9 & 2.4 & 7.6 & 64.2 \\
\hline $\mathrm{Ba}$ & 256.3 & 253.8 & 259.8 & 270.4 & 260.1 & 6.3 & 2.4 & 523.6 \\
\hline $\mathrm{Pb}$ & 28.9 & 31.5 & 33.9 & 36.3 & 32.7 & 2.8 & 8.4 & 65.8 \\
\hline $\mathrm{Bi}$ & 102.1 & 105.3 & 117.9 & 121.6 & 111.7 & 8.2 & 7.3 & 224.9 \\
\hline U-238 & 708.4 & 711.0 & 705.9 & 717.3 & 710.7 & 4.2 & 0.6 & 1430 \\
\hline
\end{tabular}

${ }^{\circ} \mathrm{OCS}=$ Original concentration in solid (glass). 


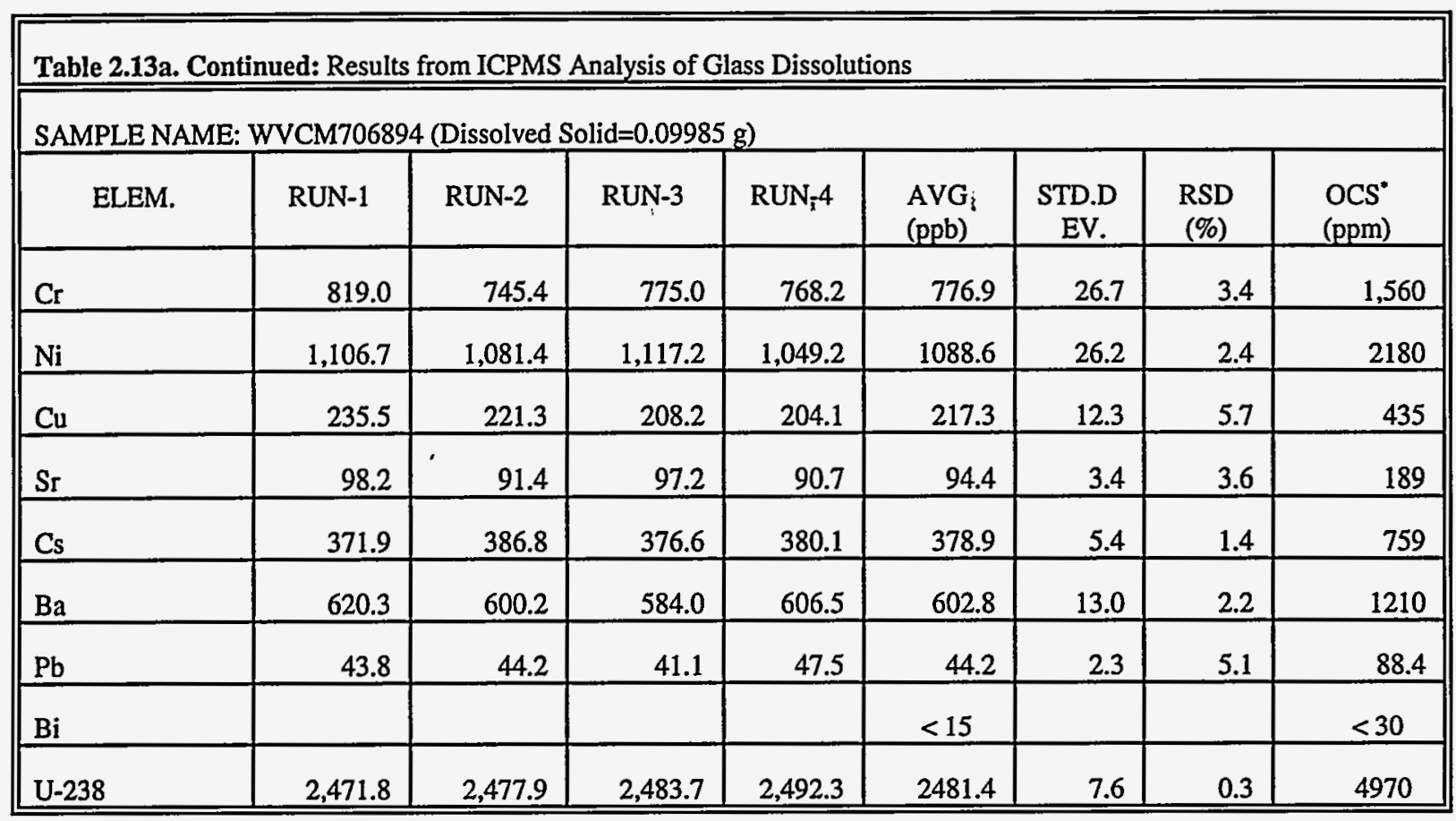

'OCS = Original concentration in solid (glass). 


\begin{tabular}{|c|c|c|c|c|c|c|c|c|}
\hline \multicolumn{9}{|l|}{ Tc-99 } \\
\hline Sample ID & RUN-1 & RUN-2 & RUN-3 & RUN-4 & $\begin{array}{l}\text { AVG } \\
(\mathrm{ppb})\end{array}$ & STD.DEV. & $\begin{array}{c}\text { RSD } \\
(\%) \\
\end{array}$ & $\begin{array}{l}\mathrm{OCS}^{*} \\
(\mathrm{ppm})\end{array}$ \\
\hline HAN24M4 & 1.36 & 1.47 & 1.2 & 1.2 & 1.31 & 0.23 & 8.74 & 2.62 \\
\hline HAN24M5 & 1.28 & 1.3 & 1.14 & 1.105 & 1.205 & 0.17 & 7.04 & 2.41 \\
\hline HAN25M4 & 1.71 & 1.75 & 1.56 & 1.55 & 1.645 & 0.18 & 5.4 & 3.29 \\
\hline HAN25M5 & 1.735 & 1.705 & 1.55 & 1.56 & 1.64 & 0.17 & 5.08 & 3.28 \\
\hline HAN26M1 & 1.635 & 1.605 & 1.63 & 1.61 & 1.62 & 0.03 & 0.79 & 3.24 \\
\hline HAN26M2 & 1.63 & 1.63 & 1.59 & 1.605 & 1.615 & 0.03 & 1.06 & 3.23 \\
\hline HAN26M3 & 1.73 & 1.675 & 1.605 & 1.605 & 1.655 & 0.1 & 3.17 & 3.31 \\
\hline HAN27M4 & 2.135 & 2.11 & 1.97 & 1.92 & 2.035 & 0.18 & 4.47 & 4.07 \\
\hline HAN27M5 & 2.12 & 2.035 & 1.93 & 1.955 & 2.01 & 0.15 & 3.7 & 4.02 \\
\hline HAN27M6 & 2.115 & 2.18 & 2.13 & 2.135 & 2.14 & 0.05 & 1.13 & 4.28 \\
\hline HAN28M4 & 1.48 & 1.425 & 1.255 & 1.26 & 1.355 & 0.2 & 7.34 & 2.71 \\
\hline HAN28M5 & 1.405 & 1.44 & 1.25 & 1.275 & 1.345 & 0.16 & 6.07 & 2.69 \\
\hline HAN28M6 & 1.415 & 1.47 & 1.415 & 1.425 & 1.43 & 0.05 & 1.59 & 2.86 \\
\hline HAN-28Am1 & 1.56 & 1.59 & 1.72 & 1.60 & 1.618 & 0.061 & 3.770 & 3.23 \\
\hline HAN-28Am2 & 1.57 & 1.57 & 1.62 & 1.60 & 1.590 & 0.021 & 1.334 & 3.16 \\
\hline HAN-28Am3 & 1.55 & 1.61 & 1.58 & 1.60 & 1.585 & 0.023 & 1.446 & 3.15 \\
\hline HAN-28Bml & 1.20 & 1.19 & 1.23 & 1.25 & 1.218 & 0.024 & 1.959 & 2.43 \\
\hline HAN-28Bm2 & 1.07 & 1.09 & 1.16 & 1.19 & 1.128 & 0.049 & 4.362 & 2.25 \\
\hline HAN-28Bm3 & 1.16 & 1.16 & 1.27 & 1.25 & 1.210 & 0.050 & 4.173 & 2.40 \\
\hline $\mathrm{HAN}-28 \mathrm{Cml}$ & 1.05 & 1.01 & 1.00 & 1.01 & 1.018 & 0.019 & 1.887 & 2.04 \\
\hline $\mathrm{HAN}-28 \mathrm{Cm} 2$ & 1.05 & 1.00 & 1.05 & 1.06 & 1.040 & 0.023 & 2.255 & 2.07 \\
\hline $\mathrm{HAN}-28 \mathrm{Cm} 3$ & 0.81 & 0.81 & 1.05 & 1.04 & 0.928 & 0.118 & 12.674 & 1.85 \\
\hline HAN-29Aml & 1.48 & 1.46 & 1.42 & 1.47 & 1.458 & 0.023 & 1.563 & 2.90 \\
\hline HAN-29Am2 & 1.46 & 1.51 & 1.48 & 1.54 & 1.498 & 0.030 & 2.024 & 3.00 \\
\hline HAN-29Am3 & 1.47 & 1.50 & 1.53 & 1.57 & 1.518 & 0.037 & 2.438 & 3.04 \\
\hline HAN29MI & 1.255 & 1.195 & 1.26 & 1.26 & 1.245 & 0.06 & 2.21 & 2.49 \\
\hline HAN29M2 & 1.16 & 1.16 & 1.17 & 1.175 & 1.165 & 0.01 & 0.56 & 2.33 \\
\hline HAN29M3 & 1.555 & 1.28 & 1.215 & 1.22 & 1.32 & 0.28 & 10.59 & 2.64 \\
\hline HAN-29FM1 & 1.06 & 1.06 & 1.04 & 1.05 & 1.05 & 0.01 & 0.76 & 2.09 \\
\hline HAN-29FM2 & 1.04 & 1.07 & 1.03 & 1.03 & 1.04 & 0.02 & 1.66 & 2.06 \\
\hline
\end{tabular}




\begin{tabular}{|c|c|c|c|c|c|c|c|c|}
\hline \multicolumn{9}{|l|}{ Tc-99 } \\
\hline Sample ID & RUN-1 & RUN-2 & RUN-3 & RUN-4 : & $\begin{array}{l}\text { AVG } \\
(\mathrm{ppb})\end{array}$ & STD.DEV. & $\begin{array}{l}\text { RSD } \\
(\%)\end{array}$ & $\begin{array}{l}\mathrm{OCS}^{\circ} \\
(\mathrm{ppm})\end{array}$ \\
\hline HAN-29FM3 & 1.05 & 1.03 & 1.05 & 1.01 & 1.03 & 0.02 & 1.49 & 2.06 \\
\hline HAN30M1 & 1.22 & 1.225 & 1.28 & 1.295 & 1.255 & 0.07 & 2.63 & 2.51 \\
\hline HAN30M2 & 1.16 & 1.18 & 1.21 & 1.245 & 1.2 & 0.06 & 2.68 & 2.4 \\
\hline HAN30M3 & 1.285 & 1.215 & 1.24 & 1.27 & 1.255 & 0.05 & 2.16 & 2.51 \\
\hline HAN-30Aml & 1.15 & 1.18 & 1.13 & 1.14 & 1.150 & 0.019 & 1.627 & 2.31 \\
\hline HAN-30Am2 & 1.21 & 1.28 & 1.17 & 1.09 & 1.188 & 0.069 & 5.785 & 2.37 \\
\hline HAN-30Am3 & 1.18 & 1.16 & 1.07 & 1.10 & 1.128 & 0.044 & 3.935 & 2.26 \\
\hline HAN-30Bm1 & 1.07 & 1.08 & 1.45 & 1.43 & 1.258 & 0.183 & 14.527 & 2.53 \\
\hline HAN-30Bm2 & 1.09 & 1.04 & 1.42 & 1.43 & 1.245 & 0.181 & 14.530 & 2.49 \\
\hline HAN-30Bm3 & 1.05 & 1.03 & 1.42 & 1.43 & 1.233 & 0.193 & 15.632 & 2.48 \\
\hline WVCM706894 & & & & & $<3.7$ & & & $<7.4$ \\
\hline
\end{tabular}

OCS = Original concentration in solid (glass).

Note: (1)All above data except the ones for Tc-99 were obtained with a dilution factor of 50; the data larger than $2000 \mathrm{ppb}$ in the column of "AVG (ppb)" should be regarded as semi-quantitative results.

(2) The data for Tc-99 were obtained with a dilution factor of 25 .

(3) Tc-99 has possible interference from Ru. However, after examining the spectra, no significant interference was found in above samples except the one of WVCM706894. (Standard glass containing Ru)

(4) $\mathrm{Ni}-60$ has possible interference from $\mathrm{Co}$ and $\mathrm{CaO}$. $\mathrm{Cu}-63$ has possible interference from $\mathrm{TiO}, \mathrm{ArNa}$, and $\mathrm{PO}$. $\mathrm{Ba}-138$ has possible interference from $\mathrm{Ce}$. Therefore, the true contents of $\mathrm{Ni}, \mathrm{Cu}$ and $\mathrm{Ba}$ could be lower than the corresponding given values here.

(5) The OCS concentrations of $C d$ are lower than $40 \mathrm{ppm}$ in above samples (data for $\mathrm{Cd}$ are not available for the HAN-28CM series and HAN-29AM series samples). 
Table 2.13b

Sodium and Technetium Retention in Crucible Melts from Tank Waste + Soil

\begin{tabular}{|c|c|c|c|c|c|}
\hline $\begin{array}{c}\text { Sample } \\
\text { Name }\end{array}$ & $\begin{array}{c}\text { Crucible } \\
\text { Type }\end{array}$ & $\begin{array}{c}\text { Retention } \\
\% \mathrm{Na}_{2} \mathrm{O}\end{array}$ & $\begin{array}{c}\text { Melt } \\
\text { Temperature } \\
\left({ }^{\circ} \mathrm{C}\right) \\
\end{array}$ & $\begin{array}{c}\text { Treatment } \\
\text { Time (min.) } \\
\left(>1200^{\circ} \mathrm{C}\right)\end{array}$ & $\begin{array}{l}\%^{99} \mathrm{Tc} \\
\text { retention }\end{array}$ \\
\hline HAN36 & $\mathrm{Pt}-\mathrm{Au}$ & $95.52 \%$ & 1200 & 70 & N.A. \\
\hline HAN37 & Pt-Au & $94.34 \%$ & 1200 & 70 & N.A. \\
\hline HAN29E & $\mathrm{Pt}-\mathrm{Au}$ & $94.00 \%$ & 1200 & 60 & N.A \\
\hline HAN29F & $\mathrm{Pt}-\mathrm{Au}$ & $93.61 \%$ & 1200 & 70 & $21.0 \%$ \\
\hline HAN28A & Quartz & $89.39 \%$ & 1200 & 70 & $32.0 \%$ \\
\hline HAN26 & Quartz & $86.81 \%$ & 1200 & 70 & $32.8 \%$ \\
\hline HAN38 & $\mathrm{Pt}-\mathrm{Au}$ & $86.47 \%$ & 1300 & 100 & N.A. \\
\hline HAN39 & $\mathrm{Pt}-\mathrm{Au}$ & $85.83 \%$ & 1300 & 65 & N.A. \\
\hline HAN25 & Quartz & $83.26 \%$ & 1200 & 75 & $32.9 \%$ \\
\hline HAN29B1 & Quartz & $82.67 \%$ & 1200 & 70 & N.A \\
\hline HAN24 & Quartz & $82.56 \%$ & 1200 & 75 & $25.2 \%$ \\
\hline HAN29A & Quartz & $81.19 \%$ & 1200 & 70 & $29.0 \%$ \\
\hline HAN28B & Quartz & $81.14 \%$ & 1300 & 100 & $24.0 \%$ \\
\hline HAN27 & Quartz & $80.03 \%$ & 1200 & 75 & $40.5 \%$ \\
\hline HAN30B & Quartz & $79.25 \%$ & 1200 & 100 & $25.0 \%$ \\
\hline HAN29B & Quartz & $77.38 \%$ & 1400 & 100 & N.A \\
\hline HAN29B2 & Quartz & $76.23 \%$ & 1300 & 100 & N.A \\
\hline HAN28 & Quartz & $73.12 \%$ & 1200 & 150 & $27.0 \%$ \\
\hline HAN30C & Quartz & $71.92 \%$ & 1400 & 190 & N.A. \\
\hline HAN29 & Quartz & $71.21 \%$ & 1200 & 120 & $49.7 \%$ \\
\hline HAN30A & Quartz & $62.17 \%$ & 1400 & 90 & $22.3 \%$ \\
\hline
\end{tabular}

N.A. $=$ Not Analyzed 
Table 2.14

Concentration of RCRA Metals (ppm) in TCLP Leachates from

Hanford Glasses

\begin{tabular}{|c|c|c|c|c|c|c|}
\hline $\begin{array}{c}\text { GLASS } \\
\text { NAME } \\
\end{array}$ & $\mathbf{B a}$ & Cd & $\mathbf{C r}$ & $\mathrm{Ni}$ & $\mathbf{P b}$ & $\mathbf{U}$ \\
\hline HAN22B & $<0.02$ & $<0.01$ & $<0.01$ & 0.001 & 0.001 & 0.005 \\
\hline HAN24 & $<0.02$ & $<0.01$ & $<0.01$ & 0.006 & 0.002 & 0.010 \\
\hline HAN25 & $<0.02$ & $<0.01$ & 0.020 & 0.018 & 0.001 & 0.004 \\
\hline HAN26 & $<0.02$ & $<0.01$ & $<0.01$ & 0.003 & 0.002 & 0.006 \\
\hline HAN27 & $<0.02$ & $<0.01$ & $<0.01$ & 0.018 & 0.001 & 0.002 \\
\hline HAN28 & $<0.02$ & $<0.01$ & $<0.01$ & 0.025 & 0.002 & 0.001 \\
\hline HAN28A & 0.36 & $<0.01$ & 0.027 & 0.014 & 0.004 & 0.025 \\
\hline HAN28B & 0.62 & $<0.01$ & 0.005 & 0.010 & 0.002 & 0.019 \\
\hline HAN29A & 0.43 & $<0.01$ & 0.022 & 0.004 & 0.003 & 0.026 \\
\hline HAN29B & 0.48 & $<0.01$ & 0.009 & 0.004 & 0.003 & 0.019 \\
\hline HAN29B1 & 0.52 & 0.002 & 0.030 & 0.011 & 0.005 & 0.079 \\
\hline HAN29B2 & 0.67 & 0.002 & $<0.01$ & 0.012 & 0.003 & 0.022 \\
\hline HAN29E & 0.14 & 0.004 & 2.42 & $<0.10$ & 0.039 & 0.065 \\
\hline HAN29F & 0.53 & 0.002 & 0.083 & $<0.10$ & 0.003 & 0.065 \\
\hline HAN30A & 0.74 & 0.003 & $<0.01$ & 0.000 & 0.015 & 0.031 \\
\hline HAN30B & 0.50 & $<0.01$ & 0.017 & 0.007 & 0.004 & 0.046 \\
\hline HAN33 & 0.78 & 0.002 & 0.000 & 0.000 & 0.059 & 0.048 \\
\hline HAN34 & 0.41 & 0.002 & 0.010 & $<0.10$ & 0.003 & 0.009 \\
\hline HAN35 & 0.77 & 0.002 & 0.000 & 0.000 & 0.027 & 0.035 \\
\hline HAN36 & 0.44 & 0.002 & 0.014 & $<0.10$ & 0.004 & 0.012 \\
\hline HAN37 & 0.47 & 0.002 & 0.010 & $<0.10$ & $<0.0035$ & 0.054 \\
\hline HAN38 & 0.47 & 0.002 & 0.010 & $<0.10$ & $<0.0035$ & 0.018 \\
\hline HAN39 & 0.46 & 0.002 & 0.010 & 0.013 & $<0.007$ & 0.021 \\
\hline ICLP Limit & 100 & 0066 & 52 & 5 & 037 & 1.78 \\
\hline
\end{tabular}


Table 2.15

\section{Concentrations of Tc-99 Determined by ICPMS in TCLP Leachates} from Hanford Glasses.

(Note: The suffix "TC" is appended to the glass name to indicate the corresponding TCLP leachate.)

\begin{tabular}{||l|l|l|l|l|l|l|l||}
\hline \hline Tc-99 (ppb) & \multicolumn{7}{|c|}{} \\
\hline SAMPLE D & RUN-1 & RUN-2 & RUN-3 & RUN-4 & $\begin{array}{c}\text { AVG } \\
\text { (ppb) }\end{array}$ & $\begin{array}{c}\text { STD. } \\
\text { DEV. }\end{array}$ & $\begin{array}{c}\text { RSD } \\
(\%)\end{array}$ \\
\hline HAN-28ATC & 2.93 & 2.94 & 2.59 & 2.59 & 2.76 & 0.17 & 6.16 \\
\hline HAN-28BTC & 0.36 & 0.35 & 0.35 & 0.35 & 0.35 & 0.00 & 0.00 \\
\hline HAN-29ATC & 1.65 & 1.65 & 1.68 & 1.69 & 1.67 & 0.02 & 1.20 \\
\hline HAN-29BTC & 0.15 & 0.13 & 0.26 & 0.25 & 0.20 & 0.06 & 30.00 \\
\hline HAN-29FTC & 0.54 & 0.52 & 0.58 & 0.57 & 0.55 & 0.02 & 3.64 \\
\hline HAN-30ATC & 1.16 & 1.14 & 1.23 & 1.24 & 1.19 & 0.04 & .3 .36 \\
\hline HAN-34TC & & & & & $<0.03$ & & \\
\hline HAN-36TC & 0.05 & 0.05 & 0.06 & 0.06 & 0.06 & 0.01 & 16.67 \\
\hline HAN-37TC & 0.06 & 0.06 & 0.07 & 0.06 & 0.06 & 0.00 & 0.00 \\
\hline HAN-38TC & 0.06 & 0.07 & 0.05 & 0.05 & 0.06 & 0.01 & 16.67 \\
\hline HAN-39TC & 0.23 & 0.24 & 0.23 & 0.23 & 0.23 & 0.00 & 0.00 \\
\hline
\end{tabular}

Note:Tc-99 has possible interference from Ru. However, after examining the spectra, no significant interference was found in these samples. 
Table 2.16

Summary of Results from PCT Leach Tests of Hanford Glasses

\begin{tabular}{|c|c|c|c|c|c|c|c|c|c|c|c|c|}
\hline & SRL-EA & HAN22B & HAN24 & HAN25 & HAN26 & HAN27 & HAN28 & HAN28A & HAN29A & HAN29B1 & HAN29E & HAN29F \\
\hline \multicolumn{13}{|c|}{ SODIUM, PPM } \\
\hline \multicolumn{13}{|c|}{\begin{tabular}{l|l}
\multicolumn{2}{|l}{ SODIUM, PPM } \\
DAYS & \\
\end{tabular}} \\
\hline 7 & 1697 & 1433 & 1134 & 305 & 383 & 695 & 178 & 585 & 1765 & 2201 & 20701 & 11441 \\
\hline 28 & 2107 & 2327 & 1756 & 487 & 604 & 1118 & 282 & 963 & 3376 & 3814 & 21040 & 16370 \\
\hline 56 & 2538 & 3437 & 2681 & 665 & 840 & 1561 & 359 & (x) & 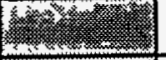 & 12470 & 21788 & 16478 \\
\hline 120 & 3791 & 13394 & 13143 & 955 & 1179 & 2368 & 483 & $y_{2}^{2}=3$ & 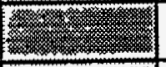 & 11745 & 19224 & 14021 \\
\hline 180 & 4456 & 12508 & 11855 & 1068 & 11466 & 11241 & 533 & (x) & X-x... & 11564 & 18456 & 15739 \\
\hline 270 & 5332 & 11485 & 11399 & 8573 & 11606 & 10550 & 600 & $4 \times 2 \times 2 \times$ & 3 & 2 & 3 & 13058 \\
\hline 365 & 5321 & 9783 & 9019 & 8212 & 9221 & 8939 & 617 & (x) & $n_{x \rightarrow 3}$ & , & 2. & \\
\hline \multicolumn{13}{|c|}{ NORMALIZED. $\mathrm{g} / \mathrm{l}$} \\
\hline 7 & 14.04 & 8.56 & 6.14 & 1.73 & 2.08 & 4.09 & 1.15 & 3.08 & 8.26 & 10.12 & 65.28 & 46.45 \\
\hline 28 & 17.43 & 13.90 & 9.52 & 2.76 & 3.28 & 6.58 & 1.81 & 5.07 & 15.80 & 17.53 & 66.35 & 66.46 \\
\hline 56 & 20.98 & 20.53 & 14.53 & 3.76 & 4.56 & 9.19 & 2.31 & 3is & 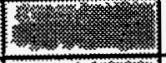 & 57.32 & 68.71 & 66.89 \\
\hline 120 & 31.34 & 80.02 & 71.22 & 5.40 & 6.40 & 13.93 & 3.11 & (2) & 2. & 53.99 & 60.63 & 56.92 \\
\hline 180 & 36.84 & 74.73 & 64.24 & 6.04 & 62.20 & 66.14 & 3.44 & 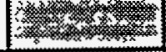 & 147 & 53.16 & 58.20 & 63.89 \\
\hline 270 & 44.10 & 68.62 & 61.77. & 48.49 & 62.96 & 62.08 & 3.87 & (l) & $\mid$ & & $\sqrt{1}$ & 53.01 \\
\hline 365 & 44.00 & 58.45 & 48.87 & 46.45 & 50.02 & 52.60 & 3.97 & 2 & $=1$ & 1 & $y^{2}-2,2 \times 2$ & \\
\hline \multicolumn{13}{|c|}{ 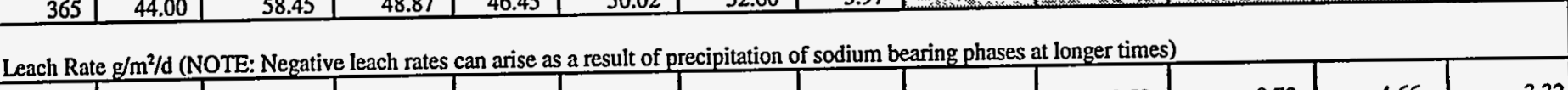 } \\
\hline 3.5 & 1.00 & 0.61 & 0.44 & 0.12 & 0.15 & 0.29 & 0.08 & 0.22 & 0.59 & 0.72 & 4.66 & 3.32 \\
\hline 17.5 & 0.08 & 0.13 & 0.08 & 0.02 & 0.03 & 0.06 & 0.02 & 0.05 & 0.18 & 0.18 & 0.03 & 0.48 \\
\hline 42 & 0.06 & 0.12 & 0.09 & 0.02 & 0.02 & 0.05 & 0.01 & 7.t. & (6) & 0.95 & 0.06 & 0.01 \\
\hline 88 & 0.08 & 0.46 & 0.44 & 0.01 & 0.01 & 0.04 & 0.01 & (i) & $1 x^{2}$ & 0.00 & -0.19 & -0.08 \\
\hline 150 & 0.05 & -0.04 & -0.06 & 0.01 & 0.47 & 0.44 & 0.00 & Fis & 1 & -0.02 & -0.06 & 0.06 \\
\hline 225 & 0.04 & -0.03 & -0.01 & 0.24 & 0.00 & -0.02 & 0.00 & 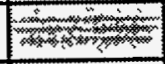 & 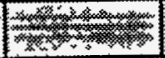 & 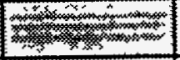 & 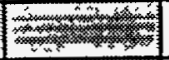 & -0.06 \\
\hline 317.5 & 0.00 & -0.05 & -0.07 & -0.01 & -0.07 & -0.05 & 0.00 & 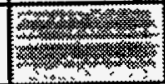 & 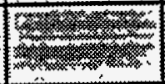 & 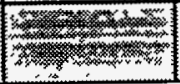 & $\sqrt{x+2}$ & \\
\hline
\end{tabular}




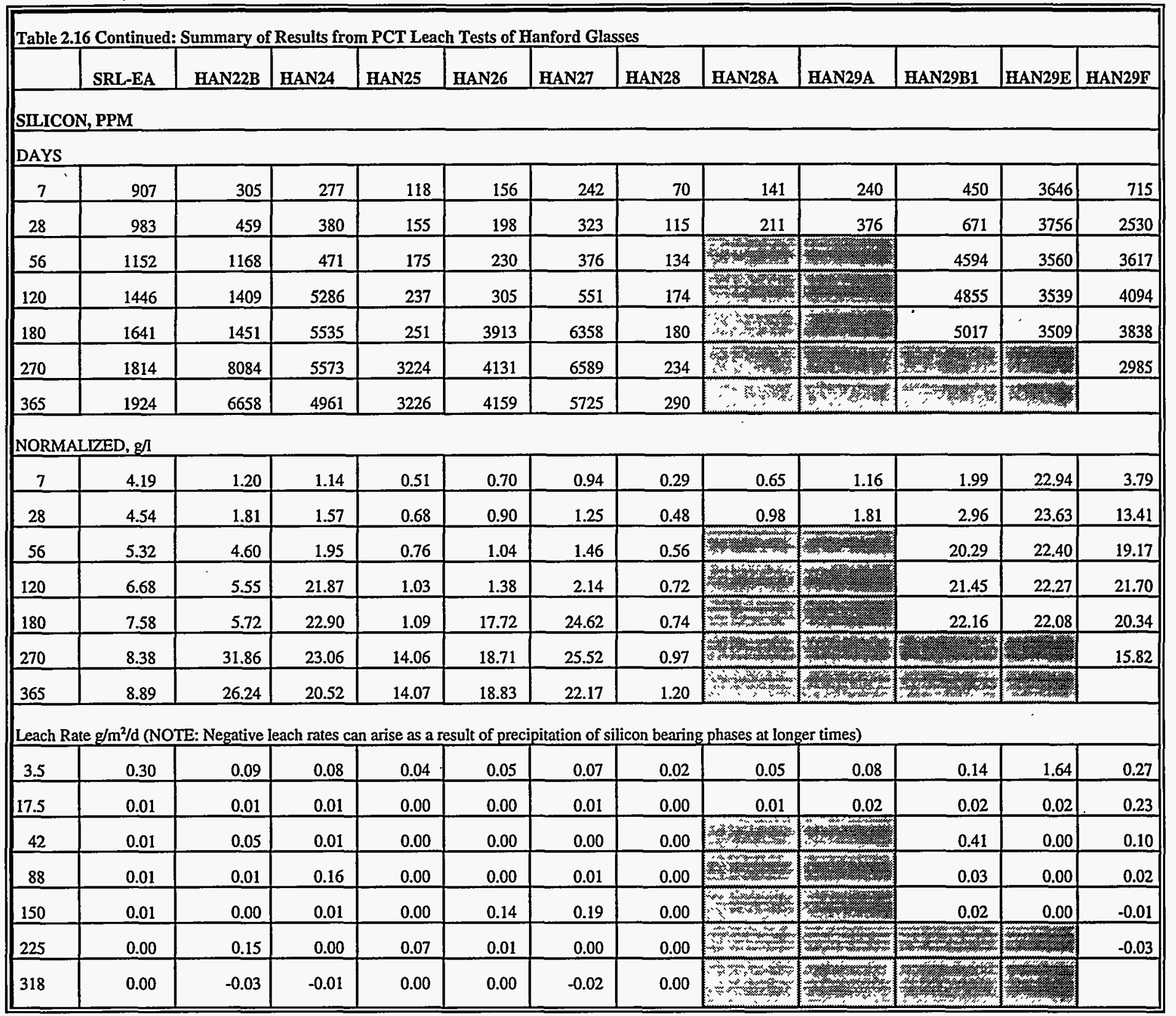




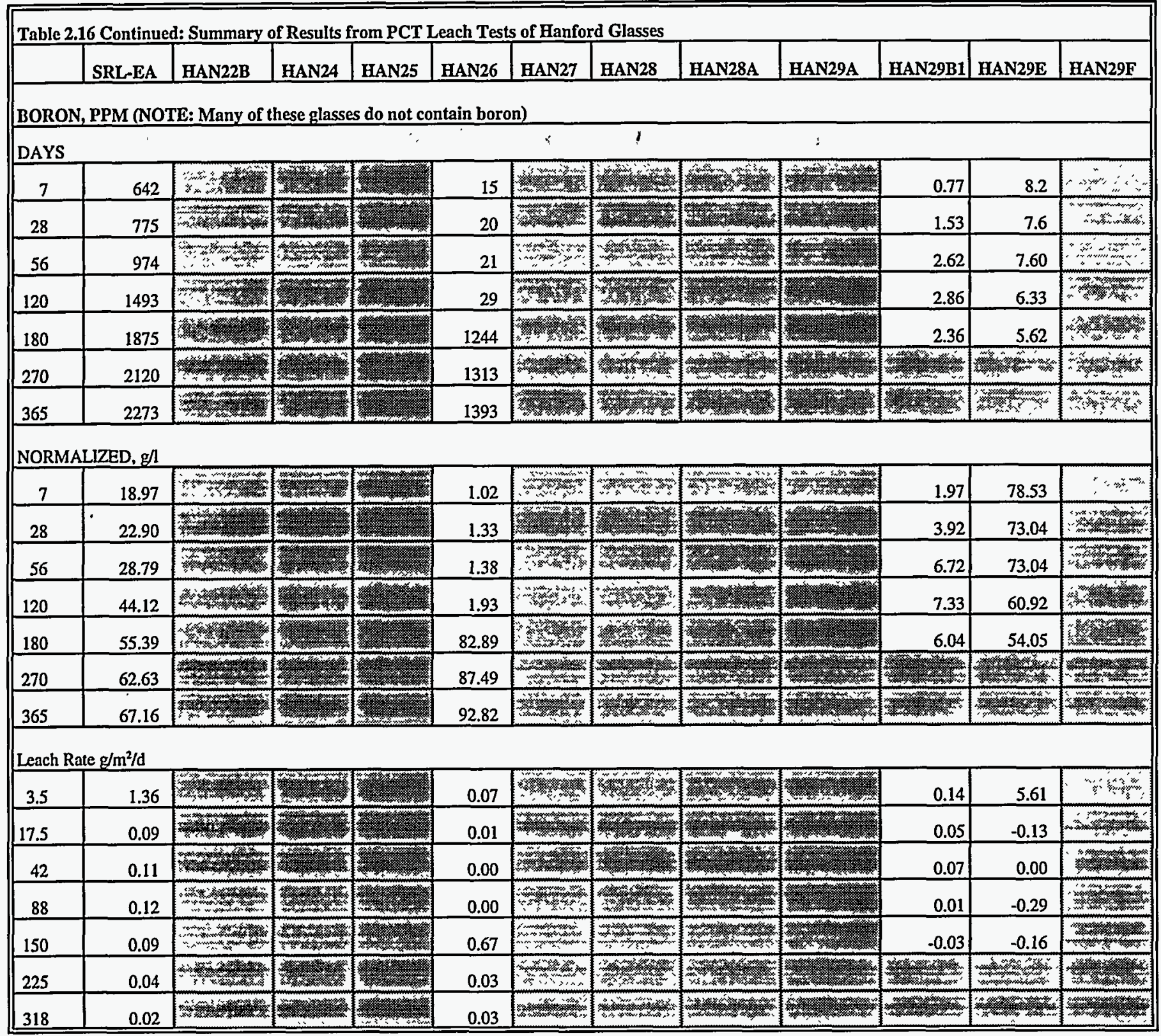




\begin{tabular}{|c|c|c|c|c|c|c|c|c|}
\hline & HAN30A & HAN33 & HAN34 & HAN35 & HAN36 & HAN37 & HAN38 & HAN39 \\
\hline \multicolumn{9}{|c|}{ SODIUM, PPM } \\
\hline \multicolumn{9}{|l|}{ DAYS } \\
\hline 7 & 107 & 26 & 20 & 18 & 1507 & 1702 . & 257 & 12507 \\
\hline 28 & 192 & 30.10 & 26 & 22 & 2698 & 3109 & 433 & 12528 \\
\hline 56 & 236.80 & 29.04 & 32 & 20.60 & 8007 & 4682 & 527 & 12422 \\
\hline 120 & 262.10 & 26.59 & 35 & 17.45 & 11823 & 11416 & 570 & 11716 \\
\hline 180 & 286.80 & 25.38 & 38 & 19.56 & 12182 & 12774 & 682 & 11407 \\
\hline 270 & ( & 2 & 38 & 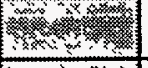 & 10783 & 10501 & 701 & 10036 \\
\hline 365 & 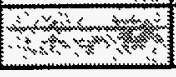 & 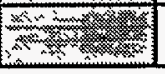 & 42 & 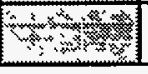 & 10218 & 8473 & 776 & 9831 \\
\hline \multicolumn{9}{|c|}{ NORMALIZED, g/1 } \\
\hline 7 & 0.66 & 1.13 & 0.34 & 0.77 & 7.43 & 8.50 & 1.48 & 74.91 \\
\hline 28 & 1.18 & 1.33 & 0.44 & 0.92 & 13.30 & 15.52 & 2.49 & 75.03 \\
\hline 56 & 1.45 & 1.28 & 0.54 & 0.87 & 39.48 & 23.37 & 3.03 & 74.40 \\
\hline 120 & 1.60 & 1.17 & 0.59 & 0.74 & 58.29 & 56.98 & 3.28 & 70.17 \\
\hline 180 & 1.75 & 1.18 & 0.64 & 0.82 & 60.06 & 63.76 & 3.92 & 68.32 \\
\hline 270 & was & $x_{2}+2+2$ & 0.63 & 列, & 53.16 & 52.42 & 4.03 & 60.11 \\
\hline 365 & 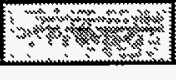 & 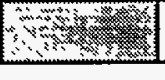 & 0.70 & (n) & 50.38 & 42.29 & 4.46 & 58.88 \\
\hline \multicolumn{9}{|c|}{$\begin{array}{l}\text { Leach Rate } g / \mathrm{m}^{2} / \mathrm{d} \text { (NOTE: Negative leach rates can arise as a result of precipitation of sodium } \\
\text { bearing phases at longer times) }\end{array}$} \\
\hline 3.5 & 0.05 & 0.08 & 0.02 & 0.05 & 0.53 & 0.61 & 0.11 & 5.35 \\
\hline 17.5 & 0.01 & 0.00 & 0.00 & 0.00 & 0.14 & 0.17 & 0.02 & 0.00 \\
\hline 42 & 0.00 & 0.00 & 0.0017 & 0.00 & 0.47 & 0.14 & 0.01 & -0.01 \\
\hline 88 & 0.00 & 0.00 & 0.0004 & 0.00 & 0.15 & 0.26 & $<0.001$ & -0.03 \\
\hline 150 & 0.00 & 0.00 & 0.0004 & 0.002 & 0.01 & 0.06 & 0.01 & -0.02 \\
\hline 225 & 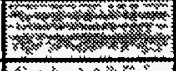 & $y$ & 0.0001 & (1) & -0.04 & -0.06 & $<0.001$ & -0.05 \\
\hline 317.5 & sing & 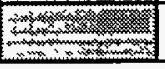 & 0.0040 & 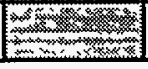 & -0.02 & -0.06 & 0.00 & $<-0.01$ \\
\hline
\end{tabular}




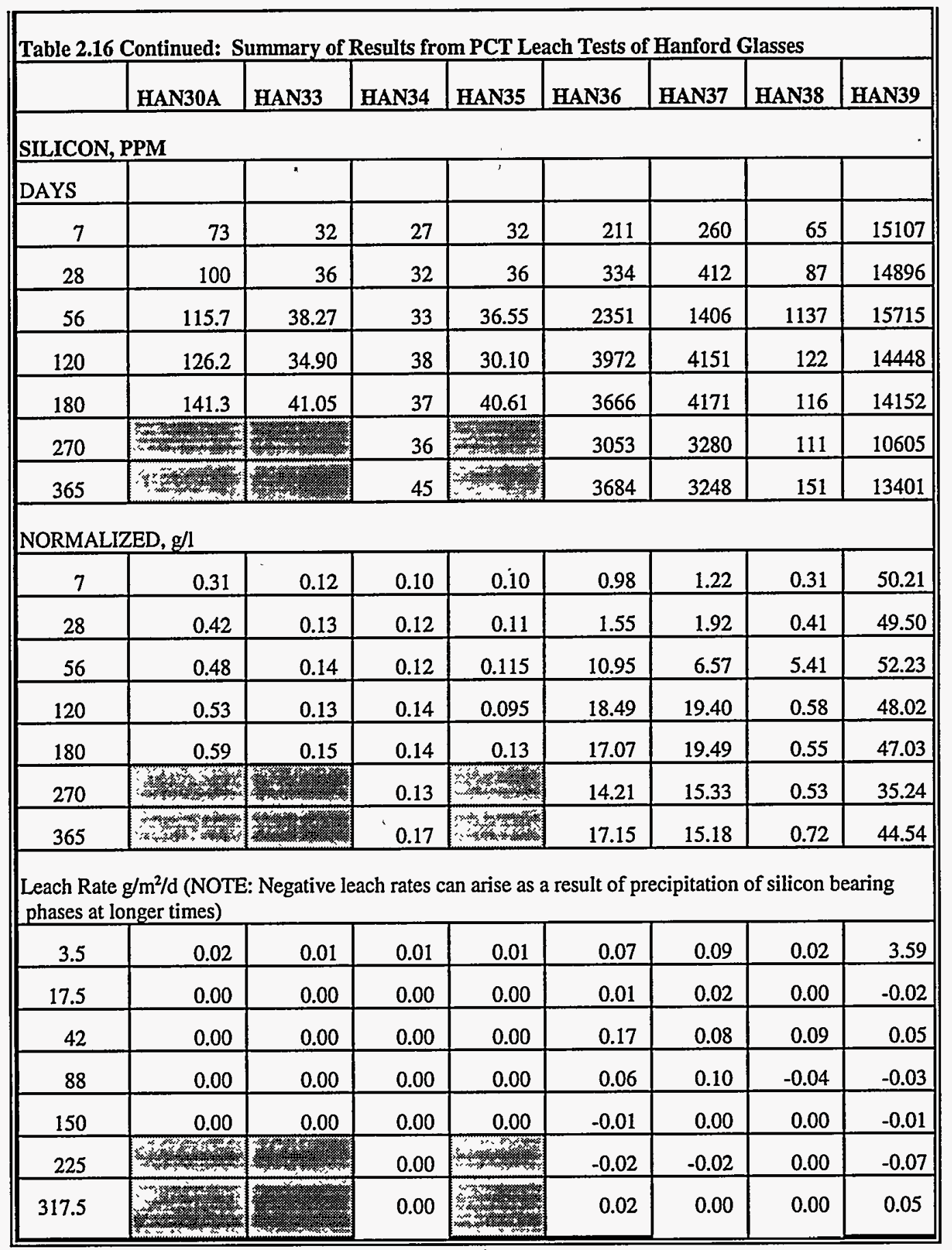




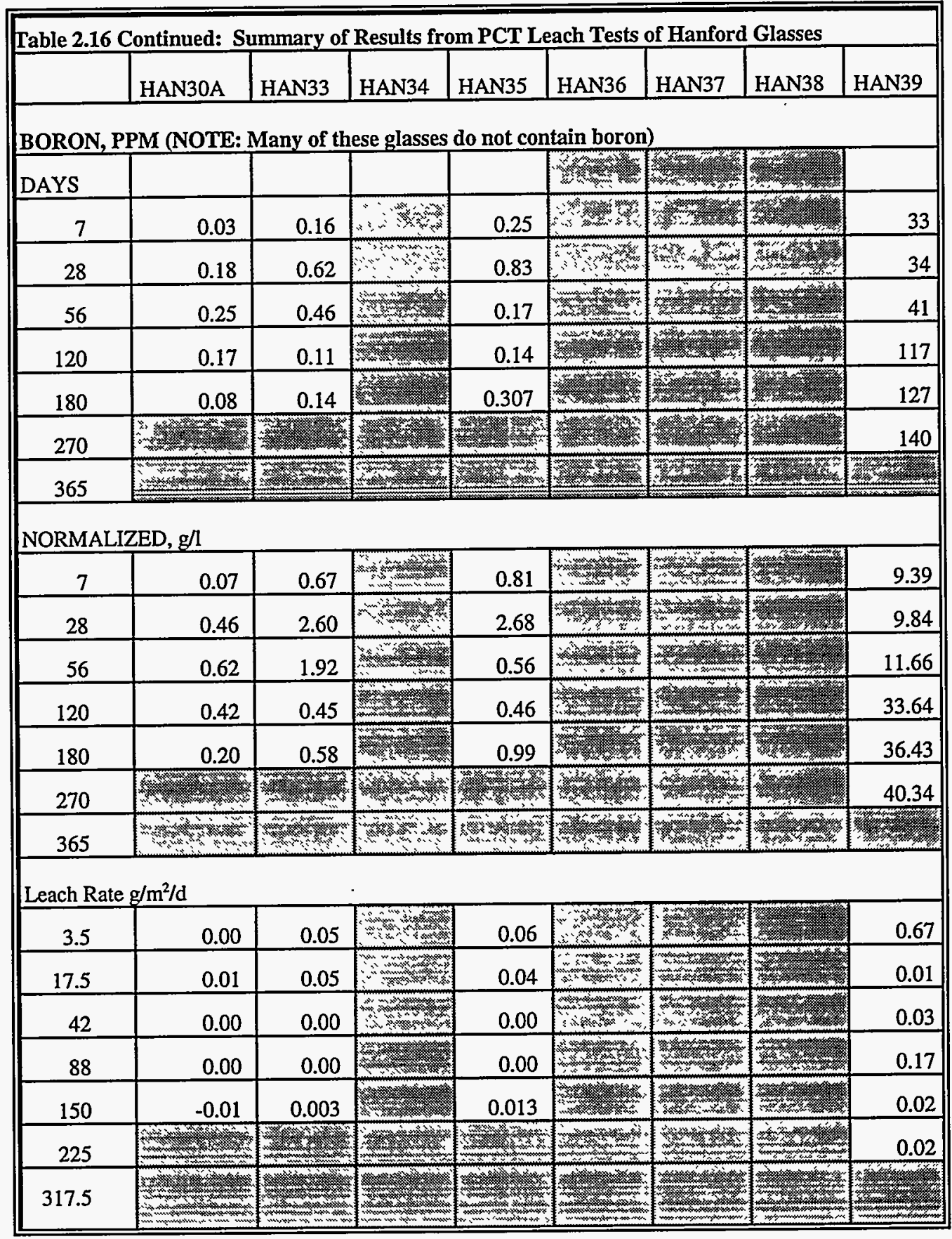


Table 2.17

Percentage of Original Glass Dissolved, Calculated from the Sodium Concentration in the PCT Leachate

\begin{tabular}{|c|c|c|c|c|c|c|c|c|}
\hline Glass Name & $\mathrm{Na}_{2} \mathrm{O}$ Content & 7-day & 28-day & 56-day & 120-day & 180-day & 270-day & 365-day \\
\hline HAN33 & 3.06 & 1.13 & 1.37 & & & & & \\
\hline HAN35 & 3.20 & 0.77 & 0.95 & $?$ & $\therefore$. & & & \\
\hline HAN34 & 8.06 & 0.34 & 0.46 & 0.56 & 0.61 & 0.66 & 0.65 & \\
\hline HAN28 & 20.93 & 1.15 & 1.86 & 2.39 & 3.21 & 3.56 & 4.01 & 4.13 \\
\hline HAN30A & 22.05 & 0.66 & 1.20 & & & & & \\
\hline HAN39 & 22.51 & 74.91 & 78.03 & 77.52 & 73.27 & 71.25 & 62.96 & \\
\hline HAN22B & 22.56 & 8.56 & 14.25 & 21.10 & 80.87 & 77.97 & 71.74 & 61.32 \\
\hline HAN27 & 22.91 & 4.09 & 6.74 & 9.45 & 14.31 & 66.72 & 64.75 & 55.19 \\
\hline HAN38 & 23.45 & 1.48 & 2.55 & 3.13 & 3.40 & 4.06 & 4.19 & \\
\hline HAN25 & 23.83 & 1.73 & 2.82 & 3.87 & 5.56 & 6.26 & 48.74 & 48.40 \\
\hline HAN26 & 24.85 & 2.08 & 3.36 & 4.69 & 6.58 & 62.46 & 65.46 & 52.64 \\
\hline HAN24 & 24.88 & 6.14 & 9.76 & 14.92 & 71.81 & 67.11 & 64.45 & 51.45 \\
\hline HAN28A & 25.59 & 3.08 & 5.20 & & & & & \\
\hline HAN37 & 27.00 & 8.50 & 15.86 & 24.01 & 57.94 & 66.08 & 55.06 & \\
\hline HAN36 & 27.34 & 7.43 & 13.60 & 40.02 & 59.89 & 62.46 & 55.66 & \\
\hline HAN29A & 28.80 & 8.26 & 16.13 & & & & & \\
\hline HAN29B1 & 29.32 & 10.12 & 17.94 & & & & & \\
\hline HAN29F & 33.20 & 46.45 & 68.31 & 69.63 & 59.70 & 66.28 & 55.66 & \\
\hline HAN29E & 42.74 & 65.28 & 68.96 & & & & & \\
\hline SRL-EA & 16.3 & 14.04 & 17.99 & 21.70 & 32.21 & 38.13 & 45.62 & 45.82 \\
\hline
\end{tabular}


Table 2.18

Crystallization Behavior in $\mathrm{Na}_{2} \mathrm{O}$ Series of Hanford Surrogate Glasses

\begin{tabular}{||c|c|c|c|c||}
\hline Glass & $\begin{array}{c}1100^{\circ} \mathbf{C} / 18 \text { or } \\
\mathbf{2 4 h r}\end{array}$ & $\begin{array}{c}1050^{\circ} \mathbf{C} / \mathbf{1} \\
\text { or } \mathbf{2 ~ h r}\end{array}$ & $\mathbf{9 7 5}^{\circ} \mathbf{C} / 2 \mathbf{~ h r}$ & $900^{\circ} \mathbf{C} / 2 \mathbf{~ h r}$ \\
\hline HAN2 & $10.3 \% \mathrm{I}$ & $\mathrm{X}$ & $\mathrm{X}$ & $\mathrm{X}$ \\
HAN3 & $11.9 \% \mathrm{I}$ & $\mathrm{X}$ & $\mathrm{X}$ & $\mathrm{X}$ \\
HAN4 & None & $\mathrm{X}$ & $\mathrm{X}$ & $\mathrm{X}$ \\
HAN5 & None & $\mathrm{X}$ & $\mathrm{X}$ & None \\
HAN6 & $\mathrm{X}$ & None & None & None \\
HAN7 & $\mathrm{X}$ & None & None & $7.8 \% \mathrm{II}, 6 \% \mathrm{III}$ \\
HAN8 & $\mathrm{X}$ & None & Trace II & $22.6 \% \mathrm{II}, 1.4 \% \mathrm{III}, 7.6 \% \mathrm{IV}$ \\
HAN9 & $\mathrm{X}$ & None & Trace II & $\mathrm{X}$ \\
HAN10 & $\mathrm{X}$ & Trace II & $\mathrm{X}$ & \\
\hline
\end{tabular}

$\mathrm{I}=$ Augite, $\mathrm{I}=$ =Aluminosilicate, $\mathrm{III}$ and $\mathrm{IV}$ unidentified. $\mathrm{X}=$ Not done. 
Table 2.19

Cristobalite-Type Phase Composition in $\mathrm{Na}_{2} \mathrm{O}$ Series of Hanford Surrogate Glasses

\begin{tabular}{||l|c|c|c|c|c||}
\hline & \multicolumn{5}{|c||}{ Glass (Heat-Treatment Temperature) } \\
\hline Element & HAN-8(900) & HAN-8(975) & HAN-9(900) & HAN-9(975) & HAN-10(1050) \\
\hline $\mathrm{Na}$ & 10.5 & 13.0 & 13.6 & 14.9 & 12.9 \\
$\mathrm{Mg}$ & 2.5 & 3.8 & 3.2 & 3.8 & 2.6 \\
$\mathrm{Al}$ & 6.1 & 8.9 & 7.2 & 8.1 & 8.6 \\
$\mathrm{Si}$ & 16.7 & 15.2 & 15.8 & 14.8 & 14.5 \\
$\mathrm{Ca}$ & 2.1 & 0.2 & 0.7 & 0.2 & 0.8 \\
$\mathrm{Fe}$ & 3.3 & 1.7 & 2.4 & 1.7 & 3.2 \\
\hline
\end{tabular}

Nominal formula of phase matched: $\left(\mathrm{Na}_{2} \mathrm{O}\right)_{50.33} \mathrm{NaAlSiO}_{4}$. (Numbers in table are not absolute concentration units, but can be used to make relative comparisons.) 
Table 2.20

Pyroxene Composition in $\mathrm{Na}_{2} \mathrm{O}$ Series of Hanford Surrogate Glasses (Atom \%)

\begin{tabular}{|l|l|l|l|l|l|l|l|l|l|l||}
\hline \hline Glass & Na & Ca & SUM & Al & Si & SUM & Mg & Fe & Ti & SUM \\
\hline HAN-2 & 1.19 & 9.28 & 10.47 & 1.51 & 17.82 & 19.33 & 6.48 & 3.37 & 0.35 & 10.20 \\
\hline HAN-3 & 1.45 & 9.23 & 10.68 & 1.18 & 18.16 & 19.34 & 6.34 & 3.36 & 0.29 & 9.99 \\
\hline
\end{tabular}


Table 2.21

Pyroxene Composition in CaO Series of Hanford Surrogate Glasses (Atom \%)

\begin{tabular}{||l|l|l|l|l|l|l|l|l|l|l||}
\hline \hline Glass & Na & Ca & SUM & Al & Si & SUM & Mg & Fe & Ti & SUM \\
\hline HAN-12 & 0.71 & 9.59 & 10.30 & 3.20 & 15.69 & 18.89 & 4.91 & 5.46 & 0.40 & 10.77 \\
\hline HAN-13 & 0.63 & 9.65 & 10.28 & 3.14 & 16.41 & 19.55 & 5.39 & 4.29 & 0.39 & 10.07 \\
\hline HAN-14 & 0.36 & 10.16 & 10.52 & 2.73 & 16.94 & 19.77 & 5.42 & 3.94 & 0.27 & 9.63 \\
\hline HAN-15 & 0.60 & 9.86 & 10.46 & 4.77 & 14.40 & 19.17 & 3.36 & 6.37 & 0.52 & 10.25 \\
\hline $\begin{array}{l}\text { HAN-15 } \\
\text { (Wol.) }\end{array}$ & 0.68 & 19.43 & & 0.19 & 19.21 & & 0.59 & 0.24 & 0.09 & \\
\hline \hline
\end{tabular}

Wol. $=$ Wollastonite is not a pyroxene 
Table 2.22

List of Hanford Glasses with Complete Viscosity, Conductivity, TCLP, and PCT Data (i.e., Glasses in the Core Data Set of $\mathbf{4 0}$ Glasses) and Representative Data for those Properties.

\begin{tabular}{|c|c|c|c|c|c|}
\hline \multirow{2}{*}{$\begin{array}{c}\text { Glass } \\
\text { Number }\end{array}$} & \multirow{2}{*}{$\begin{array}{l}\text { Glass } \\
\text { Name }\end{array}$} & \multirow{2}{*}{$\begin{array}{l}\text { Viscosity } \\
@ 1250^{\circ} \mathrm{C}\end{array}$} & \multirow{2}{*}{$\begin{array}{c}\text { Conductivity } \\
@ 1250^{\circ} \mathrm{C}\end{array}$} & TCLP & \multirow{2}{*}{$\begin{array}{l}\text { PCT-7 day } \\
\mathrm{Na} \text { (ppm) }\end{array}$} \\
\hline & & & & $\mathbf{U}(\mathbf{p p b})$ & \\
\hline 1 & HAN22B & 129 & 1.029 & 5 & 1433 \\
\hline 2 & HAN24 & 158 & 0.926 & 10 & 1134 \\
\hline 3 & HAN25 & 307 & 0.953 & 4 & 305 \\
\hline 4 & HAN26 & 74 & 0.995 & 6 & 383 \\
\hline 5 & HAN27 & 220 & 1.075 & 2 & 695 \\
\hline 6 & HAN28 & 333 & 0.752 & 1 & 178 \\
\hline 7 & HAN28A & 319 & 0.894 & 25 & 585 \\
\hline 8 & HAN29A & 101 & 1.319 & 26 & 1765 \\
\hline 9 & HAN29B1 & 61 & 1.065 & 79 & 2201 \\
\hline 10 & HAN29E & 5 & 2.377 & 65 & 20701 \\
\hline 11 & HAN29F & 24 & 1.555 & 65 & 11441 \\
\hline 12 & HAN30A & 353 & 0.418 & 31 & 107 \\
\hline 13 & HAN33 & 165 & 0.022 & 48 & 26 \\
\hline 14 & HAN34 & 1018 & 0.073 & 9 & 20 \\
\hline 15 & HAN35 & 1652 & 0.025 & 35 & 18 \\
\hline 16 & HAN36 & 157 & 0.845 & 12 & 1507 \\
\hline 17 & HAN37 & 88 & 0.779 & 54 & 1702 \\
\hline 18 & HAN38 & 197 & 0.598 & 18 & 257 \\
\hline 19 & HAN39 & 297 & 0.645 & 21 & 12507 \\
\hline
\end{tabular}




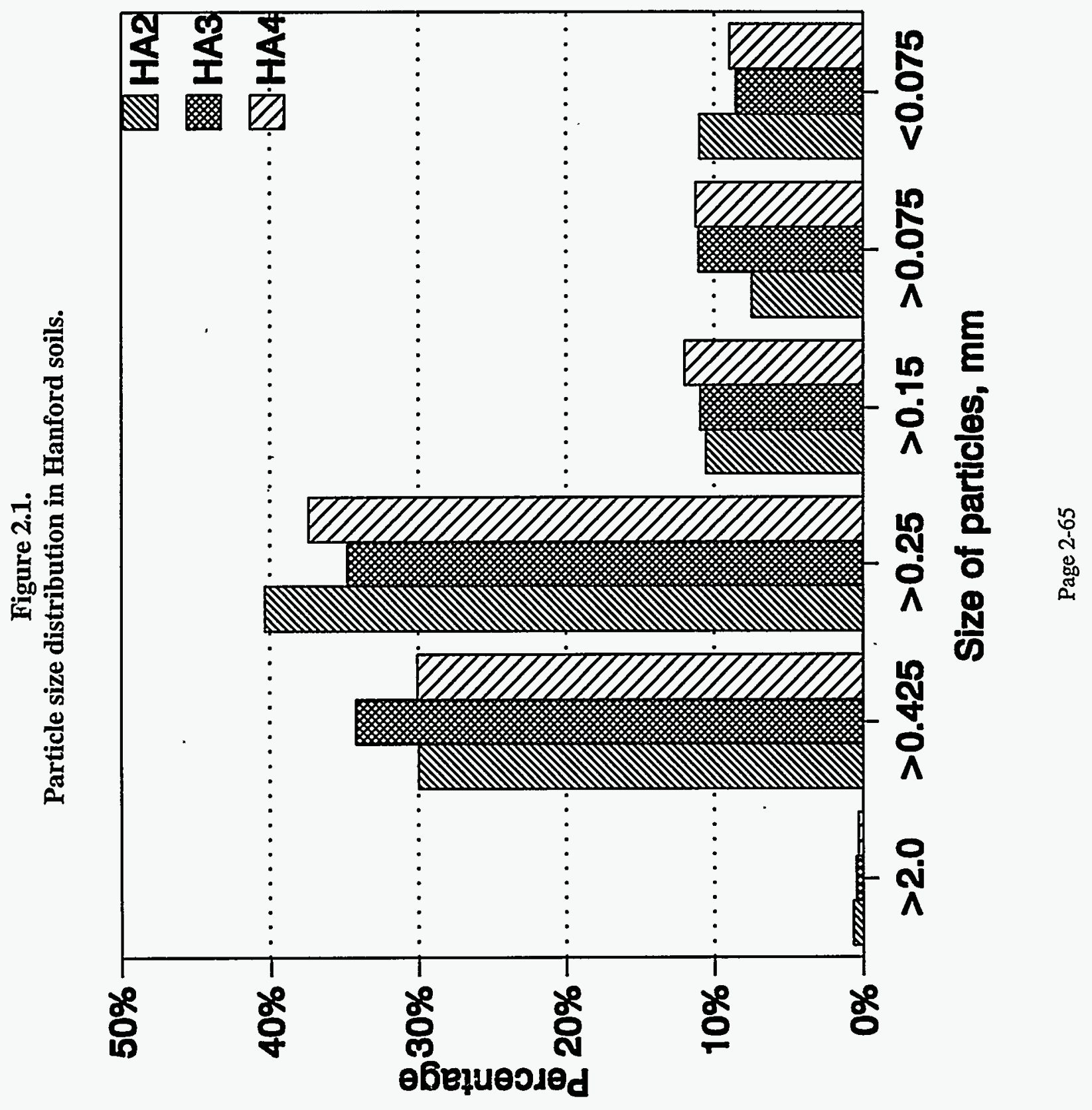




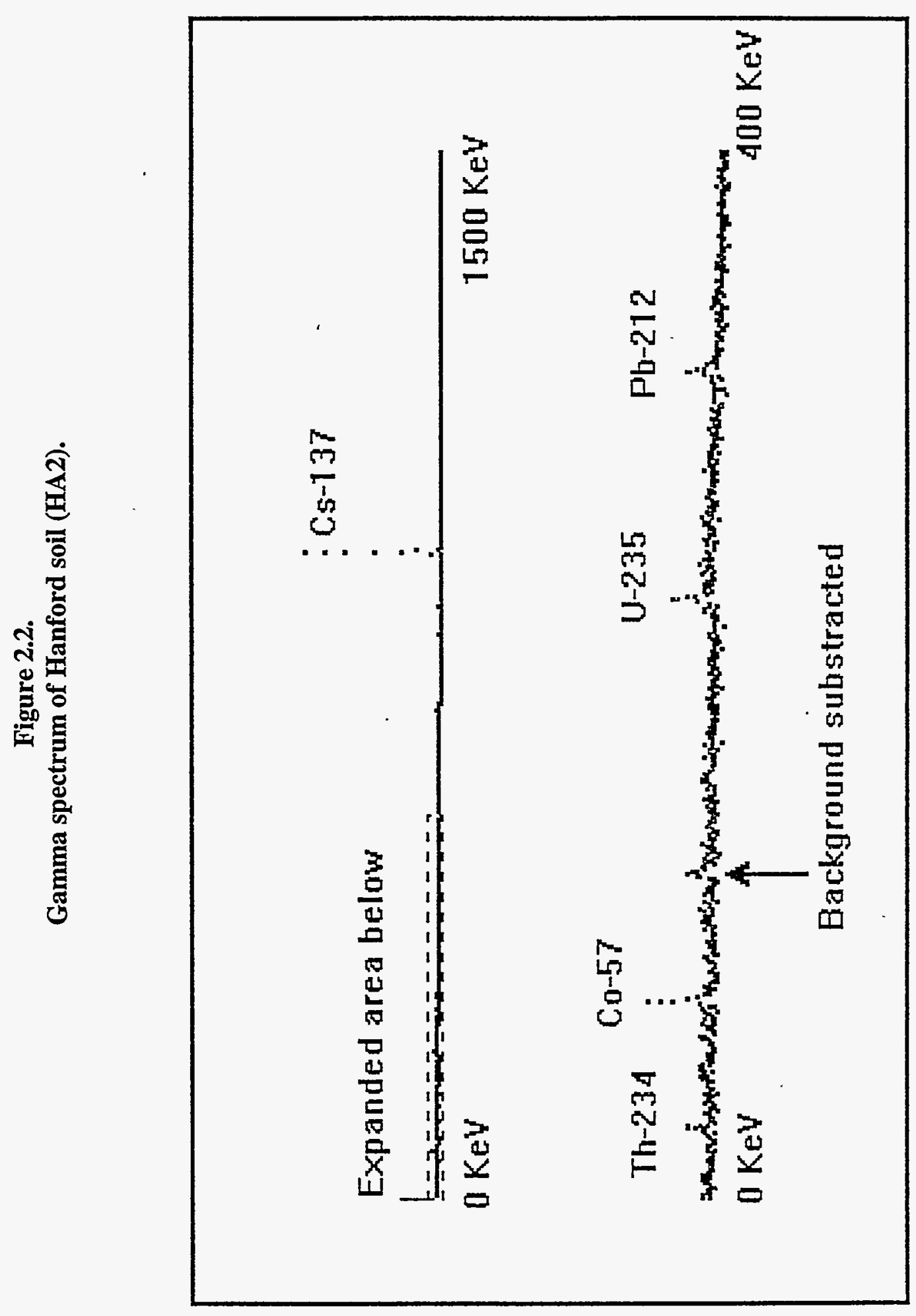

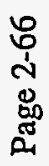




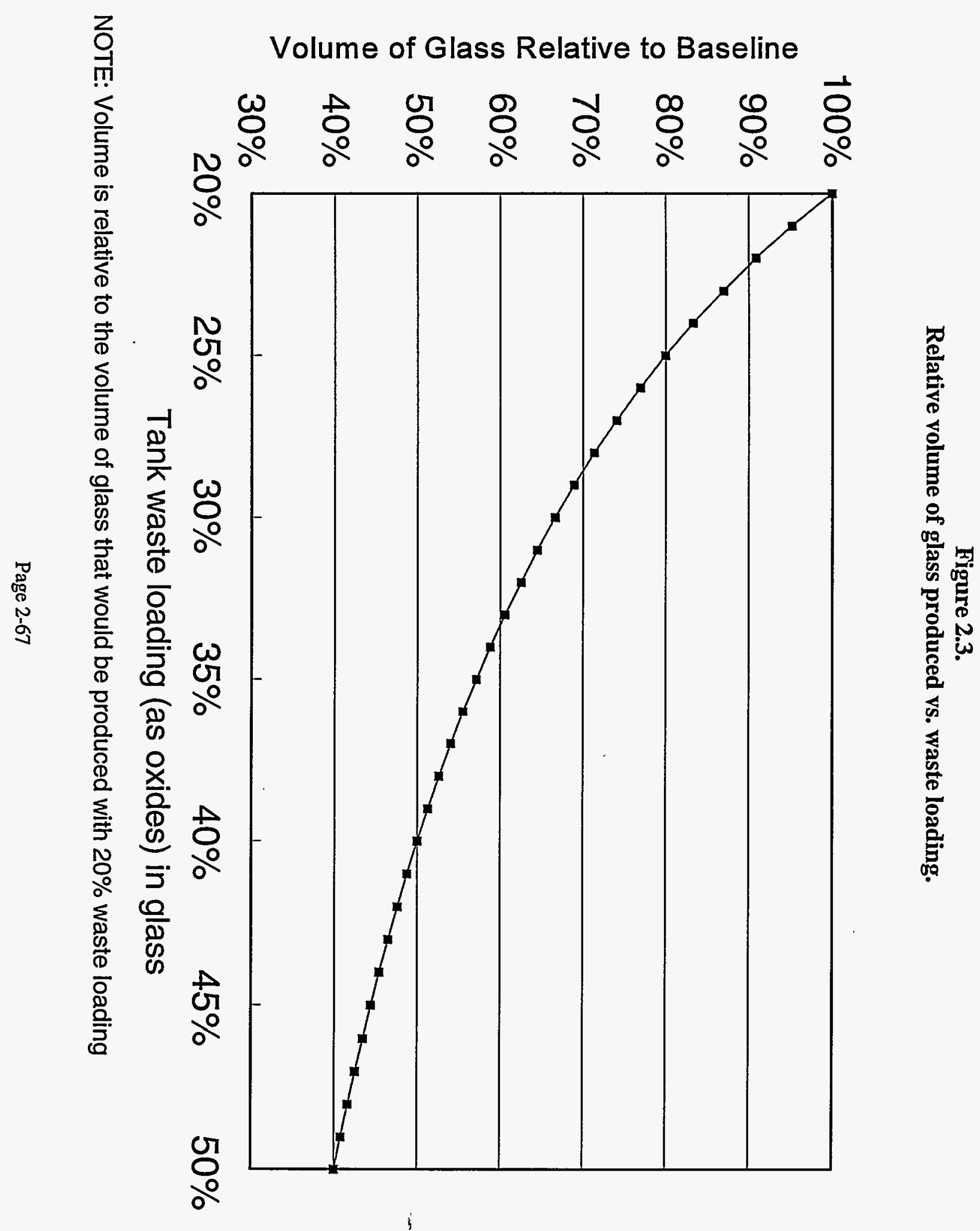


Figure 2.4.

Viscosity of Hanford tank waste glasses. Variation with temperature and sodium content.

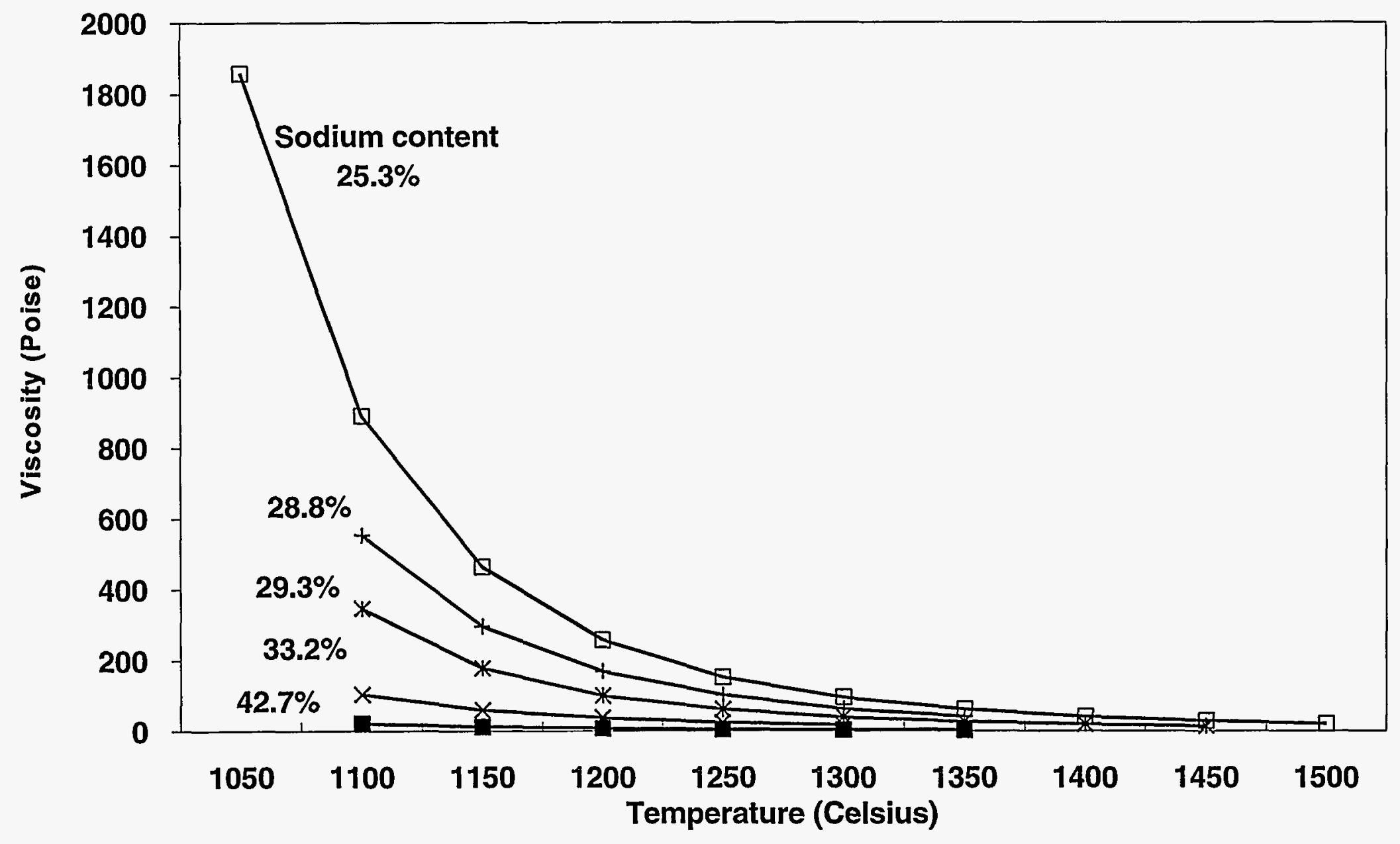

Page 2-68 
Figure 2.5.

Hanford soil - Example of use of glass property data to determine processing temperature for a given set of process constraints.

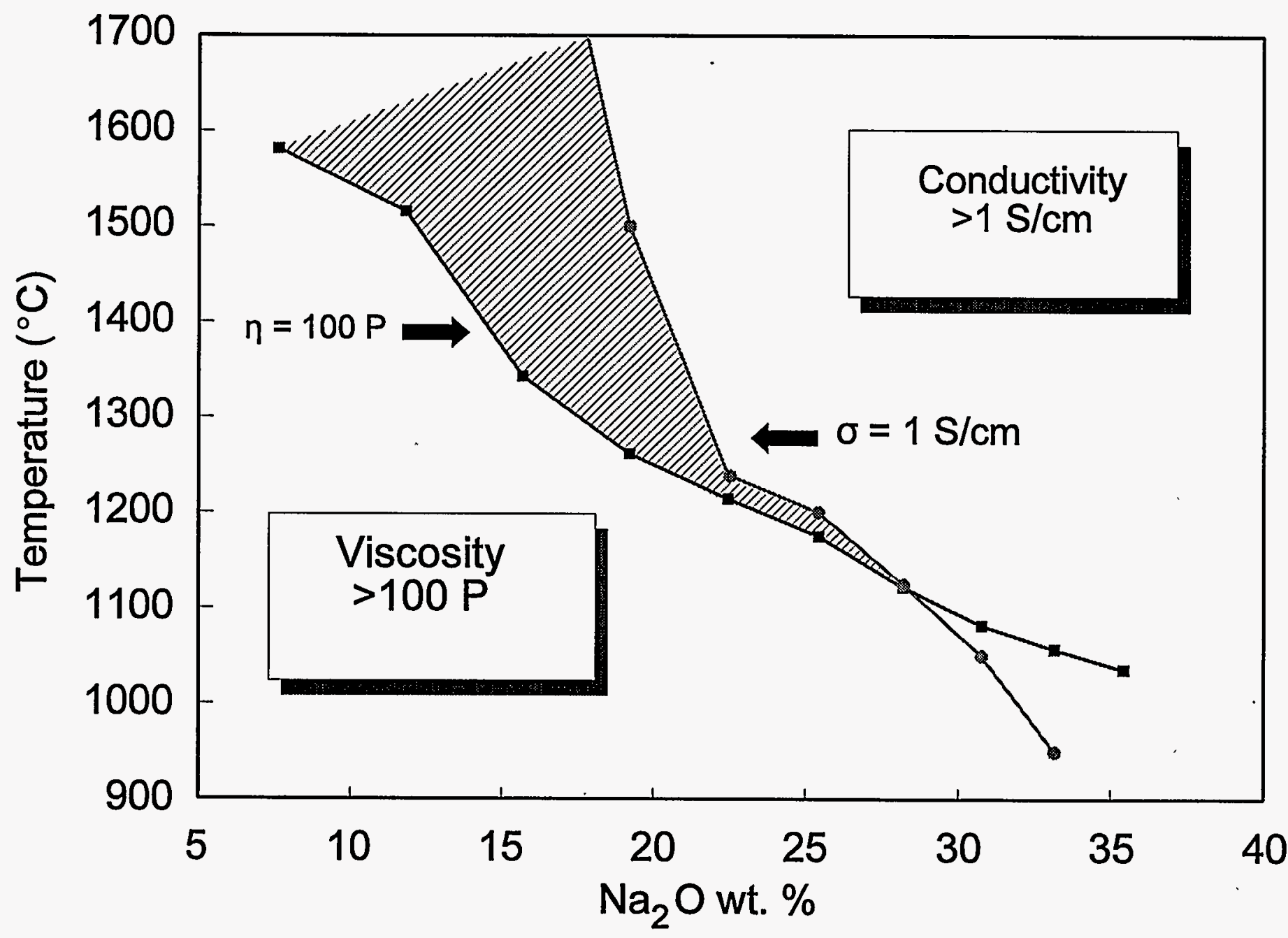

Page 2-69 
Figure 2.6.

A comparison of results of the TCLP leach test for Tc-99 with the DOE 5400.5 drinking water limit.

(Note: Concentrations in the glasses were in the range 2000-5000 ppb;

if all of the Tc-99 was released, TCLP leachate concentrates would be in the range 100-250 ppb).

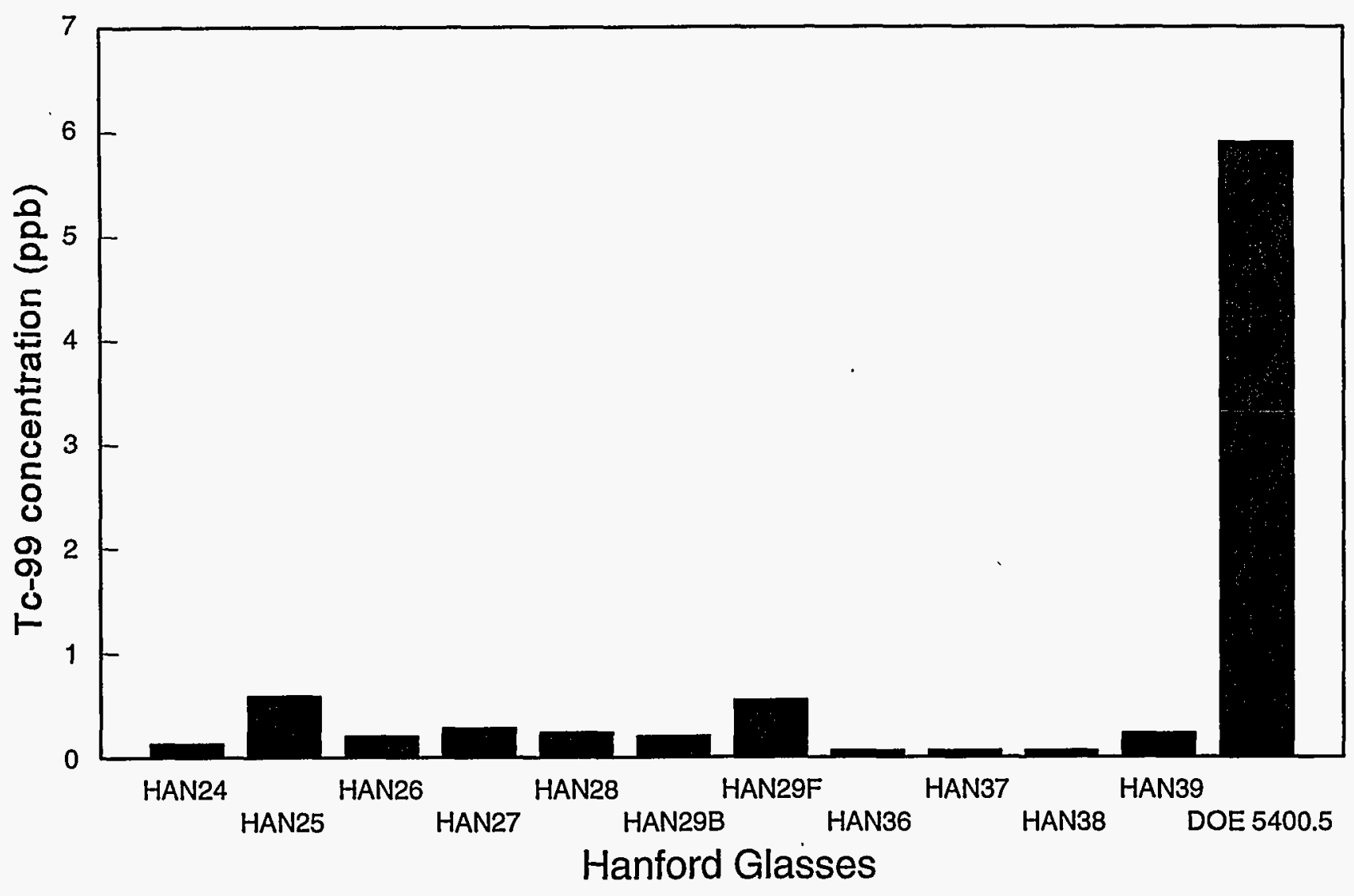

Page 2-70 
Figure 2.7.

Comparison of the effect of various additives on TCLP leaching of calcium.

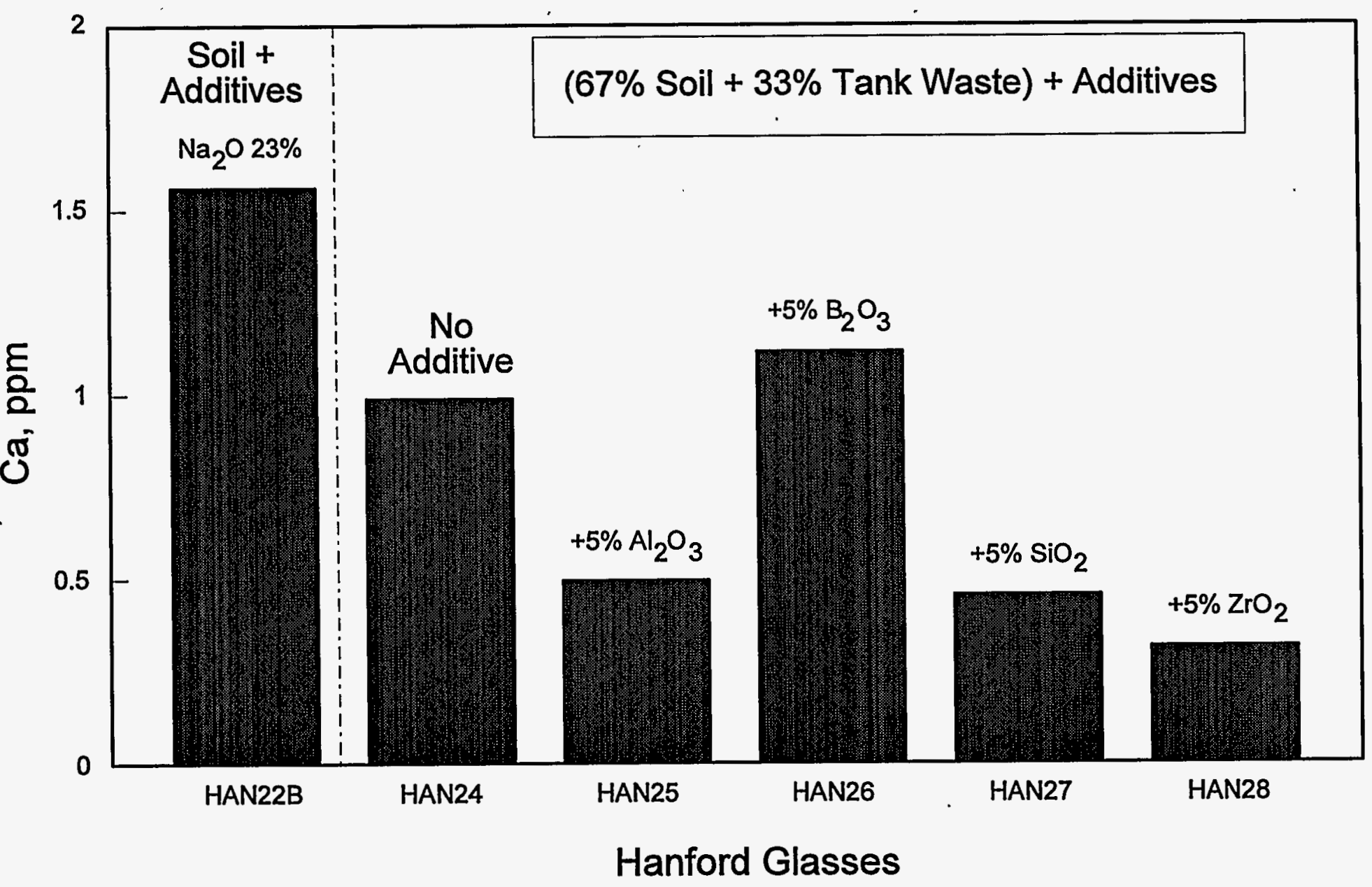

Page 2-71 


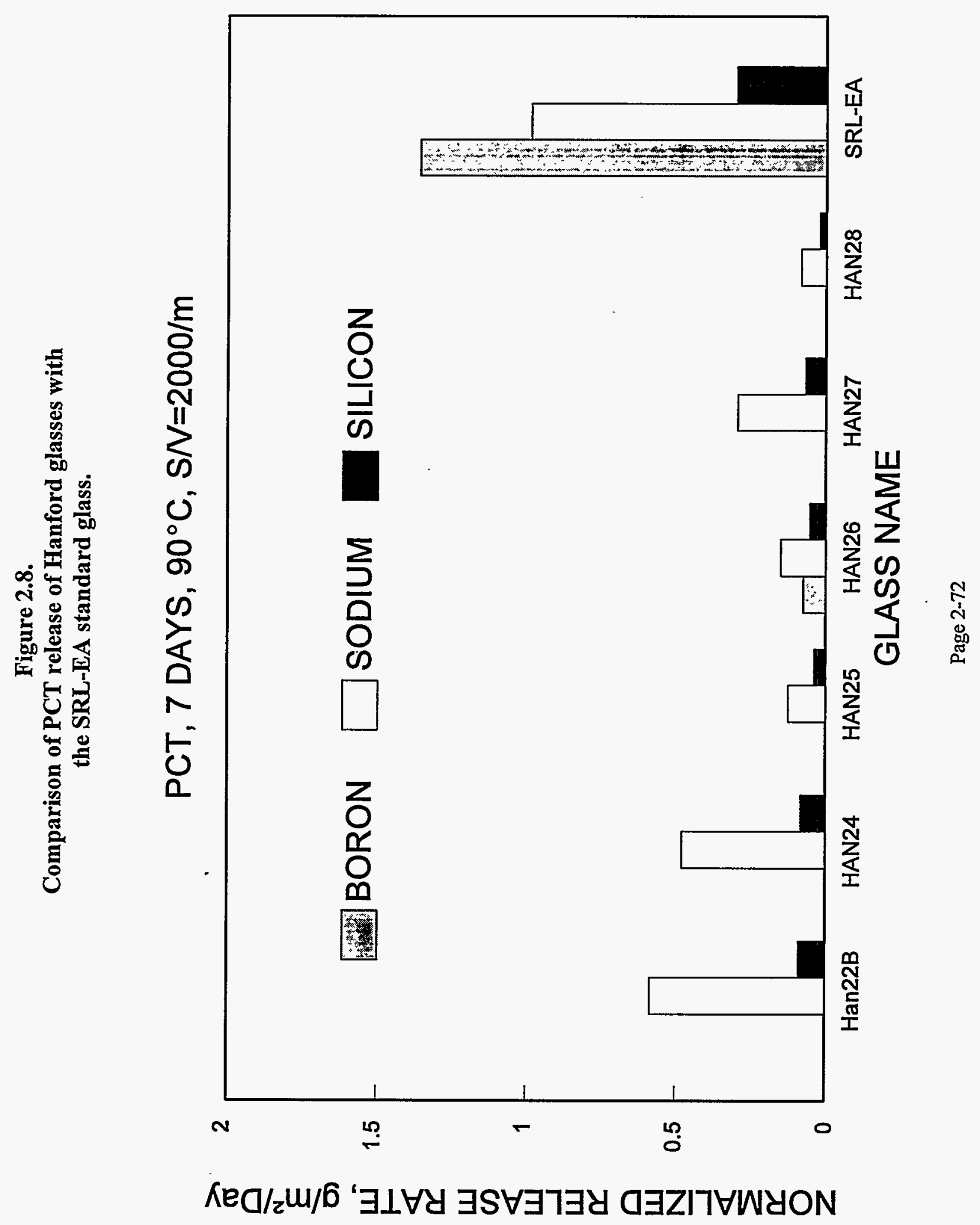




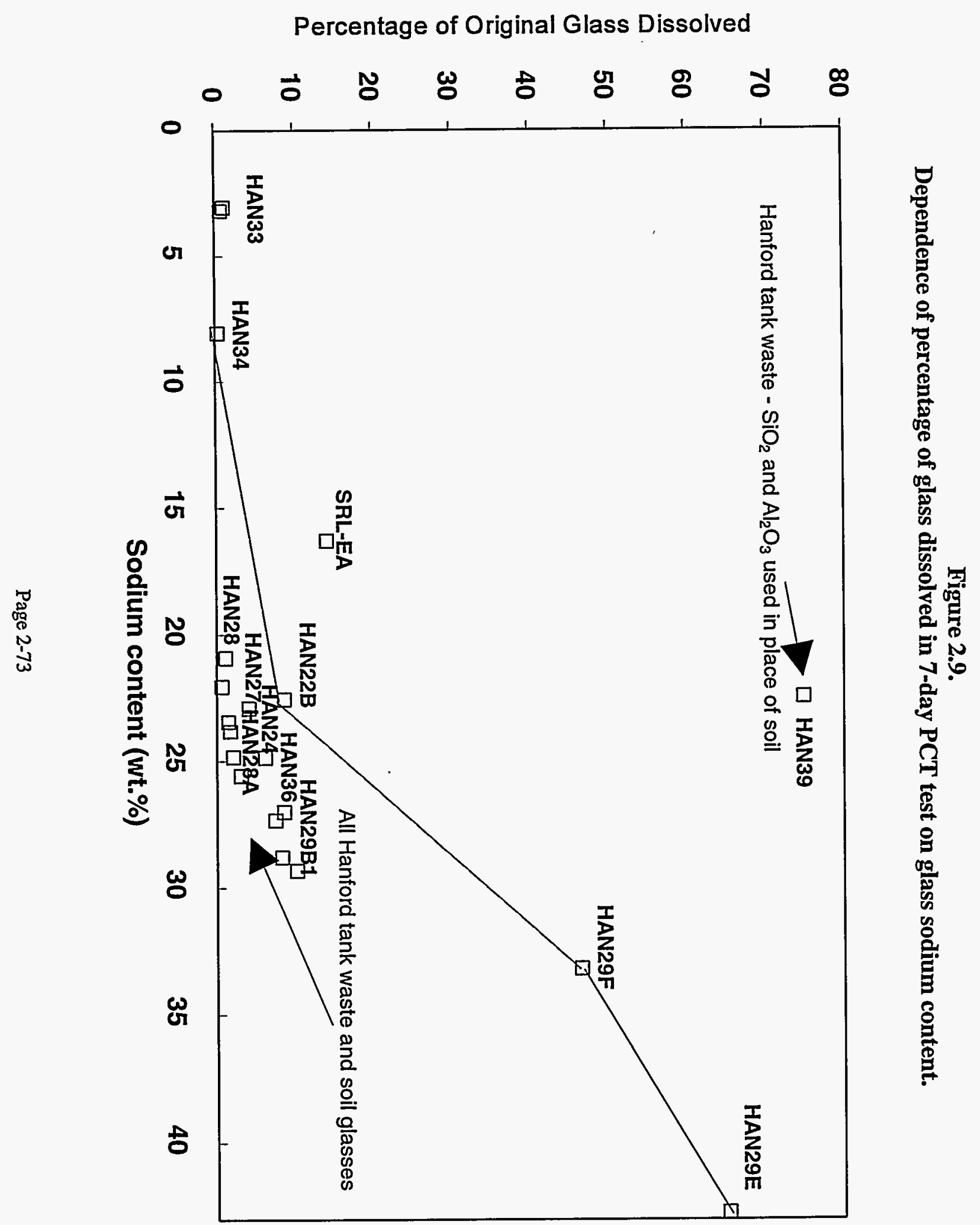


Figure 2.10.

PCT data for Hanford glasses. Silicon concentrations vs. time compared to SRL-EA glass.

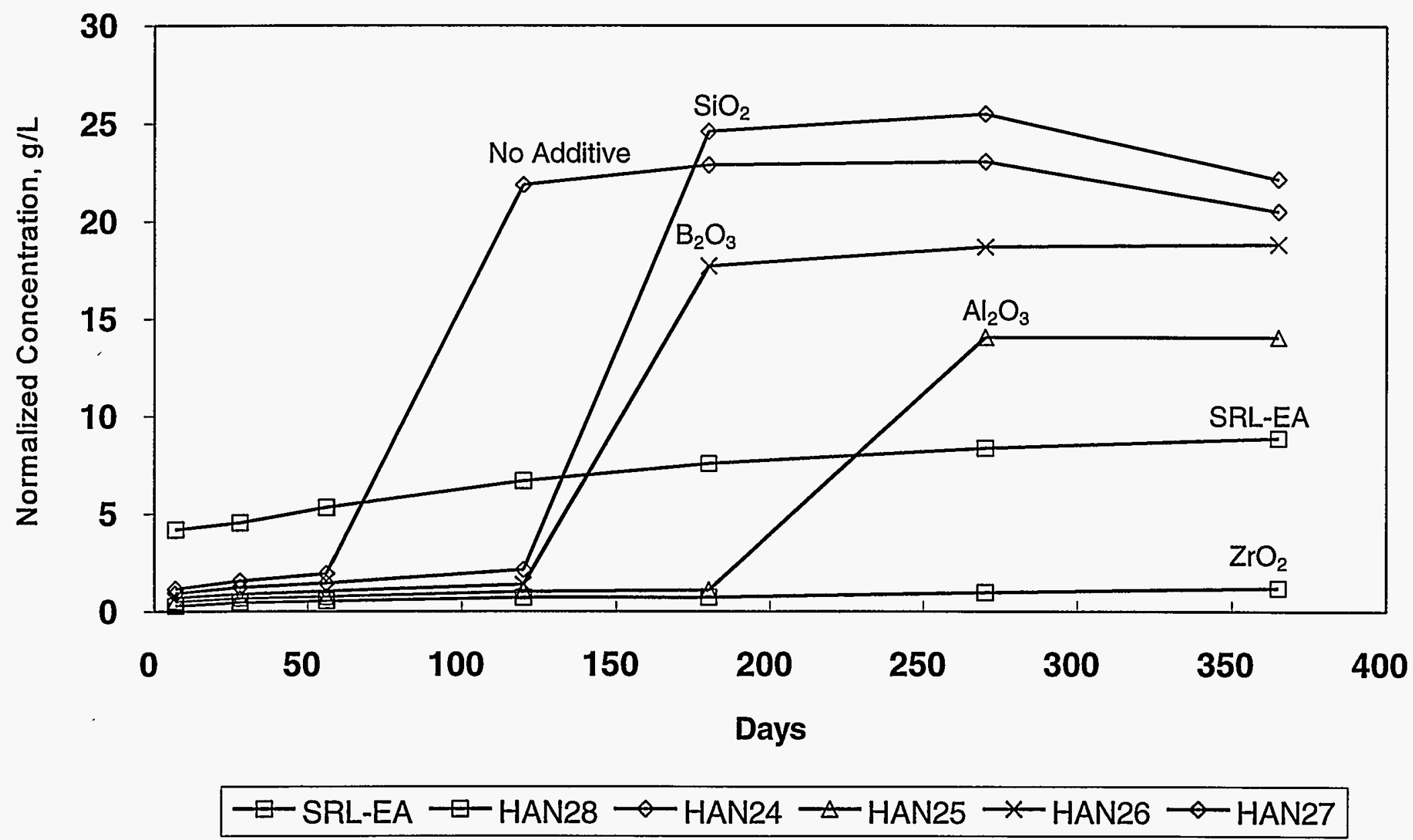




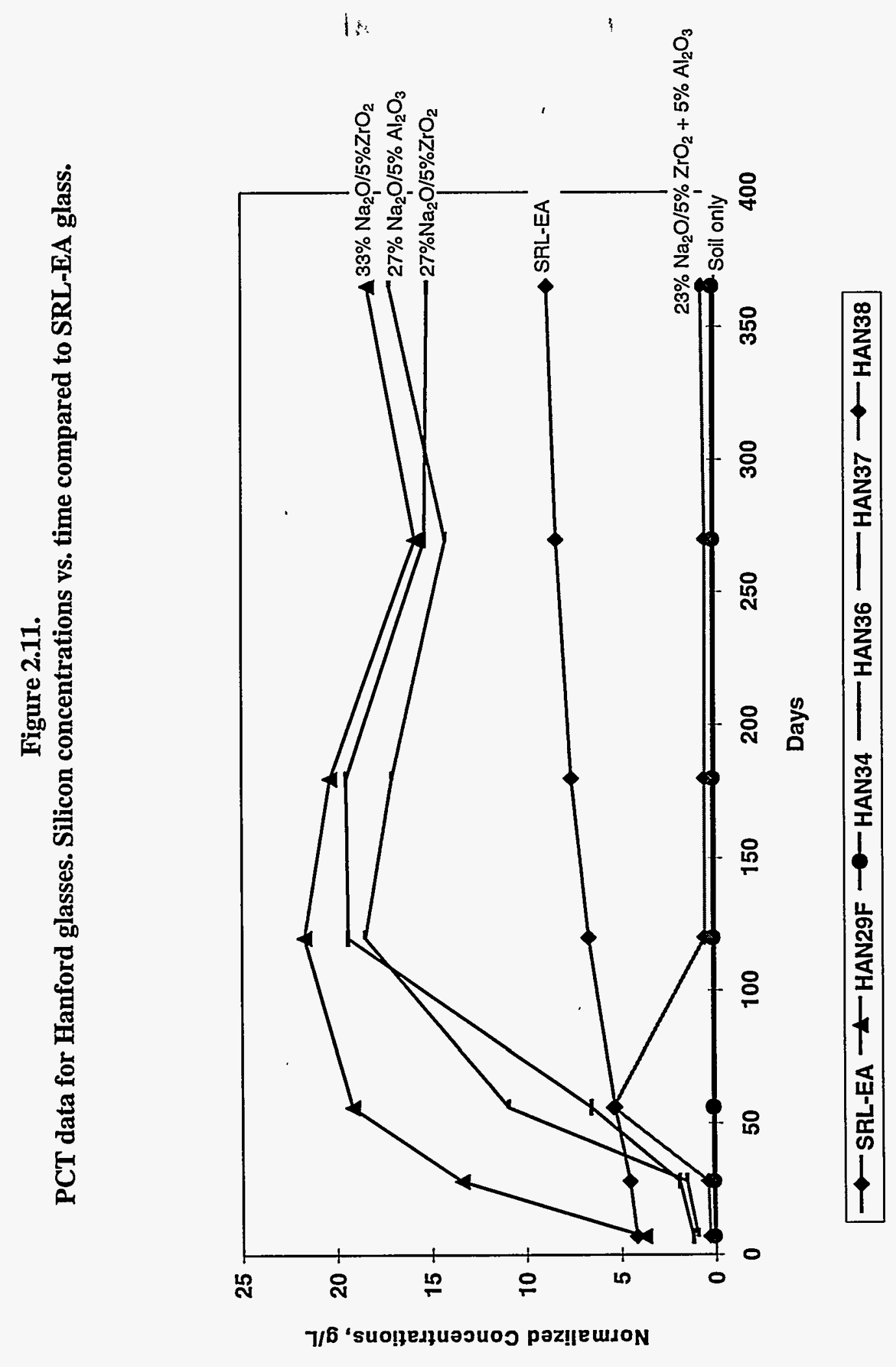

尔 


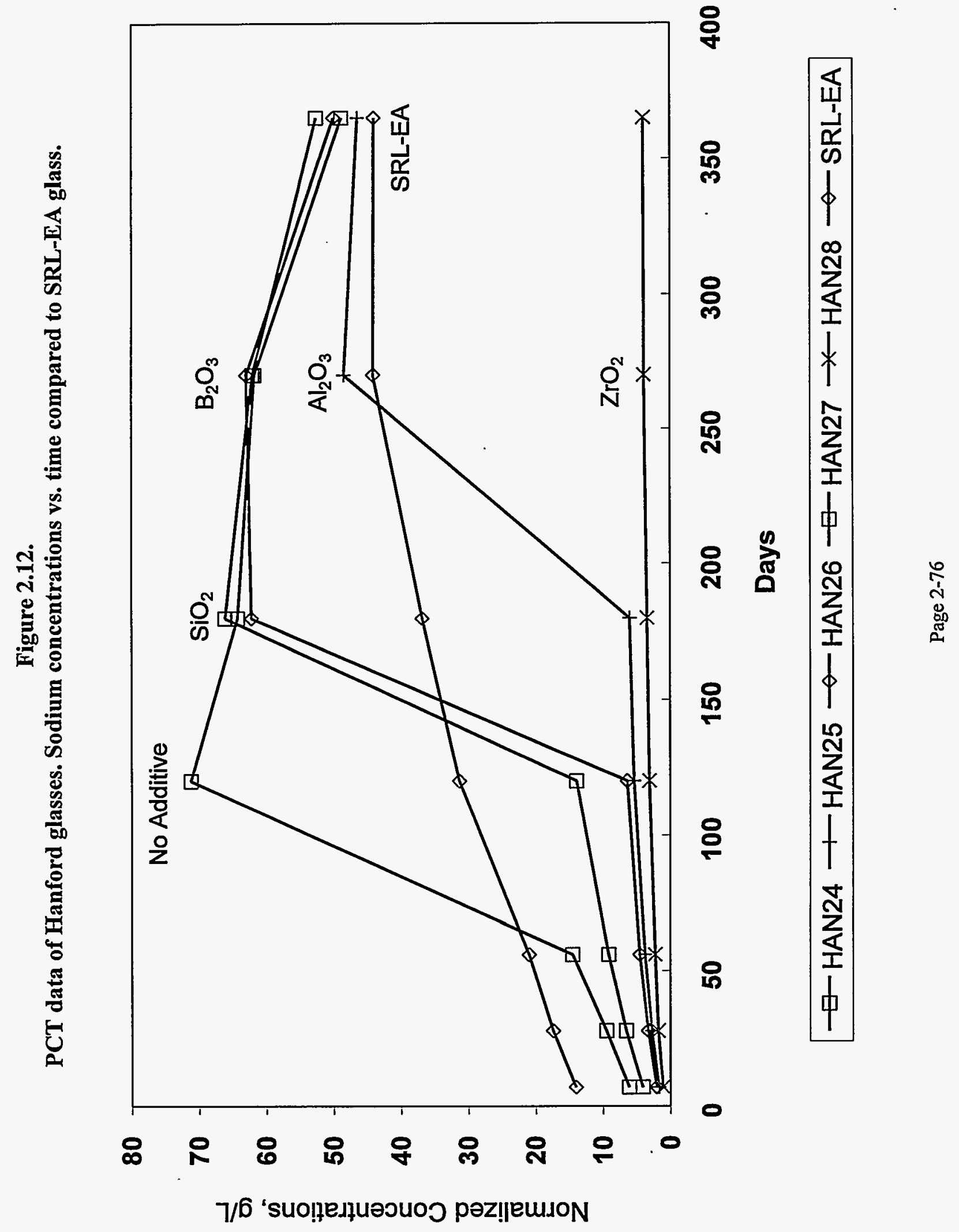


Figure 2.13.

PCT data of Hanford glasses. Sodium concentrations vs. time compared to SRL-EA glass.

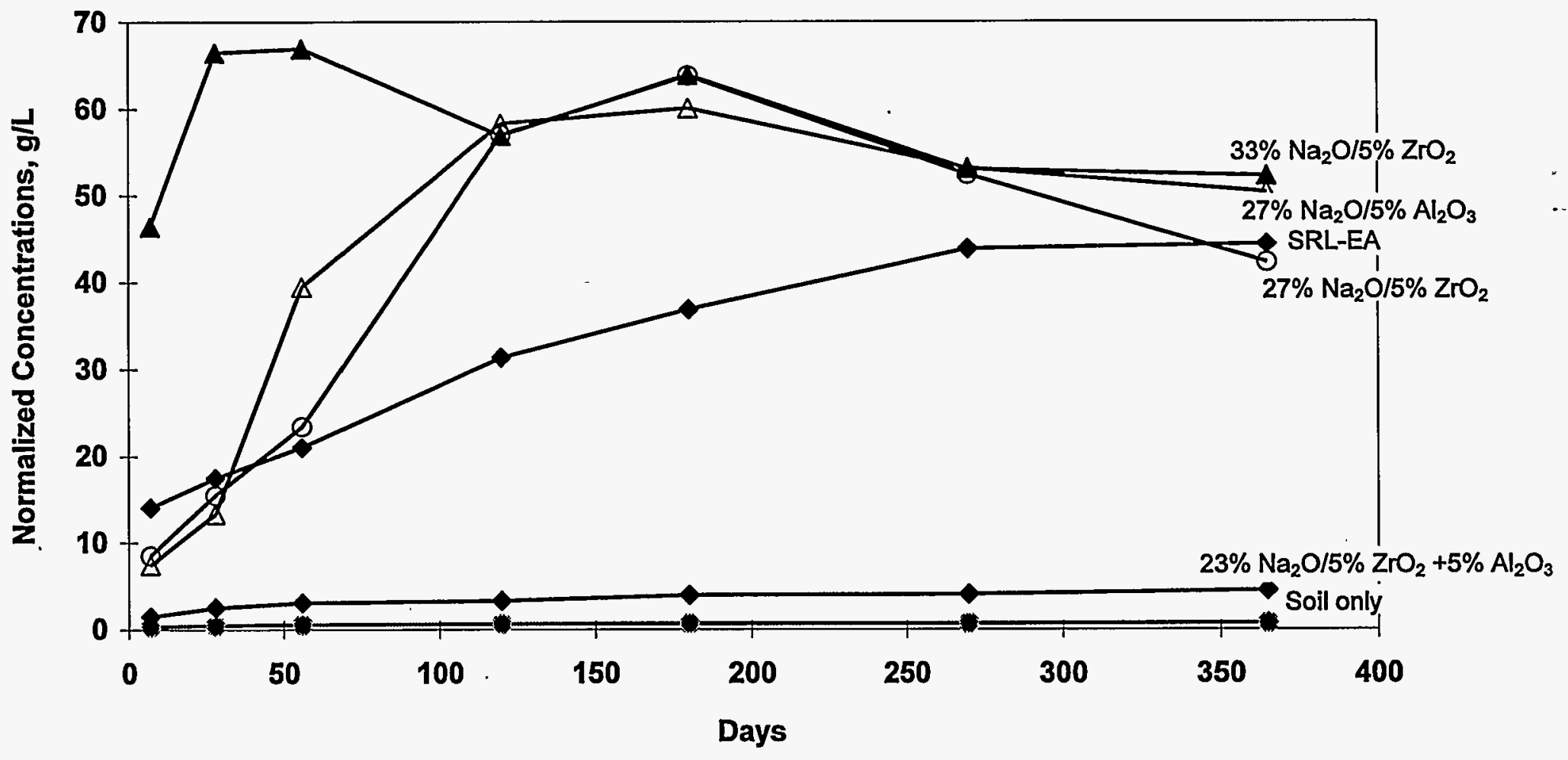

$\rightarrow-$ SRL-EA $\rightarrow$ HAN29F $-\bullet-$ HAN34 $\triangle$-HAN36 -0 -HAN37 $\rightarrow$ HAN38 


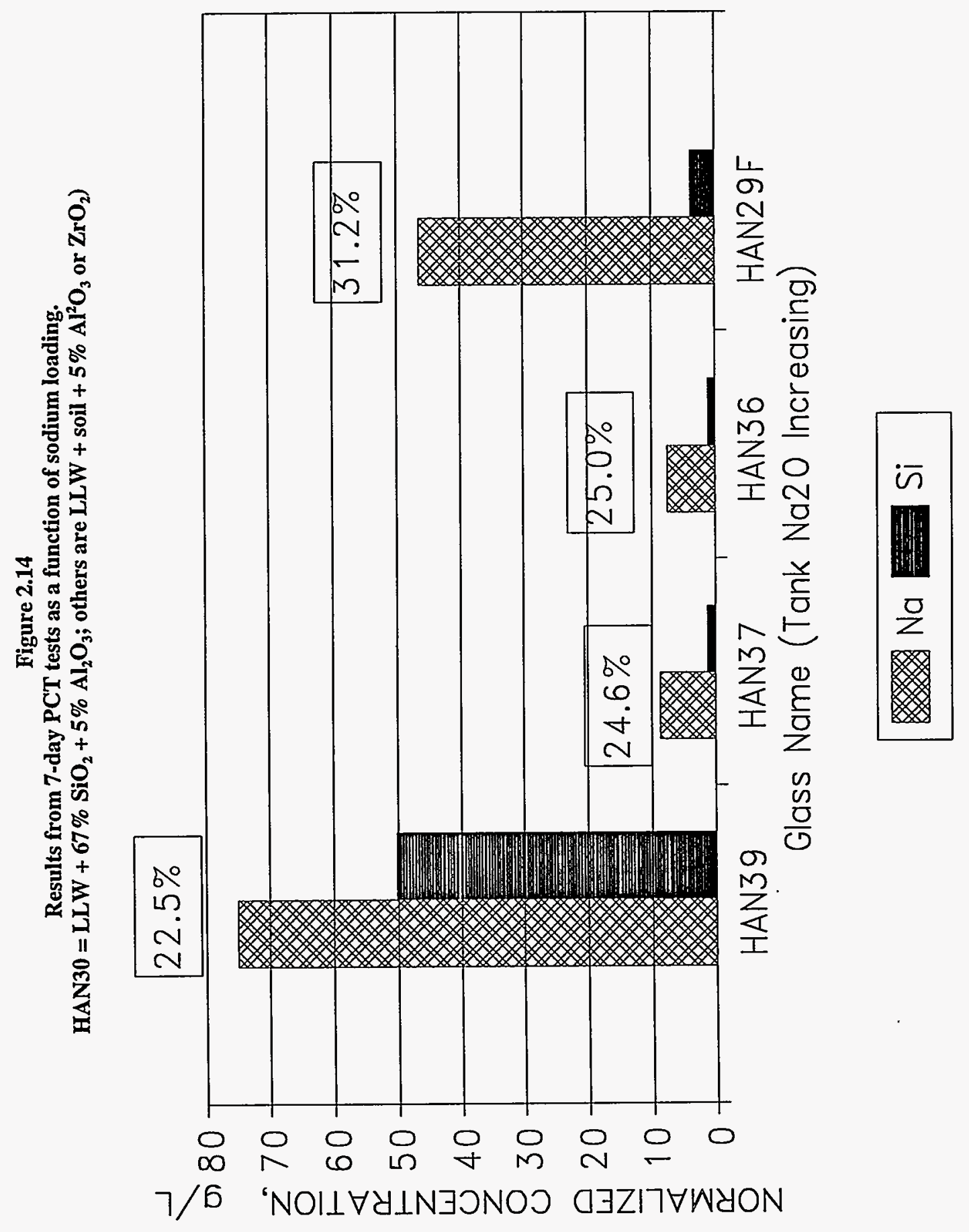

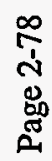


Figure 2.15

Normalized PCT leachate concentrations after 7 days for

Hanford glasses and the SRL-EA glass.

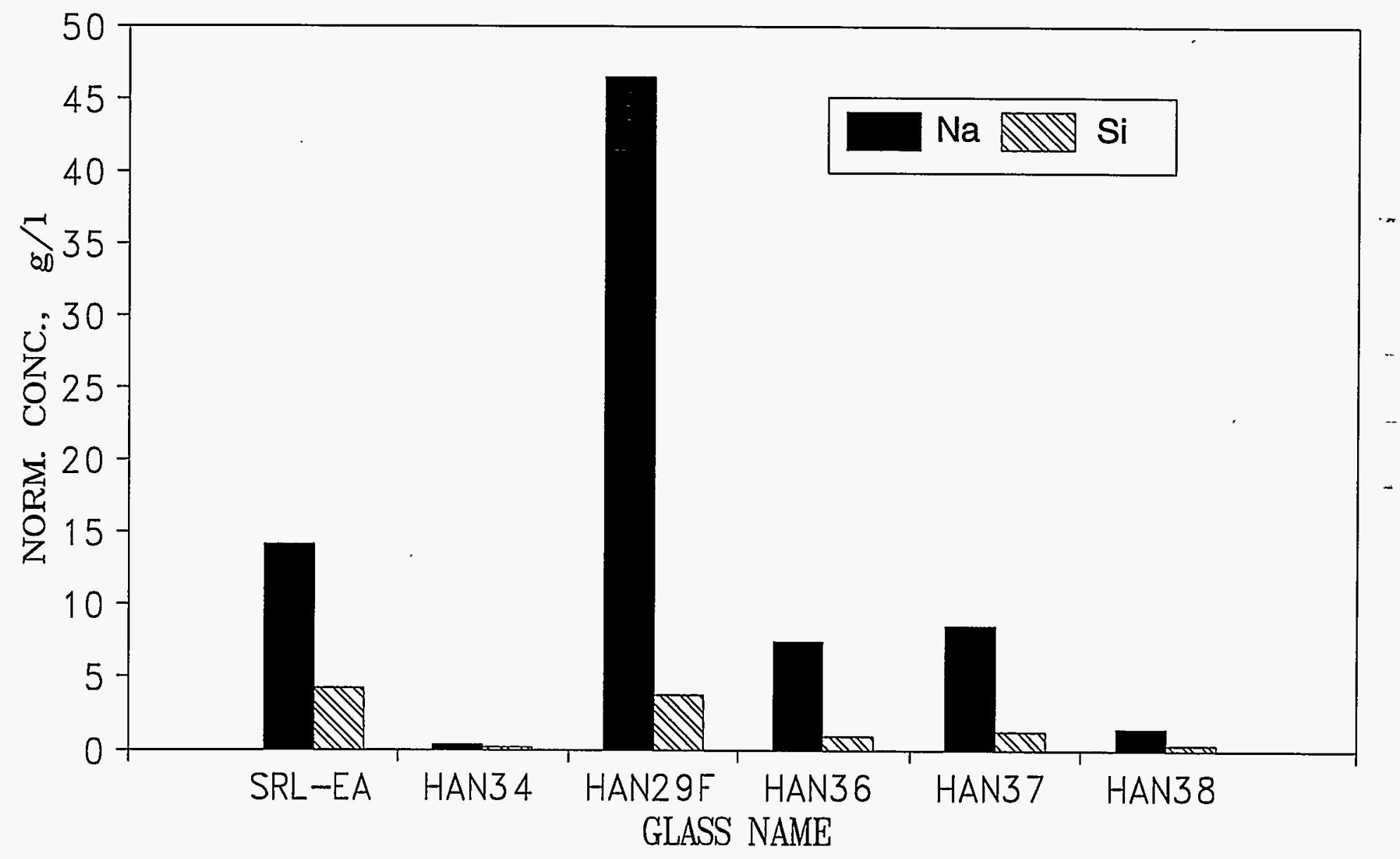

Page 2-79 
Figure 2.16.

Dehydrated, polished surface of HAN29E showing extreme sensitivity of this glass to water.

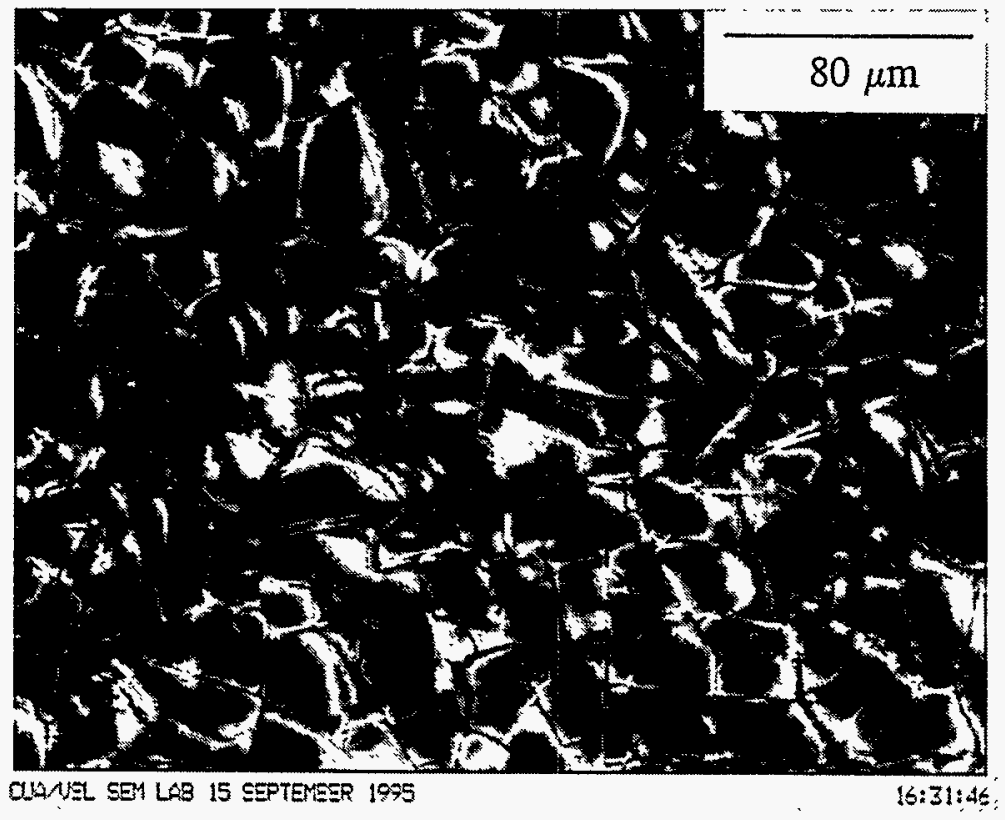


Figure 2.17.

Augite crystals in HAN2 heat treated 18 hours at $1100^{\circ} \mathrm{C}$.

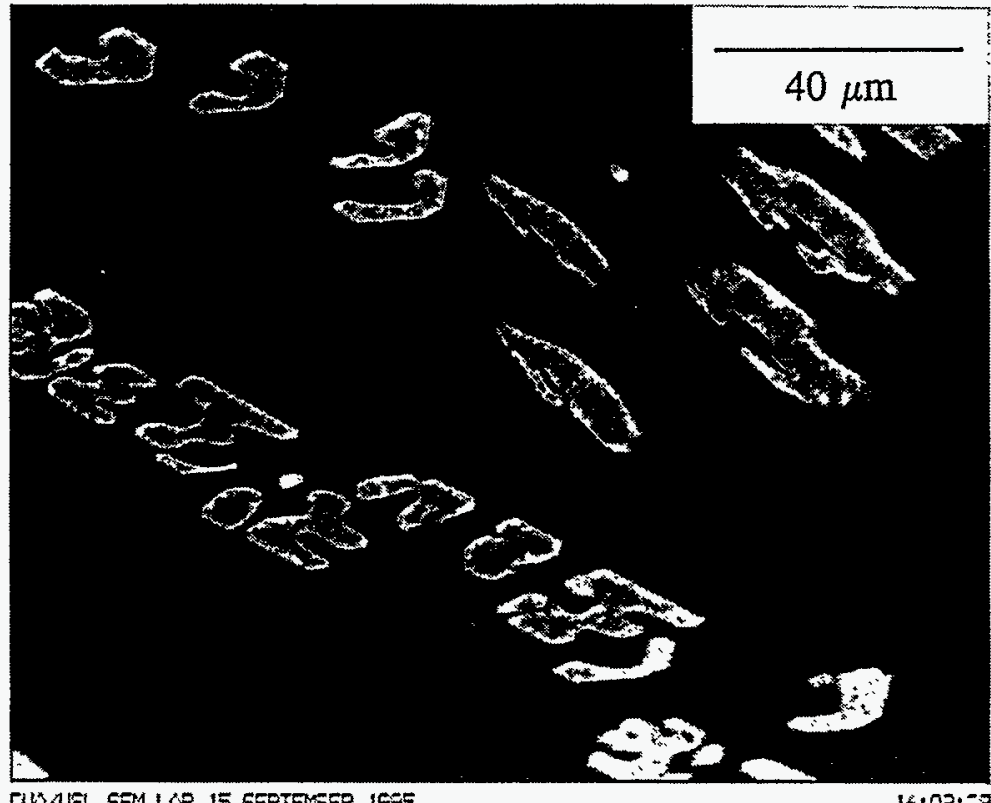

QUSAEL SBU LAB IS GEPTERER ICS5

Figure 2.18.

XRD pattern from crystals in Figure 2.17.

ID: HAN2C11H24P12H1
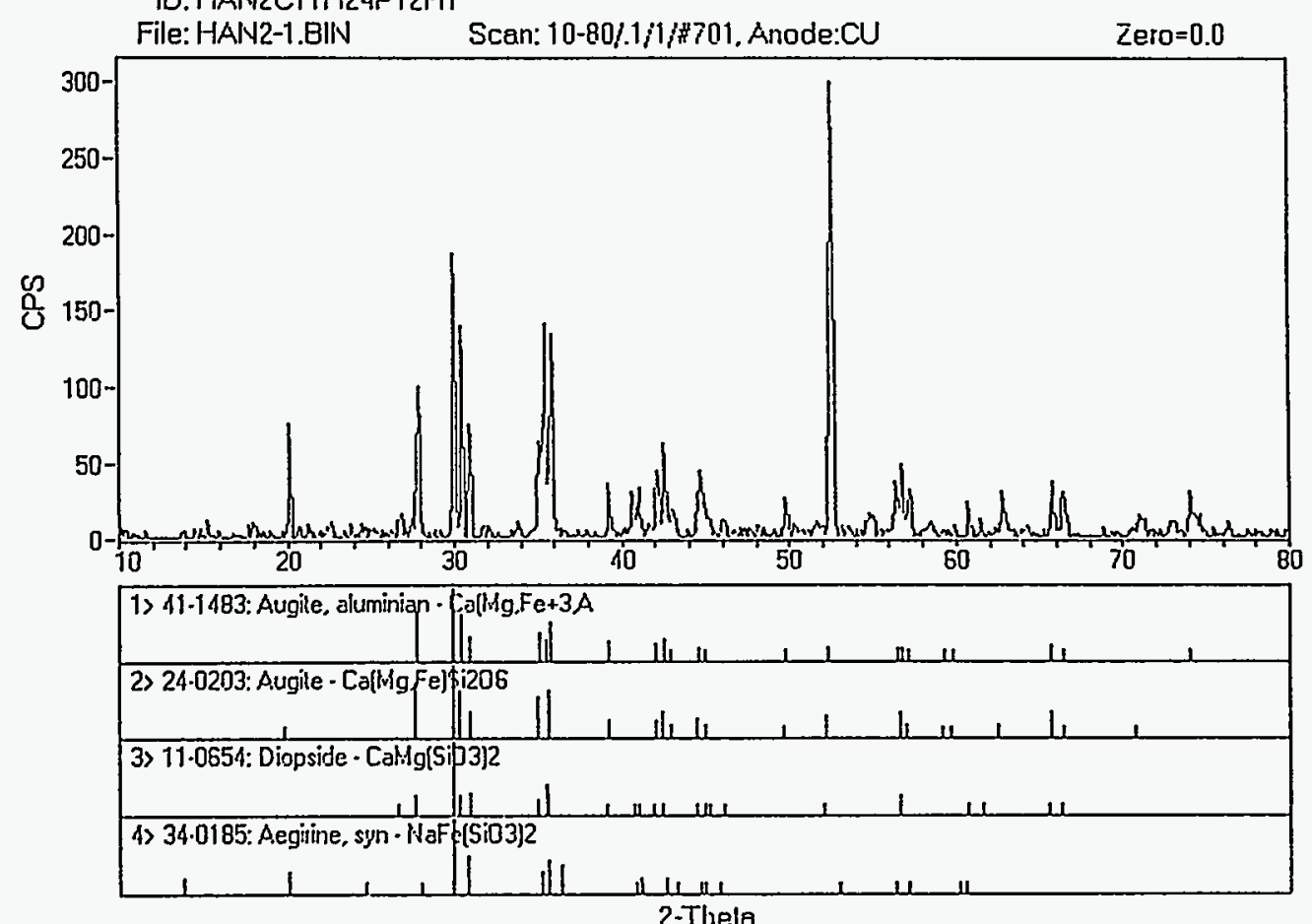
Figure 2.19.

Cristobalite-type phase in HAN9, heat treated 2 hours at $900^{\circ} \mathrm{C}$.

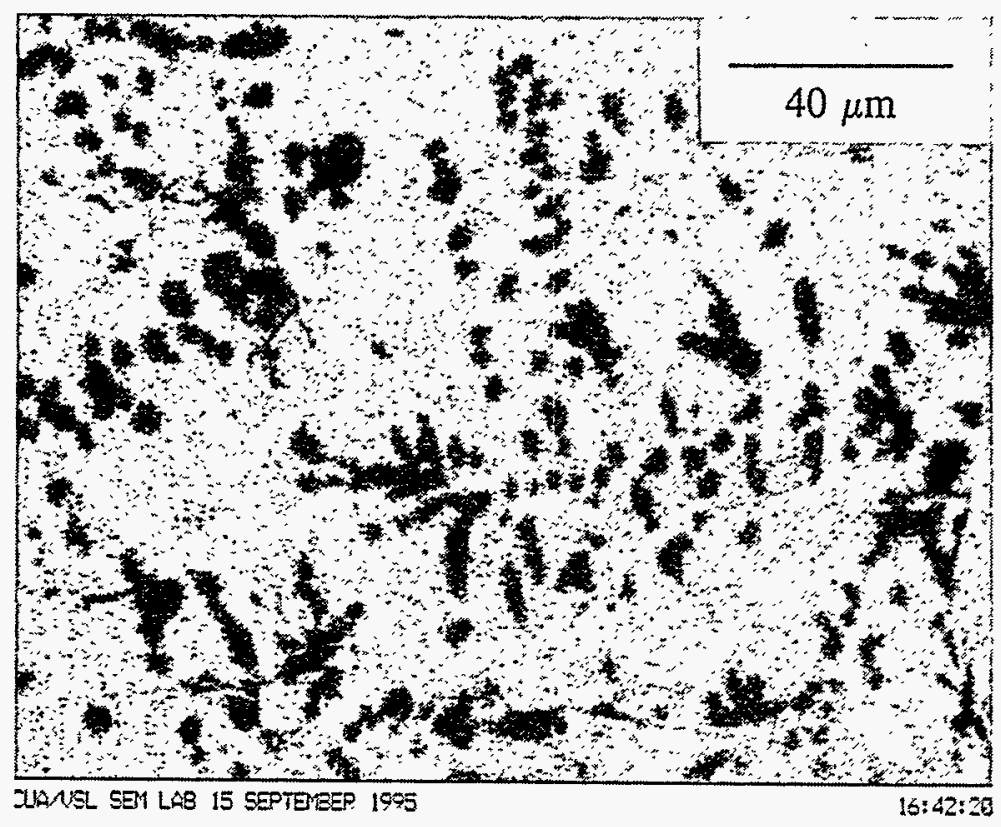

Figure 2.20.

XRD pattern from crystals in Figure 2.19.

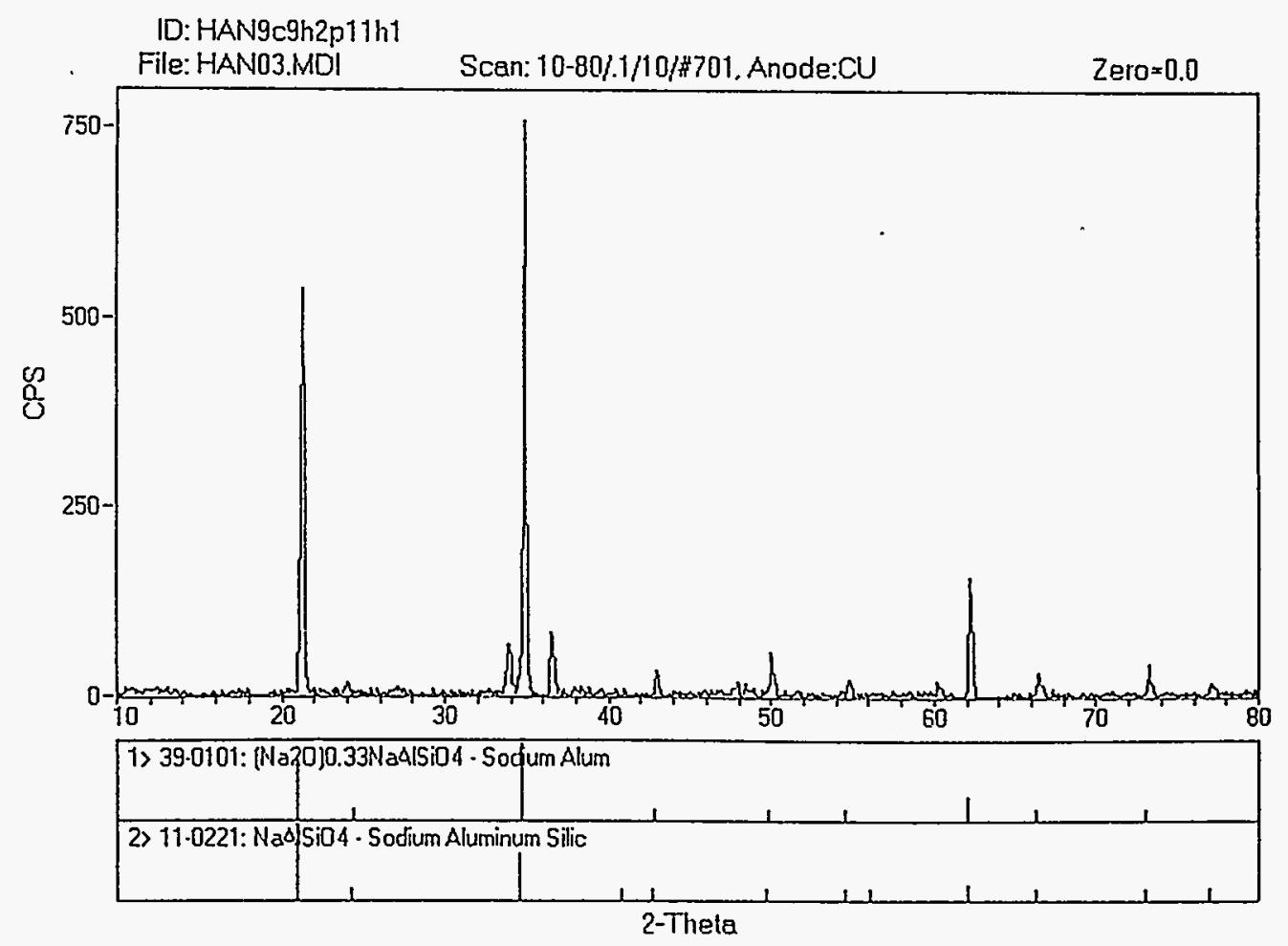

Page 2-82 
Figure 2.21.

Phase III in HAN8 heat treated for 2 hours at $900^{\circ} \mathrm{C}$. (This may actually be the remains of a water soluble phase removed during polishing).

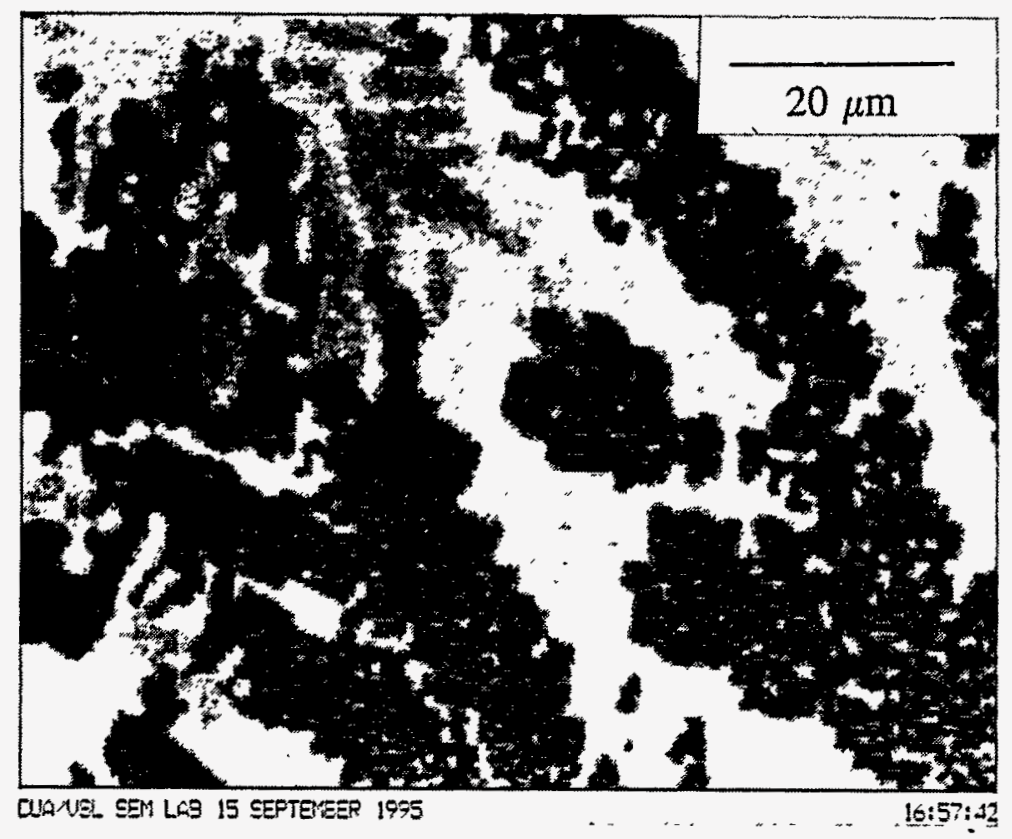

Figure 2.22.

Phase IV in HAN9, heat treated 2 hours at $900^{\circ} \mathrm{C}$. (Again, this may be the remains of a water soluble phase).

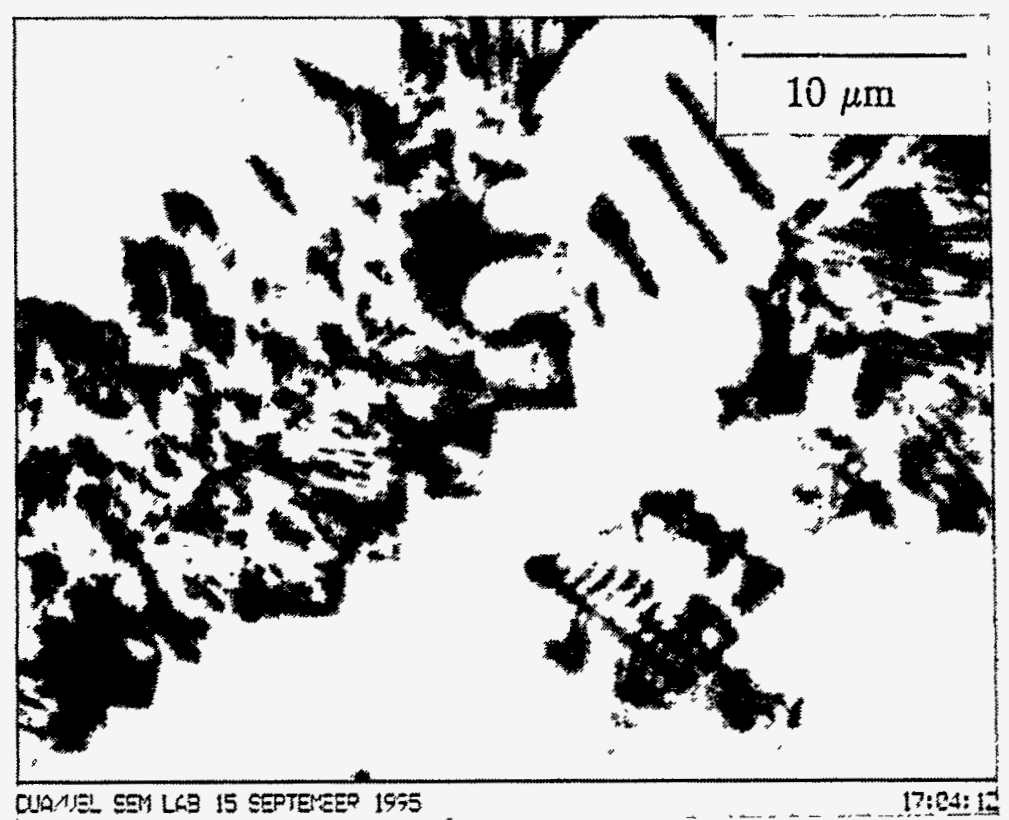


Figure 2.23.

Crystallization behavior in the $\mathrm{CaO}$ series of glasses heat treated at $1100^{\circ} \mathrm{C}$ for 24 hours. (Wollastonite $=\mathrm{CaSiO}_{3}$, triclinic, inosilicate)

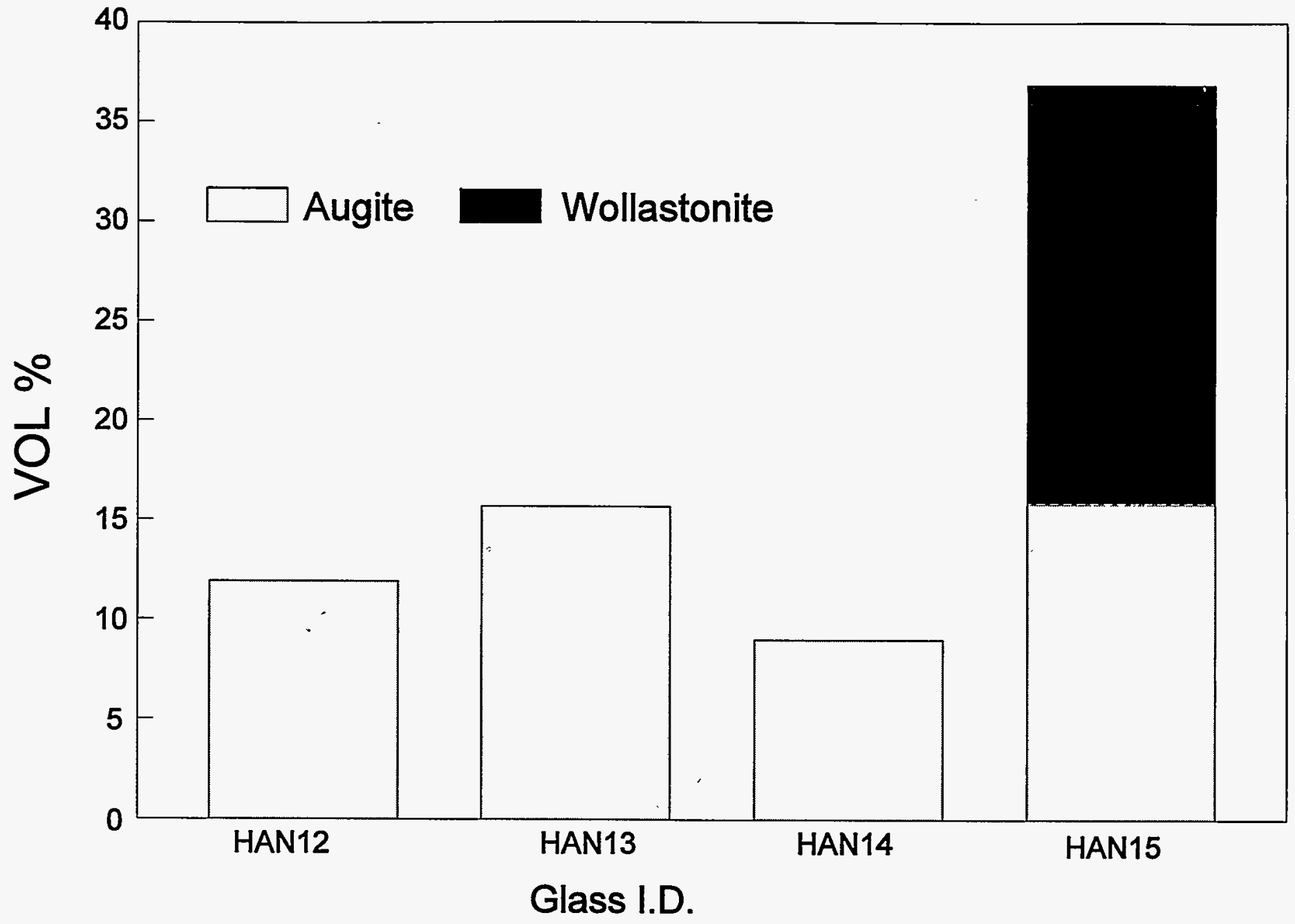


Figure 2.24.

Augite Crystals in HAN13 heat treated for 24 hours at $1100^{\circ} \mathrm{C}$.

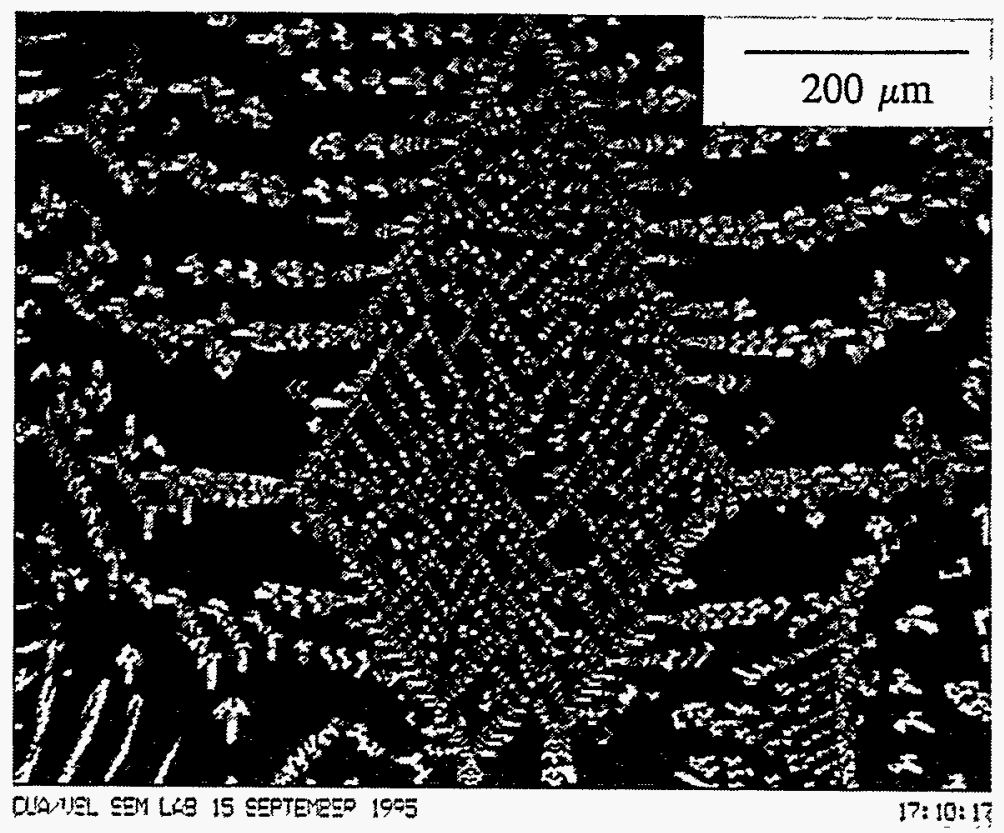

Figure 2.25.

Augite (bright polygonal) and wollastonite (grey streaks) crystals in HAN15, heat treated for 24 hours at $1100^{\circ} \mathrm{C}$.

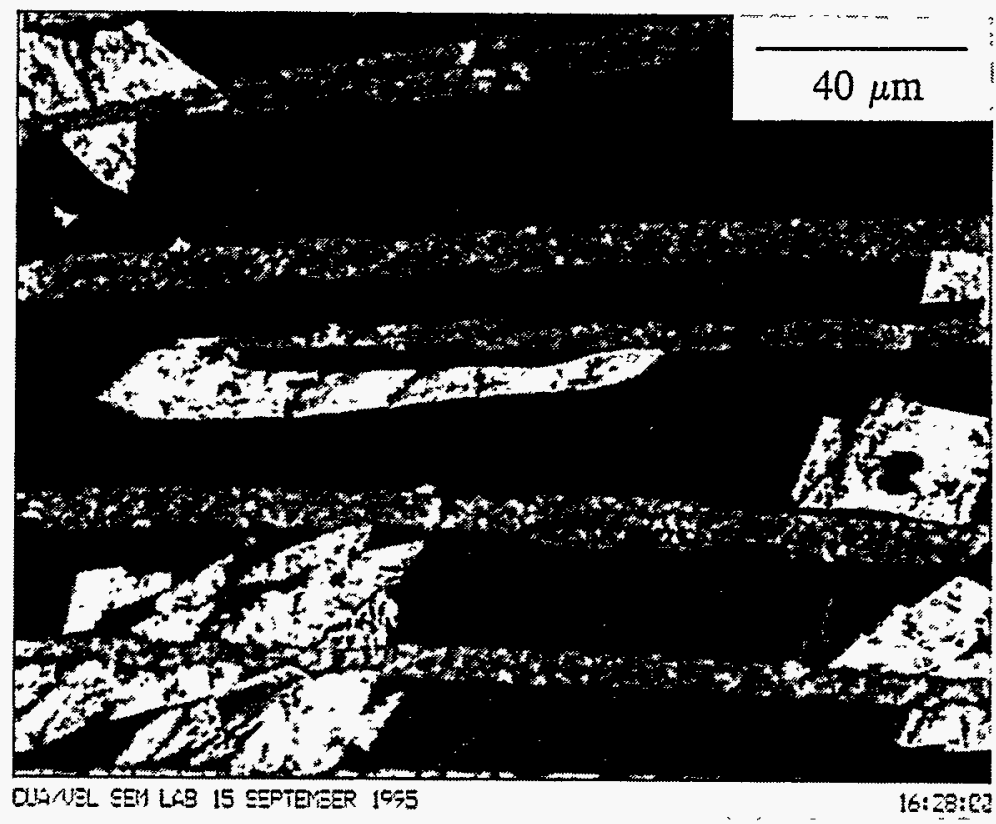


Figure 2.26.

XRD pattern from crystals in Figure 2.24.

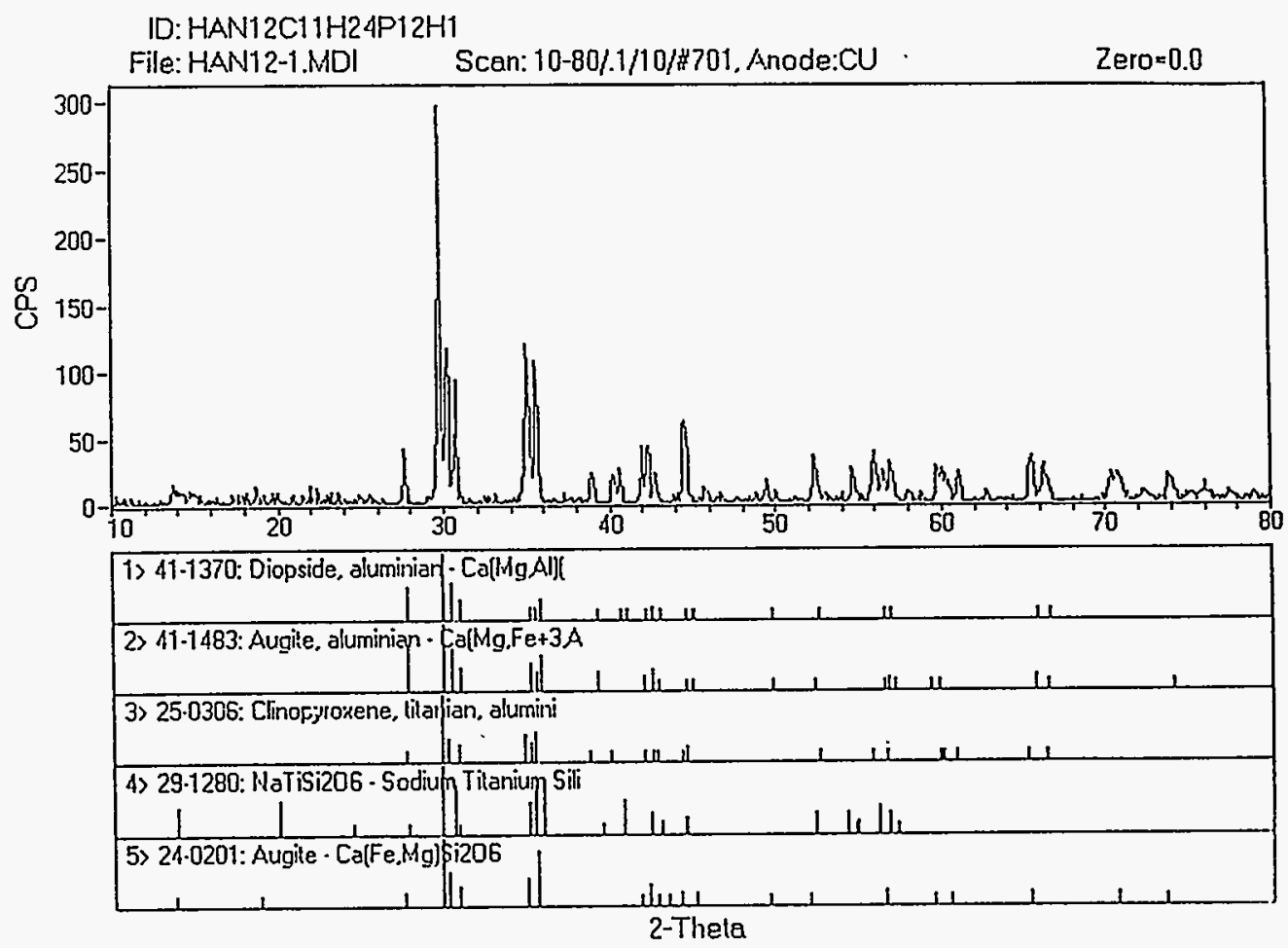

Figure 2.27.

XRD pattern from crystals in Figure 2.25.

ID: HAN15C11H24P12H1

File:HAN15-1.BIN Scan: 10-80/.1/1/\#701. Anode:CU Zero $* 0.0$
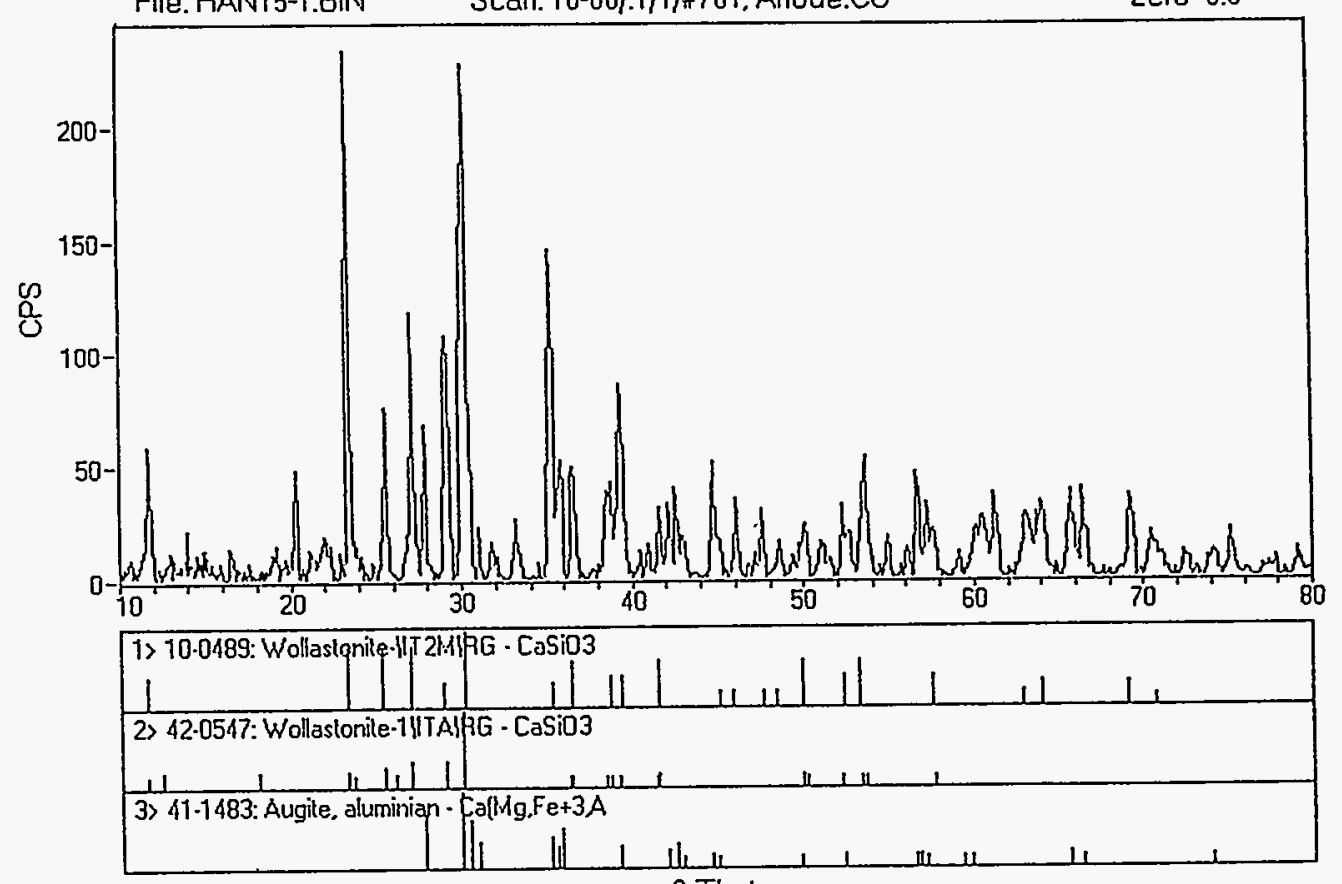

2-Thela 


\section{SECTION 3.0 \\ MAWS VITRIFICATION OF IDAHO WASTE STREAMS: CONTAMINATED ASBESTOS, EUTECTIC SALT, WERF FLY ASH, AND SOIL}

\subsection{Idaho Wastes Selected for Study}

Extensive discussions were held with representatives of the various Idaho site contractors to obtain information on Idaho site waste streams that were both suitable for, as well as available for, this work. The majority of the waste stream information data was qualitative, with most of the compositional analyses focusing on specific contaminants of concern rather than the bulk constituents. The available data were used to select a series of waste streams that were anticipated (based on origin, process history, or general descriptions) to be likely candidates for vitrification using a MAWS approach. Other factors that were considered in the selection process were: site specific interest and perceived treatment priority; volume of waste in storage and volume expected to be generated in the future, if any; whether a specific treatment technology had already been selected; whether the waste stream was representative of a bigger "class" of waste streams in the DOE complex; compositional diversity with respect to the compositional envelope objectives; compositional suitability for MAWS blending with other waste streams from the Idaho site; and availability of samples for this work in the necessary time frame.

The screening process led to the following list of waste streams which were investigated in more detail (the responsible site contractor is indicated in parentheses):

(1) Radioactive asbestos wastes (WINCO)

(2) Eutectic-salt evaporator wastes (Babcock \& Wilcox)

(3) Soils contaminated with $\mathrm{Cd}$ and $\mathrm{Pb}$ (WINCO)

(4) WERF incinerator fly ash (EG\&G)

(5) WERF incinerator bottom ash (EG\&G)

(6) TRA Warm Waste Pond sludge (EG\&G)

Samples of each of these waste streams were requested from the site for this study. However, for a variety of reasons, it was not possible to secure samples of materials (5) and (6), above; in addition, a non-radioactive soil had to be substituted for material (3). Thus, samples of three actual waste streams $(1,2$, and 4, above) and a non-radioactive site soil (referred to as a "Snake River cut") were used in this study. General descriptions and detailed characterization data, obtained on these materials from this work, are presented below. 


\subsection{Characterization of Idaho Waste Streams}

\subsubsection{Idaho Radioactive Asbestos-Containing Material (ACM)}

We have found that direct analysis of Asbestos Containing Material (ACM) generally does not provide a representative measure of the gross average composition of the material (which is relevant for vitrification) since such materials are often inhomogeneous on relatively large scales. The problem stems from the fact that most analytical techniques, particularly those involving dissolution followed by solution analysis, use a very small quantity of solid (typically, $100 \mathrm{mg}$ ), which is unlikely to be representative of the large-scale average composition. While this is, to a greater or lesser extent, a general concern in waste stream analysis, it was of particular concern for the Idaho ACM since that material was visually heterogeneous throughout the sample that was received. A technique that we have used routinely for such samples involves the conversion of a few hundred grams of material to a homogeneous glass that can then be sub-sampled on the small scale required to effect a subsequent total dissolution.

Visual sorting of the ACM sample suggested the presence of three distinct types of material and it was therefore decided to analyze samples of each of these separately. One sample of about 100 $\mathrm{g}$ of each type of material of different appearance was taken from the ACM material that was received (samples \# ID1-1, ID1-2 and D1-3). A fraction of each sample was analyzed using Scanning Electron Microscopy-Energy Dispersive X-Ray Spectroscopy (SEM-EDS) and the remainder of each sample was converted into a glass using known amounts of additives to provide a homogenized matrix for subsequent compositional analysis, as described below.

\subsubsection{Chemical Analysis and Physical Characterization}

\section{SEM-EDS Analysis}

Visual inspection of $\mathrm{D} 1-1$ characterized it as a white, very resilient fibrous material with a small amount of light brown and grey particulate matter present.

Specimen ID1-1 did not contain asbestos that could be identified by SEM-EDS analysis. The sample consisted mainly of fibers, most having aspect ratios $>10: 1$ and measuring $1-2 \mu \mathrm{m}$ in diameter, that contained only aluminum and silicon according to EDS analysis (a small amount of $\mathrm{Na}$ may also have been present). These fibers were round and appeared to be glassy in character. There were also teardrop-shaped structures present with the same composition. The only other material present was particulate matter that contained mainly $\mathrm{Ca}$ and $\mathrm{Si}$, and some $\mathrm{Mg}, \mathrm{S}, \mathrm{K}$, and $\mathrm{Fe}$. No other fibers were found in this particulate matter down to the resolution limit for this type of material $(\sim 100 \mathrm{~nm})$ in the SEM used. 
$\mathrm{X}$-ray mapping and image analysis suggest the following makeup of this material:

Aluminosilicate (possibly glass) Fibers:

Particulate Matter:

Asbestos:
$80-90$ Vol.\%

$10-20 \mathrm{Vol} . \%$

0 Vol.\%

By visual examination, specimen ID1-2 was a gray, friable material having the consistency of dry caked mud. SEM examination found that the sample contained round fibers ranging from 1-20 $\mu \mathrm{m}$ in diameter and having aspect ratios $>10: 1$. The composition of these fibers was different from those in the first sample. They contained mainly calcium and silicon with smaller quantities of $\mathrm{Al}$, $\mathrm{Mg}, \mathrm{Na}$, and $\mathrm{Fe}$. Again, the morphology of the fibers was not like that of any type of asbestos. They were generally round and showed no tendency to split nor did they give any other evidence of being bundles of fibers as is characteristic of asbestos. The only type of asbestos consistent with this elemental makeup is tremolite-actinolite, but analysis showed the aluminum and calcium content to be too high and the magnesium content too low for this form. Furthermore, there were spherical and teardrop-shaped structures of the same composition in this sample suggesting that this is some type of glassy, refractory fiber. The particulate matter contained silicon, calcium, aluminum, sodium, magnesium, iron, sulfur, potassium, titanium and a small amount of chlorine. Again, no other fibers were found down to the resolution limit.

Image analysis suggests the following approximate makeup:

Particulate Matter:

$\sim 90 \mathrm{Vol} . \%$

Non Asbestos Fibers:

$\sim 10 \mathrm{Vol} . \%$

Asbestos:

$0 \mathrm{Vol} . \%$

Sample ID 1-3 appeared by visual inspection to be mostly fibers, but well interspersed with light brown particulate matter.

In the SEM, D1-3 was similar to ID1-2 in fiber morphology and composition, but the fraction of fibers was much larger. Again, no asbestos fibers were found.

Image analysis suggests the following approximate makeup:

Particulate Matter:

20-30 Vol.\%

Fibers:

70-80 Vol.\%

Asbestos:

0 Vol.\%

Figures 3.1 and 3.2 present EDS spectra of the two types of fibers found in the samples. Because of the apparent discrepancy in the labeling of this material as asbestos-containing and our 
SEM-EDS analysis, which revealed no asbestos, as well as to ensure appropriate regulatory compliance within our facility, these three samples and five additional samplings of the parent material were sent to an independent laboratory possessing both NVLAP (National Voluntary Laboratory Accreditation Program) and AIHA (American Industrial Hygiene Association) certification for analysis by polarized light microscopy (PLM). Their findings essentially confirmed our SEM-EDS work. A few asbestos fibers were found in three of the eight samples submitted, but in every case the concentration of such fibers was below the $1 \mathrm{wt} \%$ regulatory limit; thus, for regulatory purposes, the material that we received from Idaho would not be classified as an asbestos containing material. For consistency with the waste type designation used at Idaho, however, we will continue to refer to this material as ACM.

\section{Thermogravimetry}

The weight loss of each ACM sample was determined at several temperatures. Duplicate samples of the $\mathrm{ACM}$ were weighed and then heated at $110^{\circ} \mathrm{C}$ for $18 \mathrm{hrs}$ and reweighed to determine the weight loss. The process was repeated on fresh duplicate samples at $450^{\circ} \mathrm{C}$ for $4 \mathrm{hrs}$ and at $1150^{\circ} \mathrm{C}$ for $4 \mathrm{hrs}$; the results are shown in Table 3.1. Additional indications of weight loss at $1150^{\circ} \mathrm{C}$ were obtained from fusions of the ACM samples with borax $\left(\mathrm{Na}_{2} \mathrm{~B}_{4} \mathrm{O}_{7}\right)$ that were made for the purposes of compositional analysis, as discussed above. The weight loss varied considerably between the three samples of ACM, which were of different visual appearance, as did the weight losses measured on vitrification ( $4 \%, 43 \%$, and $23 \%$ from, respectively, IDG1, IDG2 and IDG3). The primary sources of the observed weight losses from this material are probably water, both physical and chemical, carbonates, and possibly some organic materials.

\section{DCP Chemical Analysis}

For the reasons mentioned above, this rather inhomogeneous material was analyzed by first dissolving around $100 \mathrm{~g}$ in a glass melt of known initial composition to form a homogeneous material that can be sub-sampled. A small amount (typically $100 \mathrm{mg}$ ) was then subjected to total dissolution followed by solution analysis by DCP. A sample of each of the three visually different types of the ACM was fused to form a glass, typically consisting of 50-70\% waste and 30-50\% $\mathrm{Na}_{2} \mathrm{~B}_{4} \mathrm{O}_{7}$, for subsequent analysis. Additional compositional information was obtained from six other melts that were made using the Idaho eutectic salt waste stream, discussed below, in place of the borax (IDG7A, IDG7B, IDG7C, IDG8A, IDG8B and IDG8C); the salt sample was analyzed with good reproducibility (see below) which allowed subtraction of its components from the glass analysis to yield the composition of the ACM fraction that was used. The results of these analyses, together with various averages, are presented in Table 3.2. As expected on the basis of the SEM-EDS analyses, the primary constituents of this material are, on an oxide basis, alumina (11 to $20 \mathrm{wt} \%$ ), calcia (19 to $27 \mathrm{wt} \%$ ), and silica (43 to $47 \mathrm{wt} \%$ ). 


\section{Ion Chromatography and Ion Selective Electrode Analyses}

The same solutions used for the DCP analyses were also subjected to analyses to determine anion contents, with the primary interest being sulfates, fluorides, and chlorides. Ion chromatography showed sulfates and chlorides to be below their detection limits and the same result was found for fluoride determined by ion selective electrode. The detection limits for these methods as employed correspond to about $0.5 \mathrm{wt} \%$ on a solids basis.

\section{Total Carbon (TC) and Total Organic Carbon (TOC) Analysis}

In view of the small quantity of sample used in the TOC analysis together with the obvious gross inhomogeneity of the ACM, there would be little value in running this analysis unless a very large number of samples were used. TC/TOC analysis was therefore not performed on this waste stream.

\section{Gamma Spectroscopy}

The three borax glasses formed from the ACM samples were also used for determination of radionuclides by gamma spectroscopy. A one gram sample of each glass was dissolved in $500 \mathrm{ml}$ of $\mathrm{HF} / \mathrm{HNO}_{3}$ to provide the requisite quantity of liquid sample for calibrated quantitative analysis. The results, expressed in $\mathrm{nCi} / \mathrm{g}$, are shown in Table 3.4 . Essentially all of the activity is due to ${ }^{137} \mathrm{Cs}$ (peak at $661 \mathrm{KeV}$ ), as shown in Figure 3.3a.

Once again, the inhomogeneity of the ACM was evident with the gamma activity measurements falling between 0.8 and $11.3 \mathrm{nCi} / \mathrm{g}$.

\subsubsection{Idaho Radioactive Fly Ash}

\section{Thermogravimetry}

The weight losses upon heating for this material are summarized in Table 3.1. These data indicate that the material is very dry (low loss at $110^{\circ} \mathrm{C}$ ), as would be expected given its origin, but contains significant amounts of other volatile constituents that could include carbonates, hydroxides, organic or acid anion constituents. Since the weight loss at $1150^{\circ} \mathrm{C}$ was about $64 \%$, only $36 \%$ of the mass of the waste will contribute toward glass formation. This suggests that there will be a substantial volume reduction when vitrifying this waste. 


\section{Specific Gravity}

The specific gravity of the fly ash sample, as received, was measured according to the ASTM procedure $D$ 854-83 which is based on the displacement of water. The particulate density of 2.88 $\mathrm{g} / \mathrm{cm}^{3}$ is considerably higher than the dry bulk density of this material, as shown in Table 3.1

\section{Particle Size Analysis}

The particle size distribution was determined by sieving according to ASTM D 422-63 with the results shown in Table 3.1 and Figure 3.4a. The majority of the material is coarser than 60 mesh (250 microns).

\section{DCP Analysis}

The fly ash sample was analyzed directly after heating at $1150^{\circ} \mathrm{C}$ for 4 hours and also after fusion in a 50\% borax glass melt (IDG9). Table 3.3 summarizes the results from both of these analyses. The DCP analyses accounted for only $46 \%$ of the total mass of material dissolved. Most of the remainder can be accounted for once the results of ICPMS, ion chromatography, and carbon analyses are included, as described below.

\section{Ion Chromatography and Ion Selective Electrode Analysis}

The same solutions used for the DCP analyses were also subjected to analyses to determine anion contents, with the primary interest being sulfates, fluorides, and chlorides. Ion chromatography revealed a solution sulfate content of $35 \mathrm{ppm}$, which corresponds to $17.47 \mathrm{wt} \%$ in the original material. Chloride and fluoride (by ISE) were both below their detection limits, which correspond to about $0.5 \mathrm{wt} \%$ on a solids basis.

\section{ICPMS Analysis}

The analytical mass balance from DCP suggested the presence of several species that were not on the initial target analyte list. In order to identify those elements, compositional analysis was performed using ICPMS in the scanning mode (from mass 50 to 242); the results indicate high concentrations of zinc, antimony, copper, and lead. Quantitative analysis of these constituents was therefore performed by ICPMS. Zinc, copper and lead were also subsequently analyzed by DCP and good agreement was found between the two methods. Antimony was determined only by ICPMS but 
was confirmed semi-quantitatively by SEM-EDS. The overall analysis of this material is summarized in Table 3.3 .

\section{Total Organic Carbon (TOC) and Total Carbon (TC)}

The total carbon was determined by measuring the amount of carbon dioxide resulting from combustion of a known amount of the waste sample at $800^{\circ} \mathrm{C}$. The total organic carbon (TOC) was measured using the same technique; however, the waste sample was first mixed with acid and sparged to remove the inorganic carbon.

The results of the TC and TOC measurements on the fly ash are presented in Table 3.1. The sample $\mathrm{D} 3$ contains $22.9 \mathrm{wt} \%$ total carbon of which $22.4 \mathrm{wt} \%$ is organic material, indicating that the amount of carbonate is therefore very low. These results are generally consistent with the origin of this waste stream: The high temperatures have decomposed any carbonates and residual carbon is present as a product of incomplete combustion.

\section{Gamma Spectroscopy}

Table 3.4 lists the results from analysis of the fly ash using gamma spectroscopy. The predominant contribution to the overall activity of this material is due to Cs-137, as can be seen from Figure 3.3b.

\section{SEM-EDS Analysis}

The ID3 material has the appearance of a black, finely divided powder. A small quantity was mounted on an SEM stub using conductive adhesive and examined in the SEM. The typical particle size was generally $1 \mu \mathrm{m}$ and smaller. Composite EDS spectra from a rastered area approximately 50 $\mu \mathrm{m}$ square showed the presence of substantial amounts (around $10 \%$ ) of $\mathrm{Na}, \mathrm{Si}, \mathrm{S}, \mathrm{Pb}, \mathrm{Cl}$ and $\mathrm{Zn}$, and moderate amounts (1-10\%) of $\mathrm{Ca}, \mathrm{Sb}, \mathrm{Al}, \mathrm{Fe}, \mathrm{K}$ and $\mathrm{P}$. Spectra acquired by spotting the beam on individual particles indicated that the material is heterogeneous. Representative spectra are shown in Figures 3.5-3.9 to indicate the range of compositional variation as well as the average composition of the fly ash.

The fly ash was heated alone at $1150^{\circ} \mathrm{C}$ in a fireclay crucible as part of the thermogravimetric analysis, and a massive array of acicular crystals formed (Figure 3.10). Crystals toward the center of the crucible were mostly straight, long $(10-20 \mathrm{~mm})$ and dark brown to black in color. Crystals midway to the edge of the crucible were highly branched and lighter in color. Crystals growing at the crucible were short, white, acicular sprays. The major components of the crystals detected using 
SEM-EDS methods were $\mathrm{Sb}$ and $\mathrm{Zn}$. Some of the crystals contained small amounts of $\mathrm{Fe}, \mathrm{Cu}$, and small to moderate amounts of S. The crystals growing on the crucible surface also contained $\mathrm{Si}$ and Al from the crucible. Spectra in Figures 3.11-3.15 show qualitatively the range of composition variation.

A crushed, sieved specimen of a borax glass, made from the fly ash and designated IDG9, was also examined using SEM-EDS methods. The specimen was not homogeneous despite the borax fusion. The spectra (Figures 3.16-3.20) show the compositional variations that were found.

\subsubsection{Idaho Radioactive Eutectic Salt}

\section{Thermogravimetry}

Heat treatments at $110^{\circ} \mathrm{C}, 450^{\circ} \mathrm{C}$ and $1150^{\circ} \mathrm{C}$ show weight losses of $0.92,6.42$, and $46 \%$, respectively. This indicates that this material is very dry; the losses at higher temperatures are generally consistent with the presence of significant amounts of carbonates and nitrates, as determined by other methods discussed below.

\section{Specific Gravity}

The specific gravity of the salt sample was measured after drying at $110^{\circ} \mathrm{C}$ (which, as described above, led to less than 1\% weight loss). Again, the ASTM D 854-83 procedure was used but since the measurement is based on the displacement of water, the solubility of the sample in water should be considered in using the result obtained. The measured value of $2.57 \mathrm{~g} / \mathrm{cm}^{-3}$ is considerably higher than the dry bulk density, as shown in Table 3.1.

\section{Particle size Analysis}

The results of the particle size distribution analysis on this material are shown in Table 3.1 and Figure 3.4b. The salt is a very fine material with greater than $50 \%$ below $150 \mu \mathrm{m}$. This material should present little difficulty for prospective feed delivery systems and the small particle size is beneficial in that it should lead to rapid reaction in a vitrification process.

\section{DCP Chemical Analysis}

Since this material was expected to be soluble, a simple dissolution of a small quantity in water was used as well as our standard $\mathrm{HF} / \mathrm{HNO}_{3}$ dissolution procedure to produce solutions for 
subsequent analyses. The elemental analysis by DCP yields the oxide composition shown in Table 3.5. This analysis, of course, only accounts for a portion of the mass of the original material due to the presence of anions, as discussed below. For glass formation purposes, what is most relevant is the oxide composition of approximately $47 \% \mathrm{~K}_{2} \mathrm{O}, 9 \% \mathrm{Li}_{2} \mathrm{O}, 35 \% \mathrm{Na}_{2} \mathrm{O}$, and $9 \% \mathrm{U}_{3} \mathrm{O}_{8}$.

\section{Total Organic Carbon (TOC) and Total Carbon (TC)}

Results of the TC and TOC measurements are shown in Table 3.1. The eutectic salt material contains $0.9 \%$ organic carbon and $6.9 \%$ total carbon. These results indicate that $6 \%$ of the total carbon is in the form of inorganic carbon $(6.9 \%-0.9 \%=6 \%)$, which translates to $30 \mathrm{wt} \% \mathrm{CO}_{3}{ }^{2 .}$.

\section{Ion Chromatography and Fluoride electrode Analysis}

After dissolution of samples of the material, the concentration of fluoride, chloride, nitrate, and sulfate were measured using ion chromatography; fluoride was also measured by an ion selective electrode technique. The fluoride concentration was found to be below the detection limit by both methods (i.e., below about $0.5 \mathrm{wt} \%$ in the original material); the concentrations of chloride, nitrate, and sulfate were $3.3 \mathrm{wt} \%, 16 \mathrm{wt} \%$, and $0.6 \mathrm{wt} \%$ in the original material, respectively.

Combining these results with the results of the analyses discussed above, the overall composition of the eutectic salt can be summarized as follows:

METALS (fraction of total metals)

$50.8 \mathrm{wt} \%$ Potassium

$5.4 \mathrm{wt} \%$ Lithium

$33.8 \mathrm{wt} \%$ Sodium

$2.9 \mathrm{wt} \%$ Uranium

100

ANIONS (fraction of total anions)

$60.1 \mathrm{wt} \%$ Carbonates

$32.1 \mathrm{wt} \%$ Nitrate

$6.6 \mathrm{wt} \%$ Chloride

$1.2 \mathrm{wt} \%$ Sulfate

100 


\section{Gamma Spectroscopy}

One gram of the eutectic salt material was dissolved in $500 \mathrm{ml}$ of an acid solution for measurement of gamma activity (Figure 3.3c). The results indicate the presence of about $0.017 \mu \mathrm{Ci} / \mathrm{g}$ of ${ }^{234} \mathrm{Th}$ from the decay of ${ }^{238} \mathrm{U}$. Eight-tenths of a percent of the total measured gamma activity was due to ${ }^{235} \mathrm{U}$; this corresponds to about $0.2 \%$ of the total uranium content measured by DCP (Table 3.4), suggesting that the material is somewhat depleted.

\subsubsection{Idaho Non-Radioactive Soil}

The Snake River Cut soil sample received from the Idaho site was assayed for weight loss, subjected to TC/TOC measurements, and analyzed by DCP after total dissolution to obtain the compositional information required to develop formulations for crucible melts. Particle size distribution and fluoride analysis completed this characterization.

\section{Thermogravimetry}

Heat treatments at $110^{\circ} \mathrm{C}, 450^{\circ} \mathrm{C}$, and $1150^{\circ} \mathrm{C}$ led to respective weight losses of $3.1 \%$, $3.92 \%$, and $12.42 \%$. The soil evidently contains very little moisture; the level of non-glass-forming components other than free water is about $9 \mathrm{wt} \%$.

\section{Specific Gravity}

The specific gravity of the soil sample was measured after drying at $110^{\circ} \mathrm{C}$ according to the ASTM procedure D $854-83$. The value of $2.58 \mathrm{~g} / \mathrm{cm}^{3}$ is typical of soils; this is substantially higher than the dry material bulk density, as shown in Table 3.1 .

\section{Particle size Analysis}

The results from the particle size analysis are shown in Table 3.1 and Figure 3.4(c). The soil is primarily composed of particulates predominantly between 0.4 and $2 \mathrm{~mm}$ in size. 


\section{Total Organic Carbon (TOC) and Total Carbon (TC)}

The results of the TC and TOC measurements are presented in Table 3.1. The Idaho soil contains about $2.77 \%$ total carbon of which $1.11 \%$ is organic material. The difference of $1.66 \%$ inorganic carbon is equivalent to about $8 \%$ as $\mathrm{CO}_{3}{ }^{2-}$.

\section{DCP Analysis}

A sample of the Idaho soil was dried at $1150^{\circ} \mathrm{C}$ and three sub-samples of the resulting material were dissolved separately in $\mathrm{HF} / \mathrm{HNO}_{3}$; the resulting solutions were then analyzed by DCP. The results obtained are presented as an average composition in Table 3.5. The soil is basically a calcium aluminosilicate. As oxides, these three components $\left(\mathrm{CaO}, \mathrm{Al}_{2} \mathrm{O}_{3}\right.$, and $\left.\mathrm{SiO}_{2}\right)$ account for, respectively, $13 \%, 9 \%$, and $65 \%$ of the mass, with magnesium, iron, potassium, and sodium as minor components $(6 \%, 3 \%, 2 \%$, and $2 \%$, respectively). The soil is therefore well suited for use as a glassforming additive for vitrification.

\section{Ion Chromatography and Fluoride Analysis by Potentiometry}

The solution prepared for DCP analysis was also subjected to ion chromatographic analysis; no sulfate was detected by this method. The fluoride content was also examined using an ion selective electrode technique; again, none was detected. These results suggest that the concentrations of these constituents in the original soil were both below about $0.5 \mathrm{wt} \%$.

\subsubsection{Summary of Waste Stream Characterization Results}

The Idaho waste streams selected exhibit bulk densities between 0.3 to $1.3 \mathrm{~g} / \mathrm{cm}^{3}$ (though particle densities are as high as $2.9 \mathrm{~g} / \mathrm{cm}^{3}$ ) and weight losses upon heating at $1150^{\circ} \mathrm{C}$ reaching as much as $64 \%$ (see Table 3.1), which together indicate that there is the potential for large volume reductions on vitrification. The results from the chemical characterization of these wastes suggest that their compositions are amenable to the benefits of blending in a MAWS approach. Table 3.5 shows that both glass formers and fluxes are well represented among these waste streams and their compositions are generally complementary for vitrification. Furthermore, from a compositional envelope perspective, these waste streams span a wide range of compositions. As examples, silica is found at $0.4 \%$ in the eutectic salt, $5.1 \%$ in the fly ash, at $45 \%$ in the ACM, and $65 \%$ in the soil; similarly, alumina ranges from $0.1 \%$ to $13 \%$, calcia from $0.1 \%$ to $25 \%$, lithia from $0.1 \%$ to $9 \%$, and soda from $1 \%$ to $35 \%$; many other constituents show similarly wide ranges, as shown in Table 3.5. 
The concentrations of contaminant species are also large in these waste streams, both for radioactive elements and for RCRA elements; this is especially so for the eutectic salt, with 9 wt\% uranium, and the fly ash which contains cesium and six RCRA elements, some at concentrations of several percent. Characterization of the glasses made from these wastes will provide information on the ability of the waste form to contain these contaminants when subjected to aqueous leaching.

\subsection{Vitrification Testing of Idaho Waste Streams}

\subsubsection{Selection of Glass Formulations}

The target and analyzed compositions of all of the subsequent glasses prepared from the Idaho waste streams are presented in Table 3.6. Note that a given target composition (i.e., the composition based on the waste stream proportions in the batch and the nominal waste compositions [Table 3.5]) will, in general, give rise to a range of actual glass compositions due to the inherent variability of the waste streams (and particularly the $\mathrm{ACM}$ ). For instance, the single target composition, IDG8, gave rise to the three replicate melts IDG8A, IDG8B, and DG8C with the respective analyzed compositions shown in Table 3.6. As Table 3.6 shows, the analyzed compositions of the replicate melts varied considerably in their consistency with the target. The analyzed composition of each melt was used in all of the subsequent data analysis.

Also shown in Table 3.6 are data on melting time and temperature, oxidation state, and observations of visible sulfate phase separation from the melt. The presence of sulfur and carbon in the fly ash led to significant variations in the oxidation states of glasses prepared from that material depending on the waste proportions in the batch as well as the melting time and temperature. These effects are discussed further below.

In initial scoping studies, a simulated version of the ACM waste was combined with the actual eutectic salt in three crucible tests (DG4, IDG5, and IDG6). The characterization results for the ACM and the eutectic salt waste streams (ID1 and $\mathrm{D} 2$, respectively) suggest that a batch composition of about $1 / 4$ salt and $3 / 4 \mathrm{ACM}$ would provide a reasonable ratio of glass formers to fluxes and this blend was therefore used as the starting point for this study. These three glasses (IDG4, IDG5, and IDG6) contained 30\%, 20\%, and 10\% salt, respectively, and 70\%, 80\%, and 90\% ACM, respectively. All three batches formed visually homogeneous melts that poured easily. Compositional analyses of the three glasses are presented in Table 3.6.

It is likely that the salt waste stream (a "flux" from a glass forming perspective) would be available in a smaller quantity compared to all of the possible flux-poor waste streams, such as the ACM waste. It would be preferable, therefore, to optimize the glass formulations toward ACM loading with minimal use of the salt stream. Furthermore, detailed observation of the scoping melts, IDG4, IDG5, and IDG6, revealed that when the salt content in the batch reached $30 \%$, a yellow phase 
separated from the glassy phase. It is also worth noting that the salt waste contains a significant amount of carbonates which, while used routinely in commercial glass melting, can, under some circumstances, lead to foaming of the glass melt and reduced melting rates. The range of salt fractions in the batch ( $\leq 30 \mathrm{wt} \%$ ) used in the present work would not be expected to lead to such problems as was evidenced by the lack of foaming of the crucible melts.

Two series of glasses were made on this basis; the first (IDG7A, IDG7B, and IDG7C) contained $91 \%$ ACM and 9\% salt and the second (DG8A, IDG8B, and IDG8C) contained $88 \%$ $\mathrm{ACM}$ and $12 \%$ salt (percentages are on an as-received weight basis). Triplicate melts were made of each in view of the known variability of the ACM. Of these six glass melts, only one (glass IDG8A) led to an immiscible liquid phase in the $1200^{\circ} \mathrm{C}$ melt. This low viscosity, bright yellow phase is characteristic of alkali sulfate phases that tend to separate from silicate melts as the concentrations of sulfate and alkalies increase. Given the variability of the ACM and the fact that only one out of six glasses formed an immiscible phase (which constituted only about $0.3 \%$ of the total weight of glass produced), to the extent that these melts provide a representative sampling of the actual waste variability one would reasonably conclude that such a sulfate phase would not form in a large scale melt as would be characteristic of an actual process. Obviously, however, if the mean concentration of sulfur in the actual waste is higher than that inferred on the basis of the samples used for the present work, then such a sulfate phase could form in a large scale process.

Melt IDG10 was made from a blend of 60\% ACM and 40\% fly ash; all of the subsequent crucible melts (IDG11 to IDG16) were made based on formulations using blends of all four Idaho waste streams. Melts IDG10 and DG11 presented some difficulties, mostly due to the fly ash fraction of the formulations (40 and $30 \mathrm{wt} \%$, respectively) and did not produce good glassy products. The fly ash contains a significant amount of carbon that is not entirely oxidized in the course of the short residence time used in a crucible melt (60 to 90 minutes). (It is worth noting that residence times in actual vitrification processes can be many tens of hours.) The melt temperature was subsequently raised to $1400^{\circ} \mathrm{C}$ in an attempt to accelerate the oxidation process but it was still incomplete after 90 minutes. The melts that were produced also showed evidence of crystallization. The resulting materials were analyzed by SEM-EDS, which showed the presence of large amounts of sulfide crystals, mostly of $\mathrm{Zn}$ but also of $\mathrm{Pb}, \mathrm{Cu}, \mathrm{Ni}$, and $\mathrm{Sb}$; more detailed results are presented below. These results led to a reduction of the fly ash content in the subsequent formulations down to $20 \%$ and $10 \%$. Pre-oxidation of the fly ash was also investigated, as discussed below.

The fly ash content in the IDG12 formulation was reduced to $10 \%$. In addition, since the original mixture of wastes occupies such a large volume (about ten times larger than the volume of glass produced), the glass melt IDG12 was divided in two fractions. The first melt (IDG12A) was examined after one hour at $1200^{\circ} \mathrm{C}$ and since it was visibly too viscous, the temperature was increased to $1300^{\circ} \mathrm{C}$ for an additional 30 minutes. The second glass (IDG12B) was melted at a temperature of $1300^{\circ} \mathrm{C}$ for one hour. Samples of the two melts were taken for chemical, Mössbauer, 
and microstructural analyses and the two batches were then remelted together to produce a uniform pool of glass for viscosity, conductivity, and leach testing.

Another three formulations (IDG13, IDG14; and IDG15) were prepared by further varying the proportions of the four Idaho waste streams, as shown in Table 3.6. All three glasses were first melted at $1300^{\circ} \mathrm{C}$ for 1 hour. The glasses appeared to contain a separated sulfate phase as observed previously in glass $\mathrm{IDG} 12 \mathrm{~A}$, they were remelted for an extra hour at $1450^{\circ} \mathrm{C}$. The final glasses (IDG13A, IDG14A, and IDG15A) appeared shiny black and completely vitreous. Duplicate or triplicate melts of the same three formulations were preparedto evaluate some of the waste stream variability. Both the variability in the ACM composition and the oxidation-reduction effects caused by the fly ash and short melting time had significant effects on the final product, as is discussed below. The disappearance of the sulfate phase on melting at the higher temperature could have been due to evaporation or dissolution into the glass, or both. Since the sulfur contents of the final glasses were analyzed (reported as $\mathrm{SO}_{3}$ in Table 3.6), an estimate of the fraction of sulfur (as $\mathrm{SO}_{3}$ ) that was retained in the glasses can be obtained by comparison with the target composition (which, since the majority of the sulfur came from the relatively homogeneous fly ash, should be close to the true value in the batch); this calculation yields $51 \%, 87 \%$, and $23 \%$ for IDG12A,B,C, respectively; 56\%, and $47 \%$ for $\mathrm{DG} 13 \mathrm{~B}, \mathrm{C}$, respectively; $96 \%$ for IDG14B; and $84 \%$ and $75 \%$ for IDG15A,B, respectively. Thus, while a significant fraction of the sulfur evaporated, in general, the majority was retained in the glass.

\subsubsection{Glass Characterization}

\section{DCP Analysis}

The DCP analysis results, converted to an oxide basis, for all of the glasses prepared are shown in Table 3.6 and compared to their respective nominal target compositions.

In the last four formulations, we attempted to optimize the glass quality while still making use of all four waste streams to maintain zero additives. It was found after the first iteration (IDG12A, IDG13A, DG14A, and IDG15A) that the analyzed compositions were considerably different than those targeted due to waste stream variability, which obviously complicates the optimization process. The "target" was based on the average ACM analysis. However, there is some evidence to suggest that the samples used in the earlier melts (and for analysis) may have contained a greater proportion of the "gray woolly" material whereas the later samples contained a relatively larger fraction of the "white plaster-like" material. This may be the reason for the higher calcium and lower aluminum and silicon concentration measured in the later glasses (IDG13B, IDG13C, IDG14B, and DG15B). In four of the glass samples, an immiscible yellow phase separated from the glass melt; samples of this material were analyzed by DCP and ion chromatography. In all cases, the material was found to be $\left(\mathrm{K}_{0.66}, \mathrm{Na}_{0.33}\right)_{2} \mathrm{SO}_{4}$ with traces of chromium $(0.3 \mathrm{wt} \%)$ (probably the cause 
of the yellow color), and calcium (about $3 \mathrm{wt} \%$ ). The results of Mössbauer spectroscopic analyses of the associated glasses, discussed below, show that this phase formed only in the more oxidized melts.

\section{Ion Chromatography}

Ion chromatographic analyses of the $\mathrm{HF} / \mathrm{HNO}_{3}$ dissolutions of the glasses were performed for most of the glasses containing fly ash. The results generally showed relatively high sulfur contents in these glasses, in comparison to the lower solubility of sulfur in high-level nuclear waste borosilicate glasses. In the case of highly reduced glasses containing sulfides, the technique used may underestimate the sulfur content, since on acid dissolution some sulfur can then be lost as $\mathrm{H}_{2} \mathrm{~S}$ prior to analysis. This may have been the case for one set of analyses (IDG13A, IDG14A, and IDG15A) for which sulfur was not detectable in solution but was detectable by SEM-EDS.

\section{ICPMS}

ICPMS was used to supplement the compositional analysis of glasses IDG7A and IDG7B to ensure that no major constituents of the ACM had been missed in the DCP analyses. With the exception of uranium, which was expected on the basis of the salt analysis, no such constituents were found; the results are included in Table 3.6.

\section{Melt Viscosity and Electrical Conductivity}

Melt viscosity and electrical conductivity measurements were made on selected glasses formed from Idaho wastes; the data are summarized in Table 3.7. The set of glasses is divided into two groups: those made from ACM with eutectic salt (series IDG7A-B-C and IDG8A-B-C) and those made from combinations of all four waste streams (IDG12C to IDG15A). In the first series, the melt viscosities remained in the generally desirable range of below about 150 Poise for reasonable processing temperatures (below about $1300^{\circ} \mathrm{C}$ ), as shown in Figure 3.21 . As would be expected, the IDG7 formulation shows generally higher viscosities due to the lower salt content ( $9 \%$ salt - $91 \%$ ACM); IDG7C is the exception, undoubtedly due to the variability of the ACM composition. There is generally more variation between the measured viscosities of the IDG7 glasses than for the IDG8 series. The results for IDG8A show a somewhat lower viscosity and a higher conductivity than the other IDG8 glasses; this was also the only glass that formed an immiscible sulfate phase. The electrical conductivities of all of these particular melts are low, as would be expected in view of the low alkali contents. The values are toward the lower end of the range typically used for joule-heated melting. 
In the series of glasses made from all four wastes streams, large variations were observed in the measured melt viscosities and conductivities reflecting the composition variations (Table 3.6) resulting from the ACM variability. Because of the variability in composition, the property variations can therefore only be reliably related to the analyzed (rather than target) glass compositions. The same formulations were repeated using different samples of the ACM material and different oxidation conditions. The fly ash fraction of the waste normally leads to reduced glasses and the iron oxidation ratio $\left(\mathrm{Fe}^{2+} / \mathrm{Fe}_{\text {Total }}\right)$ measured by Mössbauer spectroscopy was above 0.5 in many cases (see Table 3.6). In formulations IDG13B and IDG14B, a pre-calcination of the ash was performed prior to batching the melt in order to oxidize the residual carbon. This did produce more oxidized melts and as a consequence led to the formation of small amounts of a sulfate phase; such glasses tended to show lower viscosities and higher conductivities.

An anomalous behavior of the viscosity was observed in some of these glass melts. The viscosity would change smoothly with decreasing temperature up to a point, beyond which the rotating spindle of the viscometer would freeze up. Experience has shown that this behavior can result from crystallization of the melt. Samples of two glasses exhibiting this effect, DG13C and IDG14A (see Table 3.7), were heat treated near the temperature at which the effect was observed in an attempt to induce crystallization. The heat treated glasses were examined in the SEM. In the case of IDG13C, no crystallization occurred. In the case of IDG14A, there were only a few crystals (3-10 $\mu \mathrm{m}$ dia.) present in the heat treated sample (less than 1 vol\%); these were composed of $\mathrm{Zn}, \mathrm{Al}, \mathrm{Cr}$, $\mathrm{Fe}, \mathrm{Mg}$ and $\mathrm{O}$ and appeared to be a type of spinel, based on the 2:1 ratio of trivalent to divalent cations and the hexagonal sections of the crystals. Lower heat treatment temperatures increased the amount of spinel slightly and added some small ZnS crystals as well as some star-shaped crystals of a uranium oxide (Figure 3.22); however, the total amount of all crystalline phases did not exceed 3 vol\%, which could not account for the behavior observed. The problem thus remains unsolved at present; it is, however, possible that there were oxidation state differences between the small heat treated samples and the very much larger viscosity samples, both of which were melted in air.

\section{SEM-EDS Analyses}

Samples of the IDG-10 glass, which was both the first melt prepared using the fly ash and the melt which used the largest amount of fly ash (40\%), were analyzed by SEM/EDS. The glass had a marbled appearance to the naked eye. In the glass, there were scattered crystals and globules $(<1$ vol\%, 5-15 $\mu \mathrm{m}$ dia.) of a phase containing $\mathrm{Na}, \mathrm{S}$, and $\mathrm{Zn}$ and a small amount of Fe (Figure 3.23). The larger globules sometimes showed a substructure of regions of high backscatter intensity, which were enriched in $\mathrm{Pb}$ and $\mathrm{Cu}$ compared to the rest of the nodule, as can be seen by comparing Figures 3.24 and 3.25. These were the only regions in the glass that showed significant $\mathrm{Pb}$ content, although it is possible that a low level is present throughout but is masked by the $\mathrm{K}$-peaks of $\mathrm{S}$. Also found in this sample was a crystal of a calcium aluminum silicate in a region ( $30-40 \mu \mathrm{m}$ across) with many spherical voids (Figure 3.26); sulfur was also enriched in this region. Analysis of the bulk glass by 
SEM indicated that perhaps part of the reason for the marbled appearance was the variation in the $\mathrm{Fe}$ and $\mathrm{Zn}$ content in the glass. Under backscatter imaging there were brighter streaks across the surface that were higher in $\mathrm{Fe}$ and $\mathrm{Zn}$ and lower in Si than the darker areas (Figures 3.27-3.28). On examination at higher magnification, the glass exhibited a fairly uniform distribution of small bright nodules or crystals in the size range $0.1-0.5 \mu \mathrm{m}$ which showed enrichment in $\mathrm{Na}, \mathrm{S}$, and $\mathrm{Zn}$ and are believed to be smaller crystals of the same material whose spectrum is shown in Figure 3.23. Spectra of these smaller particles are shown in Figures 3.29 and 3.30 and can be compared to those of the glass (Figures 3.27 and 3.28) to see the enrichments mentioned.

Several of the other glasses produced from Idaho waste streams were subjected to microstructural analyses: Glasses IDG13-15 and, respectively, DG13A-15A, are of the same nominal compositions, but the latter group received an additional 1 hour melting time; all of these samples contained small nodules of mixed sulfides. The size distribution was bimodal with the smaller ones having diameters $\leqslant 0.2 \mu \mathrm{m}$ and the larger ones diameters $\sim 0.5-1.0 \mu \mathrm{m}$. The content of the nodules varied, with $\mathrm{Zn}$ usually being present and one or more of the following: $\mathrm{Cu}, \mathrm{Fe}, \mathrm{Pb}, \mathrm{Ni}$, and, occasionally, Sb. The sample of IDG14 also contained some anomalously large sulfide nodules 3-10 $\mu \mathrm{m}$ in diameter. Most of these were found in clusters of uranium oxide crystals, and the nodules were large enough to reveal a highly segregated inner structure. Figure 3.31 shows a large nodule about $5 \mu \mathrm{m}$ diameter situated among uranium oxide crystals. The bright regions around the perimeter of the nodule are $\mathrm{Pb}, \mathrm{Pb}-\mathrm{S}$, the darker grey region is $\mathrm{Zn}-\mathrm{S}$ and the brighter grey is $\mathrm{Cu}-\mathrm{S}$. There is also a region on the perimeter that showed a Sb-Ni-S composition in $\mathrm{x}$-ray mapping.

Table 3.8 summarizes the observed densities of the small and larger nodules and the oxidation states (except for IDG15) of the glasses. The small magnitude of the change in oxidation state after an additional one hour of melting (at least in the glasses where "before" and "after" measurements were made) indicates that the sulfide precipitates probably buffer the redox state of the glass. The population of the larger nodules $(\sim 0.5-1 \mu \mathrm{m}$ dia.) change very little after an hour of melting. This is probably because the larger ones only erode during the increased melt time. (The large nodule count for IDG15A is considered to be atypical because of the proximity of the prepared glass specimen to a large sediment nodule; the small nodule densities are much more uniform and unaffected by such conditions.) The population of the smaller nodules decreases with longer melting time because some of them are consumed by oxidation. Densities were determined by direct counts of particles in ten randomly chosen, but representatively located, image fields in each specimen. Smaller nodules were counted in fields $25 \mu \mathrm{m}^{2}$ in area and larger nodules were counted in fields $2500 \mu \mathrm{m}^{2}$ in area.

Other features of interest in the prepared SEM specimens are as follows. Glass IDG13, IDG14, and IDG14A contained a few clusters or streaks of uranium oxide crystals. Individual crystals were $0.5-2 \mu \mathrm{m}$ dia. and regions containing them were 10 to $200 \mu \mathrm{m}$ in extent. 
Glass IDG13 contained some fragments of a Pt-Rh stirrer, which had been attacked under the reducing conditions of the melt. It showed evidence of having alloyed slightly with iron, and one globular fragment had a region adhering to its perimeter, which appeared from its EDS spectrum to be a rhodium sulfide.

A few small ( $\sim 500 \mu \mathrm{m}$ dia.) regions were found in IDG13, DG14, and IDG14A that were high in Al compared to the surrounding glass. In IDG14 and IDG14A, these were populated by some platelet-shaped aluminosilicate crystals (locally $~ 10$ vol\%) and a much smaller fraction of two other aluminum-containing crystalline species. These regions are probably present because of the difficulty of fully dissolving and homogenizing aluminosilicate fibers from the ACM into the melt under crucible conditions. This problem may not occur in a full-scale melter in which the residence time may be many hours longer than that for the crucible melts; however, large-scale systems with shorter residence times do exist (e.g., cyclone melters).

Glass IDG15 and IDG15A, which incorporated the largest fraction of flyash, contained some 10-15 mm dia. droplets of sediment of rather complex segregated microstructure (See Figure 3.32). Elements found in these included $\mathrm{Pt}, \mathrm{Pb}, \mathrm{Rh}, \mathrm{Sb}, \mathrm{Fe}, \mathrm{Zn}, \mathrm{Ni}, \mathrm{Cu}, \mathrm{Mo}$, and $\mathrm{S}$. In IDG15, there were also two non-silicate crystalline areas, each $\sim 3 \times 4 \mathrm{~mm}$, containing large platelets $(>100 \mu \mathrm{m} \mathrm{dia}$. and 5-15 $\mu \mathrm{m}$ thick) of alternately intergrown phases (see Figure 3.33). The darker phase contains $\mathrm{K}, \mathrm{Cu}$, $\mathrm{Fe}, \mathrm{Zn}$, and $\mathrm{S}$ and is $50-60$ vol\% of the total. The other contains mainly $\mathrm{Zn}$ and $\mathrm{S}$ with traces of Fe and $\mathrm{Mn}$ and is $35-45$ vol\% of the total. Also present in these regions are a few, bright globules of $\mathrm{Pb}$ or $\mathrm{Pb}-\mathrm{S}$ and a few iron oxide crystals not visible in Figure 3.33.

A sediment droplet was also recovered from IDG15C. Part of it was mounted and examined in the SEM and the rest of it was chilled with liquid nitrogen and powdered for XRD analysis. Although SEM analysis showed a substantial fraction of both $\mathrm{Pb}$ and $\mathrm{Sb}$ sulfide phases, the XRD pattern showed mainly $\mathrm{Pb}$ and additional lines, which could not be positively identified but showed some matching for $\mathrm{Sb}_{2} \mathrm{O}_{3}, \mathrm{FeS}$, and $\mathrm{Cu}_{3} \mathrm{SbS}_{4}$; none of these, except perhaps $\mathrm{Sb}_{2} \mathrm{O}_{3}$, seem very likely to be present in large quantity from the SEM/EDS data. It is possible that the lead coated the other phase during grinding and partially obscured it during the XRD run.

\section{Mössbauer Spectroscopy and SEM}

The fly ash waste from Idaho contain more carbon (23\%) than any of the other waste streams used in this study. While small amounts of carbon in a crucible melt batch that is melted in air typically still produce oxidized glasses (depending on the melt time and temperature, composition, and kinetic factors,) this becomes less likely as the carbon content is increased. Thus, reductionoxidation (redox) reactions would be expected to play a greater role in melts that include the fly ash waste. The is particularly so in view of the presence of significant amounts of other reducible species in the fly ash, notably sulfur, zinc, lead, antimony, iron, copper, and chromium. For these reasons, 
measurements of the oxidation state were made for the glasses that were produced from formulations that included the fly ash waste since this is an important additional compositional variable that characterizes the glass and, therefore, also the properties of the glass.

The oxidation state of each of the glasses in the IDG12 to IDG15 series (containing all four wastes) was determined from the Mössbauer spectrum of the iron which is present in concentrations of about $3 \mathrm{wt} \%$ in these glasses. As shown in Table 3.6 and Figure 3.34, the oxidation ratio (expressed as $\mathrm{Fe}^{2+} / \mathrm{Fe}_{\text {total }}$ ) increases with the fraction of fly ash and decreases with melting time. Increasing the fly ash content increases the carbon content of the batch and the following reactions ensue on melting:

$$
\begin{gathered}
\mathrm{C}_{(\mathrm{s})}+1 / 2 \mathrm{O}_{2} \rightarrow \mathrm{CO} \text { (gas) } \\
\mathrm{CO}{ }_{\text {(gas) }}+1 / 2 \mathrm{O}_{2} \rightarrow \mathrm{CO}_{2 \text { (gas) }} \\
\mathrm{Fe}_{\text {(glass) }}^{2+}+1 / 4 \mathrm{O}_{2 \text { (gas) }} \neq \mathrm{Fe}_{\text {(glass) }}^{3+}+1 / 2 \mathrm{O}_{\text {(glass) }}^{2-}
\end{gathered}
$$

Increased melting times will increase the extent of the reaction with oxygen at the glass/air interface, which will consume the carbon and eventually reoxidize the glass with sufficiently long melt durations.

In most of the melts prepared from as-received (i.e., not pre-calcined) fly ash, the iron oxidation ratio of the resultant glass was around $\mathrm{Fe}^{2+} / \mathrm{Fe}_{\text {total }}=0.75$. In an attempt to decrease the oxidation ratio, the melting time was prolonged. Unfortunately, in a small crucible melt, the melt time cannot be increased indefinitely due to the evaporation of some of the glass components. In particular, sulfur, an important constituent in these wastes, also undergoes a series of oxidation reactions that convert it from sulfides (e.g., $\mathrm{ZnS}, \mathrm{PbS}, \mathrm{CuS}$, etc.), which are present as secondary phases in the glass, to $\mathrm{SO}_{2}$, which has very limited solubility in the glass melt, and eventually to $\mathrm{SO}_{3}$, which has much greater but still limited solubility and which may lead to separation of sulfur as an alkali sulfates phase.

To better simulate the extent of the oxidation process that might be more typical of many large-scale vitrification processes, without using extremely long melt times, the fly ash was precalcined for four hours at $850^{\circ} \mathrm{C}$ prior to incorporation in the melt batch. In the case of IDG13B, IDG13C, and IDG14B, 100\% of the fly ash used was pre-calcined; in the case of IDG15B, 1/3 precalcined and $2 / 3$ as-received fly ash was used. The Mössbauer analysis of the first three of those glasses confirmed that they were significantly more oxidized with $\mathrm{Fe}^{2+} / \mathrm{Fe}_{\text {total }}$ ratios of around 0.25 . The change in glass oxidation state was found to have a significant influence on the crystallization behavior; in particular, the zinc-nickel-copper sulfide phase found in the reduced glass by SEM-EDS 
analysis was not present in the oxidized glass. Instead, the oxidized glasses, when imaged by backscattered electrons in the SEM, appeared nonuniform, being divided into light and dark regions and striae. The difference in image intensity between light and dark areas was small, but brighter areas were higher in $\mathrm{Ca}$ and lower in $\mathrm{Al}, \mathrm{K}$, and $\mathrm{Si}$. They also contained small amounts of $\mathrm{S}, \mathrm{Cl}$, and possibly $\mathrm{Pb}$, which seemed to be absent in the EDS spectra of the darker areas. No additional microstructure could be observed except for an occasional included pocket of $\mathrm{K}_{2} \mathrm{SO}_{4} / \mathrm{Na}_{2} \mathrm{SO}_{4}$ in fracture specimens (see Figure 3.35; the solubility of sulfates precluded their observation in normally prepared specimens). In the oxidized glasses, the sulfate indeed separated as a yellow phase; the yellow material was analyzed by DCP to be $2 / 3$ potassium sulfate and $1 / 3$ sodium sulfate with about $0.2 \%$ chromium, which is enough to create the yellow coloration. The fraction of the separated sulfate phase in relation to the mass of glass recovered appears to vary significantly with the length of time of the melt. In the case of the mixture of as received and pre-calcined fly ash used for IDG15B, the oxidation ratio was measured at $\mathrm{Fe}^{2+} / \mathrm{Fe}_{\text {total }}=0.34$, still significantly more oxidized than in the case of the as-received fly ash, and again, some sulfate phase separated.

The height of the sulfur peak in the EDS spectra of oxidized and reduced glasses shows considerable variability, even from place to place in a single specimen. The maximum heights in the reduced glasses are slightly higher on average than those observed for oxidized glasses, but this finding is on the basis of limited data.

A classic treatment of the dissolution of sulfur in melts was performed by Goldman (Goldman-1985). His work is quoted by Schreiber (Schreiber-1987) in an experimental study of the solubility of sulfur as a function of oxygen fugacity in an alkali borosilicate glass typical of those proposed for high-level nuclear waste disposal. A minimum is found in the solubility of sulfur as a function of oxygen fugacity at the point at which the sulfate ion $\mathrm{SO}_{4}^{-2}$ is reduced to the sulfide ion $\mathrm{S}^{-2}$, which is expected from the treatment by Goldman. Other observations that Schreiber et al. confirm in their study are that as the melt temperature increases, the solubility of sulfur as the sulfide ion increases, while its solubility as the sulfate ion decreases which leads to the minimum in solubility moving in the oxidizing direction.

However, since the Idaho glasses are not borosilicates, but somewhat more like soda-lime silicate glasses, the work of Papadopoulos (Papadopoulos-1973) may also be relevant. He worked close to the lime-silica eutectic under oxidizing conditions and found that when all other variables are held constant, the solubility of $\mathrm{SO}_{3}$ the glass increases (i) As the silica content is reduced; (ii) As soda replaces lime at a given silica level; and (iii) as melt temperature decreases. It should be noted, however, that point (ii) isinconsistent with previous work on Weldon Spring waste glasses (Pegg1993a) and work on Fernald K-65 waste glasses (Merrill-1994).

The EDS data accumulated on the Idaho waste glasses seems to support the greater solubility of sulfur in the reduced glasses; however, the sulfur peaks observed may not be entirely due to 
sulfide ions accommodated in the glass network since the possible presence of very small sulfide precipitates cannot be excluded.

\section{PCT Leach Testing}

Results of the 7-day PCT tests for all of the Idaho glasses that were tested are included in Table 3.9 together with the results of longer-time testing through 365 days, as available at the time of writing. The normalized sodium, and silicon concentrations in the leachates (Figure 3.36 and 3.37) compare well with those of the SRL-EA glass (boron concentrations are typically reported for the PCT test but there is no boron in most of the Idaho glasses). In particular, all of the normalized silicon concentrations are 20 to 40 times lower than those of SRL-EA standard glass, with the lowest concentrations for the more reduced glasses. The oxidized glasses (IDG13B, IDG13C and IDG14B) show values consistently about $60 \%$ higher than the reduced compositions for which the normalized silicon concentration was around $0.1 \mathrm{~g} / \mathrm{l}$ throughout the series. Conversely, the sodium concentrations in the leachates do not appear to be sensitive to the oxidation state of the glass. It was not possible to examine the oxidation effect on the PCT leaching of iron since its solubility is extremely low at the high $\mathrm{pH}$ values of the leachates; in all cases the iron concentrations are below the detection limit (about $0.1 \mathrm{ppm}$ ) of the DCP instrument used for analysis. While it is possible that the differences in silicon leaching are due to the oxidation state differences, they are more likely a consequence of the differences in composition, notably the higher alumina and lower calcia contents of the reduced glasses (Table, 3.6).

The general PCT results tests that were performed on the Idaho glasses suggest that these glasses exhibit low PCT leachability and low and stable leach rates for the duration of the tests conducted (Figure 3.38 and 3.39). All of the glasses leached less than the SRL-EA glass.

\section{TCLP}

The TCLP was also performed on the Idaho glasses, and both DCP and ICPMS techniques were used for analysis of the leachates; the results for all of the glasses tested are presented in Table 3.10 and Figure 3.40. For comparison purposes, the TCLP was also performed on two of the waste streams themselves (the fly ash and the eutectic salt wastes since these contain the majority of the contaminants); the results are summarized in Table 3.10a. As expected, the vitrified materials show considerable reductions in TCLP releases compared to the untreated wastes.

In general, all of the Idaho waste glasses leach less than the current regulatory limits; for most elements $(\mathrm{Ni}, \mathrm{Cr}, \mathrm{Ba}, \mathrm{Cd})$, the glass leachate concentrations are two orders of magnitude below their respective limits. Mercury, which was found in the fly ash in the 10 to $70 \mathrm{ppm}$ range, is found in the leachate at levels of about one order of magnitude below the TCLP limit; however, the extent of 
mercury loss during melting is unknown. Lead is present at about $10 \mathrm{wt} \%$ on an oxide basis in the fly ash; for this element, the regulatory limit is met only by glasses IDG13A and IDG14A, in which the ash fraction is $10 \%$. The limit is exceeded in the case of IDG15A, in which the ash fraction is $20 \%$ and also in instances in which the formulations with $10 \%$ ash are repeated under oxidizing conditions (IDG13B). A high lead leachate concentration was found for glass IDG12C; this result does not appear to be consistent with the analysis of the glass, which showed the lead content to be only $0.38 \mathrm{wt} \%$. We believe that this result was due to the presence of beads of metallic lead in the sample used in the TCLP.

As shown in Table 3.10, there are also indications that the TCLP leaching concentrations of chromium are slightly increased in the cases where the glass was more oxidized (IDG13B and IDG14B compared to IDG13A and IDG 14A, respectively); this is consistent with the much greater solubility of hexavalent chromium compared to di- or tri-valent chromium in aqueous solutions. Figure 3.40 also shows that the presence of a potassium-sodium sulfate phase, which is both quite soluble and chromium-containing, can also increase the concentration of chromium in the leachate.

In the case of uranium (Figure 3.41), the TCLP leachate concentrations are compared to the "Ingested Water Limit", described in the DOE Order 5400.5 , of $1.8 \mathrm{ppm}$ for ${ }^{238} \mathrm{U}$; the measured leachate concentrations are 3 to 7 times lower than this limit for most glasses. The results suggest that the leaching of uranium is also increased in the oxidized glasses over their respective reduced glasses (e.g., when comparing IDG13B to IDG13A or IDG14B and IDG14A) but, as with the PCT results discussed above, this difference may be more a result of the compositional differences between these glasses (Table 3.6).

\subsection{Summary and Conclusions}

The results from the chemical characterization of the Idaho waste streams selected for this study suggest that their compositions are amenable to blending in a MAWS approach. From a compositional envelope perspective, these waste streams span a wide range of compositions (Table 3.5), both in terms of their bulk constituents and the hazardous and radioactive contaminants that are present.

The Idaho waste streams exhibit bulk densities between 0.3 to $1.3 \mathrm{~g} / \mathrm{cm}^{3}$ (though particle densities are as high as $2.9 \mathrm{~g} / \mathrm{cm}^{3}$ ) and weight losses upon heating at $1150^{\circ} \mathrm{C}$ reaching as much as $64 \%$. These data indicate the potential for large volume reductions on vitrification if high waste loading can be achieved. Glasses formulated from blends of these waste streams with $100 \%$ waste loadings have been prepared and characterized; the typical volume reductions observed for all of these formulations were about a factor of 9-12 reduction from the waste blend to the final vitrified product. 
The results also show that the four waste streams studied can be effectively blended and vitrified without additives as either binary or quaternary mixtures; combinations other than those investigated are certainly possible, however. In the binary approach, mixtures of salt and ACM were studied. The glass formulations investigated, containing around $10 \mathrm{wt} \%$ salt and $90 \mathrm{wt} \% \mathrm{ACM}$, produced viable glass products in replicate melts despite the composition variability found in the ACM waste stream. A series of formulations was also investigated using all four waste streams.

The leach resistance of the waste forms produced are uniformly high on the basis of the PCT results. Many of the glasses met all of the TCLP release limits, generally by a wide margin; the high concentration of lead in the fly ash was the primary factor for glasses that exceeded the TCLP release limits.

The Idaho glasses that have been subjected to viscosity, conductivity, TCLP, and PCT testing (i.e., those glasses in the core data set of 40 glasses, as discussed in Section 1.3) are listed in Table 3.11. For convenience, Table 3.11 also lists representative data for each characteristic, consisting of the viscosity and conductivity values at $1350^{\circ} \mathrm{C}$, the uranium concentrations in the TCLP leachates, and the sodium concentration in the PCT leachates after seven days.

Overall, the results suggest that viable glass formulations can be developed for a wide range of combinations of the waste streams investigated with no additions of chemical additives, i.e., $100 \%$ waste loadings. While the limited number of formulations investigated in this study would be unlikely to lead to fully optimized compositions, the results show the general ranges of glass and melt properties that are achievable with no chemical additives. The melt properties would be suitable for processing in a variety of vitrification technologies.

The most problematic (from a vitrification perspective) of the waste streams investigated in this study was the fly ash due to the high concentrations of carbon, sulfur, and heavy metals, many of which are relatively redox sensitive. As would be expected, the use of the fly ash waste was found to have significant impacts on the oxidation state of the glass melts that are produced due to the high carbon content of that material. The oxidation state variations are, in turn, coupled to the speciation of the redox sensitive species in the glass and therefore lead to variations in both the physical and chemical properties of the product and the melt. As a further complication, there are important kinetic effects that must be considered since the melts are produced in a finite time under an oxidizing atmosphere but with reductants in the batch. In this area, some caution should be exercised in using the crucible melt observations to predict what might be expected to occur in a full-scale process since factors such as residence time and oxygen availability are highly dependent on the specific vitrification process concerned. For example, it has recently been demonstrated that oxidized glasses could be produced from a commercial radioactive waste stream composed of $97 \%$ organic materials in a prolonged pilot-scale joule-heated melter demonstration (Brandys-1995) though this would not be possible in typical 1-2 hour crucible melt. 
Thus, while selection and control of the oxidation state range is an important consideration for full scale processing of wastes, such as the Idaho fly ash, the specific preferred values must be determined on the basis of the particular process characteristics and limitations. As an example, the production of reduced glasses has the advantage of preventing the formation of an immiscible sulfate phase (at the expense of sending the sulfur to the off-gas system) but promotes the formation of molten metal phases (particularly lead in this case) from the melt. This may be an acceptable tradeoff for some processes while the reverse may be preferred for others; for yet other processes, neither may be acceptable. Clearly, these decisions cannot be made on the basis of crucible melts alone.

In summary, vitreous waste forms have been produced with large volume reductions from blends of four Idaho waste streams using a MAWS vitrification approach. Since these waste streams are of diverse chemical compositions, it is likely that other Idaho waste streams could also be incorporated. The diversity of the waste streams selected make the data obtained of additional value with respect to the compositional envelope modelling objectives of this project in that a relatively wide range of glass composition space is spanned; these implications are discussed further in Section 5. 
Table 3.1

Summary of Idaho Waste Physical Characterization

\begin{tabular}{|c|c|c|c|c|}
\hline $\begin{array}{c}\text { Idaho } \\
\text { Samples }\end{array}$ & $\begin{array}{c}\text { Asbestos } \\
\text { Sample ID1 }\end{array}$ & $\begin{array}{c}\text { Eutectic Salt } \\
\text { Sample ID2 }\end{array}$ & $\begin{array}{c}\text { Fly Ash } \\
\text { Sample ID3 }\end{array}$ & $\begin{array}{c}\text { Soil } \\
\text { Sample ID4 }\end{array}$ \\
\hline \multicolumn{5}{|c|}{ Thermogravimetric Analysis (weight loss \% as a function of treatments) } \\
\hline $110^{\circ} \mathrm{C} / 18 \mathrm{hr}$ & 2.59 to $13.42 \%$ & $0.92 \%$ & $2.56 \%$ & $3.1 \%$ \\
\hline $450^{\circ} \mathrm{C} / 4 \mathrm{hr}$ & 0 to $19.42 \%$ & $6.42 \%$ & $19.54 \%$ & $3.92 \%$ \\
\hline $1150^{\circ} \mathrm{C} / 4 \mathrm{hr}$ & 3.85 to $30.82 \%$ & $46.00 \%$ & $64.33 \%$ & $12.42 \%$ \\
\hline \multicolumn{5}{|c|}{ Specific Gravity, $\mathrm{g} \mathrm{cm}^{-3}$} \\
\hline ASTM D854-83 & 2.50 & 2.57 & 2.88 & 2.58 \\
\hline Bulk dry density & 0.28 & 1.19 & 0.66 & 1.30 \\
\hline \multicolumn{5}{|c|}{ Particle Size Analysis: retained particles \% as a function of U.S. Mesh values } \\
\hline Mesh 10 & N.A. & $0.00 \%$ & $21.91 \%$ & $19.62 \%$ \\
\hline Mesh 40 & N.A. & $4.04 \%$ & $32.79 \%$ & $61.97 \%$ \\
\hline Mesh 60 & N.A. & $11.81 \%$ & $39.25 \%$ & $2.64 \%$ \\
\hline Mesh 100 & N.A. & $28.03 \%$ & $4.83 \%$ & $4.35 \%$ \\
\hline Mesh 200 & N.A. & $32.97 \%$ & $0.71 \%$ & $8.00 \%$ \\
\hline Passing 200 & N.A. & $23.15 \%$ & $0.51 \%$ & $3.42 \%$ \\
\hline \multicolumn{5}{|c|}{ Total Carbon (TC) and Total Organic Carbon (TOC), wt\% } \\
\hline $\mathrm{TC} *$ & N.A. & $6.9 \pm 0.2$ & $23 \pm 0.3$ & $2.8 \pm 0.03$ \\
\hline TOC * & N.A. & $0.9 \pm 0.03$ & $22 \pm 0.3$ & $1.2 \pm 0.03$ \\
\hline
\end{tabular}

* Average of 9 measurements.

N.A. $=$ Not Analyzed. 
Table 3.2

Summary of the Asbestos Containing Material Analyses (wt\%)

\begin{tabular}{|c|c|c|c|c|c|c|c|c|}
\hline & $\begin{array}{c}\text { ID1 } \\
\text { from } \\
\text { IDG1 } \\
\end{array}$ & $\begin{array}{c}\text { ID1 } \\
\text { from } \\
\text { IDG2 } \\
\end{array}$ & $\begin{array}{l}\text { ID1 } \\
\text { from } \\
\text { DG3 } \\
\end{array}$ & $\begin{array}{l}\text { ID1 }{ }^{* *} \\
\text { from } \\
\text { IDG7 } \\
\end{array}$ & $\begin{array}{l}\text { ID1** } \\
\text { from } \\
\text { IDG8 } \\
\end{array}$ & $\begin{array}{c}\text { DD1*** } \\
\text { Average } \\
\text { ACM }\end{array}$ & $\underset{\text { Minimum }}{\text { ID1 }}$ & $\underset{\text { Maximum }}{\text { ID1 }}$ \\
\hline $\mathrm{Al}_{2} \mathrm{O}_{3}$ & 20.14 & 11.11 & 18.81 & 13.69 & 11.85 & 12.77 & 11.11 & 20.14 \\
\hline $\mathrm{B}_{2} \mathrm{O}_{3}$ & 0.12 & 0.13 & 0.12 & 0.13 & 0.11 & 0.12 & 0.11 & 0.13 \\
\hline $\mathrm{BaO}$ & 0.06 & 0.05 & 0.05 & 0.07 & 0.08 & 0.07 & 0.05 & 0.08 \\
\hline $\mathrm{CaO}$ & 19.57 & 23.16 & 21.03 & 23.41 & 27.00 & 25.21 & 19.57 & 27.00 \\
\hline $\mathrm{Cr}_{2} \mathrm{O}_{3}$ & 0.06 & 0.03 & 0.04 & 0.03 & 0.07 & 0.05 & 0.03 & 0.07 \\
\hline $\mathrm{Fe}_{2} \mathrm{O}_{3}$ & 4.72 & 1.78 & 3.45 & 3.61 & 5.40 & 4.50 & 1.78 & 5.40 \\
\hline $\mathrm{K}_{2} \mathrm{O}$ & 0.67 & 1.31 & 1.18 & 2.02 & 0.45 & 1.24 & 0.45 & 2.02 \\
\hline $\mathrm{Li}_{2} \mathrm{O}$ & 0.14 & 0.18 & 0.19 & 0.36 & 0.16 & 0.26 & 0.14 & 0.36 \\
\hline $\mathrm{MgO}$ & 6.00 & 4.67 & 5.45 & 5.47 & 8.26 & 6.86 & 4.67 & 8.26 \\
\hline $\mathrm{MnO}_{2}$ & 0.33 & 0.11 & 0.21 & 0.26 & 0.57 & 0.42 & 0.11 & 0.57 \\
\hline $\mathrm{Na}_{2} \mathrm{O}$ & 1.88 & 11.28 & 3.38 & 1.93 & $\begin{array}{l}\cdot \quad 72 \\
\end{array}$ & 1.33 & 0.72 & 11.28 \\
\hline $\mathrm{NiO}$ & 0.03 & 0.03 & 0.02 & 0.03 & 0.02 & 0.02 & 0.02 & 0.03 \\
\hline $\mathrm{P}_{2} \mathrm{O}_{5}$ & 0.24 & 0.29 & 0.21 & 0.22 & 0.17 & 0.20 & 0.17 & 0.29 \\
\hline $\mathrm{SO}_{3}$ & 0.00 & 0.00 & 0.00 & 0.39 & 0.38 & 0.38 & 0.00 & 0.39 \\
\hline $\mathrm{SiO}_{2}$ & 45.15 & 45.10 & 45.02 & 47.38 & 43.77 & 45.57 & 43.77 & 47.38 \\
\hline $\mathrm{SrO}$ & 0.05 & 0.08 & 0.06 & 0.08 & 0.07 & 0.07 & 0.05 & 0.08 \\
\hline $\mathrm{TiO}_{2}$ & 0.62 & 0.40 & 0.53 & 0.48 & 0.74 & 0.61 & 0.40 & 0.74 \\
\hline $\mathrm{UO}_{2}$ & 0.20 & 0.25 & 0.20 & 0.41 & 0.16 & 0.28 & 0.16 & 0.41 \\
\hline $\mathrm{ZrO}_{2}$ & 0.03 & 0.03 & 0.03 & 0.03 & 0.03 & 0.03 & 0.03 & 0.03 \\
\hline Sum & 100.00 & 100.00 & 100.00 & 100.00 & 100.00 & 100.00 & & \\
\hline
\end{tabular}

** This is an average of three compositions listed in Table 3.6.

*** The average used in our target glass formulation was the average on the six glasses IDG7 and IDG8 only. 
Table 3.3

Summary of Analysis on Idaho Fly Ash

\begin{tabular}{|c|c|c|c|c|c|}
\hline & Element & $\mathrm{ppm}$ & Oxide & D3 wt\% & ID3 Normalized \\
\hline DCP & $\begin{array}{l}\mathrm{Al} \\
\mathrm{B} \\
\mathrm{Ba} \\
\mathrm{Ca} \\
\mathrm{Cd} \\
\mathrm{Cr} \\
\mathrm{Cu} \\
\mathrm{Fe} \\
\mathrm{Hg} \\
\mathrm{K} \\
\mathrm{Li} \\
\mathrm{Mg} \\
\mathrm{Mn} \\
\mathrm{Na} \\
\mathrm{Ni} \\
\mathrm{P} \\
\mathrm{Pb} \\
\mathrm{Si} \\
\mathrm{Sr} \\
\mathrm{Ti} \\
\mathrm{U} \\
\mathrm{Zn} \\
\mathrm{Zr} \\
\end{array}$ & $\begin{array}{c}1.2 \\
0.5 \\
0.2 \\
5.6 \\
1.10 \\
0.4 \\
4.7 \\
4.3 \\
0.03 \\
7.6 \\
0.2 \\
0.6 \\
0.3 \\
14.9 \\
0.2 \\
1.3 \\
30.7 \\
8.0 \\
0.02 \\
1.0 \\
0.1 \\
90.0 \\
0.1 \\
\end{array}$ & $\begin{array}{l}\mathrm{Al}_{2} \mathrm{O}_{3} \\
\mathrm{~B}_{2} \mathrm{O}_{3} \\
\mathrm{BaO} \\
\mathrm{CaO} \\
\mathrm{CdO} \\
\mathrm{Cr}_{2} \mathrm{O}_{3} \\
\mathrm{CuO} \\
\mathrm{Fe}_{2} \mathrm{O}_{3} \\
\mathrm{HgO} \\
\mathrm{K}_{2} \mathrm{O} \\
\mathrm{Li}_{2} \mathrm{O} \\
\mathrm{MgO} \\
\mathrm{MnO}_{2} \\
\mathrm{Na}_{2} \mathrm{O} \\
\mathrm{NiO} \\
\mathrm{P}_{2} \mathrm{O}_{5} \\
\mathrm{PbO} \\
\mathrm{SiO}_{2} \\
\mathrm{SrO} \\
\mathrm{TiO}_{2} \\
\mathrm{UO}_{2} \\
\mathrm{ZnO}_{2} \\
\mathrm{ZrO}_{2} \\
\end{array}$ & $\begin{array}{r}0.45 \\
0.32 \\
0.05 \\
1.57 \\
0.26 \\
1.11 \\
1.19 \\
1.23 \\
0.01 \\
1.83 \\
0.09 \\
0.19 \\
0.10 \\
4.01 \\
0.05 \\
0.61 \\
6.61 \\
3.43 \\
0.01 \\
0.34 \\
0.03 \\
22.36 \\
0.03 \\
\end{array}$ & $\begin{array}{r}0.67 \\
0.48 \\
0.07 \\
2.35 \\
0.39 \\
1.66 \\
1.78 \\
1.84 \\
0.01 \\
2.74 \\
0.13 \\
0.28 \\
0.15 \\
6.00 \\
0.07 \\
0.91 \\
9.89 \\
5.13 \\
0.01 \\
0.51 \\
0.04 \\
33.45 \\
0.04 \\
\end{array}$ \\
\hline ICPMS & $\begin{array}{l}\mathrm{Cu} \\
\mathrm{Pb} \\
\mathrm{Sb} \\
\mathrm{Zn}\end{array}$ & $\begin{array}{r}5 \\
30 \\
5 \\
90 \\
\end{array}$ & $\begin{array}{l}\mathrm{CuO} \\
\mathrm{PbO} \\
\mathrm{SbO} \\
\mathrm{ZnO} \\
\end{array}$ & 3.5 & 5.24 \\
\hline \multicolumn{2}{|c|}{$\begin{array}{l}\text { Ion Chromatography* } \\
\mathrm{SO}_{3}\end{array}$} & 35.00 & $\mathrm{SO}_{3}$ & 17.47 & 26.13 \\
\hline \multicolumn{2}{|c|}{ SUM (Oxide) } & & & 66.85 & 100.00 \\
\hline
\end{tabular}

* $\mathrm{Cl}$ less than $1 \%$ by Ion Chromatography.

$\mathrm{F}$ less than $0.5 \%$ by ion selective electrode. 
Table 3.4

Summary of Gamma Spectroscopy on Idaho Wastes in Ci/g

\begin{tabular}{||l|l|l|l||}
\hline \multicolumn{1}{|c|}{ Radionuclide } & \multicolumn{1}{|c|}{$\begin{array}{c}\text { Asbestos Material } \\
\text { (Sample ID1)* }\end{array}$} & \multicolumn{1}{|c|}{$\begin{array}{c}\text { Salt Cake } \\
\text { (Sample ID2) }\end{array}$} & \multicolumn{1}{|c|}{$\begin{array}{c}\text { Fly Ash } \\
\text { (Sample ID3) }\end{array}$} \\
\hline Co-60 & Below MDA & Below MDA & $3.6 \mathrm{E}-10$ \\
\hline Sb-125 & Below MDA & Below MDA & $4.4 \mathrm{E}-10$ \\
\hline Cs-134 & Below MDA & Below MDA & $1.1 \mathrm{E}-10$ \\
\hline Cs-137 & $\begin{array}{l}0.8 \mathrm{E}-9 \text { from IDG2 } \\
9.6 \mathrm{E}-9 \text { from IDG3 } \\
1.1 \mathrm{E}-8 \text { from IDG1 }\end{array}$ & Below MDA & $1.3 \mathrm{E}-8$ \\
\hline \hline Th-234 & $\begin{array}{l}\text { <MDA from IDG2 } \\
2.1 \mathrm{E}-10 \text { from IDG3 } \\
1.3 \mathrm{E}-10 \text { from IDG1 }\end{array}$ & $1.7 \mathrm{E}-8$ & $2.1 \mathrm{E}-11$ \\
\hline \hline U-235 & Below MDA & $1.8 \mathrm{E}-10$ & Below MDA \\
\hline \hline
\end{tabular}

* Three glass dissolution solutions were counted.

MDA $=$ Minimum Detectable Activity

List of MDA for the counting time used (72000 seconds):

Isotopes

Co-60

$\mathrm{Sb}-125$

Cs-134

Cs-137

Th-234

U-235
MDA (in $\mathrm{Ci} / \mathrm{g}$ )

$6.3 \mathrm{E}-12$

$2.6 \mathrm{E}-13$

$3.2 \mathrm{E}-12$

$3.0 \mathrm{E}-12$

9.7E-11

4.0E-12 
Table 3.5

Summary of Waste Composition as Oxides Used in Glass Formulations (wt\%)

\begin{tabular}{|c|c|c|c|c|}
\hline & $\begin{array}{c}\text { ID1 } \\
\text { Asbestos } \\
\end{array}$ & $\begin{array}{c}\mathrm{ID2} \\
\text { Eutectic Salt } \\
\end{array}$ & $\begin{array}{c}\text { ID3 } \\
\text { Fly Ash } \\
\end{array}$ & $\begin{array}{l}\text { ID4 } \\
\text { Soil } \\
\end{array}$ \\
\hline $\mathrm{Al}_{2} \mathrm{O}_{3}$ & 12.77 & 0.08 & 0.67 & 8.72 \\
\hline $\mathrm{B}_{2} \mathrm{O}_{3}$ & 0.12 & 0.32 & 0.48 & 0.20 \\
\hline $\mathrm{BaO}$ & 0.07 & 0.00 & 0.07 & 0.07 \\
\hline $\mathrm{CaO}$ & 25.21 & 0.12 & 2.35 & 12.84 \\
\hline $\mathrm{CdO}$ & . & & 0.39 & \\
\hline $\mathrm{Cr}_{2} \mathrm{O}_{3}$ & 0.05 & 0.01 & 1.66 & 0.02 \\
\hline $\mathrm{CuO}$ & & & 1.78 & \\
\hline $\mathrm{Fe}_{2} \mathrm{O}_{3}$ & 4.50 & 0.04 & 1.84 & 3.09 \\
\hline $\mathrm{HgO}$ & & & 0.01 & \\
\hline $\mathrm{K}_{2} \mathrm{O}$ & 1.24 & 46.70 & 2.74 & 1.86 \\
\hline $\mathrm{Li}_{2} \mathrm{O}$ & 0.26 & 8.63 & 0.13 & 0.06 \\
\hline $\mathrm{MgO}$ & 6.86 & 0.11 & 0.28 & 5.51 \\
\hline $\mathrm{MnO}_{2}$ & 0.42 & 0.00 & 0.15 & 0.10 \\
\hline $\mathrm{Na}_{2} \mathrm{O}$ & 1.33 & 34.80 & 6.00 & 1.74 \\
\hline $\mathrm{NiO}$ & 0.02 & 0.04 & 0.07 & 0.01 \\
\hline $\mathrm{P}_{2} \mathrm{O}_{5}$ & 0.20 & 0.14 & 0.91 & 0.47 \\
\hline $\mathrm{PbO}$ & & & 9.89 & \\
\hline $\mathrm{SO}_{3}$ & 0.38 & 0.60 & 26.13 & \\
\hline $\mathrm{SiO}_{2}$ & 45.57 & 0.40 & 5.13 & 64.79 \\
\hline $\mathrm{SbO}$ & & & 5.24 & \\
\hline $\mathrm{SrO}$ & 0.07 & 0.00 & 0.01 & 0.03 \\
\hline $\mathrm{TiO}_{2}$ & 0.61 & 0.02 & 0.51 & 0.38 \\
\hline $\mathrm{UO}_{2}$ & 0.28 & 9.02 & 0.04 & 0.03 \\
\hline $\mathrm{ZnO}$ & & & 33.45 & \\
\hline $\mathrm{ZrO}_{2}$ & 0.03 & 0.00 & 0.04 & 0.09 \\
\hline Sum & 100.00 & 100.00 & 100.00 & 100.00 \\
\hline
\end{tabular}


Table 3.6

Summary of Compositions of Glass Made from Idaho Wastes (wt\%)

\begin{tabular}{|c|c|c|c|c|c|c|c|c|}
\hline & \multicolumn{2}{|c|}{ IDG4 } & \multicolumn{2}{|c|}{ IDG5 } & \multirow{2}{*}{$\frac{\text { IDG5A }}{\text { Analysis }}$} & \multicolumn{2}{|c|}{ IDG6 } & \multirow{2}{*}{$\begin{array}{l}\text { IDG7 } \\
\text { Target }\end{array}$} \\
\hline & Target & Analysis & Target & Analysis & & Target & Analysis & \\
\hline $\begin{array}{l}\text { Asbestos\% } \\
\text { Eutectic Salt\% } \\
\text { Fly Ash\% } \\
\text { Soil \% } \\
\end{array}$ & \multicolumn{2}{|c|}{$\begin{array}{l}70 \% \\
30 \%\end{array}$} & \multicolumn{3}{|c|}{$\begin{array}{l}80 \% \\
20 \%\end{array}$} & \multicolumn{2}{|c|}{$\begin{array}{l}90 \% \\
10 \%\end{array}$} & $\begin{array}{c}90.91 \% \\
9.09 \%\end{array}$ \\
\hline $\begin{array}{l}\text { Melt Temp. }\left({ }^{\circ} \mathrm{C}\right) \\
\text { Melt time (min.) }\end{array}$ & & $\begin{array}{r}1200 \\
75 \\
\end{array}$ & & $\begin{array}{r}1200 \\
75 \\
\end{array}$ & $\begin{array}{r}1200 \\
135 \\
\end{array}$ & & $\begin{array}{r}1200 \\
135 \\
\end{array}$ & \\
\hline $\begin{array}{l}\mathrm{Fe}^{2+} / \text { Total } \\
\text { Visible Sulfate \% }\end{array}$ & & $\begin{array}{c}\text { Oxidized } \\
\text { Yes }\end{array}$ & & $\begin{array}{c}\text { Oxidized } \\
0.0 \% \\
\end{array}$ & $\begin{array}{c}\text { Oxidized } \\
0.0 \% \\
\end{array}$ & & $\begin{array}{c}\text { Oxidized } \\
0.0 \% \\
\end{array}$ & \\
\hline $\mathrm{Al}_{2} \mathrm{O}_{3}$ & 8.96 & 13.51 & 10.23 & 14.07 & 13.05 & 11.50 & 16.16 & 11.62 \\
\hline $\mathrm{B}_{2} \mathrm{O}_{3}$ & 0.18 & 3.24 & 0.16 & 4.53 & 4.14 & 0.14 & 4.36 & 0.14 \\
\hline $\mathrm{BaO}$ & 0.05 & 0.04 & 0.06 & 0.00 & 0.00 & 0.06 & 0.00 & 0.06 \\
\hline $\mathrm{CaO}$ & 17.68 & 13.13 & 20.19 & 15.68 & 16.37 & 22.70 & 18.37 & 22.93 \\
\hline $\mathrm{CdO}$ & 0.00 & & 0.00 & & & 0.00 & & 0.00 \\
\hline $\mathrm{Cr}_{2} \mathrm{O}_{3}$ & 0.04 & 0.04 & 0.04 & 0.01 & 0.00 & 0.04 & 0.00 & 0.04 \\
\hline $\mathrm{CuO}$ & 0.00 & & 0.00 & & & 0.00 & & 0.00 \\
\hline $\mathrm{Fe}_{2} \mathrm{O}_{3}$ & 3.16 & 3.17 & 3.61 & 3.60 & 3.57 & 4.06 & 4.11 & 4.10 \\
\hline $\mathrm{HgO}$ & 0.00 & & 0.00 & & & 0.00 & & 0.00 \\
\hline $\mathrm{K}_{2} \mathrm{O}$ & 14.66 & 14.25 & 10.19 & 9.79 & 9.68 & 5.71 & 4.88 & 5.30 \\
\hline $\mathrm{Li}_{2} \mathrm{O}$ & 2.77 & 2.68 & 1.94 & 1.78 & 1.86 & 1.10 & 0.87 & 1.02 \\
\hline $\mathrm{MgO}$ & 4.84 & 4.05 & 5.51 & 5.61 & 5.73 & 6.19 & 6.24 & 6.25 \\
\hline $\mathrm{MnO}_{2}$ & 0.29 & 0.22 & 0.33 & 0.01 & 0.01 & 0.38 & 0.01 & 0.38 \\
\hline $\mathrm{Na}_{2} \mathrm{O}$ & 11.43 & 11.76 & 8.06 & 7.13 & 6.96 & 4.69 & 3.44 & 4.39 \\
\hline $\mathrm{NiO}$ & 0.03 & 0.03 & 0.03 & 0.02 & 0.01 & 0.02 & 0.01 & 0.02 \\
\hline $\mathrm{P}_{2} \mathrm{O}_{5}$ & 0.18 & 0.20 & 0.19 & 0.24 & 0.21 & 0.19 & 0.20 & 0.19 \\
\hline $\mathrm{PbO}$ & 0.00 & & 0.00 & & & 0.00 & & 0.00 \\
\hline $\mathrm{SO}_{3}$ & 0.27 & & 0.31 & & & 0.35 & & 0.35 \\
\hline $\mathrm{SiO}_{2}$ & 32.02 & 30.34 & 36.54 & 35.44 & 36.34 & 41.06 & 40.25 & 41.47 \\
\hline Sbo & 0.00 & & 0.00 & & & 0.00 & & 0.00 \\
\hline SrO & 0.05 & 0.04 & 0.06 & 0.01 & 0.01 & 0.06 & 0.01 & 0.06 \\
\hline $\mathrm{TiO}_{2}$ & 0.43 & 0.42 & 0.49 & 0.03 & 0.03 & 0.55 & 0.02 & 0.56 \\
\hline $\mathrm{UO}_{2}$ & 2.92 & 2.85 & 2.04 & 2.05 & 2.02 & 1.16 & 1.04 & 1.08 \\
\hline $\mathrm{ZnO}$ & 0.00 & & 0.00 & & & 0.00 & & 0.00 \\
\hline $\mathrm{ZrO}_{2}$ & 0.02 & 0.02 & 0.03 & 0.01 & 0.00 & 0.03 & 0.01 & 0.03 \\
\hline Sum & 100.00 & 100.00 & 100.00 & 99.99 & 100.00 & 100.00 & 99.99 & 100.00 \\
\hline
\end{tabular}




\begin{tabular}{|c|c|c|c|c|c|c|c|}
\hline & IDG7A & IDG7B & IDG7C & IDG8 & IDG8A & IDG8B & IDG8C \\
\hline & Analysis & Analysis & Analysis & Target & Analysis & Analysis & Analysis \\
\hline $\begin{array}{l}\text { Asbestos\% } \\
\text { Eutectic Salt\% } \\
\text { Fly Ash\% } \\
\text { Soil \% }\end{array}$ & \multicolumn{3}{|c|}{$\begin{array}{r}90.91 \% \\
9.09 \%\end{array}$} & \multicolumn{3}{|c|}{$\begin{array}{l}88.00 \% \\
12.00 \%\end{array}$} & \\
\hline $\begin{array}{l}\text { Melt Temp. }\left({ }^{\circ} \mathrm{C}\right) \\
\text { Melt time (min.) }\end{array}$ & $\begin{array}{r}1300 \\
90\end{array}$ & $\begin{array}{r}1300 \\
75\end{array}$ & $\begin{array}{r}1300 \\
70\end{array}$ & & $\begin{array}{r}1300 \\
75\end{array}$ & $\begin{array}{r}1300 \\
70\end{array}$ & $\begin{array}{r}1300 \\
75\end{array}$ \\
\hline $\begin{array}{l}\mathrm{Fe}^{2+} / \mathrm{Total} \\
\text { Visible Sulfate }\end{array}$ & $\begin{array}{l}\text { Oxidized } \\
0.0 \%\end{array}$ & $\begin{array}{l}\text { Oxidized } \\
0.0 \%\end{array}$ & $\begin{array}{l}\text { Oxidized } \\
0.0 \%\end{array}$ & & $\begin{array}{l}\text { Oxidized } \\
0.3 \%\end{array}$ & $\begin{array}{l}\text { Oxidized } \\
0.0 \%\end{array}$ & $\begin{array}{c}\text { Oxidized } \\
0.0 \%\end{array}$ \\
\hline $\mathrm{Al}_{2} \mathrm{O}_{3}$ & 13.06 & 13.11 & 13.06 & 11.25 & 10.70 & 11.26 & 11.42 \\
\hline $\mathrm{B}_{2} \mathrm{O}_{3}$ & 0.14 & 0.15 & 0.12 & 0.14 & 0.09 & 0.12 & 0.14 \\
\hline $\mathrm{BaO}$ & 0.06 & 0.06 & 0.06 & 0.06 & 0.07 & 0.07 & 0.07 \\
\hline $\mathrm{CaO}$ & 22.23 & 22.31 & 22.54 & 22.20 & 26.67 & 24.85 & 24.53 \\
\hline $\mathrm{CdO}$ & & & & 0.00 & & & \\
\hline $\mathrm{Cr}_{2} \mathrm{O}_{3}$ & 0.03 & 0.02 & 0.03 & 0.04 & 0.06 & 0.06 & 0.07 \\
\hline $\mathrm{CuO}$ & . & & & 0.00 & & & \\
\hline $\mathrm{Fe}_{2} \mathrm{O}_{3}$ & 3.79 & 3.65 & 2.91 & 3.97 & 5.40 & 4.86 & 4.94 \\
\hline $\mathrm{HgO}$ & & & & 0.00 & & & . \\
\hline $\mathrm{K}_{2} \mathrm{O}$ & 3.87 & 4.22 & 4.28 & 6.61 & 3.25 & 3.40 & 3.27 \\
\hline $\mathrm{Li}_{2} \mathrm{O}$ & 0.72 & 0.76 & 0.79 & 1.27 & 0.74 & 0.64 & 0.69 \\
\hline $\mathrm{MgO}$ & 5.24 & 5.05 & 5.40 & 6.05 & 8.24 & 7.49 & 7.55 \\
\hline $\mathrm{MnO}_{2}$ & 0.27 & 0.24 & 0.24 & 0.37 & 0.60 & 0.45 & 0.55 \\
\hline $\mathrm{Na}_{2} \mathrm{O}$ & 3.30 & 3.35 & 3.87 & 5.37 & 2.79 & 2.78 & 3.07 \\
\hline NiO & 0.05 & 0.03 & 0.02 & 0.02 & 0.02 & 0.02 & 0.02 \\
\hline $\mathrm{P}_{2} \mathrm{O}_{5}$ & 0.31 & 0.18 & 0.16 & 0.19 & 0.17 & 0.18 & 0.16 \\
\hline $\mathrm{PbO}$ & & & & 0.00 & & & \\
\hline $\mathrm{SO}_{3}$ & & & 0.37 & 0.34 & 0.37 & 0.34 & 0.37 \\
\hline $\mathrm{SiO}_{2}$ & 45.57 & 45.47 & 44.74 & 40.15 & 39.27 & 42.02 & 42.01 \\
\hline Sbo & & & & 0.00 & & & \\
\hline SrO & 0.07 & 0.07 & 0.08 & 0.06 & $0.06^{\circ}$ & 0.06 & 0.06 \\
\hline $\mathrm{TiO}_{2}$ & 0.47 & 0.45 & 0.47 & 0.54 & 0.69 & 0.68 & 0.72 \\
\hline $\mathrm{UO}_{2}$ & 0.79 & 0.84 & 0.83 & 1.34 & 0.77 & 0.69 & 0.70 \\
\hline $\mathrm{ZnO}$ & & & & 0.00 & & & \\
\hline $\mathrm{ZrO}_{2}$ & 0.05 & 0.03 & 0.02 & 0.03 & 0.03 & 0.03 & 0.03 \\
\hline Sum & 99.95 & 99.98 & 99.98 & 100.00 & 99.97 & 99.97 & 100.34 \\
\hline
\end{tabular}

Page 3-31 


\begin{tabular}{|c|c|c|c|c|c|c|c|}
\hline & IDG10 & IDG10 & IDG11 & IDG12 & IDG12A & IDG12B & IDG12C \\
\hline & Target & Analysis & Target & Target & Analyzed & Analyzed & Analyzed \\
\hline $\begin{array}{l}\text { Asbestos\% } \\
\text { Eutectic Salt\% } \\
\text { Fly Ash\% } \\
\text { Soil \% }\end{array}$ & \multicolumn{2}{|c|}{$\begin{array}{l}60 \% \\
40 \%\end{array}$} & $\begin{array}{l}45.00 \% \\
10.00 \% \\
30.00 \% \\
15.00 \%\end{array}$ & & \multicolumn{3}{|c|}{$\begin{array}{l}70.00 \% \\
10.00 \% \\
10.00 \% \\
10.00 \%\end{array}$} \\
\hline $\begin{array}{l}\text { Melt Time }\left({ }^{\circ} \mathrm{C}\right) \\
\text { Melt time (min.) }\end{array}$ & & $\begin{array}{r}1400 \\
115\end{array}$ & $\begin{array}{r}1350 \\
75\end{array}$ & & $\begin{array}{r}1300 \\
70\end{array}$ & $\begin{array}{r}1300 \\
60\end{array}$ & $\begin{array}{r}1400 \\
130\end{array}$ \\
\hline $\begin{array}{l}\mathrm{Fe}^{2+} / \text { Total } \\
\text { Visible Sulfate \% }\end{array}$ & & $\begin{array}{c}\text { Reduced } \\
0.0 \%\end{array}$ & $\begin{array}{c}\text { Reduced } \\
0.0 \%\end{array}$ & & $\begin{array}{l}0.60 \\
\text { Trace }\end{array}$ & $\begin{array}{c}0.94 \\
0.0 \%\end{array}$ & $\begin{array}{c}0.64 \\
0.0 \%\end{array}$ \\
\hline $\mathrm{Al}_{2} \mathrm{O}_{3}$ & 7.93 & 13.27 & 7.27 & 9.89 & 10.41 & 10.14 & 11.19 \\
\hline $\mathrm{B}_{2} \mathrm{O}_{3}$ & 0.26 & 0.10 & 0.26 & 0.18 & 0.14 & 0.07 & 0.00 \\
\hline $\mathrm{BaO}$ & 0.07 & 0.08 & 0.07 & 0.06 & 0.06 & 0.06 & 0.06 \\
\hline $\mathrm{CaO}$ & 16.06 & 18.91 & 13.98 & 19.17 & 19.70 & 22.75 & 20.87 \\
\hline $\mathrm{CdO}$ & 0.16 & 0.00 & 0.12 & 0.04 & N.A. & N.A. & 0.00 \\
\hline $\mathrm{Cr}_{2} \mathrm{O}_{3}$ & 0.69 & 0.22 & 0.52 & 0.20 & 0.09 & 0.08 & 0.35 \\
\hline $\mathrm{CuO}$ & 0.71 & 1.28 & 0.53 & 0.18 & 0.10 & 0.06 & 0.09 \\
\hline $\mathrm{Fe}_{2} \mathrm{O}_{3}$ & 3.44 & 6.15 & 3.05 & 3.65 & 5.57 & 3.65 & 5.49 \\
\hline $\mathrm{HgO}$ & 0.01 & & 0.00 & 0.00 & N.A. & 0.00 & \\
\hline $\mathrm{K}_{2} \mathrm{O}$ & 1.84 & 0.41 & 6.26 & 5.92 & 3.10 & 3.62 & 2.22 \\
\hline $\mathrm{Li}_{2} \mathrm{O}$ & 0.21 & 0.09 & 1.03 & 1.07 & 0.80 & 0.75 & 0.72 \\
\hline $\mathrm{MgO}$ & 4.23 & 5.68 & 4.01 & 5.40 & 5.88 & 6.49 & 6.16 \\
\hline $\mathrm{MnO}_{2}$ & 0.31 & 0.47 & 0.25 & 0.32 & 0.28 & 0.27 & 0.26 \\
\hline $\mathrm{Na}_{2} \mathrm{O}$ & 3.19 & 1.61 & 6.16 & 5.20 & 3.72 & 3.73 & 3.26 \\
\hline $\mathrm{NiO}$ & 0.04 & 0.14 & 0.04 & 0.03 & 0.03 & 0.01 & 0.15 \\
\hline $\mathrm{P}_{2} \mathrm{O}_{5}$ & 0.48 & 0.46 & 0.45 & 0.29 & 0.19 & 0.14 & 0.22 \\
\hline $\mathrm{PbO}$ & 3.96 & 0.15 & 2.97 & 0.99 & 0.65 & 0.07 & 0.38 \\
\hline $\mathrm{SO}_{3}$ & 10.68 & & 8.01 & 2.88 & 1.48 & 2.50 & 0.66 \\
\hline $\mathrm{SiO}_{2}$ & 29.40 & 39.87 & 31.81 & 38.93 & 41.34 & 42.26 & 42.72 \\
\hline $\mathrm{SbO}$ & 2.10 & & 1.57 & 0.52 & N.A. & N.A. & \\
\hline $\mathrm{SrO}$ & 0.05 & 0.05 & 0.04 & 0.05 & 0.05 & 0.05 & 0.05 \\
\hline $\mathrm{TiO}_{2}$ & 0.57 & 0.91 & 0.49 & 0.52 & 0.61 & 0.58 & 0.58 \\
\hline $\mathrm{UO}_{2}$ & 0.19 & 0.07 & 1.05 & 1.11 & 0.87 & 0.80 & 0.81 \\
\hline $\mathrm{ZnO}$ & 13.38 & 10.02 & 10.03 & 3.34 & 5.34 & 2.37 & 3.70 \\
\hline $\mathrm{ZrO}_{2}$ & 0.04 & 0.06 & 0.04 & 0.04 & 0.04 & 0.03 & 0.03 \\
\hline Sum & 100.00 & 100.00 & 100.00 & 100.00 & 100.00 & 100.00 & 100.00 \\
\hline
\end{tabular}

Page 3-32 


\begin{tabular}{|c|c|c|c|c|c|c|c|}
\hline & IDG13 & IDG13A & IDG13B & IDG13C & IDG14 & IDG14A & IDG14B \\
\hline & Target & Analyzed & Analyzed & Analyzed & Target & Analyzed & Analyzed \\
\hline $\begin{array}{l}\text { Asbestos\% } \\
\text { Eutectic Salt\% } \\
\text { Fly Ash\% } \\
\text { Soil \% }\end{array}$ & & $\begin{array}{l}30.00 \% \\
20.00 \% \\
10.00 \% \\
40.00 \%\end{array}$ & & & & $\begin{array}{l}50.00 \% \\
20.00 \% \\
10.00 \% \\
20.00 \%\end{array}$ & \\
\hline $\begin{array}{l}\text { Melt Temp }\left({ }^{\circ} \mathrm{C}\right) \\
\text { Melt time (min.) }\end{array}$ & & $\begin{array}{r}1450 \\
80\end{array}$ & $\begin{array}{r}1400 \\
70\end{array}$ & $\begin{array}{r}1400 \\
70\end{array}$ & & $\begin{array}{r}1450 \\
75\end{array}$ & $\begin{array}{r}1400 \\
70\end{array}$ \\
\hline $\begin{array}{l}\mathrm{Fe}^{2+} / \mathrm{Total} \\
\text { Visible Sulfate \% }\end{array}$ & & $\begin{array}{r}0.75 \\
0.0 \% \\
\end{array}$ & $\begin{array}{r}0.27 \\
0.73 \% \\
\end{array}$ & $\begin{array}{r}0.25 \\
2.30 \% \\
\end{array}$ & & $\begin{array}{r}0.81 \\
0.0 \%\end{array}$ & $\begin{array}{r}0.20 \\
5.63 \%\end{array}$ \\
\hline $\mathrm{Al}_{2} \mathrm{O}_{3}$ & 7.40 & 12.70 & 8.69 & 8.61 & 8.21 & . $\quad 23.19$ & 9.25 \\
\hline $\mathrm{B}_{2} \mathrm{O}_{3}$ & 0.23 & 0.19 & 0.00 & 0.00 & 0.21 & 0.12 & 0.06 \\
\hline $\mathrm{BaO}$ & 0.06 & 0.05 & 0.06 & 0.05 & 0.06 & 0.04 & 0.05 \\
\hline $\mathrm{CaO}$ & 12.95 & 9.61 & 12.04 & 9.90 & 15.43 & 6.46 & 12.26 \\
\hline $\mathrm{CdO}$ & 0.04 & 0.00 & 0.00 & 0.19 & 0.04 & 0.00 & 0.00 \\
\hline $\mathrm{Cr}_{2} \mathrm{O}_{3}$ & 0.19 & 0.29 & 0.26 & 0.21 & 0.20 & 0.25 & 0.23 \\
\hline $\mathrm{CuO}$ & 0.18 & 0.18 & 0.18 & 0.27 & 0.18 & 0.18 & 0.30 \\
\hline $\mathrm{Fe}_{2} \mathrm{O}_{3}$ & 2.78 & 3.00 & 4.46 & 2.50 & 3.06 & 2.32 & 3.40 \\
\hline $\mathrm{HgO}$ & 0.00 & 0.00 & & & 0.00 & 0.00 & \\
\hline $\mathrm{K}_{2} \mathrm{O}$ & 10.59 & 6.55 & 8.25 & 8.70 & 10.46 & 5.76 & 7.07 \\
\hline $\mathbf{L i}_{2} \mathrm{O}$ & 1.84 & 1.57 & 1.45 & 1.54 & 1.88 & 1.37 & 1.59 \\
\hline $\mathrm{MgO}$ & 4.31 & 2.67 & 3.65 & 2.29 & 4.59 & 1.86 & 3.14 \\
\hline $\mathrm{MnO}_{2}$ & 0.18 & 0.17 & 0.17 & 0.09 & 0.24 & 0.14 & 0.13 \\
\hline $\mathrm{Na}_{2} \mathrm{O}$ & 8.69 & 6.72 & 7.07 & 7.29 & 8.61 & 6.00 & 7.01 \\
\hline $\mathrm{NiO}$ & 0.02 & 0.13 & 0.12 & 0.10 & 0.03 & 0.12 & 0.11 \\
\hline $\mathrm{P}_{2} \mathrm{O}_{5}$ & 0.37 & 0.29 & 0.23 & 0.29 & 0.31 & 0.23 & 0.31 \\
\hline $\mathrm{PbO}$ & 0.99 & 0.30 & 1.06 & 1.21 & 0.99 & 0.34 & 1.52 \\
\hline $\mathrm{SO}_{3}$ & 2.73 & N.A. & 1.54 & 1.28 & 2.81 & N.A. & 2.77 \\
\hline $\mathrm{SiO}_{2}$ & 40.18 & 49.76 & 45.22 & 48.94 & 36.34 & 46.17 & 42.15 \\
\hline Sbo & 0.52 & N.A. & & & 0.52 & N.A. & N.A. \\
\hline SrO & 0.03 & 0.02 & 0.03 & 0.03 & 0.04 & 0.02 & 0.04 \\
\hline $\mathrm{TiO}_{2}$ & 0.39 & 0.38 & 0.45 & .0 .39 & 0.44 & 0.30 & 0.47 \\
\hline $\mathrm{UO}_{2}$ & 1.92 & 1.64 & 1.59 & 1.76 & 1.97 & 1.51 & 2.09 \\
\hline $\mathrm{ZnO}$ & 3.34 & 3.74 & 3.43 & 4.32 & 3.34 & 3.60 & 6.01 \\
\hline $\mathrm{ZrO}_{2}$ & 0.05 & 0.04 & 0.03 & 0.03 & 0.04 & 0.03 & 0.02 \\
\hline Sum & 100.00 & 100.00 & 100.00 & 100.00 & 100.00 & 100.00 & 100.00 \\
\hline
\end{tabular}




\begin{tabular}{|c|c|c|c|c|}
\hline & IDG15 & IDG15A & IDG15B & IDG15C \\
\hline & Target & Analyzed & Analyzed & Analyzed \\
\hline $\begin{array}{l}\text { Asbestos\% } \\
\text { Eutectic Salt\% } \\
\text { Fly Ash\% } \\
\text { Soil \% }\end{array}$ & & $\begin{array}{l}30.00 \% \\
20.00 \% \\
20.00 \% \\
30.00 \% \\
\end{array}$ & & \\
\hline $\begin{array}{l}\text { Melt Temp }\left({ }^{\circ} \mathrm{C}\right) \\
\text { Melt time (min.) }\end{array}$ & & $\begin{array}{r}1450 \\
70 \\
\end{array}$ & $\begin{array}{r}1400 \\
75 \\
\end{array}$ & $\begin{array}{r}1400 \\
60 \\
\end{array}$ \\
\hline $\begin{array}{l}\mathrm{Fe}^{2+} / \text { Total } \\
\text { Visible Sulfate \% }\end{array}$ & & $\begin{array}{r}0.72 \\
0.0 \% \\
\end{array}$ & $\begin{array}{r}0.34 \\
4.54 \% \\
\end{array}$ & $\begin{array}{r}0.80 \\
0.0 \% \\
\end{array}$ \\
\hline $\mathrm{Al}_{2} \mathrm{O}_{3}$ & 6.60 & 8.69 & 8.91 & 9.30 \\
\hline $\mathrm{B}_{2} \mathrm{O}_{3}$ & 0.26 & 0.07 & 0.22 & 0.10 \\
\hline $\mathrm{BaO}$ & 0.06 & 0.07 & 0.06 & 0.07 \\
\hline $\mathrm{CaO}$ & 11.91 & 13.06 & 9.08 & 9.36 \\
\hline $\mathrm{CdO}$ & 0.08 & 0.00 & 0.00 & 0.00 \\
\hline $\mathrm{Cr}_{2} \mathrm{O}_{3}$ & 0.35 & 0.35 & 0.14 & 0.29 \\
\hline $\mathrm{CuO}$ & 0.36 & & 0.37 & 0.25 \\
\hline $\mathrm{Fe}_{2} \mathrm{O}_{3}$ & 2.65 & 3.71 & 2.40 & 3.80 \\
\hline $\mathrm{HgO}$ & 0.00 & & & \\
\hline $\mathrm{K}_{2} \mathrm{O}$ & 10.68 & 4.23 & 6.80 & 6.23 \\
\hline $\mathrm{Li}_{2} \mathrm{O}$ & 1.85 & 1.57 & 1.46 & 1.61 \\
\hline $\mathrm{MgO}$ & 3.79 & 3.64 & 2.34 & 2.30 \\
\hline $\mathrm{MnO}_{2}$ & 0.18 & 0.25 & 0.11 & 0.15 \\
\hline $\mathrm{Na}_{2} \mathrm{O}$ & 9.12 & 6.90 & 7.06 & 7.70 \\
\hline $\mathrm{NiO}$ & 0.03 & 0.12 & 0.08 & 0.04 \\
\hline $\mathrm{P}_{2} \mathrm{O}_{5}$ & 0.41 & 0.41 & 0.46 & 0.42 \\
\hline $\mathrm{PbO}$ & 1.98 & 0.28 & 1.93 & 0.26 \\
\hline $\mathrm{SO}_{3}$ & 5.34 & & 4.50 & 4.03 \\
\hline $\mathrm{SiO}_{2}$ & 34.22 & 50.14 & 41.52 & 45.48 \\
\hline $\mathrm{SbO}$ & 1.05 & & N.A. & N.A. \\
\hline SrO & 0.03 & 0.03 & 0.04 & 0.03 \\
\hline $\mathrm{TiO}_{2}$ & 0.40 & 0.56 & 0.48 & 0.51 \\
\hline $\mathrm{UO}_{2}$ & 1.92 & 1.65 & 1.87 & 2.05 \\
\hline $\mathrm{ZnO}$ & 6.69 & 4.20 & 10.12 & 5.98 \\
\hline $\mathrm{ZrO}_{2}$ & 0.05 & 0.06 & 0.07 & 0.04 \\
\hline Sum & 100.00 & 100.00 & 100.00 & 100.00 \\
\hline
\end{tabular}

Page 3-34 
Table 3.7

Summary of Viscosity and Conductivity of Idaho Glasses

\begin{tabular}{|c|c|c|c|c|c|c|c|c|c|}
\hline & IDG7A & IDG7B & IDG7C & IDG8A & IDG8B & IDG8C & IDG12C & IDG13A & IDG13B \\
\hline Asbestos\% & $90.91 \%$ & $90.91 \%$ & $90.91 \%$ & $88.00 \%$ & $88.00 \%$ & $88.00 \%$ & $70.00 \%$ & $30.00 \%$ & $30.00 \%$ \\
\hline $\begin{array}{l}\text { Eutectic } \\
\text { Salt\% }\end{array}$ & $9.09 \%$ & $9.09 \%$ & $9.09 \%$ & $12.00 \%$ & $12.00 \%$ & $12.00 \%$ & $10.00 \%$ & $20.00 \%$ & $20.00 \%$ \\
\hline Fly Ash\% & & & & & & & $10.00 \%$ & $10.00 \%$ & $10.00 \%$ \\
\hline Soil \% & & & & & & & $10.00 \%$ & $40.00 \%$ & $40.00 \%$ \\
\hline $\mathrm{Fe}^{2+} / \mathrm{Total}$ & & & & & & & 0.64 & 0.75 & 0.27 \\
\hline \multicolumn{10}{|c|}{ Viscosity (Poise) versus Temperature (in Celsius) } \\
\hline$@ 1100 \mathrm{C}$ & & & 139.34 & & & & & & 342.73 \\
\hline @.1150C & 379.09 & 287.77 & 79.27 & & SOLID & SOLID & 120.35 & 512.32 & 201.68 \\
\hline$@ 1200 \mathrm{C}$ & 184.46 & 146.25 & 48.23 & & 59.69 & 60.51 & 63.21 & 318.43 & 123.03 \\
\hline$@ 1250 \mathrm{C}$ & 98.81 & 81.35 & 31.02 & 16.73 & 31.38 & 33.92 & 36.60 & 210.88 & 77.53 \\
\hline (a,1300C & 57.22 & 48.69 & 20.91 & 10.30 & 17.87 & 20.45 & 22.57 & 147.03 & 50.31 \\
\hline (a.1350C & 35.34 & 30.96 & 14.67 & 6.71 & 10.88 & 13.08 & 14.73 & 106.96 & 33.53 \\
\hline @ $1400 \mathrm{C}$ & 23.02 & 20.70 & 10.65 & 4.59 & 7.00 & 8.79 & 10.08 & 80.61 & 22.89 \\
\hline @ $1450 \mathrm{C}$ & 15.69 & 14.44 & 19.57 & 3.26 & 4.71 & 6.16 & 7.18 & 62.59 & 15.98 \\
\hline \multicolumn{10}{|c|}{ Measurement Range } \\
\hline $\begin{array}{l}\text { Lower } \\
\text { Higher }\end{array}$ & 1223 & 1157 & 1128 & 1191 & 1243 & 1213 & 1158 & 1173 & 1169 \\
\hline \multicolumn{10}{|c|}{ Conductivity (S/cm) versus Temperature (in Celsius) } \\
\hline$@ 1100 \mathrm{C}$ & & & 0.010 & & & & & & 0.069 \\
\hline$@ 1150 \mathrm{C}$ & 0.020 & 0.022 & 0.017 & & 0.017 & 0.050 & 0.025 & & 0.091 \\
\hline$@ 1200 \mathrm{C}$ & 0.030 & 0.036 & 0.028 & 0.055 & 0.026 & 0.072 & 0.038 & & 0.116 \\
\hline$@ 1250 \mathrm{C}$ & 0.044 & 0.057 & 0.045 & 0.078 & 0.040 & 0.101 & 0.057 & 0.099 & 0.147 \\
\hline$@ 1300 \mathrm{C}$ & 0.063 & 0.086 & 0.068 & 0.108 & 0.059 & 0.140 & 0.084 & 0.129 & 0.183 \\
\hline$@ 1350 \mathrm{C}$ & 0.088 & 0.129 & 0.102 & 0.146 & 0.085 & 0.189 & 0.121 & 0.166 & 0.225 \\
\hline$@ 1400 \mathrm{C}$ & 0.120 & 0.187 & 0.150 & 0.195 & 0.120 & 0.251 & 0.169 & 0.210 & 0.272 \\
\hline$@ 1450 \mathrm{C}$ & 0.162 & & & & & & & 0.262 & 0.327 \\
\hline \multicolumn{10}{|c|}{ Measurement Range } \\
\hline $\begin{array}{l}\text { Lower } \\
\text { Higher }\end{array}$ & 1199 & 1191 & 1120 & 1238 & 1186 & 1193 & 1160 & 1289 & 1159 \\
\hline
\end{tabular}




\begin{tabular}{|c|c|c|c|c|c|c|}
\hline & IDG13C & IDG14A & IDG14B & IDG15A & IDG15B & IDG15C \\
\hline Asbestos\% & $30.00 \%$ & $50.00 \%$ & $50.00 \%$ & $30.00 \%$ & $30.00 \%$ & 0.3 \\
\hline Eutectic Salt\% & $20.00 \%$ & $20.00 \%$ & $20.00 \%$ & $20.00 \%$ & $20.00 \%$ & \\
\hline Fly Ash\% & $10.00 \%$ & $10.00 \%$ & $10.00 \%$ & $20.00 \%$ & $20.00 \%$ & \\
\hline Soil \% & $40.00 \%$ & $20.00 \%$ & $20.00 \%$ & $30.00 \%$ & $30.00 \%$ & \\
\hline $\mathrm{Fe}^{2+} / \mathrm{Total}$ & 0.25 & 0.81 & 0.20 & 0.72 & 0.34 & 0.80 \\
\hline \multicolumn{7}{|c|}{ Viscosity (Poise) versus Temperature (in Celsius) } \\
\hline @.1100C & & & 163.45 & & 238.02 & 185.37 \\
\hline$@ 1150 \mathrm{C}$ & & & 96.37 & & 145.39 & 114.90 \\
\hline @.1200C & SOLID & & 59.72 & 295.25 & 91.84 & 75.63 \\
\hline$@ 1250 \mathrm{C}$ & 144.55 & & 38.64 & 167.10 & 59.79 & 52.29 \\
\hline$@ 1300 c$ & 96.49 & SOLID & 25.95 & 101.55 & 40.00 & 37.68 \\
\hline$@ 1350 \mathrm{C}$ & 66.04 & 427.12 & 18.01 & 65.44 & 23.43 & 28.11 \\
\hline @1400C & 46.23 & 237.27 & 12.87 & 44.28 & 19.24 & 21.59 \\
\hline @ $1450 \mathrm{C}$ & 33.04 & 140.21 & 9.43 & 31.22 & 13.78 & 17.01 \\
\hline \multicolumn{7}{|l|}{ Measurement range } \\
\hline $\begin{array}{l}\text { Lower } \\
\text { Higher }\end{array}$ & $\begin{array}{l}1294 \\
1440 \\
\end{array}$ & $\begin{array}{l}1381 \\
1432 \\
\end{array}$ & $\begin{array}{l}1203 \\
1404 \\
\end{array}$ & $\begin{array}{l}1245 \\
1392 \\
\end{array}$ & $\begin{array}{l}1148 \\
1391 \\
\end{array}$ & $\begin{array}{l}1139 \\
1429 \\
\end{array}$ \\
\hline \multicolumn{7}{|c|}{ Conductivity $(\mathrm{S} / \mathrm{cm})$ versus Temperature (in Celsius) } \\
\hline$@ 1100 \mathrm{C}$ & & \multirow{7}{*}{$\begin{array}{c}\text { Too } \\
\text { Viscous }\end{array}$} & & & 0.028 & \multirow{6}{*}{$\begin{array}{c}\text { Too } \\
\text { Much } \\
\text { Metallic } \\
\text { Lead } \\
\text { for } \\
\text { Measurement }\end{array}$} \\
\hline (a) $1150 \mathrm{C}$ & & & 0.108 & & 0.042 & \\
\hline$@ 1200 \mathrm{C}$ & 0.114 & & 0.144 & 0.081 & 0.062 & \\
\hline$@ 1250 \mathrm{C}$ & 0.146 & & 0.189 & 0.110 & 0.089 & \\
\hline$@ 1300 \mathrm{C}$ & 0.183 & & 0.243 & 0.146 & 0.124 & \\
\hline @1350c & 0.227 & & 0.308 & 0.191 & 0.170 & \\
\hline$@ 1400 \mathrm{C}$ & 0.278 & & 0.386 & 0.246 & 0.229 & \\
\hline$@ 1450 c$ & & $\sim 0.3$ & & & & \\
\hline \multicolumn{7}{|l|}{ Measurement range } \\
\hline $\begin{array}{l}\text { Lower } \\
\text { Higher }\end{array}$ & $\begin{array}{l}1232 \\
1387\end{array}$ & 1542 & $\begin{array}{l}1192 \\
1387\end{array}$ & $\begin{array}{l}1243 \\
1377\end{array}$ & $\begin{array}{l}1149 \\
1389\end{array}$ & 1388 \\
\hline
\end{tabular}


Table 3.8

Summary of SEM Observations on Idaho Reduced Glasses

\begin{tabular}{|c|c|c|c||}
\hline \multirow{2}{*}{ Glass } & \multicolumn{2}{|c|}{ Concentration of Sulfide Nodules } & \multirow{2}{*}{$\mathrm{Fe}^{+2} / \mathrm{Fe}^{\mathrm{Tot}}$} \\
\cline { 2 - 3 } & $s 0.2 \mu \mathrm{m}$ diameter & $\sim 0.5-1 \mu \mathrm{m}$ diameter & \\
\hline IDG13 & $0.23 \mu \mathrm{m}^{-2}$ & $1.8 \cdot 10^{-3} \mu \mathrm{m}^{-2}$ & 0.80 \\
IDG13A & $0.12 \mu \mathrm{m}^{-2}$ & $2.2 \cdot 10^{-3} \mu \mathrm{m}^{-2}$ & 0.75 \\
IDG14 & $0.28 \mu \mathrm{m}^{-2}$ & $1.8 \cdot 10^{-3} \mu \mathrm{m}^{-2}$ & 0.88 \\
IDG14A & $0.1 \mu \mathrm{m}^{-2}$ & $1.4 \cdot 10^{-3} \mu \mathrm{m}^{-2}$ & 0.81 \\
IDG15 & $0.48 \mu \mathrm{m}^{-2}$ & $7.9 \cdot 10^{-3} \mu \mathrm{m}^{-2}$ & Not Measured \\
IDG15A & $0.18 \mu \mathrm{m}^{-2}$ & $0.12 \cdot 10^{-3} \mu \mathrm{m}^{-2}$ & 0.72 \\
\hline
\end{tabular}


Table 3.9

Summary of Analysis of PCT Leachates of Idaho Glasses

\begin{tabular}{|c|c|c|c|c|c|c|c|c|c|c|c|c|c|}
\hline & \multirow[b]{2}{*}{ SRL-EA } & \multicolumn{12}{|c|}{ IDG- } \\
\hline & & $7 \mathrm{~A}$ & $7 \mathrm{~B}$ & $7 \mathrm{C}$ & $8 \mathrm{~A}$ & $8 \mathrm{C}$ & $12 \mathrm{C}$ & $13 \mathrm{~A}$ & $13 \mathrm{~B}$ & $13 \mathrm{C}$ & $14 \mathrm{~A}$ & $14 \mathrm{~B}$ & $15 \mathrm{~A}$ \\
\hline \multicolumn{14}{|c|}{ SODIUM, PPM } \\
\hline Days 7 & 1700 & 4.62 & 28.21 & 4.75 & 5.47 & 2.96 & 5.35 & 15.22 & 19.71 & 19.98 & 11.21 & 15.73 & 15.50 \\
\hline 28 & 2110 & 5.39 & 33.00 & 5.65 & 6.34 & 14.70 & 5.18 & 19.30 & 22.37 & 25.06 & 12.20 & 18.23 & 19.50 \\
\hline 56 & 2540 & 6.02 & 31.30 & 6.49 & 6.88 & 14.60 & 4.76 & 22.50 & 23.85 & 30.23 & 13.40 & 20.61 & 22.00 \\
\hline 120 & 3790 & 5.53 & 31.80 & 5.99 & 6.13 & 16.30 & 5.06 & 24.66 & 29.96 & 38.64 & 13.04 & 25.09 & 23.93 \\
\hline 180 & 4460 & 6.17 & 34.80 & 6.48 & 6.65 & 18.40 & , & 25.13 & 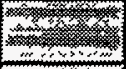 & 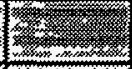 & 12.24 & 型 & 24.60 \\
\hline 270 & 5330 & $(x)$ & 38.40 & (x) & (3) & 19.15 & 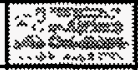 & $x_{3}$ & 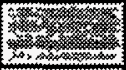 & 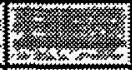 & $\mathrm{C}_{2}$ & 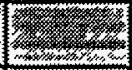 & 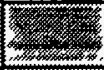 \\
\hline 365 & 5320 & N & 40.24 & For & $\mathrm{l}_{2}$ & 19.81 & 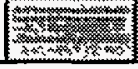 & 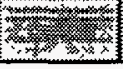 & (x) & $\mathrm{C}^{\mathrm{h}}$ & 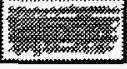 & 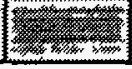 & 16 \\
\hline \multicolumn{14}{|c|}{ NORMALIZED, $\mathrm{g} / \mathbf{1}$} \\
\hline Days 7 & 14.0 & 0.19 & 1.14 & 0.17 & 0.26 & 0.13 & 0.22 & 0.31 & 0.38 & 0.37 & 0.25 & 0.30 & 0.30 \\
\hline 28 & 17.4 & 0.22 & 1.33 & 0.20 & 0.31 & 0.65 & 0.21 & 0.39 & 0.43 & 0.46 & 0.27 & 0.35 & 0.38 \\
\hline 56 & 21 & 0.25 & 1.26 & 0.23 & 0.33 & 0.64 & 0.20 & 0.45 & 0.45 & 0.56 & 0.30 & 0.40 & 0.43 \\
\hline 120 & 31.3 & 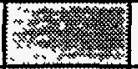 & 1.28 & 0.21 & 0.30 & 0.72 & 0.21 & 0.49 & 0.57 & 0.71 & 0.29 & 0.48 & 0.47 \\
\hline 180 & 36.8 & 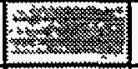 & 1.40 & 0.23 & 0.32 & 0.81 & $m_{x}$ & 0.50 & (3) & $\mathrm{l}_{\mathrm{H}}$ & 0.28 & (3) & 0.48 \\
\hline 270 & 44.1 & 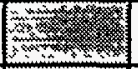 & 1.55 & mon & $x_{23}$ & 0.84 & 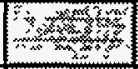 & $f_{1}$ & (3) & $\mathrm{I}_{\mathrm{S}}$ & (3) & I & trits \\
\hline 365 & 44.0 & 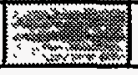 & 1.62 & (4) & I & 0.87 & \begin{tabular}{|c|} 
\\
\end{tabular} & $b_{2}$ & 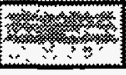 & $\mathrm{f}_{x, 2}$ & $x_{2 x}$ & $\rho_{1}$ & 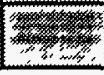 \\
\hline \multicolumn{14}{|c|}{ Leach Rate, $\mathrm{g} / \mathrm{m}^{2} / \mathrm{day}$} \\
\hline Days 3.5 & 1.00 & 0.01 & 0.08 & 0.01 & 0.02 & 0.01 & 0.02 & 0.02 & 0.03 & 0.03 & 0.02 & 0.02 & 0.02 \\
\hline 17.5 & 0.08 & 0.00 & 0.01 & 0.00 & 0.00 & 0.01 & 0.00 & 0.00 & 0.00 & 0.01 & 0.00 & 0.00 & 0.00 \\
\hline 42 & 0.06 & 0.00 & 0.00 & 0.00 & 0.00 & 0.00 & 0.00 & 0.00 & 0.00 & 0.01 & 0.00 & 0.00 & 0.00 \\
\hline 88 & 0.08 & 0.00 & 0.00 & 0.00 & 0.00 & 0.00 & 0.00 & 0.00 & 0.00 & 0.01 & 0.00 & $y=$ & 0.00 \\
\hline 150 & 0.05 & 0.00 & 0.00 & 0.00 & 0.00 & 0.00 & - & 0.00 & $\begin{array}{l}m-1 \\
x\end{array}$ & $\int$ & 0.00 & 4 & 0.00 \\
\hline 225 & 0.04 & 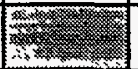 & 0.00 & - & y. & 0.00 & $\frac{1}{3 \times 2}$ & (x) & (3) & 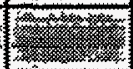 & ( & 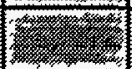 & 綝 \\
\hline 317.5 & -0.0005 & 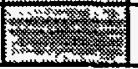 & 0.00 & mox & , & 0.00 & ( & $\beta_{\infty}$ & ( & $\mathrm{ln}_{\mathrm{s}}$ & 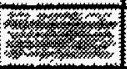 & $y_{3}$ & rest \\
\hline
\end{tabular}




\begin{tabular}{|c|c|c|c|c|c|c|c|c|c|c|c|c|c|}
\hline & & & & & & & & QG- & & & & & \\
\hline & SRL-EA & $7 \mathrm{~A}$ & $7 \mathrm{~B}$ & $7 \mathrm{C}$ & $8 \mathrm{~A}$ & $8 \mathrm{C}$ & $12 \mathrm{C}$ & $13 \mathrm{~A}$ & 13B & $13 \mathrm{C}$ & $14 \mathrm{~A}$ & $14 \mathrm{~B}$ & $15 \mathrm{~A}$ \\
\hline \multicolumn{14}{|c|}{ SILICON, PPM } \\
\hline Days 7 & 910 & 21.0 & 21.7 & 22.7 & 21.4 & 20.4 & 22.8 & 28.2 & 37.1 & 41.4 & 20.3 & 35.2 & 34.8 \\
\hline 28 & 983 & 23.7 & 21.8 & 25.1 & 22.7 & 22.5 & 22.87 & 30.1 & 38.61 & 44.14 & 19.7 & 37.70 & 38.1 \\
\hline 56 & 1150 & 23.51 & 21.6 & 25.31 & 25.55 & 22.4 & 21.20 & 30.6 & 37.86 & 46.28 & 19.5 & 38.75 & 37.7 \\
\hline 120 & 1450 & 20.48 & 22.6 & 22.16 & 22.47 & 25.9 & 19.97 & 38.28 & 40.82 & 47.89 & 24.55 & 39.89 & 48.47 \\
\hline 180 & 1640 & 25.33 & 26.0 & 26.86 & 27.48 & 29.2 & 10 & 34.52 & .2 & 3.3 & 20.21 & $1=0$ & 43.92 \\
\hline 270 & 1810 & 1 & 34.3 & 2 & 2.2 & 33.2 & & 2 & & 2 & & 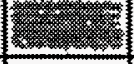 & 2 \\
\hline 365 & 1920 & $3+2$ & 33.53 & 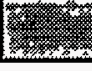 & 23 & 32.58 & 2 & $2 x$ & & 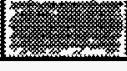 & 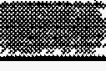 & 18 & 3.8 \\
\hline \multicolumn{14}{|c|}{ NORMALIZED, $\mathrm{g} / \mathrm{l}$} \\
\hline Days 7 & 4.19 & 0.10 & 0.10 & 0.11 & 0.12 & 0.10 & 0.11 & 0.12 & 0.18 & 0.18 & 0.09 & 0.18 & 0.15 \\
\hline 28 & 4.54 & 0.11 & 0.10 & 0.12 & 0.12 & 0.1 & 0.11 & 0.13 & 0.18 & 0.19 & 0.09 & 0.19 & 0.16 \\
\hline 56 & 5.32 & 0.11 & 0.10 & 0.12 & 0.14 & 0.11 & 0.11 & 0.13 & 0.18 & 0.20 & 0.09 & 0.20 & 0.16 \\
\hline 120 & 6.68 & 0.10 & 0.11 & 0.11 & 0.12 & 0.13 & 0.10 & 0.16 & 0.19 & 0.21 & 0.11 & 0.20 & 0.21 \\
\hline 180 & 7.58 & 0.12 & 0.12 & 0.13 & 0.15 & 0.15 & 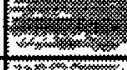 & 0.15 & 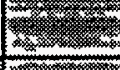 & 4 & 0.09 & -2 & 0.19 \\
\hline 270 & 8.38 & 3 & 0.16 & 2 & & 0.17 & 1328 & 4 & 1 & $(203$ & & 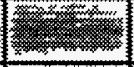 & 3 \\
\hline 365 & 8.89 & 4, & 0.16 & 20 & 23 & 0.17 & 2 & 2.2 & $y_{-2}$ & $y=2$ & 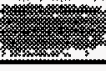 & $12=2$ & 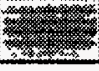 \\
\hline \multicolumn{14}{|c|}{ Leach Rate, $g / \mathrm{m}^{2} /$ day } \\
\hline Days 3.5 & 0.30 & 0.01 & 0.01 & 0.01 & 0.01 & 0.01 & 0.01 & 0.01 & 0.01 & 0.01 & 0.01 & 0.01 & 0.01 \\
\hline 17.5 & 0.01 & 0.00 & 0.00 & 0.00 & 0.00 & 0.00 & 0.00 & 0.00 & 0.00 & 0.00 & 0.00 & 0.00 & 0.00 \\
\hline 42 & 0.013 & 0.00 & 0.00 & 0.00 & 0.00 & 0.00 & 0.00 & 0.00 & 0.00 & 0.00 & 0.00 & 0.00 & 0.00 \\
\hline 88 & 0.01 & 0.00 & 0.00 & 0.00 & 0.00 & 0.00 & 0.00 & 0.00 & 0.00 & 0.00 & 0.00 & 0.00 & 0.00 \\
\hline 150 & 0.01 & 0.00 & 0.00 & 0.00 & 0.00 & 0.00 & 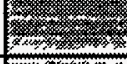 & 0.00 & t. & 4 & 0.00 & $1=2$ & 0.00 \\
\hline 225 & 0.004 & 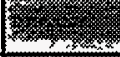 & 0.00 & & & 0.00 & 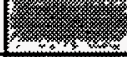 & 2 & 12.2 & 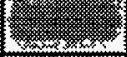 & 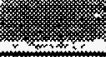 & $=-1$ & 2. \\
\hline 317.5 & 0.003 & $x$ & 0.00 & 3 & 3 & 0.00 & 283 & 2 & 10 & 4 & 2 & $1=$ & 3 \\
\hline
\end{tabular}




\begin{tabular}{|c|c|c|c|c|c|c|c|c|c|c|c|c|c|}
\hline & \multirow[b]{2}{*}{ SRL-EA } & \multicolumn{12}{|c|}{ IDG- } \\
\hline & & $7 \mathrm{~A}$ & $7 \mathrm{~B}$ & $7 \mathrm{C}$ & $8 \mathrm{~A}$ & $8 \mathrm{C}$ & $12 \mathrm{C}$ & $13 \mathrm{~A}$ & $13 \mathrm{~B}$ & $13 \mathrm{C}$ & $14 \mathrm{~A}$ & $14 \mathrm{~B}$ & $15 \mathrm{~A}$ \\
\hline \multicolumn{14}{|c|}{ BORON, PPM } \\
\hline Days 7 & 642 & 0.24 & 21.2 & 0 & 0.01 & 3.06 & 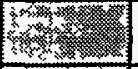 & 0.67 & s. & (m) & 0.69 & 0.14 & 0.39 \\
\hline 28 & 775 & 0.54 & 20.6 & 0.34 & 0 & 2.84 & (x) & 0.30 & $\begin{array}{l}y_{x} \\
y_{2}\end{array}$ & (y, & 0.75 & 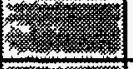 & 1.05 \\
\hline 56 & 974 & 0.70 & 20.1 & 0.73 & 0.33 & 2.78 & (2) & 0.19 & (2) & $y_{0}$ & 0.84 & & 0.21 \\
\hline 120 & 1490 & 0.41 & 20.0 & 0.07 & 0.43 & 2.98 & r & 0.11 & s. & 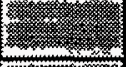 & 0.53 & - & 0.25 \\
\hline 180 & 1870 & 0.32 & 22.1 & 0.23 & 0.24 & 3.45 & (x) & 0.14 & 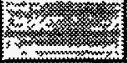 & 12 & 0.66 & . & 0.15 \\
\hline 270 & 2120 & 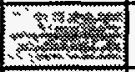 & 23.7 & 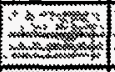 & mox & 3.97 & 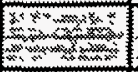 & 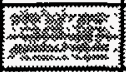 & ext & $y_{2}$ & (3) & 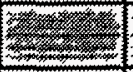 & 3 \\
\hline 365 & 2270 & send & 20.74 & m & $x_{2}$ & 2.91 & mine & (3) & क & m & क्रा & metrty & $x_{2}$ \\
\hline \multicolumn{14}{|c|}{ NORMALIZED, g/ } \\
\hline Days 7 & 19.0 & 0.53 & 44.60 & 0.00 & 0.02 & 7.05 & - & 1.14 & 20. & 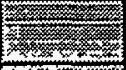 & 1.80 & 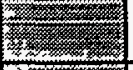 & 1.80 \\
\hline 28 & 22.9 & 1.21 & 43.40 & 0.92 & 0.00 & 6.55 & (2) & 0.50 & 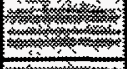 & $\frac{1}{4}$ & 1.96 & 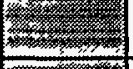 & 4.81 \\
\hline 56 & 28.8 & 1.56 & 42.20 & 1.95 & 1.15 & 6.40 & $(x)$ & 0.32 & (2) & 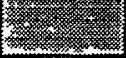 & 2.19 & 32 & 0.98 \\
\hline 120 & 44.1 & 0.91 & 41.90 & 0.18 & 1.48 & 6.87 & 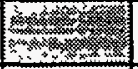 & 0.19 & 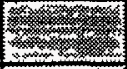 & $y_{2}$ & 1.37 & 1 & 1.13 \\
\hline 180 & 55.4 & 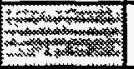 & 46.60 & 0.61 & 0.82 & 7.94 & 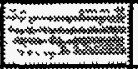 & 0.24 & Ex & 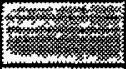 & 1.71 & m. & 0.67 \\
\hline 270 & 62.6 & itis & 49.70 & 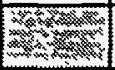 & prom & 9.15 & 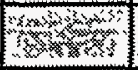 & 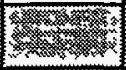 & ( & (y) & $y_{2}$ & (Y) & (2) \\
\hline 365 & 67.2 & 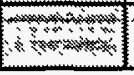 & ons & a & 서요 & 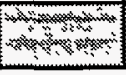 & 1 & or & $\mathrm{C}^{2}$ & sis & $\mathrm{b}_{3}$ & 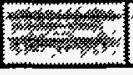 & 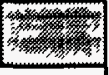 \\
\hline \multicolumn{14}{|c|}{ Leach Rate, $\mathrm{g} / \mathrm{m}^{2} / \mathrm{d}$} \\
\hline Days 3.5 & 1.36 & 0.04 & 3.19 & 0.00 & 0.00 & 0.50 & (n) & 0.08 & 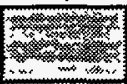 & 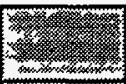 & 0.13 & (n) & 0.13 \\
\hline 17.5 & 0.09 & 0.02 & -0.03 & 0.02 & 0.00 & -0.01 & 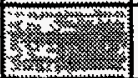 & -0.02 & (2) & $\sqrt{6-25}$ & 0.00 & my & 0.07 \\
\hline 42 & 0.11 & 0.01 & -0.02 & 0.02 & 0.03 & 0.00 & 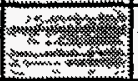 & 0.00 & 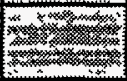 & 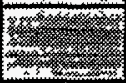 & 0.00 & 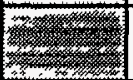 & -0.07 \\
\hline 88 & 0.12 & -0.02 & 0.00 & -0.04 & 0.01 & 0.00 & 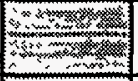 & 0.00 & 2 & $x^{2}$ & -0.01 & 2 & 0.00 \\
\hline 150 & 0.10 & 0.00 & 0.04 & 0.01 & -0.02 & 0.01 & and & 0.00 & 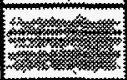 & $\sqrt{2}$ & 0.01 & 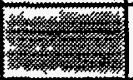 & -0.01 \\
\hline 225 & 0.04 & 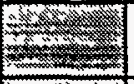 & 0.02 & +2 & 10 & 0.01 & 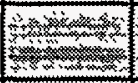 & $f_{x}$ & 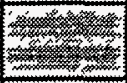 & 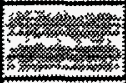 & (x) & $y_{y}$ & 3 \\
\hline 317.5 & 0.02 & (3) & -0.03 & ( & (3) & -0.01 & (3) & $l_{2}$ & 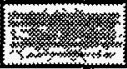 & (3) & 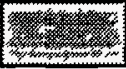 & $y_{1}$ & (6) \\
\hline
\end{tabular}


Table 3.10a

Results of TCLP Leaching Tests on Untreated Idaho Wastes (ppm)

\begin{tabular}{|l|c|c|c|c|c|c|c||}
\hline WASTE & $\mathbf{B a}$ & $\mathbf{C d}$ & $\mathbf{C r}$ & $\mathrm{Hg}$ & $\mathrm{Ni}$ & $\mathbf{P b}$ & $\mathbf{U}$ \\
\hline Eutectic Salt & 0.7 & 0.04 & 1.3 & 1 & 7 & 11 & 2100 \\
\hline Fly Ash & 0.01 & 73 & 0.05 & 0.1 & 6 & 11 & 1.1 \\
\hline Regulatory Limit & 100 & 0.066 & 5.2 & 0.2 & 5 & 0.37 & 1.78 \\
\hline
\end{tabular}

Table 3.10

Summary of TCLP Test Results on Idaho Glasses (ppm)

\begin{tabular}{||l|r|r|r|r|r|r|r||}
\hline GLASS NAME & \multicolumn{1}{c|}{$\mathrm{Ba}$} & $\mathrm{Cd}$ & $\mathrm{Cr}$ & \multicolumn{1}{l|}{$\mathrm{Hg}$} & \multicolumn{1}{c|}{$\mathrm{Ni}$} & \multicolumn{1}{c|}{$\mathrm{Pb}$} & \multicolumn{1}{c|}{$\mathrm{U}$} \\
\hline IDG7A & 0.570 & 0.003 & 0.010 & $* *$ & 0.019 & 0.011 & 0.400 \\
\hline IDG7B & 0.589 & 0.003 & 0.010 & $* *$ & 0.010 & 0.010 & 0.452 \\
\hline IDG7C & 0.718 & 0.000 & 0.000 & 0.032 & 0.015 & 0.048 & 0.506 \\
\hline IDG8A & 0.655 & 0.000 & 0.134 & 0.024 & 0.042 & 0.159 & 1.879 \\
\hline IDG8B & 0.487 & 0.003 & 0.011 & $* *$ & 0.010 & 0.011 & 0.798 \\
\hline IDG8C & 0.483 & 0.003 & 0.010 & $* *$ & 0.010 & 0.007 & 0.612 \\
\hline IDG12C & 0.598 & 0.004 & 0.003 & 0.010 & 0.047 & 9.484 & 0.664 \\
\hline IDG13A & 0.632 & 0.004 & 0.004 & 0.071 & 0.004 & 0.171 & 0.244 \\
\hline IDG13B & 0.524 & 0.004 & 0.043 & 0.010 & 0.017 & 0.924 & 1.117 \\
\hline IDG13C & 0.460 & 0.004 & 0.003 & 0.010 & 0.003 & 0.685 & 0.707 \\
\hline IDG14A & 0.627 & 0.004 & 0.006 & 0.025 & 0.013 & 0.158 & 0.474 \\
\hline IDG14B & 0.677 & 0.005 & 0.012 & 0.102 & 0.056 & 2.060 & 2.339 \\
\hline IDG15A & 0.507 & 0.004 & 0.006 & 0.015 & 0.026 & 0.526 & 0.341 \\
\hline IDG15B & 0.650 & 0.010 & 0.030 & 0.080 & 0.080 & 3.190 & 1.440 \\
\hline IDG15C & 0.670 & 0.000 & 0.030 & 0.060 & 0.360 & 5.940 & 0.930 \\
\hline Regulatory Limit & 100 & 0.066 & 5.2 & 0.20 & 5 & 0.37 & 1.78 \\
\hline
\end{tabular}

** Not Analyzed. 
Table 3.11

List of Idaho Glasses with Complete Viscosity, Conductivity, TCLP, and PCT Data (i.e., Glasses in the Core Data Set of $\mathbf{4 0}$ Glasses) and Representative Data for those Properties.

\begin{tabular}{|c|c|c|c|c|c|}
\hline \multirow{2}{*}{$\begin{array}{l}\text { Glass } \\
\text { Number }\end{array}$} & \multirow{2}{*}{$\begin{array}{l}\text { Glass } \\
\text { Name }\end{array}$} & \multirow{2}{*}{$\begin{array}{l}\text { Viscosity } \\
@ 1350^{\circ} \mathrm{C}\end{array}$} & \multirow{2}{*}{$\begin{array}{l}\text { Conductivity } \\
\text { @ } 1350^{\circ} \mathrm{C}\end{array}$} & TCLP & PCT-7 day \\
\hline & & & & $\mathrm{U}(\mathrm{ppb})$ & $\mathrm{Na}(\mathrm{ppm})$ \\
\hline 1 & IDG7A & 36 & 0.088 & 400 & 5 \\
\hline 2 & IDG7B & 31 & 0.129 & 452 & 28 \\
\hline 3 & IDG7C & 15 & 0.102 & 506 & 5 \\
\hline 4 & IDG8A & 7 & 0.146 & 1879 & 5 \\
\hline 5 & IDG8C & 13 & 0.189 & 612 & 3 \\
\hline 6 & IDG12C & 15 & 0.121 & 664 & 5 \\
\hline 7 & IDG13A & 107 & 0.166 & 244 & 15 \\
\hline 8 & IDG13B & 34 & 0.225 & 1117 & 20 \\
\hline 9 & IDG13C & 66 & 0.227 & 707 & 20 \\
\hline 10 & IDG14A & 427 & $>0.3$ & 474 & 11 \\
\hline 11 & IDG14B & 18 & 0.308 & 2339 & 16 \\
\hline 12 & IDG15A & 65 & 0.191 & 341 & 16 \\
\hline
\end{tabular}




\section{Figure 3.1.}

Type I fiber in Idaho ACM.

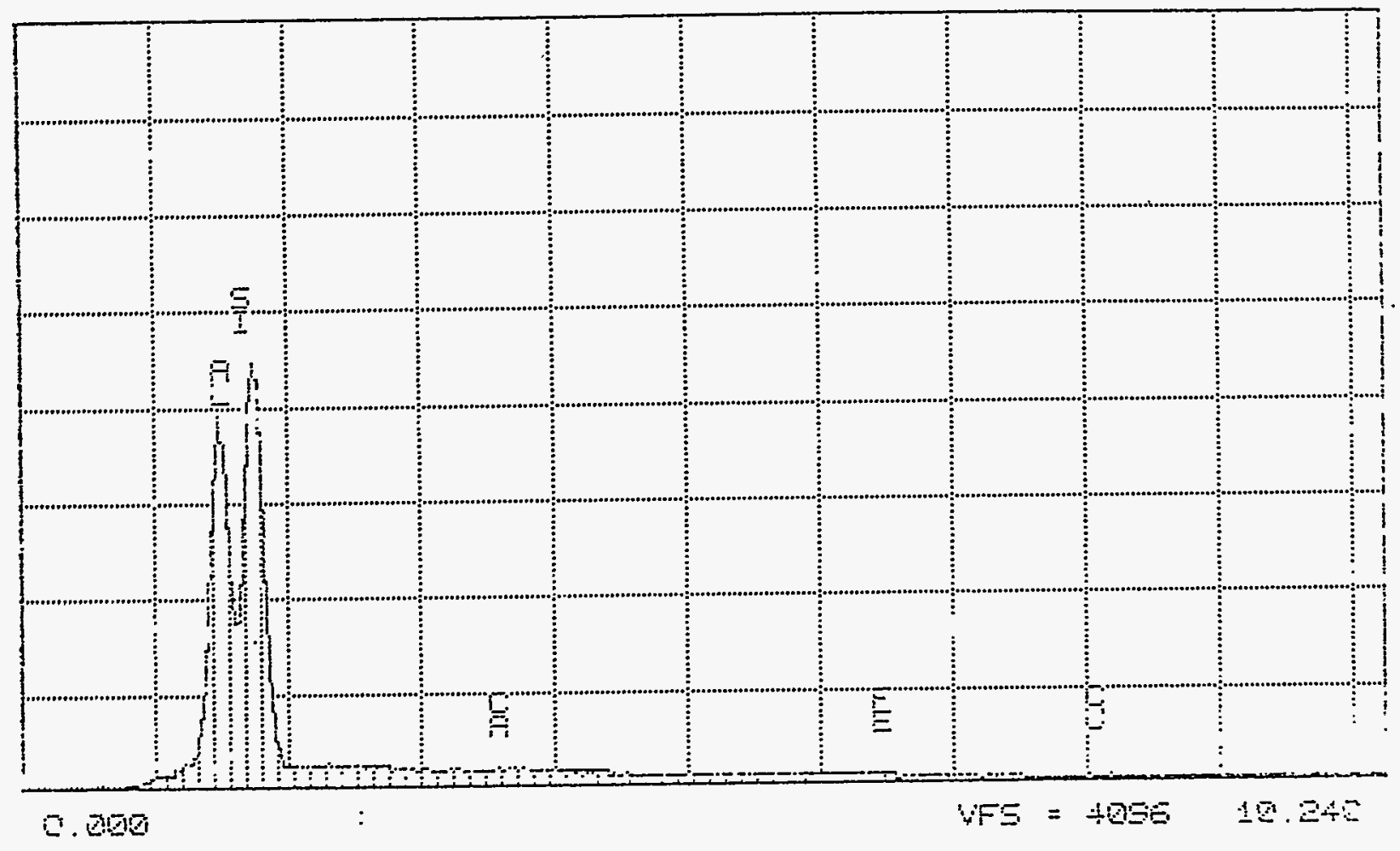

Figure 3.2.

Type II fiber in Idaho ACM.

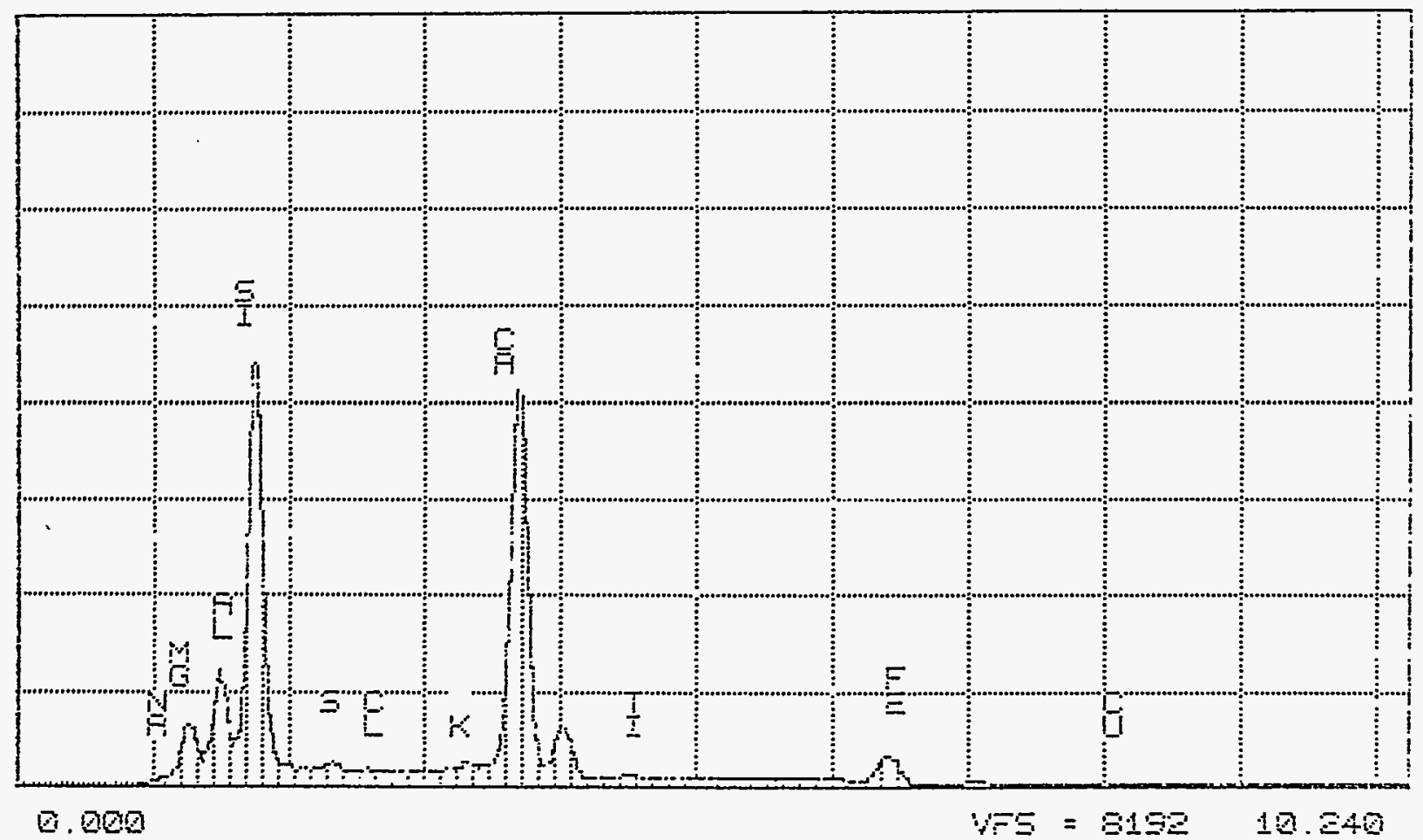


Figure 3.3a.

Results of gamma spectroscopy on ID1 (Asbestos).

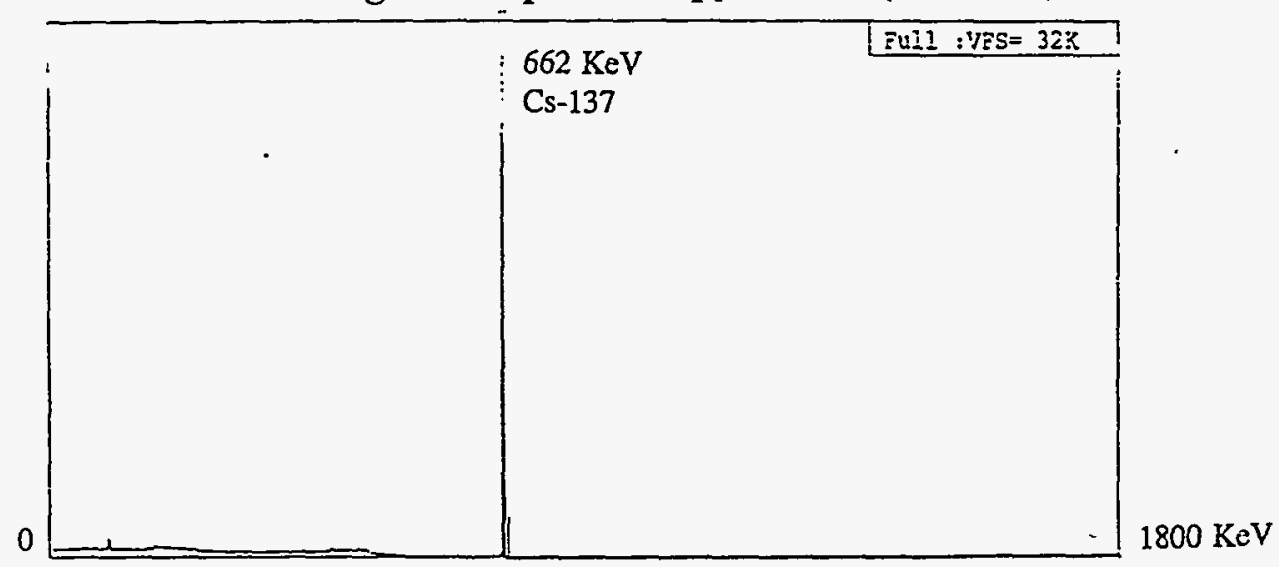

Figure 3.3b.

Results of gamma spectroscopy on ID3 (Fly ash).

(The qualitative spectrum of the raw Fly ash shows a higher level of ${ }^{137} \mathrm{Cs}$ than in the quantitative measurements made on the borax fusion made from that material for the analysis. The much lower level of ${ }^{137} \mathrm{Cs}$ compared to the other isotopes is probably due to evaporation during the fusion preparation)

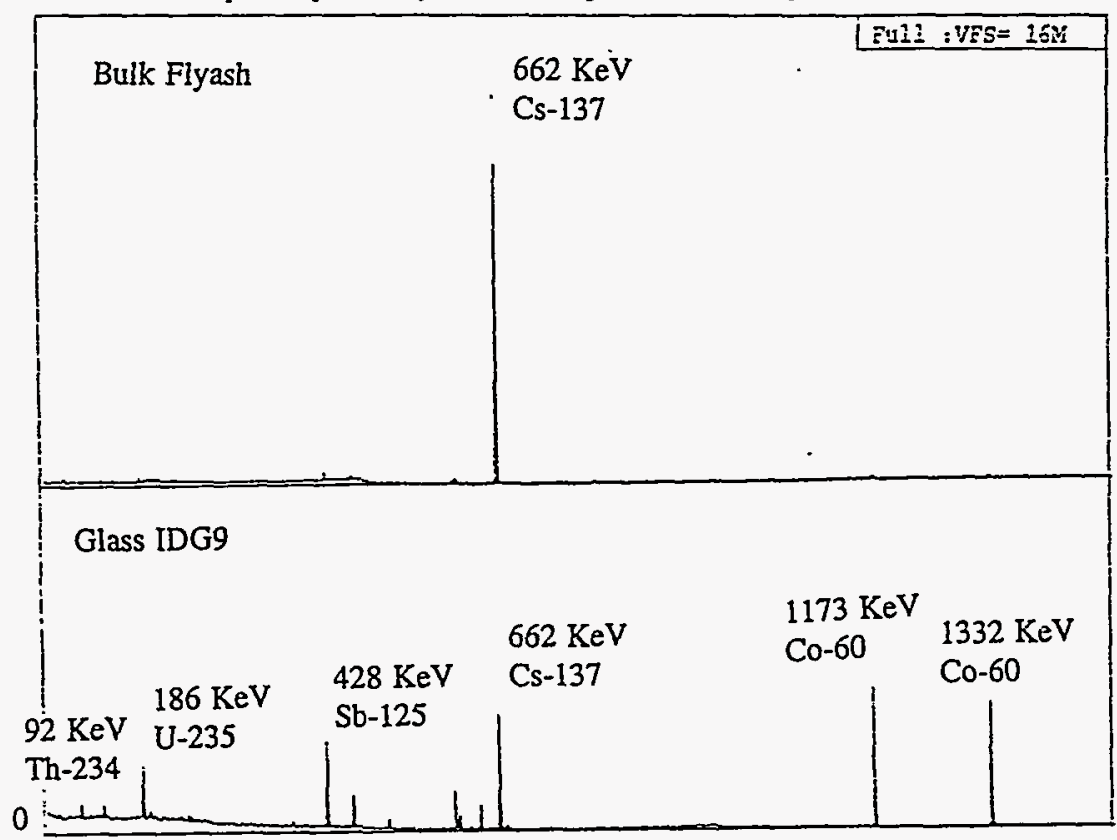

$1800 \mathrm{KeV}$

Figure 3.3c.

Results of gamma spectroscopy of ID2 (Eutectic Salt).

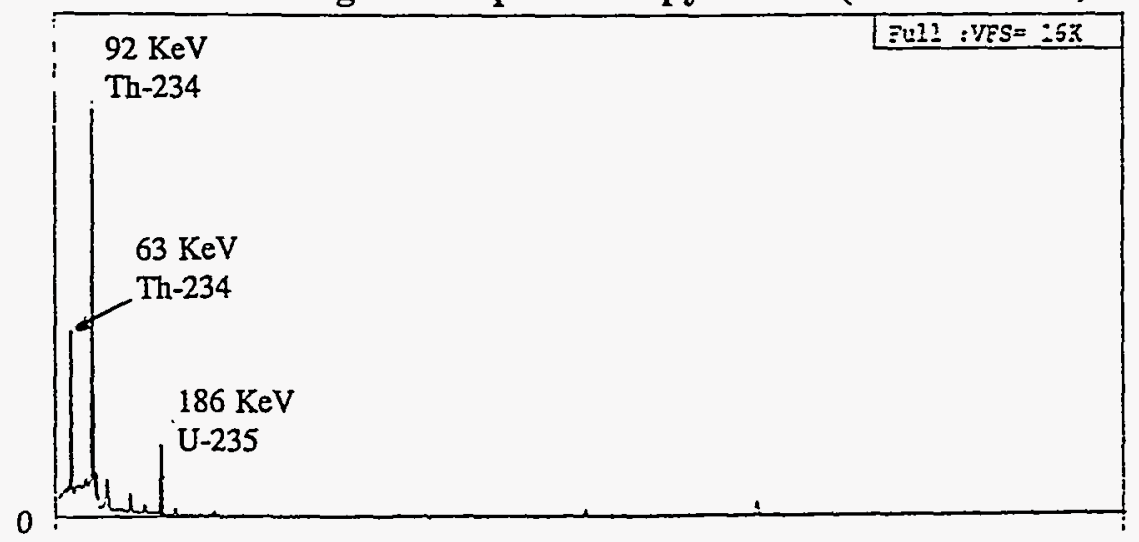


Figure 3.4.

Results of particle size analysis of Idaho wastes.

(a) Idaho Salt

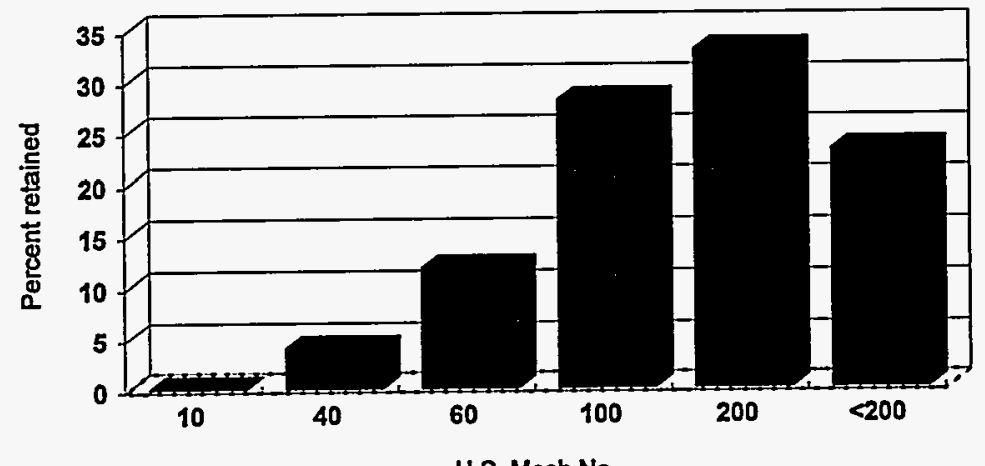

(b) Idaho Fly Ash

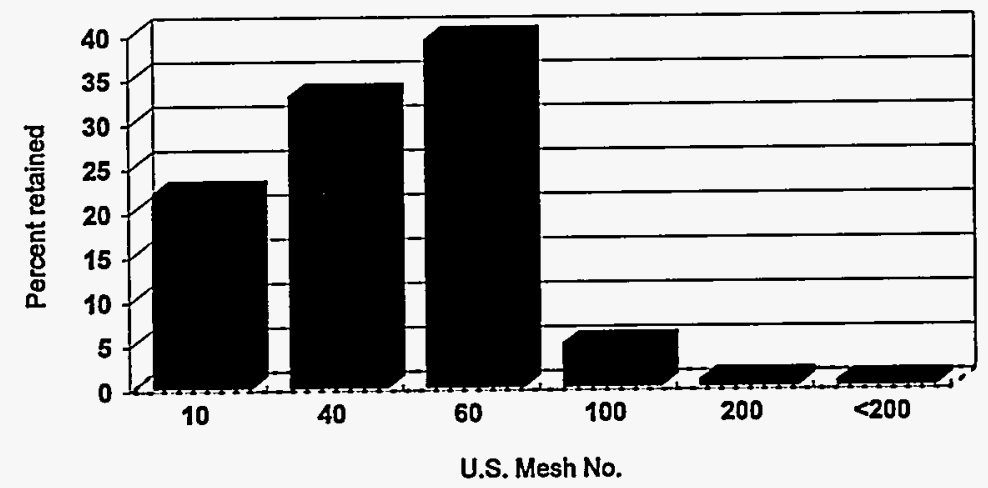

(c) Idaho Soil

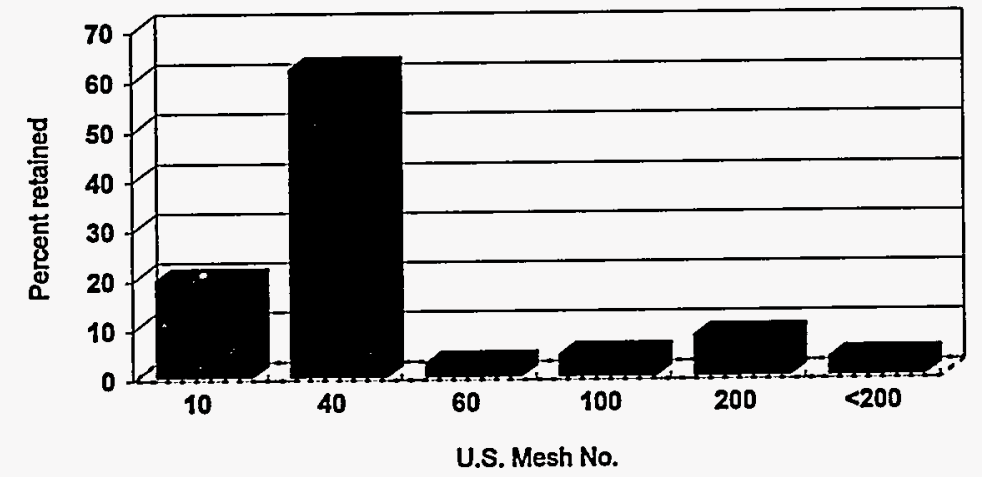


Figure 3.5.

EDS spectrum of Idaho flyash (ID3), average

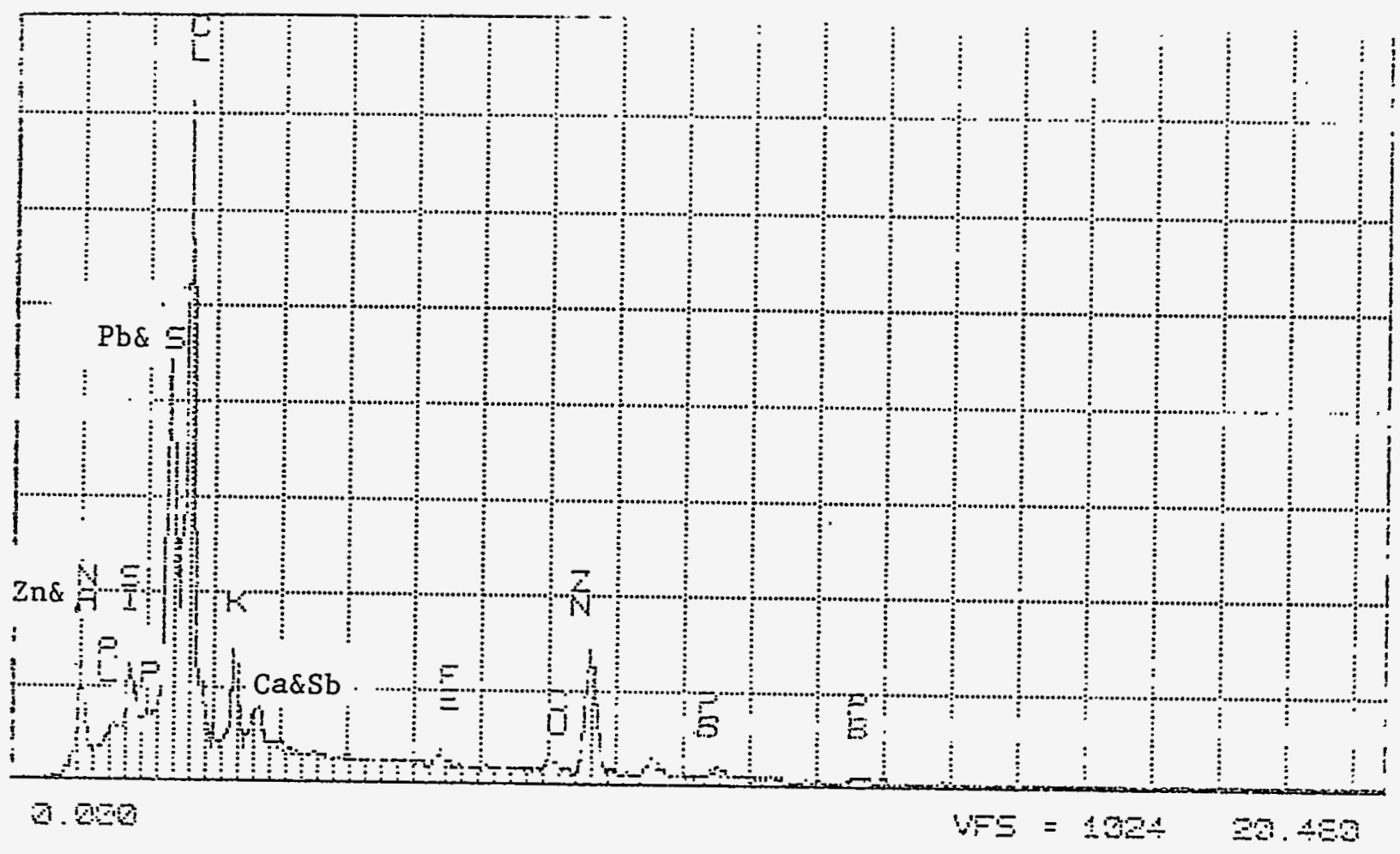

Figure 3.6.

EDS spectrum of Idaho flyash (ID3), particle \#1.

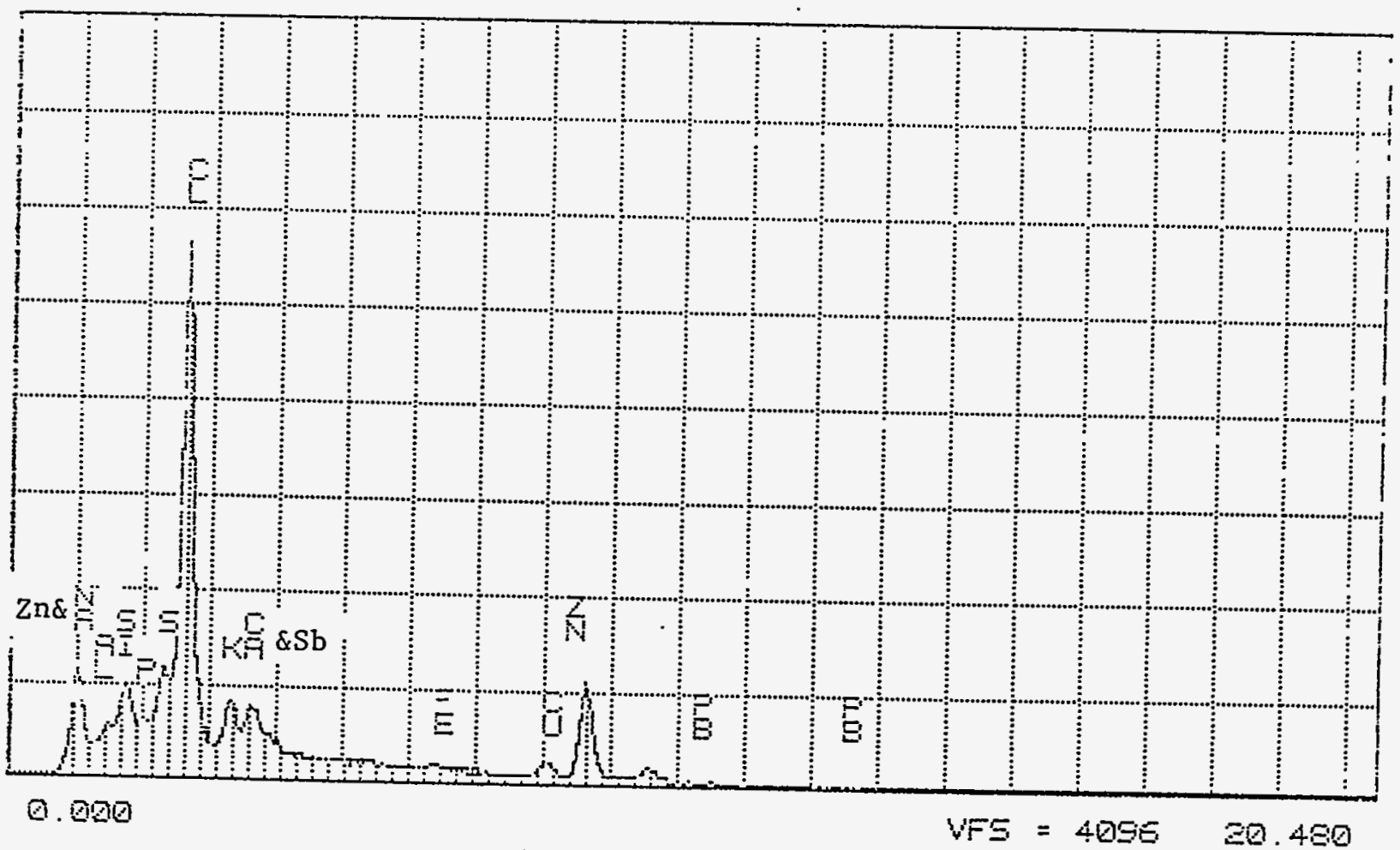


Figure 3.7.

EDS spectrum of Idaho flyash (ID3), particle \#2.

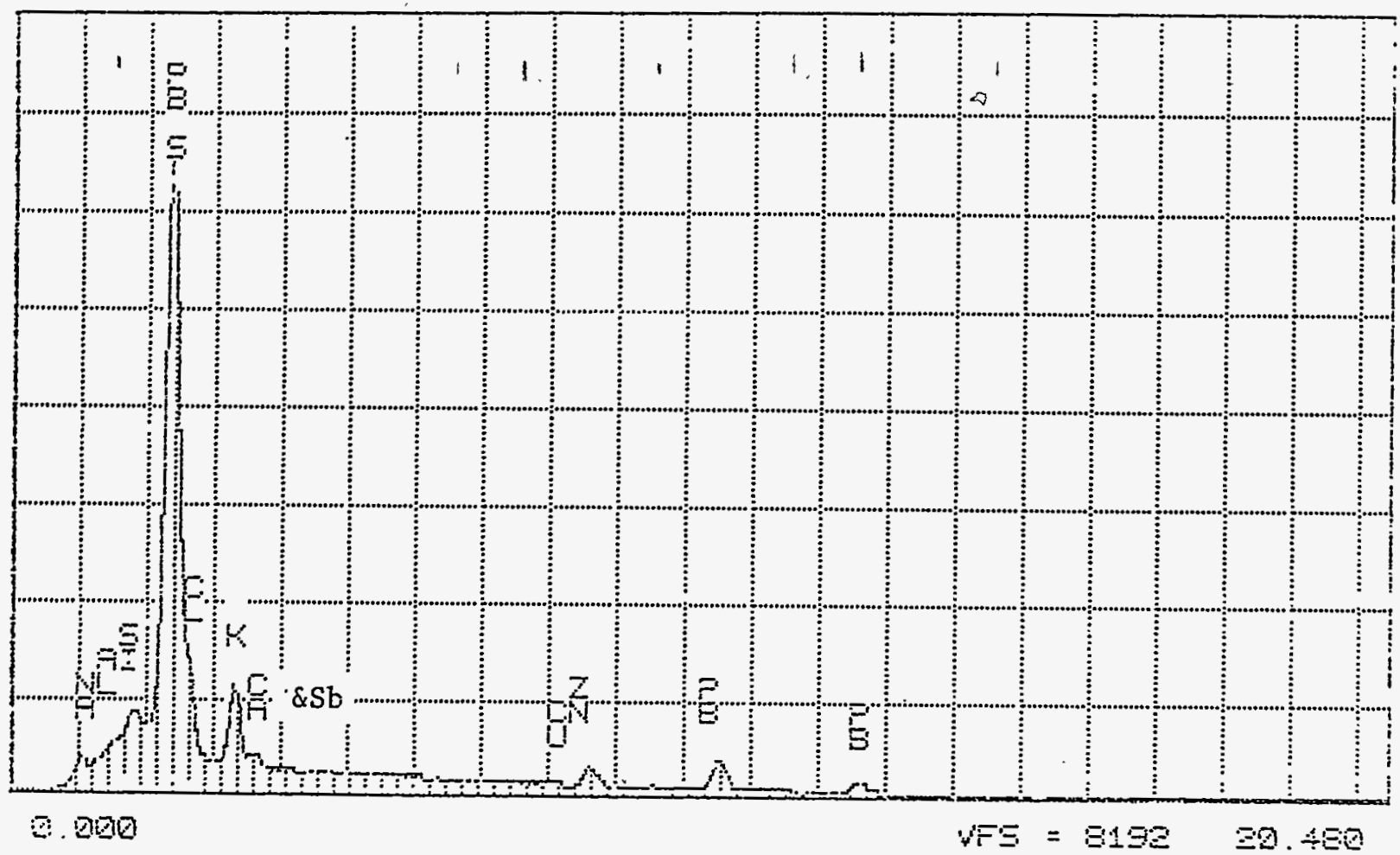

Figure 3.8.

EDS spectrum of Idaho flyash (ID3), particle \#3.

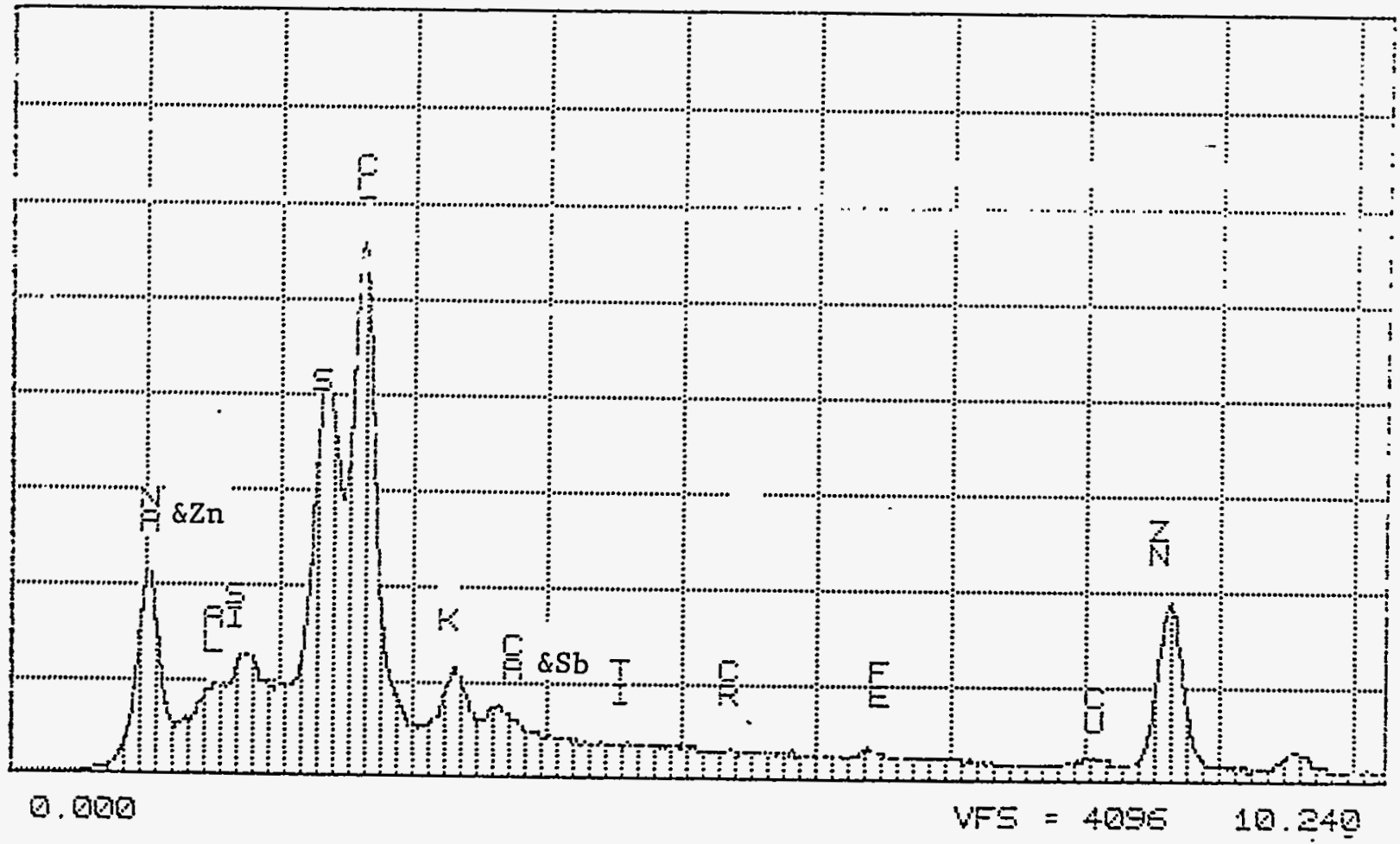


Figure 3.9.

EDS spectrum of Idaho flyash (ID3), particle \#4.

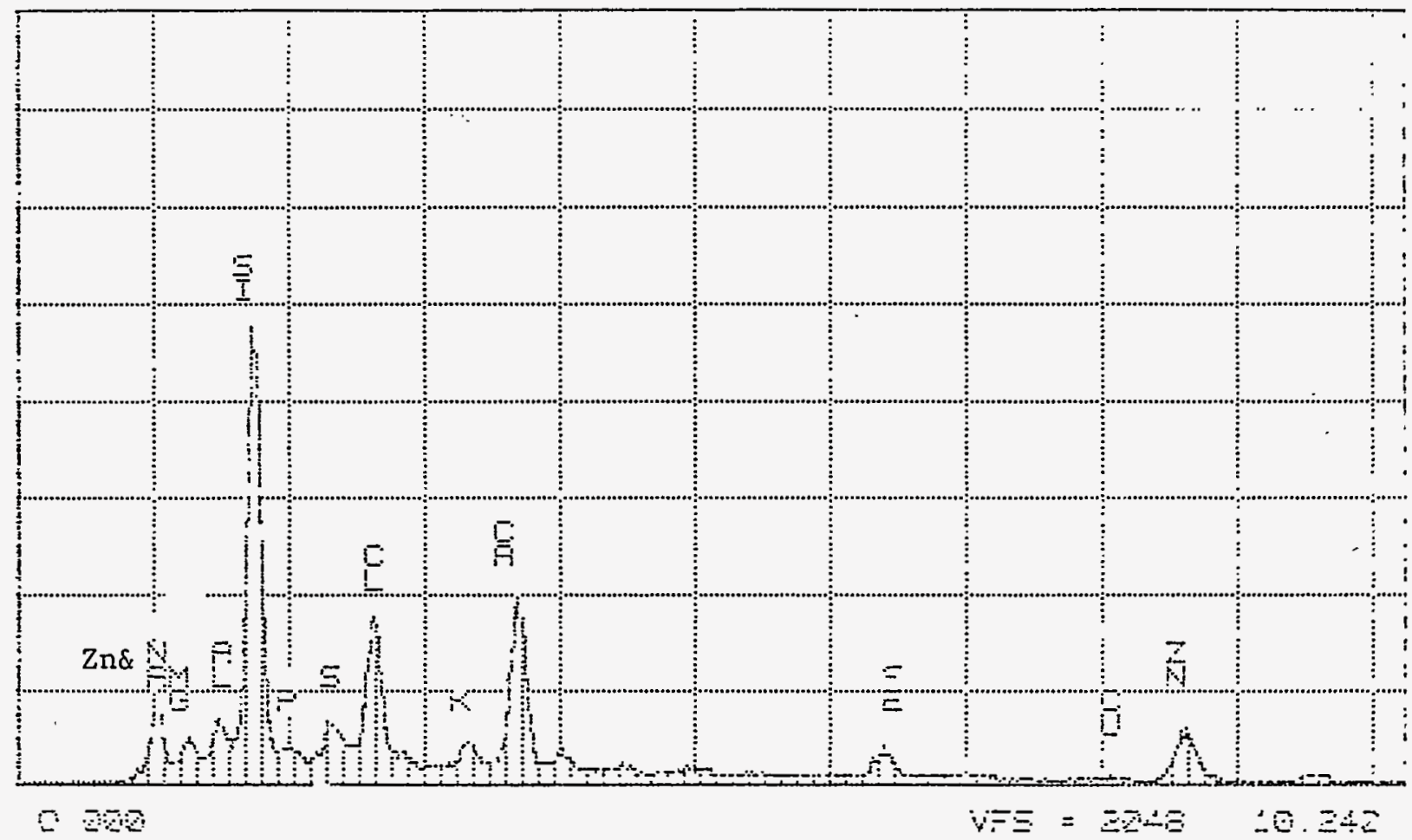

Page 3-48 
DTE OT $\quad$ ETS $=5 \pm A$

$D O B \cdot \mathrm{B}$

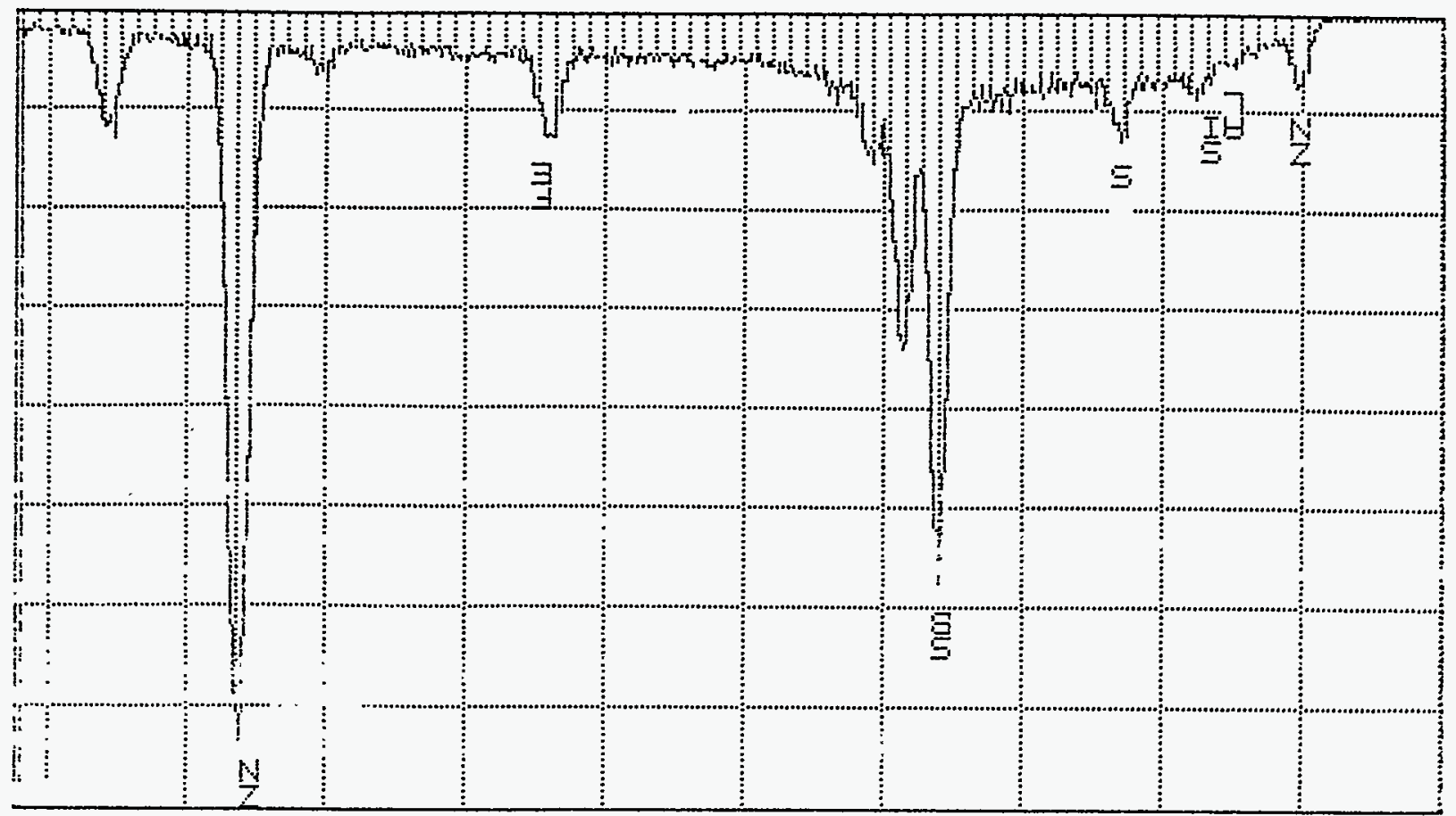

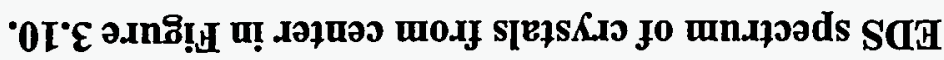
'II'E ว.Inoิ!!

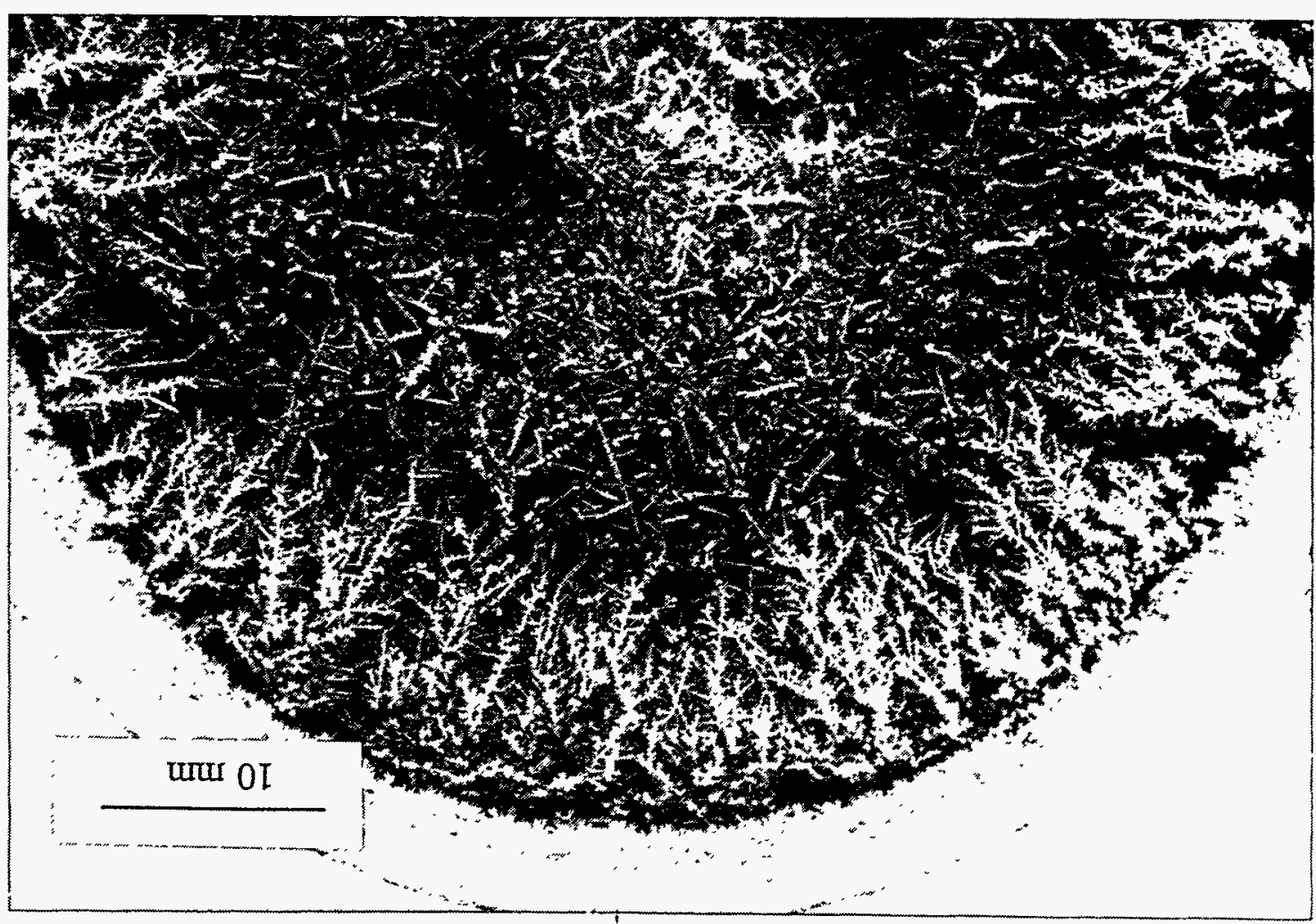

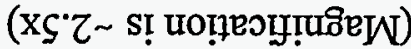

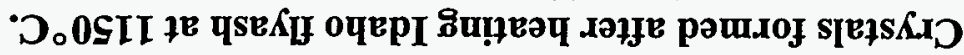

० $0{ }^{\circ} \varepsilon$ ว.mô! 
Figure 3.12.

EDS spectrum of long blade-shaped crystals from Figure 3.10.

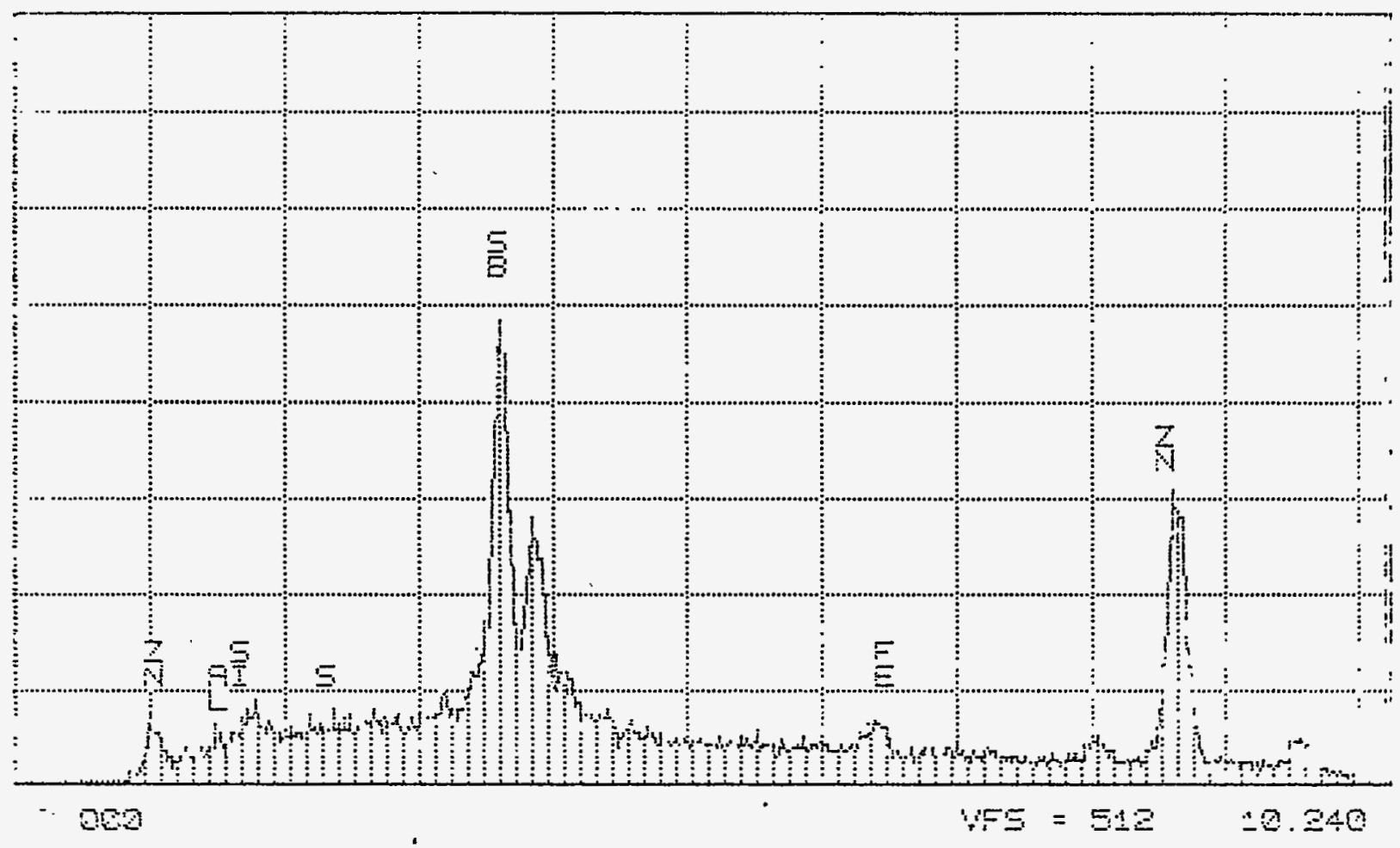

Figure 3.13.

EDS spectrum of small polyhedral crystals from Figure 3.10.

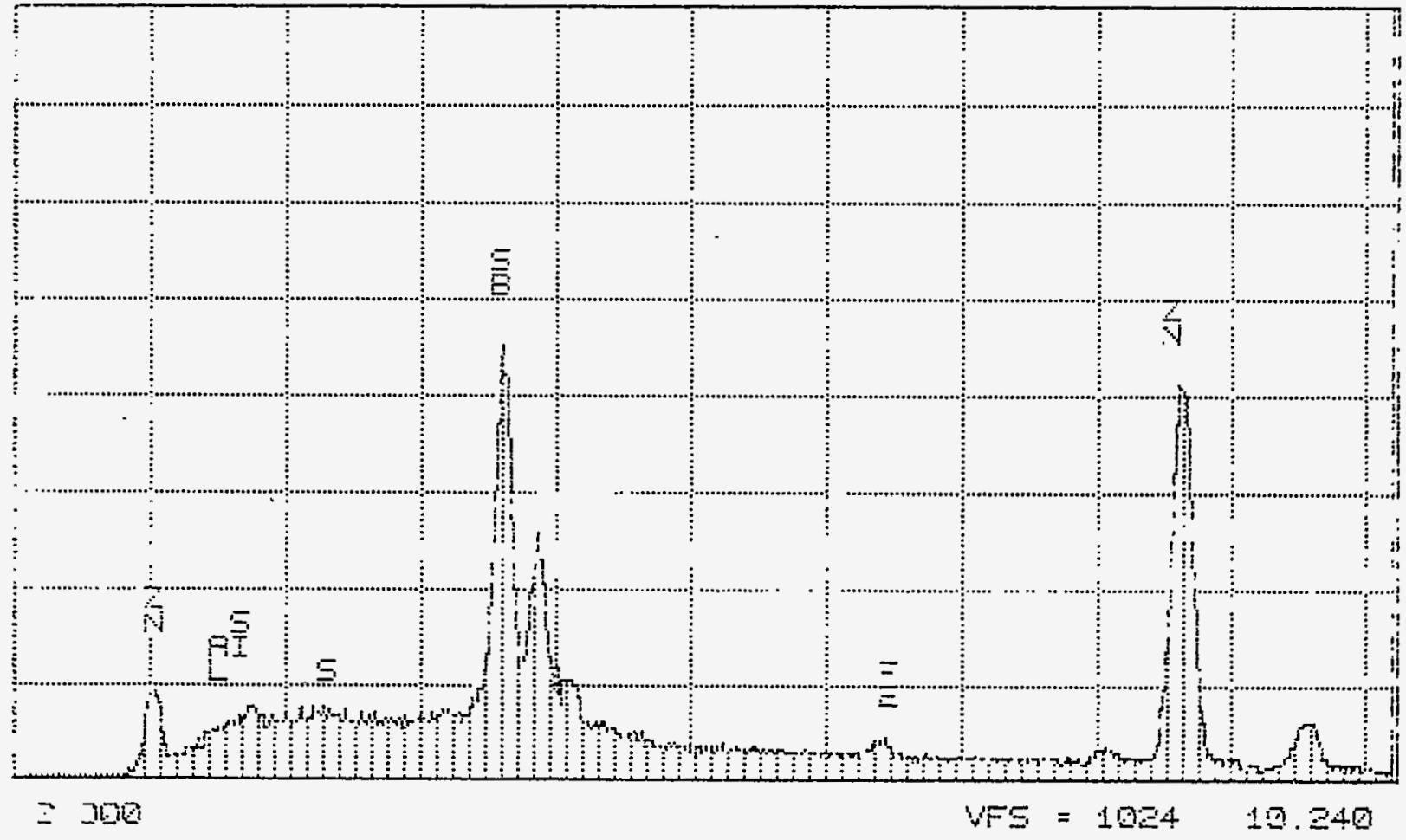


Figure 3.14.

EDS spectrum of small acicular crystals from Figure 3.10.

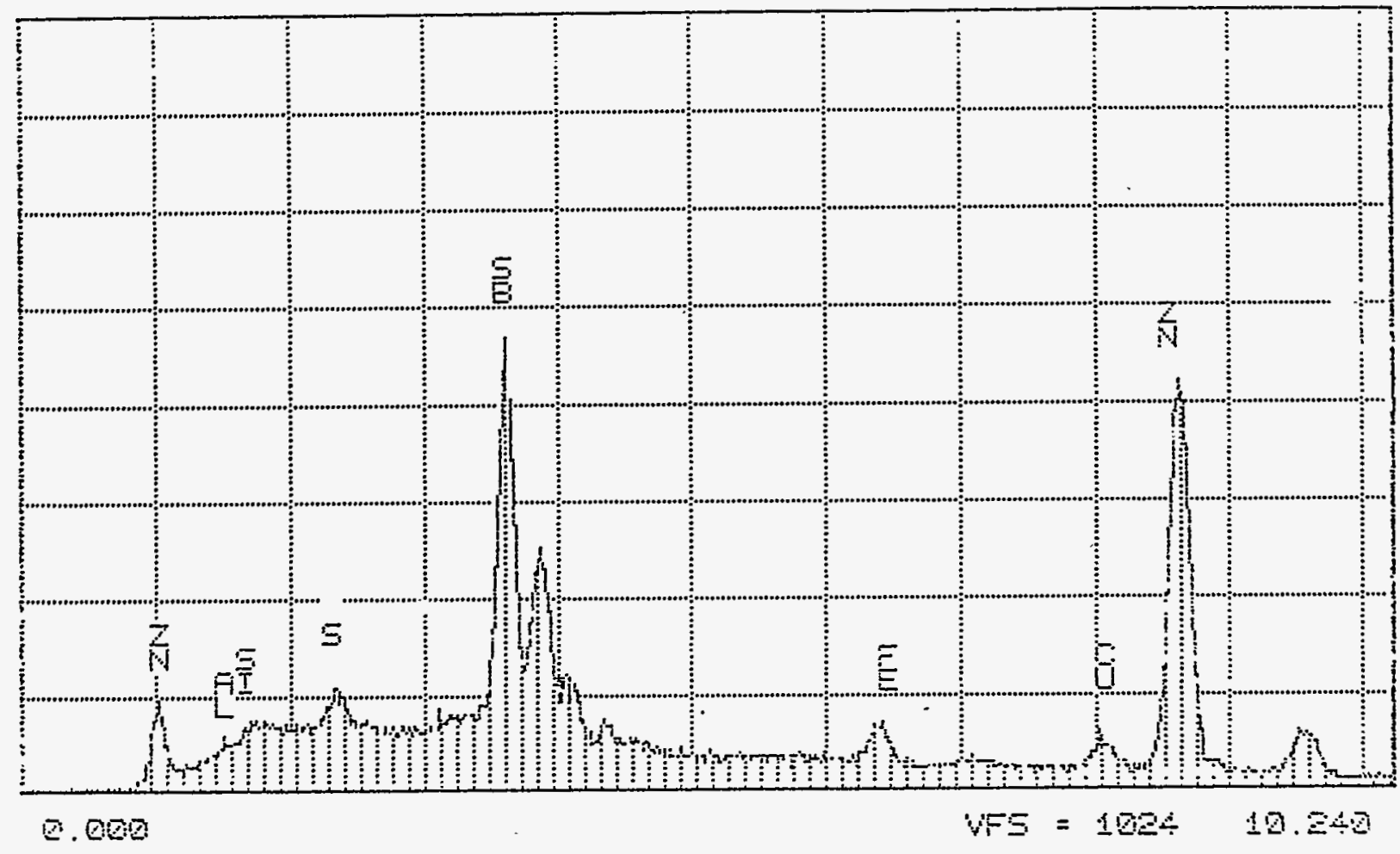

Figure 3.15.

EDS spectrum of crystals at edge of crucible in Figure 3.10.

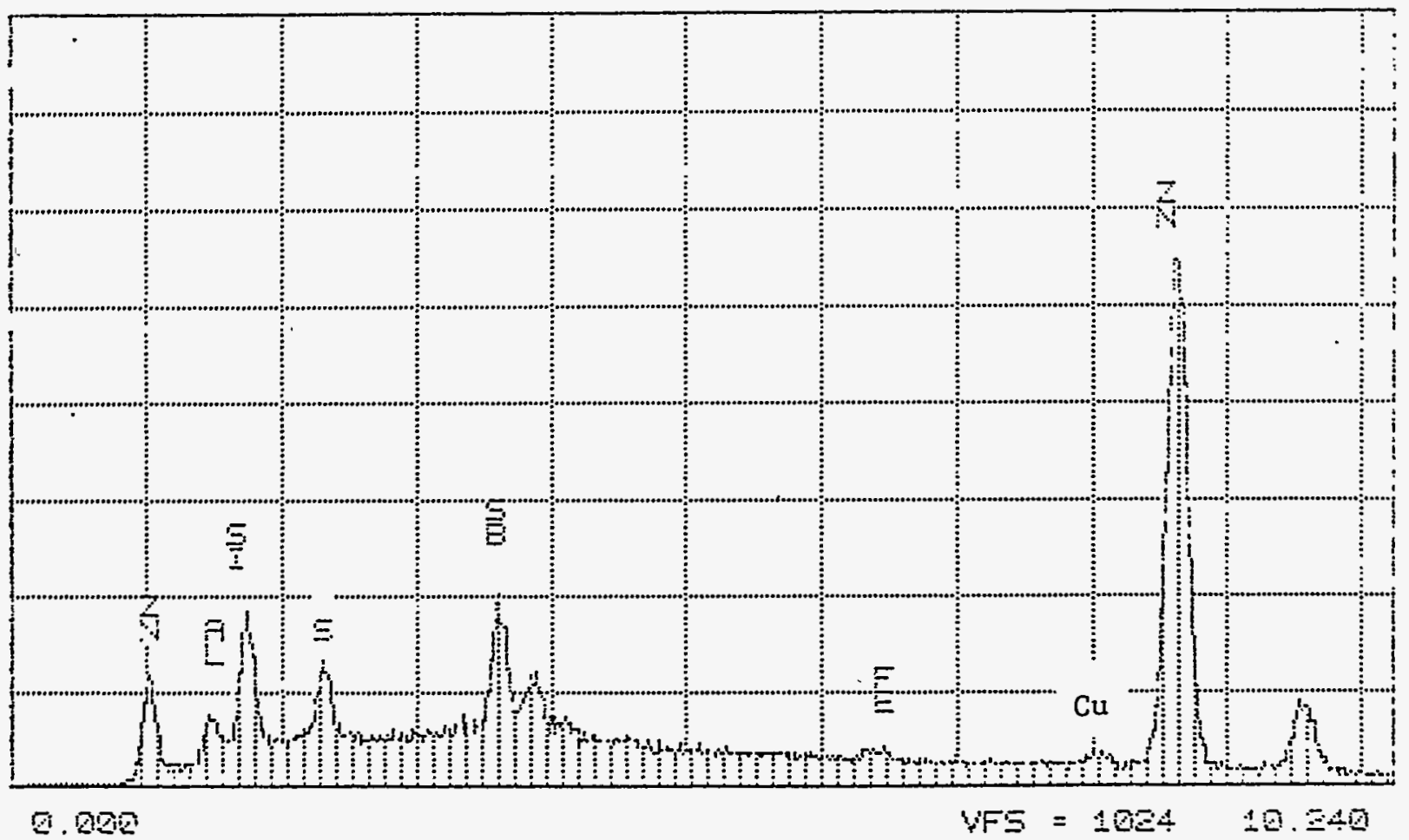


Figure 3.16.

EDS spectrum of crushed borax glass (IDG9), average.

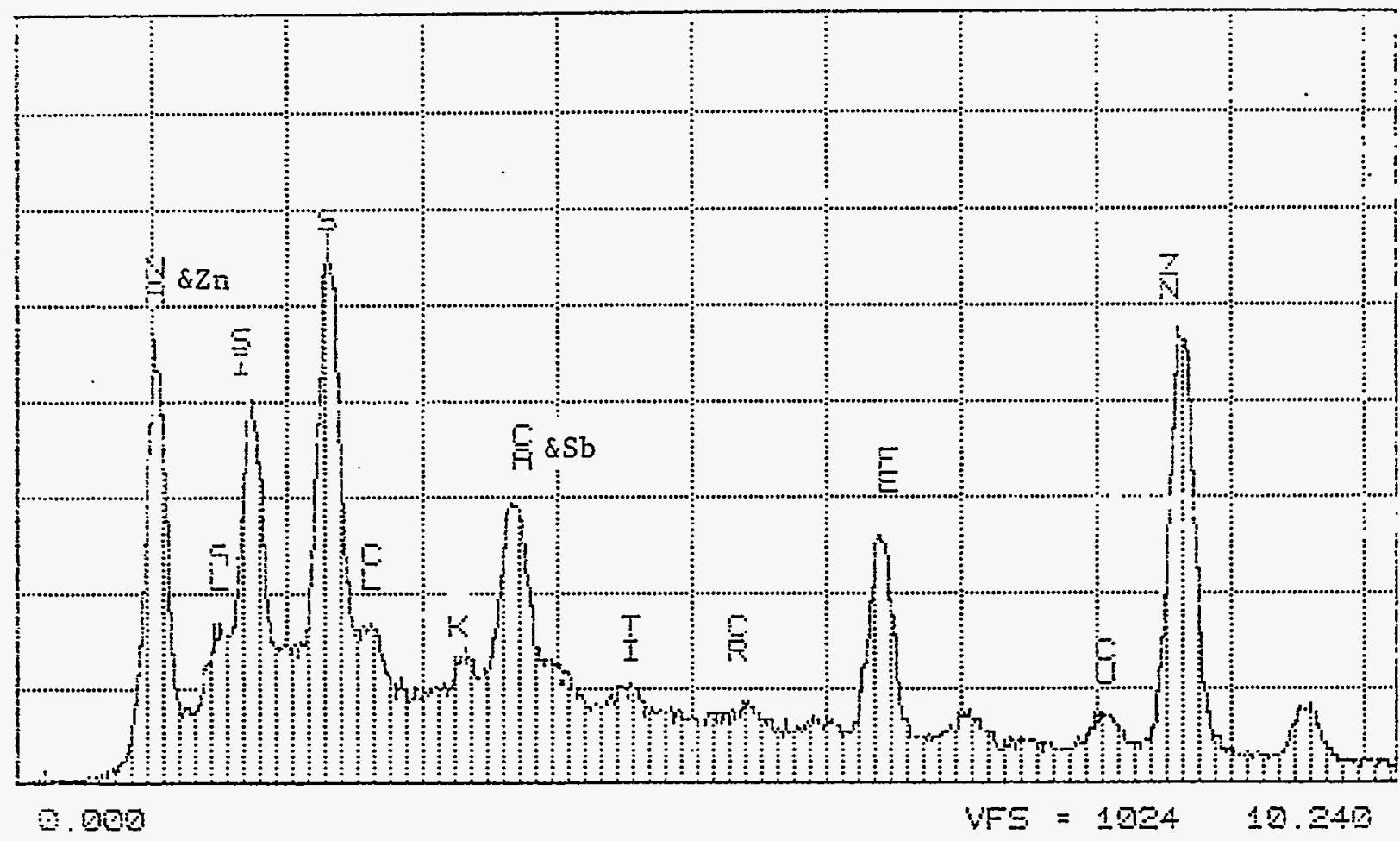

Figure 3.17.

EDS spectrum of crushed borax glass (IDG9), particle \#1.

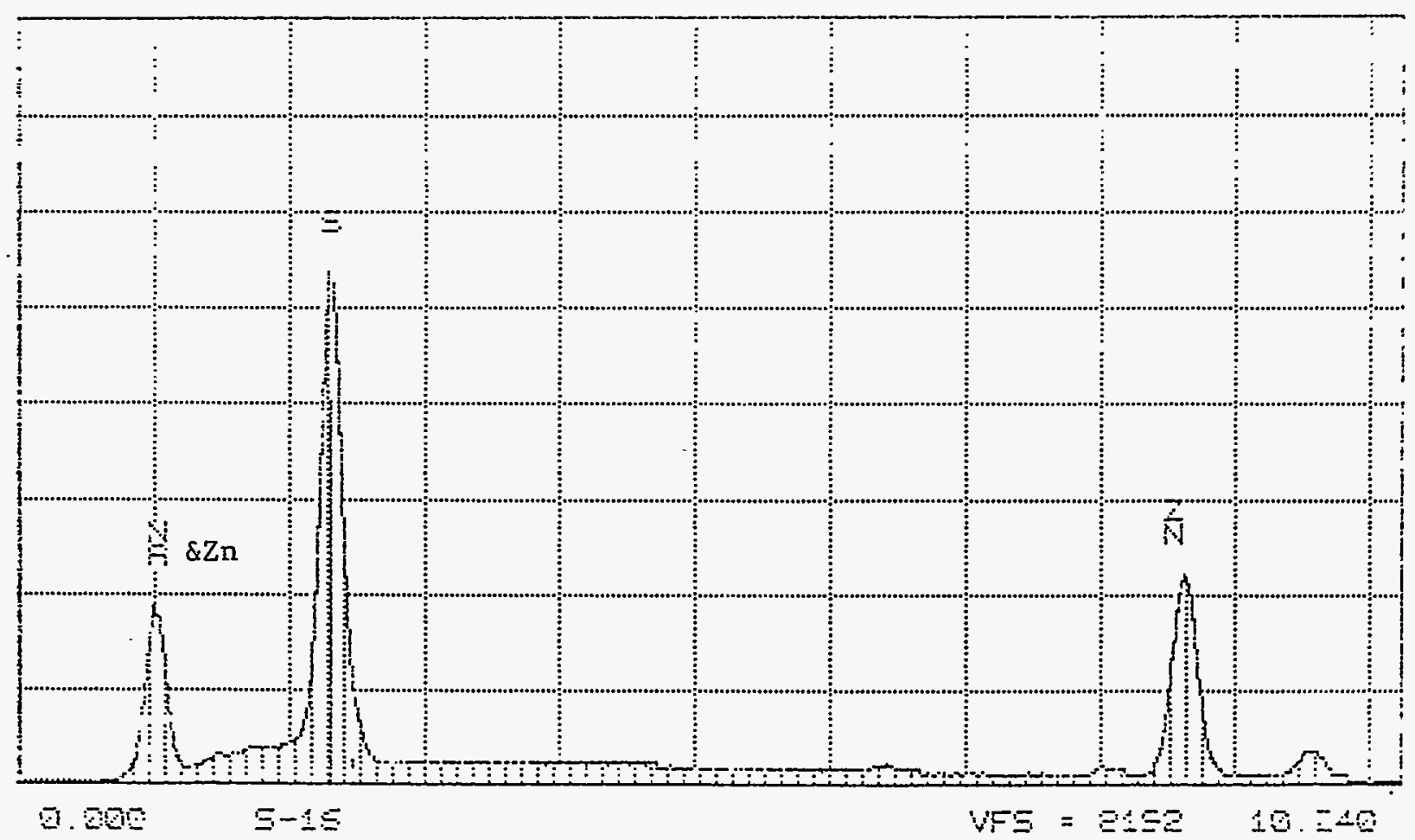


Figure 3.18.

EDS spectrum of crushed borax glass (IDG9), particle \#2.

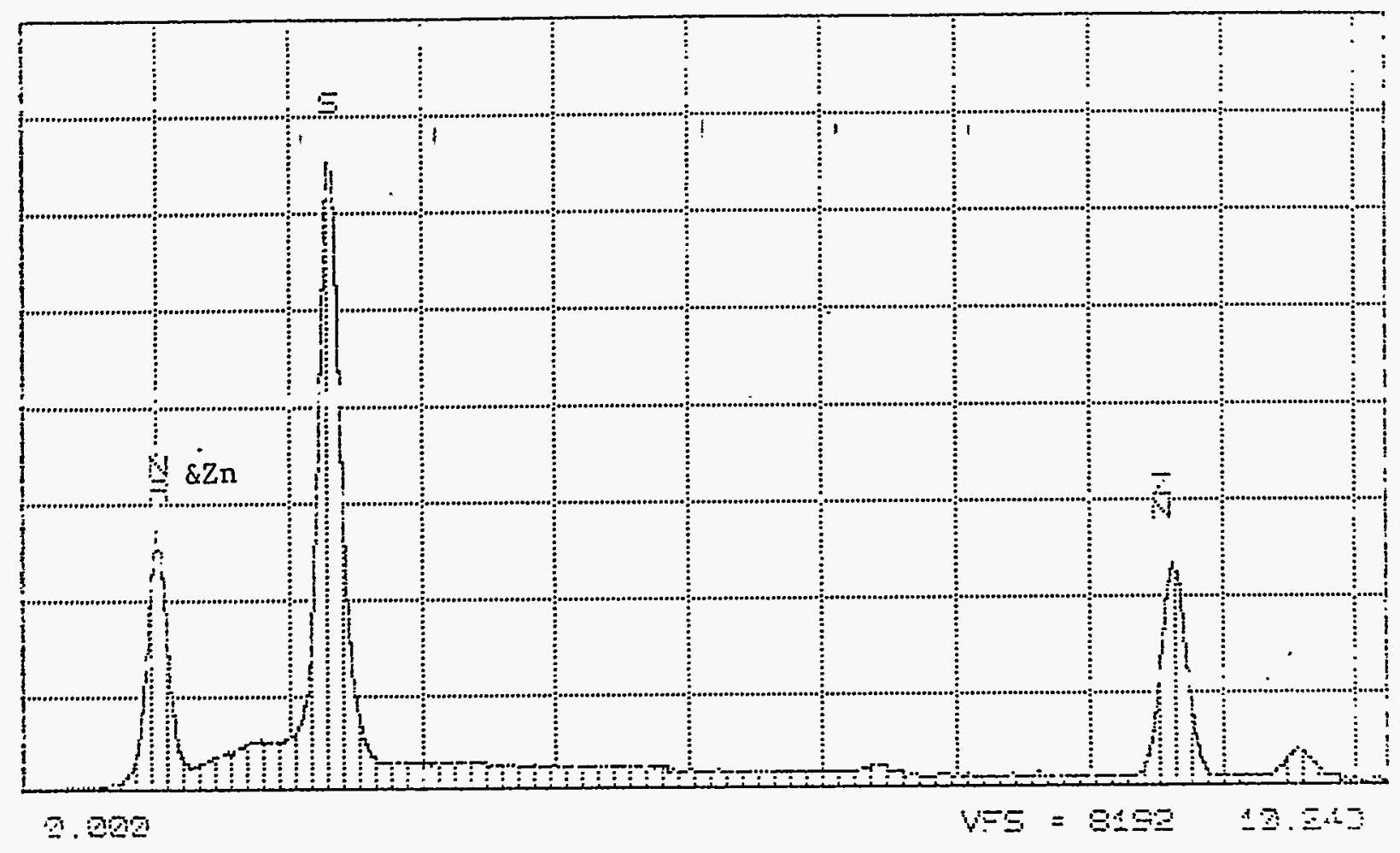

Figure 3.19.

EDS spectrum of crushed borax glass (IDG9), particle \#3.

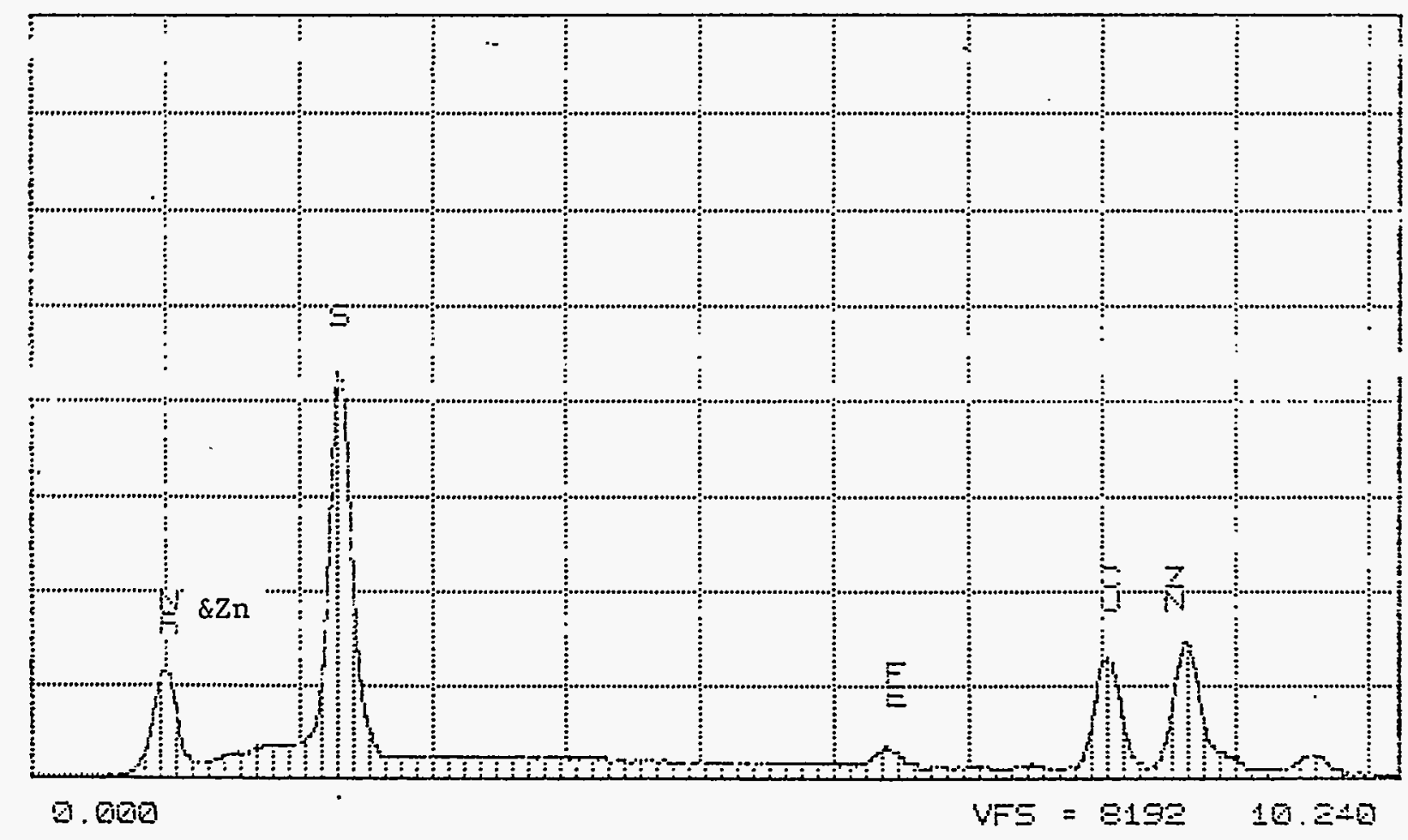


Figure 3.20.

EDS spectrum of crushed borax glass (IDG9), particle \#4.

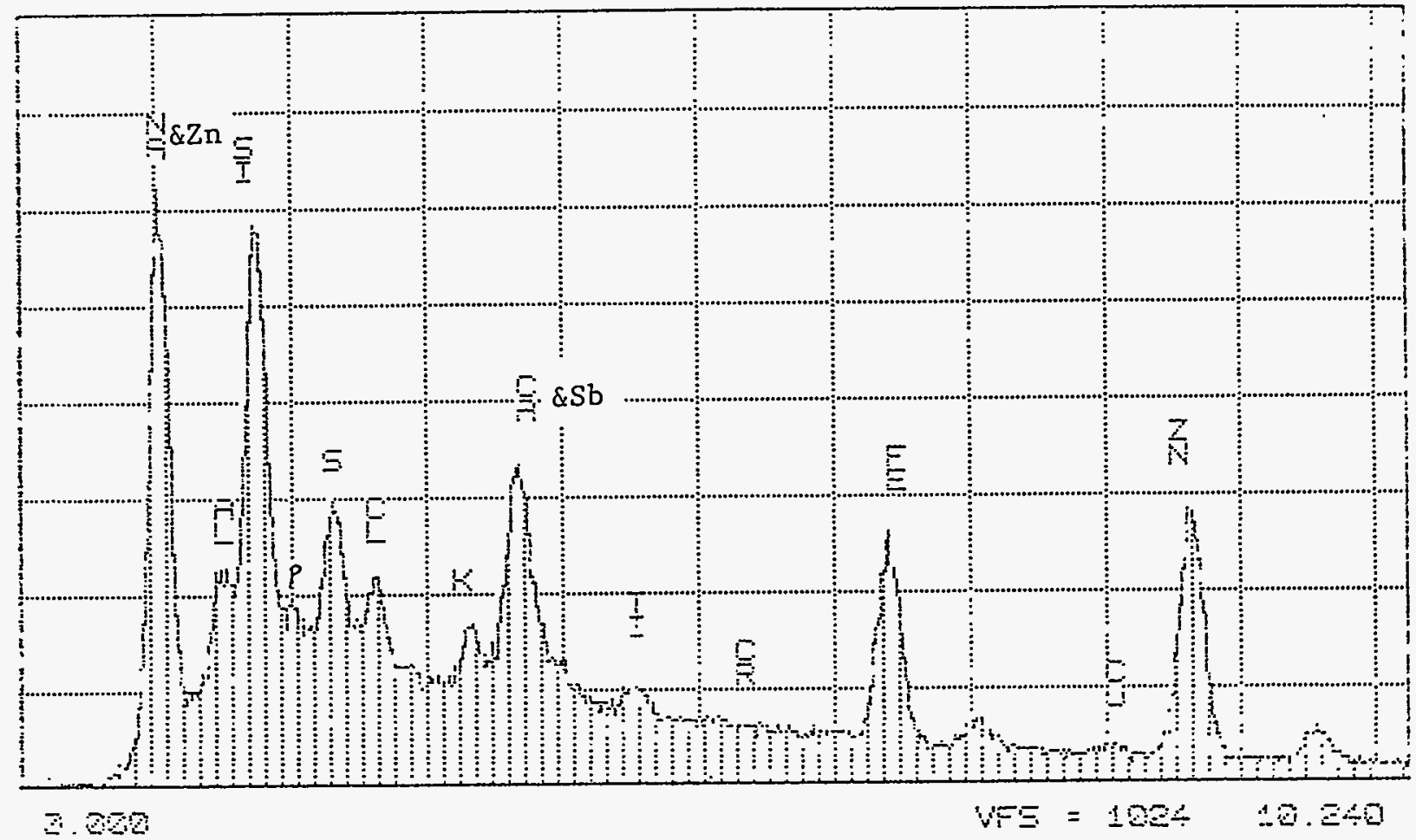


Figure 3.21.

Viscosity of Idaho asbestos and eutectic salt glasses:

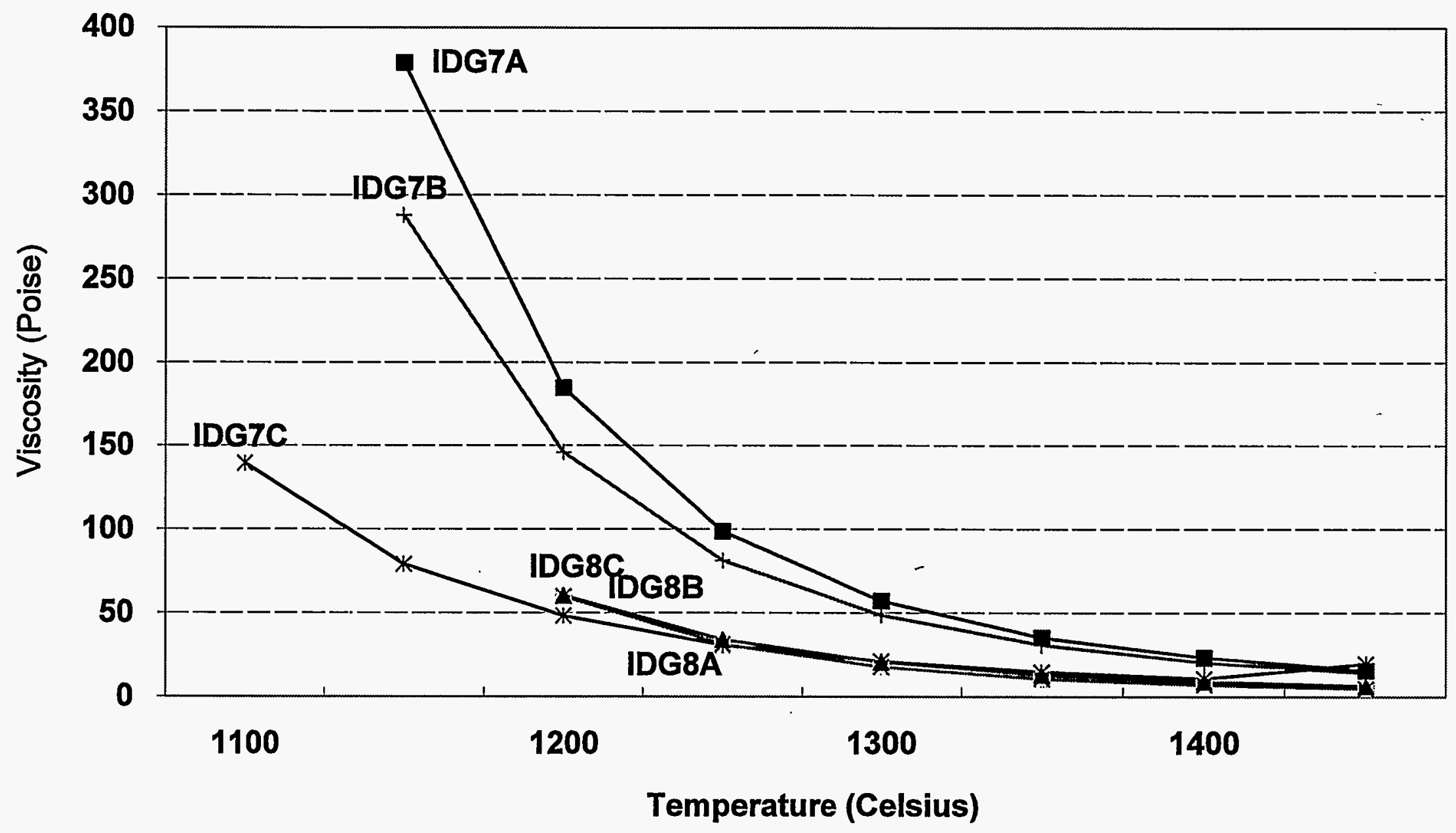


Figure 3.22.

Crystallization in IDG14A heat treated at $1100^{\circ} \mathrm{C}$ for $20 \mathrm{hrs}$.

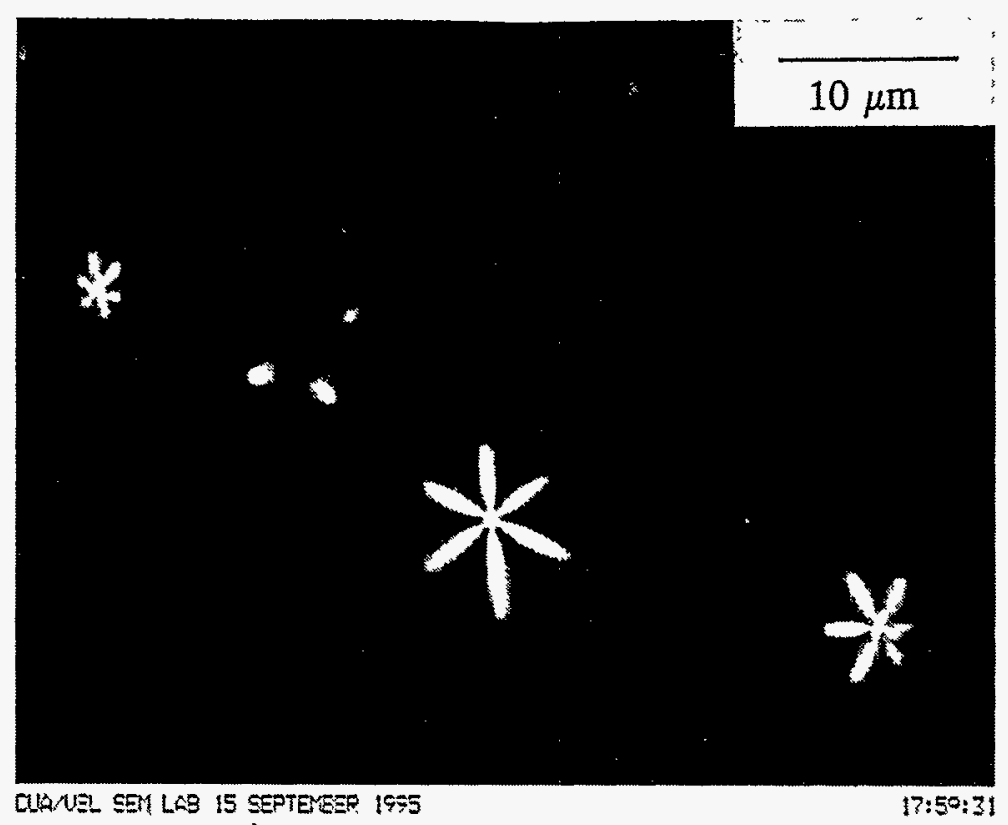

Page 3-56 
Figure 3.23.

EDS spectrum of crystal in IDG10 glass.

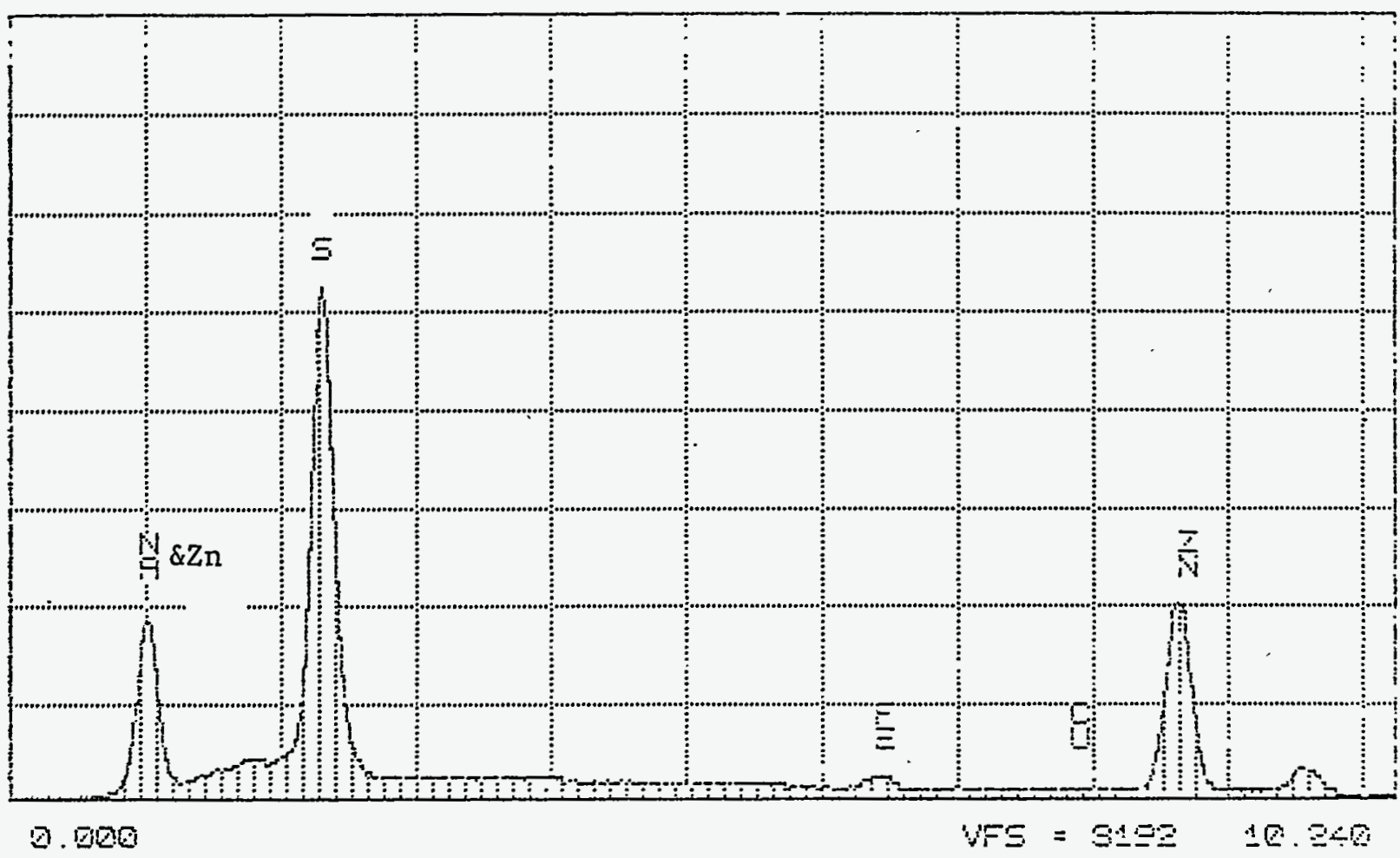

Figure 3.24.

EDS spectrum of $5 \mu \mathrm{m}$ dia. Nodule in IDG10 glass.

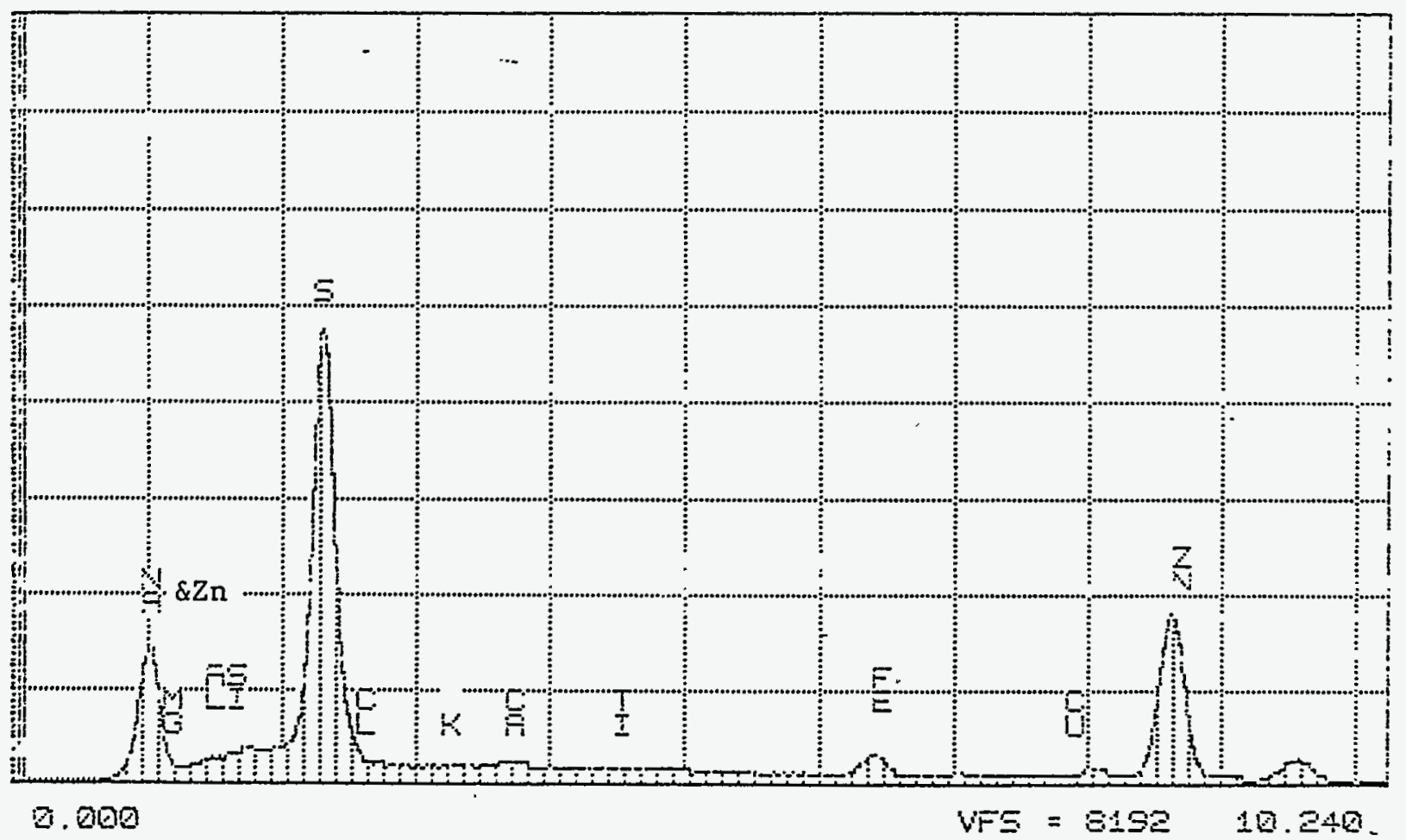


Figure 3.25.

EDS spectrum of $15 \mu \mathrm{m}$ dia. Nodule in IDG10 glass.

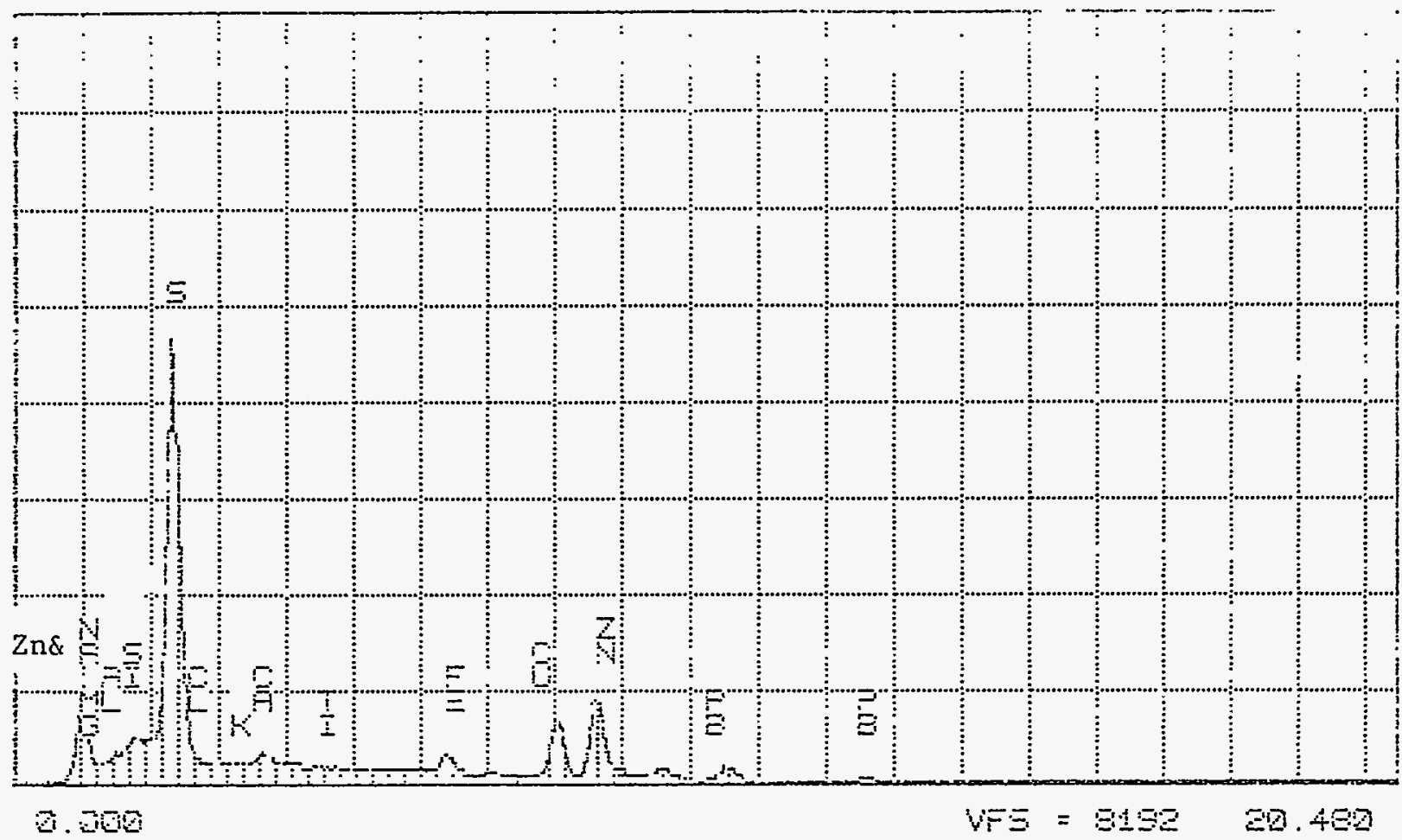

Figure 3.26.

EDS spectrum of region containing many spherical voids in IDG10 glass.

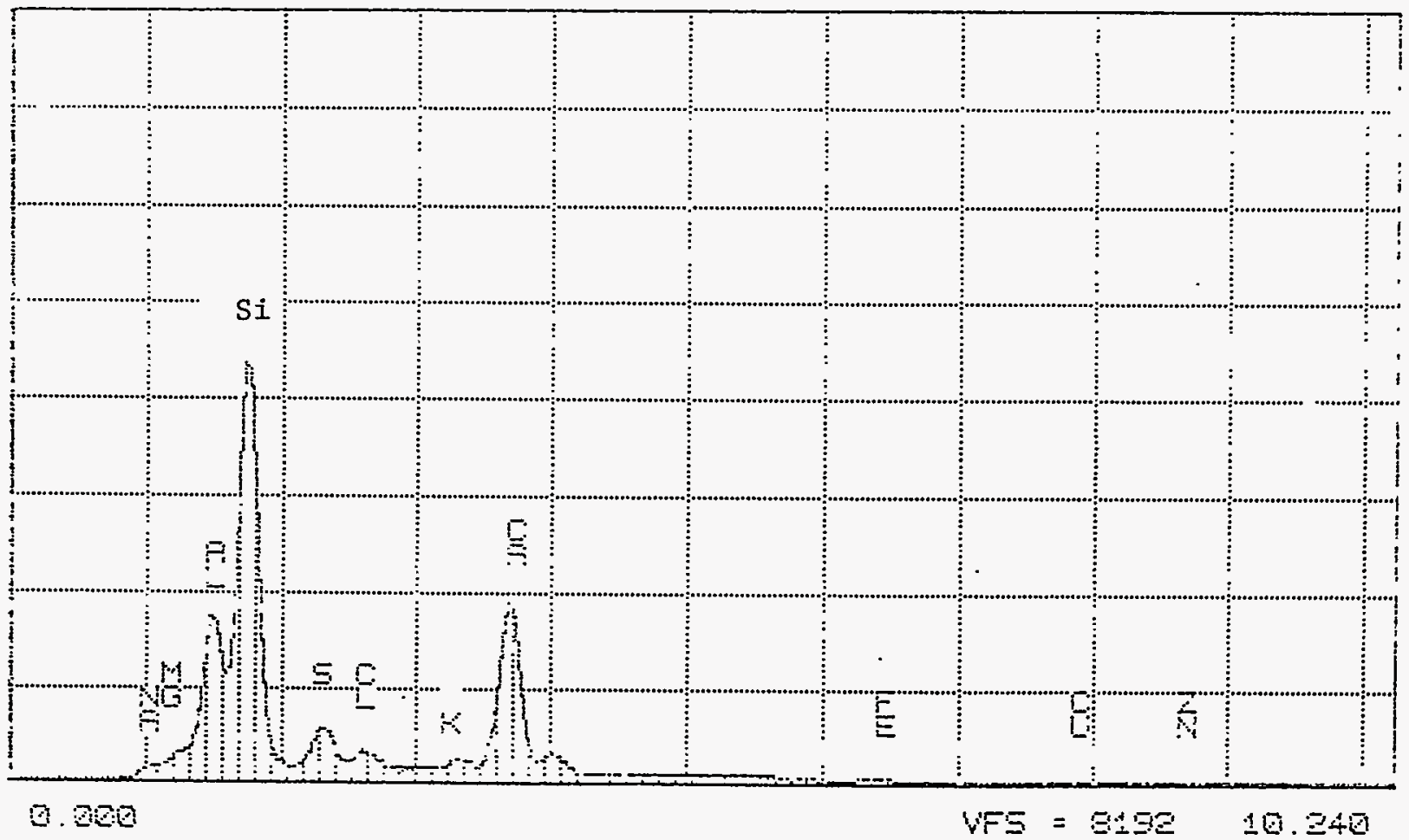


Figure 3.27.

EDS spectrum from high-backscatter-intensity region IDG10 glass.

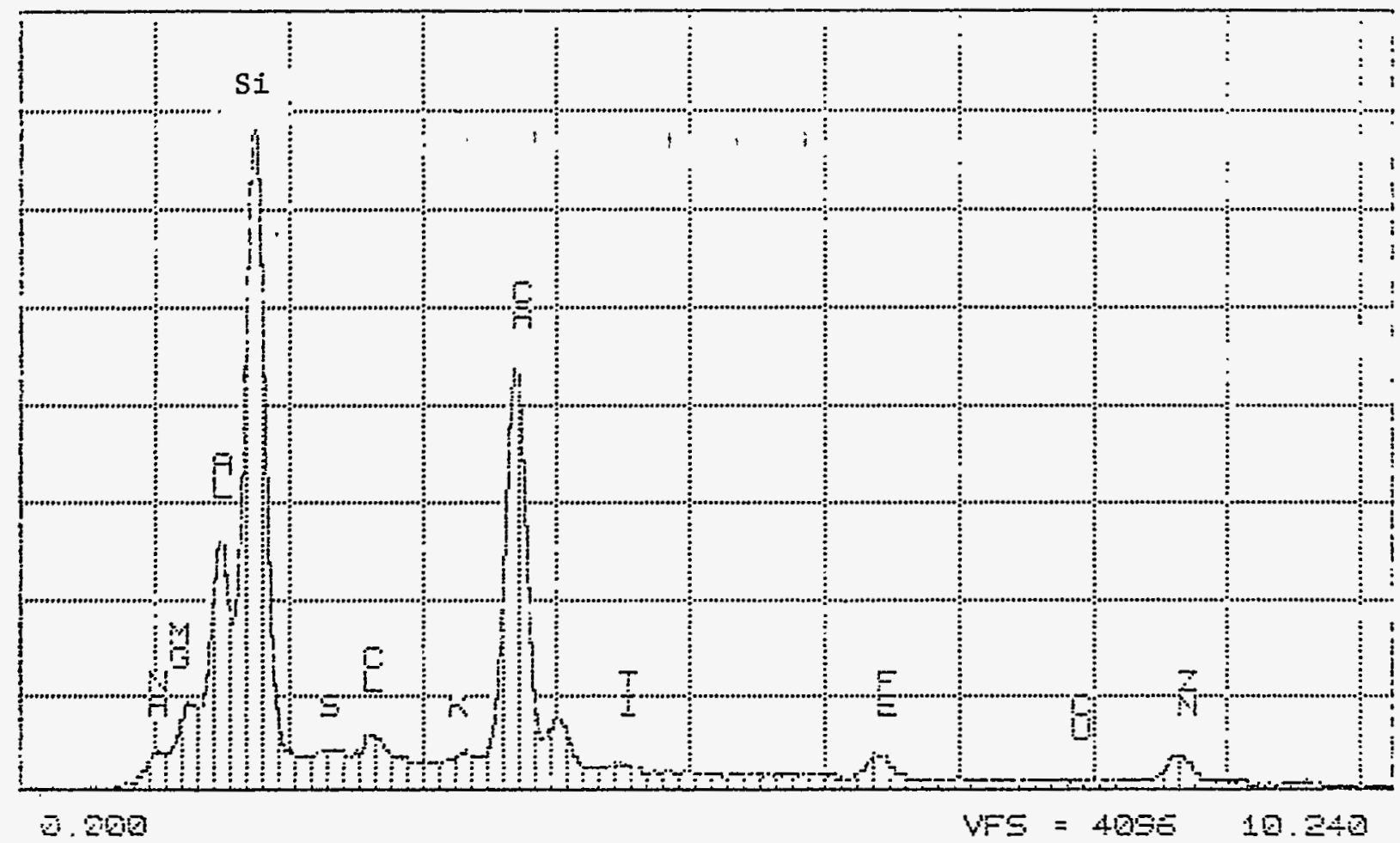

Figure 3.28.

EDS spectrum from low-backscatter-intensity region IDG10 glass.

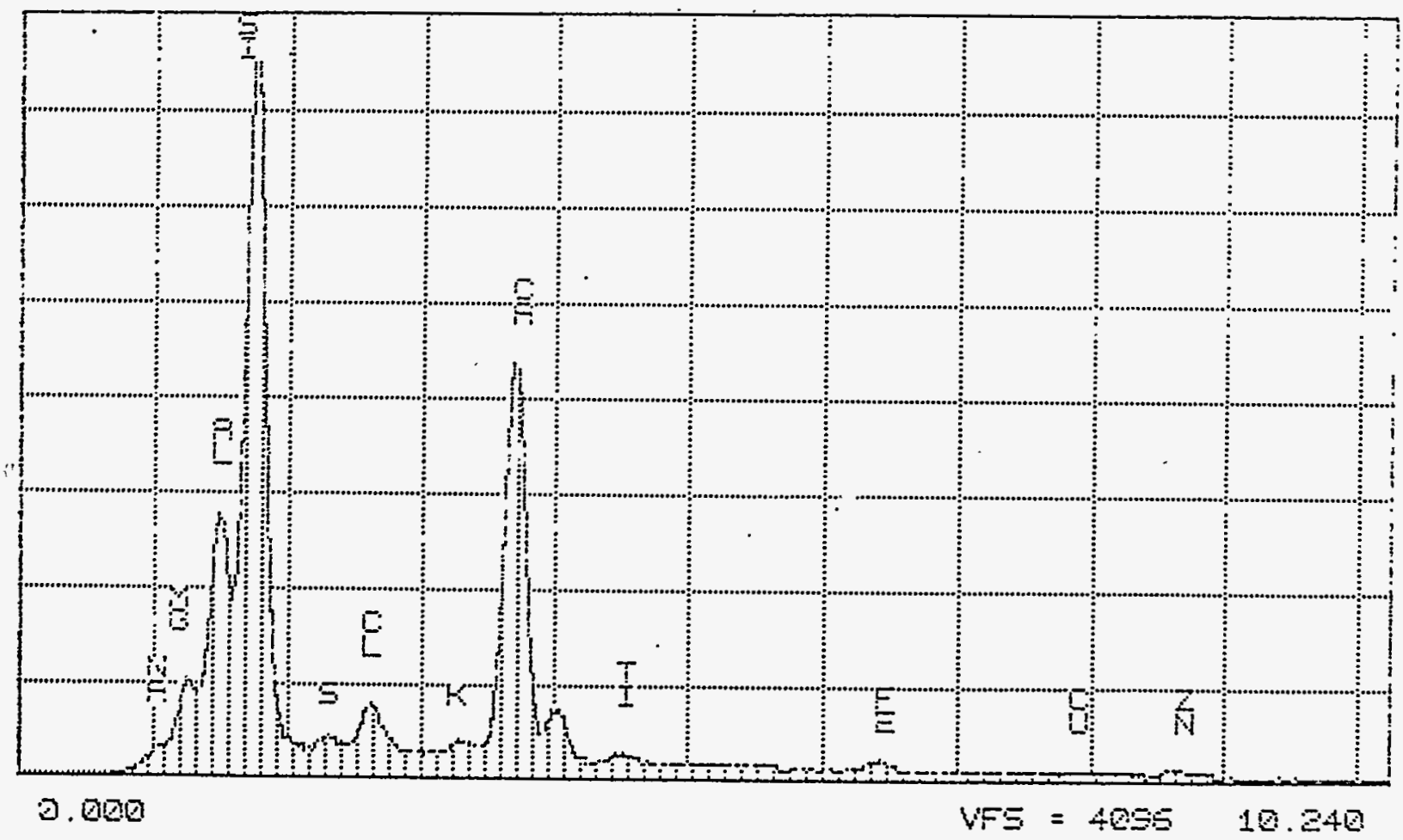


Figure 3.29.

EDS spectrum of $0.5 \mu \mathrm{m}$ dia. Nodule in IDG10 glass.

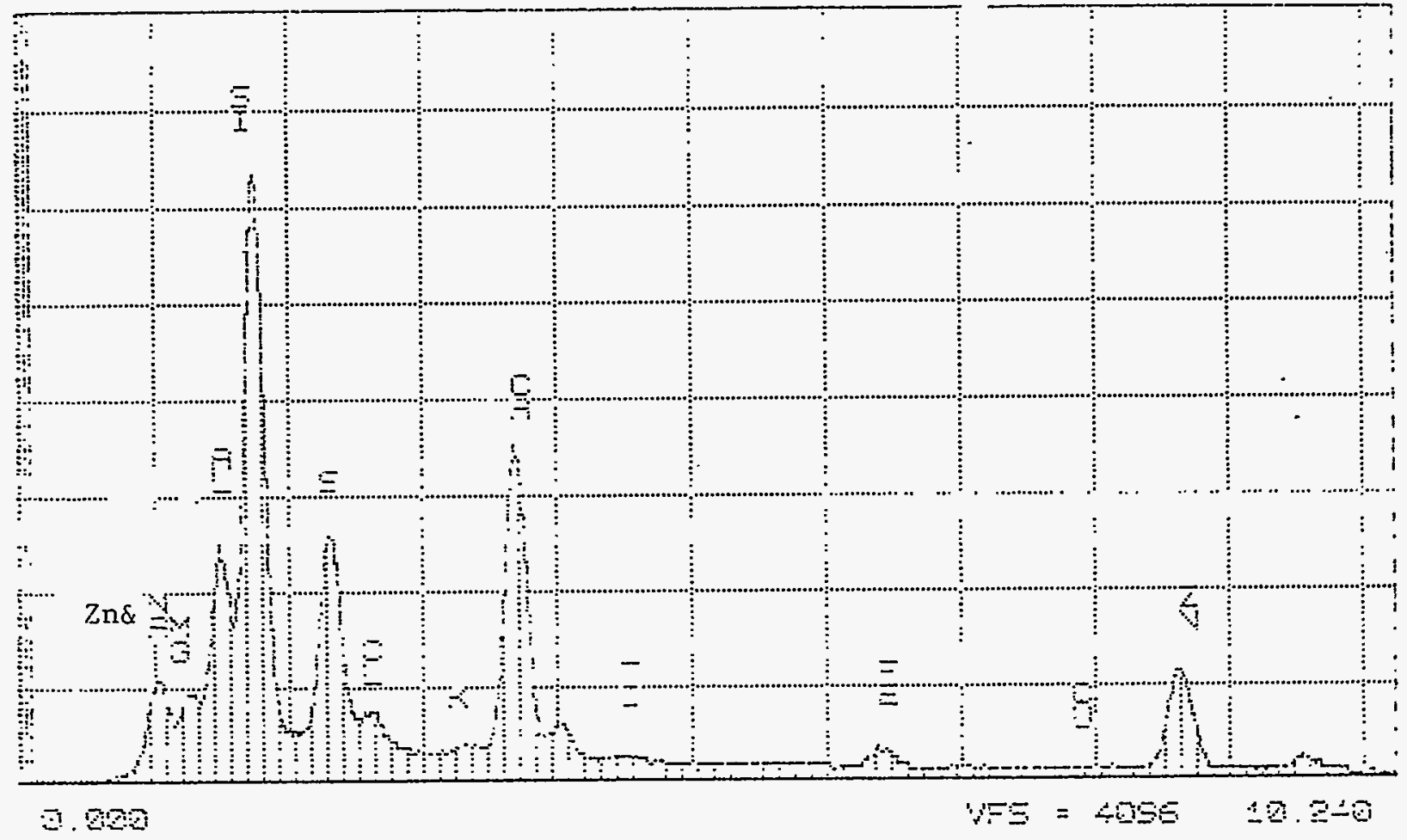

Figure 3.30.

EDS Spectrum of $0.2 \mu \mathrm{m}$ dia. Nodule in IDG10 Glass.

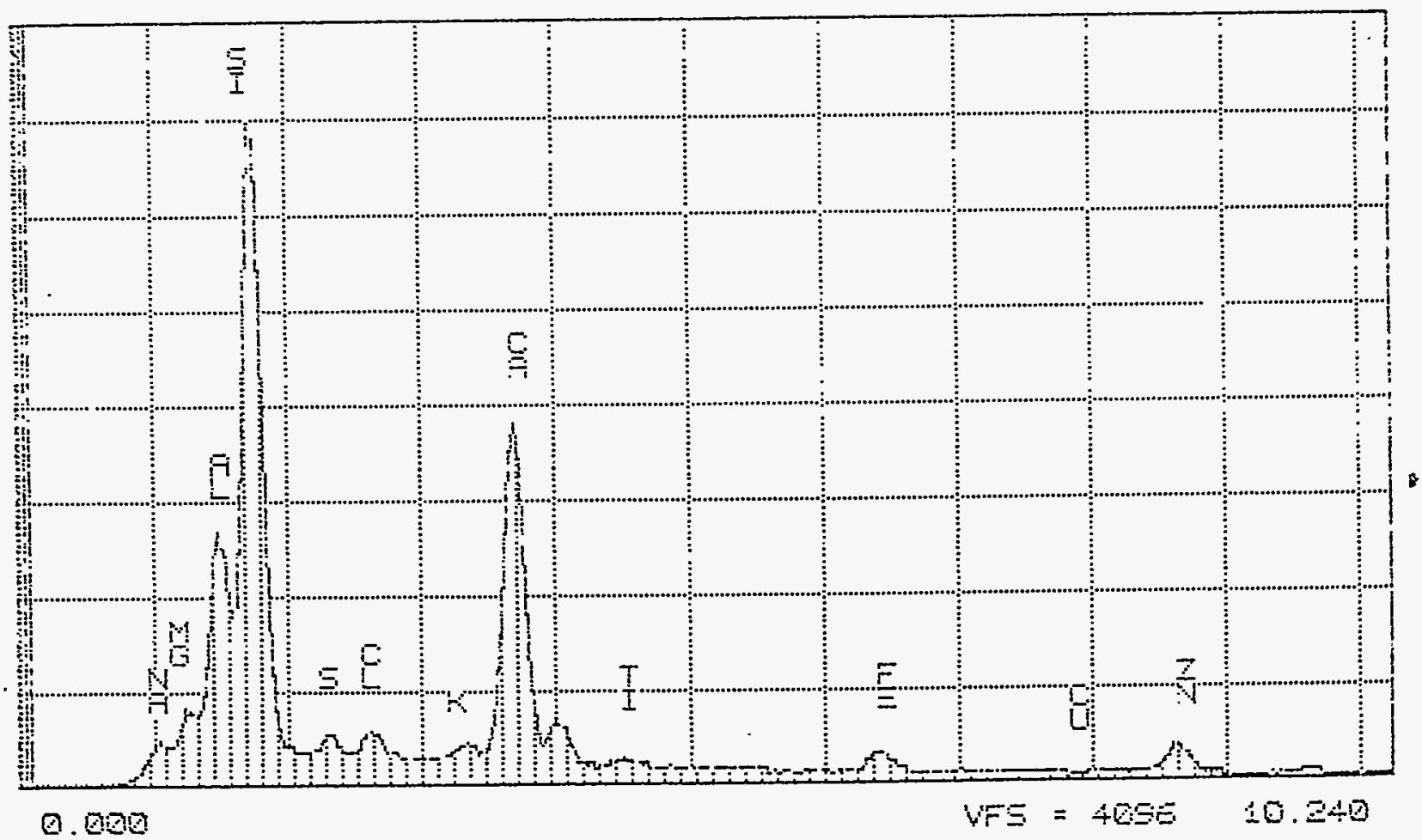


Figure 3.31.

Sulfide nodule among smaller uranium oxide crystals in IDG14 glass.

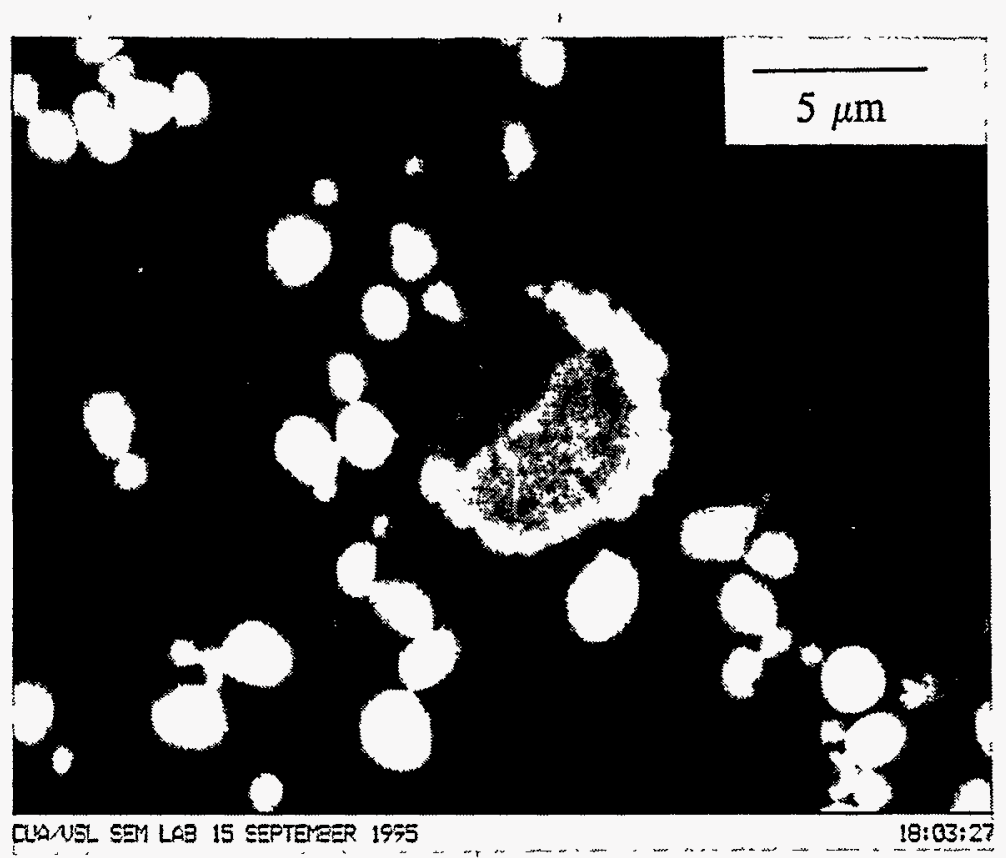


Figure 3.32.

Microstructure of large sediment nodule from IDG15 glass. Elemental distribution is highly varied.

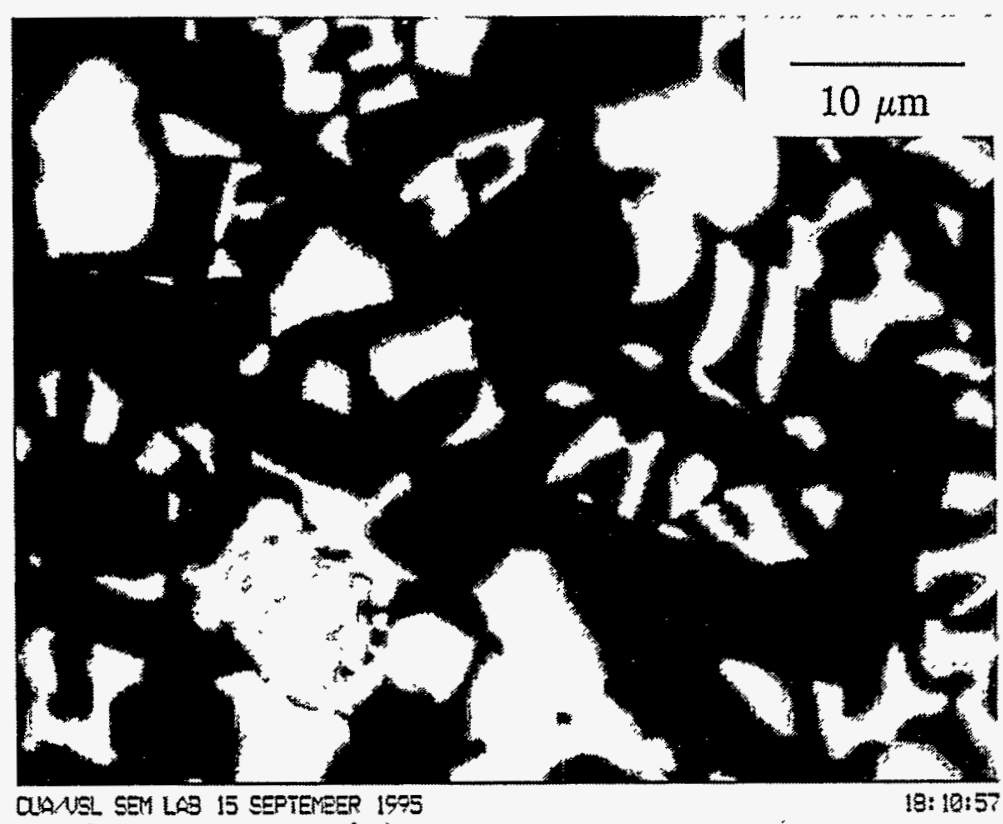

Figure 3.33.

Microstructure of crystalline region in IDG15 glass.

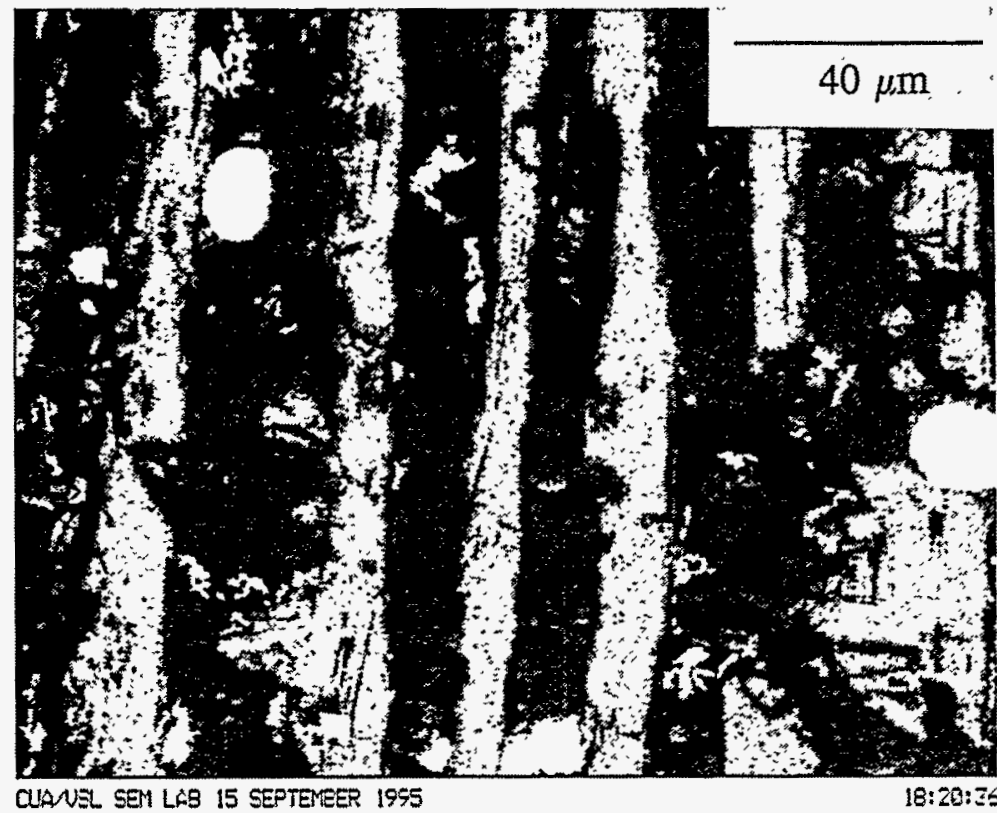


Figure 3.34.

Mössbauer spectrum of Idaho glasses.

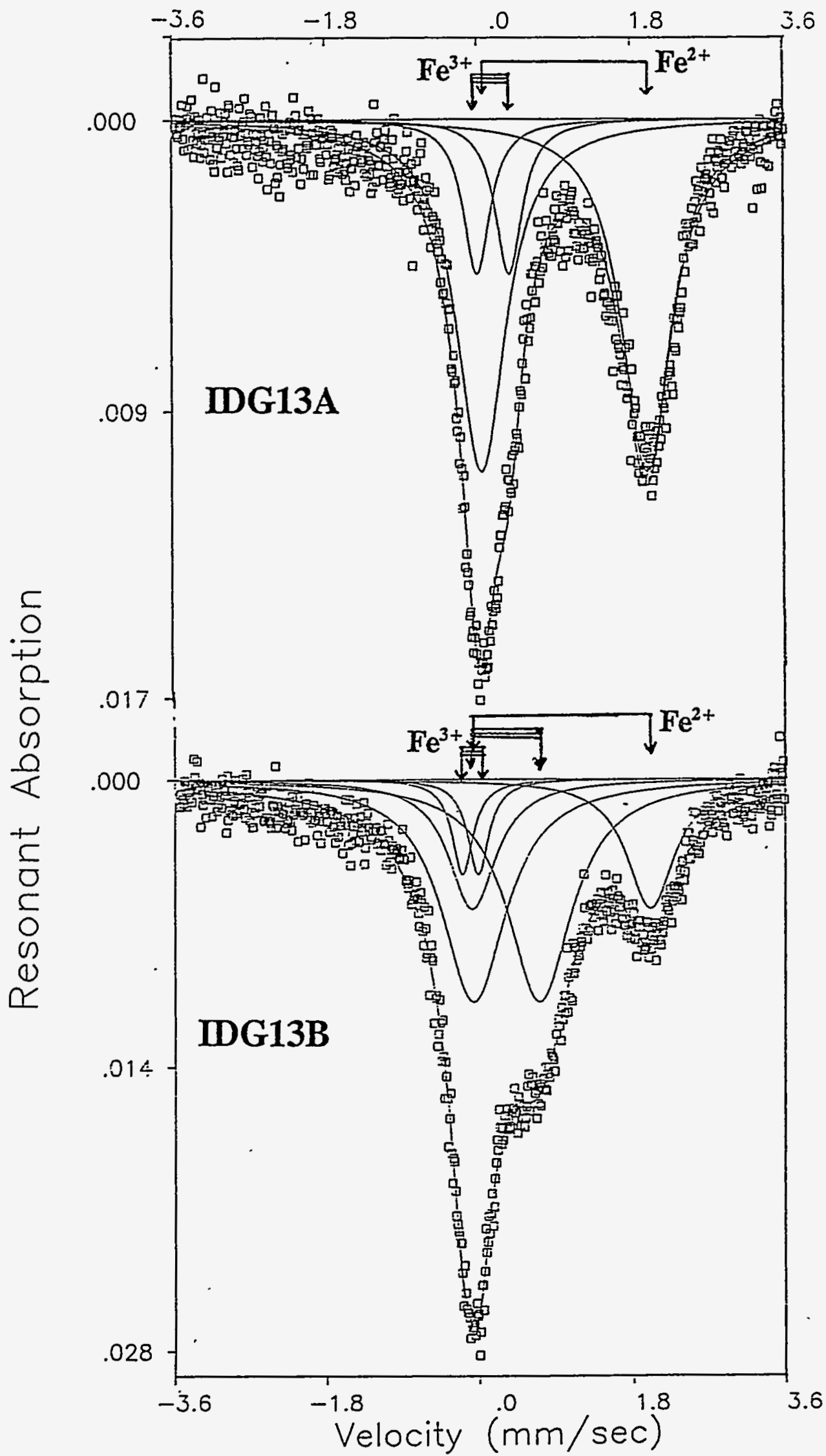


Figure 3.35.

EDS spectrum of sulfate inclusion in IDG13B glass.

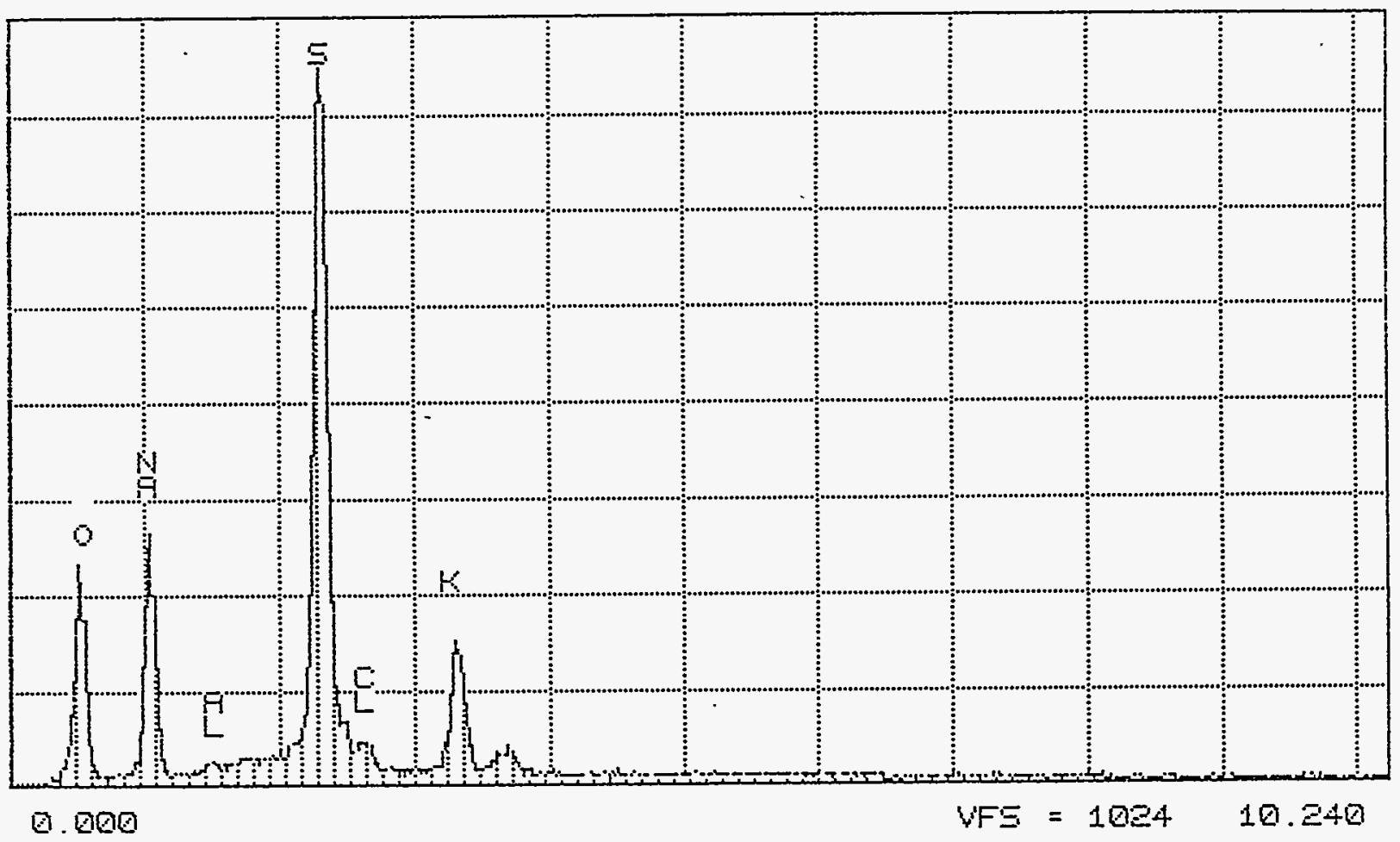


Figure 3.36.

Results from PCT testing of Idaho glasses. Sodium concentration in leachate normalized to that in the glass.

(Compared with Savannah River EA reference glass).

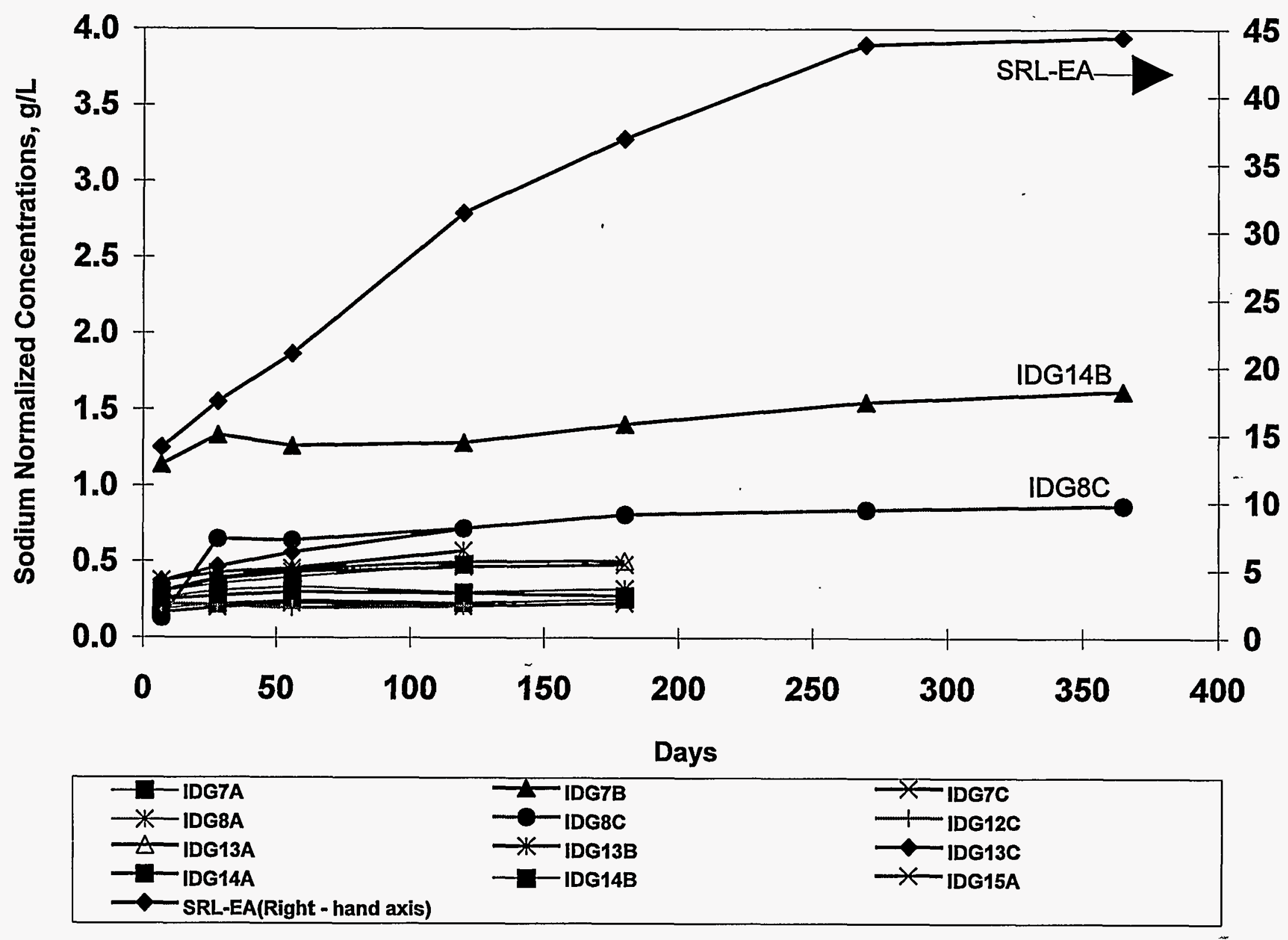


Figure 3.37.

Results from PCT testing of Idaho glasses. Silicon concentration in leachate normalized to that in the glass.

(Compared with the Savannah River EA reference glass.)

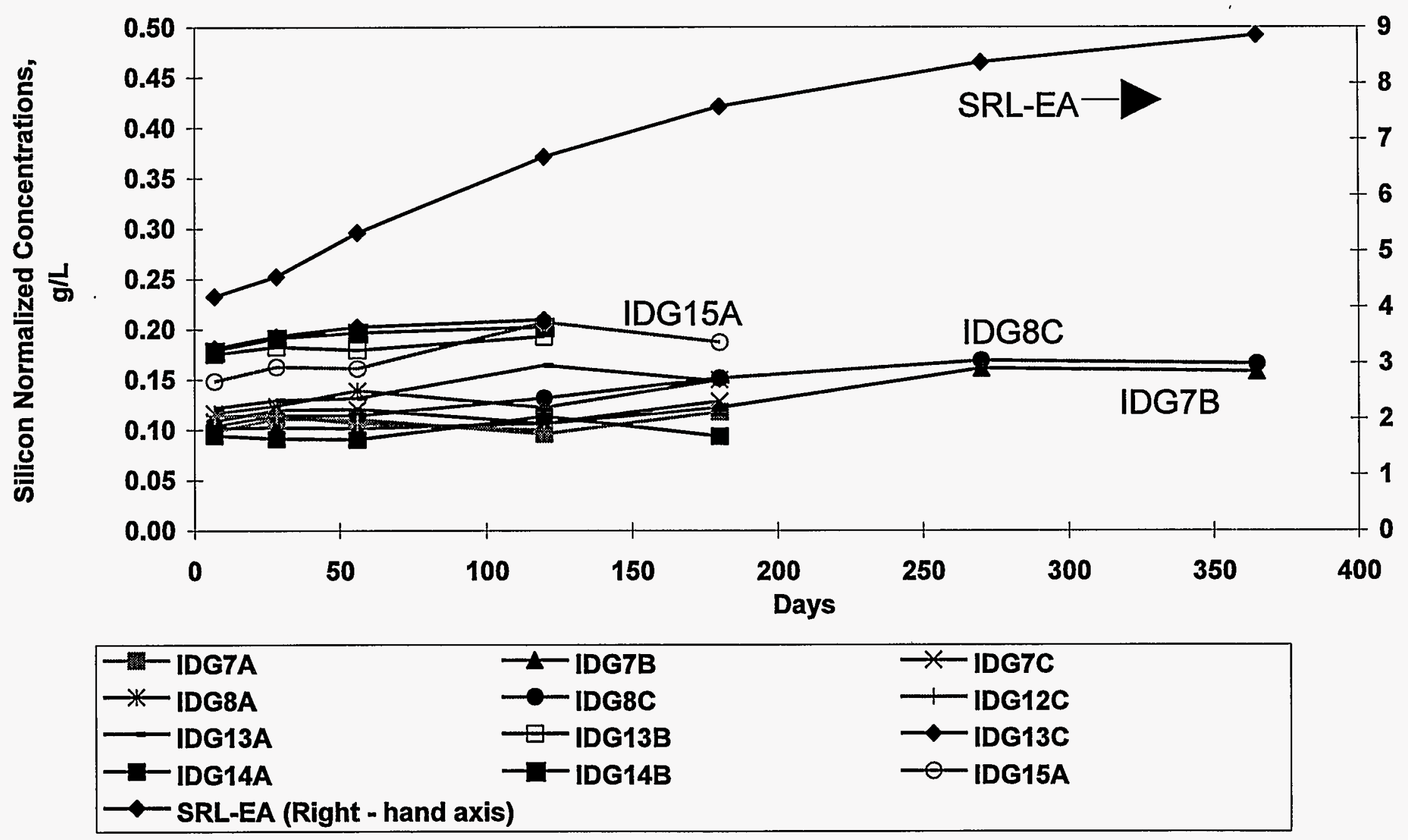


Figure 3.38.

Sodium PCT leach rate of Idaho glasses over one year of testing.

(Compared to the Savannah River EA Reference glass.)

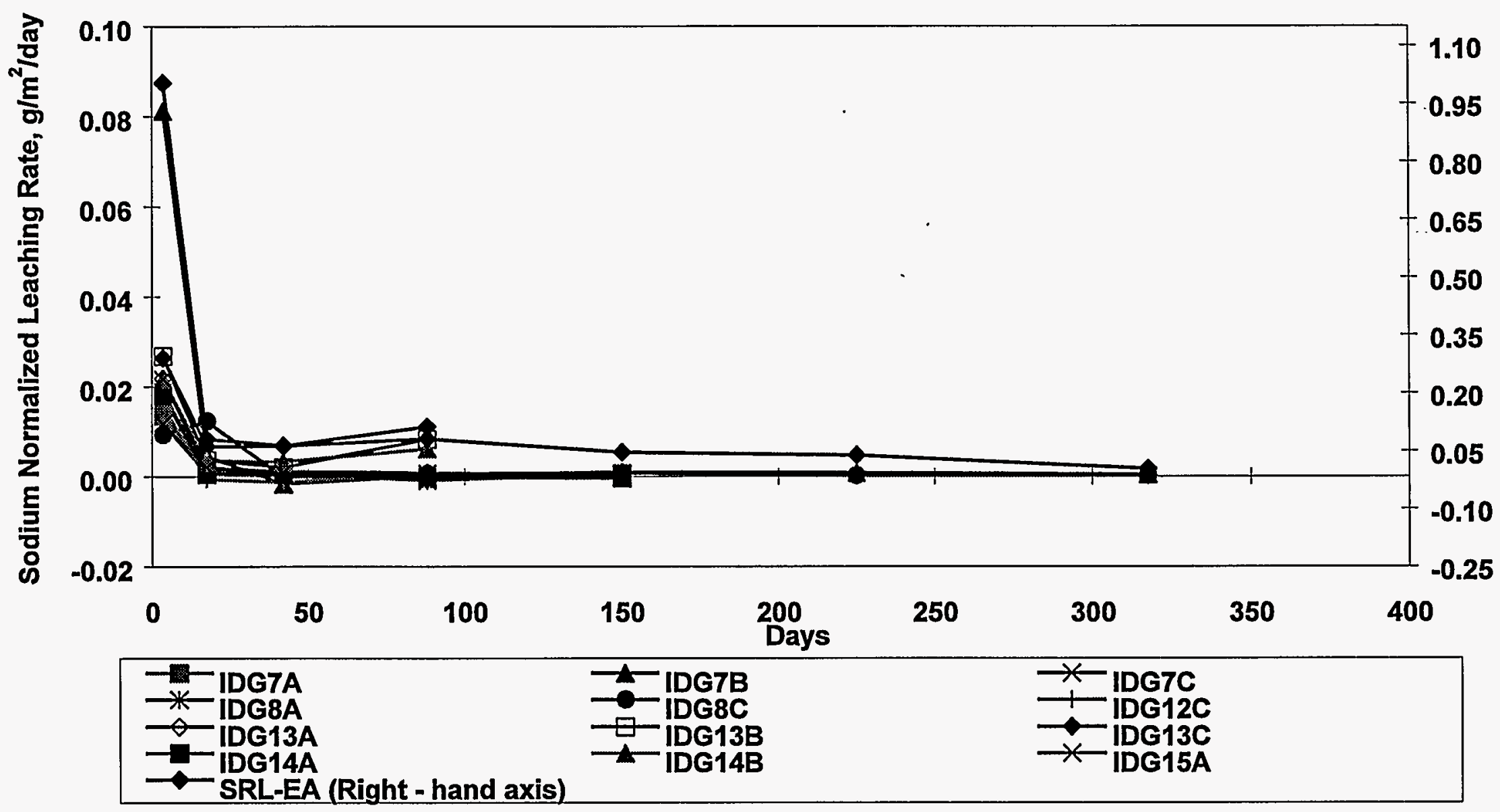


Figure 3.39.

Silicon PCT leach rate of Idaho glasses over one year of testing.

(Compared to the Savannah River EA Reference glass.)

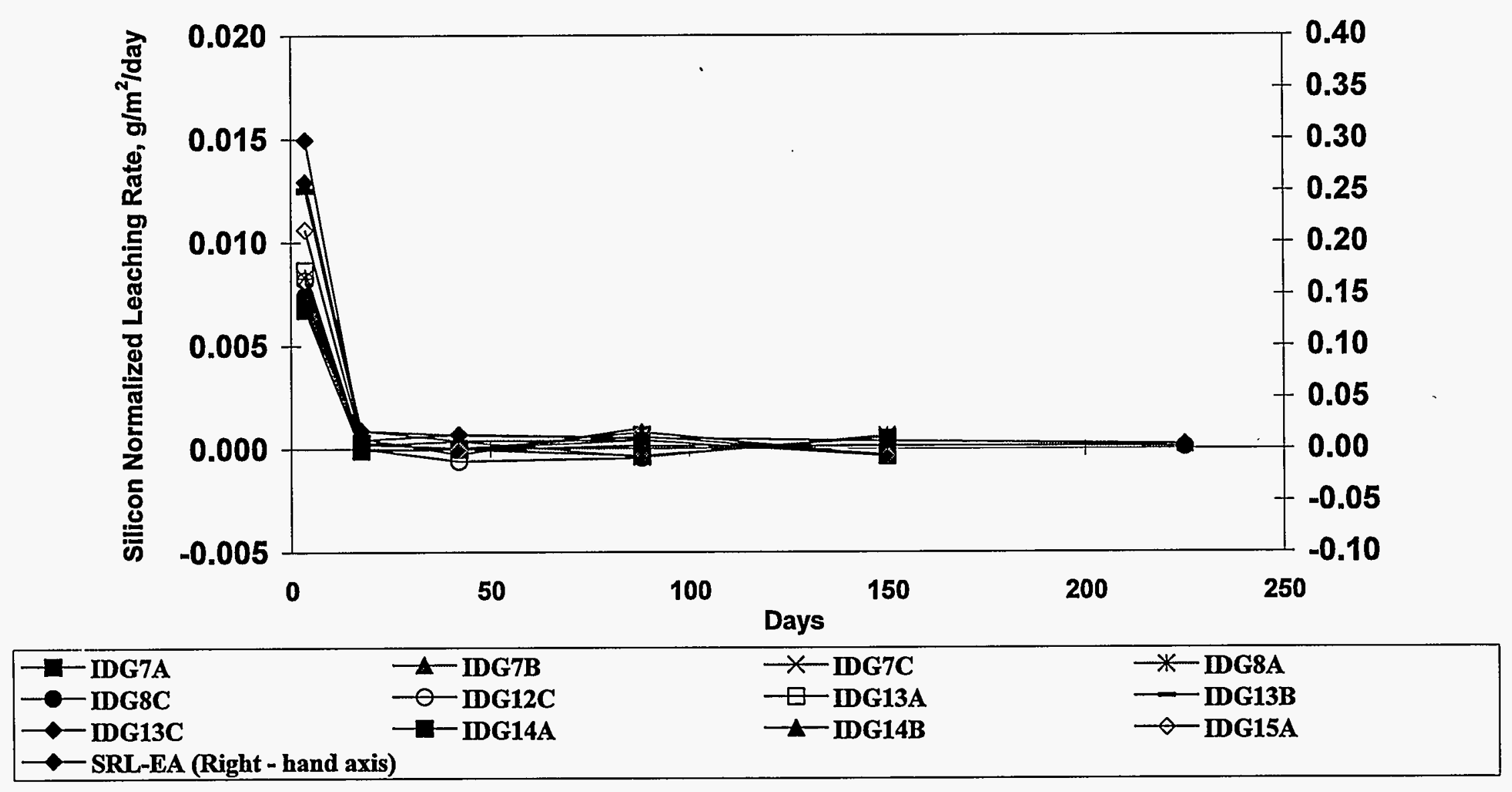




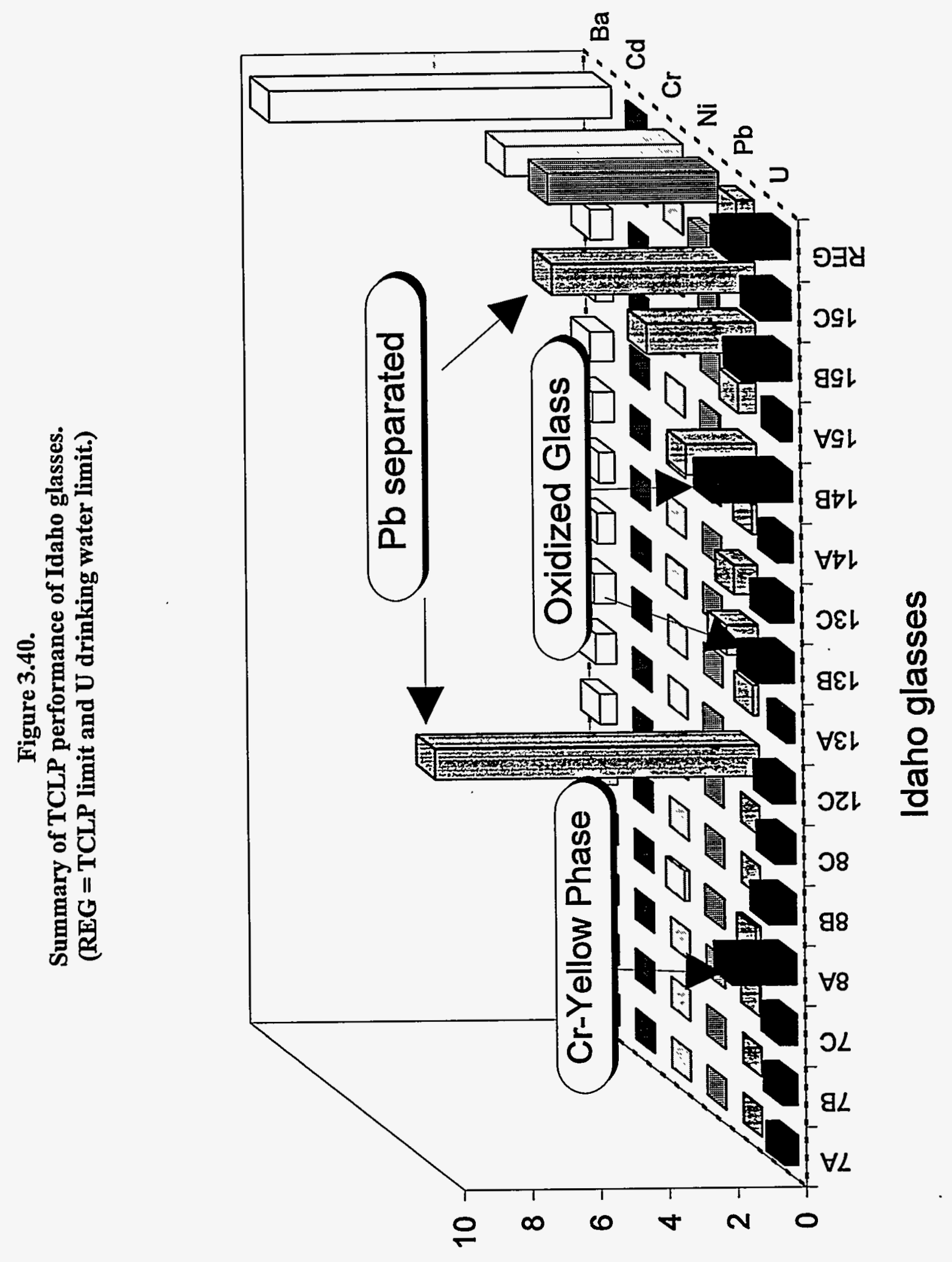

udd u! uo!̣eגłuəouos

әңеบวеәา 
Figure 3.41.

Uranium leaching in TCLP testing of Idaho glasses compared to the drinking water standard in DOE Order 5400.5

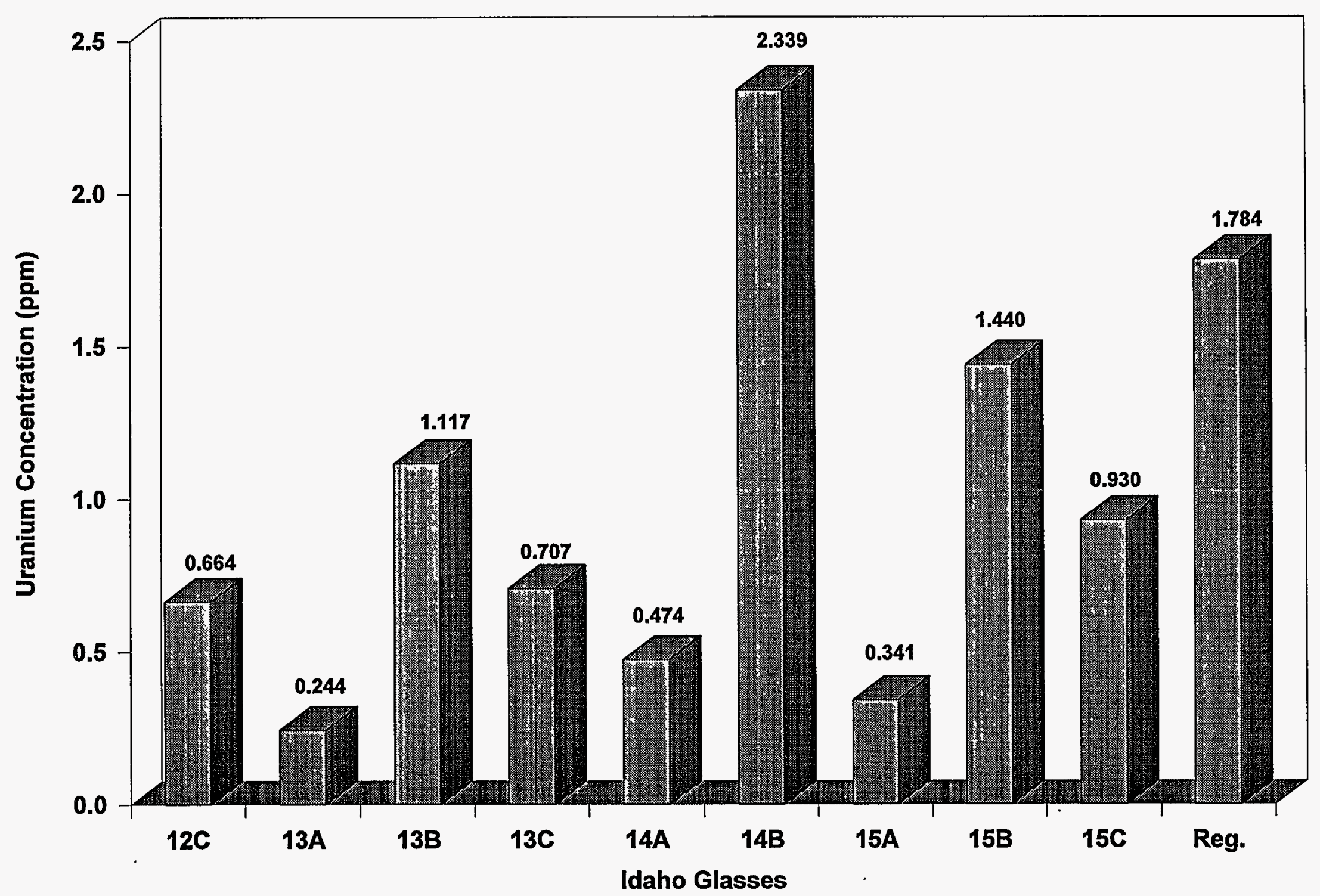




\section{SECTION 4.0 \\ MAWS VITRIFICATION OF OAK RIDGE K-1407 POND WASTE, WEST END TREATMENT FACILITY SLUDGE, TSCA ASH, AND SOIL}

\subsection{Oak Ridge Wastes Selected for Study}

Extensive discussions were held with representatives of the Oak Ridge site contractor (Martin Marietta Energy Systems, Inc.) and appropriate DOE Offices to obtain information on Oak Ridge site waste streams that may be both suitable for, as well as available for, this work. Requests for samples of several suitable waste streams were made on the basis of these discussions. The available data were used to select a series of waste streams anticipated (based on available characterization data, origin, process history, or general descriptions) to be likely candidates for vitrification using a MAWS approach. Other factors that were considered in the selection process were: Site specific interest and perceived treatment priority; volume of waste in storage and volume expected to be generated in the future, if any; whether a specific treatment technology had already been selected; whether the waste stream was representative of a bigger "class" of waste streams in the DOE complex; compositional diversity with respect to the compositional envelope objectives; compositional suitability for MAWS blending with other waste streams from the Idaho site; and availability of samples.

The screening process led to the following list of waste streams that were investigated in more detail:

(1) West End Treatment Facility (WETF) sludge

(2) Contaminated soils

(3) TSCA incinerator ash

(4) TSCA incinerator blowdown sludge

(5) Central Neutralization Facility (CNF) sludge

(6) K-1407 Pond Waste

Samples of each of these waste streams were requested from the site for this study. Samples of the WETF sludge were received along with samples of soils collected from outside the Oak Ridge site boundary, and surrogate formulations, used for the TSCA ash and the Pond Waste; four of the six waste streams listed above were included in the study. 


\subsection{Characterization of Oak Ridge Wastes}

\subsubsection{Oak Ridge K-1407 Pond Waste}

The surrogate formulation developed at the Oak Ridge K-25 facility (Bostick-1994, Table 15) was used for the Oak Ridge K-1407 Pond Waste. This formulation requires the use of a pottery clay to improve the suspension characteristics of the simulant. However, since good suspension of the simulated slurry is not a major concern in crucible melt preparations and the use of the clay would increase the uncertainty of the chemical composition, the recipe was revised to replace the Indian Red Pottery Clay (IRPC) with a combination of silica, $\mathrm{Al}(\mathrm{OH})_{3}$, and $\mathrm{Fe}(\mathrm{OH})_{3}$. This material, spiked with contaminants and radionuclides as determined on the basis of the available characterization data, was used in the crucible melt studies. The blend of dry components required to form the surrogate composition (described in Table 4.1) was weighed out for each crucible melt and additives or other waste streams were used as necessary to form an acceptable glass waste form. Note that the hazardous and radioactive constituents were included in the formulation.

\subsubsection{TSCA Bottom Ash}

The surrogate for the TSCA Bottom Ash was prepared based on the Waste Information Sheet provided by the site; the composition developed on this basis was very close to that cited by Whittington et al. (Whittington-1994). The blend of chemicals used, detailed in Table 4.1, included the hazardous and radioactive constituents (nickel, lead, and uranium) as part of the formulation.

\subsubsection{Oak Ridge Soil}

A non-radioactive soil sample from an area close to the Oak Ridge site was obtained in place of the contaminated soil samples. The soil was subjected to the characterization procedures described below.

\section{Thermogravimetry}

The weight losses after drying at $110^{\circ} \mathrm{C}$ for 18 hours, $450^{\circ} \mathrm{C}$ for 4 hours, and $1150^{\circ} \mathrm{C}$ for 4 hours were $9.69 \%, 21.1 \%$, and $22.9 \%$, respectively. The results show that there are some volatile losses beyond simply free water in the samples. These losses are probably due to chemical water, carbonates, and organics. 


\section{Specific Gravity}

The specific gravity was measured according to the ASTM procedure D 854-83 which is based on the displacement of water. The measured value of $3.04 \mathrm{~g} / \mathrm{cm}^{-3}$ is somewhat higher than values measured for several other site soils across the country, which tend to average around $2.8 \mathrm{~g} / \mathrm{cm}^{-3}$.

\section{Particle Size Analysis}

The ASTM D 422-63 procedure was used for particle size analysis. The results of sieving into six particle size fractions above 10 and below 200 U.S. Mesh (Table 4.2) showed that the soil was quite coarse with the majority of the soil above the $2 \mathrm{~mm}$ particle size, as shown in Table 4.2 and Figure 4.1.

\section{DCP Analysis}

DCP analysis was performed on a solution of the soil formed by first drying a sample at $1150^{\circ} \mathrm{C}$ and then dissolving a $0.1 \mathrm{~g}$ sub-sample in an $\mathrm{HF} / \mathrm{HNO}_{3}$ mixture to effect a total dissolution. Analysis revealed the soil to be of a rather refractory type since it was composed of $77 \% \mathrm{SiO}_{2}$, $15.5 \% \mathrm{Al}_{2} \mathrm{O}_{3}$, and $6 \% \mathrm{Fe}_{2} \mathrm{O}_{3}$ with traces of $\mathrm{K}_{2} \mathrm{O}$ and $\mathrm{MgO}$ present at less than $1 \%$. Given the large particle size and refractory nature of the soil, it was determined that some size reduction would be necessary to use this material in the crucible melts in view of the short residence times; this was confirmed in a few test melts using the soil directly. The soil was therefore simulated using a combination of powdered chemicals to achieve the same composition.

\section{Ion Chromatography and Ion Selective Electrode Analysis}

The solution prepared by dissolution of a sample of the soil for DCP analysis was also used for analysis by ion chromatography and ion selective electrode. No sulfate was detected by the former and no fluoride was detected by the latter, indicating that the amounts of these species in the original soil were below about $0.5 \mathrm{wt} \%$. 


\subsubsection{Oak Ridge West End Treatment Facility Sludge}

A sample of about $100 \mathrm{~kg}$ of WETF sludge (from Tank 7) was used in this study. The sludge was homogenized using a hand-held electric mixer for about one hour. Six samples were collected and duplicate weight loss assays at $110^{\circ} \mathrm{C}, 450^{\circ} \mathrm{C}$, and $1150^{\circ} \mathrm{C}$ were performed, as described below.

\section{Thermogravimetry}

Heat treatments at $110^{\circ} \mathrm{C}$ for 18 hours, $450^{\circ} \mathrm{C}$ for 4 hours, and $1150^{\circ} \mathrm{C}$ for 4 hours led to weight losses of $69.95 \%, 72.69 \%$, and $81.93 \%$, respectively. These results indicate that this sludge contains about $70 \%$ moisture and about $18 \%$ glass forming components; this suggests that there is the potential for large volume reductions through vitrification.

\section{Total Organic Carbon (TOC) and Total Carbon (TC)}

The results from the total carbon content (TC) and total organic content (TOC) measurements of seven replicates are presented in Table 4.2. The WETF sludge, after drying at $110^{\circ} \mathrm{C}$, contains $9.17 \%$ total carbon of which $1.13 \%$ is organic carbon.

\section{DCP Analysis}

Samples of the sludge were first dried (and converted to oxides) by heating at $1150^{\circ} \mathrm{C}$ and then subjected to microwave dissolution; results from DCP analysis of the resulting solutions are reported in Table 4.3. The composition is significantly different from that given for this material by Whittington et al. (Whittington-1994). Our analysis indicates that the presence of about 19\% alumina, $5 \%$ magnesium oxide, $2 \%$ iron oxide, $1 \%$ phosphorus oxide, and $1.2 \%$ uranium decreases the calcium oxide content by about $30 \%$ from the value cited in Whittington et al. A review of the Waste Information Sheet, provided by Oak Ridge in February 1994, showed that our analysis is very close to that of the average waste sampled on site, especially for the major glass components. The contaminants, however, showed a large variation in the site study. 


\section{Ion Chromatography and Ion Selective Electrode Analysis}

The solution of the WETF sludge prepared for DCP analysis was also subjected to analyses by ion chromatography, which revealed the presence of $13 \mathrm{ppm}$ of sulfate in the solution which converts to $2.36 \mathrm{wt} \%$ of $\mathrm{SO}_{3}$ in the solid. Fluoride was also measured in the same solution using an ion selective electrode technique. The measured fluoride content was only slightly above the detection limit and a maximum fluoride content in the original solid material of about $1 \mathrm{wt} \%$ is estimated.

\section{Gamma Spectroscopy}

- The radionuclide content of WETF sludge was examined using gamma spectrometry. A TCLP extract of the WETF sludge was used for this purpose. As shown in Figure 4.2, the gamma spectrum of the extract shows the presence of both ${ }^{235} \mathrm{U}(6.2 \mathrm{E}-06 \mu \mathrm{Ci} / \mathrm{g})$ and ${ }^{137} \mathrm{Cs}(3.2 \mathrm{E}-06 \mu \mathrm{Ci} / \mathrm{g})$, as well as many daughter products of ${ }^{238} \mathrm{U}$.

\subsubsection{Summary of Waste Stream Characterization Results}

The selection of the four Oak Ridge waste streams studied introduced new compositional areas into the study as compared to the waste streams of the other two sites previously presented. The WETF sludge is a relatively large volume waste stream and an example of a large family of wastes generated by the precipitation of heavy metals from various process wastes (in this case, a biodenitration process) with calcium. The TSCA ash is another example of a high-calcium waste, although otherwise of quite different composition and origin from the WETF sludge. After calcium, the next most abundant cation in the WETF sludge is aluminum that, as the oxide, constitutes about $20 \mathrm{wt} \%$. The soil has large amounts of silica. Together, these observations suggest that the glasses formed will be of the general silica-lime-alumina type. Both the TSCA ash and the Pond Waste contain significant amounts of phosphorus that can lead to phase separation in silicate melts.

The primary contaminants in these wastes are uranium and nickel, which are present in significant amounts in both the Pond Waste and the WETF sludge. Barium, chromium and lead are present at the tenth of a percent concentration while arsenic, silver, and mercury are present at the tens of ppm concentration. The expected volume reduction of these wastes is large in view of the water contents of these materials ( $70 \%$ measured for the WETF sludge, $90 \%$ reported in site analysis for the TSCA bottom ash sludge). 


\subsection{Oak Ridge Vitrification Testing}

A total of 14 glasses were melted from the K-1407 Pond Waste surrogate, the WETF sludge, and the TSCA Ash and soil surrogates. The objectives of these melts and the properties of the resulting glasses are discussed below.

\subsubsection{Melts to Maximize K-1407 Pond Waste Loading}

The major difficulty with regard to the contaminants in the Pond Waste is likely to be due to nickel, which is found at a concentration of $2.72 \%$ on an oxide basis. $\mathrm{The}^{\mathrm{in}} \mathrm{i}^{2+}$ ion has a propensity to form spinels with the oxides of iron and chromium that are also found in the Pond Waste. While nickel-containing glasses can be formulated to pass the TCLP requirements, nickel leaching can lead to limitations on the waste loading for waste streams containing appreciable amounts of nickel. A further difficulty presented by the Pond Waste is the combination of the high concentrations of both calcium and phosphate, which is likely to create an immiscible phase upon melting that also may limit waste loadings.

As summarized in Table 4.4, three different glass melting series were prepared for the Oak Ridge Pond Waste. These correspond to the following objectives:

1) The Pond Waste could be vitrified alone in the event that no other waste streams could be made available; minimum additives should be used.

2) The Pond Waste could be vitrified using contaminated soil. The formulations need not have the highest Pond Waste loadings since a second waste stream is also used.

3) The Pond Waste could also be vitrified as a secondary waste stream with the soil and one of the other two waste streams (WETF sludge or TSCA ash). This option is addressed in the following section.

Three glasses (ORNL1, ORNL2, and ORNL3) were prepared based on objective 1. They correspond, respectively, to $100 \%$ Pond Waste, $89.3 \%$ Pond Waste+ $2.8 \% \mathrm{Na}_{2} \mathrm{O}+8.0 \% \mathrm{SiO}_{2}$, and 82.7\% Pond Waste+5.1\% $\mathrm{Na}_{2} \mathrm{O}+12.2 \% \mathrm{SiO}_{2}$ (Table 4.4).

The second series of four glasses (ORNL4, ORNL5, ORNL6, and ORNL7) were melted with soil and Pond Waste surrogate, according to the proportions described in Table 4.4 and correspond to the second objective, above. Their respective waste loadings (Pond Waste + soil) correspond to $85 \%, 95 \%, 75 \%$, and $75 \%$. 
When melting glass composition ORNL6 (shown in Table 4.4), it was determined that the soil was very difficult to melt due to its refractory nature and large particle size. This may be a kinetic effect that is unique to crucible melts due to the relatively short melting time. Glass ORNL7 was therefore batched to produce the same composition as ORNL6 using chemicals (surrogate soil using the analyzed composition in Table 4.3) in place of the actual soil. The DCP compositional analysis of the ORNL6 glass showed that the silica content was low because the soil did not dissolve completely, whereas the compositional analyses of ORNL7 were very close to the target composition. Surrogate soil was used in all subsequent melts.

The following three glasses formulations (Table 4.4) were selected after review of the preliminary work done previously with the surrogate Pond Waste and soil; all of these glasses were spiked with $\mathrm{Tc}-99$ as well as $\mathrm{As}, \mathrm{Cr}, \mathrm{Cu}, \mathrm{Ni}, \mathrm{Pb}, \mathrm{Zn}$, and $\mathrm{U}-238$.

Glass ORNL8 was produced from the Pond Waste surrogate directly without additives. Glass ORNL9 was prepared with $50 \%$ Pond Waste, $25 \%$ soil, and $25 \%$ additives, whereas ORNL10 contained $40 \%$ Pond Waste, $25 \%$ soil, and $35 \%$ additives. All three glasses were melted at $1300^{\circ} \mathrm{C}$.

\subsubsection{Melts to Maximize WETF Sludge or TSCA Bottom Ash}

Both the WETF sludge and the TSCA bottom ash wastes are predominantly calcia (with $66 \%$ and $63 \%$, respectively) and some alumina (19\% and $12 \%$, respectively). Due to this similarity, they are not themselves particularly complementary compositions for use in a MAWS glass formulation. If these wastes are considered separately, they would be unlikely to constitute more than about $50 \%$ or so of the glass formulation due to their low glass-former contents. Formulations of glasses using these wastes were therefore split into two sets of three glasses and a similar approach was taken for the two series:

(i) A mixture in equal proportions with the alumino-silicate Oak Ridge soil;

(ii) Addition of Pond Waste to (i); and,

(iii) Addition of additives in exchange for a fraction of the soil.

Six glass formulations (ORNL11 to ORNL16) based on this approach were prepared. The first three (with WETF sludge as the primary waste) were all melted in one hour at $1400^{\circ} \mathrm{C}$, while the last three (with TSCA ash as the primary waste) were melted at $1450^{\circ} \mathrm{C}$ for an additional half hour since the first hour at $1400^{\circ} \mathrm{C}$ did not result in complete melting. 


\subsubsection{Glass Testing}

\section{DCP Glass Analysis}

Samples of all of the glasses in these series were dissolved in $\mathrm{HF} / \mathrm{HNO}_{3}$ solutions and analyzed by DCP. The analyzed and targeted compositions are presented in Table 4.4; the overall agreement was good for these glasses except for those prepared using the site soil directly (ORNL5, ORNL6, ORNL7), for the reasons discussed above. These results are generally as would be expected since little compositional variability was expected for WETF waste since this sludge is relatively easily homogenized and the other waste streams were simulated.

\section{ICPMS}

The results from ICPMS analyses of glasses ORNL8, ORNL9A, and ORNL10 are shown in Table 4.5. The four contaminants that could not be detected by DCP are confirmed to be at the concentration targeted. Note that the value listed in the table ( $\mathrm{ppb}$ in solution) must be multiplied by 2000 to convert to the concentration in the glass ( 0.1 g glass dissolved into $200 \mathrm{ml}$ ), as is shown in the column headed "OCS" (original concentration in solid).

\section{Microstructure of Oak Ridge Glasses}

Three glasses, ORNL1, ORNL2, and ORNL3 were subjected to microstructural analysis by SEM-EDS methods. Melt ORNL1 produced a black semi-vitreous substance. SEM examination revealed a complex and varied microstructure containing occasional small clusters of spinels with individual crystals about $5-10 \mu \mathrm{m}$ in diameter, widespread crystallization of smaller dendritic spinels, and a background of dark and light lamellar regions, the light regions being higher in $\mathrm{Ca}$, $\mathrm{P}$, and $\mathrm{Ni}$, and the dark regions being higher in $\mathrm{Si}, \mathrm{Al}$, and $\mathrm{K}$. Some $\mathrm{Cu}$ always seemed to incorporate into the spinels and occasionally one would observe a region very high in copper (spherical, $<1 \mu \mathrm{m}$ diameter). XRD performed on ORNL1 produced results consistent with whitlockite $(\mathrm{Ca}, \mathrm{Mg})_{3}\left(\mathrm{PO}_{4}\right)_{2}$, spinels (trevorite, $\left(\mathrm{FeNiFeO}_{4}^{-}\right)$, and others) and possibly a $\mathrm{Cu}-\mathrm{Ni}$ compound. There are a few small glassy regions near the surface in ORNL1.

Figures 4.3 and 4.4 show the microstructure of ORNL1. Figure 4.3 shows one of the few regions in ORNL1 that did not contain massive dendritic spinels or highly differentiated microstructure; a few spinels have nucleated, and dendrites are evident at some of the corners of these crystals. These larger spinel crystals contain $\mathrm{Al}$ and $2-5 \mathrm{wt} \% \mathrm{Cr}$; the $\mathrm{Cr}$ concentration is greater towards the center of the crystal, as is also true for $\mathrm{Fe}$ and $\mathrm{Ni}$ which are the principal constituents of the dendritic spinels. The texture of the glass is modified near the spinel, but there 
is no major difference in the composition. Figure 4.4 shows the usual structure of this sample. It has the massive dendritic spinel and lamellar light and dark regions mentioned above. The spinels constitute $25-35$ vol\% of the bulk.

Figure 4.5 shows a low magnification view in ORNL2 of the extensive type of dendritic spinel growth seen in both ORNL1 and 2. This was at the edge of the sample. At some places in the background, the division into lighter and darker lamellar regions is still just discernible at this magnification. Again, the fraction of spinels in this material is $25-35 \mathrm{vol} \%$.

Figures 4.6, 4.7, 4.8, and 4.9 show the microstructure of ORNL3. Figure 4.6 shows a region of phase separation accompanied by small spinel growth. The dendritic crystals are poor in $\mathrm{Ni}$, unlike the large dendritic spinels seen in ORNL1 and ORNL2. Note that they are in the Si-Al rich (dark) regions where there is normally less Ni. The small bright dots in the lighter phase-separated regions are higher in nickel. Figure 4.7 shows a region of fine grained phase separation and possibly incipient spinel growth (regions rich in $\mathrm{Ni}$ and $\mathrm{Fe}$ ). Figure 4.8 shows a cluster of high-Cr spinels surrounded by a small amount of dendritic spinel growth, both of which are rare in this sample. Figure 4.9 is a low magnification micrograph that shows the extent and variation in spatial scale of phase separation in ORNL3. Although the large-scale structure is that of amorphous phase separation, the fine-scale graininess visible here indicates considerable devitrification. 'Overall, ORNL3 shows a great deal of variety in the microstructure, but not the massive dendritic spinel growth of ORNL1 and 2. Phase separation is evident on a variety of spatial scales, and there are some small $(<1 \mu \mathrm{m})$ spherical voids in one area.

A sample of ORNL 7 prepared for the SEM contained about 0.3 vol.\% Cr-Fe-Ni spinel with one large cluster about $50 \times 100 \mu \mathrm{m}$ in dimension. This latter cluster lacked the angular facets normally seen on smaller crystals.

Samples of ORNL9 and ORNL10, prepared for the SEM, revealed only a uniform vitreous microstructure with a very few spinels.

The microstructure of the SEM sample of ORNL12 exhibited several areas enriched in $\mathrm{Si}$, which indicate undissolved or partially dissolved silica, but it is nonetheless vitreous and free from crystallization. 


\section{Melt Viscosity and Electrical Conductivity}

As discussed above the high Pond Waste glasses tend to be subject to considerable devitrification. The effective viscosity of a glass melt can increase dramatically as crystal growth occurs, which can lead to additional processing difficulties. Melt viscosity and electrical conductivity measurements were made on samples ORNL1 to ORNL10. The results for ORNL3 are included in Table 4.6. The impact of crystallization starting at around $1250^{\circ} \mathrm{C}$ is clear from the dramatic change between 17 Poise at $1300^{\circ} \mathrm{C}$ and 490 Poise at $1250^{\circ} \mathrm{C}$. On this basis, it would appear that a processing temperature of above about $1300^{\circ} \mathrm{C}$ would be acceptable. Other glasses in the series appeared to be more stable with respect to crystallization.

As shown in Figure 4.10, increases in the amount of Pond Waste in the formulations tended to increase the melt viscosity. The general effect of the additives used is most apparent in ORNL10 where the viscosity-temperature curve is considerably flattened with the result that the glass working range is moved to lower temperatures (down to about $1100^{\circ} \mathrm{C}$ for ORNL10). The electrical conductivities of these melts are low but are within the necessary range to make Joule-heated vitrification a viable alternative, even at temperatures as low as $1200^{\circ} \mathrm{C}$.

Of the remaining glasses, many show rather higher viscosities due to the increased soil contents (Figure 4.11). In contrast, the two formulations with the lowest soil fractions, ORNL13 and ORNL16, show that small amounts of additives can be used when a lower melt temperature is needed; these are also the only formulations containing a significant amount of sodium (respectively, $7.4 \%$ and $5.4 \%$ ). Note that since these glass formulations also tended to crystallize at the lower end of the measurement range, the results are reported as "Unstable" at those temperatures. The results also indicate that the liquidus temperature is reduced as the waste loading is decreased for these glasses. Together, these results suggest that higher temperature processing may be the preferred option in order to maximize waste loading for these wastes; however, trade-offs, such as increased volatilization of contaminants and increased process complexity, should also be considered.

The electrical conductivities of these glasses remained generally low despite the low viscosity. However, the additional effect of the density of charge carriers (primarily the alkali elements and, to a much lesser extent, the alkaline earth elements) must also be considered. These glass compositions generally contain a total of only about $2 \mathrm{wt} \%$ of the three alkali metal oxides; the two exceptions, ORNL13 and ORNL16, which used soda as an additive, showed lower viscosities and higher electrical conductivities. 


\section{PCT Leach Testing}

Results for all of the glasses that were subjected to PCT leach testing are presented in Table 4.7, which includes data from the standard seven-day PCT test as well as for subsequent samplings extending up to 365 days. The results are also shown in Figures 4.12 to 4.17 where they are compared to the corresponding results for the SRL-EA glass. The seven-day normalized leachate concentrations are between 16 and 100 times lower than those for SRL-EA glass (Table 4.7).

\section{TCLP}

Results for all of the glasses that were subjected to leach testing using the TCLP are presented in Table 4.8. The results for the nickel and uranium release from samples ORNL1-3 are shown in Figure 4.18. The uranium release from these materials varies approximately in proportion to its concentration in the solid whereas this is clearly not the case for the nickel release. Nickel release from ORNL1 and ORNL2, both of which contained high levels (25-35 vol\%) of nickelbearing spinels, is ten times lower than for ORNL3, which is more vitreous and contains less spinel ( $<1 \mathrm{vol} \%)$. The release level is below the RCRA limit for all elements, including nickel for the two first glasses, but the nickel concentration in the leachates of the third glass exceeded the TCLP LDR limit

The TCLP leachate concentrations for glasses ORNL7, ORNL8, ORNL9, and ORNL10 were also generally low. These leachates were also analyzed by ICPMS for ${ }^{99} \mathrm{Tc}$. The leachate concentrations were lower $(<0.3 \mathrm{ppb})$ than the Drinking Water Standard (5.9 ppb; DOE Order 54-00.5).

Since the WETF waste and the TSCA waste are listed under different waste codes, the TCLP leachates of the glasses produced from them should be compared to the corresponding regulatory limits (see Table 4.8). Accordingly, glasses ORNL11 to ORNL13 are compared to the limits for the WETF listed waste (F006/F007), while ORNL14 to ORNL16 are compared to the TSCA Ash characteristic waste limits (D004-D011), as is shown for lead in Figure 4.19.

The concentrations of most elements $(\mathrm{Cr}, \mathrm{As}, \mathrm{Ag}, \mathrm{Ba})$ in the glass leachates are two orders of magnitude lower than their respective limits. Cadmium is lower by 10 to 16 times in the glass leachates, the largest reduction being when the Pond Waste is used and the WETF waste fraction is decreased. Similar results are found in the case of the TSCA ash with lead, where the ORNL15 has a leachate concentration, a factor of 80 below the limit. Mercury from the WETF waste stream was found to leach from a glass such as ORNL12 by a factor of 40 below the $0.2 \mathrm{ppm}$ limit. The best glass leaching results are found when the Pond Waste was included as part of the waste blend. 
At present there is no regulatory TCLP limit for uranium. For the purposes of comparison we have used the "Ingested water limit" in DOE Order 5400.5 (1.78 ppm for $\left.{ }^{238} \mathrm{U}\right)$. This limit is about 7 times higher than the concentrations found in the glass leachates, except for ORNL16 which leached $2.25 \mathrm{ppm}$ of uranium. This latter glass formulation has the lowest level of glass formers (including silica and alumina), and poorer leach resistance for all of the analytes.

Of the series of glasses ORNL11-13, ORNL12 performs significantly better on the TCLP test than the other two. The major composition difference is due to the incorporation of the Pond Waste in ORNL12, which primarily increases the iron and phosphorus (and nickel) contents; in most other respects, this glass composition is intermediate between the other two. We believe the improvement to be due primarily to the increased iron content, but the increased level of phosphorus may also be important. It is also worth noting that the change from ORNL11 to ORNL13, at the cost of $20 \%$ in waste loading due to the additions of soda, boria, and silica, gave only a marginal improvement.

For comparative purposes, the TCLP was also performed on the dried, but otherwise untreated, WETF sludge. The leachate analysis is included in Table 4.8 and shows that contaminant levels were 10 to 20 times higher than those for the MAWS glasses reported here with values as high as 37 times greater for lead.

\subsubsection{Summary and Conclusions}

Of the waste streams selected for this study, one sample of actual waste (WETF) was obtained from the Oak Ridge site and three other waste streams were simulated. The simulants did include the radioactive and hazardous constituents of concern but it is unknown whether these added constituents are representative of that in the actual wastes. The waste streams selected introduced new compositional areas into this study as compared to the waste streams from Hanford and Idaho discussed earlier. The WETF sludge is both a relatively large volume waste stream and an example of a large family of wastes, generated by the precipitation of heavy metals from various process wastes (in this case, a biodenitration process) with calcium. The TSCA ash is another example of a high-calcium waste, although otherwise of quite different composition and origin from the WETF sludge. After calcium, the next most abundant cation in the WETF sludge is aluminum that, as the oxide, constitutes about $20 \mathrm{wt} \%$. The soil is rich in silica. Together these observations suggest that high waste loading glasses would be of the general silica-lime-alumina type. Both the TSCA ash and the Pond Waste contain significant amounts of phosphorus that can lead to phase separation in silicate melts. The primary contaminants in these wastes are uranium and nickel, which are present in significant amounts in both the Pond Waste and the WETF sludge. Barium, chromium and lead are present at the tenth of a percent level while arsenic, silver, and mercury are present at the tens of ppm level. 
A variety of waste blends were investigated to demonstrate the flexibility of the general MAWS formulation approach. It was found that both the WETF sludge and the TSCA ash wastes can be blended with contaminated soil and minimal amounts of additives to produce glasses with good leach resistance as well as acceptable melt characteristics for processing. The ternary systems investigated were the WETF/Pond Waste/Soil system and the TSCA ash/Pond Waste/Soil system. Both of these combinations can be used to produce glasses and melts with acceptable characteristics for vitrification; in addition, the glasses formed from these blends (which tend to be higher in both iron and phosphorus) show a significant reduction in the measured TCLP leachate concentrations.

The results of this study suggest that viable glass formulations can be developed for a wide range of combinations of Oak Ridge wastes with high waste loading (in some cases $100 \%$ ). The results show the general ranges of glass and melt properties that are achievable and would be suitable for processing in a variety of vitrification technologies. The leach resistance of the waste forms produced are uniformly high on the basis of the PCT. All but one of the glasses produced leached considerably less than any of the RCRA TCLP release limits. Since only one actual waste was used (all of the others were chemically simulated), the volume reductions could not be directly measured for the glasses that were produced (although a lower bound on the basis of the WETF sludge alone could be obtained).

The Oak Ridge glasses that have been subjected to viscosity, conductivity, TCLP, and PCT testing (i.e., those glasses in the core data set of 40 glasses, as discussed in Section 1.3) are listed in Table 4.9. Table 4.9 also lists representative data for each characteristic, consisting of the viscosity and conductivity values at $1250^{\circ} \mathrm{C}$, the uranium concentrations in the TCLP leachates, and the sodium concentration in the PCT leachates after seven days.

In summary, vitreous waste forms have been produced with large volume reductions from blends of four Oak Ridge waste streams using a MAWS vitrification approach. Since these waste streams are of quite diverse chemical compositions, it is certainly likely that other Oak Ridge waste streams could also be incorporated. 
Table 4.1

Oak Ridge Surrogates Waste Formulations

\begin{tabular}{|c|c|c|c|c|c|c|}
\hline & \multicolumn{3}{|c|}{ K-1407 Pond Waste Recipe } & \multicolumn{3}{|c|}{ TSCA Ash Recipe } \\
\hline & $\begin{array}{l}\text { Oxide } \\
\text { Wt\% }\end{array}$ & Chemical & Grams & $\begin{array}{l}\text { Oxide } \\
\text { Wt } \%\end{array}$ & Chemical & Grams \\
\hline $\mathrm{Al}_{2} \mathrm{O}_{3}$ & 13.381 & $\mathrm{Al}_{2} \mathrm{O}_{3}$ & 13.381 & 11.575 & $\mathrm{Al}_{2} \mathrm{O}_{3}$ & 11.575 \\
\hline $\mathrm{As}_{2} \mathrm{O}_{3}$ & 0.029 & $\mathrm{As}_{2} \mathrm{O}_{3}$ & 0.029 & 0.000 & $\mathrm{As}_{2} \mathrm{O}_{3}$ & \\
\hline $\mathrm{CaO}$ & 16.618 & $\mathrm{CaCO}_{3}$ & 29.659 & 62.474 & $\mathrm{CaCO}_{3}$ & 111.501 \\
\hline $\mathrm{Cr}_{2} \mathrm{O}_{3}$ & 0.330 & $\mathrm{Cr}_{2} \mathrm{O}_{3}$ & 0.330 & 0.030 & $\mathrm{Cr}_{2} \mathrm{O}_{3}$ & 0.030 \\
\hline $\mathrm{CuO}$ & 0.273 & $\mathrm{CuO}$ & 0.273 & 0.522 & $\mathrm{CuO}$ & 0.522 \\
\hline $\mathrm{Fe}_{2} \mathrm{O}_{3}$ & 19.216 & $\mathrm{Fe}_{2} \mathrm{O}_{3}$ & 19.216 & 4.337 & $\mathrm{Fe}_{2} \mathrm{O}_{3}$ & 4.337 \\
\hline $\mathrm{K}_{2} \mathrm{O}$ & 4.083 & $\mathrm{~K}_{2} \mathrm{CO}_{3}$ & 5.990 & 0.552 & $\mathrm{~K}_{2} \mathrm{CO}_{3}$ & 0.810 \\
\hline $\mathrm{MgO}$ & 3.108 & $\mathrm{MgCO}_{3}(84 \%)$ & 6.501 & 2.881 & $\mathrm{MgCO}_{3}(84 \%)$ & 6.027 \\
\hline $\mathrm{MnO}_{2}$ & 0.894 & $\mathrm{MnO}_{2}$ & 0.894 & 0.100 & $\mathrm{MnO}_{2}$ & 0.100 \\
\hline $\mathrm{Na}_{2} \mathrm{O}$ & 1.751 & $\mathrm{NaHCO}_{3}$ & 3.098 & 0.883 & $\mathrm{NaHCO}_{3}$ & 2.395 \\
\hline $\mathrm{NiO}$ & 2.718 & $\mathrm{NiO}$ & 2.718 & 0.141 & $\mathrm{NiO}$ & 0.141 \\
\hline $\mathrm{P}_{2} \mathrm{O}_{5}$ & 16.688 & $\left(\mathrm{NH}_{4}\right)_{2} \mathrm{HPO}_{4}$ & 31.050 & 11.696 & $\left(\mathrm{NH}_{4}\right)_{2} \mathrm{HPO}_{4}$ & 21.762 \\
\hline $\mathrm{PbO}$ & 0.022 & $\mathrm{PbO}$ & 0.022 & 0.351 & $\mathrm{PbO}$ & 0.351 \\
\hline $\mathrm{SO}_{3}$ & 0.637 & $\mathrm{Na}_{2} \mathrm{SO}_{4}$ & 0.565 & 0.000 & $\mathrm{Na}_{2} \mathrm{SO}_{4}$ & \\
\hline $\mathrm{SiO}_{2}$ & 17.968 & $\mathrm{SiO}_{2}$ & 17.968 & 2.450 & $\mathrm{SiO}_{2}$ & 2.450 \\
\hline $\mathrm{TiO}_{2}$ & 1.634 & $\mathrm{TiO}_{2}$ & 1.634 & 0.301 & $\mathrm{TiO}_{2}$ & 0.301 \\
\hline $\mathrm{U}_{3} \mathrm{O}_{8}$ & 0.444 & $\mathrm{U}_{3} \mathrm{O}_{8}$ & 0.444 & 1.436 & $\mathrm{U}_{3} \mathrm{O}_{8}$ & 1.436 \\
\hline $\mathrm{ZnO}$ & 0.103 & $\mathrm{ZnO}$ & 0.103 & 0.268 & $\mathrm{ZnO}$ & 0.268 \\
\hline $\mathrm{Cl}$ & 0.069 & $\mathrm{NaCl}$ & 0.113 & 0.000 & $\mathrm{NaCl}$ & \\
\hline $\mathrm{F}$ & 0.034 & $\mathrm{NaF}$ & 0.075 & 0.000 & $\mathrm{NaF}$ & \\
\hline Sum \% & 100.000 & Sum (g) & 134.065 & 100.000 & Sum (g) & 164.005 \\
\hline
\end{tabular}


Table 4.2

Summary of Oak Ridge Waste Physical Characterization

\begin{tabular}{|c|c|c|c|c|}
\hline $\begin{array}{c}\text { Idaho } \\
\text { Samples }\end{array}$ & $\begin{array}{c}\text { Soil } \\
\text { Sample OR1 }\end{array}$ & $\begin{array}{l}\text { WETF sludge } \\
\text { Sample OR2 }\end{array}$ & $\begin{array}{c}\text { K-1407 Pond } \\
\text { Waste } \\
\text { Surrogate }\end{array}$ & $\begin{array}{l}\text { TSCA Ash } \\
\text { Surrogate }\end{array}$ \\
\hline \multicolumn{5}{|c|}{ Thermogravimetric Analysis (weight loss \% as a function of treatments) } \\
\hline $110^{\circ} \mathrm{C} / 18 \mathrm{hr}$ & $9.69 \%$ & $69.95 \%$ & N.A. & N.A. \\
\hline $450^{\circ} \mathrm{C} / 4 \mathrm{hr}$ & $20.1 \%$ & $72.69 \%$ & N.A. & N.A. \\
\hline $1150^{\circ} \mathrm{C} / 4 \mathrm{hr}$ & $22.9 \%$ & $81.93 \%$ & $25.41 \%^{\mathrm{d}}$ & $39.03 \%^{\mathrm{a}}$ \\
\hline \multicolumn{5}{|c|}{ Specific Gravity, $\mathrm{g} / \mathrm{cm}^{3}$} \\
\hline ASTM D854-83 & 3.04 & 2.5 & N.A. & N.A. \\
\hline Bulk dry density & 1.13 & N.A. & N.A. & N.A. \\
\hline \multicolumn{5}{|c|}{ Particle size Analysis: retained particles \% as a function of U.S. Mesh values } \\
\hline Mesh 10 & 97.59 & N.A. & N.A. & N.A. \\
\hline Mesh 40 & 1.67 & N.A. & N.A. & N.A. \\
\hline Mesh 60 & 0.16 & N.A. & N.A. & N.A. \\
\hline Mesh 100 & 0.23 & N.A. & N.A. & N.A. \\
\hline Mesh 200 & 0.23 & N.A. & N.A. & N.A. \\
\hline Passing 200 & 0.13 & N.A. & N.A. & N.A. \\
\hline \multicolumn{5}{|c|}{ Total Carbon (TC) and Total Organic carbon (TOC) in ppm } \\
\hline $\mathrm{TC}^{\mathrm{b}}$ & N.A. & $91764 \pm 943$ & N.A. & $86000^{\circ}$ \\
\hline TOC $^{b}$ & N.A. & $11333 \pm 288$ & 0 for surrogate & 0 for surrogate \\
\hline
\end{tabular}

a This value would be as high as $90 \%$ with the real waste according to site's waste characterization data.

${ }^{b}$ Average of 9 measurements on material dried at $100^{\circ} \mathrm{C}$.

' The total carbon value is taken from the site's waste characterization data.

${ }^{d}$ Does not include weight loss from water present in the real waste.

N.A. $=$ Not Available or Not Applicable. 
Table 4.3

Composition of Wastes Used in Oak Ridge Vitrification (wt\%)

\begin{tabular}{|c|c|c|c|c|}
\hline Sample Name & Soil & WETF & Pond Waste & TSCA Ash \\
\hline Oxides & Analyzed & Analyzed & Target & Target \\
\hline $\mathrm{Al}_{2} \mathrm{O}_{3}$ & 15.22 & 18.76 & 13.38 & 11.58 \\
\hline $\mathrm{AgO}$ & & 0.003 & & $2 \mathrm{ppm}^{\mathrm{a}}$ \\
\hline $\mathrm{As}_{2} \mathrm{O}_{3}$ & & & 0.03 & \\
\hline $\mathrm{B}_{2} \mathrm{O}_{3}$ & 0.04 & & & \\
\hline $\mathrm{BaO}$ & & 0.07 & & \\
\hline $\mathrm{CaO}$ & 0.07 & 65.71 & 16.62 & 62.47 \\
\hline $\mathrm{CdO}$ & & 0.006 & & $2 \mathrm{ppm}^{\mathrm{a}}$ \\
\hline $\mathrm{Cr}_{2} \mathrm{O}_{3}$ & 0.01 & 0.13 & 0.33 & 0.03 \\
\hline $\mathrm{CuO}$ & & 0.27 & 0.27 & 0.52 \\
\hline $\mathrm{Fe}_{2} \mathrm{O}_{3}$ & 5.70 & 1.97 & 19.22 & 4.34 \\
\hline $\mathrm{HgO}$ & & $<10 \mathrm{ppm}$ & & \\
\hline $\mathrm{K}_{2} \mathrm{O}$ & 0.81 & 0.07 & 4.08 & 0.55 \\
\hline $\mathrm{Li}_{2} \mathrm{O}$ & & 0.53 & & \\
\hline $\mathrm{MgO}$ & 0.49 & 4.67 & 3.11 & 2.88 \\
\hline $\mathrm{MnO}_{2}$ & 0.06 & & 0.89 & 0.10 \\
\hline $\mathrm{Na}_{2} \mathrm{O}$ & 0.04 & 0.70 & 1.75 & 0.88 \\
\hline $\mathrm{NiO}$ & 0.01 & 0.34 & 2.72 & 0.14 \\
\hline $\mathrm{P}_{2} \mathrm{O}_{5}$ & 0.16 & 1.06 & 16.69 & 11.70 \\
\hline $\mathrm{PbO}$ & & 0.09 & 0.02 & 0.35 \\
\hline $\mathrm{SO}_{3}$ & & 2.36 & 0.64 & \\
\hline $\mathrm{SiO}_{2}$ & 76.68 & 1.99 & 17.97 & 2.45 \\
\hline $\mathrm{TiO}_{2}$ & 0.67 & & 1.63 & 0.30 \\
\hline $\mathrm{U}_{3} \mathrm{O}_{8}$ & 0.00 & 1.19 & 0.44 & 1.44 \\
\hline $\mathrm{ZnO}$ & & 0.07 & 0.10 & 0.27 \\
\hline $\mathrm{Cl}$ & & traces & 0.07 & \\
\hline $\mathrm{F}$ & & $\sim 1.0$ & 0.03 & \\
\hline Sum & 100.00 & 100.00 & 100.00 & 100.0 \\
\hline
\end{tabular}

${ }^{a}$ These values were reported in the site's waste characterization data sheet; they were not included in glass formulation. 
Table 4.4

Summary of Oak Ridge Glass Compositions (wt\%)

\begin{tabular}{|c|c|c|c|c|c|c|c|c|}
\hline \multirow[t]{2}{*}{ Glass } & \multicolumn{2}{|c|}{ ORNL1 } & \multicolumn{2}{|c|}{ ORNL2 } & \multicolumn{2}{|c|}{ ORNL3 } & \multicolumn{2}{|c|}{ ORNLA } \\
\hline & Target & Analysis & Target & Analysis & Target & Analysis & Target & Analysis \\
\hline Soil \% & & & & & 1 & & $15.00 * *$ & \\
\hline Pond Waste \% & 100.00 & & 89.29 & & 82.68 & & 70.00 & \\
\hline TSCA Ash \% & & & & & & & & \\
\hline WETF \% & & & & & & & & \\
\hline Added $\mathrm{B}_{2} \mathrm{O}_{3}$ & & & & & & & 10.38 & \\
\hline Added $\mathrm{Na}_{2} \mathrm{O}$ & & & 2.68 & & 5.11 & & 4.62 & \\
\hline Added $\mathrm{SiO}_{2}$ & & & 8.04 & & 12.20 & & 0.00 & \\
\hline $\mathrm{Al}_{2} \mathrm{O}_{3}$ & 13.38 & 13.04 & 11.95 & 11.22 & 11.06 & 10.42 & 11.65 & 5.05 \\
\hline $\mathrm{AgO}$ & & & 0.00 & & 0.00 & & 0.00 & \\
\hline $\mathrm{As}_{2} \mathrm{O}_{3}$ & 0.03 & 0.03 & 0.03 & 0.03 & 0.02 & 0.01 & 0.02 & 0.03 \\
\hline $\mathrm{B}_{2} \mathrm{O}_{3}$ & 0.00 & 0.47 & 0.00 & 0.21 & 0.00 & 0.08 & 10.39 & 11.31 \\
\hline $\mathrm{BaO}$ & & & 0.00 & & 0.00 & & 0.00 & \\
\hline $\mathrm{CaO}$ & 16.62 & 16.83 & 14.84 & 14.88 & 13.74 & 13.47 & 11.64 & 11.81 \\
\hline$\dot{\mathrm{CdO}}$ & & & 0.00 & & 0.00 & & 0.00 & \\
\hline $\mathrm{Cr}_{2} \mathrm{O}_{3}$ & 0.33 & 0.45 & 0.29 & 0.29 & 0.27 & 0.21 & 0.23 & 0.24 \\
\hline $\mathrm{CuO}$ & 0.27 & 0.19 & 0.24 & 0.19 & 0.23 & 0.14 & 0.19 & 0.14 \\
\hline $\mathrm{Fe}_{2} \mathrm{O}_{3}$ & 19.22 & 18.78 & 17.16 & 16.25 & 15.89 & 15.12 & 14.31 & 14.70 \\
\hline $\mathrm{K}_{2} \mathrm{O}$ & 4.08 & 2.91 & 3.65 & 2.37 & 3.38 & 2.81 & 2.98 & 2.25 \\
\hline $\mathrm{Li}_{2} \mathrm{O}$ & & & 0.00 & & 0.00 & & 0.00 & \\
\hline $\mathrm{MgO}$ & 3.11 & 2.92 & 2.78 & 2.59 & 2.57 & 2.24 & 2.25 & 2.06 \\
\hline $\mathrm{MnO}_{2}$ & 0.89 & 0.94 & 0.80 & 0.84 & 0.74 & 0.71 & 0.63 & 0.64 \\
\hline $\mathrm{Na}_{2} \mathrm{O}$ & 1.75 & 2.12 & 4.24 & 4.34 & 6.56 & 6.72 & 5.85 & 6.86 \\
\hline $\mathrm{NiO}$ & 2.72 & 2.64 & 2.43 & 2.40 & 2.25 & 1.44 & 1.90 & 1.82 \\
\hline $\mathrm{P}_{2} \mathrm{O}_{5}$ & 16.69 & 16.99 & 14.90 & 14.97 & 13.80 & 15.31 & 11.70 & 12.30 \\
\hline $\mathrm{PbO}$ & 0.02 & 0.017 & 0.02 & 0.025 & 0.02 & 0.016 & 0.02 & 0.021 \\
\hline $\mathrm{SO}_{3}$ & 0.64 & & 0.57 & N.A. & 0.53 & N.A. & 0.45 & \\
\hline $\mathrm{SiO}_{2}$ & 17.97 & 19.42 & 24.08 & 26.94 & 27.06 & 29.47 & 24.08 & 28.96 \\
\hline $\mathrm{TiO}_{2}$ & 1.63 & 1.74 & 1.46 & 1.64 & 1.35 & 1.38 & 1.24 & 1.37 \\
\hline $\mathrm{U}_{2} \mathrm{O}_{8}$ & 0.44 & 0.43 & 0.40 & 0.43 & 0.37 & 0.33 & 0.31 & 0.35 \\
\hline $\mathrm{ZnO}$ & 0.10 & 0.09 & 0.09 & 0.38 & 0.09 & 0.11 & 0.07 & 0.09 \\
\hline $\mathrm{Cl}$ & 0.07 & 0.07 & 0.06 & N.A. & 0.06 & N.A. & 0.05 & \\
\hline F & 0.03 & 0.03 & 0.03 & N.A. & 0.03 & N.A. & 0.02 & \\
\hline Sum & 100.00 & 100.00 & 100.00 . & 100.00 & 100.00 & 100.00 & 100.00 & 100.00 \\
\hline
\end{tabular}

**The soil did not dissolve properly in these melts. It was replaced in ORNL7 and subsequent melts by a surrogate formulation. 


\begin{tabular}{|c|c|c|c|c|c|c|c|c|}
\hline \multirow[t]{2}{*}{ Glass } & \multicolumn{2}{|c|}{ ORNL5 } & \multicolumn{2}{|c|}{ ORNL6 } & \multicolumn{2}{|c|}{ ORNL7 } & \multicolumn{2}{|c|}{ ORNL8 } \\
\hline & Target & Analysis & $\mathrm{wt} \%$ & Analyzed & Target & Analyzed & Target & Analyzed \\
\hline Soil \% & $25.00^{* *}$ & & $25.00^{* *}$ & & 25.00 & & & \\
\hline Pond Waste \% & 70.00 & & 50.00 & & 50.00 & & 100.00 & \\
\hline TSCA Ash \% & & & & & & & & \\
\hline WETF \% & & & & & & & & \\
\hline Added $\mathrm{B}_{2} \mathrm{O}_{3}$ & 0.00 & & 12.46 & & 12.46 & & & \\
\hline Added $\mathrm{Na}_{2} \mathrm{O}$ & 5.00 & & 5.54 & & 5.54 & & & \\
\hline Added $\mathrm{SiO}_{2}$ & 0.00 & & 7.00 & & 7.00 & & & \\
\hline $\mathrm{Al}_{2} \mathrm{O}_{3}$ & 13.17 & 14.10 & 10.50 & 12.37 & 10.50 & 10.25 & 13.38 & 13.13 \\
\hline $\mathrm{AgO}$ & 0.00 & & 0.00 & & 0.00 & & 0.00 & \\
\hline $\mathrm{As}_{2} \mathrm{O}_{3}$ & 0.02 & 0.02 & 0.01 & & 0.01 & & 0.03 & \\
\hline $\mathrm{B}_{2} \mathrm{O}_{3}$ & 0.01 & 0.14 & 12.47 & 13.92 & 12.47 & 13.07 & 0.00 & 0.54 \\
\hline $\mathrm{BaO}$ & 0.00 & & 0.00 & & 0.00 & & 0.00 & \\
\hline $\mathrm{CaO}$ & 11.65 & 10.30 & 8.33 & 8.98 & 8.33 & 8.52 & 16.62 & 15.47 \\
\hline $\mathrm{CdO}$ & 0.00 & & 0.00 & & 0.00 & & 0.00 & \\
\hline $\mathrm{Cr}_{2} \mathrm{O}_{3}$ & 0.23 & 0.18 & 0.17 & 0.15 & 0.17 & 0.12 & 0.33 & 0.26 \\
\hline $\mathrm{CuO}$ & 0.19 & 0.12 & 0.14 & & 0.14 & & 0.27 & \\
\hline $\mathrm{Fe}_{2} \mathrm{O}_{3}$ & 14.88 & 13.23 & 11.03 & 13.03 & 11.03 & 11.29 & 19.22 & 17.02 \\
\hline $\mathrm{K}_{2} \mathrm{O}$ & 3.06 & 2.41 & 2.24 & 1.76 & 2.24 & 1.69 & 4.08 & 2.72 \\
\hline $\mathrm{Li}_{2} \mathrm{O}$ & 0.00 & & 0.00 & & 0.00 & & 0.00 & \\
\hline $\mathrm{MgO}$ & 2.30 & 1.93 & 1.68 & 1.54 & 1.68 & 1.56 & 3.11 & 3.09 \\
\hline $\mathrm{MnO}_{2}$ & 0.64 & 0.59 & 0.46 & 0.49 & 0.46 & 0.48 & 0.89 & 0.88 \\
\hline $\mathrm{Na}_{2} \mathrm{O}$ & 6.24 & 2.39 & 6.43 & 6.86 & 6.43 & 6.82 & 1.75 & 1.97 \\
\hline $\mathrm{NiO}$ & 1.90 & 1.64 & 1.36 & 1.31 & 1.36 & 1.31 & 2.72 & 2.04 \\
\hline $\mathrm{P}_{2} \mathrm{O}_{5}$ & 11.72 & 10.80 & 8.38 & 8.67 & 8.38 & 8.89 & 16.69 & 15.57 \\
\hline $\mathrm{PbO}$ & 0.02 & 0.017 & 0.01 & & 0.01 & & 0.02 & \\
\hline $\mathrm{SO}_{3}$ & 0.45 & & 0.32 & & 0.32 & & 0.64 & 0.06 \\
\hline $\mathrm{SiO}_{2}$ & 31.75 & 40.40 & 35.15 & 29.48 & 35.15 & 34.56 & 17.97 & 25.00 \\
\hline $\mathrm{TiO}_{2}$ & 1.31 & 1.27 & 0.99 & 1.08 & 0.99 & 1.03 & 1.63 & 1.69 \\
\hline $\mathrm{U}_{2} \mathrm{O}_{8}$ & 0.31 & 0.37 & 0.22 & 0.21 & 0.22 & 0.23 & 0.44 & 0.42 \\
\hline $\mathrm{ZnO}$ & 0.07 & 0.09 & 0.05 & & 0.05 & & 0.10 & \\
\hline $\mathrm{Cl}$ & 0.05 & & 0.03 & & 0.03 & & 0.07 & \\
\hline$F$ & 0.02 & & 0.02 & & 0.02 & & 0.03 & \\
\hline Sum & 100.00 & 100.00 & 100.00 & 100.00 & 100.00 & 100.00 & 100.00 & 100.00 \\
\hline
\end{tabular}

**The soil did not dissolve properly in these melts. It was replaced in ORNL7 and subsequent melts by a surrogate formulation. 


\begin{tabular}{|c|c|c|c|c|c|c|c|c|}
\hline \multirow[t]{2}{*}{ Glass } & \multicolumn{2}{|c|}{ ORNL9A } & \multicolumn{2}{|c|}{ ORNL10 } & \multicolumn{2}{|c|}{ ORNLI1 } & \multicolumn{2}{|c|}{ ORNL12 } \\
\hline & Target & Analyzed & Target & Analyzed & Target & Analysis & Target & Analysis \\
\hline Soil \% & 25.00 & & 25.00 & & $50.00 \%$ & & $45.00 \%$ & \\
\hline Pond Waste \% & 50.00 & & 40.00 & & $0.00 \%$ & & $20.00 \%$ & \\
\hline TSCA Ash \% & & & & & $0.00 \%$ & & $0.00 \%$ & \\
\hline WETF \% & & & & & $50.00 \%$ & & $35.00 \%$ & \\
\hline Added $\mathrm{B}_{2} \mathrm{O}_{3}$ & 12.46 & & 12.46 & & & & & \\
\hline Added $\mathrm{Na}_{2} \mathrm{O}$ & 5.54 & & 10.54 & & & & & \\
\hline Added $\mathrm{SiO}_{2}$ & 7.00 & & 12.00 & & & & $0.00 \%$ & \\
\hline $\mathrm{Al}_{2} \mathrm{O}_{3}$ & 10.50 & 10.06 & 9.16 & 9.10 & 16.99 & 16.59 & 16.09 & 16.32 \\
\hline $\mathrm{AgO}$ & & & & & 0.0015 & 0.0008 & 0.0011 & 0.0010 \\
\hline $\mathrm{As}_{2} \mathrm{O}_{3}$ & 0.01 & & 0.01 & & 0.0000 & N.A. & 0.0058 & N.A. \\
\hline $\mathrm{B}_{2} \mathrm{O}_{3}$ & 12.47 & 13.03 & 12.47 & 12.48 & 0.02 & 0.39 & 0.02 & 0.11 \\
\hline $\mathrm{BaO}$ & & & & & 0.0350 & N.A. & 0.0245 & N.A. \\
\hline $\mathrm{CaO}$ & 8.33 & 8.49 & 6.67 & 7.00 & 32.89 & 30.60 . & 26.36 & 28.21 \\
\hline CdO & & & & & 0.003 & 0.00 & 0.002 & 0.00 \\
\hline $\mathrm{Cr}_{2} \mathrm{O}_{3}$ & 0.17 & 0.26 & 0.13 & 0.33 & 0.07 & 0.23 & 0.12 & 0.36 \\
\hline $\mathrm{CuO}$ & 0.14 & & 0.11 & & 0.14 & 0.15 & 0.15 & 0.17 \\
\hline $\mathrm{Fe}_{2} \mathrm{O}_{3}$ & 11.03 & 11.25 & 9.11 & 9.66 & 3.84 & 5.07 & 7.10 & 6.69 \\
\hline $\mathrm{K}_{2} \mathrm{O}$ & 2.24 & 1.79 & 1.83 & 1.50 & 0.44 & 0.65 & 1.20 & 0.99 \\
\hline $\mathrm{Li}_{2} \mathrm{O}$ & & & & & 0.27 & N.A. & 0.19 & N.A. \\
\hline $\mathrm{MgO}$ & 1.68 & 1.61 & 1.37 & 1.31 & 2.58 & 2.21 & 2.48 & 2.29 \\
\hline $\mathrm{MnO}_{2}$ & 0.46 & 0.52 & 0.37 & 0.41 & 0.03 & 0.22 & 0.20 & 0.35 \\
\hline $\mathrm{Na}_{2} \mathrm{O}$ & 6.43 & 6.32 & 11.26 & 10.41 & 0.37 & 1.71 & 0.61 & 1.61 \\
\hline $\mathrm{NiO}$ & 1.36 & 1.32 & 1.09 & 1.09 & 0.17 & 0.31 & 0.67 & 0.50 \\
\hline $\mathrm{P}_{2} \mathrm{O}_{5}$ & 8.38 & 7.75 & 6.71 & 6.91 & 0.61 & 2.12 & 3.78 & 3.64 \\
\hline $\mathrm{PbO}$ & 0.01 & & 0.01 & & 0.05 & 0.04 & 0.04 & 0.04 \\
\hline $\mathrm{SO}_{3}$ & 0.32 & 0.00 & 0.25 & 0.01 & 1.18 & N.A. & 0.95 & N.A. \\
\hline $\mathrm{SiO}_{2}$ & 35.15 & 36.12 & 38.36 & 38.61 & 39.33 & 38.68 & 38.80 & 37.41 \\
\hline $\mathrm{TiO}_{2}$ & 0.99 & 1.16 & 0.82 & 0.92 & 0.34 & 0.21 & 0.63 & 0.35 \\
\hline $\mathrm{U}_{2} \mathrm{O}_{8}$ & 0.22 & 0.24 & 0.18 & 0.19 & 0.60 & 0.53 & 0.51 & 0.51 \\
\hline $\mathrm{ZnO}$ & 0.05 & & 0.04 & & 0.04 & 0.29 & 0.05 & 0.42 \\
\hline $\mathrm{Cl}$ & 0.03 & & 0.03 & & 0.00 & N.A. & 0.01 & N.A. \\
\hline$F$ & 0.02 & & 0.01 & & 0.00 & N.A. & 0.01 & N.A. \\
\hline Sum & 100.00 & 100.00 & 100.00 & 100.00 & 100.00 & 100.00 & 100.00 & 100.00 \\
\hline
\end{tabular}

**'The soil did not dissolve properly in these melts. It was replaced in ORNL7 and subsequent melts by a surrogate formulation. 


\begin{tabular}{|c|c|c|c|c|c|c|c|c|}
\hline Glass & OR & L13 & OR & IL14 & OR & L15 & OR & IL16 \\
\hline$\%$ & Target & Analysis & Target & Analysis & Target & Analysis & Target & Analysis \\
\hline Soil \% & $40.00 \%$ & & $50.00 \%$ & & $45.00 \%$ & & $30.00 \%$ & \\
\hline Pond Waste \% & $0.00 \%$ & & $0.00 \%$ & & $10.00 \%$ & & $0.00 \%$ & \\
\hline TSCA Ash $\%$ & $0.00 \%$ & & $50.00 \%$ & & $45.00 \%$ & & $50.00 \%$ & \\
\hline WETF \% & $40.00 \%$ & & $0.00 \%$ & & $0.00 \%$ & & $0.00 \%$ & \\
\hline Added $\mathrm{B}_{2} \mathrm{O}_{3}$ & $7.00 \%$ & & & & & & $5.00 \%$ & \\
\hline Added $\mathrm{Na}_{2} \mathrm{O}$ & $7.00 \%$ & & & & & & $5.00 \%$ & \\
\hline Added $\mathrm{SiO}_{2}$ & $6.00 \%$ & & & & & & $10.00 \%$ & \\
\hline $\mathrm{Al}_{2} \mathrm{O}_{3}$ & 13.59 & 13.99 & 13.40 & 13.24 & 13.40 & 13.32 & 10.35 & 10.96 \\
\hline $\mathrm{AgO}$ & 0.0012 & 0.0010 & 0.0000 & 0.0014 & 0.0000 & 0.0007 & 0.0000 & 0.0004 \\
\hline $\mathrm{As}_{2} \mathrm{O}_{3}$ & 0.0000 & N.A. & 0.0000 & N.A. & 0.0029 & N.A. & 0.0000 & N.A. \\
\hline $\mathrm{B}_{2} \mathrm{O}_{3}$ & 7.02 & 6.05 & 0.02 & 0.07 & 0.02 & 0.03 & 5.01 & 4.88 \\
\hline $\mathrm{BaO}$ & 0.0280 & N.A. & 0.0000 & N.A. & 0.0000 & N.A. & 0.0000 & N.A. \\
\hline $\mathrm{CaO}$ & 26.31 & 27.70 & 31.27 & 30.43 & 29.81 & 29.56 & 31.26 & 32.39 \\
\hline $\mathrm{CdO}$ & 0.002 & 0.00 & 0.000 & 0.00 & 0.000 & 0.00 & 0.000 & 0.00 \\
\hline $\mathrm{Cr}_{2} \mathrm{O}_{3}$ & 0.06 & 0.26 & 0.02 & 0.23 & 0.05 & 0.27 & 0.02 & 0.25 \\
\hline $\mathrm{CuO}$ & 0.11 & 0.08 & 0.26 & 0.29 & 0.26 & 0.27 & 0.26 & 0.22 \\
\hline $\mathrm{Fe}_{2} \mathrm{O}_{3}$ & 3.07 & 3.46 & 5.02 & 4.62 & 6.44 & 5.76 & 3.88 & 3.93 \\
\hline $\mathrm{K}_{2} \mathrm{O}$ & 0.35 & 0.29 & 0.68 & 0.39 & 1.02 & 0.77 & 0.52 & 0.37 \\
\hline $\mathrm{Li}_{2} \mathrm{O}$ & 0.21 & N.A. & 0.00 & N.A. & 0.00 & N.A. & 0.00 & N.A. \\
\hline $\mathrm{MgO}$ & 2.06 & 1.86 & 1.69 & 1.39 & 1.83 & 1.58 & 1.59 & 1.52 \\
\hline $\mathrm{MnO}_{2}$ & 0.02 & 0.10 & 0.08 & 0.16 & 0.16 & 0.28 & 0.07 & 0.16 \\
\hline $\mathrm{Na}_{2} \mathrm{O}$ & 7.30 & 7.52 & 0.46 & 0.60 & 0.59 & 0.64 & 5.45 & 5.44 \\
\hline $\mathrm{NiO}$ & 0.14 & 0.19 & 0.08 & 0.17 & 0.34 & 0.40 & 0.07 & 0.17 \\
\hline $\mathrm{P}_{2} \mathrm{O}_{5}$ & 0.49 & 0.60 & 5.93 & 5.39 & 7.00 & 6.81 & 5.89 & 6.12 \\
\hline $\mathrm{PbO}$ & 0.04 & 0.04 & 0,18 & 0.17 & 0.16 & 0.14 & 0.18 & 0.14 \\
\hline $\mathrm{SO}_{3}$ & 0.94 & N.A. & 0.00 & N.A. & 0.06 & N.A. & 0.00 & N.A. \\
\hline $\mathrm{SiO}_{2}$ & 37.47 & 37.18 & 39.56 & 41.55 & 37.40 & 38.72 & 34.23 & 32.13 \\
\hline $\mathrm{TiO}_{2}$ & 0.27 & 0.05 & 0.49 & 0.15 & 0.60 & 0.29 & 0.35 & 0.16 \\
\hline $\mathrm{U}_{2} \mathrm{O}_{8}$ & 0.48 & 0.45 & 0.72 & 0.77 & 0.69 & 0.72 & 0.72 & 0.82 \\
\hline $\mathrm{ZnO}$ & 0.03 & 0.18 & 0.13 & 0.39 & 0.13 & 0.42 & 0.13 & 0.34 \\
\hline $\mathrm{Cl}$ & 0.00 & N.A. & 0.00 & N.A. & 0.01 & N.A. & 0.00 & N.A. \\
\hline $\mathrm{F}$ & 0.00 & N.A. & 0.00 & N.A. & 0.00 & N.A. & 0.00 & N.A. \\
\hline Sum & 100.00 & 100.00 & 100.00 & 100.00 & 100.00 & 100.00 & 100.00 & 100.00 \\
\hline
\end{tabular}

**The soil did not dissolve properly in these melts. It was replaced in ORNL7 and subsequent melts by a surrogate formulation. 
Table 4.5

ICPMS Analysis of Glasses Made from Spiked Oak Ridge K-1407 Pond Waste (Note: Values Presented are Solution Concentration (ppb) After Dissolution;

Values for the Solid are Listed Under OCS)

\begin{tabular}{|c|c|c|c|c|c|c|c|c|}
\hline ORNL8M1 & RUN-1 & RUN-2 & RUN-3 & RUN-4 & AVG(ppb) & STD.DEV. & $\mathrm{RSD}(\%)$ & $\begin{array}{l}\mathbf{O C S}^{\star} \\
(\mathrm{ppm})\end{array}$ \\
\hline $\mathrm{Cu}$ & 1048.0 & 1042.2 & 1026.2 & 1022.0 & 1034.6 & 10.8 & 1.0 & 2069.0 \\
\hline $\mathrm{Zn}$ & 280.1 & 314.4 & 310.3 & 283.7 & 297.1 & 15.3 & 5.2 & 594.0 \\
\hline As & 58.7 & 66.0 & 58.8 & 80.3 & 66.0 & 8.8 & 13.3 & 132.0 \\
\hline $\mathrm{Pb}$ & 82.4 & 87.8 & 85.6 & 88.7 & 86.1 & 2.4 & 2.8 & 172.0 \\
\hline ORNL8M2 & RUN-1 & RUN-2 & RUN-3 & RUN-4 & AVG(ppb) & STD.DEV. & $\mathrm{RSD}(\%)$ & \\
\hline $\mathrm{Cu}$ & 1066.6 & 1024.6 & 1024.7 & 984.5 & 1025.1 & 29.0 & 2.8 & 2050.0 \\
\hline $\mathrm{Zn}$ & 307.9 & 307.8 & 332.8 & 328.4 & 319.2 & 11.5 & 3.6 & 638.0 \\
\hline As & 67.8 & 63.3 & 76.7 & 61.2 & 67.3 & 6.0 & 8.9 & 134.0 \\
\hline $\mathrm{Pb}$ & 84.4 & 86.1 & 93.9 & 90.0 & 88.6 & 3.7 & 4.1 & 177.0 \\
\hline ORNL9AM1 & RUN-1 & RUN-2 & RUN-3 & RUN-4 & AVG(ppb) & STD.DEV. & $\operatorname{RSD}(\%)$ & \\
\hline $\mathrm{Cu}$ & 624.1 & 556.0 & 548.9 & 540.7 & 567.4 & 33.2 & 5.8 & 1135.0 \\
\hline $\mathrm{Zn}$ & 172.2 & 155.2 & 166.6 & 169.7 & 165.9 & 6.5 & 3.9 & 332.0 \\
\hline As & 46.5 & 38.3 & 57.7 & 41.0 & 45.9 & 7.4 & 16.2 & 92.0 \\
\hline $\mathrm{Pb}$ & 50.6 & 50.9 & 50.1 & 57.6 & 52.3 & 3.1 & 5.9 & 105.0 \\
\hline ORNL9AM2 & RUN-1 & RUN-2 & RUN-3 & RUN-4 & AVG(ppb) & STD.DEV. & RSD(\%) & \\
\hline $\mathrm{Cu}$ & 563.0 & 564.0 & 573.9 & 525.6 & 556.6 & 18.4 & 3.3 & 1114.0 \\
\hline $\mathrm{Zn}$ & 158.6 & 165.6 & 177.1 & 190.8 & 173.0 & 12.2 & 7.1 & 346.0 \\
\hline As & 54.6 & 41.9 & 48.6 & 48.2 & 48.3 & 4.5 & 9.3 & 97.0 \\
\hline $\mathrm{Pb}$ & 53.6 & 51.7 & 54.2 & 54.9 & 53.6 & 1.2 & 2.2 & 107.0 \\
\hline ORNL10M1 & RUN-1 & RUN-2 & RUN-3 & RUN-4 & AVG(ppb) & STD.DEV. & $\mathrm{RSD}(\%)$ & \\
\hline $\mathrm{Cu}$ & 460.3 & 444.8 & 424.8 & 438.5 & 442.1 & 12.8 & 2.9 & 884.0 \\
\hline $\mathrm{Zn}$ & 106.4 & 113.3 & 124.8 & 124.5 & 117.3 & 7.8 & 6.6 & 235.0 \\
\hline As & 39.6 & 34.1 & 52.5 & 47.3 & 43.4 & 7.1 & 16.3 & 87.0 \\
\hline $\mathrm{Pb}$ & 46.3 & 45.6 & 47.2 & 47.2 & 46.6 & 0.7 & 1.4 & 93.0 \\
\hline ORNL10M2 & RUN-1 & RUN-2 & RUN-3 & RUN-4 & AVG(ppb) & STD.DEV. & $\mathrm{RSD}(\%)$ & \\
\hline $\mathrm{Cu}$ & 456.9 & 449.8 & 445.2 & 436.3 & 447.1 & 7.5 & 1.7 & 894.0 \\
\hline $\mathrm{Zn}$ & 103.9 & 117.2 & 112.8 & 140.2 & 118.5 & 13.4 & 11.3 & 237.0 \\
\hline As & 36.2 & 44.6 & 31.4 & 44.8 & 39.3 & 5.7 & 14.5 & 79.0 \\
\hline $\mathrm{Pb}$ & 45.7 & 50.8 & 48.9 & 51.2 & 49.2 & 2.2 & 4.4 & 98.0 \\
\hline
\end{tabular}

(1) All above data were obtained with a dilution factor of 25 .

(2) $\mathrm{Cu}-63$ has possible interference from $\mathrm{TiO}, \mathrm{ArNa}$, and $\mathrm{PO} 2 ; \mathrm{Zn}-68$ has possible interference from $\mathrm{Ba}++\mathrm{Ce}++$ and $\mathrm{SiF} 2$.

As-75 has possible interference from $\mathrm{ArCl}$ and $\mathrm{Si2F}$. Therefore, the true values for $\mathrm{Cu}, \mathrm{Zn}$ and As could be lower.

* OCS = Original Concentration in Solid. 


\begin{tabular}{|c|c|c|c|c|c|c|c|c|}
\hline Tc-99 analysis & RUN-1 & RUN-2 & RUN-3 & RUN-4 & AVG(ppb) & STD.DEV. & $\operatorname{RSD}(\%)$ & $\begin{array}{l}\text { OCS }^{\star} \\
\text { (ppm) }\end{array}$ \\
\hline ORNL-8M1 & & & & & $<0.1$ & & & $<0.2$ \\
\hline ORNL-8M2 & & & & & $<0.1$ & & & $<0.2$ \\
\hline ORNL-9AM1 & 0.35 & 0.40 & 0.25 & 0.23 & 0.31 & 0.07 & 22.56 & 0.61 \\
\hline ORNL-9AM2 & 0.41 & 0.36 & 0.25 & 0.26 & 0.32 & 0.07 & 21.48 & 0.62 \\
\hline ORNL-10M1 & 0.20 & 0.19 & 0.23 & 0.24 & 0.22 & 0.02 & 9.24 & 0.44 \\
\hline ORNL-10M2 & 0.22 & 0.18 & 0.21 & 0.20 & 0.20 & 0.01 & 7.30 & 0.40 \\
\hline
\end{tabular}

* OCS = Original Concentration in Solid. 
Table 4.6

Viscosity and Conductivity of Oak Ridge Glasses

\begin{tabular}{|c|c|c|c|c|c|}
\hline Glass & ORNL3 & ORNL7 & ORNL8 & ORNL9A & ORNL10 \\
\hline Soil \% & r & $25.00 \%$ & $=$ & $25.00 \%$ & $25.00 \%$ \\
\hline Pond Waste $\%$ & $82.69 \%$ & $50.00 \%$ & $100.00 \%$ & $50.00 \%$ & $40.00 \%$ \\
\hline \multicolumn{6}{|l|}{ TSCA Ash \% } \\
\hline \multicolumn{6}{|l|}{ WETF \% } \\
\hline Added $\mathrm{B}_{2} \mathrm{O}_{3}$ & $5.11 \%$ & $12.46 \%$ & & $12.46 \%$ & $12.46 \%$ \\
\hline Added $\mathrm{Na}_{2} \mathrm{O}$ & $12.20 \%$ & $5.54 \%$ & & $5.54 \%$ & $10.54 \%$ \\
\hline Added $\mathrm{SiO}_{2}$ & & $7.00 \%$ & & $7.00 \%$ & $12.00 \%$ \\
\hline Viscosity & \multicolumn{5}{|c|}{ (Poise) versus Temperature (in Celsius) } \\
\hline$@ 1100 \mathrm{C}$ & & 350.23 & & 207.65 & 166.09 \\
\hline @1150C & & 157.96 & 352.92 & 106.25 & 98.82 \\
\hline$@ 1200 \mathrm{C}$ & & 83.95 & 129.32 & 59.82 & 61.43 \\
\hline$@ 1250 \mathrm{C}$ & 490.34 & 48.54 & 54.17 & 36.37 & 39.69 \\
\hline$@ 1300 \mathrm{C}$ & 17.61 & 30.05 & 25.30 & 23.52 & 26.53 \\
\hline$@ 1350 \mathrm{C}$ & 10.11 & 19.69 & 12.92 & 16.02 & 18.28 \\
\hline$@ 1400 \mathrm{C}$ & 8.05 & 13.52 & 7.11 & 11.38 & 12.94 \\
\hline$@ 1450 \mathrm{C}$ & 7.11 & 9.66 & 4.17 & 8.39 & 9.38 \\
\hline \multicolumn{6}{|l|}{ Measurement range } \\
\hline Lower & 1256 & 1188 & 1306 & 1148 & 1149 \\
\hline Higher & 1360 & 1383 & 1392 & 1405 & 1378 \\
\hline \multicolumn{6}{|c|}{$\begin{array}{ll}\text { Conductivity } & \text { (S/cm) versus Temperature (in Celsius) }\end{array}$} \\
\hline$@ 1100 \mathrm{C}$ & & 0.033 & & 0.041 & 0.098 \\
\hline$@ 1150 \mathrm{C}$ & & 0.041 & & 0.054 & 0.120 \\
\hline @1200C & 0.105 & 0.050 & & 0.071 & 0.145 \\
\hline$@ 1250 \mathrm{C}$ & 0.150 & 0.061 & 0.083 & 0.090 & 0.173 \\
\hline @1300c & 0.208 & 0.073 & 0.115 & 0.114 & 0.204 \\
\hline @1350c & 0.283 & 0.087 & 0.157 & 0.142 & 0.238 \\
\hline @ $1400 \mathrm{C}$ & 0.379 & 0.102 & 0.211 & 0.174 & 0.275 \\
\hline @1450c & 0.498 & 0.119 & 0.278 & 0.211 & 0.316 \\
\hline \multicolumn{6}{|l|}{ Measurement range } \\
\hline Lower & 1259 & 1174 & 1271 & 1148 & 1127 \\
\hline Higher & 1384 & 1362 & 1475 & 1380 & 1383 \\
\hline
\end{tabular}




\begin{tabular}{|c|c|c|c|c|c|c|}
\hline Glass & ORNL11 & ORNL12 & ORNL13 & ORNL14 & ORNL15 & ORNL16 \\
\hline Soil \% & $50.00 \%$ & $45.00 \%$ & $40.00 \%$ & $25.00 \%$ & $45.00 \%$ & $30.00 \%$ \\
\hline Pond Waste \% & & $20.00 \%$ & & & $10.00 \%$ & $0.00 \%$ \\
\hline TSCA Ash \% & & & & $50.00 \%$ & $45.00 \%$ & $50.00 \%$ \\
\hline WETF \% & $50.00 \%$ & $35.00 \%$ & $40.00 \%$ & & & $50.00 \%$ \\
\hline Added $\mathrm{B}_{2} \mathrm{O}_{3}$ & & & $7.00 \%$ & & & $5.00 \%$ \\
\hline Added $\mathrm{Na}_{2} \mathrm{O}$ & & & $7.00 \%$ & & & $5.00 \%$ \\
\hline Added $\mathrm{SiO}_{2}$ & & & $6.00 \%$ & & & $10.00 \%$ \\
\hline Viscosity & (Poise) versus Te & nperature (in Cels & ius) & & & \\
\hline$@ 1100 \mathrm{C}$ & & & 85.26 & & & 172.65 \\
\hline$@ 1150 \mathrm{C}$ & 245.02 & & 46.00 & 625.55 & 376.38 & 69.77 \\
\hline$@ 1200 \mathrm{C}$ & 115.92 & 310.45 & 26.37 & 242.93 & 163.32 & 33.00 \\
\hline$@ 1250 \mathrm{C}$ & 62.12 & 57.42 & 15.93 & 109.34 & 81.44 & 17.60 \\
\hline$@ 1300 \mathrm{C}$ & 36.65 & 26.84 & 10.07 & 55.24 & 45.20 & 10.30 \\
\hline$@ 1350 \mathrm{C}$ & 23.31 & 17.42 & 6.62 & 30.60 & 27.29 & 6.49 \\
\hline$@ 1400 \mathrm{C}$ & 15.75 & 13.17 & 4.50 & 18.27 & 17.62 & 4.34 \\
\hline$@ 1450 \mathrm{C}$ & 11.18 & 4.74 & 3.16 & 11.59 & 12.02 & 3.05 \\
\hline \multicolumn{7}{|l|}{ Measurement range } \\
\hline Lower & 1241 & 1198 & 1060 & 1192 & 1230 & 1125 \\
\hline Higher & 1391 & 1361 & 1285 & 1345 & 1361 & 1402 \\
\hline \multicolumn{7}{|c|}{ Conductivity $\quad(\mathrm{S} / \mathrm{cm})$ versus Temperature (in Celsius) } \\
\hline$@ 1100 \mathrm{c}$ & & & 0.037 & & & \\
\hline$@ 1150 \mathrm{C}$ & & & 0.055 & & & 0.040 \\
\hline$@ 1200 \mathrm{C}$ & 0.026 & & 0.080 & & & 0.064 \\
\hline$@ 1250 c$ & 0.042 & 0.036 & 0.113 & 0.068 & 0.044 & 0.100 \\
\hline$@ 1300 \mathrm{C}$ & 0.065 & 0.059 & 0.157 & 0.091 & 0.065 & 0.151 \\
\hline$@ 1350 \mathrm{C}$ & 0.099 & 0.092 & 0.212 & 0.120 & 0.094 & 0.223 \\
\hline$@ 1400 \mathrm{C}$ & 0.147 & 0.141 & 0.283 & 0.154 & 0.132 & 0.322 \\
\hline$@ 1450 \mathrm{C}$ & 0.214 & 0.212 & 0.370 & 0.196 & 0.181 & 0.454 \\
\hline \multicolumn{7}{|l|}{ Measurement range } \\
\hline Lower & 1216 & 1257 & 1073 & 1280 & 1255 & 1164 \\
\hline Higher & 1437 & 1398 & 1351 & 1475 & 1466 & 1425 \\
\hline
\end{tabular}


Table 4.7

Summary of PCT Results for Oak Ridge Glasses

\begin{tabular}{|c|c|c|c|c|c|c|c|c|c|c|}
\hline Glass & SRL-EA & ORNL3 & ORNL9A & ORNL10 & ORNL11 & ORNL12 & ORNL13 & ORNL14 & ORNL15 & ORNL16 \\
\hline \multicolumn{11}{|c|}{ SODIUM, PPM } \\
\hline \multicolumn{11}{|l|}{ DAYS } \\
\hline 7 & 1700 & 35.7 & 8.64 & 25.8 & 1.87 & 2.1 & 10.1 & 0.56 & 0.747 & 8.69 \\
\hline 28 & 2110 & 78.3 & 22 & 44.6 & 2.01 & 2.4 & 11.8 & 1 & 1.29 & 9.97 \\
\hline 56 & 2540 & 148.7 & 24.5 & 56.7 & 2 & 2.53 & 12 & 0.85 & 1.19 & 10.5 \\
\hline 120 & 3790 & 200.2 & 43.7 & 78.9 & 2.9 & 2.747 & 13.54 & 0.947 & 1.447 & 11.47 \\
\hline 180 & 4460 & 233.8 & 65.4 & 91.7 & 4.543 & 2.913 & 21.11 & 1.113 & 1.58 & 12.16 \\
\hline 270 & 5330 & 3 & 73.7 & 95.5 & & & 8 & & 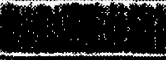 & \\
\hline 365 & 5320 &. & 83.78 & 92.55 & & & 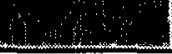 & & 8 & \\
\hline \multicolumn{11}{|c|}{ NORMALIZED, $g / 1$} \\
\hline 7 & 14.04 & 0.7 & 0.185 & 0.334 & 0.149 & 0.175 & 0.183 & 0.127 & 0.156 & 0.215 \\
\hline 28 & 17.4 & 1.54 & 0.47 & 0.577 & 0.16 & 0.2 & 0.213 & 0.226 & 0.27 & 0.247 \\
\hline 56 & 21 & 2.9201 & 0.524 & 0.735 & 0.16 & 0.211 & 0.217 & 0.192 & 0.25 & 0.259 \\
\hline 120 & 31.3 & 3.9314 & 0.933 & 1.02 & 0.2312 & 0.2293 & 0.2456 & 0.2144 & 0.3028 & 0.2841 \\
\hline 180 & 36.8 & 4.59 & 1.4 & 1.19 & 0.3622 & 0.2432 & 0.383 & 0.252 & 0.3306 & 0.3012 \\
\hline 270 & 44.1 & 78 & 1.58 & 1.24 & & & & & & H \\
\hline 365 & 44 & & 1.7894 & 1.997 & 3 & & & $\because$ & & 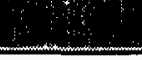 \\
\hline \multicolumn{11}{|c|}{ Leach $\mathrm{g} / \mathrm{m}^{2} / \mathrm{d}$} \\
\hline 3.5 & 1 & 0.05 & 0.0132 & 0.0238 & 0.0107 & 0.0125 & 0.01304 & 0.0091 & 0.0112 & 0.0154 \\
\hline 17.5 & 0.081 & 0.0199 & 0.00679 & 0.0058 & 0.000266 & 0.00059 & 0.000734 & 0.00236 & 0.00269 & 0.000753 \\
\hline 42 & 0.0635 & 0.0329 & 0.000969 & 0.00281 & -0.00001 & 0.000198 & 0.0000713 & -0.000618 & -0.000351 & 0.000223 \\
\hline 88 & 0.0809 & 0.0241 & 0.0032 & 0.00224 & 0.0013 & 0.0003 & 0.0005 & 0.0004 & 0.0009 & 0.0004 \\
\hline 150 & 0.0458 & 0.016 & 0.00387 & 0.00139 & 0.0023 & 0.0002 & 0.0025 & 0.0007 & 0.0005 & 0.0003 \\
\hline 225 & 0.0403 & & 0.000986 & 0.000274 & & & 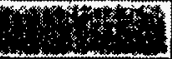 & & & $7 \%$ \\
\hline 3175 & $=0$ & & 00012 & . & & & & & & \\
\hline
\end{tabular}




\begin{tabular}{|c|c|c|c|c|c|c|c|c|c|c|}
\hline Glass & SRL-EA & ORNL3 & ORNL9A & ORNL10 & ORNL11 & ORNL12 & ORNL13 & ORNL14 & ORNL15 & ORNL16 \\
\hline \multicolumn{11}{|c|}{ SILICON, PPM } \\
\hline \multicolumn{11}{|l|}{ DAYS } \\
\hline 7 & 907 & 38.1 & 27.1 & 31 & 16.8 & 15.4 & 18.4 & 18 & 17.7 & 19.2 \\
\hline 28 & 983 & 57.4 & 30.2 & 40.7 & 13.8 & 13 & 16.2 & 15.9 & 15.8 & 16.9 \\
\hline 56 & 1150 & 57.12 & 30.4 & 43.3 & 18.8 & 18.5 & 21.2 & 20.6 & 21.4 & 23 \\
\hline 120 & 1450 & 49.4 & 36.4 & 46.7 & 17.68 & 17.78 & 20.44 & 19.76 & 20.59 & 22.12 \\
\hline 180 & 1640 & 60.8 & 45.5 & 56.1 & 25.72 & 23.34 & 28.6 & 24.65 & 25.21 & 25.7 \\
\hline 270 & 1810 & 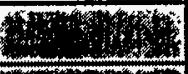 & 50.6 & 58.4 & ways & 6 & 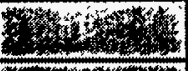 & 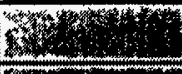 & 4 & 6 \\
\hline 365 & 1920 & 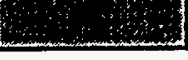 & 53.13 & 53.97 & 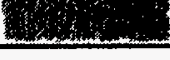 & & & & 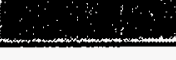 & $\therefore$ \\
\hline \multicolumn{11}{|c|}{ NORMALIZED, g/l } \\
\hline 7 & 4.19 & 0.27 & 0.16 & 0.172 & 0.09 & 0.088 & 0.107 & 0.093 & 0.0977 & 0.128 \\
\hline 28 & 4.54 & 0.408 & 0.179 & 0.226 & 0.08 & 0.074 & 0.095 & 0.082 & 0.0874 & 0.113 \\
\hline 56 & 5.32 & 0.4053 & 0.18 & 0.24 & 0.11 & 0.106 & 0.12 & 0.11 & 0.119 & 0.153 \\
\hline 120 & 6.68 & 0.3505 & 0.216 & 0.26 & 0.0989 & 0.1017 & 0.1191 & 0.1018 & 0.1139 & 0.1474 \\
\hline 180 & 7.58 & 0.431 & 0.27 & 0.311 & 0.1439 & 0.1278 & 0.1666 & 0.127 & 0.1395 & 0.1712 \\
\hline 270 & 8.38 & & 0.3 & 0.324 & 6 & & 5 & 1 & & 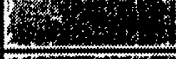 \\
\hline 365 & 8.89 & & 0.3156 & 0.2997 & & & & & & \\
\hline \multicolumn{11}{|c|}{ Leach $\mathrm{g} / \mathrm{m}^{2} / \mathrm{d}$} \\
\hline 3.5 & 0.299 & 0.019 & 0.0115 & 0.01229975 & 0.0067195 & 0.0062949 & 0.00764 & 0.00663 & 0.00698 & 0.0091146 \\
\hline 17.5 & 0.00834 & 0.0033 & 0.00043414 & 0.00129 & -0.000397 & -0.000332 & -0.0002927 & -0.0002588 & -0.000245 & -0.000351 \\
\hline 42 & 0.0139 & -0.001 & 0.00001909 & 0.00024889 & 0.0004937 & 0.0005651 & 0.00051396 & 0.00043243 & 0.00055525 & 0.0007175 \\
\hline 88 & 0.0106 & -0.0013 & 0.00028165 & 0.00014924 & -0.0001 & -0.0001 & -0.0001 & -0.0001 & -0.0001 & -0.0001 \\
\hline 150 & 0.0075 & 0.002 & 0.00044991 & 0.00043498 & 0.0008 & 0.0005 & 0.0004 & 0.0005 & 0.0005 & 0.0004 \\
\hline 225 & 0.00445 & $8+3$ & 0.00016729 & 0.00007003 & 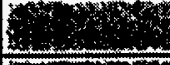 & & & & & \\
\hline 317.5 & 0.00268 & & 0.0001 & -0.0001 & & & & & & \\
\hline
\end{tabular}




\begin{tabular}{|c|c|c|c|c|c|c|c|c|c|c|}
\hline Glass & SRL-EA & ORNL3 & ORNL9A & ORNL10 & ORNLI1 & ORNL12 & ORNL13 & ORNL14 & ORNL15 & ORNL16 \\
\hline \multicolumn{11}{|c|}{ BORON, PPM } \\
\hline \multicolumn{11}{|l|}{ DAYS } \\
\hline 7 & 642 & 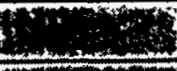 & 10.12 & 13.12 & 0.08 & 0.203 & 2.987 & 0.203 & 0.313 & 2.957 \\
\hline 28 & 775 & & 15.17 & 21.16 & 0.32 & 0.383 & 4.26 & 0.317 & 0.56 & 4.43 \\
\hline 56 & 974 & & 19.48 & 26.94 & 0.077 & 0.463 & 4.237 & 0.273 & 0.74 & 4.45 \\
\hline 120 & 1500 & & 41.44 & 37.23 & 0.017 & 0.013 & 3.727 & 0.42 & 0.327 & 3.693 \\
\hline 180 & 1870 & & 68.12 & 48.62 & 0.047 & 0.043 & 6.16 & 0.537 & 0.227 & 3.763 \\
\hline 270 & 2120 & & 82.17 & 53.74 & & & & & & 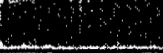 \\
\hline 365 & 2270 & & 95.59 & 51.95 & 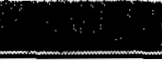 & & & & & \\
\hline \multicolumn{11}{|c|}{ NORMALIZED, g/l } \\
\hline 7 & 19 & 6 & 0.25 & 0.339 & 1.3 & 0.6 & 0.161 & 0.883 & 3.14 & 0.194 \\
\hline 28 & 22.9 & $1+$ & 0.376 & 0.547 & 5.21 & 1.13 & 0.23 & 1.38 & 5.62 & 0.292 \\
\hline 56 & 28.8 & 8 & 0.482 & 0.696 & 1.25 & 1.37 & 0.228 & 1.19 & 7.43 & 0.294 \\
\hline 120 & 44.1 & 2 & 1.03 & 0.96 & 0.2768 & 0.0385 & 0.2008 & 1.827 & 3.2815 & 0.2436 \\
\hline 180 & 55.4 & & 1.69 & 1.26 & 0.7653 & 0.1274 & 0.3319 & 2.336 & 2.278 & 0.1482 \\
\hline 270 & 62.6 & 6 & 2.04 & 1.39 & 18 & & 4 & & 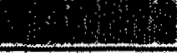 & 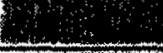 \\
\hline 365 & 67.2 & $\because$ & 2.3676 & 1.3424 & & $\because$ & & & +1 & \\
\hline \multicolumn{11}{|c|}{ Leach $\mathrm{g} / \mathrm{m}^{2} / \mathrm{d}$} \\
\hline 3.5 & 1.36 & & 0.0179 & 0.0242 & 0.0931 & 0.043 & 0.0115 & 0.0631 & 0.224 & 0.0139 \\
\hline 17.5 & 0.0935 & & 0.00298 & 0.00495 & 0.0931 & 0.0127 & 0.00163 & 0.0118 & 0.059 & 0.002324 \\
\hline 42 & 0.105 & na & 0.00191 & 0.00267 & -0.070659 & 0.0042311 & -0.0000221 & -0.0034179 & 0.0323 & 0.0000306 \\
\hline 88 & 0.12 & La: & 0.00425 & 0.00208 & -0.0174 & -0.0238 & -0.0005 & 0.0114 & -0.074 & -0.0009 \\
\hline 150 & 0.094 & 1 & 0.0055 & 0.00245 & 0.0087 & 0.0016 & 0.0023 & 0.0091 & -0.0179 & 0.0001 \\
\hline 225 & 0.0402 & $\ldots$ & 0.0019 & 0.00073501 & 6 & & & & & 3 \\
\hline 75 & 00238 & & م0018. & . & & & & & & \\
\hline
\end{tabular}


Table 4.8

Summary of TCLP Results for Oak Ridge Glasses and Waste (ppm)

\begin{tabular}{|c|c|c|c|c|c|c|c|c|c|}
\hline $\begin{array}{l}\text { GLASS } \\
\text { NAME }\end{array}$ & Ag & As & $\mathbf{B a}$ & Cd & $\mathrm{Cr}$ & $\mathbf{H g}$ & $\mathbf{N i}$ & $\mathbf{P b}$ & $\mathbf{U}$ \\
\hline ORNL1 & & 0.022 & 0.53 & $<0.002$ & 0.002 & & 0.18 & 0.001 & 0.15 \\
\hline ORNL2 & & 0.020 & 0.54 & $<0.002$ & 0.002 & & 0.13 & 0.001 & 0.11 \\
\hline ORNL3 & & 0.011 & 0.19 & $<0.002$ & 0.002 & & 1.76 & 0.001 & 0.08 \\
\hline ORNL7 & & $<0.07$ & 0.35 & $<0.002$ & 0.000 & & 0.90 & 0.002 & 0.11 \\
\hline ORNL8 & & $<0.07$ & 0.37 & $<0.002$ & 0.010 & & 0.19 & 0.001 & 0.15 \\
\hline ORNL9A & . & $<0.07$ & 0.42 & $<0.002$ & 0.010 & & 0.63 & 0.003 & 0.11 \\
\hline ORNL10 & & $<0.07$ & 0.40 & $<0.002$ & 0.010 & & 0.32 & 0.003 & 0.04 \\
\hline ORNL11 & 0.008 & $<0.07$ & 0.78 & 0.004 & 0.052 & 0.098 & 0.19 & 0.21 & 1.86 \\
\hline ORNL12 & 0.001 & $<0.07$ & 0.73 & 0.005 & 0.019 & 0.012 & 0.42 & 0.075 & 0.58 \\
\hline ORNL13 & 0.002 & $<0.07$ & 0.71 & 0.007 & 0.030 & 0.035 & 0.18 & 0.21 & 1.45 \\
\hline ORNL14 & & $<0.07$ & & & 0.006 & & 0.04 & 0.068 & 0.27 \\
\hline ORNL15 & & $<0.07$ & & & 0.002 & & 0.13 & 0.064 & 0.28 \\
\hline ORNLI6 & & $<0.07$ & & & 0.018 & & 0.68 & 0.52 & 2.25 \\
\hline $\begin{array}{l}\text { Standard } \\
\text { D004/D011 }\end{array}$ & 5 & 5 & 100 & 1 & 5 & 0.2 & 5 & 5 & $1.78^{*}$ \\
\hline $\begin{array}{l}\text { Standard } \\
\text { F006/F007 }\end{array}$ & 0.3 & 5 & 100 & 0.19 & 0.86 & 0.2 & 5 & 0.37 & $1.78^{\circ}$ \\
\hline
\end{tabular}

\begin{tabular}{||c|c|c|c|c|c|c|c|c|c||}
\hline $\begin{array}{l}\text { SAMPLE } \\
\text { NAME }\end{array}$ & $\mathrm{Ag}$ & $\mathrm{As}$ & $\mathrm{Ba}$ & $\mathrm{Cd}$ & $\mathrm{Cr}$ & $\mathrm{Hg}$ & $\mathrm{Ni}$ & $\mathrm{Pb}$ & $\mathrm{U}$ \\
\hline $\begin{array}{l}\text { WETF } \\
\text { Sludge }\end{array}$ & 0.067 & $<0.07$ & 2.07 & 0.9 & 0.15 & 0.2 & 1.66 & 2.83 & 30.2 \\
\hline $\begin{array}{l}\text { Standard } \\
\text { F006/F007 }\end{array}$ & 0.3 & 5 & 100 & 0.19 & 0.86 & 0.2 & 5 & 0.37 & $1.78^{*}$ \\
\hline
\end{tabular}

Note: $\mathrm{Ag}$ and $\mathrm{Hg}$ are only present in the WETF waste.

$\mathrm{Ba}$ and $\mathrm{Cd}$ were not reported in the TSCA surrogate; they were not used in these glasses.

* Ingested water limit for uranium from DOE Order 5400.5 . 
Table 4.9

List of Oak Ridge Glasses with Complete Viscosity, Conductivity, TCLP, and PCT Data (i.e., Glasses in the Core Data Set of $\mathbf{4 0}$ Glasses) and Representative Data for those Properties.

\begin{tabular}{|c|c|r|r|r|r||}
\hline \multirow{2}{*}{$\begin{array}{c}\text { Glass } \\
\text { Number }\end{array}$} & \multirow{2}{*}{$\begin{array}{c}\text { Glass } \\
\text { Name }\end{array}$} & \multirow{2}{*}{$\begin{array}{c}\text { Viscosity } \\
\text { @ } \mathbf{1 2 5 0 ^ { \circ } \mathbf { C }}\end{array}$} & $\begin{array}{c}\text { Conductivity } \\
\text { @ } \mathbf{1 2 5 0} \mathbf{C}\end{array}$ & \multicolumn{1}{c|}{ TCLP } & PCT-7 day \\
\cline { 5 - 7 } & & & U (ppb) & Na (ppm) \\
\hline \hline 1 & ORNL3 & 490 & 0.150 & 84 & 36 \\
\hline 2 & ORNL9A & 36 & 0.090 & 110 & 9 \\
\hline 3 & ORNL10 & 40 & 0.173 & 39 & 26 \\
\hline 4 & ORNL11 & 62 & 0.042 & 1864 & 2 \\
\hline 5 & ORNL12 & 57 & 0.036 & 576 & 2 \\
\hline 6 & ORNL13 & 16 & 0.113 & 1454 & 10 \\
\hline 7 & ORNL14 & 109 & 0.068 & 272 & 1 \\
\hline 8 & ORNL15 & 81 & 0.044 & 279 & 1 \\
\hline 9 & ORNL16 & 18 & 0.100 & 2252 & 9 \\
\hline
\end{tabular}




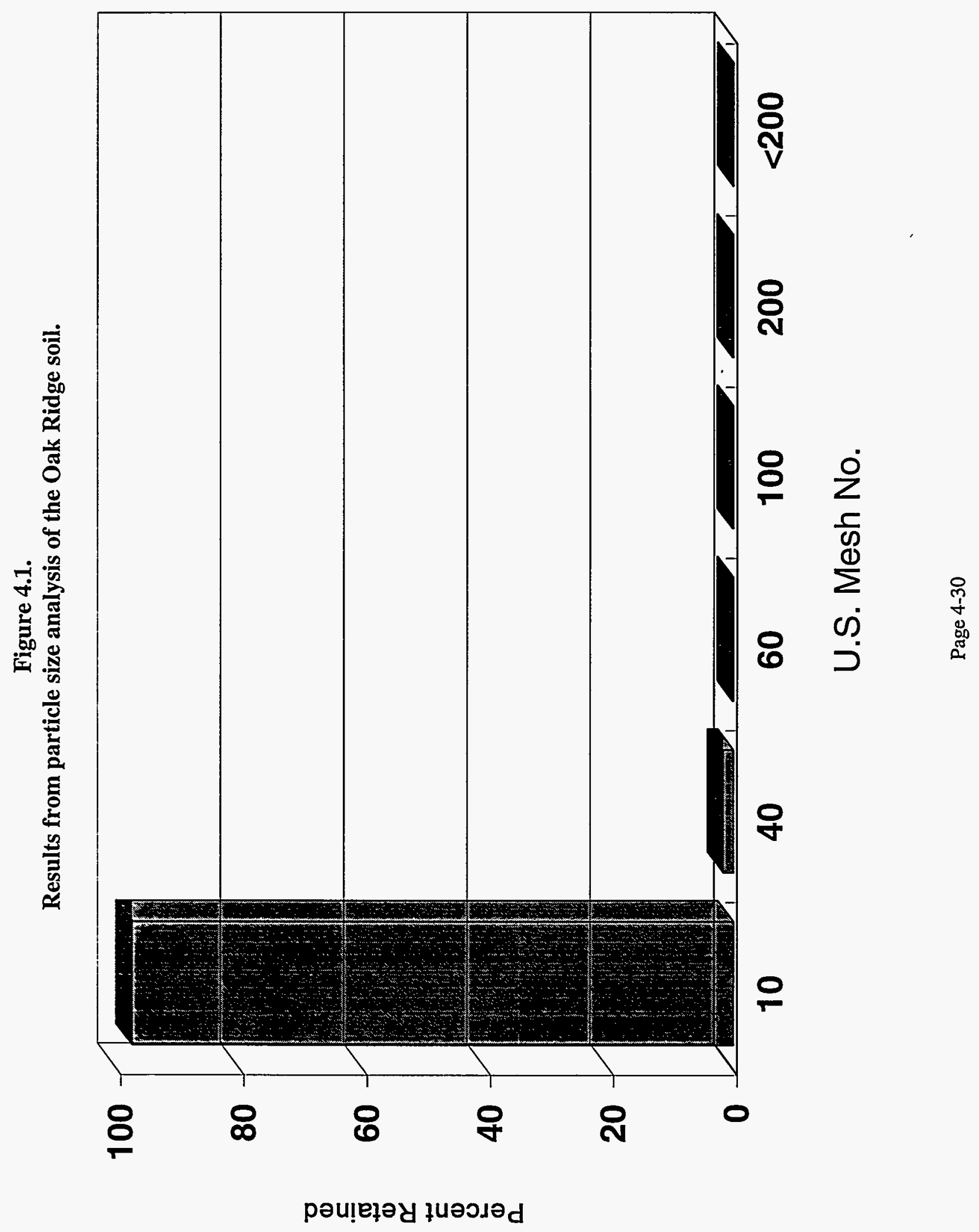


Figure 4.2.

Results from gamma spectrometry of WETF sludge.
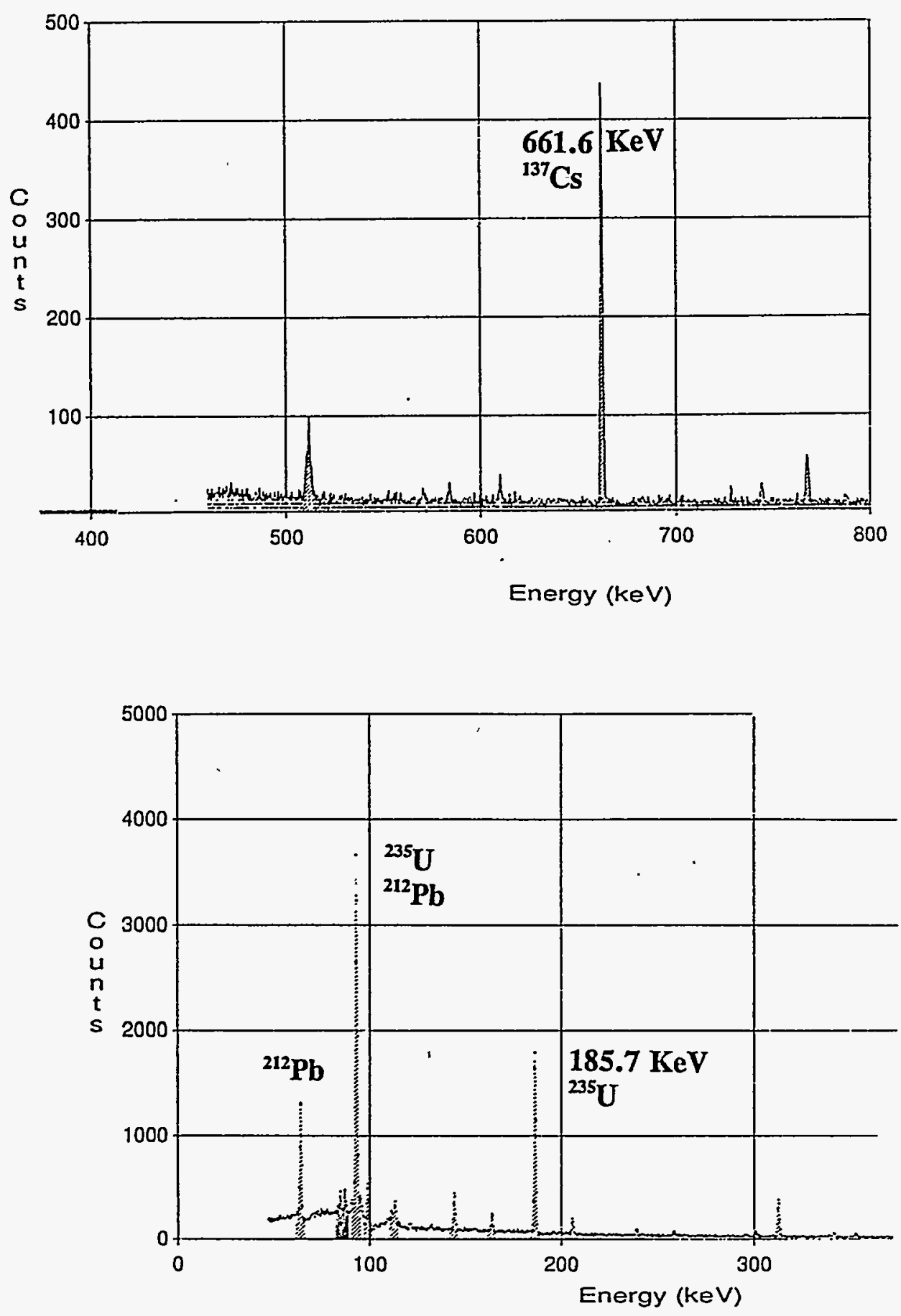

Page 4-31 
Figure 4.3.

Blocky Ni-bearing spinel crystals in one of the few glassy regions of ORNL1.

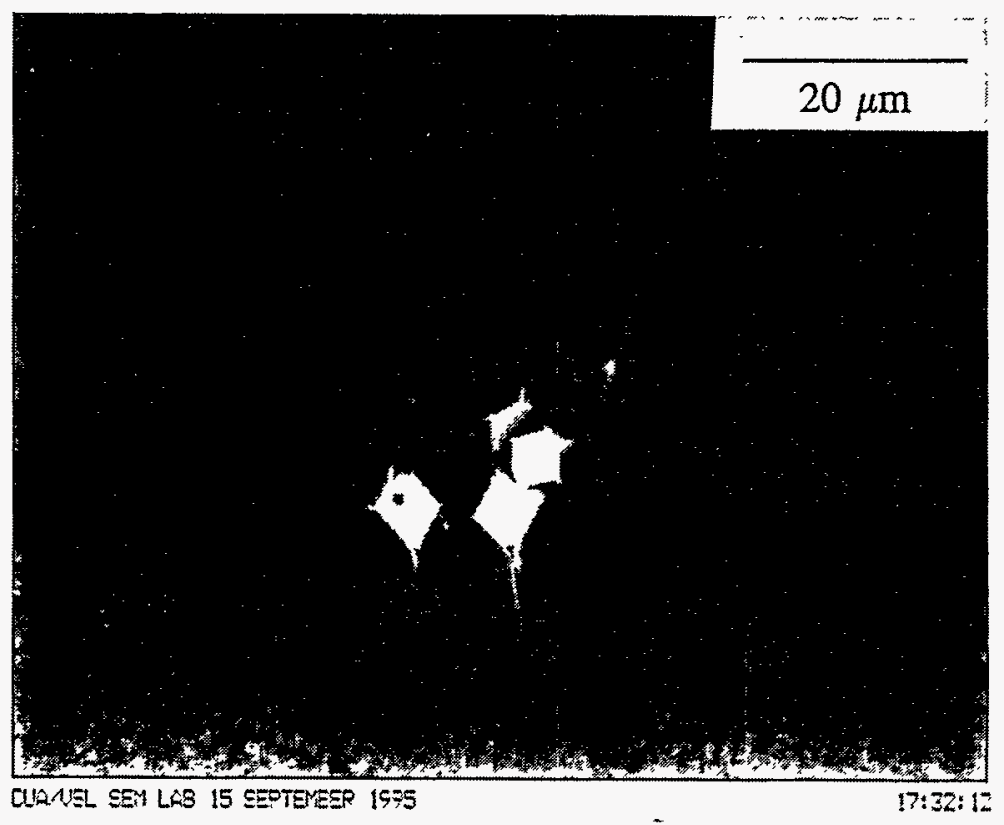

Figure 4.4.

Structure found in the majority of ORNL1.

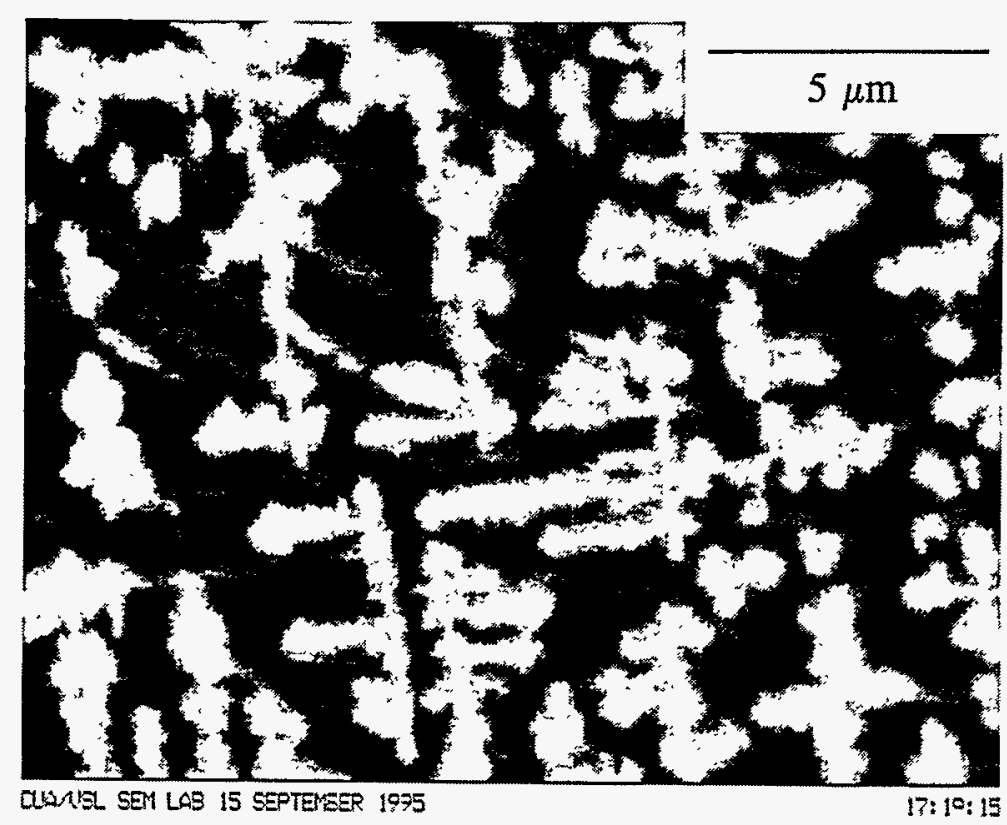


Figure 4.5.

Massive dendritic spinel growth in ORNL2.

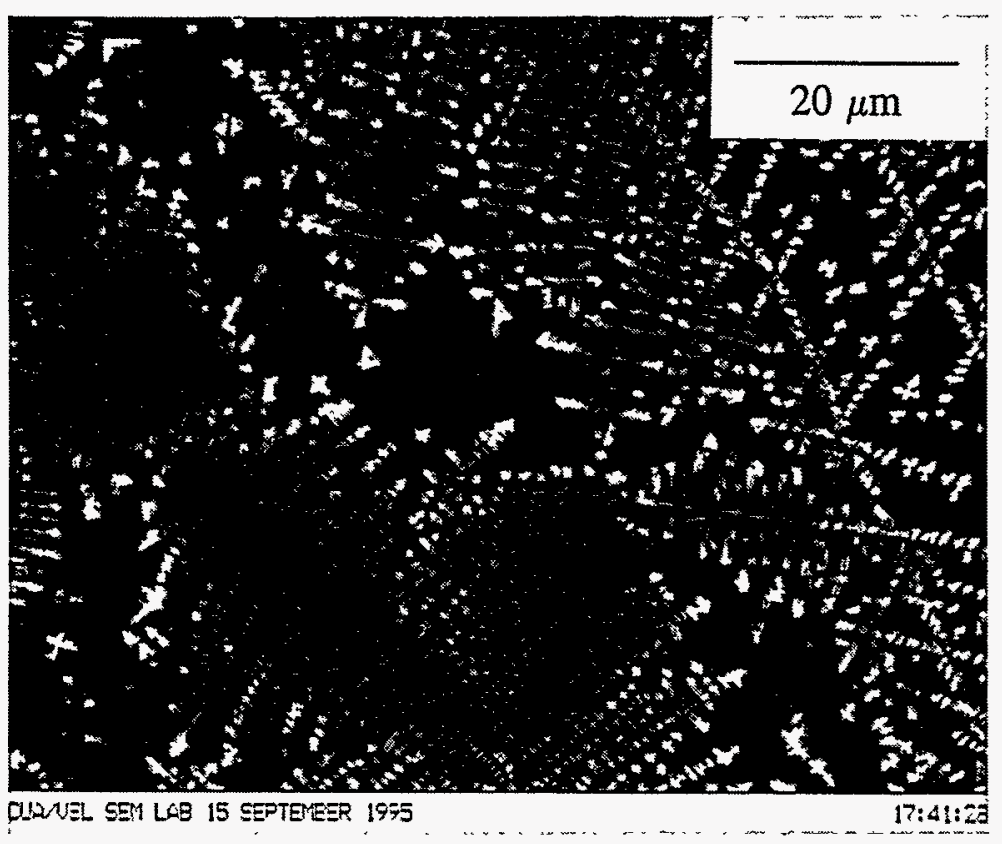


Figure 4.6.

Region in ORNL3 showing liquid-liquid phase separation and small crystals of a variety of phases.

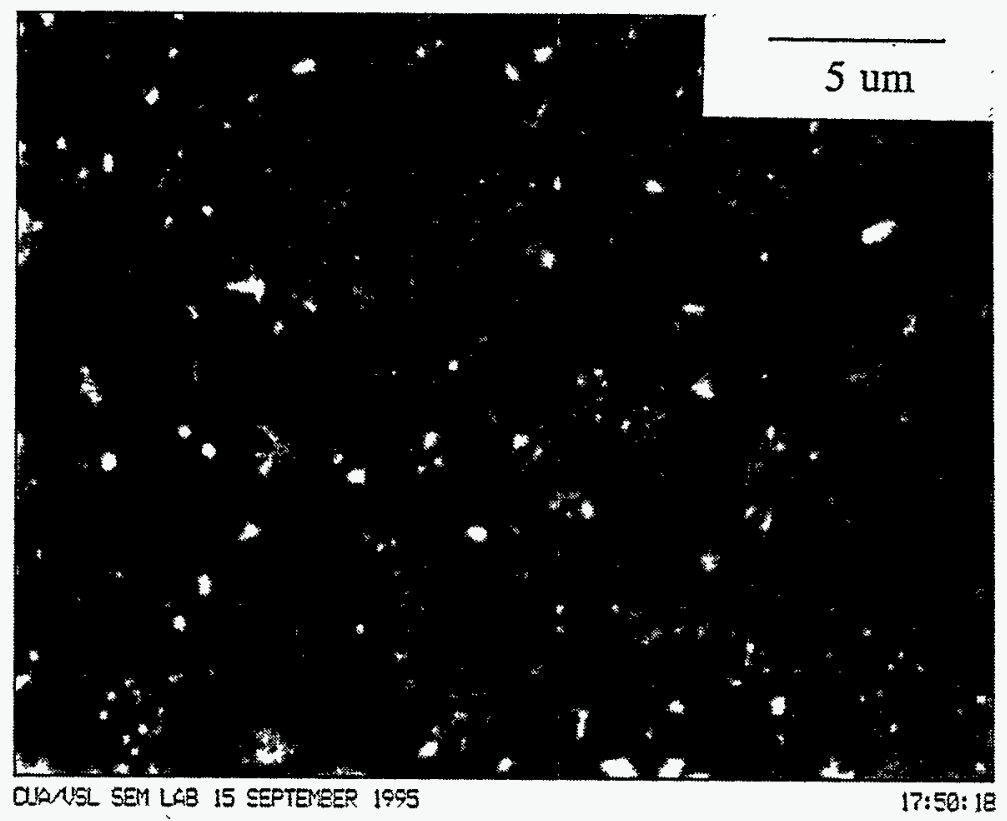

Figure 4.7.

Very fine scale liquid-liquid phase separation in ORNL3. Bright regions are Ni-rich, and may be nucleation centers of spinel crystallization.

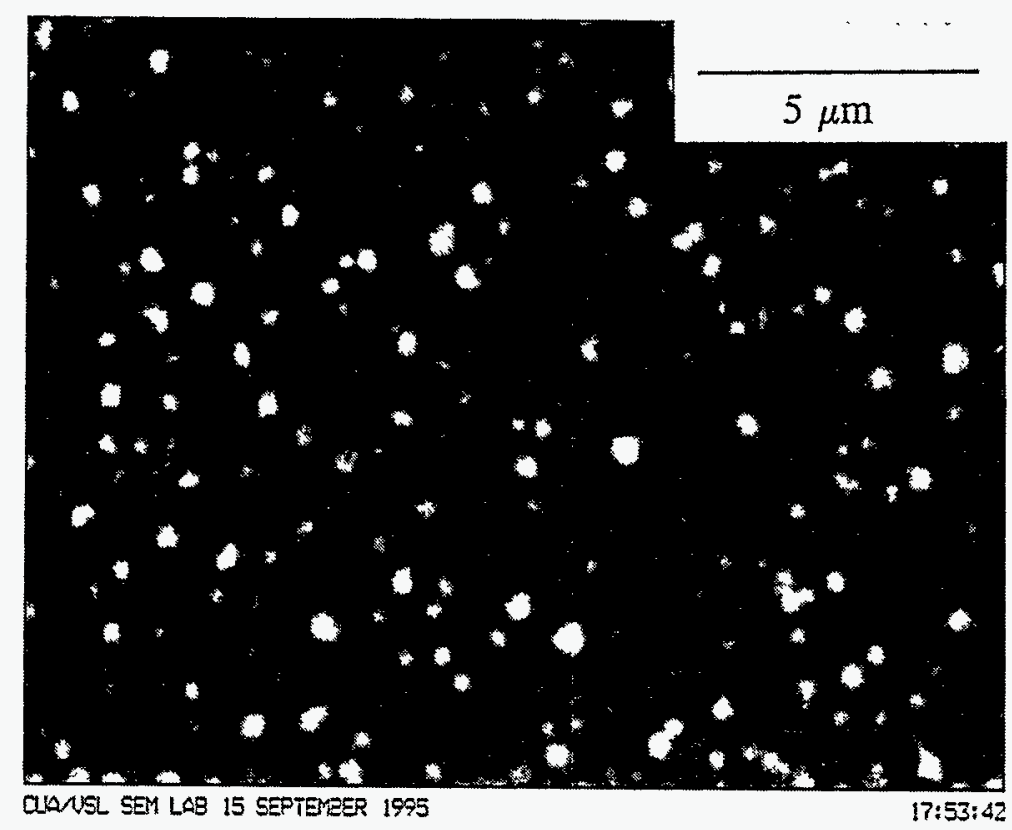


Figure 4.8.

A cluster of high $\mathrm{Cr}$ spinel crystals surrounded by some dendritic growth of Ni-Fe spinel.

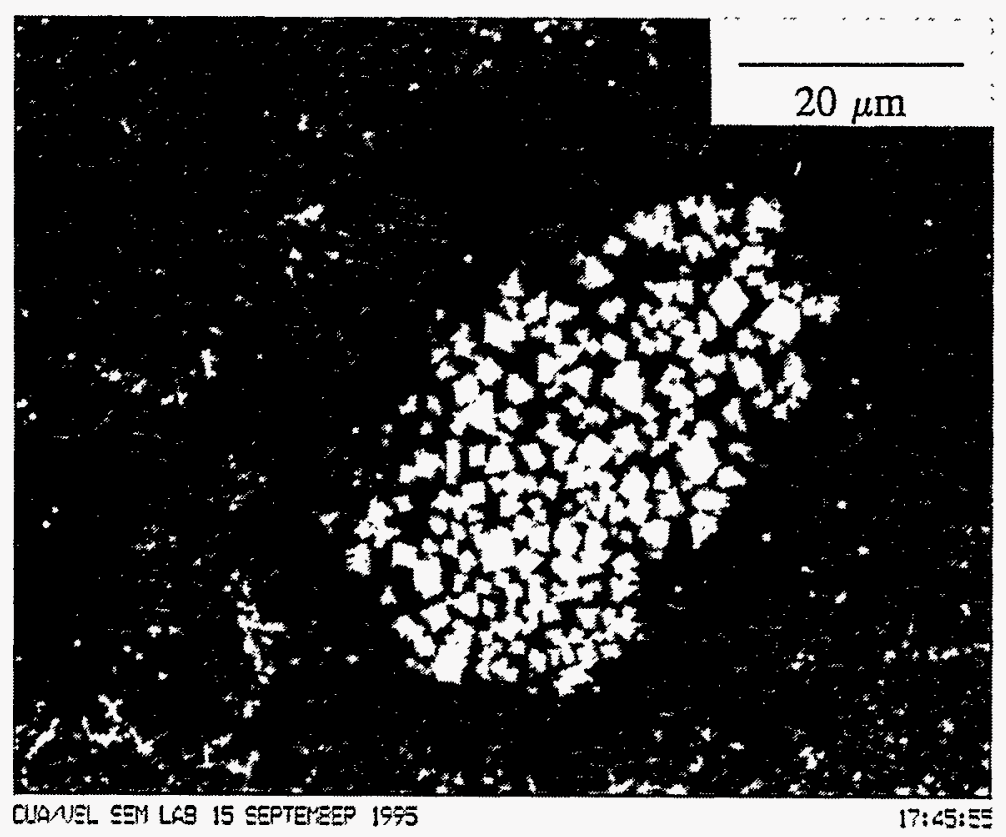

Figure 4.9.

The wide range of spatial scale typical of liquid-liquid phase separation in ORNL3 is evident in this micrograph.

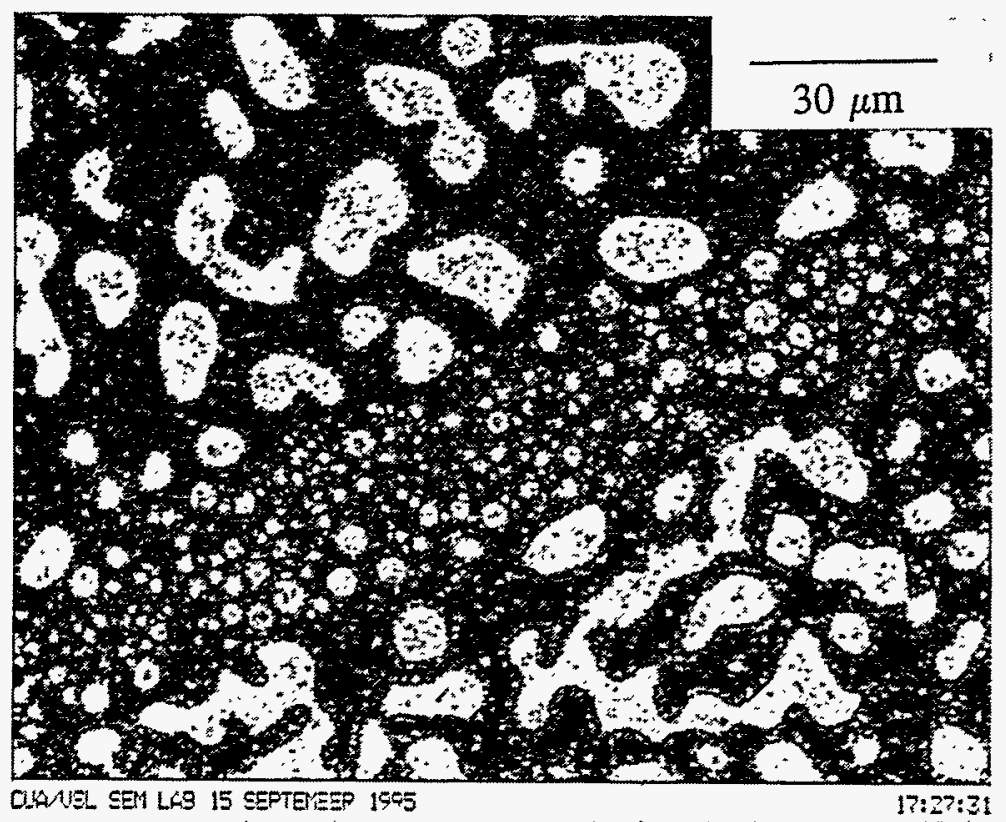


Figure 4.10.

Viscosity of some Oak Ridge pond waste glasses. The lower pond waste loading glasses display the lowest viscosity.

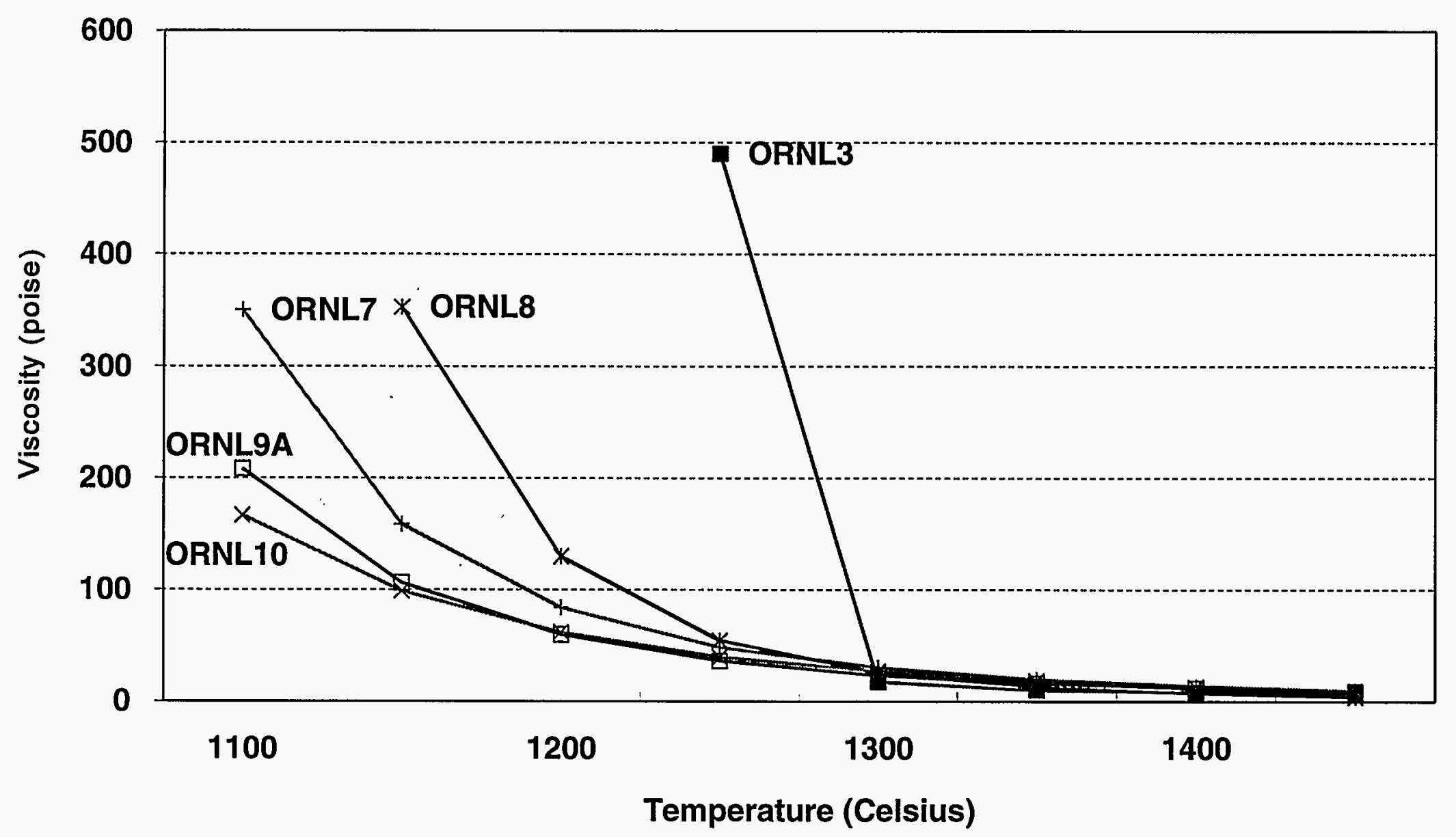

Page 4-36 
Figure 4.11.

Viscosity of the WETF and TSCA waste glasses as a function of temperature.

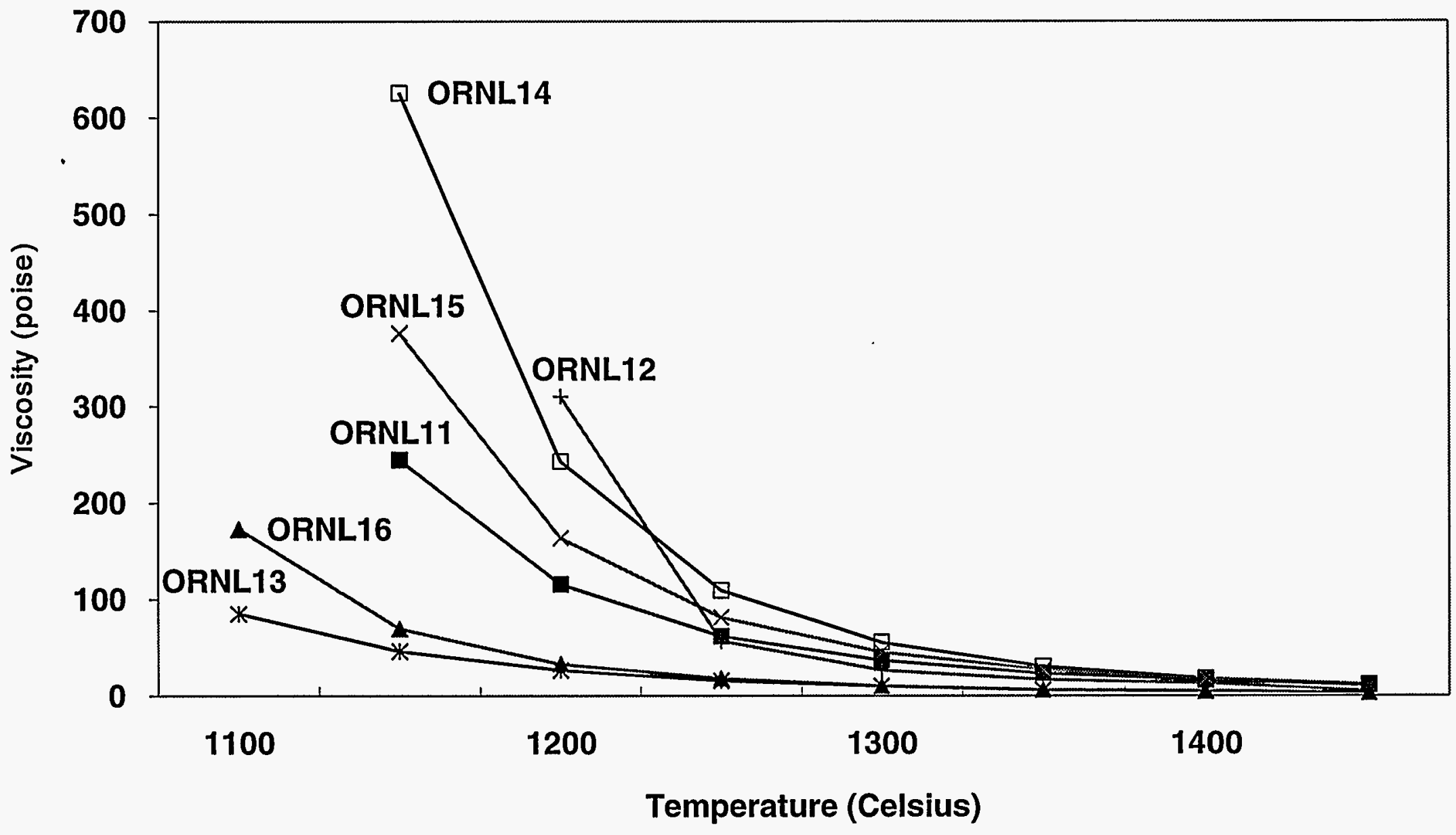

Page 4-37 
Figure 4.12.

PCT leaching of Oak Ridge waste glasses over one year. Normalized boron concentration compared to the Savannah River Reference Glass.

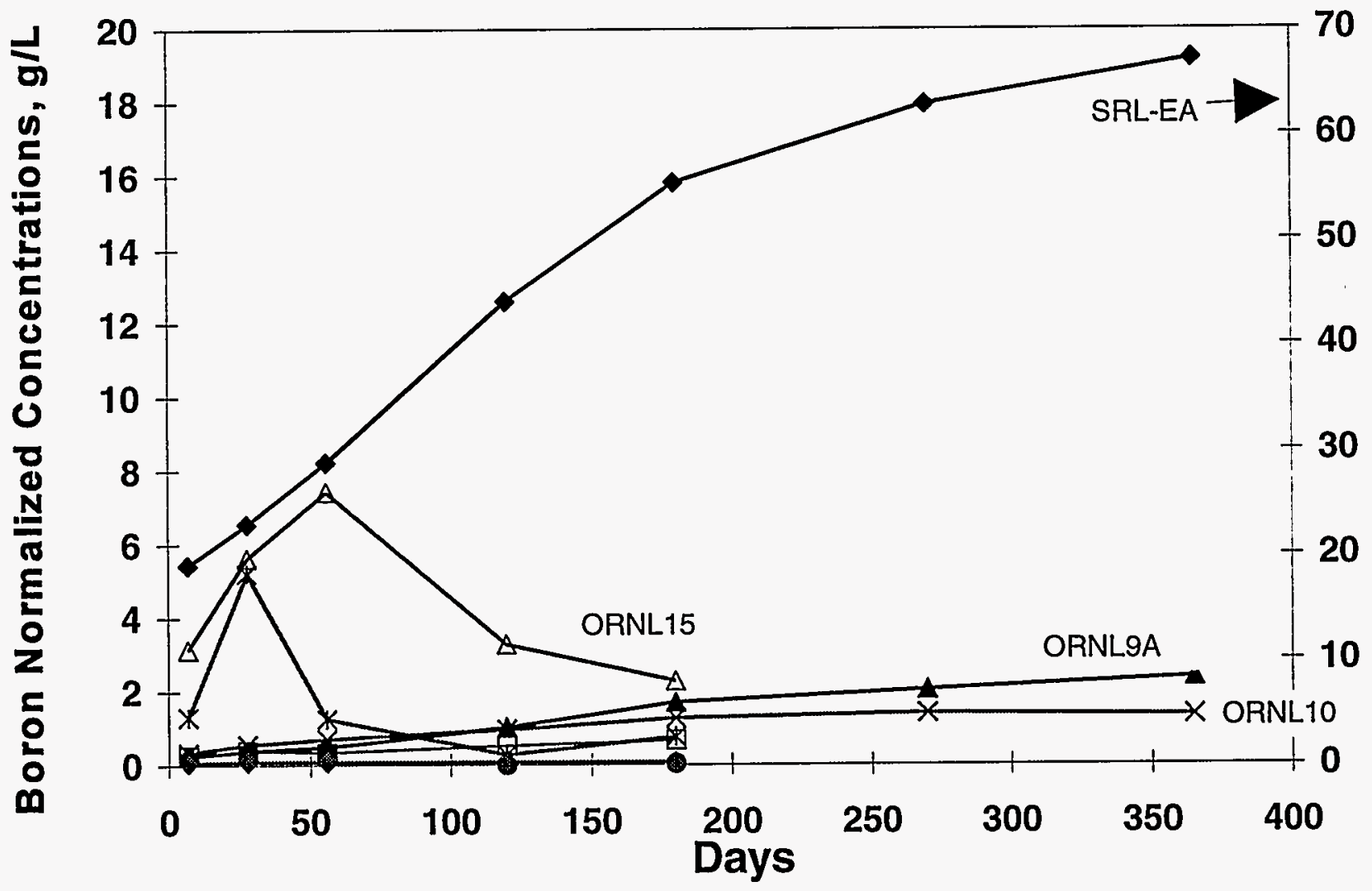


Figure 4.13.

PCT leaching of Oak Ridge waste glasses over one year. Normalized boron leach rate compared to the Savannah River Reference Glass.

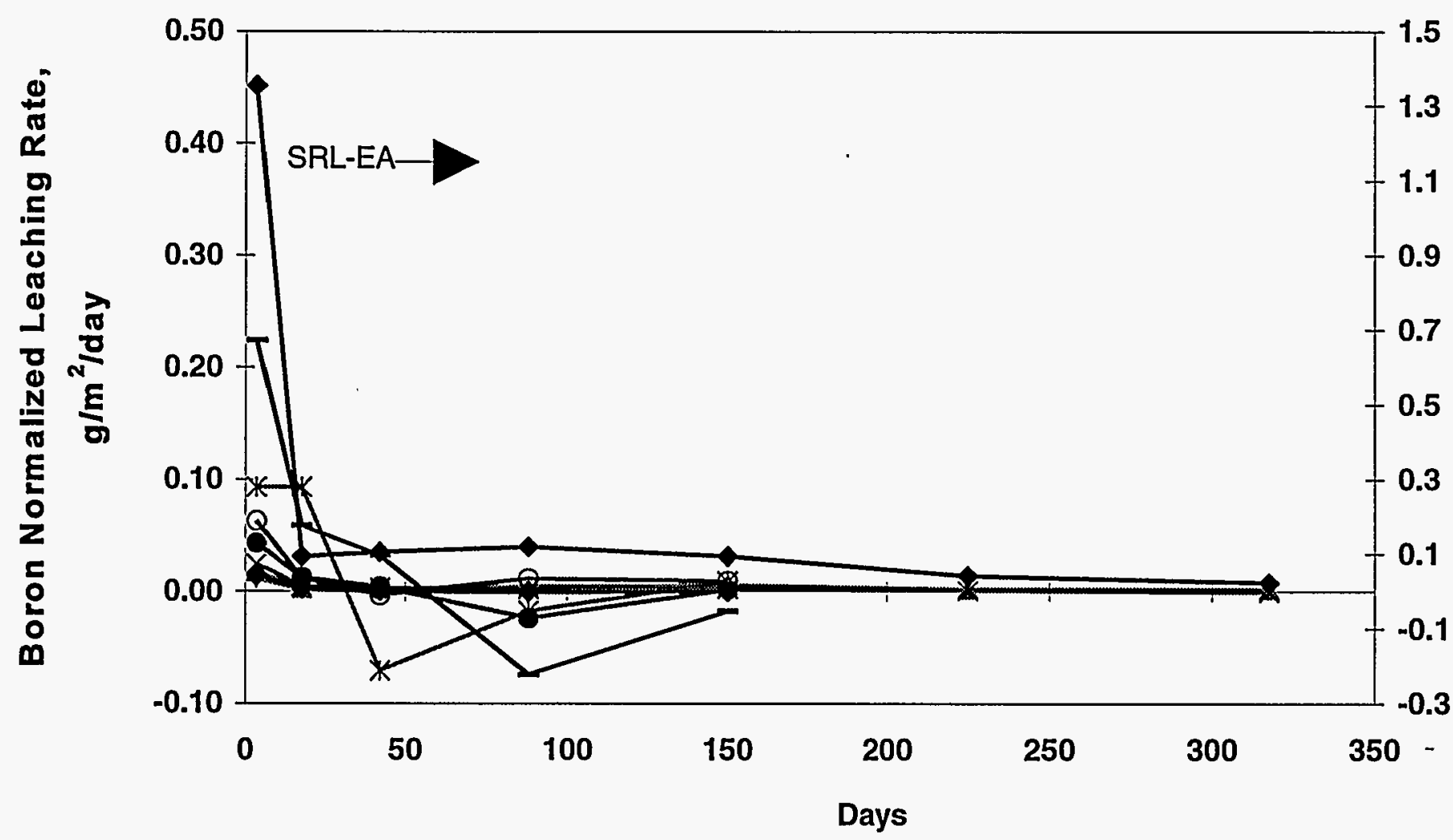

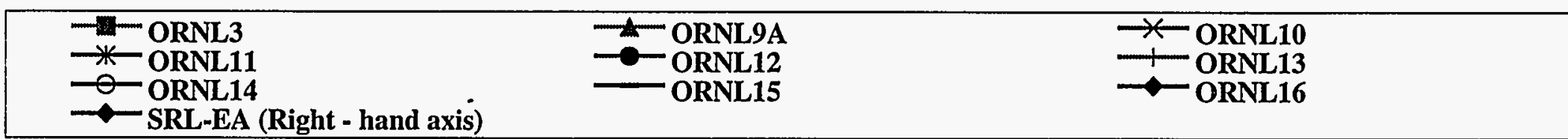


Figure 4.14.

PCT leaching of Oak Ridge waste glasses over one year. Normalized silicon concentration compared to the Savannah River Reference Glass.
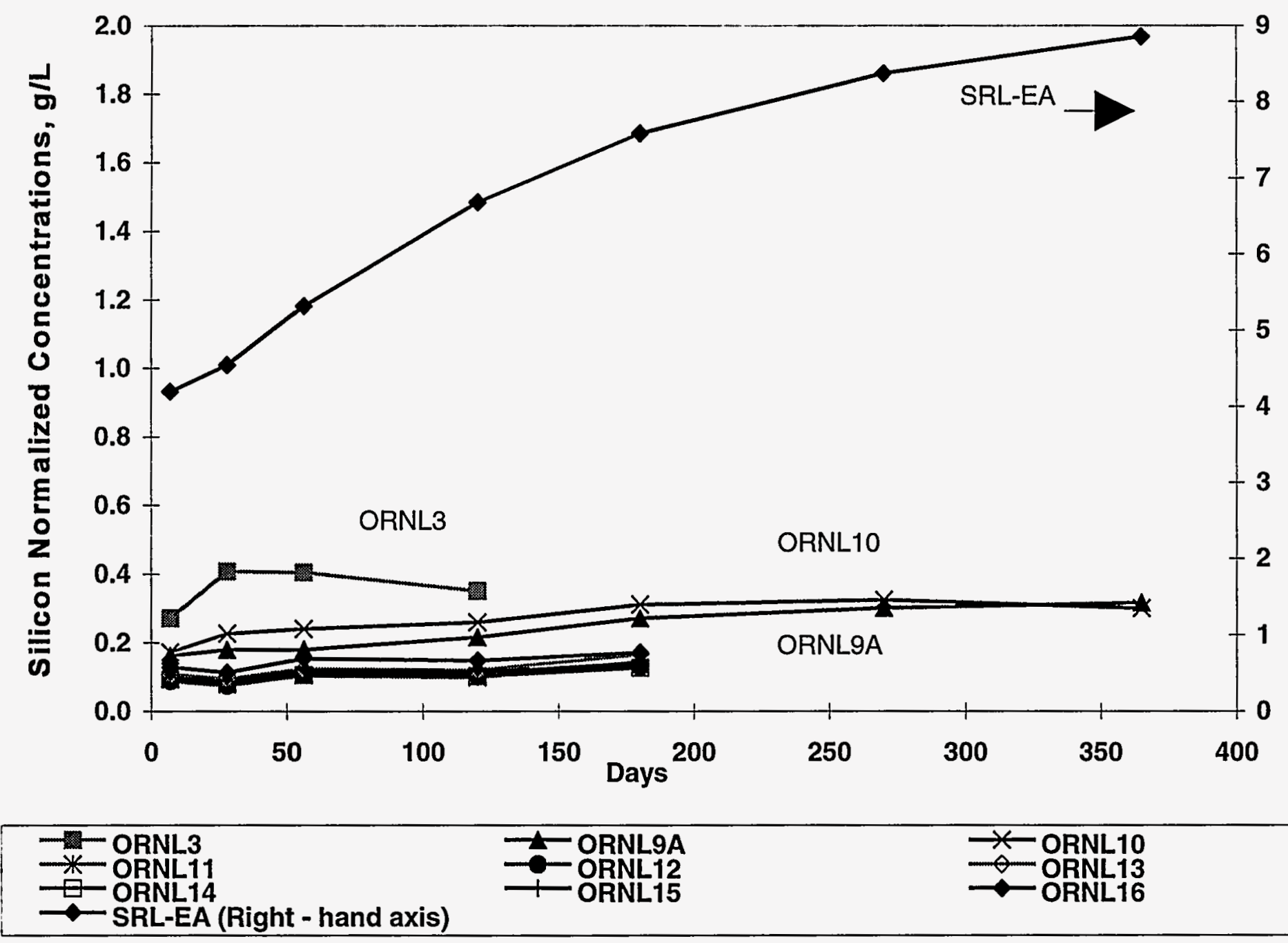

Page 4-40 
Figure 4.15.

PCT leaching of Oak Ridge waste glasses over one year. Normalized silicon leach rate compared to the Savannah River Reference Glass.

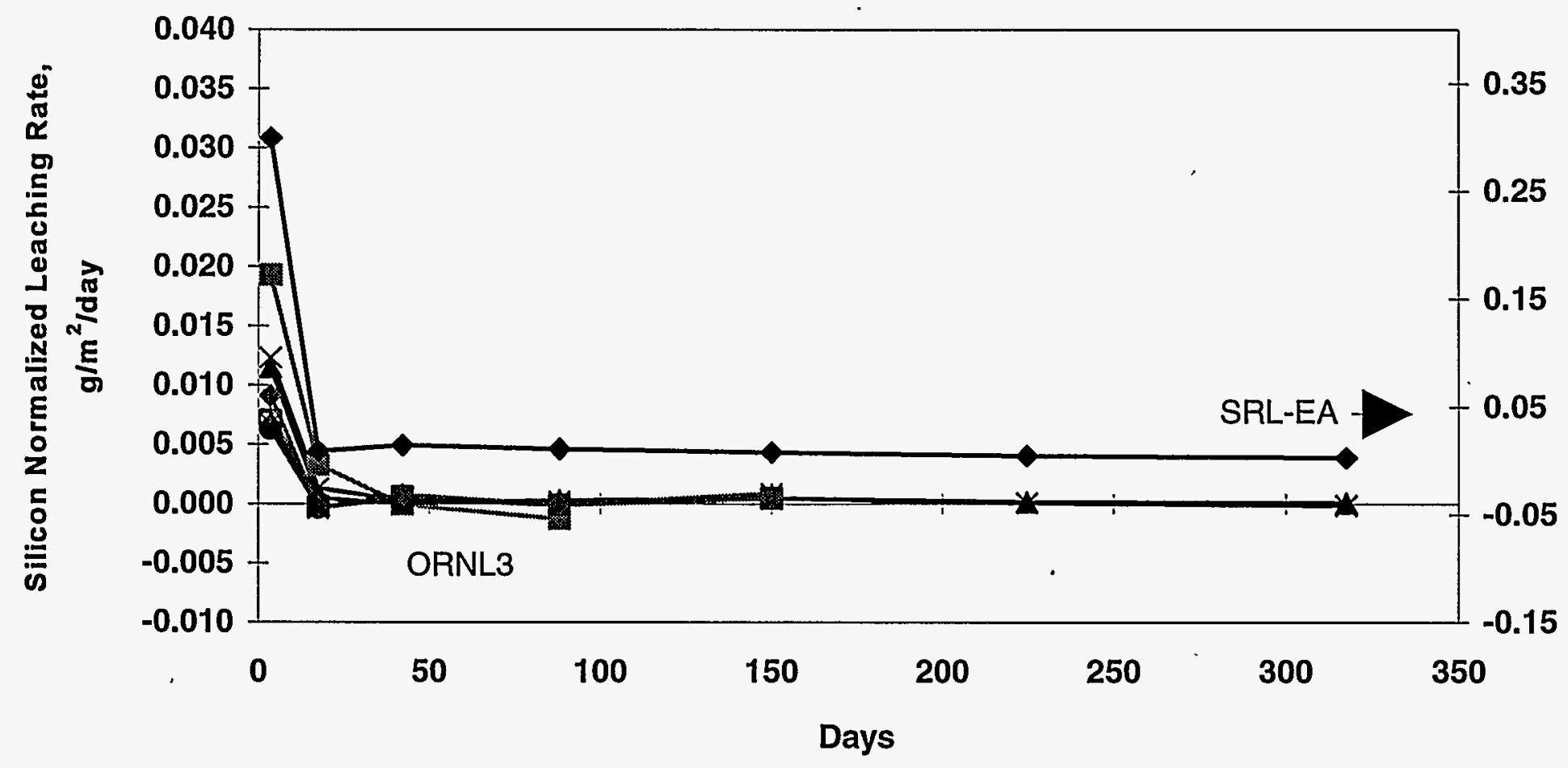

\begin{tabular}{|c|c|c|}
\hline $\begin{array}{l}-1-\text { ORNL3 } \\
-1-\text { ORNL11 } \\
\rightarrow \text { ORNL14 } \\
\sim \text { SRL-EA (Right - hand axis) }\end{array}$ & $\begin{array}{l}- \text { ORNL9A } \\
- \text { ORNL12 } \\
- \text { ORNL15 }\end{array}$ & $\begin{array}{l}\rightarrow \text { ORNL10 } \\
\text { ORNL13 } \\
\text { ORNL16 }\end{array}$ \\
\hline
\end{tabular}


Figure 4.16.

PCT leaching of Oak Ridge waste glasses over one year. Normalized sodium concentration compared to the Savannah River Reference Glass.

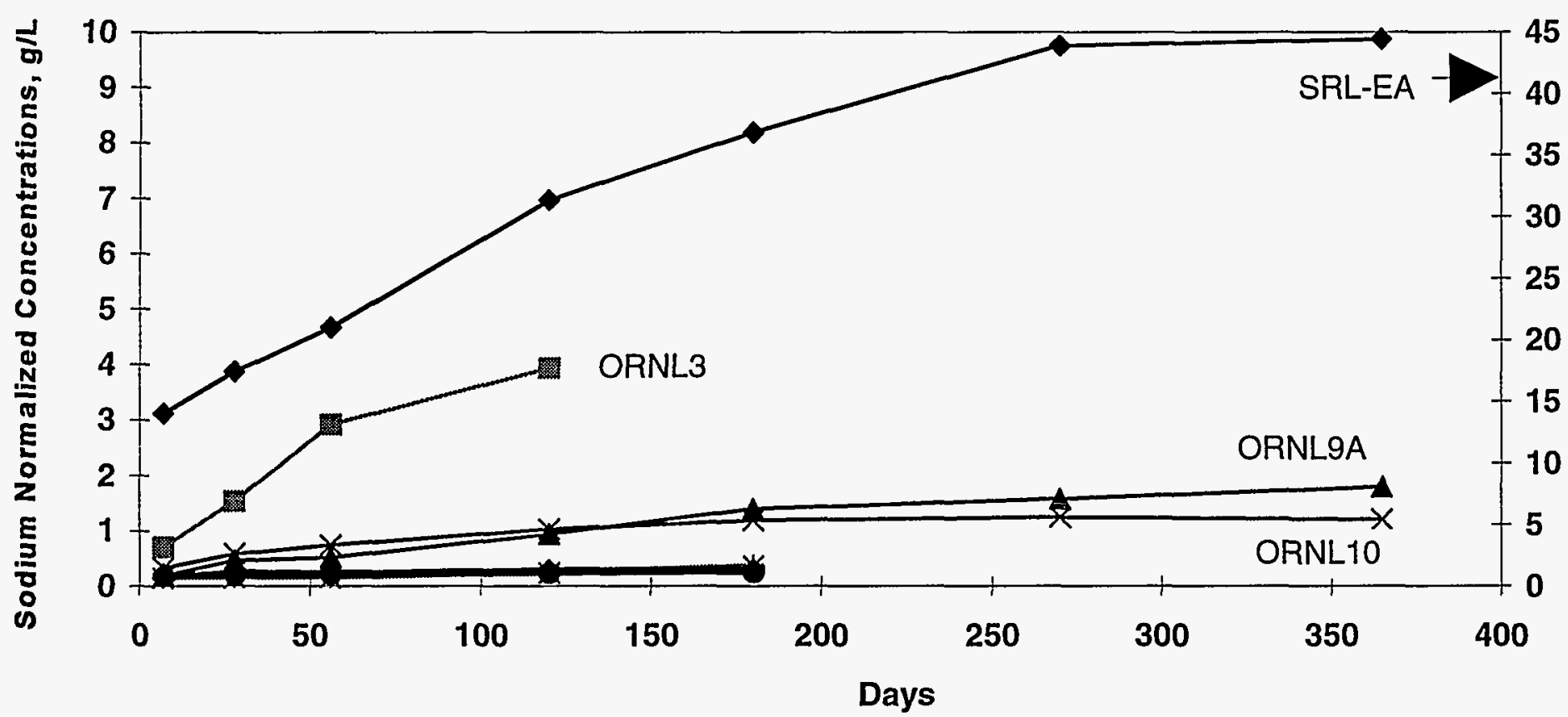

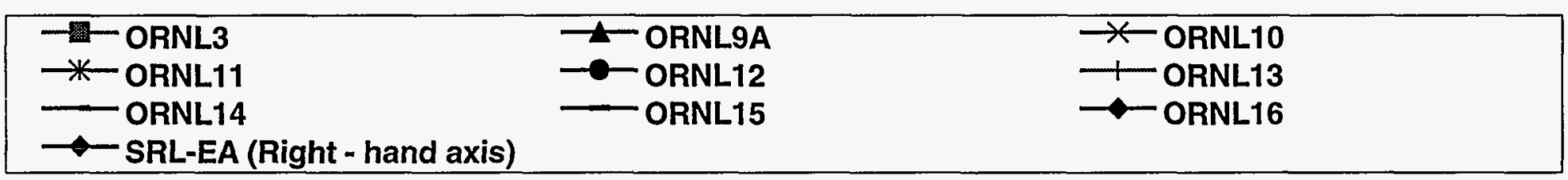


Figure 4.17.

PCT leaching of Oak Ridge waste glasses over one year. Normalized sodium leach rate compared to the Savannah River Reference Glass.

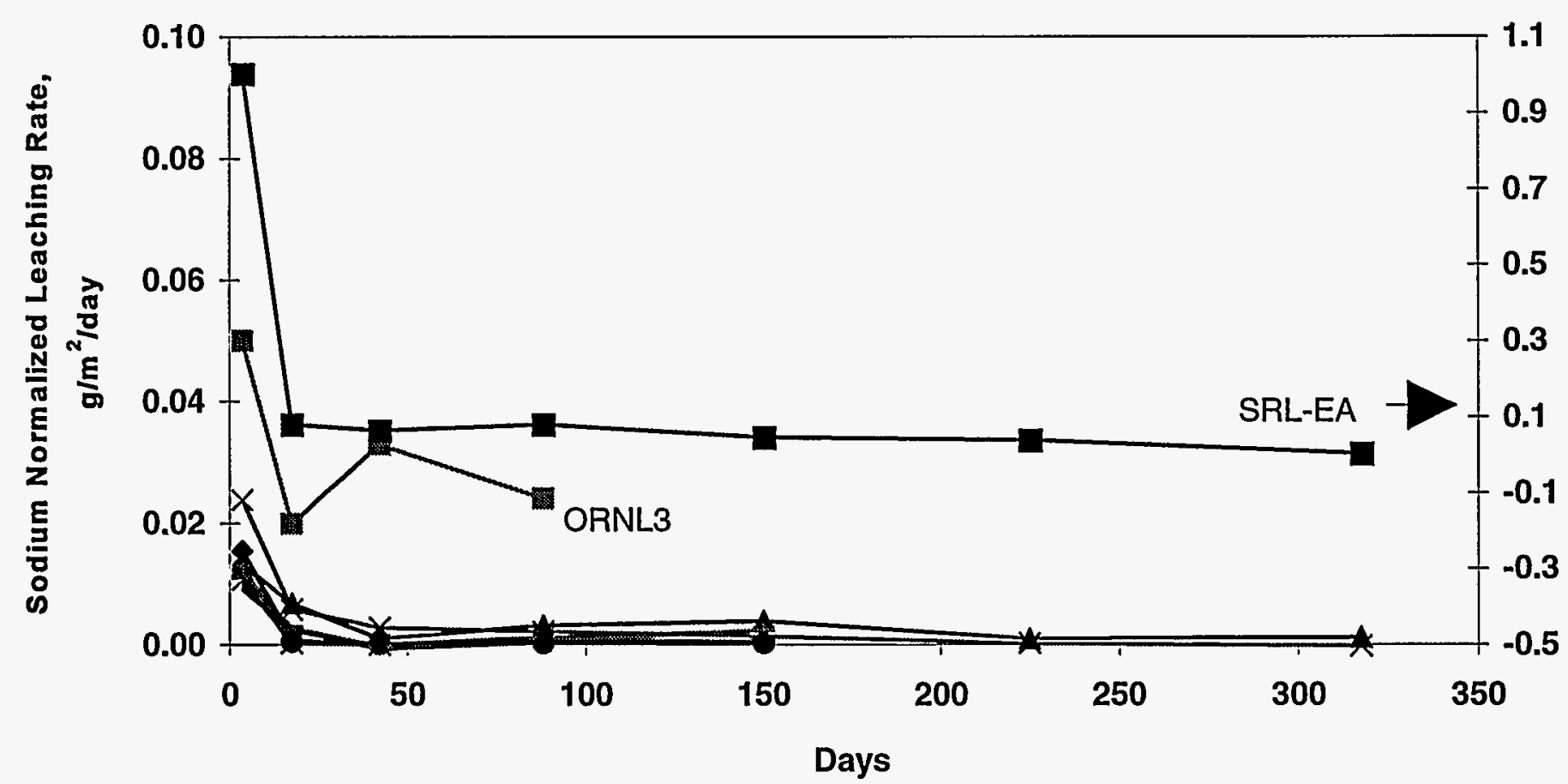

\begin{tabular}{|c|c|c|}
\hline $\begin{array}{l}\Rightarrow \text {-ORNL3 } \\
\rightarrow \text {-ORNL11 } \\
\Rightarrow \text { ORNL14 } \\
\rightarrow \text { - SRL-EA (Bight - hand axis) }\end{array}$ & $\begin{array}{l}\text { 드-ORNL9A } \\
- \text { ORNL12 } \\
- \text { ORNL15 }\end{array}$ & $\begin{array}{l}\rightarrow \leftarrow \text { ORNL10 } \\
+ \text { ORNL13 } \\
\rightarrow \text { ORNL16 }\end{array}$ \\
\hline
\end{tabular}


Figure 4.18.

TCLP performance of selected Oak Ridge pond waste glasses for nickel and uranium.

Uranium follows the concentration in the glass while nickel is very nonlinear due to the decrease of spinel content and leaches higher in ORNL3 in which nickel is lowest.

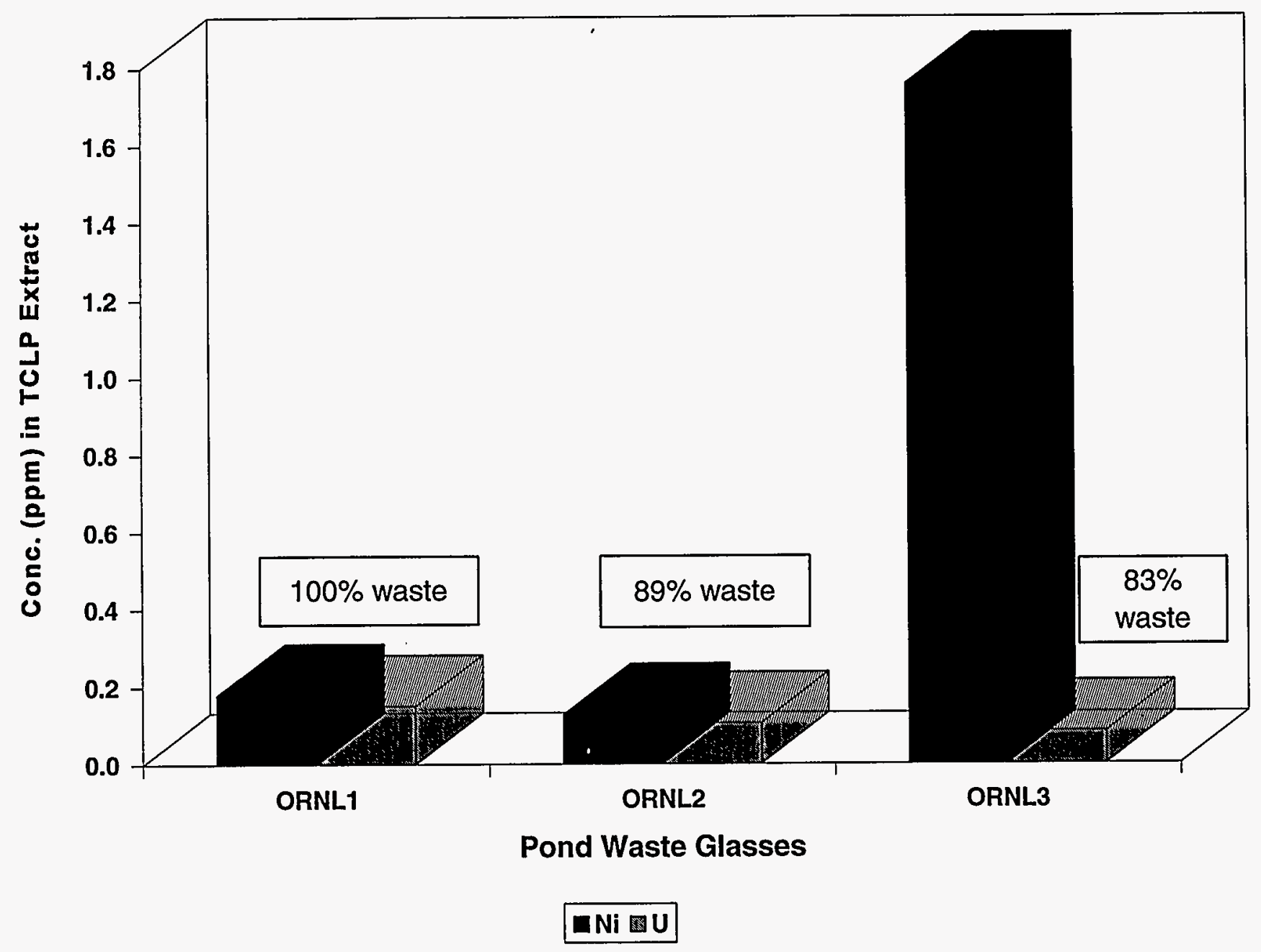

Page 4-44 
Figure 4.19.

TCLP performance of the WETF and TSCA waste glasses for lead.

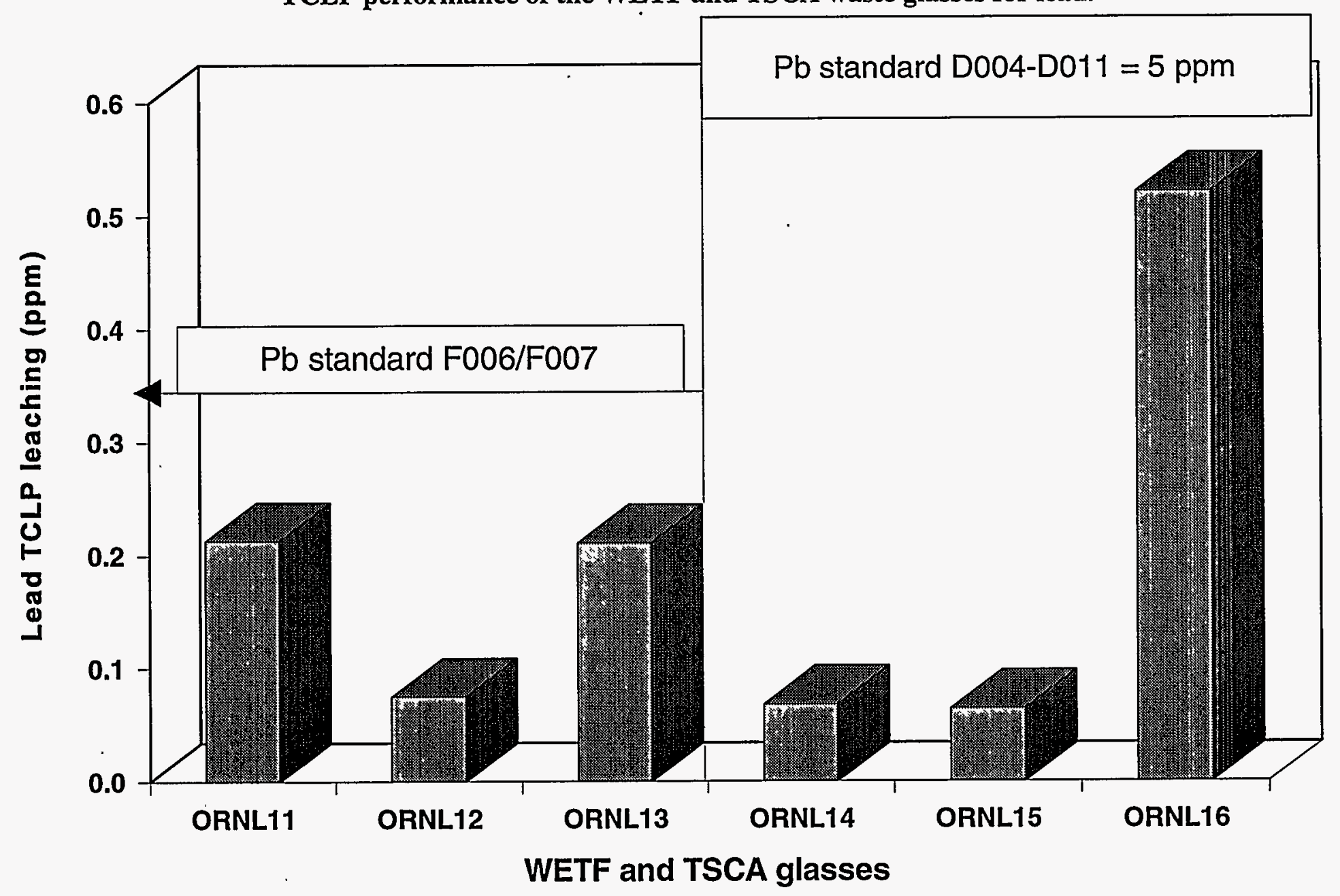

$\mathrm{QPb}$

Page 4-45 



\section{SECTION 5.0 \\ DEVELOPMENT OF COMPOSITIONAL ENVELOPE DATABASE AND MODELING CAPABILITIES}

\subsection{Introduction}

This section discusses the development of a computer-based, integrated glass propertycomposition database and predictive modeling facility. The major objective of this facility is to support the design and formulation of glass compositions using the MAWS approach to maximize waste loading. The longer term objective is that this modeling capability will allow glasses to be formulated from single waste streams or combinations of waste streams subject to a variety of userimposed constraints including waste stream usage priorities, process related constraints (e.g., melt viscosity, electrical conductivity, etc.), and performance related constraints (e.g., TCLP and PCT leaching characteristics). This capability will be useful in guiding future glass formulation studies to support any future applications of vitrification technologies.

In this proposed modeling approach, the data contained in the database form the starting point and the glass composition property models are the tools that permit useful predictions to be made based on these data; once a specific waste treatment problem is defined, a range of viable solutions of acceptable glass formulations and waste stream blends can be proposed by the model and serve as a staring point for laboratory vitrification.

The basic element required to fulfill the objectives discussed in the Introduction is a well organized glass property-composition database, encompassing data relevant to a wide range of DOE waste streams and models. In the following, we discuss the development of the Glass CompositionProperty Database (GCPD) and modeling capabilities, and evaluate the performance of the propertycompositional models.

\subsection{Glass Composition-Property Database}

\subsubsection{Database Management System}

The Oracle7 Client/Server Database Management System was selected as the platform for the development of this system. Oracle7 has all of the advantages of an advanced relational database, particularly in its simple, tabular data structures and powerful data manipulation operations. Moreover, Oracle7 software provides full support for a multi-user working environment on a PC- 
platform, thus allowing the direct integration of the glass database into a local area network. The client/server architecture of Oracle7 can simplify the procedure of developing custom client applications and reduce the cost of database construction and operation by using widely available, inexpensive, low-end client workstations to access the database server via a computer network (Figure 5.1).

\subsubsection{Glass Composition-Property Database: Contents and Structure}

The Oracle7 relational database management system stores data in the form of tables. The table, in a relational database, is a focused collection of related information, i.e., a set of data records that all have the same attributes. The tables and their relationship to each other represent the logical structure of the database. A list of all of the attributes of the tables thus describes the contents and structure of the data stored in the database. Figure 5.2 shows tables we have created in the GCPD. Each of these is linked to others, directly or indirectly, by a unique relationship, or entity code (in this case, a unique glass identification number).

Table 5.1 describes the major contents of the GCPD tables. SYSTEM.G_VIS stores all of the viscosity data, which contains, at the time of writing, 1375 viscosity measurements on 299 molten glasses with complete compositional data (the total number is larger if glasses with incomplete compositional data are included). PCT data, which actually consist of large numbers of different test parameters and data types, were grouped and stored in several tables (such as, SYSTEM.PCTINDEX and SYSTEM.PCTRAWDATA); many experimental parameters are controlled (or varied) in PCT experiments, but frequently only part of the information is needed, depending upon the requirements of specific users. The data stored in each table is linked through the unique glass numbers that are allocated and strictly controlled by the database system administrator. This means that users can retrieve data pertaining to, for instance, glass name, waste site or project name, glass composition, and corresponding viscosity data at all temperatures, although they are stored in separate tables. The typical interactions between end users and the Database server (e.g., enter, retrieve, or analyze data), rely on various client application user interfaces.

\subsubsection{Database User Interface: Client Application Development}

The client application, as the front end component of a database system, determines how users enter/retrieve information to/from the database. Technically, a client application uses a Structured Query Language (SQL) statement to send information to and request information from the database server. Oracle7 provides some useful client applications and many client application development 
tools. Table 5.2 lists the client applications and application development tools leased from Oracle, Inc. for this work. Oracle Data Browser and SQL* Plus are interactive data access or query applications for end users. Oracle Forms, Oracle Reports and Oracle Graphics are useful tools to develop the custom client application interfaces. "Forms" is suitable for data entry and on-line transaction; "Reports" and "Graphics" are suitable for text and visual reporting applications. All of these tools were employed in developing user interfaces for the GCPD.

Table 5.3 gives a list of client applications created for the GCPD, and describes briefly the major functions of each application from the view point of an end user. To illustrate the functions of these intuitively designed graphical user interface applications, we have included two computer screen captures from the Glass Entry Form application (Figures 5.3 and 5.4). Any authorized user linked to the Oracle7 server can use this Form application to view and enter glass data in the associated windows of composition, viscosity, and conductivity. Another versatile custom client application is the Glass Modeling Tool application which integrates various Oracle tools (e.g., Form, Reports, and Graphics) and allows users to analyze data in various ways and prepare data for modeling work (Figure 5.5). For example, a user can select any group of glasses by any class from the GCPD, select properties such as viscosity, conductivity, or 7-day PCT data, then display the selected data in 2-D scatter plots, or in tables of rows and columns. For users who wish to do more sophisticated statistical analysis or modeling, the Glass Modeling Tool application has a separate menu under which data can be prepared for property compositional models. The selection query will then be stored as a view in the database server, and later sent to a user-preferred modeling program that can be completely independent from the Oracle products. In this way, the modeling work is integrated dynamically into the database. As will be discussed later in the modeling section, the direct link between end users and the database allows us to develop a data-driven modeling-prediction application giving users greater flexibility in selecting the data on which any particular model is based.

Quality Assurance considerations were also taken into account and were factored into the database development process. All of the data were carefully checked both before and after entry into the database; the information was also subjected to systematic random sampling and verification checks. By using the data integrity procedures provided by the Oracle7 database server, multiple field constraints were enforced for every table created in the GCPD. This feature allows a variety of logical validity checks; an example is the check for valid values in the compositional range fields for a glass component which cannot be less than zero or more than $100 \%$. An entered value that does not satisfy the data integrity constraints will not be accepted by the database server. 


\subsection{Glass Composition-Property Model Development Results}

The development and refinement of useful predictive models or correlations that represent the compositional dependence of the key properties of glasses and their melts, based on the information stored in the database, is an essential aspect of the overall system development. In the following sections, we first describe the compositional coverage of the data that were used in the modeling work; obviously the broader and denser the coverage, the better should be the prediction capability of the system in the identification of the composition envelope for any specified set of constraints (i.e., vitrification application). We then discuss the basic considerations in the selection of the models, and the evaluation of the performance of these correlations in reproducing experimental data and making predictions.

\subsubsection{Sample Distribution in Compositional Space}

The primary data source for this work was the experimental data reported in Sections 2-4. However, these data were supplemented with data from other vitrification studies that have been conducted at Catholic University; this increased the quantity and scope of the data. The individual data sets are grouped by glass designator prefixes that relate to the waste origin or project. The additional data sets used (beyond those in Sections 2-4), their corresponding designators, and their sources are as follows:

100U = Fernald (Fu-1994a); ANLG = Oak Ridge (Pegg-1993a); CM0 = Fernald (Fu1994a); DIG = Bavaria (Muller-1993a); F-4 = Fernald Pit 4 (Fu-1994b); F-5 = Fernald Pit 5 (Fu-1994a, Fu-1993); MIC2, 3 = (Fu-1994a); MM1 = Medical Waste (Yan-1994); SMG = Savannah River M-Area (Muller-1993b); WETW = Wet Wastes (Brandys-1995); WS = Weldon Spring (Pegg-1993a); WV = West Valley (Adel-Hadadi-1988, Pegg-1991, Pegg1993b, Pegg-1994c).

\subsubsection{Sample Distribution of Viscosity Table}

The viscosity table, which is the largest table in the glass composition-property database, contains complete experimental characterization records of 299 glasses including all of the experimental data discussed in the previous sections.

The glasses from the viscosity table cover a wide range in compositional space. Figures 5.6a to $6 \mathrm{~d}$ show data distributions for $\mathrm{Al}_{2} \mathrm{O}_{3}, \mathrm{~B}_{2} \mathrm{O}_{3}, \mathrm{SiO}_{2}$, and $\mathrm{Na}_{2} \mathrm{O}$ wt\%. About $30 \%$ of the glasses do not contain $\mathrm{B}_{2} \mathrm{O}_{3} ; \mathrm{Al}_{2} \mathrm{O}_{3}$ is not generally higher than $15 \mathrm{wt} \%$; the $\mathrm{SiO}_{2}$ content clusters around 30 to 50 
wt\%; and $\mathrm{Na}_{2} \mathrm{O}$ ranges from 0 to $40 \mathrm{wt} \%$. The composition ranges and the median values for all major components (mostly oxides) are given in Figure 5.7.

For a clear picture of the compositional range that is covered by the present data set, we explored various ways of projecting the compositional data into two dimensional diagrams. The objective is to show the coverage of our GCPD, particularly in relation to distinguishing the characteristics of the underlying waste streams and DOE sites (as applicable). Ideally, the axes of the projection diagrams should also indicate boundaries of making stable or durable glasses to facilitate discussions of the interrelationships between glasses from different locations and rationalization of their properties using structural information on the microscopic scale.

Three parameters were selected to show the distribution of glasses in such a "reduced" compositional space: NBOT, M3T and M1M12. These parameters were constructed in terms of the prevailing or characteristic short-range structures or configurations in the glasses:

$\begin{array}{ll}\text { NBOT }= & \begin{array}{l}\text { Average number of non-bridging oxygens per network forming tetrahedral } \\ \text { site; }\end{array} \\ \text { M3T }= & \begin{array}{l}\text { Occupancy fraction of network forming trivalent cations }\left(\mathrm{Al}^{3+}, \mathrm{B}^{3+} \text { and } \mathrm{Fe}^{3+}\right) \\ \text { in network forming tetrahedral sites; }\end{array} \\ \text { M1M12 }= & \begin{array}{l}\text { Charge contribution of alkali cations in regard to alkali and alkaline earth } \\ \text { cations. }\end{array}\end{array}$

The number of total non-bridging oxygens is defined as being equal to the number of charges carried by the alkali and alkaline earth cations not used to charge-balance tetrahedrally coordinated $\mathrm{Al}, \mathrm{B}$, and $\mathrm{Fe}^{3+}$, since none of the glasses in the GCPD contains free oxygens. For a simplified picture, we assume that, with sufficient charge-balancing alkali and alkaline earth cations, all of the $\mathrm{Al}, \mathrm{B}$, and $\mathrm{Fe}^{3+}$ will reside in tetrahedral sites copolymerizing with $\mathrm{Si}^{4+}$ tetrahedra as part of the network (Mysen-1981, Mysen-1985, Engelhardt-1985, Kirkpatrick-1986, Domine-1986, Dickenson-1981, Dyar-1985, Brown-1988, Mysen-1989,.Park-1979, Dell-1983, and Gan-1994). Since almost all glasses in the viscosity table have more than enough, or approximately equal, $\mathrm{M}^{1+}$ and $\mathrm{M}^{2+}$ to neutralize the tetrahedrally coordinated $\mathrm{M}^{3+}$ species, the total number of non-bridging oxygens can be simply calculated as the difference between the sum of the charges of $\mathrm{M}^{1+}$ and $\mathrm{M}^{2+}$, and the sum of $\mathrm{M}^{3+}$ cations. M3T is the ratio of the sum of $\mathrm{Al}^{3+}, \mathrm{B}^{3+}$, and $\mathrm{Fe}^{3+}$ to the sum of all network forming cations $\left(\mathrm{Al}^{3+}, \mathrm{B}^{3+}, \mathrm{Fe}^{3+}\right.$ and $\left.\mathrm{Si}^{4+}\right) . \mathrm{M} 1 \mathrm{M} 12$ is the ratio of the sum of alkali cations to the sum of alkali cations and two times the alkaline earth cations. NBOT is an index for the degree of polymerization in silicate melts because the number of the non-bridging oxygens associated with a network forming cation (e.g., $\mathrm{Si}^{4+}$ or $\mathrm{Al}^{3+}$ ) controls, on average, the connectivity of this cation to other network forming cations surrounding it as the next nearest neighbors. M3T indicates the fraction, on average, of networking tetrahedral sites occupied by $\mathrm{M}^{3+}$, and $\mathrm{M} 1 \mathrm{M} 12$ gives the fraction of alkali cation 
among $\mathrm{M}^{1+}$ and $\mathrm{M}^{2+}$ on an equal charge basis. Functionally, as presentation variables, M3T can differentiate glasses with a similar degree of polymerization (i.e., similar NBOT), and, M1M12 can further set apart glasses with similar NBOT and M3T. All three parameters have reasonably well defined structural significance for silicate melts and glasses. The number of non-bridging oxygen atoms and its distribution among various network forming tetrahedra can be measured directly by various spectroscopic techniques (McMillan-1984, Schramm-1984, Dupree-1984, Dupree-1986, and Maekawa-1991). The occupancy fraction of $\mathrm{M}^{3+}$ cations in networking tetrahedra influences the intermediate range structure and the overall stability (Taylor-1979, Geisinger-1988 and Gan-1994); in particular, phase separation usually occurs when the ratio is too high. In addition to the wellknown (Paul-1990) strong effects on the melt viscosity, conductivity, and glass leach resistance, the abundance of the alkali cations relative to that of the alkaline earths, from a structural point of view, influences the bond strength in the glass network and controls the $\mathrm{Q}^{\mathrm{n}}$ species distribution at a given degree of polymerization (in this notation, $Q$ refers to a tetrahedral site and (4-n) is the number of non-bridging oxygens associated with one $\mathrm{SiO}_{4}$ tetrahedron) (Navrotsky-1984, DeJong-1981, Stebbins-1988, Maekawa-1991, Bockris-1954, Bockris-1955, Tickle-1967, Ingram-1987).

While the parameters selected have underlying structural implications, our primary use for these three parameters was to permit the display of sample distributions in reduced composition space, to compare general differences between them, and to evaluate the scope of the data used for the subsequent analyses. It is not the intention here to assign a definite physical or chemical meaning to all of the glasses projected in these diagrams on the basis of these parameters since the parameters themselves represent simplified structural interpretations. More importantly, the radioactive waste glasses under consideration contain far more components than the simple binary, ternary, or even commercial glasses upon which most of the structural studies have been focused. Furthermore, for this representation we did not take into account many of the minor components present in the waste glasses, nor did we consider the role of fluorine (as a major glass component in some of the glasses) in affecting NBOT, simply because of the lack of systematic and conclusive structural studies in that composition region. As a result, the distribution for some of the glasses, particularly the F-rich glasses from the Fernald site, may not bear quite the meaning that may be suggested by the facevalues of the axes. Nevertheless, the essential characteristics of the prevailing structure types should still be reasonably approximated for most of the glasses in the database.

Figure 5.8 shows the sample distribution in the space of NBOT versus M3T. It is interesting to note that glasses from the same DOE site cluster within a narrow region. Generally, the glasses in the viscosity table range from a $\mathrm{M}^{3+}$-rich, fully polymerized region (West Valley high-level and Savannah River high-level and $\mathrm{M}$-Area mixed waste glasses) to a $\mathrm{M}^{3+}$-poor (in a relative sense) rather depolymerized region (some very high sodium Hanford glasses and some from Weldon Spring glasses) bounded by four straight lines labeled $Q^{4}, Q^{2}, A n$ and $A b$, respectively. The An and $A b$ lines are at M3T $=0.25$ and 0.5 , which are coincident with the values observed in the two end members of 
the most abundant natural aluminosilicate mineral, plagioclase $(0.25$ for albite $(\mathrm{Ab})$ and 0.5 for anorthite (An).The sample distribution within this rectangular area is rather dense and well spread although not very uniform in coverage (Figure 5.9, note the maximum around West Valley glasses). This indicates that, on average, the glasses in the viscosity table vary considerably in the degree of polymerization from a fully polymerized framework $\left(Q^{4}\right.$ type) to a rather depolymerized single-chain type $\left(Q^{2}\right.$ type). For similar levels of NBOT, the trivalent cation occupancy varies from 0.25 (Ab type with one quarter of the tetrahedral sites occupied by $\mathrm{M}^{3+}$ ) up to around 0.5 (An type with equal $\mathrm{M}^{3+}$ and $\mathrm{Si}$ in tetrahedral sites in the network). If we neglect the F-rich glasses and a few very depolymerized glasses (between $\mathrm{Q}^{2}$ and $\mathrm{Q}^{3}$ type), most of the remaining glasses fall between the $\mathrm{Q}^{4}$ to $\mathrm{Q}^{3}$ and $\mathrm{An}$ to $\mathrm{Ab}$ boundaries. This observation should not be surprising. As is known, the melt viscosity, conductivity, and glass leach resistance are all very sensitive to the content of glass formers; a glass with fewer formers will be less durable, and a melt with high flux contents tends to have high conductivity and low viscosity. This understanding is consistent with the fact that $\mathrm{Q}^{4}$ type glasses (like West Valley) tend to have high M3T, and low-M3T glasses are in the less polymerized region (e.g., Hanford glasses). It is reasonable that most of the glasses along the M3T axis fall within the $\mathrm{An}$ and $\mathrm{Ab}$ boundaries. $\mathrm{Q}^{4}$ and $\mathrm{Q}^{3}$ types, representing a fully connected framework and a twodimensional sheet respectively, are still rather polymerized melts, and a $Q^{3}$ melt of $M 3 T=0$ is probably too viscous to process. Increasing M3T while holding NBOT fixed will decrease the melt viscosity significantly, but not drastically affect the conductivity and glass leach resistance. This is probably the reason why no glasses are present near the line of M3T $=0$. On the other hand, few glasses are projected beyond M3T $>0.5$ and it is known that silicate melts tend to be unstable in that region since the glass forming region will then either disappear or shift to a higher temperature (e.g., as in the $\mathrm{SiO}_{2}-\mathrm{Al}_{2} \mathrm{O}_{3}-\mathrm{Na}_{2} \mathrm{O}$ ternary) (Osborn-1960).

In summary, we find that:

(1) The glasses in the viscosity table cover a wide area in reduced compositional space. Glasses from the same site tend to cluster, differing in the space of NBOT versus M3T from those of different waste stream origins. Overall, the data fall between $0<\mathrm{NBOT}<2$ and $0.25<\mathrm{M} 3 \mathrm{~T}<0.5$, and are concentrated in the region of $0<\mathrm{NBOT}<1$ and $0.25<\mathrm{M} 3 \mathrm{~T}<0.5$.

(2) Data in the viscosity table map most of the possible composition regions suitable for radioactive waste vitrification (for silicate-based glasses). Regions outside of $Q^{4}-Q^{2}$ and $\mathrm{An}-\mathrm{Ab}$ limits are not likely to meet criteria for processability and leach resistance (this does not apply to F-rich glasses).

As a note in passing, it is interesting to find that the overall distribution of the trivalent occupancy parameter (Figure 5.10) shows a clear bimodal frequency distribution with peaks around 
$\mathrm{M} 3 \mathrm{~T}=0.25$ and 0.5 , which are coincident with the values observed in the two end members of the most abundant natural aluminosilicate mineral, plagioclase ( 0.25 for albite $(\mathrm{Ab})$ and 0.5 for anorthite (An)).

\subsubsection{Sample Distribution for Conductivity Table}

The distribution of samples in the conductivity table is quite similar to the sample distribution of the viscosity table (Figure 5.11) The major difference is the absence of any conductivity data for the West Valley glasses.

\subsubsection{Sample Distribution for Hanford, Oak Ridge, and Idaho Glasses}

Hanford glasses (using the data discussed in Section 2.0) represent the largest group of glasses studied in this work. Hanford glasses cover a wide range of NBOT and M1M12 (Figures 5.8 and 5.12). The degree of polymerization varies from nearly fully polymerized $Q^{4}$ type to a much more depolymerized $\mathrm{Q}^{1.5}$ (resembling double-chain tetrahedra structure, on average). However, the trivalent occupancy in tetrahedral sites (as described by M3T) is relatively low and varies little around M3T $=0.25$ (i.e., on average, $25 \%$ of the tetrahedral sites are occupied by trivalent network forming cations). ORNL (Oak Ridge) glasses vary considerably in both polymerization and trivalent cation occupancy. In contrast, IDG (Idaho) glasses show relatively little variation in both NBOT and M3T.

\subsubsection{Sample Distribution for 7-day PCT Table}

Glasses in the 7-day PCT table have a similar distribution pattern in the NBOT versus M3T plot (Figure 5.13); the data again fall generally within the $\mathrm{Q}^{4}-\mathrm{Q}^{2}$ and $\mathrm{An}-\mathrm{Ab}$ boundaries. However, in view of the somewhat sparser coverage (less data on this property exists) the predictive capability would be expected to be corresponding poorer for this property, especially for target compositions which fall into empty regions. Despite this caveat, the results presented below show that the models provide a good representation of the data in the database and produce reasonable predictions for glasses that are not included in the data set used to generate the correlation. 


\subsubsection{Property-Composition Models and Optimization Algorithm}

\subsubsection{Property-Composition Models for Viscosity, Conductivity, and 7-day PCT Data}

The aim of this modeling work is to develop the capability to predict properties of melts and glasses from their compositions. The key properties of concern are the melt viscosity, melt conductivity, and the 7-day PCT performance. The melt viscosity and conductivity are critical glass melter processing parameters, and 7-day PCT results provide an important measure of glass leach resistance. Unfortunately, there exist no practical fundamental methods of calculating the dependence of these properties on composition; the problem is especially complicated in view of the inherent multi-component nature of the glasses of interest. We have limited our modeling efforts to the use of the best possible empirical equations that can both reasonably reproduce the experimental data and give stable predictions for the glasses whose composition are encompassed by the compositional envelope of the underlying data set.

\section{Empirical Models for Melt Viscosity and Conductivity}

The dependence of melt viscosity on temperature can generally be represented by the Fulcher equation

$$
\ln \eta=A_{f} /\left(T-T_{0}\right)+B
$$

where $\eta$ is viscosity (herein, in poise), $T$ is absolute temperature in Kelvin, $A_{f}, B$, and $T_{0}$ are adjustable parameters. $A_{f}, B$, and $T_{o}$ vary considerably with melt composition. If the temperature is far above glass transition, an Arrhenius type of equation

$$
\ln \eta=A / T+B
$$

can usually describe viscosity data adequately, where $\mathrm{A}$ and $\mathrm{B}$ are adjustable parameters. The parameter A in (5-2) is proportional to the activation energy for viscous flow, and B is usually referred to as the pre-exponential factor. Since the viscosity data of interest relate to temperatures of several hundred degrees above the glass transition temperature, empirically, an Arrhenius equation is found to provide an adequate representation of the temperature dependence of the viscosity. We will therefore focus only upon empirical models of an Arrhenius form.

To build an empirical property-compositional model, the difficulty lies in how to introduce compositional variables appropriately. For a predictive model, it is preferable to incorporate the 
compositional variables in the simplest possible form, and hopefully in a way which is physically and chemically reasonable.

It is expected that the activation energy for viscous flow, $A$ in $(5-2)$, is influenced by the strengths of the prevailing chemical bonds in the melt (MacKenzie-1960 and Bockris-1970). For silicate-dominated melts, the flow process involves the breaking and rearrangement of the Si-O bonds as well as ionic effects (Farnan-1990). The effects due to configurational entropy tend to be evident only in very viscous melts at temperatures close to the glass transition and should be relatively unimportant in the temperatures of interest here (Adam-1965). Numerous theoretical and experimental studies have concluded that the Si-O bonds are subject to influences by other melt components as well as by temperature (Navrotsky-1985). For example, increasing alkali oxide in $\mathrm{M}_{2} \mathrm{O}-\mathrm{SiO}_{2}$ binary melts breaks more Si-O-Si bonds (creating non-bridging oxygens), thus making viscous flow easier (i.e., lowering the activity energy). Even for systems with the same level of polymerization (e.g., all $\mathrm{Q}^{3}$ ), different metal cations would affect $\mathrm{Si}-\mathrm{O}$ bond strength differently (Navrotsky-1985). The apparent activation energy A and pre-exponential factor B could be reasonably viewed as the sum of contributions from all melt components. In the simplest approximation, we therefore consider a linear combination of these composition effects:

$$
\ln \eta=\Sigma a_{i} X i / T+\Sigma b_{i} X i
$$

where $a_{i}$ and $b_{i}$ are coefficients describing the contribution from component $i$, and $X_{i}$ is the concentration of component $i$ in the melt. Equation (5-3) is, of course, an over-simplified expression for the complex viscous flow process in multi-component silicate melts. But there is good evidence, for example, that high-temperature viscosity data along some pseudo-binary aluminosilicate melts are indeed additive (Ritchet-1984). Moreover, if we wish to include a consideration of the interactions among various melt components, we can further include ad hoc compositional variables of higher orders. For example, we can let

$$
A=\Sigma a_{i} X_{i}+\Sigma a_{i j} X_{i} X_{j}
$$

However, as we will show later, a higher-order empirical model does not significantly improve the model performance in making predictions.

Ionic conduction, as measured by electrical conductivity, is not controlled by the same mechanism that controls viscous flow. However, DC conductivity data are also found to follow the Arrhenius equation quite well. The activation energy for conduction will depend on the size and charges of the charge carriers as well as their concentration (MacKenzie-1960) and many factors imposed by other melt components which may or may not be efficient charge-carriers themselves. 
We again assume an empirical model for the DC conductivity involving linear combinations of the contributions from the various melt components:

$$
\ln \epsilon=\Sigma \mathrm{a}_{\mathrm{i}} \mathrm{Xi} / \mathrm{T}+\Sigma \mathrm{b}_{\mathrm{i}} \mathrm{Xi}
$$

where $\epsilon$ is the DC conductivity in $\mathrm{S} / \mathrm{cm}$, and the other variables are defined in the same way as in the viscosity model (5-3).

\section{Empirical Models for 7-Day PCT Data}

A similar empirical model was also adopted for the 7-day PCT data involving a linear combination of composition variables. There are obvious complications involved, however, such as the fact that the glass is not a thermodynamically stable phase under standard PCT conditions and that 7 days is not long enough to establish even a transient steady state for partitioning of elements between a test glass and solution. Nevertheless, if we assume that the thermodynamic "driving force" plays an important role in determining the 7-day PCT results (again remembering that equilibrium is not actually reached) and that the elemental concentrations in solution are at least influenced by the fictitious "equilibrium constant" for the "equilibrium" of element $Z$ between glasses and solution, we can write the reaction as

$$
\mathrm{Z} \text { (in glass) }=\mathrm{Z} \text { (in solution), }
$$

and then,

$$
R T \ln a_{Z \text { (in solution) }} / a_{Z \text { (in glass) }}=-\Delta G .
$$

where $a_{z}$ is the activity of element $Z$ in solution or glass. The value of $\Delta G$ is composed of a number of contributions including the effects of bonding, structure, composition, and entropy of mixing in the glass as well as the thermodynamics of the aqueous solution. In the "hydration thermodynamic" model of Newton and Paul (Newton-1980) and later Jantzen and Plodinec (Jantzen-1984), the glass is treated as a mechanical mixture of somewhat arbitrary units and $\Delta G$ is assumed to be the molefraction-weighted sum of the hydration free energies of these units. If one assumes that $\Delta G$ is a linear combination of the contributions from each glass component, one obtains

$$
R T \ln \mathrm{a}_{Z_{\text {(in solution) }}} / a_{Z \text { (in glass) }}=\Sigma g_{i} X_{i}+C
$$


where the $g_{\mathrm{i}}$ are fictitious free-energy coefficients and $\mathrm{C}$ is a constant. At constant temperature, if one assumes a constant ratio of the activity coefficient of $Z$ in solution to the product of the activity coefficient of $Z$ in the glass and the concentration of $Z$ in glass, we obtain

$$
\ln [Z]_{\text {(in solution) }}=\Sigma a_{\mathrm{i}} \mathrm{X}_{\mathrm{i}}+\mathrm{C}^{\prime}
$$

where $\mathrm{C}^{\prime}$ is a constant and $\mathrm{a}_{\mathrm{i}}$ is simply proportional to $\mathrm{g}_{\mathrm{i}}$; pragmatically (especially in view of all of the caveats noted above), these are parameters to be determined by statistical regression of actual data.

While the heuristic argument above may have some merit, it is an empirical fact that, from a statistical point of view, for a linear mixture model like (5-9), the goodness of fit as measured by $\mathrm{R}^{2}$ (total variance of the dependent variable explained by the model) improves significantly after transforming [Z] to $\ln [Z]$. Thus, we have used equations of the form (5-9) in our analysis of the PCT data in this work.

\subsubsection{Weighted Least Squares Regression}

An empirical model obviously only provides an approximation to the true unknown mechanism and always performs better in fitting the data than in making predictions. In general, it is unlikely that linear mixture models, as proposed above, would provide adequate representations of the behavior of the properties of interest over a wide composition range; their performance should improve, however, as the composition range is limited. Given the importance of the ability to make reliable predictions, as opposed to fitting the data, we have avoided mathematical equations of higher-order which tend to be less stable in nature. As an alternative, we have investigated methods of emphasizing interpolation rather than extrapolation.

In this approach, a weighting factor is used for each observation, the value of which depends on the distance of the observation from the point of interest. The weight function we have used takes a Gaussian form which decays away from the center point depending on the Euclidean distance defined in the multi-dimensional glass composition space:

$$
\mathrm{w}_{\mathrm{i}}\left(\mathbf{X}_{\mathrm{i}}, \mathbf{X}_{0}\right)=\exp \left(-\mathrm{D}_{\mathrm{i}}^{2} / 2 \sigma^{2}\right)
$$

where $\mathrm{w}_{\mathrm{i}}\left(\mathbf{X}_{\mathrm{i}}, \mathbf{X}_{0}\right)$ is the weight function for the $\mathrm{i}$-th observation, $\mathbf{X}_{\mathrm{i}}$ and $\mathbf{X}_{0}$ are composition vectors for the $i$-th observation and for the center of weight respectively [i.e., $X_{i}=\left(X_{i 1}, X_{i 2}, \ldots X_{i n}\right), X_{0}=\left(X_{01}\right.$, $\mathrm{X}_{02}, \ldots \mathrm{X}_{0 \mathrm{n}}$ ), and $\mathrm{n}$ is the dimension of the composition space], $\sigma$ is the parameter controlling the 
dispersion of the weight function, and $\mathrm{D}_{\mathrm{i}}$ is the Euclidean distance in n-dimensional compositional space:

$$
\mathrm{D}_{\mathrm{i}}^{2}=\Sigma_{\mathrm{j}}\left(\mathrm{X}_{\mathrm{ij}}-\mathrm{X}_{0 \mathrm{j}}\right)^{2}
$$

To perform the weighted least-squares regression, an integer form of the weights is required by the statistical analysis software that we used (Statistica ${ }^{\mathrm{TM}}$ by Statsoft). The weights calculated by $(5-10)$ were therefore truncated (toward zero) to the nearest integer before being used in the leastsquares regression.

A major advantage of using an integrated database-modeling system to provide the desired predictive capability is that we obtain a "data-driven" model optimized in the region of interest rather than a supposedly universal set of coefficients resulting from a global fit. In the weighted regression approach, the center of the weight function is moved to the point of interest depending on the input of the user. The application of the weighted least squares regression method will be discussed in detail below.

\subsubsection{Variable Selection and Model Performance Evaluation}

\section{Variable Selection}

Selecting the appropriate set of independent variables is as important as choosing models and devising optimization algorithms. An empirical model will usually fit the data better as the number of adjustable parameters is increased. However, fitting data is not nearly as important as making reliable predictions in this work. A good $\mathrm{R}^{2}$ value for an empirical model does not assure good performance in prediction. Similarly, a model with smaller uncertainties in its coefficients does not necessarily give more accurate predictions than do models with larger uncertainties. Generally there is no simple analytical way to prejudge or characterize the prediction performance of an empirical model. The objectives of this work imply that the empirical correlations should be optimized for their prediction performance. This consideration influences many aspects of the modeling processes from model-construction and optimization algorithm, to variable-selection. In selecting variables sensibly, we first need to understand the structure or interrelations between prospective compositional variables.

Pairwise correlation analysis is the easiest and most effective way to detect relationships between variables. We explored interrelations among the eleven most abundant compositional variables in the viscosity table which, when the temperature is included, leads to the following 22 variables: 

$\mathrm{Al}, \mathrm{B}, \mathrm{Ca}, \mathrm{F}, \mathrm{Fe}, \mathrm{K}, \mathrm{Li}, \mathrm{Mg}, \mathrm{Na}, \mathrm{O}, \mathrm{Si}, \mathrm{Al} / \mathrm{T}, \mathrm{B} / \mathrm{T}, \mathrm{Ca} / \mathrm{T}, \mathrm{F} / \mathrm{T}, \mathrm{Fe} / \mathrm{T}, \mathrm{K} / \mathrm{T}, \mathrm{Li} / \mathrm{T}, \mathrm{Mg} / \mathrm{T}$,
$\mathrm{Na} / \mathrm{T}, \mathrm{O} / \mathrm{T}, \mathrm{Si} / \mathrm{T}$.

Figure 5.14 displays correlations between these 22 variables in the viscosity table, and Figure 5.15 shows the interrelations among the eleven elements and the eleven composition-temperature composite variables (mole \% / T(K)) in the same table. Several features stand out:

1) $\mathrm{F}$ and $\mathrm{O}$ show a clear negative correlation;

2) $\mathrm{F}$ and $\mathrm{Mg}$ are positively correlated because of the inherent high $\mathrm{F}$ and $\mathrm{Mg}$ contents in Fernald glasses;

3) $\mathrm{Mg}$ decreases with increasing $\mathrm{O}$ due to the correlations of 1) and 2);

4) $\mathrm{O}$ increases with $\mathrm{Si}$ since $\mathrm{SiO}_{2}$ is the most abundant glass component in our silicate-based glasses;

5) There is a weak trend between increasing $\mathrm{Ca}$ and decreasing alkali elements;

6) There are evident correlations between $X_{i}$ (molar percent of element $i$ ) and the corresponding $X_{\mathrm{i}} / \mathrm{T}$ variables for most elements except $\mathrm{O}$ and $\mathrm{Si}$.

Generally speaking, highly correlated independent variables are unfavorable for any model. A least-squares solution from a very ill-conditioned matrix (due to correlations or multicollinearity between variables) would be unstable or may totally fail. An effective remedy is to remove statistically insignificant, or redundant variables. The revised models will usually have a similar $\mathrm{R}^{2}$, but smaller standard errors for estimated model parameters. Statistically, the modified model is better because it obtains approximately the same fit with fewer parameters and smaller errors. However, there arises a serious concern if the model is set up to make predictions. Because the independent variables are not orthogonal to each other, the coefficients for corresponding variables could lose their utility in describing the marginal effect for each individual independent variable. For a model using only statistically significant variables (selected from a pool of "independent variables" with some level of correlation), for instance, the coefficient of variable i may not actually describe the effect of $\mathrm{i}$ alone, but of both $\mathrm{i}$ and $\mathrm{j}$, since variable $\mathrm{j}$ was dropped because of the redundancy caused by the interrelation of $j$ and $i$.

Of course, variable $\mathrm{j}$ can be deleted for other reasons; for example, if the variance of the dependent variable, as explained by the independent variable $\mathrm{j}$, is considered not significant for a given statistical criterion. In both cases (redundant ${ }^{1}$ or insignificant), variable $\mathrm{j}$ (if still included) tends to have a relatively large standard error in its coefficient. Excluding variables with large errors and small t-values is commonly practiced in data reduction. However, it is not particularly suitable for models intended to make prediction because of the forever problem of data deficiency. For

${ }^{1}$ : Strictly, redundancy is also a form of lack of significance. We treat the two differently only in above context. 
example, a variable correlation observed in the data sample may not hold for glasses whose properties we will predict in the future. Moreover, a variable which is not very significant in the previous data set (e.g., due to its relatively smaller variation) could be a key player in other samples. This can lead, in the case of strong correlations with serious data deficiency, to unreliable predictions for which the only solution is to include more data in the data set. However, there is a grey zone between strong correlation and orthogonality. In practice, statistical factors are only one of the concerns, and in fact, there is no substitute for experience and intuition in the development and evaluation of such models.

Our approach, in general, is to balance both sets of considerations in order to find sets of variables that are physically sound and not statistically redundant. Thus, in practice, we may retain some variables that are not significant on the basis of a pure statistical criterion but that, in our judgement, could still be physically important. Since the observed variable correlation is not severe in such situations, we believe the estimated coefficients for such compositional variables, in spite of their relatively large statistical uncertainties, contain meaningful information. The prediction tests, which we conducted (presented below), have shown that this approach works quite well with respect to the intended objectives.

For the linear mixture models (equations 5-3,5-5 and 5-9), we selected a compositional variable $\left(\mathrm{X}_{\mathrm{i}}\right)$ based on its concentration in the glasses (a physical criterion) and its variation (a statistical criterion). Because viscosity and conductivity are both bulk properties that are not particularly sensitive to elements at the trace level, in spite of their variation on a relative scale (thus, potentially significant by that measure), only the most abundant elements which also show considerable variations were selected for our empirical models. Figure 5.16 compares the standard deviations of the sixteen most abundant elements and their products with reciprocal temperature. For a stable regression solution, we limited the total number of compositional variables to around 20 so that we can maintain an approximately 1:10 ratio of variables to samples (i.e., glasses with different composition, not observations, since several observations were typically made for each sample). Thus, only variables whose standard deviations are above the dashed line in Figure 5.16 are selected for the modeling work. The elements $\mathrm{P}, \mathrm{S}, \mathrm{Ti}, \mathrm{U}$, and $\mathrm{Zr}$ are excluded because of their overall lower concentrations in the glasses and consequently much smaller deviations. Nevertheless, the effects of these elements are often not critical even for glasses relatively rich in these elements. We found that the predictions made by models without these variables and with them are identical within the prediction uncertainties. For the variables $X_{i} / T$, we selected only those whose corresponding $X_{i}$ were selected (see Figure 5.16).

The overall situation becomes more complicated if composition variables of higher order are considered. For example, including quadratic terms (i.e., $\mathrm{X}_{\mathrm{i}} \mathrm{X}_{\mathrm{j}}$, or $\mathrm{X}_{\mathrm{i}}^{2}$ ) opens up a wide range of selection possibilities of new independent variables which are often highly correlated. Although 
stepwise regression methods can be helpful in reducing independent variables systematically, the accompanying internal correlations can lead to a model which performs rather poorly in making predictions. Such methods were tested in this project; however, it was found that the viscosity predicted (i.e., for data not included in the original data set) by a quadratic mixture model (using stepwise regression to select the independent variables, and therefore resulting in smaller errors for each coefficient) can deviate by an order of magnitude, in contrast to the much better prediction (deviating by $50-70 \%$ ) by a simple linear mixture model (using the above proposed 11 major elements and their products with $1 / \mathrm{T}$ ). We have therefore used only linear compositional variables in this modeling work.

For 7-day PCT correlations, we used additional compositional variables, including Ti and $\mathrm{Zr}$ in light of their important roles in improving glass leach resistance. Because the temperature dependence is not a consideration (at this point) for these data, the variable-to-glass ratio is still below 1:10.

\section{Model Evaluation}

As a routine part of the statistical analysis, all modeling results were examined to assure the validity of the underlying least-squares premises. The residuals frequency distributions for models proposed in this task are all very close to a standard normal distribution. The residuals do not show any evident dependence on any of the independent variables. In addition to the standard $R^{2}$ and $F$ tests, we assess the models on the basis of their ability to make accurate predictions for data not included in modeling processes (e.g., the "Test Group" glasses listed in Table 5.4). The details are given in the following sections.

\subsubsection{Modeling Results and Performance Evaluation}

\subsubsection{Conductivity-Composition Model}

\section{Model}

A linear mixture model (5-5) was selected to correlate the 917 conductivity measurements on 220 molten glasses. The conductivity model has 23 adjustable parameters to describe the dependence of $A$ and $B$ (as in equation 5-2) on 11 major elements:

$$
\mathrm{Al}, \mathrm{B}, \mathrm{Ca}, \mathrm{F}, \mathrm{Fe}, \mathrm{K}, \mathrm{Li}, \mathrm{Mg}, \mathrm{Na}, \mathrm{O}, \mathrm{Si} \text {. }
$$


We elected to represent the composition variables as the elements to avoid as arbitrarily chosen fluorides. The 11 linear-form variables ( $\mathrm{Al}, \mathrm{B}, \mathrm{Ca}$, etc.) entered the equation on the basis of their concentrations and variations. The variables of higher order (e.g., quadratic terms) were not included because:

1) A mixture model containing quadratic compositional variables did not improve the fit significantly; and

2) Although quadratic models can usually fit our data slightly better or with fewer parameters than a linear model, significant multi-collinearity was detected among the variables of quadratic models which, as a result, tend to perform rather poorly in prediction.

\section{Weighted Least Squares Regression}

The GCPD allows us to model conductivity-composition relationship in a convenient and interactive fashion. Model-prediction tests demonstrated that a model optimized by weighted leastsquares method (Section 5.2.2.2) outperforms those obtained by ordinary least-squares. The weight factor (Equation 5-10) is a function of all 11 selected composition variables plus $\mathrm{P}, \mathrm{Ti}, \mathrm{Zr}, \mathrm{S}$, and $\mathrm{U}$. To predict conductivity for an "imagined" molten glass at a temperature $\mathrm{T}$, we let the composition of this glass be at the center of the weight function (i.e., at $X_{o}$ in Equation 5-11). Weight factors for each sample in the database are then determined by the Euclidean distance, $D$ relative to $X_{0}$, and the dispersion parameter, $\sigma$. Obviously, a smaller $\mathrm{D}$ means a larger weight, and a larger $\sigma$ means a slower decrease of the weight with increasing $\mathrm{D}$. The optimal value of $\sigma$ was found to be around 7.5 for our conductivity data. A typical weight as a function of distance is shown in Figure 5.17 using the HAN5 glass as the center of weight. Before weighted least-squares regression, we multiply the calculated weight factors by 10 and truncate them to integers (shown as open boxes in Figure 5.17).

Different sample frequency distributions on a weight axis, with respect to different test glasses (HAN5, WS18, and DIG7 in Figure 5.18), are the result of inhomogeneous sample density in the multi-dimensional composition space. WS18 and DIG7 in our testing group were randomly selected from the Weldon Spring site group and the "Bavaria" group respectively. These glasses make up only a small fraction of the conductivity data set and, their compositions are not well bracketed by other data points. Consequently, we find only a small number of data points surrounding them since most of the data points have a weight smaller than 6 (Figure 5.18). In contrast, the sample frequency distribution with HAN5 as the center (i.e., at $X_{0}$ ) shows a considerable number of data points with a weight of 6 or larger (Figure 5.18).

Figure 5.19 shows the weighted sample frequency distribution for each of HAN5, WS18, and DIG7 as the centers. As expected, the weighted data would favor the points near the target glass 
composition (note that all the observations with a zero weight are dropped in the weighted distribution). The effectiveness of the weighted algorithm depends not only on the form of the property-composition relationship, but also on the way the distance is defined. For the empirical models used here, the Euclidean distance, with its simple and straightforward meaning, is a logical starting point.

Model prediction results for glasses in the Test Group are summarized in Table 5.4. Figure 5.20 compares the predictions of conductivity data around $1150^{\circ} \mathrm{C}$ by the weighted and unweighted regression methods. The predicted values by the weighted conductivity model are always better than those by the unweighted model. The advantage of adding the weighting factor is most evident for WS18 and DIG7. Overall, the normalized prediction residuals by the weighted model are less than $\pm 30 \%$ as opposed to around $50 \%$ by the unweighted model (Figure 5.21 ). The only point with $70 \%$ deviation has a low conductivity which tends to cause the relative deviation to be large for only small absolute deviations. In conclusion, we selected the weighted least-squares regression method as the basis for prediction of the conductivity of a unknown glass. As a tradeoff, the weighted least-squares method does not produce a single globally valid equation between conductivity and composition but rather an algorithm for making "data-driven" predictions.

\section{Step-Weight Function: Conductivity-Composition Model for Hanford Data}

As the simplest way of adding a weight function, we can multiply each data point in the database by 0 or 1 subject to simple or complex conditions. Detailed inspection of data distributions have indicated that glasses from the same parents tend to cluster in compositional space. It is, therefore, logical to select samples on the basis of their origin, i.e., determining the value of the weight ( 0 or 1 ) on the basis of site or waste streams origin. The structure of the GCPD managed by Oracle 7 makes this very straightforward; the conductivity data relating to a specific DOE site (and later, waste stream type) can be easily retrieved and analyzed by using our client application interfaces. As a working example, we present a conductivity-composition model for the Hanford glasses.

The Hanford glasses in the GCPD have their own characteristic compositions. As a group, the distribution in, for example, NBOT-M3T-M1M12 space is narrow in M3T but wide in both NBOT and M1M12 (see Section 5.3.1). This means that the variability of major glass components in Hanford glasses may differ in important ways from the overall average pattern of the data in the whole conductivity or viscosity tables. We would like, therefore, to consider a set of independent compositional variables for the Hanford glasses only. On the other hand, we have to limit the total number of independent variables used for the Hanford model simply because of the smaller number of data points (179 conductivity measurements on 38 Hanford glasses). 
Using the same physical and statistical criterion (as described in Section 5.3.2.3), we selected six oxide components on the basis of their standard deviations: $\mathrm{Al}_{2} \mathrm{O}_{3}$ wt\%, $\mathrm{CaO}$ wt\%, $\mathrm{Fe}_{2} \mathrm{O}_{3}$ wt\%, $\mathrm{Na}_{2} \mathrm{O} w t \%, \mathrm{SiO}_{2} \mathrm{wt} \%$, and $\mathrm{ZrO}_{2}$ wt\% (Figure 5.22). Since Hanford glasses do not contain fluorine, we have used oxide weight percent instead of element molar percent for convenience.

A linear model (5-5) with 13 parameters (for 6 compositional variables) was used to model the compositional dependence of conductivity for Hanford glasses. The modeling results are listed in Table 5.5, and the fitted points are plotted against the observed values in Figure 5.23. Generally, the fit of the Hanford data using this model is not significantly better than that by the models using 23 parameters for all of the conductivity data.

To show how each composition variable would affect the conductivity of the melt, on the basis of the fitted models, we calculated the conductivity of the melt after progressively adding or subtracting small amounts of one oxide to or from that of HAN7 (a sodium-rich glass). The calculated isothermal conductivity values of six series are plotted in Figure 5.24. Adding $\mathrm{CaO}$ to $\mathrm{HAN7}$ is predicted to significantly decrease the melt conductivity at $1200^{\circ} \mathrm{C}$, but produces no effect at $1500^{\circ} \mathrm{C}$ (Figure 5.25). This is probably due, in part, to the mixed cation effect since HAN7 is an alkali-rich glass. Adding $\mathrm{CaO}$ would decrease the electrical conductivity before the minimum is reached. The mixed cation effect tends to be stronger at lower temperature, and disappears with increasing temperature.

\subsubsection{Viscosity Compositional Model}

\section{Model}

A linear mixture model (Equation 5-3) was selected to model 1352 viscosity measurements of 299 molten glasses. The viscosity model has 23 adjustable parameters to describe the dependence of $\mathrm{A}$ and $\mathrm{B}$ (Equation 5-2) on 11 major elements:

$\mathrm{Al}, \mathrm{B}, \mathrm{Ca}, \mathrm{F}, \mathrm{Fe}, \mathrm{K}, \mathrm{Li}, \mathrm{Mg}, \mathrm{Na}, \mathrm{O}, \mathrm{Si}$.

As described in the conductivity-composition model, we chose to represent composition variables in element form by which fluorine enters as the element $F$ rather than as different fluorides chosen arbitrarily. The 11 linear-form variables ( $\mathrm{Al}, \mathrm{B}, \mathrm{Ca}$, etc.) entered the equation because of their concentrations and variations. The variables of higher order (e.g., quadratic terms) are not included for the reasons explained earlier. 


\section{Modeling Results}

Ordinary least-squares and weighted least-squares regression methods were both used to fit the experiment data and to predict viscosities for a group of "unknown" glasses in the Test Group. The correlations obtained by either method satisfactorily describe more than $85 \%$ of the variation of viscosity (Table 5.6) and performed almost equally well in making predictions (Figure 5.26, Table 5.6). Since adding weights to observations on the basis of their compositional "distance" from the point of interest does not improve the model's predictions, we decided to model the viscosity data by ordinary least-squares regression. The estimated model coefficients are given in Table 5.7. The fitted viscosities are plotted versus those observed in Figure 5.27.

Judging by the results from the testing group, the unweighted viscosity model predicts with an relative uncertainty of $\pm 50 \%$ (residual/observation). For very low viscosity values, the normalized residual can be larger (e.g., $150 \%$ in Figure 5.28). Considering the range of viscosity values (very low up to 670 poise) and compositions represented by the Test Group, the results show that the unweighted viscosity model can predict the viscosity for "unknown" glasses with a reasonable uncertainty.

\section{Viscosity Model for Hanford Glasses}

In a similar way to the conductivity model for Hanford glasses, we have modeled the 186 viscosity measurements on 38 Hanford glasses with the same six compositional variables: $\mathrm{Al}_{2} \mathrm{O}_{3}$ wt $\%, \mathrm{CaO} w t \%, \mathrm{Fe}_{2} \mathrm{O}_{3} \mathrm{wt} \%, \mathrm{Na}_{2} \mathrm{O} \mathrm{wt} \%, \mathrm{SiO}_{2} \mathrm{wt} \%$, and $\mathrm{ZrO}_{2} \mathrm{wt} \%$. The modeling results are listed in Table 5.8, and the fitted points are plotted versus the observed values in Figure 5.29. Generally, the fit of Hanford data by the 13-parameter-model is significantly better than that by the model with 23 parameters for all of the viscosity data. The model estimated from Hanford-only data is, in this case, therefore likely to give better predictions for samples which originated from the same site.

The Hanford viscosity model can also be used to show how a melt component affects the viscosity at a given temperature. Using the same method described for the conductivity modeling, we calculated the viscosity as a function of adding and removing in small increments each melt's component (Figure 5.30). It is interesting to note that, for HAN7 glass, the calculated effect of $\mathrm{CaO}$ relative to $\mathrm{Na}_{2} \mathrm{O}$ varies little from $1200^{\circ} \mathrm{C}$ to $1400^{\circ} \mathrm{C}$ (Figure 5.31). Generally, however, it is often found that the actual effect of $\mathrm{CaO}$ on melt viscosity relative to that of $\mathrm{Na}_{2} \mathrm{O}$ increases with temperature. 


\subsubsection{7-Day PCT Compositional Models}

\section{Models}

Linear mixture models (Equation 5-9) were used to model 197 sets of 7-day PCT data for the elements $\mathrm{Si}, \mathrm{B}, \mathrm{Na}$, and $\mathrm{Li}$ individually. We selected the elements $\mathrm{Si}, \mathrm{B}, \mathrm{Na}$ and $\mathrm{Li}$ because of their different roles in the glass-forming process, glass-water interactions, and solution chemistry. The $\mathrm{pH}$ value, as one of most important parameters in aqueous solution chemistry, was also modeled in this work. All PCT composition models were treated as functions of 15 major glass components in forms of oxides or fluorides:

$$
\mathrm{Al}_{2} \mathrm{O}_{3}, \mathrm{~B}_{2} \mathrm{O}_{3}, \mathrm{CaO}, \mathrm{FeO}, \mathrm{Fe}_{2} \mathrm{O}_{3}, \mathrm{~K}_{2} \mathrm{O}, \mathrm{Li}_{2} \mathrm{O}, \mathrm{MgO}, \mathrm{MgF}_{2}, \mathrm{NaF}, \mathrm{Na}_{2} \mathrm{O}, \mathrm{P}_{2} \mathrm{O}_{5}, \mathrm{SiO}_{2}, \mathrm{TiO}_{2}, \mathrm{ZrO}_{2} \text {. }
$$

Weight percent variables were used in the calculation for convenience. Note that data extending to longer times as well as data for many other elements are available from the PCT data.

\section{Modeling Results}

Overall, the linear form of Equation 5-9 worked reasonably well. The correlation coefficients of multiple regressions are around 0.9 for all five models. This means that the model correlation reasonably corresponds with about $80 \%$ of the observed variances for $\mathrm{Si}, \mathrm{B}, \mathrm{Na}$, and $\mathrm{Li} 7$-day PCT results and $\mathrm{pH}$ values (Table 5.9). In Table 5.10, we list the model coefficients for all four elements and the pH. A detailed illustration of goodness of fit is given in Figure 5.32. The residuals for approximately 6 to 13 observations are larger than two standard deviations. Adding second order variables does not improve the fit significantly. Considering the empirical nature of the PCT model (Equation 5-9) and the detectable multi-collinearity for mixture models of higher orders, we decided to use linear mixture models (Equation 5-9) to predict 7-day PCT data for unknown glasses.

The predicted 7-day PCT results are plotted in Figure 5.33a to 5.33d with incremental changes of major glass components. The component effects, as predicted by our models, are consistent with observations from compositionally more constrained systems. Generally, element $\mathrm{PCT}$ values would increase with their concentrations in glasses. Increasing $\mathrm{Na}_{2} \mathrm{O}$ or $\mathrm{Li}_{2} \mathrm{O}$ enhances leaching while increasing $\mathrm{TiO}_{2}$ and $\mathrm{Al}_{2} \mathrm{O}_{3}$ appears to make the glasses more durable. For HAN38 glass, the predicted PCT values do not shift significantly with $\mathrm{CaO}, \mathrm{B}_{2} \mathrm{O}_{3}, \mathrm{Fe}_{2} \mathrm{O}_{3}$ and $\mathrm{SiO}_{2}$. The effect of $\mathrm{ZrO}_{2}$ tends to be larger for PCT Si data than for the PCT values for B and Na. 


\section{Evaluation of 7-Day PCT Model: Prediction Performance}

Our PCT Test Group consists of 12 sets of 7-Day PCT data representing a wide range of glass composition (see Table 5.11 for glass name, experimental results and model's predictions for $\mathrm{Si}, \mathrm{B}$, $\mathrm{Na}, \mathrm{Li}$ 7-day PCT and $\mathrm{pH}$ ). The errors for the majority of the predictions are less than $\pm 50 \%$ if normalized to the observed values (Figure 5.34). Ninety-five percent of the predictions deviate by less than a factor of two. Among three true outliers, two Li points are associated with the glasses containing little $\mathrm{Li}_{2} \mathrm{O}$, hence the deviation in ppm is actually very small. The third point, a Na point off by a factor of 8 , is associated with a F-rich Fernald glass. Judging by our testing results, the 7day PCT models for $\mathrm{Si}, \mathrm{B}, \mathrm{Na}$ and $\mathrm{Li}$ can give reasonable predictions from glass composition with relative errors of about $50 \%$ to $200 \%$.

\section{Comparison with "Hydration Free-Energy" Model}

Claims have been made in the literature (Jantzen-1991, Jantzen-1984) as to the correlatory and predictive capabilities of the hydration free-energy model for various types of glass leaching data including $\mathrm{MCC} 1$ and PCT results. In view of the discussion in Section 5.3.2.1, such results would be surprising. Recent studies have failed to support these contentions on Hanford high-level nuclear waste glasses by Hrma et al. (Hrma-1994). We have examined the performance of this model for the PCT data set discussed above which includes the data on the diverse mixed waste glasses from the Hanford, Idaho, and Oak Ridge sites generated in this study. The results are shown in Figures 5.35a to $5.35 \mathrm{~d}$ where the logarithms of the normalized leachate concentrations of sodium, lithium, silicon, and boron are plotted against the hydration free energy calculated from the composition of each glass. (Note that the lines in these graphs are simply regression lines; the model predicts neither their location nor their slopes, only that a linear correlation should be observed). The results show uniformly poor correlations throughout.

\subsection{Example Model Results for Hanford, Idaho, and Oak Ridge Glasses}

The correlations that have been developed were evaluated further by comparison of the predictions to the experimental data that were discussed in Sections 2-4. Specifically, the ability of the models to predict the core data set of 40 glasses (see Table 1.1) was investigated. The procedure used was as follows:

(i) The set of 40 glasses listed in Table 1.1 was divided into three groups with each group containing approximately the same number of glasses from each site (see Table 5.12); the division into groups was otherwise random. 
(ii) All of the glasses in Group 1 were removed from the database and the coefficients for the property models were recalculated.

(iii) The property data for the glasses in Group 1 were then predicted using the models and the predictions were then compared with the measured values.

(iv) The Group 1 data were then returned to the database and the Group 2 data were removed and a similar prediction and comparison procedure performed. Finally, the Group 3 data were treated similarly.

This procedure provides an indication of the ability of the models to predict the properties of glasses that were not included in the database from which the models were generated. The glasses involved have high loadings of a diverse set of waste streams.

The results of these tests are shown in Figures 5.36a-c and 5.37a-c for the viscosity and for the PCT sodium release after seven days; respectively; note that the viscosity plots include more than one data point for each glass since measurements were made at multiple temperatures.

The general trends in the data are correctly predicted by the current models and the quantitative agreement is reasonable considering the wide range of compositions that these glasses cover and the similarly wide range of the property values.

\subsection{Conclusions}

An integrated glass property composition database and modeling facility has been designed, a substantial portion of the development work has been completed, and evaluation tests of the system have been conducted. The results obtained to date are encouraging; the system should be a useful tool in the design and optimization of glass compositions for waste vitrification activities. While other glass composition databases exist, particularly those developed by the commercial glass industry and some efforts in support of high-level nuclear waste vitrification, these typically relate to narrow composition ranges in comparison to those of the present work. The diversity of mixed and low-level waste streams in the DOE complex requires a set of data and modeling capability based firmly on data from vitrification studies conducted predominantly on of actual waste streams.

The predictive capability of the system relies on the underlying data and the quality of the composition-property models that are employed. The models presented in this section for conductivity, viscosity, and 7-Day PCT results are only reasonable approximations to the true complex composition-property relationships of multicomponent waste glasses. As stated previously, 
Table 5.1

Table Contents of Glass Composition-Property Database (GCPD).

\begin{tabular}{|c|c|c|c|}
\hline Table Database Name & Table Title & $\begin{array}{l}\text { Table } \\
\text { Size }\end{array}$ & Data Categary \\
\hline SYSTEM.PROJECTS & Project Names & 22 & $\begin{array}{l}\text { Project name and the numbers of the associated glass } \\
\text { samples. }\end{array}$ \\
\hline SYSTEM.GLASSES & Glass Names & 745 & $\begin{array}{l}\text { Glass names, glass melting temperature, date, and flags for } \\
\text { all glass properties in GCPDB. }\end{array}$ \\
\hline SYSTEM.GCOMP_พ่T & Glass Composition (wt \%) & 556 & Glass chemical composition in wt\%. \\
\hline SYSTEM.GCOMP_MOL & Glass Composition (mol\%) & 556 & Glass chemical composition in mol\%. \\
\hline SYSTEM.G_COND & Conductivity of Molten Glasses & 298 & $\begin{array}{l}\text { Conductivity, experimental temperature and experiment } \\
\text { measurement number. }\end{array}$ \\
\hline SYSTEM.G_VIS & Viscosity of Molten Glasses & 393 & $\begin{array}{l}\text { Viscosity, experimental temperature and experiment } \\
\text { measurement number. }\end{array}$ \\
\hline SYSTEM.PCTINDEX & PCT Experimental & 143 & $\begin{array}{l}\text { Experimental records (mass, area, volume, period, starting } \\
\text { and sampling data, etc.). }\end{array}$ \\
\hline SYSTEMPCTRAWDATA & PCT Raw Data & 143 & $\begin{array}{l}\text { Elements analyzed and their concentration in ppm (before } \\
\text { normalization). }\end{array}$ \\
\hline SYSTEM.7DAYPCT & PCT Raw Data at 7 Day & 229 & $\begin{array}{l}\text { 7-day PCT results of } \mathrm{Si}, \mathrm{B}, \mathrm{Na} \text { and } \mathrm{Li} \text { in } \mathrm{ppm} \text {, and } \mathrm{pH} \\
\text { values of solution. }\end{array}$ \\
\hline SYSTEM.TCLP & TCLP Raw Data & 108 & Elements analyzed and their concentration in ppm. \\
\hline
\end{tabular}


Table 5.2

Oracle7 Client Applications

\begin{tabular}{|c|l|l|}
\hline Name & \multicolumn{1}{|c|}{ Major Function } & \multicolumn{1}{c|}{ Potential User } \\
\hline Oracle Data Browser & $\begin{array}{l}\text { Graphical interactive data access tool. Able to build and send ad } \\
\text { hoc queries to Oracle 7 server. }\end{array}$ & End User ( SQL not required) \\
\hline Oracle SQL*Plus & $\begin{array}{l}\text { Interactive query tool. Able to issue SQL statement to server in } \\
\text { simple command-line interface. }\end{array}$ & End User and Developer (SQL required) \\
\hline Oracle Forms & $\begin{array}{l}\text { An Oracle tool to prepare screens with fields for entering and } \\
\text { updating data. }\end{array}$ & Developer and end user \\
\hline Oracle Reports & $\begin{array}{l}\text { An Oracle tool to prepare reports with fields filled by the selected } \\
\text { data from the database. }\end{array}$ & Developer and end user \\
\hline Oracle Graphics & $\begin{array}{l}\text { An Oracle tool to prepare graphs to represent selected data on } \\
\text { screens or printer. }\end{array}$ & Developer and end user \\
\hline
\end{tabular}


Table 5.3

Custom Client Applications for Glass-Composition-Property Database.

\begin{tabular}{|l|l|l|}
\hline Custom Client Application. & Major Functions & Developed by Oracle Tools of \\
\hline Glass Entry Form & $\begin{array}{l}\text { An application for entering and updating the data of glass compositions and } \\
\text { properties. }\end{array}$ & Oracle Forms \\
\hline Glass Modeling Tool & $\begin{array}{l}\text { An application for selecting and displaying data in form of reports and graphs, } \\
\text { and preparing data for statistical analysis. }\end{array}$ & $\begin{array}{l}\text { Oracle Forms, Oracle Reports, } \\
\text { and Oracle Graphics }\end{array}$ \\
\hline Glass Search Tool & $\begin{array}{l}\text { An applicaiton for searching glass in the database within given compositional } \\
\text { ranges. }\end{array}$ & Oracle Forms \\
\hline
\end{tabular}




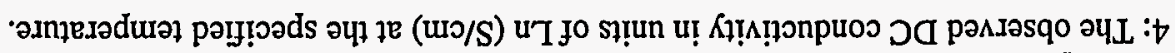

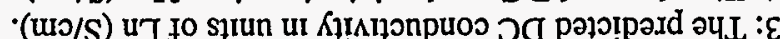

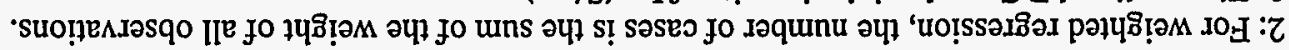
'นо!

\begin{tabular}{|c|c|c|c|c|c|c|c|c|}
\hline$\forall / N$ & e6Z UеH & $\forall / N$ & 6I UеH & $\forall / N$ & $\angle$ UEH & $\forall / N$ & $\varsigma$ ueH & 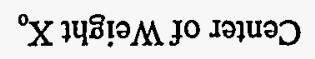 \\
\hline$\forall / N$ & $S L$ & $\forall / \mathbb{N}$ & $S L$ & $\forall / \mathbb{N}$ & $S L$ & $\forall / N$ & $S^{*} L$ & ڤ uoỊsıəds!̣ \\
\hline$\varepsilon 8 \mathrm{II}$ & $\varepsilon 8 I I$ & $8 L I I$ & $8 L I I$ & 69 II & 69II & $6 \mathrm{IZI}$ & 6IZI & 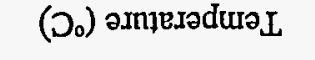 \\
\hline IEI๋0 & IEI'0 & I9S I- & I9S' I- & $\varepsilon 80^{\circ} 0^{-}$ & $\varepsilon 80^{\circ} 0^{-}$ & StS'O- & $S t S^{*} 0^{-}$ & ${ }_{\text {p}}$ pəa.rəsqO \\
\hline$\angle 9 Z^{\circ} 0$ & $120^{\circ} 0^{-}$ & $\$ 9 L^{\circ} I^{-}$ & E9S' I- & $8 \angle E^{\circ} 0^{-}$ & $281^{\circ} 0^{-}$ & $9 \varepsilon I^{\circ} I^{-}$ & $688^{\circ} 0^{-}$ & ${ }_{\varepsilon} \mathrm{p} \partial \jmath \operatorname{lol}$ \\
\hline IeuroN & [EUION & [eumoN & [eшroN & IPUION & โEuLION & [euíoN & jeuroN & uo!̣nq!ns! ब ə|np!səy \\
\hline દโ6 & $\not I S I$ & $L 06$ & $06 I z$ & $\varepsilon I 6$ & $968 I$ & $\varepsilon[6$ & $6 \mathrm{IIZ}$ & z \\
\hline$乙 乙$ & $\tau 乙$ & $\tau \tau$ & $\tau \tau$ & $\tau 乙$ & $\tau \tau$ & $\tau 乙$ & Z乙 & səqqe!̣e $\Lambda$ Jo \# \\
\hline$\angle Z 8^{\circ} 0$ & $9 \varepsilon 8^{\circ} 0$ & $\varepsilon \varepsilon 8^{\circ} 0$ & SI6.0 & $828^{\circ} 0$ & $\angle S 8^{\circ} 0$ & $628^{\circ} 0$ & $888^{\circ} 0$ & ${ }_{2} \mathrm{~d}$ \\
\hline I6.0 & $t I 6^{\circ} 0$ & $\varepsilon\left[6^{\circ} 0\right.$ & $\angle S 6^{\circ} 0$ & I6.0 & $926^{\circ} 0$ & $\operatorname{II} 6^{\circ} 0$ & $\varepsilon \nabla 6^{\circ} 0$ & y \\
\hline$M \cap$ & $M$ & $M \cap$ & $M$ & $\mathrm{Mn}$ & $M$ & $M \cap$ & $M$ & ${ }_{\mathrm{I}} M \cap{ }^{10} \mathrm{M}$ \\
\hline V6ZNVH & VGZNVH & 6INVH & 6INVH & LNVH & LNVH & SNVH & SNPH & ग्रdues \\
\hline
\end{tabular}

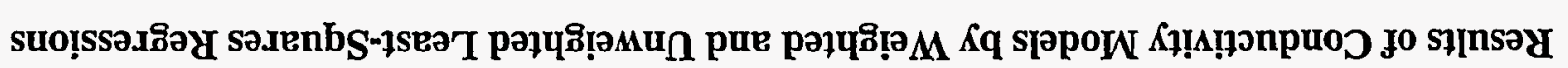

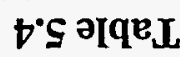




\begin{tabular}{|c|c|c|c|c|c|c|c|c|c|c|}
\hline Sample & HAN33 & HAN33 & HAN38 & HAN38 & HAN39 & HAB39 & DIG7 & DIG7 & WS18 & WS18 \\
\hline W or UW ${ }^{1}$ & W & UW & W & UW & W & UW & W & UW & W & UW \\
\hline R & 0.943 & 0.91 & 0.93 & 0.91 & 0.933 & 0.911 & 0.918 & 0.911 & 0.915 & 0.913 \\
\hline $\mathrm{R}^{2}$ & 0.89 & 0.829 & 0.866 & 0.829 & 0.871 & 0.829 & 0.843 & 0.829 & 0.838 & 0.833 \\
\hline \# of Variables & 22 & 22 & 22 & 22 & 22 & 22 & 22 & 22 & 22 & 22 \\
\hline \# of Cases ${ }^{2}$ & 1227 & 912 & 2017 & 891 & 1384 & 913 & 1194 & 913 & 950 & 913 \\
\hline Residule Distribution & Normal & Normal & Normal & Normal & Normal & Normal & Normal & Normal & Normal & Normal \\
\hline Predicted $^{3}$ & -2.9588 & -2.872 & -0.523 & -0.536 & -0.639 & -1.162 & -3.930 & -3.163 & -3.144 & -3.854 \\
\hline Observed $^{4}$ & -3.507 & -3.507 & -0.777 & -0.777 & -0.777 & -0.777 & -3.912 & -3.912 & -2.813 & -2.813 \\
\hline Temperature $\left({ }^{\circ} \mathrm{C}\right)$ & 1343 & 1343 & 1165 & 1165 & 1165 & 1165 & 1190 & 1190 & 1000 & 1000 \\
\hline Dispersion $\sigma$ & 7.5 & $\mathrm{~N} / \mathrm{A}$ & 7.5 & N/A & 7.5 & N/A & 7.5 & N/A & 7.5 & N/A \\
\hline Center of Weight $\mathrm{X}_{\mathrm{o}}$ & Han 33 & N/A & Han 38 & N/A & Han 39 & N/A & DIG 7 & N/A & WS 18 & N/A \\
\hline
\end{tabular}




\begin{tabular}{|c|c|c|c|c|c|c|c|c|}
\hline Sample & IDG7A & IDG7A & IDG8B & IDG8B & ORNL10 & ORNL10 & ORNL11 & ORNL11 \\
\hline $\mathrm{W}$ or $\mathrm{UW}^{1}$ & W & UW & $\mathrm{W}$ & UW & W & UW & W & UW \\
\hline $\mathbf{R}$ & 0.928 & 0.91 & 0.918 & 0.911 & 0.939 & 0.911 & 0.917 & 0.917 \\
\hline $\mathrm{R}^{2}$ & 0.861 & 0.829 & 0.843 & 0.83 & 0.882 & 0.829 & 0.841 & 0.841 \\
\hline \# of Variables & 22 & 22 & 22 & 22 & 22 & 22 & 22 & 22 \\
\hline \# of Cases ${ }^{2}$ & 1642 & 913 & 1521 & 913 & 1937 & 913 & 1109 & 913 \\
\hline Residule Distribution & Normal & Normal & Normal & Normal & Normal & Normal & Normal & Normal \\
\hline Predicted $^{3}$ & -3.244 & -2.843 & -3.072 & -2.601 & -1.95 & -1.845 & -2.570 & -2.607 \\
\hline Observed $^{4}$ & -3.507 & -3.507 & -3.912 & -3.912 & -1.833 & -1.833 & -2.659 & -2.659 \\
\hline Temperature $\left({ }^{\circ} \mathrm{C}\right)$ & 1199 & 1199 & 1186 & 1186 & 1228 & 1228 & 1302 & 1302 \\
\hline Dispersion $\sigma$ & 7.5 & N/A & 7.5 & N/A & 7.5 & $\mathrm{~N} / \mathrm{A}$ & 7.5 & N/A \\
\hline Center of Weight $X_{0}$ & IDG7A & N/A & IDG8B & N/A & ORNL10 & $\mathrm{N} / \mathrm{A}$ & ORNL11 & N/A \\
\hline
\end{tabular}


Table 5.5

Results of Conductivity Model of Hanford Glasses by Unweighted Least-Squares Regression

$\mathrm{R}=0.956$

$\mathrm{R}^{2}=0.913$

Number of cases $=179$

Standard error of estiminate $=0.437$

\begin{tabular}{|l|r|r|}
\hline \multicolumn{1}{|c|}{ Variable } & Coefficient & \multicolumn{1}{c|}{$\begin{array}{c}\text { Standard } \\
\text { Error }\end{array}$} \\
\hline Intercept & -1.1230 & 3.2576 \\
\hline $\mathrm{Al}_{2} \mathrm{O}_{3}$ wt\% & -0.1040 & 0.2287 \\
\hline $\mathrm{CaO} w t \%$ & 0.4640 & 0.1494 \\
\hline $\mathrm{Fe}_{2} \mathrm{O}_{3} \mathrm{wt} \%$ & -0.2090 & 0.2105 \\
\hline $\mathrm{Na}_{2} \mathrm{O} w t \%$ & 0.3390 & 0.0684 \\
\hline $\mathrm{SiO}_{2} \mathrm{wt} \%$ & -0.0650 & 0.0670 \\
\hline $\mathrm{ZrO}_{2} \mathrm{wt} \%$ & -0.1000 & 0.2872 \\
\hline $\mathrm{Al}_{2} \mathrm{O}_{3} \mathrm{wt} \% / \mathrm{T}(\mathrm{K})$ & 100.4380 & 370.3280 \\
\hline $\mathrm{CaO}_{\mathrm{wt}} / \mathrm{T}(\mathrm{K})$ & -789.1990 & 221.6160 \\
\hline $\mathrm{Fe}_{2} \mathrm{O}_{3} \mathrm{wt} \% / \mathrm{T}(\mathrm{K})$ & 334.5390 & 310.4660 \\
\hline $\mathrm{Na}_{2} \mathrm{O} \mathrm{wt} \% / \mathrm{T}(\mathrm{K})$ & -347.4320 & 77.3360 \\
\hline $\mathrm{SiO}_{2} \mathrm{wt} \% / \mathrm{T}(\mathrm{K})$ & 64.3980 & 86.7190 \\
\hline $\mathrm{ZrO}_{2} \mathrm{wt} \% / \mathrm{T}(\mathrm{K})$ & 58.1190 & 429.5300 \\
\hline
\end{tabular}


Table 5.6

Results of Viscosity Models by Weighted and Unweighted Least-Squares Regressions.

\begin{tabular}{|l|c|c|c|c|c|}
\hline & Unweighted & Weighted 1 & Weighted 2 & Weighted 3 & Weighted 4 \\
\hline $\mathrm{R}$ & 0.936 & 0.941 & 0.94 & 0.919 & 0.907 \\
\hline $\mathrm{R}^{2}$ & 0.875 & 0.886 & 0.0883 & 0.844 & 0.822 \\
\hline $\begin{array}{l}\text { Number of } \\
\text { Variables }\end{array}$ & 22 & 22 & 22 & 22 & 22 \\
\hline $\begin{array}{l}\text { Number of } \\
\text { Samples* }\end{array}$ & 1375 & 4059 & 5392 & 3246 & 2450 \\
\hline $\begin{array}{l}\text { Center of weight } \\
\text { (Weighted only) }\end{array}$ & N/A & HAN7 & WVUTH202 & DIG7 & WS18 \\
\hline
\end{tabular}

* For weighted least-squares regression, this number refers the nominal sample number which is the sum of weight for each observation. 
Table 5.7

Viscosity Model by Unweighted Least-Squares Regression.

$\mathrm{R}=0.936$

$\mathrm{R}^{2}=0.875$

Number of cases $=1375$

Standard error of estiminate $=0.554$

\begin{tabular}{|l|l|l|}
\hline Variables & \multicolumn{1}{|c|}{ Coefficients } & Standard Error \\
\hline Intercept & -18.8690 & 6.1741 \\
\hline $\mathrm{Al}$ & -0.1065 & 0.2725 \\
\hline $\mathrm{B}$ & 0.1214 & 0.2098 \\
\hline $\mathrm{Ca}$ & -0.4309 & 0.1411 \\
\hline $\mathrm{F}$ & -0.1766 & 0.1424 \\
\hline $\mathrm{Fe}$ & -1.0291 & 0.3408 \\
\hline $\mathrm{K}$ & -0.7571 & 0.4850 \\
\hline $\mathrm{Li}$ & 0.1850 & 0.1511 \\
\hline $\mathrm{Mg}$ & 0.1020 & 0.2119 \\
\hline $\mathrm{Na}$ & 0.0617 & 0.0838 \\
\hline $\mathrm{O}$ & 0.1402 & 0.1615 \\
\hline $\mathrm{Si}$ & 0.3983 & 0.2407 \\
\hline $\mathrm{Al} / \mathrm{T} * 10^{3}$ & 0.5218 & 0.3899 \\
\hline $\mathrm{B} / \mathrm{T} * 10^{3}$ & -0.3667 & 0.3050 \\
\hline $\mathrm{Ca} / \mathrm{T} * 10^{3}$ & 0.4274 & 0.1969 \\
\hline $\mathrm{F} / \mathrm{T} * 10^{3}$ & 0.4874 & 0.1536 \\
\hline $\mathrm{Fe} / \mathrm{T} * 10^{3}$ & 1.2809 & 0.4768 \\
\hline $\mathrm{K} / \mathrm{T} * 10^{3}$ & 1.1513 & 0.6714 \\
\hline $\mathrm{Li} / \mathrm{T} * 10^{3}$ & -0.3876 & 0.2034 \\
\hline $\mathrm{Mg} / \mathrm{T} * 10^{3}$ & -0.1241 & 0.2985 \\
\hline $\mathrm{Na} / \mathrm{T} * 10^{3}$ & -0.0668 & 0.1033 \\
\hline $\mathrm{O} / \mathrm{T} * 10^{3}$ & 0.3371 & 0.1758 \\
\hline $\mathrm{Si} / \mathrm{T} * 10^{3}$ & -0.4110 & 0.3552 \\
\hline & & \\
\hline
\end{tabular}

Page 5-32 
Table 5.8

Viscosity Model of Hanford Glasses by Unweighted Least-Squares Regression.

$\mathrm{R}=0.988$

$\mathrm{R}^{2}=0.977$

Number of cases $=172$

Standard error of estiminate $=0.1898$

\begin{tabular}{|l|r|r|}
\hline \multicolumn{1}{|c|}{ Variable } & Coefficient & Standard Error \\
\hline Intercept & -1.9628 & 1.3805 \\
\hline $\mathrm{Al}_{2} \mathrm{O}_{3} \mathrm{wt} \%$ & -0.2915 & 0.1032 \\
\hline $\mathrm{CaO} w t \%$ & -0.0997 & 0.0762 \\
\hline $\mathrm{Fe}_{2} \mathrm{O}_{3} \mathrm{wt} \%$ & -0.6307 & 0.1062 \\
\hline $\mathrm{Na}_{2} \mathrm{O} w t \%$ & -0.1731 & 0.035 \\
\hline $\mathrm{SiO}_{2} \mathrm{wt} \%$ & 0.0745 & 0.0312 \\
\hline $\mathrm{ZrO}_{2} \mathrm{wt} \%$ & -0.3568 & 0.1427 \\
\hline $\mathrm{Al}_{2} \mathrm{O}_{3} \mathrm{wt} \% / \mathrm{T}(\mathrm{K})$ & 799.2619 & 166.123 \\
\hline $\mathrm{CaO}_{\mathrm{w}} \mathrm{wt} / \mathrm{T}(\mathrm{K})$ & 51.2016 & 118.5831 \\
\hline $\mathrm{Fe}_{2} \mathrm{O}_{3} \mathrm{wt} \% / \mathrm{T}(\mathrm{K})$ & 885.8955 & 158.6489 \\
\hline $\mathrm{Na}_{2} \mathrm{O} \mathrm{wt} \% / \mathrm{T}(\mathrm{K})$ & 160.1108 & 46.7136 \\
\hline $\mathrm{SiO}_{2} \mathrm{wt} \% / \mathrm{T}(\mathrm{K})$ & 73.5143 & 41.0916 \\
\hline $\mathrm{ZrO}_{2} \mathrm{wt} \% / \mathrm{T}(\mathrm{K})$ & 764.9550 & 211.0122 \\
\hline
\end{tabular}


Table 5.9

Results of Modeling 7-Day PCT of Si, B, Na, Li, and pH

by Unweighted Least Squares Regression.

\begin{tabular}{|l|c|c|c|c|c|}
\hline Model of & $\mathbf{S i}$ & $\mathrm{B}$ & $\mathbf{N a}$ & $\mathbf{L i}$ & $\mathbf{p H}$ \\
\hline $\mathrm{R}$ & 0.910 & 0.907 & 0.873 & 0.929 & 0.909 \\
\hline $\mathrm{R}^{2}$ & 0.829 & 0.824 & 0.762 & 0.864 & 0.826 \\
\hline $\begin{array}{l}\text { Number of } \\
\text { Varibles }\end{array}$ & 15 & 15 & 15 & 15 & 15 \\
\hline $\begin{array}{l}\text { Number of } \\
\text { Samples }\end{array}$ & 185 & 185 & 185 & 184 & 185 \\
\hline $\begin{array}{l}\text { Residual } \\
\text { Distribution* }\end{array}$ & $\mathrm{N}$ & $\mathrm{N}$ & $\mathrm{N}$ & $\mathrm{N}$ & $\mathrm{N}$ \\
\hline
\end{tabular}

*: ' $\mathrm{N}$ ' indicates that the residual distribution is very close to standard normal distribution. 
Table 5.10

7-Day PCT Model Coefficients for $\mathrm{Si}, \mathrm{B}, \mathrm{Na}, \mathrm{Li}$, and $\mathrm{pH}$.

\begin{tabular}{|c|c|c|c|c|c|}
\hline Oxide Name & . & _aisithium & aisodium & aisilicon & $\mathbf{a}_{\mathrm{ipH}}$ \\
\hline Intercept & 5.67443 & 3.961145 & 7.297923 & 1.094473 & 8.236481 \\
\hline $\mathrm{SiO}_{2}$ & -0.04378 & -0.062726 & -0.036100 & 0.068312 & -0.006247 \\
\hline $\mathrm{Al}_{2} \mathrm{O}_{3}$ & -0.24770 & -0.222493 & -0.250977 & -0.090015 & -0.028130 \\
\hline $\mathrm{B}_{2} \mathrm{O}_{3}$ & 0.14894 & -0.033193 & -0.067815 & -0.033350 & -0.042179 \\
\hline $\mathrm{Fe}_{2} \mathrm{O}_{3}$ & -0.06228 & -0.029665 & -0.095559 & -0.009284 & 0.008457 \\
\hline $\mathrm{FeO}$ & 0.01981 & 0.093760 & -0.036784 & 0.035006 & 0.029490 \\
\hline $\mathrm{MgO}$ & 0.13739 & -0.015909 & 0.133711 & -0.087629 & -0.012005 \\
\hline $\mathrm{CaO}$ & -0.04157 & -0.031388 & -0.049743 & -0.007886 & 0.073096 \\
\hline $\mathrm{Na}_{2} \mathrm{O}$ & 0.03520 & -0.011338 & 0.195910 & 0.093742 & 0.167516 \\
\hline $\mathrm{K}_{2} \mathrm{O}$ & 0.02086 & 0.109245 & 0.024878 & -0.010681 & 0.134817 \\
\hline $\mathrm{Li}_{2} \mathrm{O}$ & 0.26948 & 0.749565 & 0.213796 & 0.232233 & 0.288172 \\
\hline $\mathrm{TiO}_{2}$ & -1.37188 & 0.846109 & -0.858445 & -0.158224 & -0.327866 \\
\hline $\mathrm{P}_{2} \mathrm{O}_{5}$ & -0.01606 & 0.013517 & -0.005091 & 0.024716 & 0.002447 \\
\hline $\mathrm{MgE}_{2}$ & 0.07845 & -0.021180 & 0.063183 & -0.009109 & -0.015939 \\
\hline $\mathrm{NaF}$ & 0.13255 & 0.052263 & 0.215649 & 0.022065 & 0.098916 \\
\hline $7 \times f$ & -0.12476 & -0149945 & -0.101991 & -0.073607 & $-0,003151$ \\
\hline
\end{tabular}


Table 5.11

Prediction of 7-Day PCT of Si, B, Na, Li, and pH.

\begin{tabular}{|c|c|c|c|c|c|c|c|c|c|c|}
\hline & B_ppm & B_ppm & & Li_ppm & Li_ppm & & Na_ppm & Na_ppm & & \\
\hline Glass Name & Predicted & Observed & Residue & Predicted & Observed & Residue & Predicted & Observed & Residue & Project \\
\hline $\mathrm{F} 4-22$ & 213.60 & 270.00 & -56.40 & 0.63 & 1.71 & -1.08 & 259.40 & 304.70 & -45.30 & MAWS \\
\hline F4-4 & 294.57 & 275.20 & 19.37 & 0.82 & 0.80 & 0.002 & 379.40 & 43.45 & 335.95 & MAWS \\
\hline F5-23 & 35.89 & 28.01 & 7.88 & 0.20 & 0.20 & 0.00 & 81.22 & 71.95 & 9.27 & FERNALD \\
\hline F5-40 & 244.58 & 315.00 & -70.42 & 1.02 & 1.05 & -0.03 & 372.49 & 597.00 & -224.51 & FERNALD \\
\hline HAN25 & 0.75 & 1.04 & -0.31 & 0.10 & 0.01 & 0.09 & 276.97 & 305.10 & -28.13 & DOE \\
\hline HAN38 & 0.97 & 2.64 & -1.67 & 0.12 & 0.22 & -0.10 & 344.95 & 257.20 & 87.75 & DOE \\
\hline ORNL10 & 9.92 & 13.12 & -3.20 & 0.73 & 0.02 & 0.71 & 34.22 & 25.75 & 8.47 & OAK \\
\hline $\begin{array}{l}\text { UTH } 218 \text { D - } \\
\text { R40-10 }\end{array}$ & 60.05 & 35.47 & 24.58 & 43.52 & 15.56 & 27.96 & 57.89 & 59.49 & -1.60 & WV \\
\hline WVCM62 & 27.17 & 17.61 & 9.56 & 5.94 & 8.33 & -2.39 & 52.81 & 44.44 & 8.37 & WV \\
\hline WVCM70 & 43.46 & 23.82 & 19.64 & 19.56 & 9.85 & 9.71 & 56.96 & 30.24 & 26.72 & WV \\
\hline WVCM70R28 & 57.21 & 41.02 & 16.19 & 28.87 & 16.48 & 12.39 & 25.49 & 52.03 & -26.54 & WV \\
\hline WVITH180 & 2541 & 14.36 & 1105 & 13.37 & 7.77 & 5.60 & 4071 & 22.80 & 1791 & wV \\
\hline
\end{tabular}




\begin{tabular}{|c|c|c|c|c|c|c|c|}
\hline & Si_ppm & Si_ppm & & $\mathrm{pH}$ & $\mathrm{pH}$ & & \\
\hline Glass Name & Predicted & Observed & Residue & Predicted & Observed & Residue & Project \\
\hline $\mathrm{F} 4-22$ & 11.26 & 9.90 & 1.36 & 9.02 & 8.50 & 0.52 & MAWS \\
\hline F4-4 & 10.08 & 3.42 & 6.66 & 9.56 & 8.65 & 0.91 & MAWS \\
\hline F4-23 & 18.65 & 21.93 & -3.28 & 9.58 & 9.45 & 0.13 & FERNALD \\
\hline F4-40 & 6.90 & 6.64 & 0.26 & 9.78 & 9.34 & 0.44 & FERNALD \\
\hline HAN25 & 159.94 & 118.00 & 41.94 & 11.75 & 11.87 & -0.12 & DOE \\
\hline HAN38 & 108.24 & 65.36 & 42.88 & 11.91 & 10.38 & 1.53 & DOE \\
\hline ORNL10 & 26.50 & 31.01 & -4.51 & 9.40 & 9.52 & -0.12 & OAK \\
\hline $\begin{array}{l}\text { UTH218D- } \\
\text { R40-10 }\end{array}$ & 86.04 & 50.30 & 35.74 & 10.82 & 10.24 & 0.58 & wV \\
\hline WVCM62 & 50.07 & 58.66 & -8.59 & 9.95 & 10.07 & -0.12 & WV \\
\hline WVCM70 & 60.01 & 44.88 & 15.13 & 10.21 & 10.04 & 0.17 & WV \\
\hline WVCM70R28 & 70.37 & 58.29 & 12.08 & 10.29 & 10.10 & 0.19 & WV \\
\hline WVUTH 180 & 5705 & 4107 & 1598 & 1015 & 10.58 & -0.43 & wy \\
\hline
\end{tabular}


Table 5.12

Division of 40 Glasses into Three Groups; Each Group was Sequentially Removed from the Database to Examine the Predictive Capabilities of the Models for "Unknown" Glasses.

\begin{tabular}{|c|c|c|c|c|c|c|c|c||}
\hline \multicolumn{3}{|c|}{ GROUP 1. } & \multicolumn{3}{c|}{ GROUP 2 } & \multicolumn{3}{c||}{ GROUP 3 } \\
\hline HANFORD & IDAHO & OAK RIDGE & HANFORD & IDAHO & OAK RIDGE & HANFORD & DAHO & OAK RIDGE \\
\hline HAN22B & IDG7A & ORNL3 & HAN24 & IDG7C & ORNL10 & HAN25 & IDG8A & ORNL9A \\
\hline HAN26 & IDG7B & ORNL13 & HAN27 & IDG8C & ORNL15 & HAN28 & IDG13C & ORNL11 \\
\hline HAN28A & IDG13A & ORNL14 & HAN29A & IDG13B & ORNL16 & HAN30A & IDG14B & ORNL12 \\
\hline HAN29B1 & IDG14A & & HAN29F & IDG13C & & HAN34 & IDG15A & \\
\hline HAN29E & & & HAN33 & & & HAN38 & & \\
\hline HAN35 & & & HAN37 & & & HAN39 & & \\
\hline HAN36 & & & & & & & & \\
\hline
\end{tabular}


Figure 5.1

The Oracle7 Client/Server Database Management System. SPX and TCP/IP are Communication Protocols.

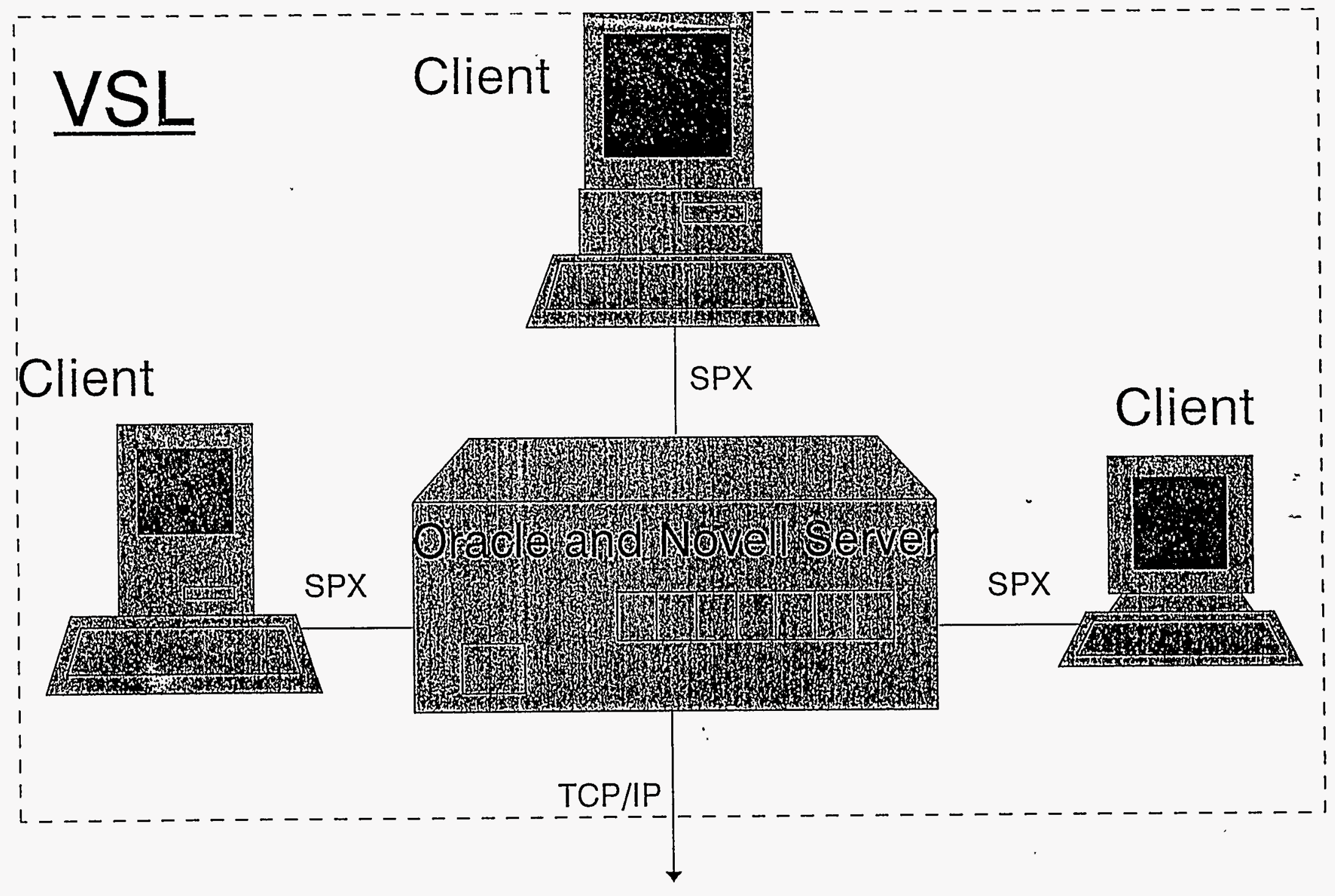

To Internet 
Figure 5.2

Table structure of the Glass Composition-Property Database. Titles of each table are abbreviated and highlighted in title bars. The first column of each table lists the contents of a table, and the second column specifies the categories of the table contents. ("A" refers to text variables, "789" refers to numerical variables, and " 31 " in a box indicates date variables.)

\section{Table Structure of the Database of Glasses}

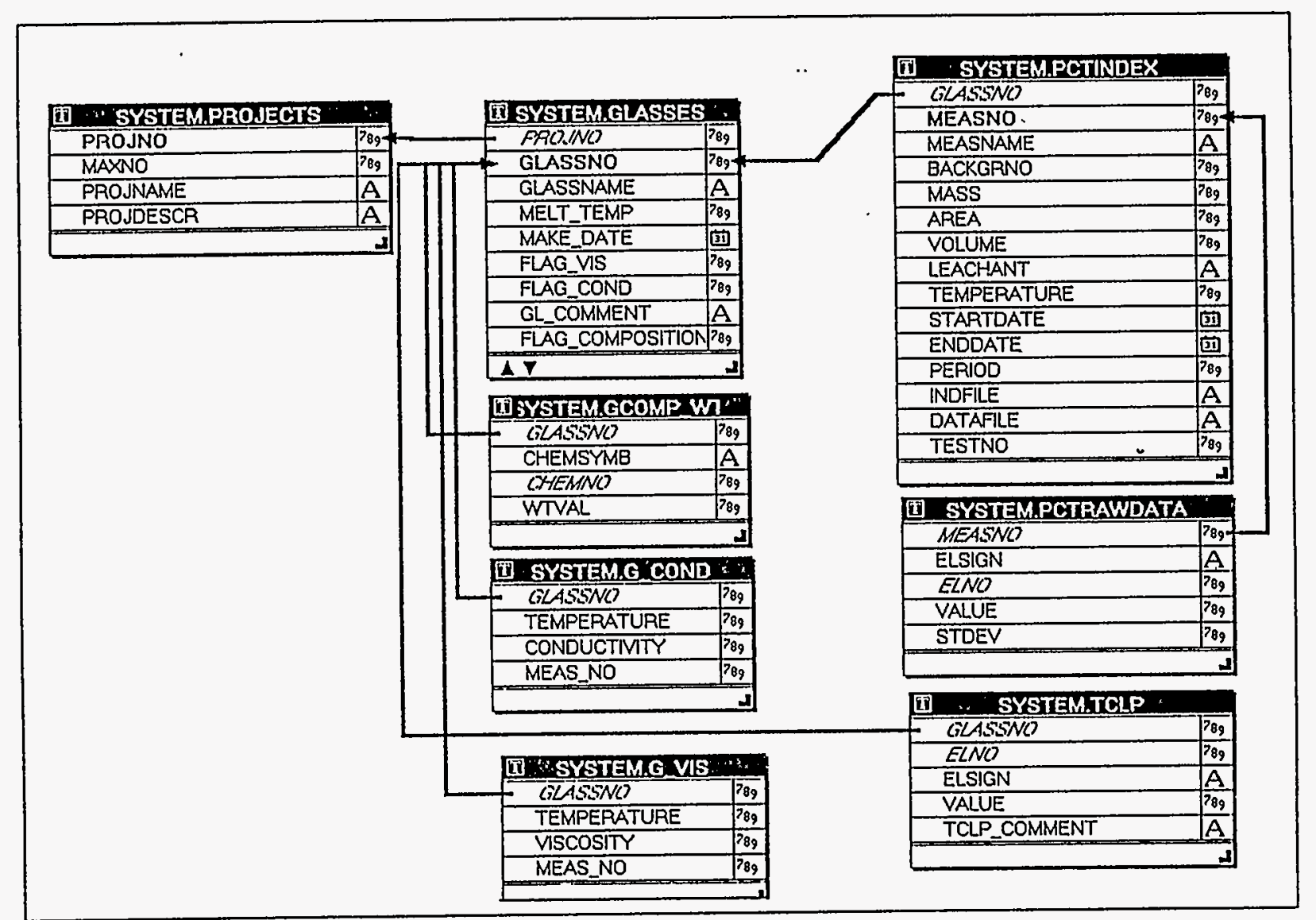


Figure 5.3

Example of Glass Entry Form: a list of samples from the Hanford project. The boxes under the columns of "Cmp", "Vis", "Cond", "Tclp", and "PCT7d" summarize the information associated with each glass. Cmp=composition data, Vis=viscosity data, Cond=conductivity data, Tclp=TCLP data, and PCT7d=7-Day PCT for Si, B, Na, and Li. Experimental data included in the GCPD are marked with a check sign in corresponding rows (glassname) and columns (data type).

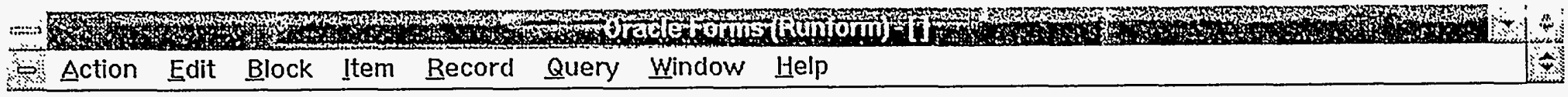
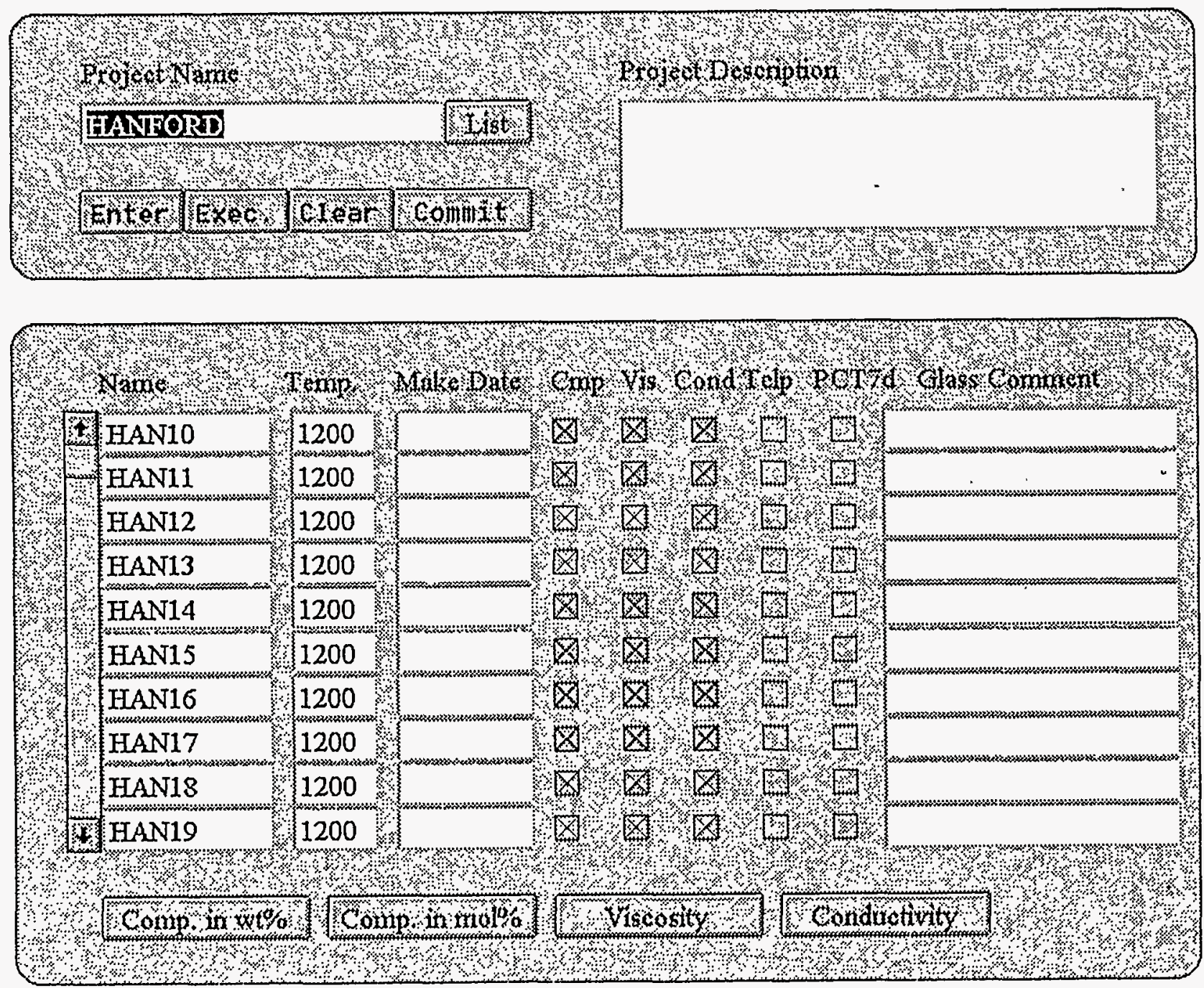

Page 5-41 


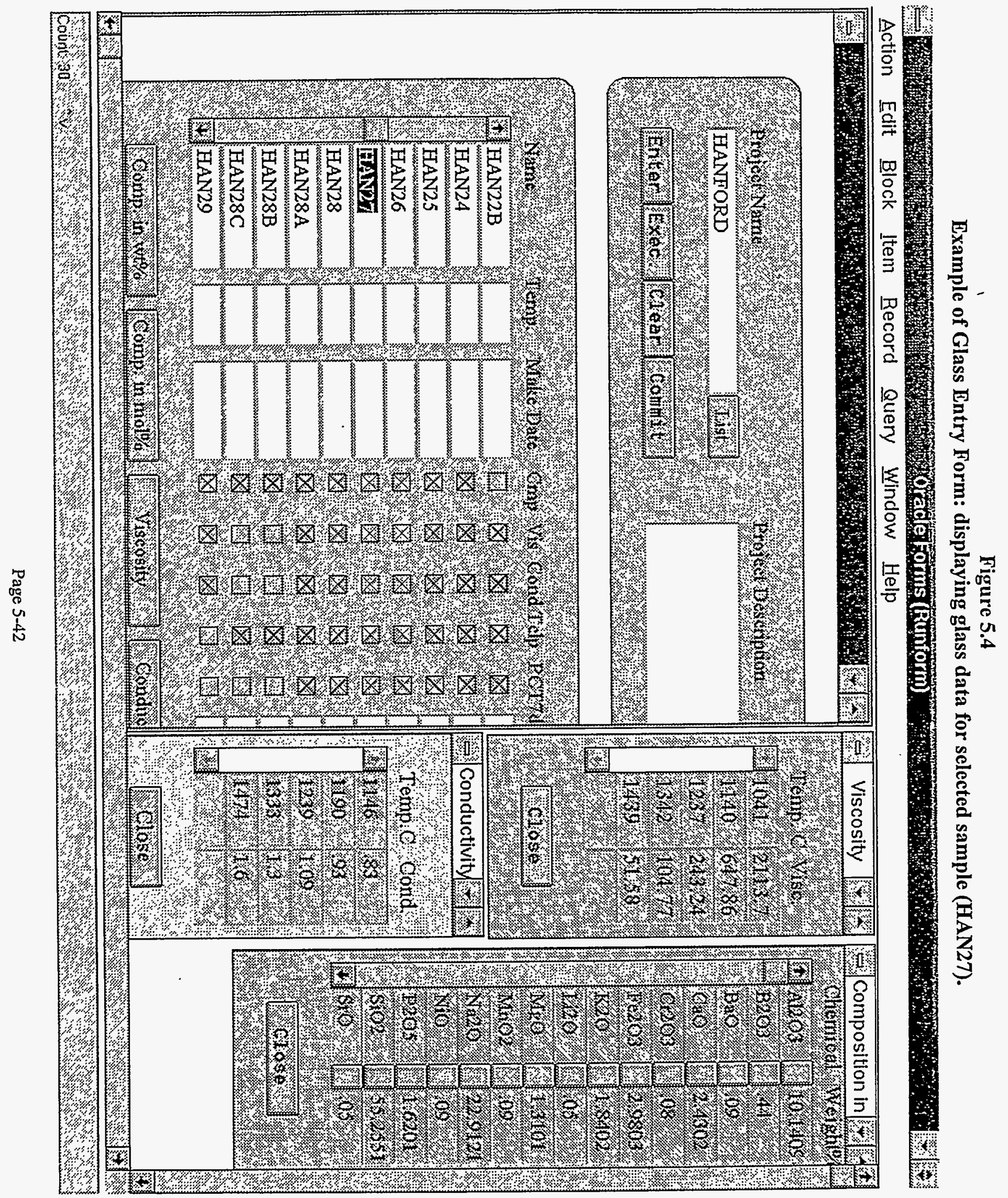


Figure 5.5

Example of Glass Modelling Tool. Menu "Select" is highlighted on the screen. After selecting Projects, Glasses, a user retrieves molar compositional data of the selected glasses. The collected information can then be used in a report, displayed in various 2-D scatter plots, or prepared as a data file for modelling work.

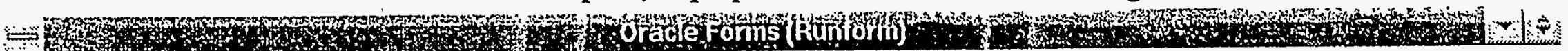

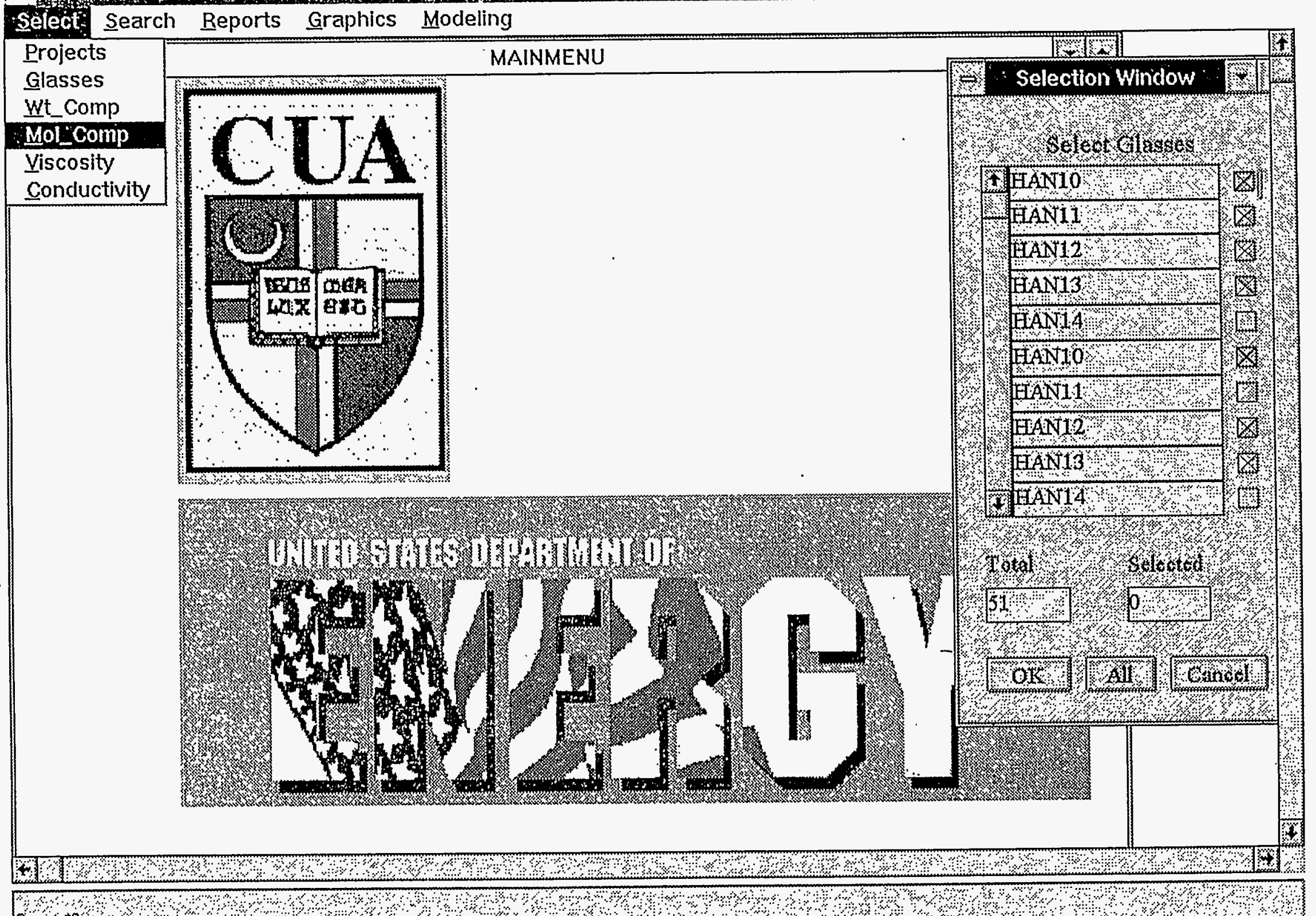

Cont

Page 5-43 


\section{Figure 5.6}

Data frequency distribution along $\mathrm{SiO}_{2}, \mathrm{Al}_{2} \mathrm{O}_{3}, \mathrm{~B}_{2} \mathrm{O}_{3}$ and $\mathrm{Na}_{2} \mathrm{O}$ wt\% (Data from viscosity table). On a relative scale, the distribution patterns approximate very closely the sample distribution on the same axis because normally four viscosity measurements would be taken for each glass (in molten state) with few exceptions.

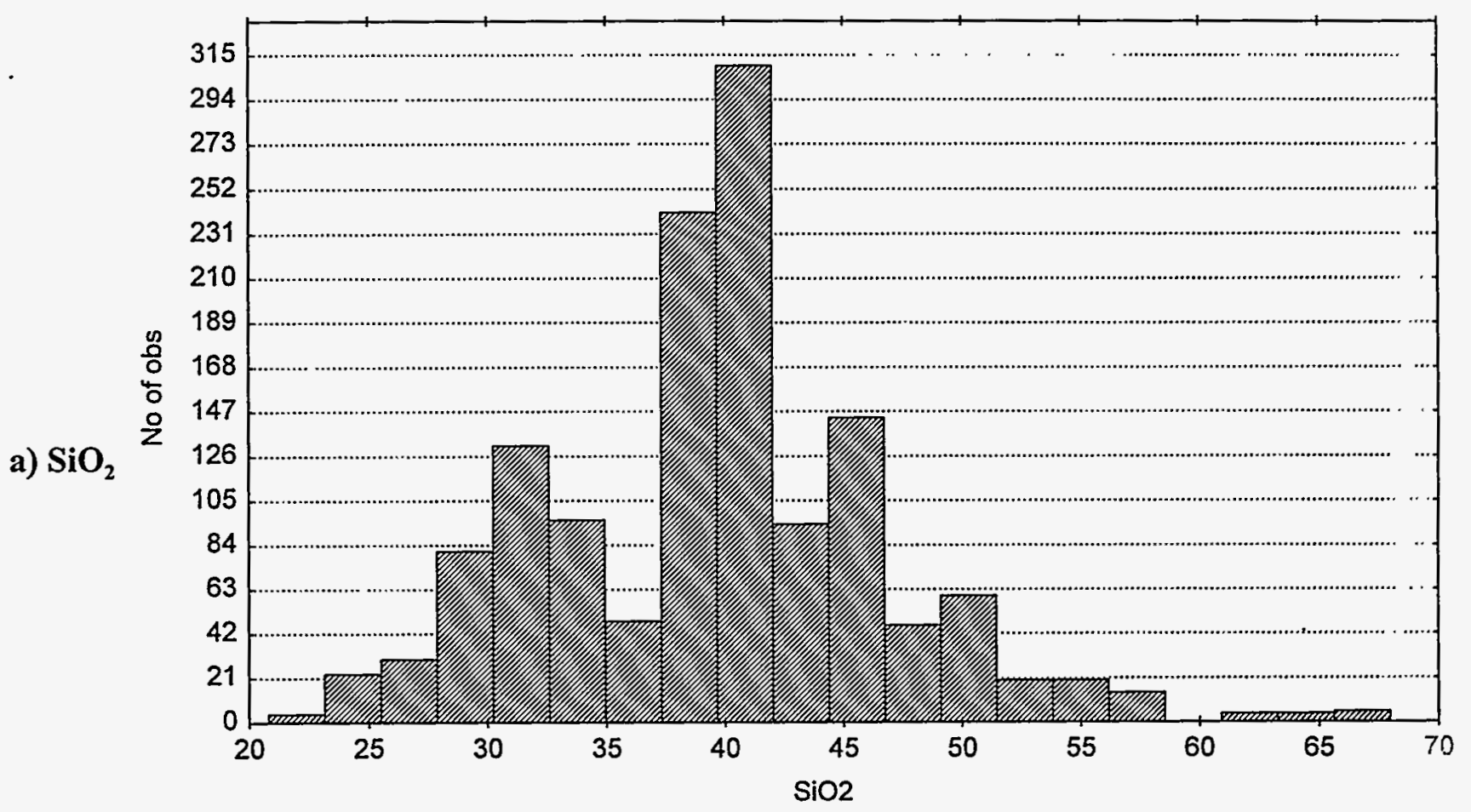

b) $\mathrm{Al}_{2} \mathrm{O}_{3}$

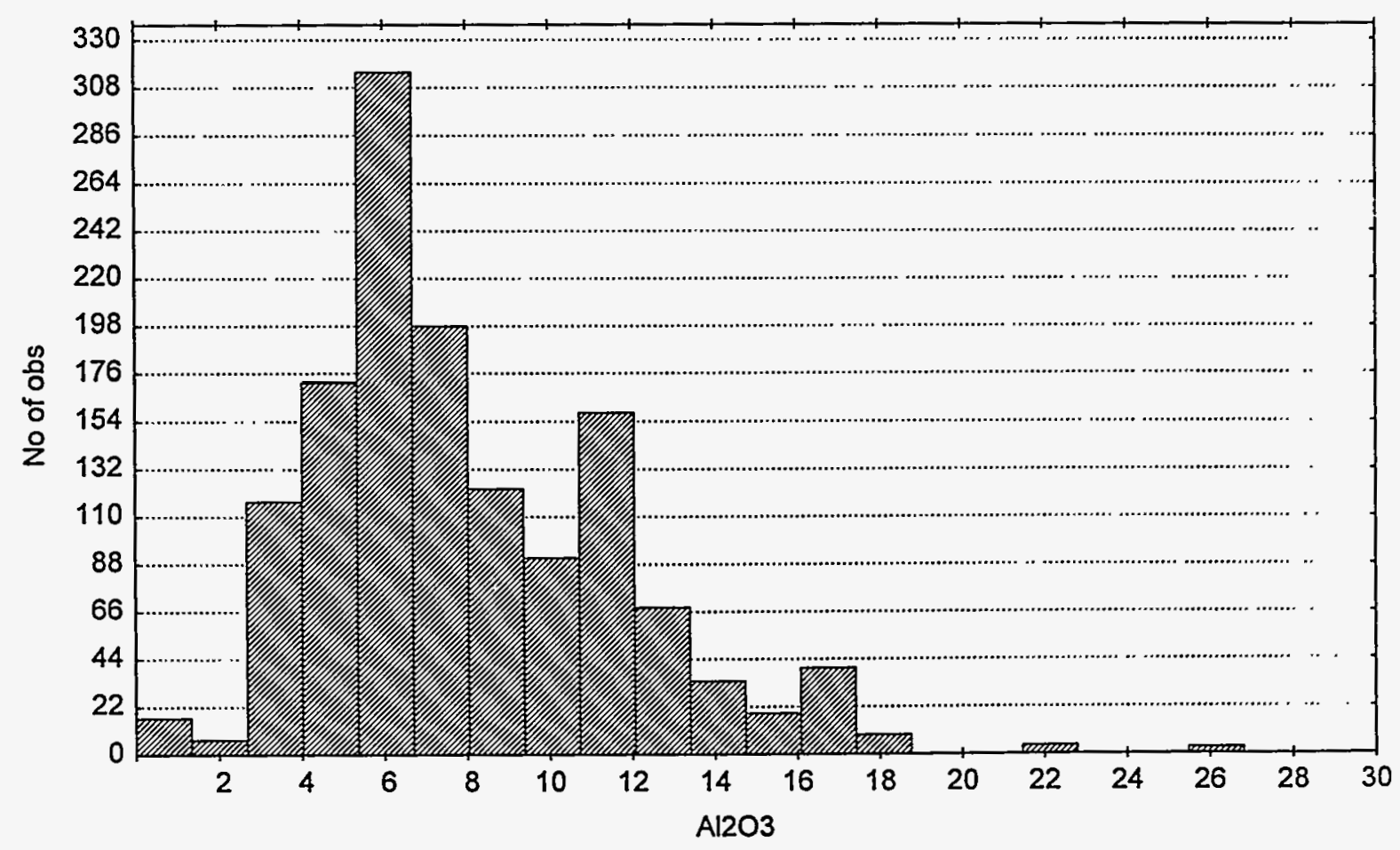


Figure 5.6 continued

Data frequency distribution along $\mathrm{SiO}_{2}, \mathrm{Al}_{2} \mathrm{O}_{3}, \mathrm{~B}_{2} \mathrm{O}_{3}$ and $\mathrm{Na}_{2} \mathrm{O}$ wt\% (Data from viscosity table). On a relative scale, the distribution patterns approximate very closely the sample distribution on the same axis because normally four viscosity measurements would be taken for each glass (in molten state) with few exceptions.

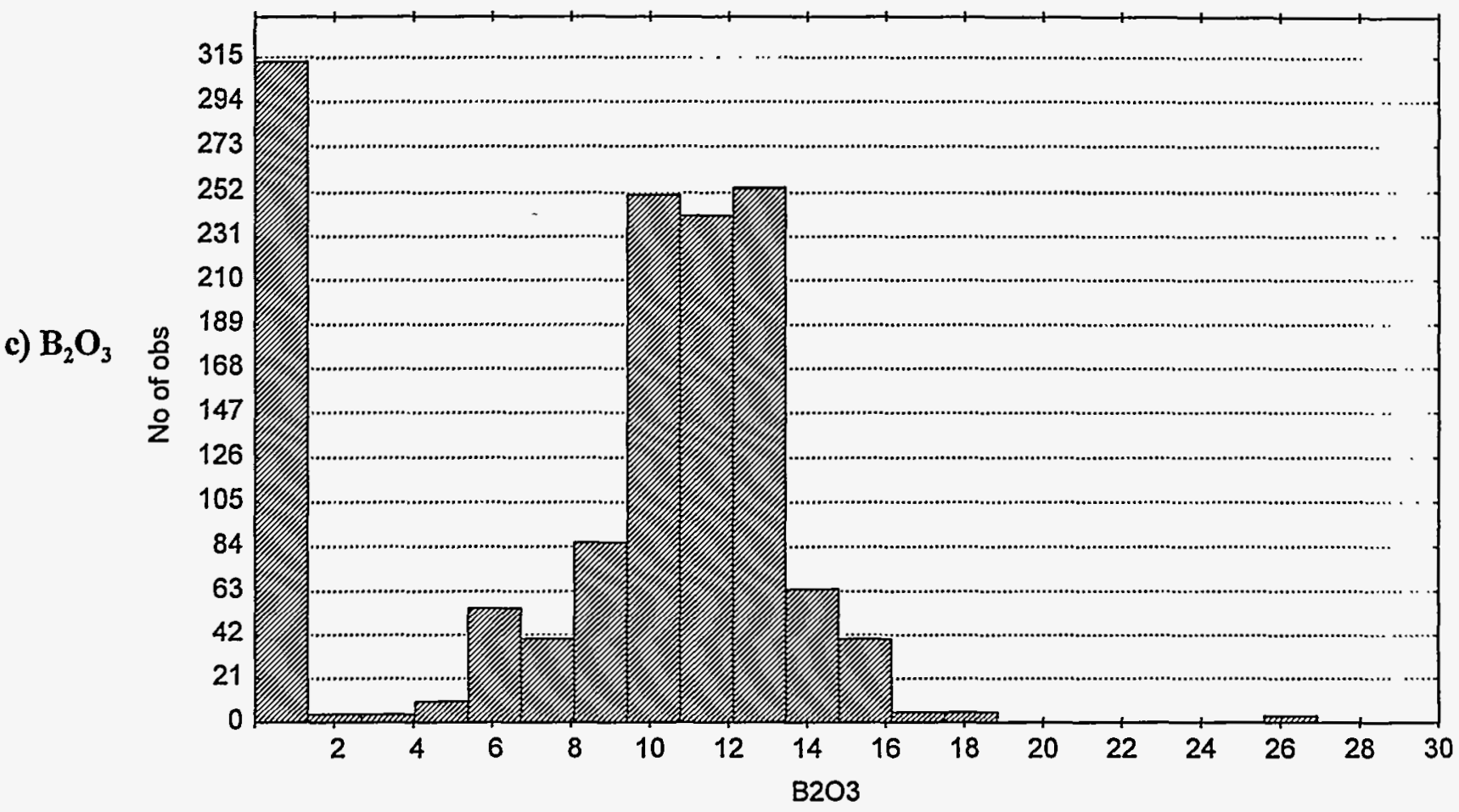

d) $\mathrm{Na}_{2} \mathrm{O}$

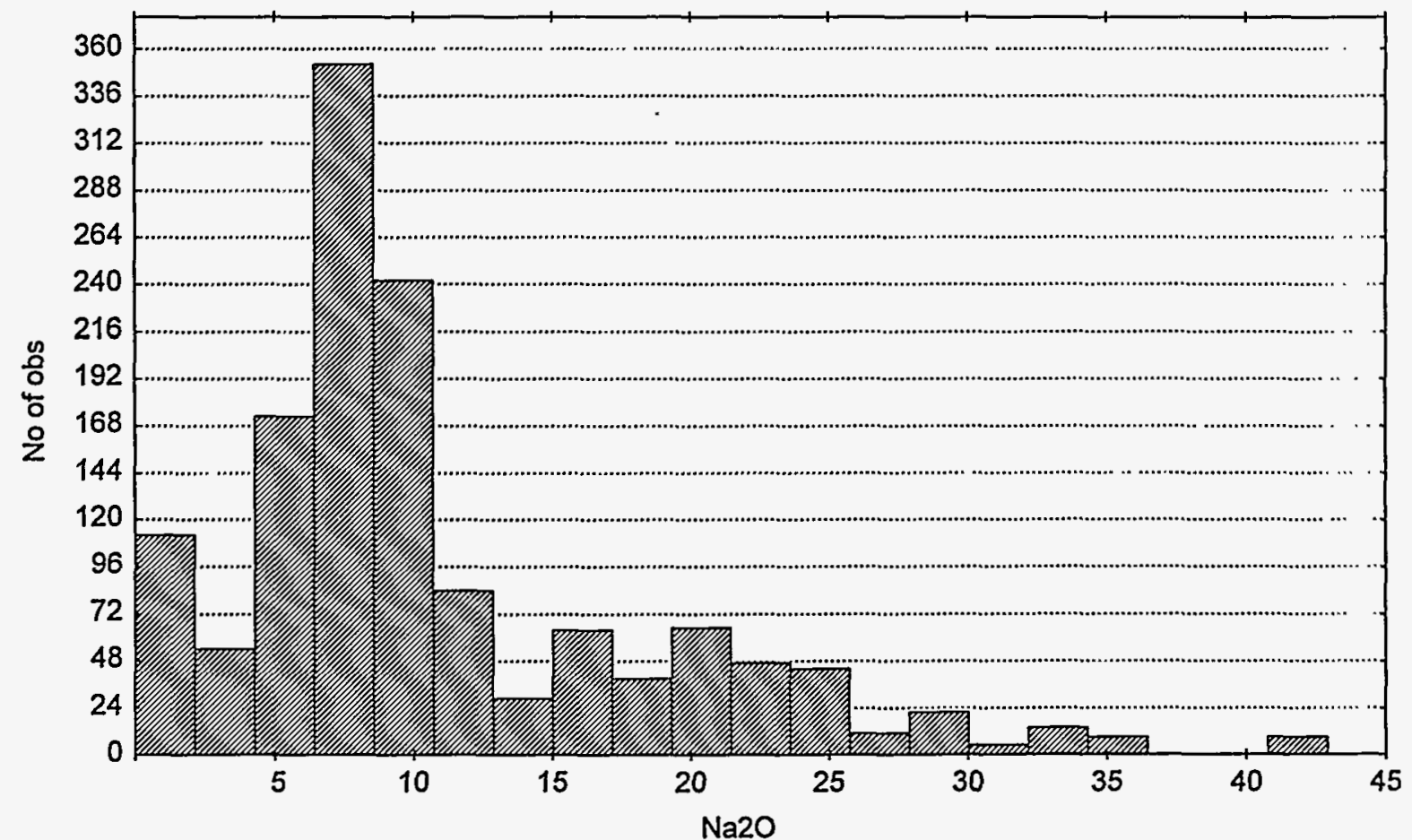




\section{Figure 5.7}

Box and whisker plot of glass compositions in viscosity table. The box and whisker plot describes the central tendency of the variable in terms of the median of the values (represented by the smallest boxes in the plot). The spread (variability) in compositional variables (in wt\%) is represented by the quartiles (the 25th and 75th percentiles, larger boxes in the plot) and the minimum and maximum values of the variables (the 'whiskers' in the plot).

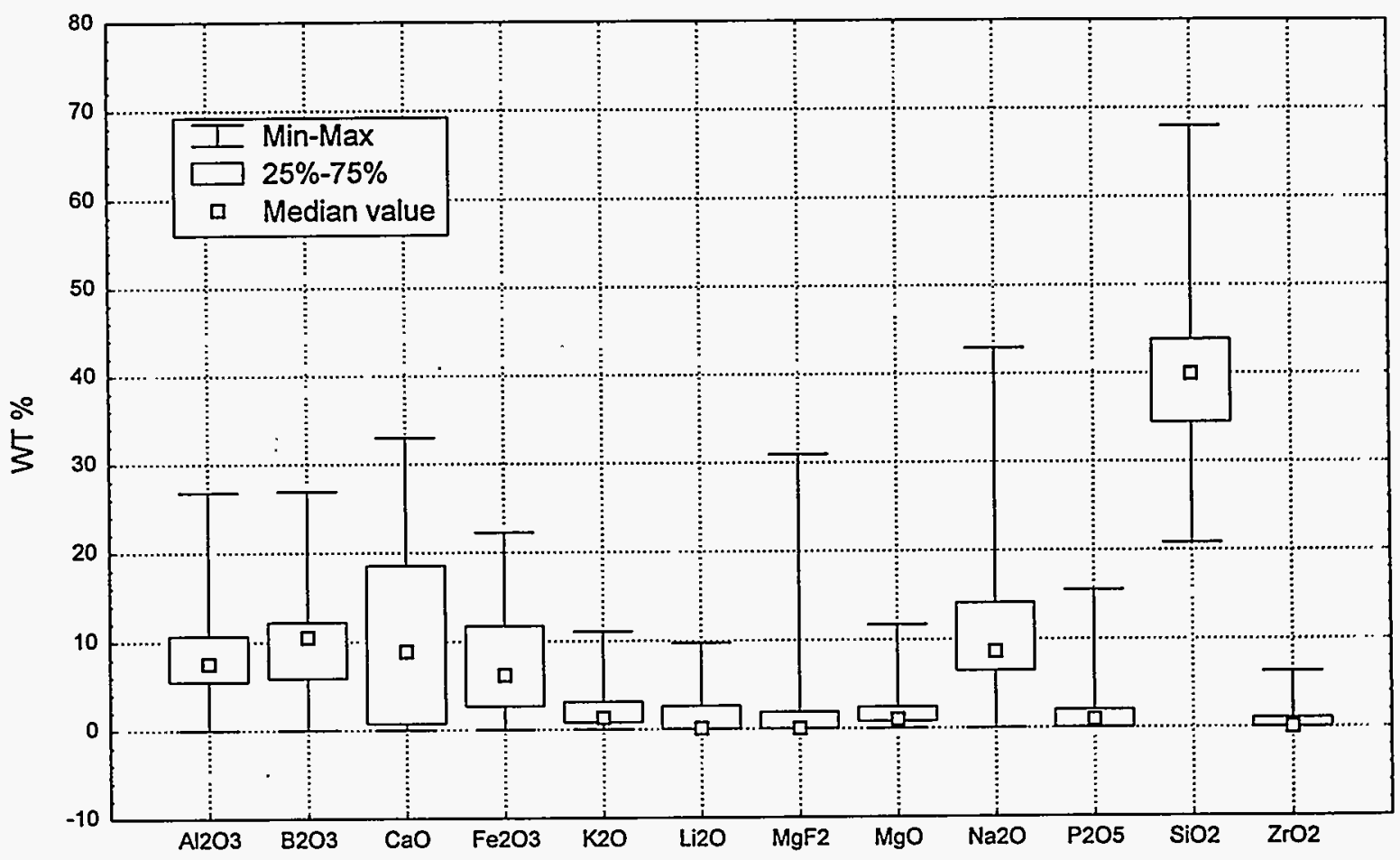




\section{Figure 5.8}

Sample distribution in reduced compositional space of NBOT versus M3T. Each symbol in the figure represents the samples from the same project. Straight lines $\mathrm{Ab}$ and $\mathrm{An}$ represent the locations where $M 3 T$ is 0.25 or 0.5 , respectively, and straight lines $Q^{4}, Q^{3}$, and $Q^{2}$ represent 0,1 or 2 non-bridging oxygens (NBOT), respectively. Generally a $Q^{n}$ refers to a tetrahedral site with (4-n) non-bridging oxygens. Note that the glasses with NBOT $<0$ have slightly more trivalent cations than the sum of charges from alkali and alkaline earth cations. $(100 \mathrm{U}=100 \mathrm{Kg}$ melter project; $\mathrm{ANLG}=$ samples from Argonne project; CM0=samples from Fernald site; DIG=Bavaria project; F-4=samples from Fernald Pit 4; F-5=samples from Fernald Pit 5; HAN=samples from Hanford site; IDG=samples from Idaho site; MIC2, 3=Micromelter project; $M M 1=$ Medical Waste project; $O R N L=$ samples from Oak Ridge site; SMG=samples from Savannah River site; WETW=Wet Waste project; WS=samples from Weldon Spring site; WV=samples from West Valley site).

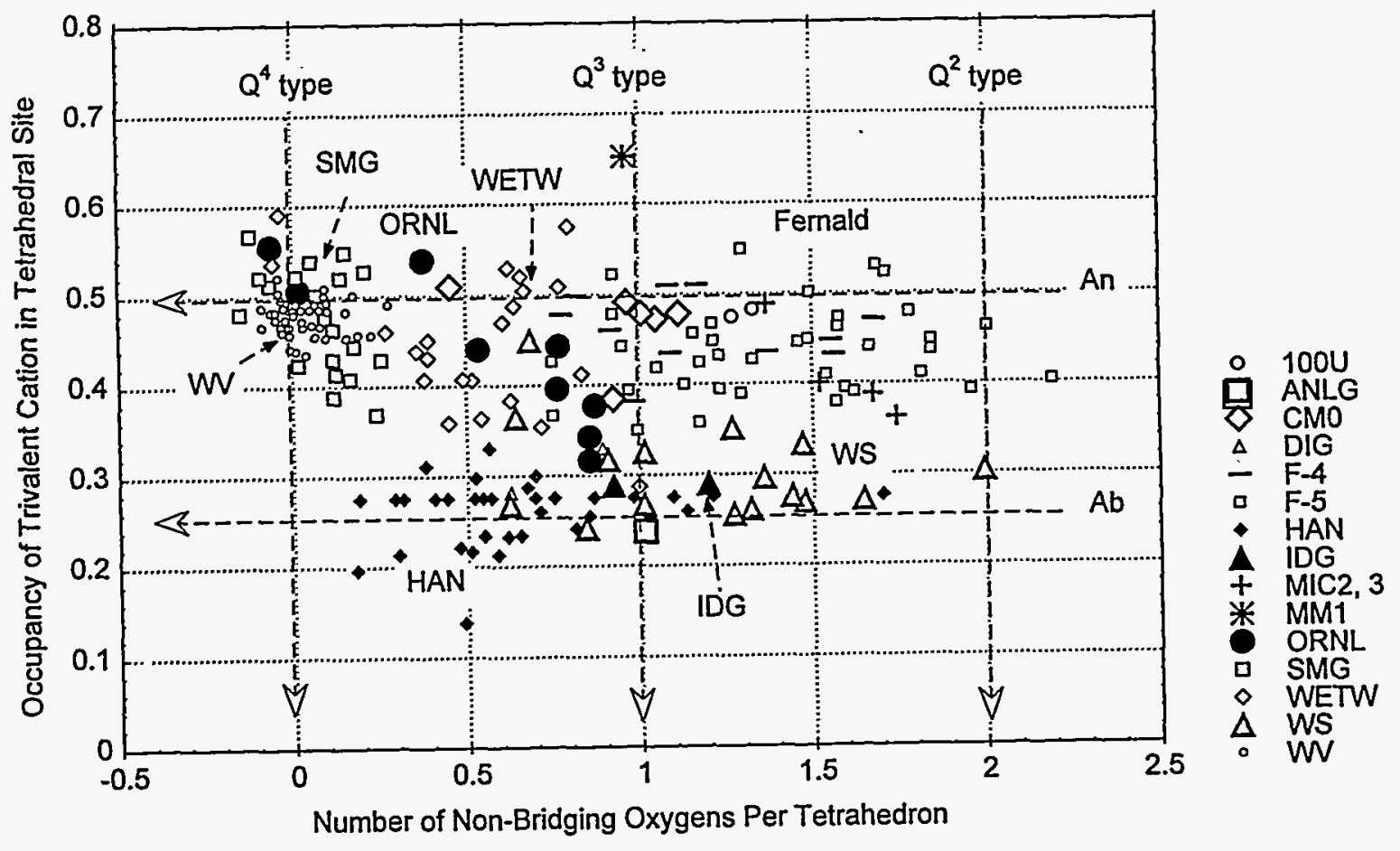


Figure 5.9

Data frequency distribution in reduced compositional space of NBOT versus M3T (Data are from viscosity table).

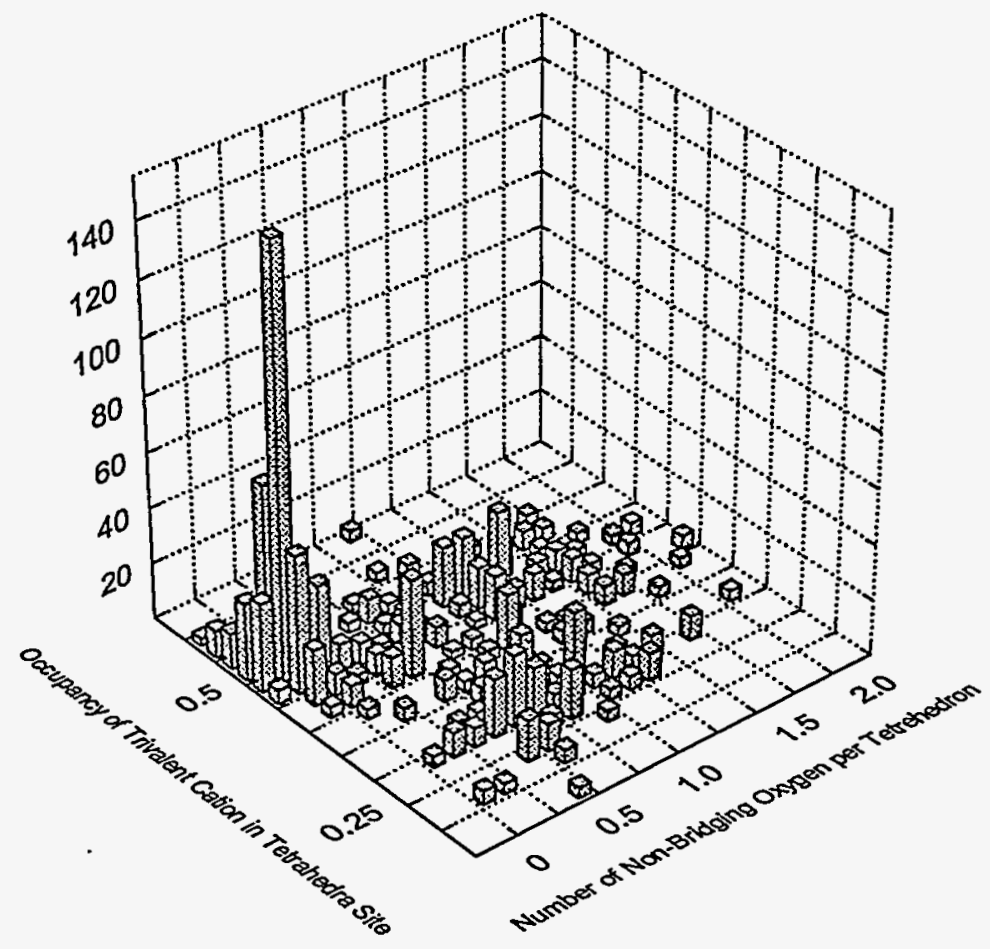

Figure 5.10

Data frequency distribution on M3T axis (from viscosity table). The symbols Ab, and An indicate the points of $\mathrm{M} 3 \mathrm{~T}=0.25$ and 0.5 , respectively.

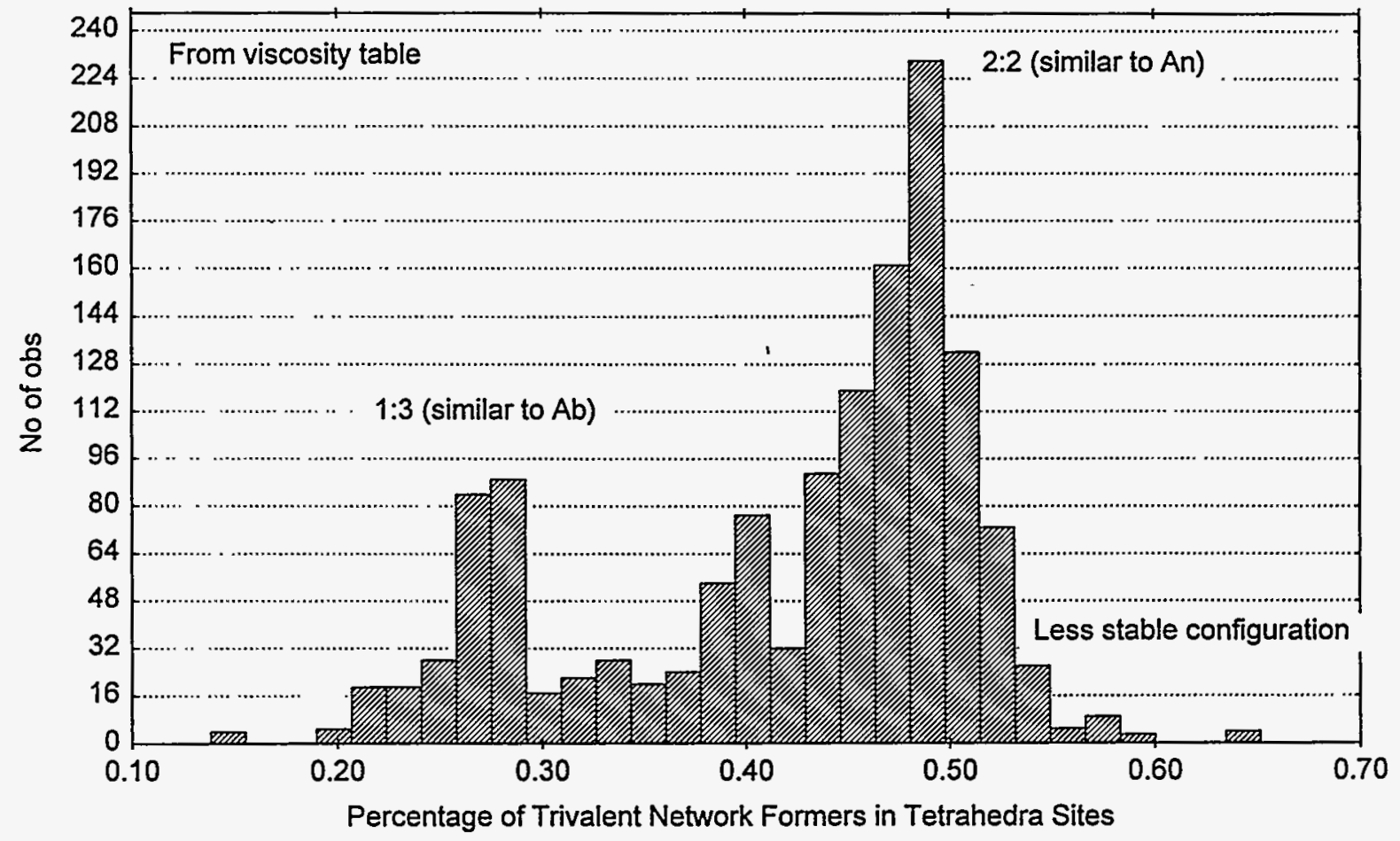

Page 5-48 
Figure 5.11

Sample distribution in reduced compositional space of NBOT versus M3T (Data from conductivity table). (100U $=100 \mathrm{Kg}$ melter project; $\mathrm{ANLG}=$ samples from Argonne project; $\mathrm{CM} 0=$ samples from Fernald site; DIG=Bavaria project; F-4=samples from Fernald Pit 4; F-5=samples from Fernald Pit 5; HAN=samples from Hanford site; IDG=samples from Idaho site; MIC2, 3=Micromelter project; MM1=Medical Waste project; ORNL=samples from Oak Ridge site; SMG=samples from Savannah River site; WETW=Wet Waste project; WS=samples from Weldon Spring site).

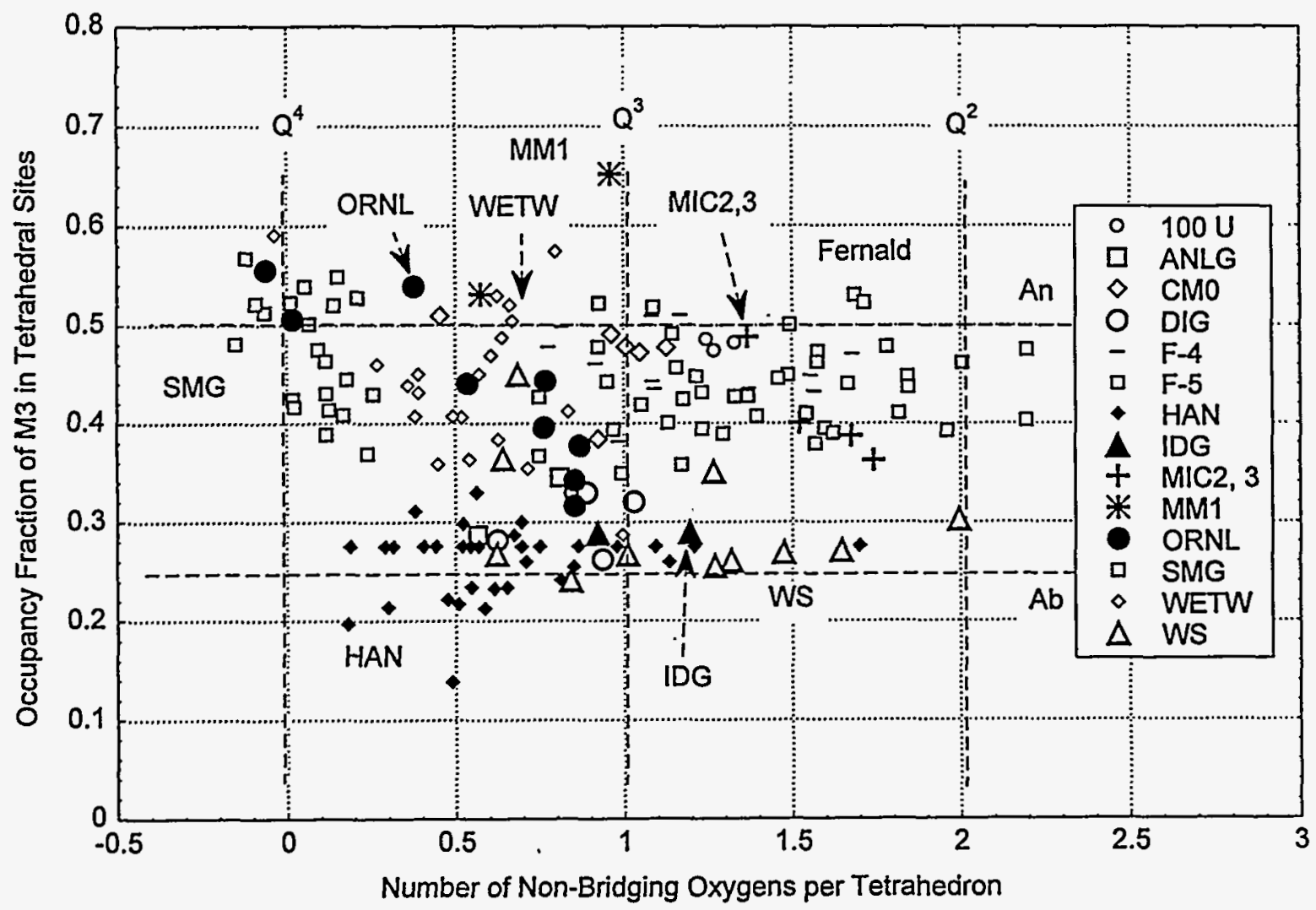


Figure 5.12

Sample distribution in reduced compositional space of NBOT versus M1M12 (from viscosity table). Note the narrow range of M1M12 for West Valley data, and wide coverage by Hanford samples. ( $100 \mathrm{U}=100 \mathrm{Kg}$ melter project; $\mathrm{ANLG}=$ samples from Argonne Project; CM0=samples from Fernald site; DIG=Bavaria project; F-4=samples from Fernald Pit 4; F-5=samples from Fernald Pit 5; HAN=samples from Hanford site; IDG=samples from Idaho site; MIC2, 3=Micromelter project; $M M 1=$ Medical Waste project; $O R N L=$ samples from Oak Ridge site; SMG=samples from Savannah River site; WETW=Wet Waste project; WS=samples from Weldon Spring site; WV=samples from West Valley site).

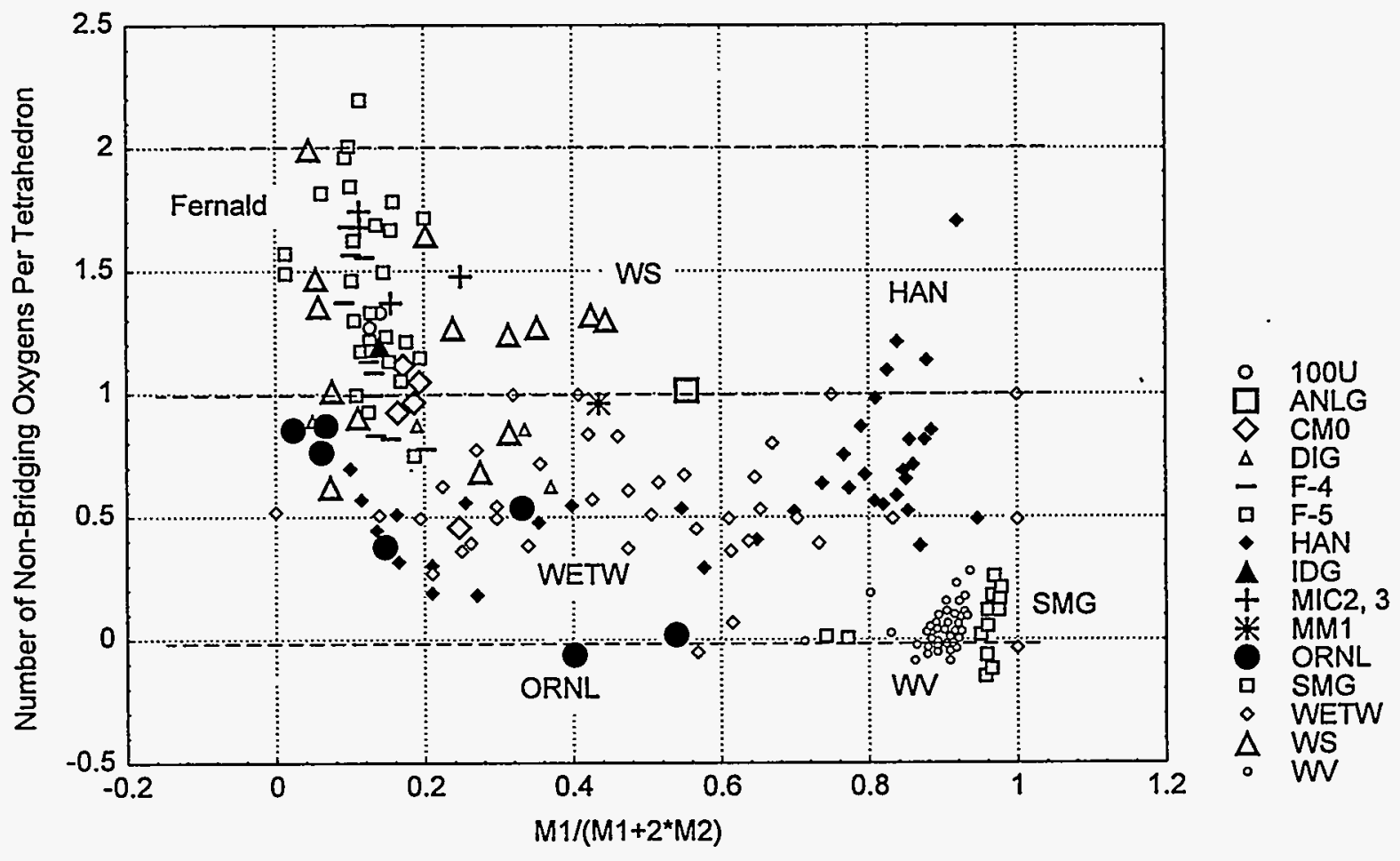


Figure 5.13

Sample distribution in reduced compositional space of NBOT versus M3T (Data from 7-

Day PCT Table for element $\mathrm{Si}, \mathrm{B}, \mathrm{Na}$ and $\mathrm{Li}) .(100 \mathrm{U}=100 \mathrm{Kg}$ melter project;

ANLG=samples from Argonne project; F-4=samples from Fernald Pit 4; F-5=samples from

Fernald Pit 5; HAN=samples from Hanford site; IDG=samples from Idaho site; MIC2,

3=Micromelter project; ORNL=samples from Oak Ridge site; WETW=Wet Waste project;

WS=samples from Weldon Spring site; WV=samples from West Valley site).

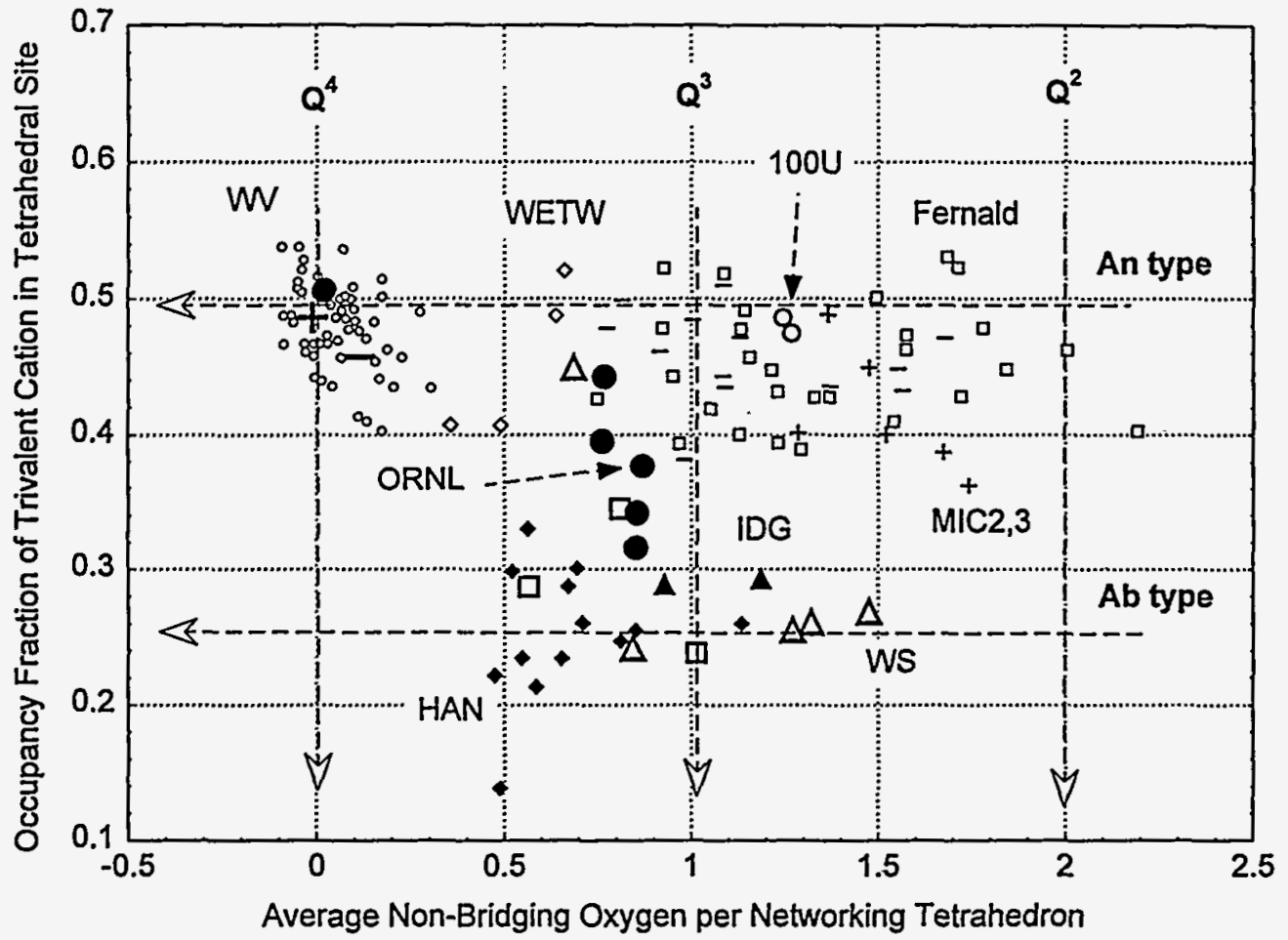

\begin{tabular}{ll}
$\circ$ & $100 U$ \\
$\square$ & ANLG \\
- & F-4 \\
$\square$ & F-5 \\
H & HAN \\
IDG & MIC2,3 \\
+ & ORNL \\
$\circ$ & WETW \\
$\triangle$ & WS \\
\hline & WNCM59 \\
+ & WCM62 \\
& WN
\end{tabular} 
Figure 5.14

Scatter plot matrix of 11 of the most abundant elements in the viscosity table. The elements in mol\% are plotted against each other as in a correlation matrix. Straight lines in each cell are by least-squares regression.

\begin{tabular}{|c|c|c|c|c|c|c|c|c|c|c|}
\hline & 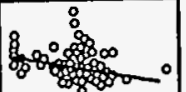 & 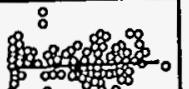 & 总 & \begin{tabular}{|c|c|} 
\\
9
\end{tabular} & \begin{tabular}{|ll} 
\\
8
\end{tabular} & 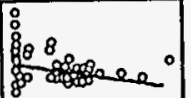 & 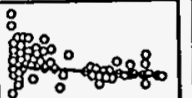 & ${ }^{\circ}{ }^{\circ}$ & \% & 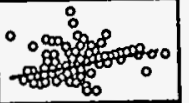 \\
\hline \% & 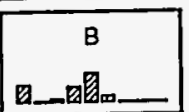 & 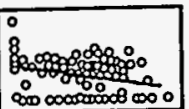 & 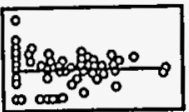 & 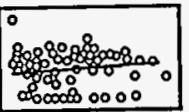 & 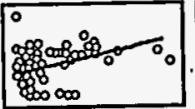 & {$\left[\begin{array}{llll}0 & 8 & & \\
800 & 0 & 0 & 0\end{array}\right.$} & $\begin{array}{lll}0 \\
8 \\
8 \\
8\end{array}$ & 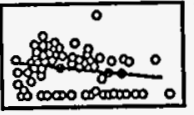 & क०ष: & \\
\hline$\frac{\infty}{\infty}$ & 188 & $\begin{array}{r}\mathrm{CA} \\
\mathrm{C}\end{array}$ & : : & 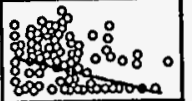 & 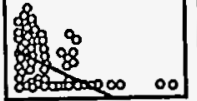 & 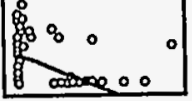 & 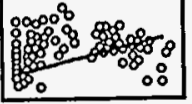 & 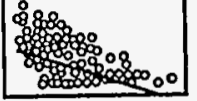 & 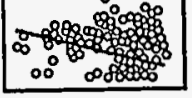 & \\
\hline 웅 & & (0) & & 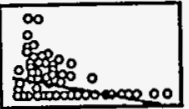 & 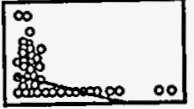 & 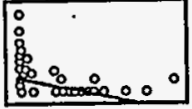 & 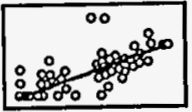 & 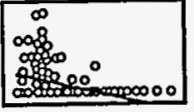 & & \\
\hline$\frac{1}{80}$ & 8 & 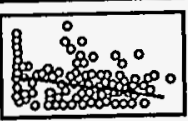 & $880^{\circ}$ & $\begin{array}{r}\text { FE } \\
\text { E四四因 } \\
\end{array}$ & & & & 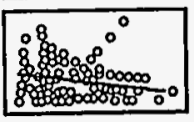 & & \\
\hline & \% & \begin{tabular}{|lll}
8 & \\
8 & 80 \\
& 8 & 0 \\
\end{tabular} & 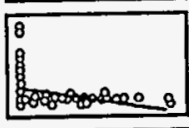 & 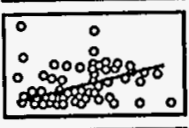 & K & 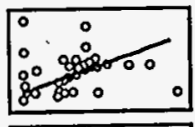 & \begin{tabular}{|l|l|}
8 \\
8 \\
$8_{9} \circ 0$ \\
8 & 0 \\
\end{tabular} & \begin{tabular}{|c|}
0 \\
0 \\
0 \\
0 \\
0
\end{tabular} & nest & ए్య० \\
\hline & \begin{tabular}{|lll}
0 & 8 \\
0 & 8 \\
0 & 800 \\
8 & 0 \\
\end{tabular} & 8 & 1 & 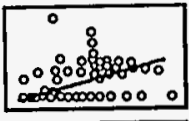 & 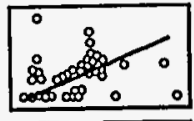 & LI & 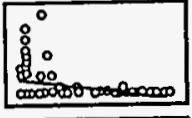 & 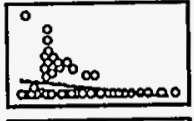 & & $\overline{\sigma a}$ \\
\hline 然 & 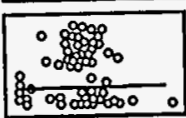 & 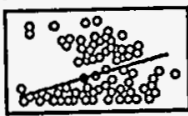 & $\begin{array}{l}00 \% \\
8 \% 8 \\
0 \%\end{array}$ & 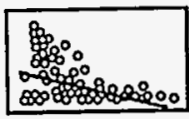 & 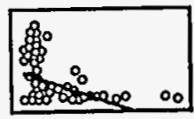 & 铝 & MG & 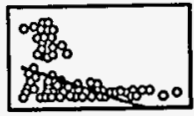 & & \\
\hline 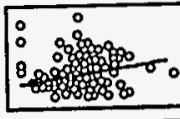 & 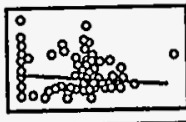 & 䢰: & 80 & 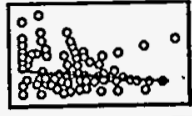 & 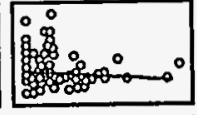 & \begin{tabular}{|lll}
8 & \\
8 & 0 & \\
8 & 0 & 0
\end{tabular} & 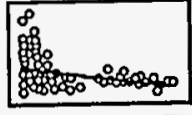 & 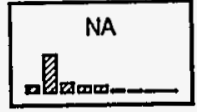 & $\infty$ & $0^{\circ}$ \\
\hline 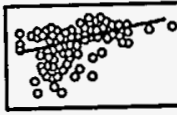 & 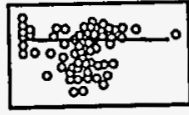 & $\begin{array}{l} \\
8000 \\
\end{array}$ & & 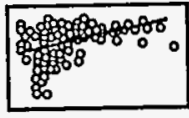 & 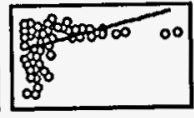 & 8 & $\begin{array}{c}3_{8}^{80} \\
00 \\
0\end{array}$ & 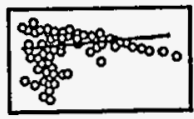 & 0 & 翟 \\
\hline 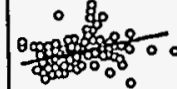 & : & 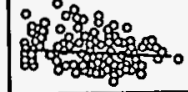 & 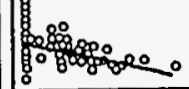 & 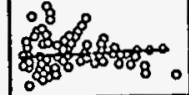 & ${ }^{0}$ & $\frac{8}{8} 800000$ & 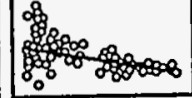 & 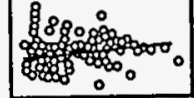 & 2080 & 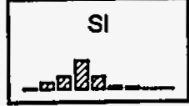 \\
\hline
\end{tabular}


Figure 5.15

Scatter plots of 11 of the most abundant elements versus their products with $1 / T$ (from viscosity table). The element in mol\% is plotted against the product of element $\mathrm{mol} \%$ and $1 / \mathrm{T}$ in the form of a correlation table. Straight lines in each cell are by leastsquares regression.

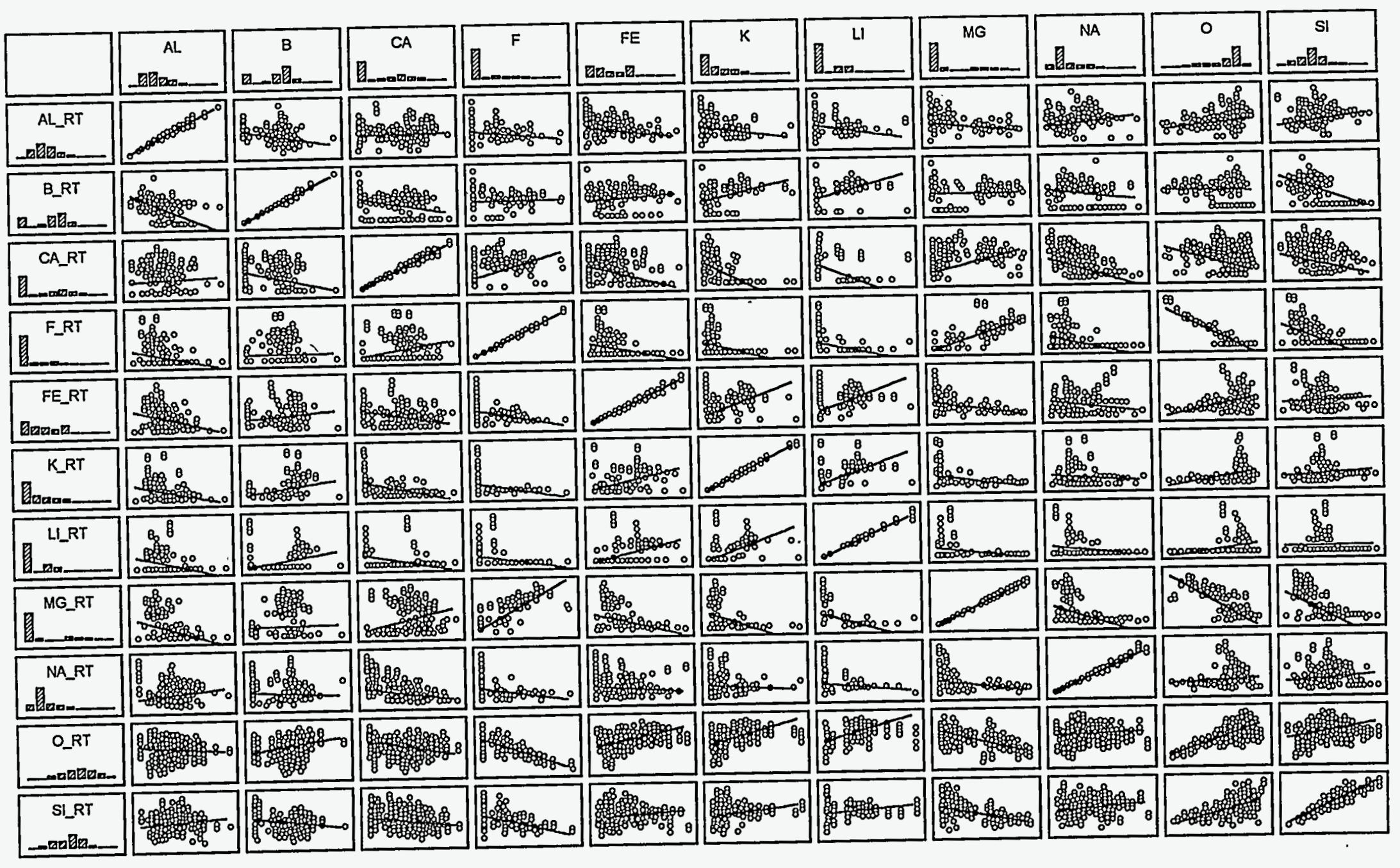

Page 5-53 
Figure 5.16

Standard deviations of 16 of the most abundant elements and their products with $1 / T$ (data from viscosity table).

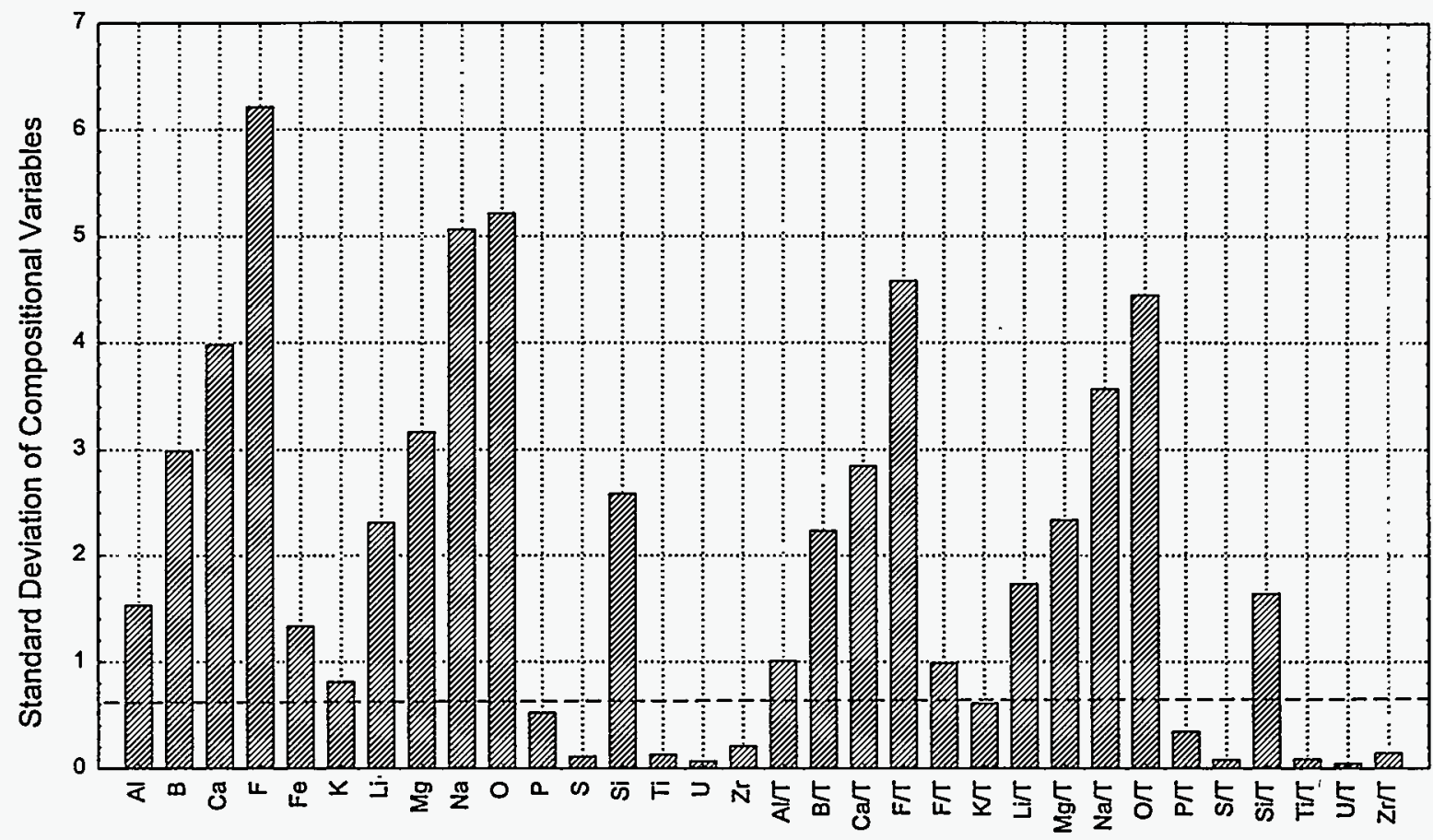

Elements (in mole \% or $1000^{\star}$ mole $\% / K$ )

Figure 5.17

Weight is a function of Euclidean distance between two sample points in multi-dimensional compositional space (with HAN5 at $\mathrm{X}_{0}$ ). The calculated weight in continuous form (filled circles and the curve) is truncated to integers (boxes in the figure) before it is used in weighted least-squares regression.

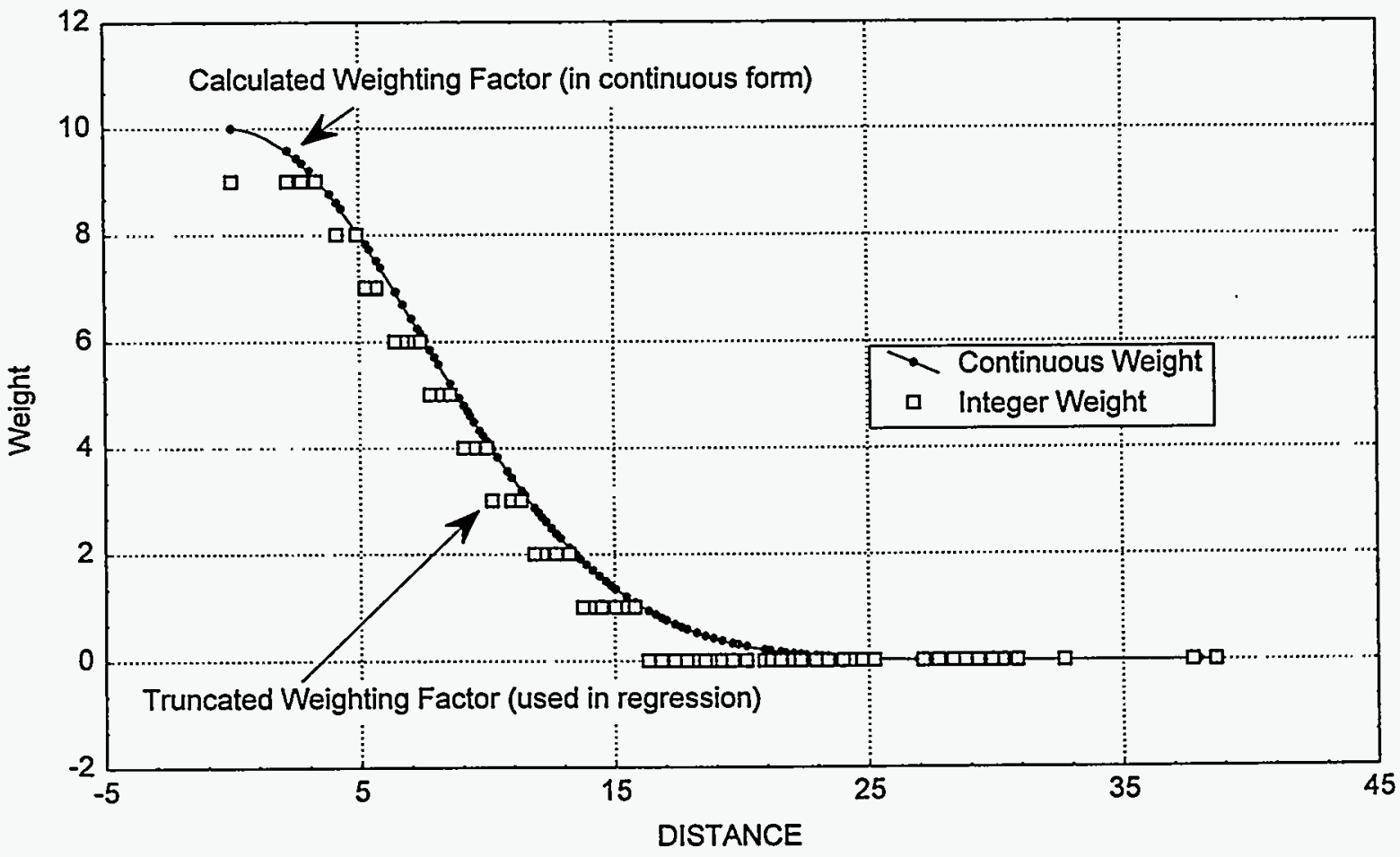

Page 5-54 
Figure 5.18

Frequency distributions of raw conductivity observations along weight axis. a) HAN5 is the center of weight function $\left(X_{0}\right)$, b) WS18 is the center, and c) DIG7 is the center. Note that a large weight (e.g., 8, or 9) indicates a small distance to the center of weight function (i.e.,

HAN5, WS18, or DIG7).

a)

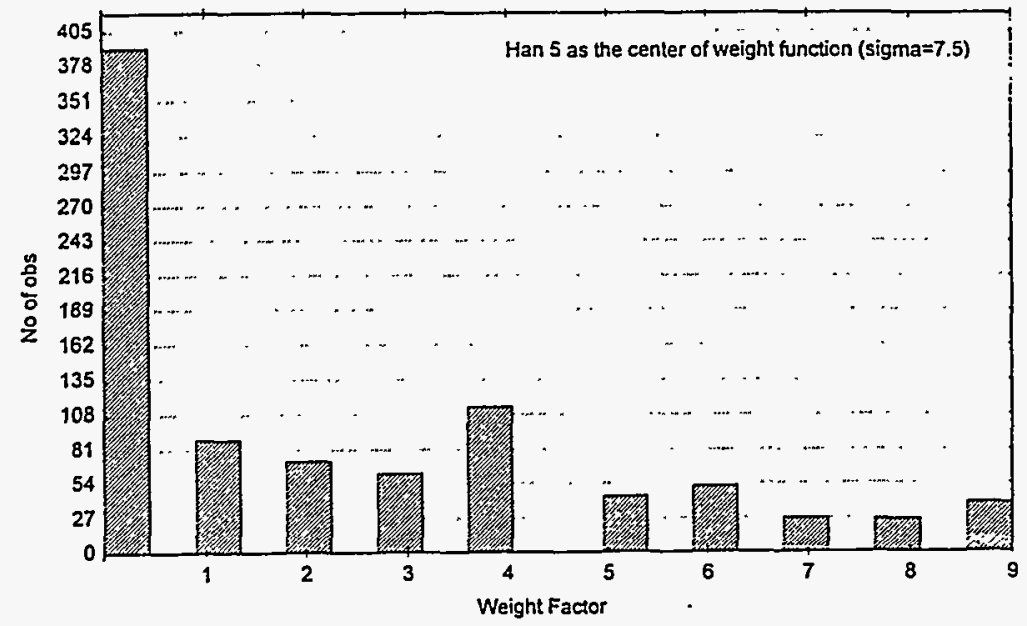

b)

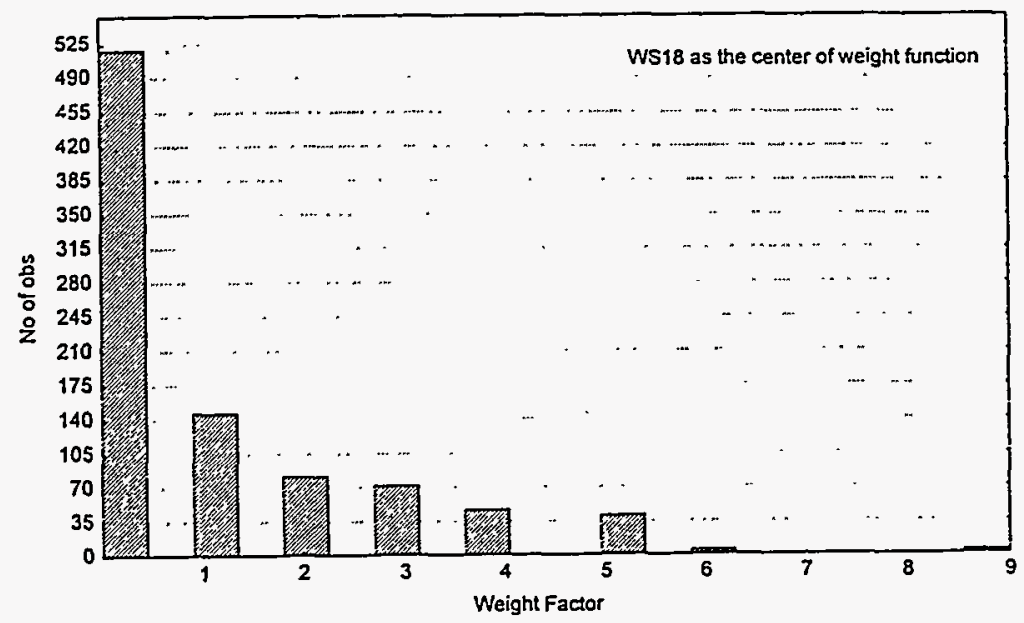

c)

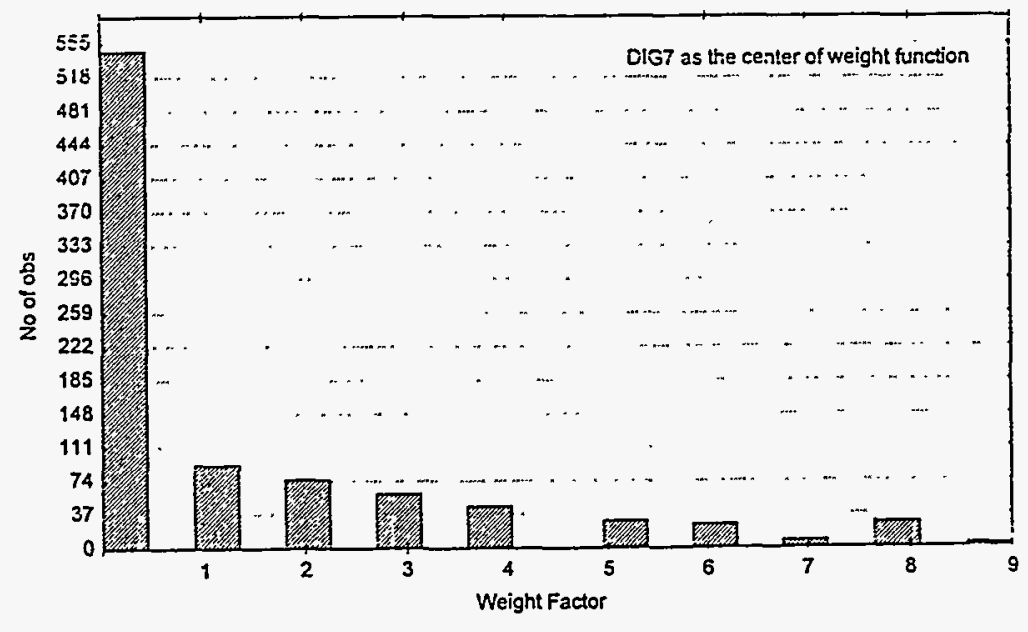

Page 5-55 
Figure 5.19

Frequency distributions of weighted conductivity observations along weight axis. The apparent data points are sums of weights for each original data point in the conductivity table. Proportions of data points with common weights (i.e., at the same Euclidean distance to the reference glass composition) would influence the final results of least-squares regression. a) HAN5 is the center of weight function $\left.\left(X_{0}\right), b\right)$ WS18 is the center, and c) DIG7 is the center. Note that a large weight (e.g., 8, or 9) indicates a small Euclidean distance to the center of weight function (i.e., HAN5, WS18 or DIG7).

a)

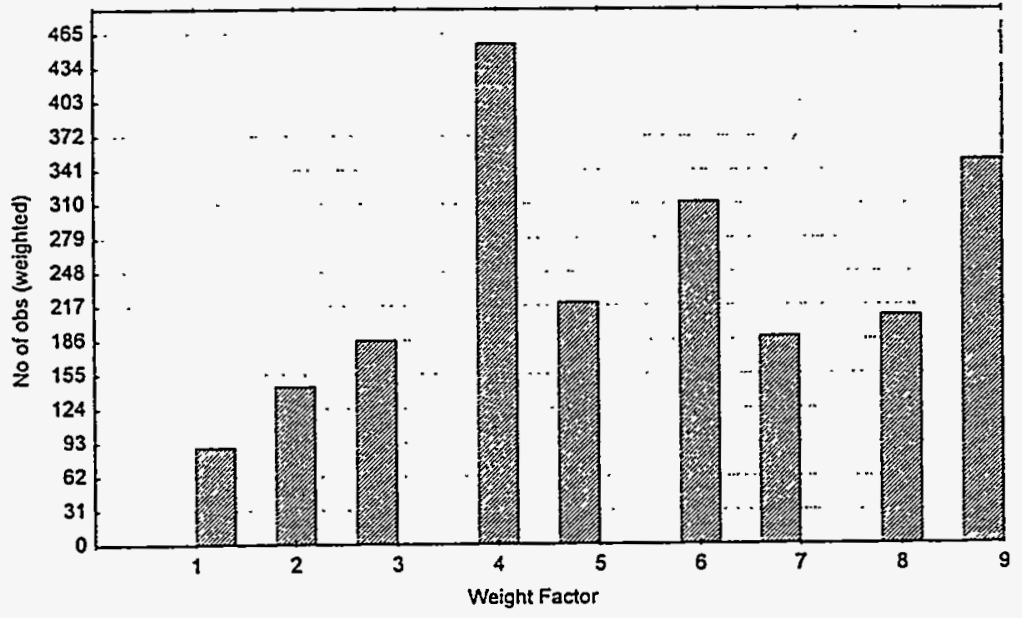

b)

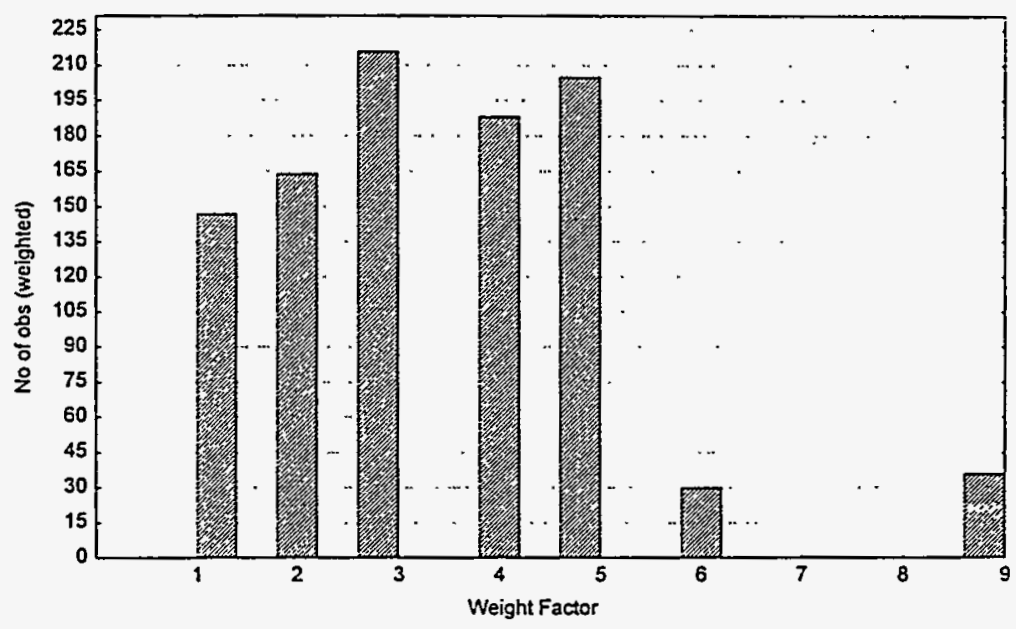

c)

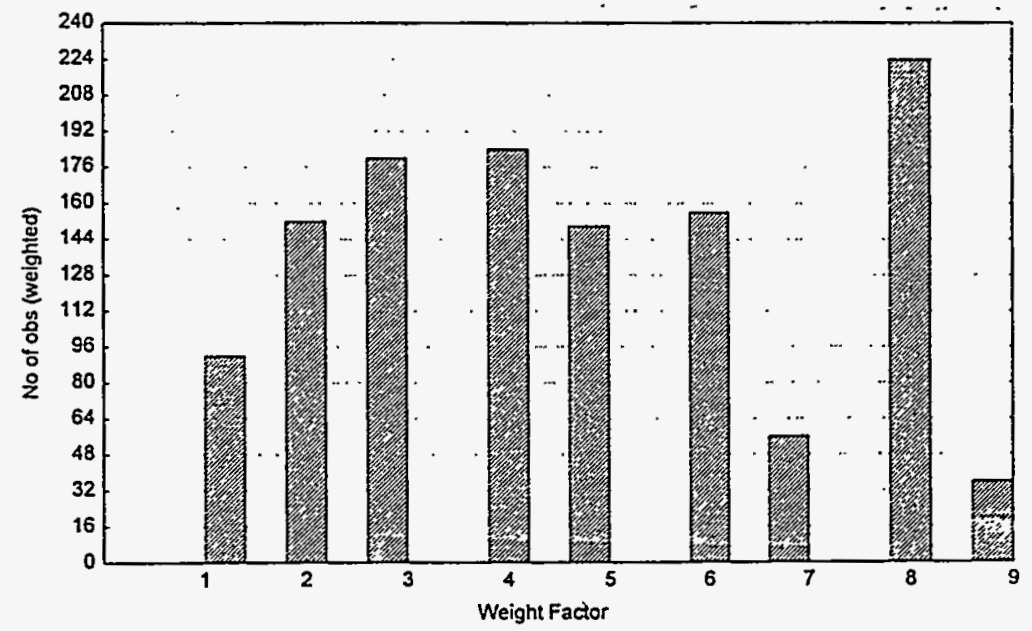




\section{Figure 5.20}

Conductivities predicted by models of the weighted and unweighted least-squares regressions. Measurement temperature is from $1150^{\circ} \mathrm{C}$ to $1300^{\circ} \mathrm{C}$.

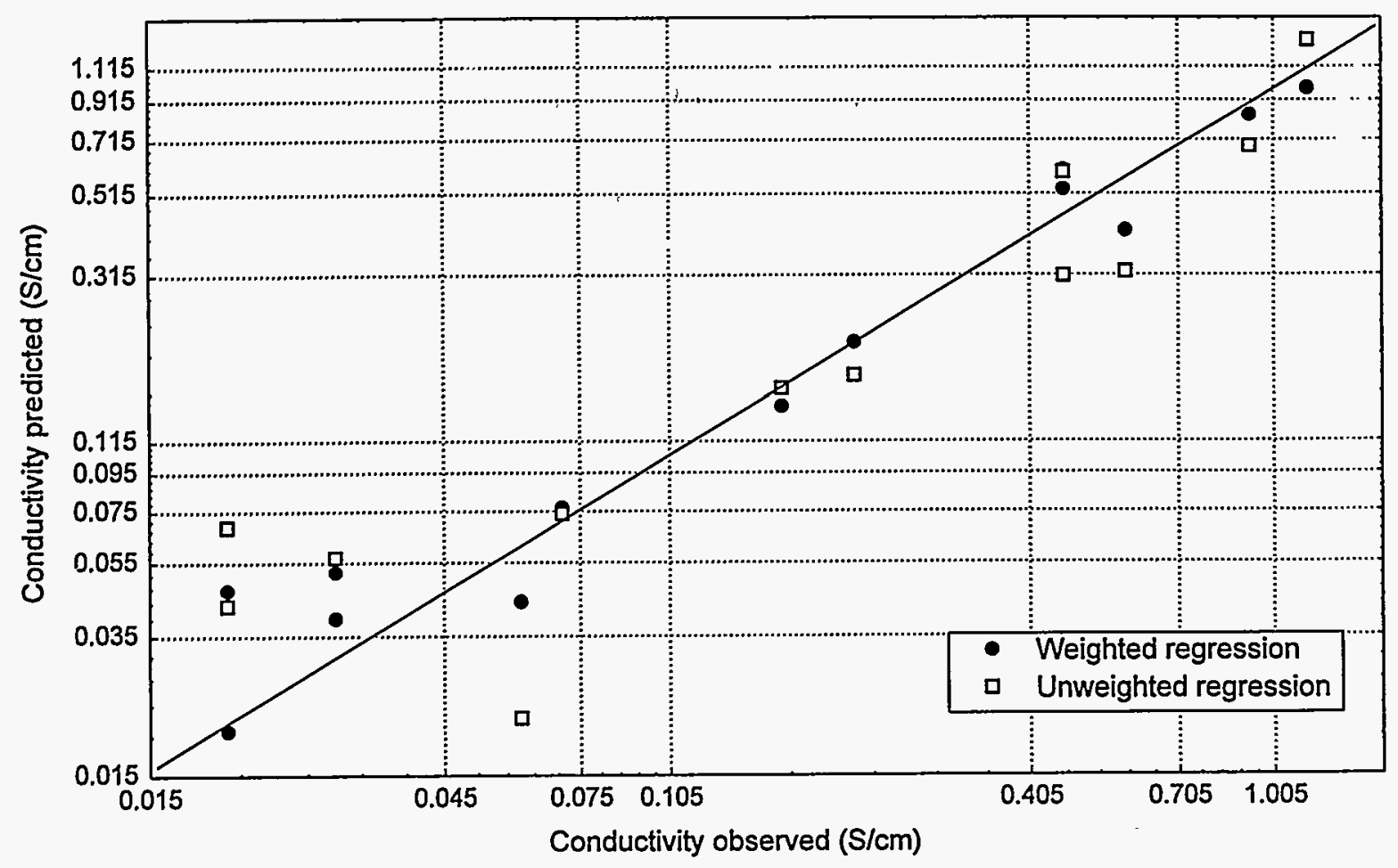

Figure 5.21

Normalized residuals (residual/observed) of conductivities predicted by models of weighted and unweighted least-squares regression. Measurement temperature is from $1150^{\circ} \mathrm{C}$ to

$1300^{\circ} \mathrm{C}$.

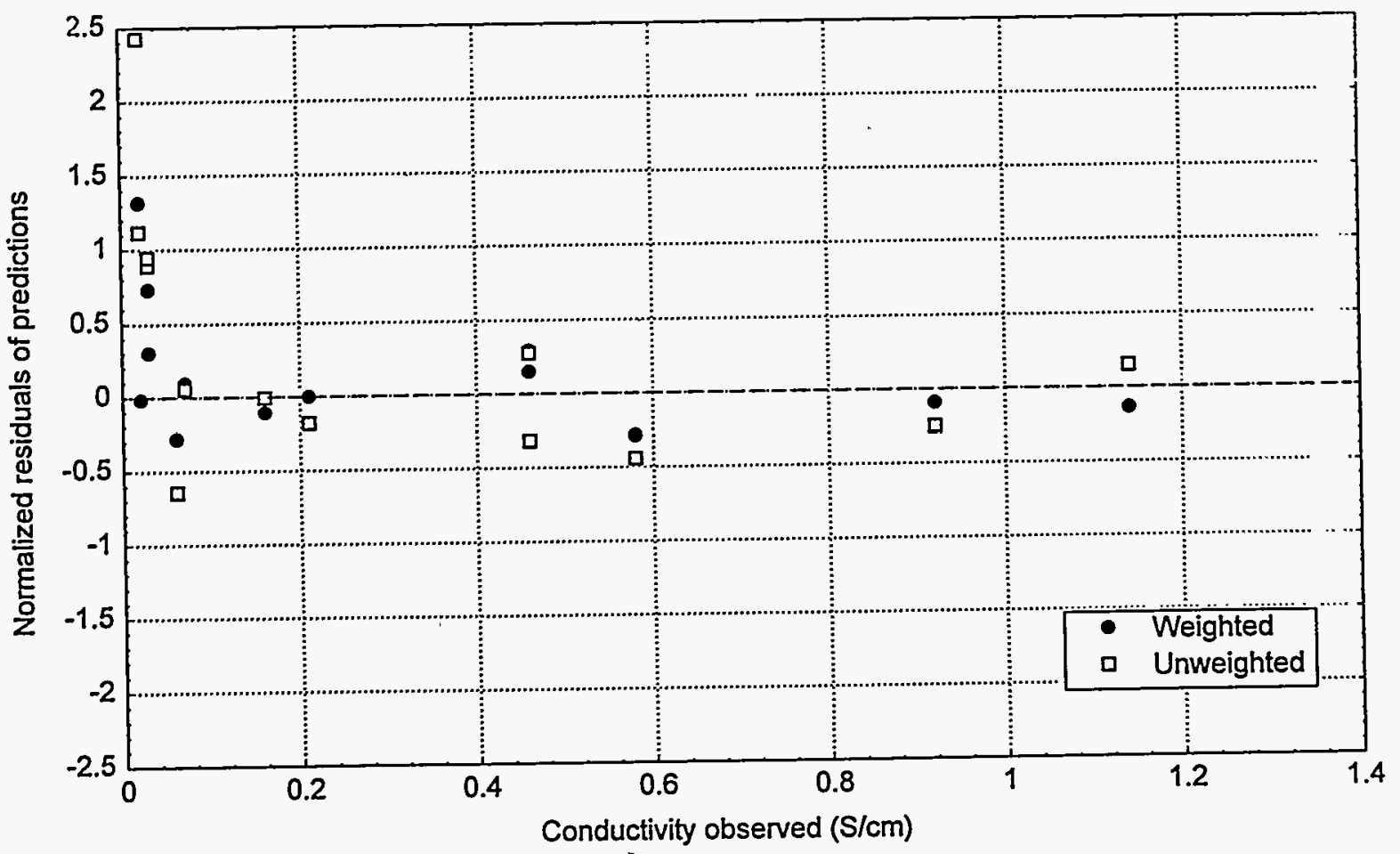


Standard deviations of 13 most abundant oxides (wt\%) of Hanford Glasses.

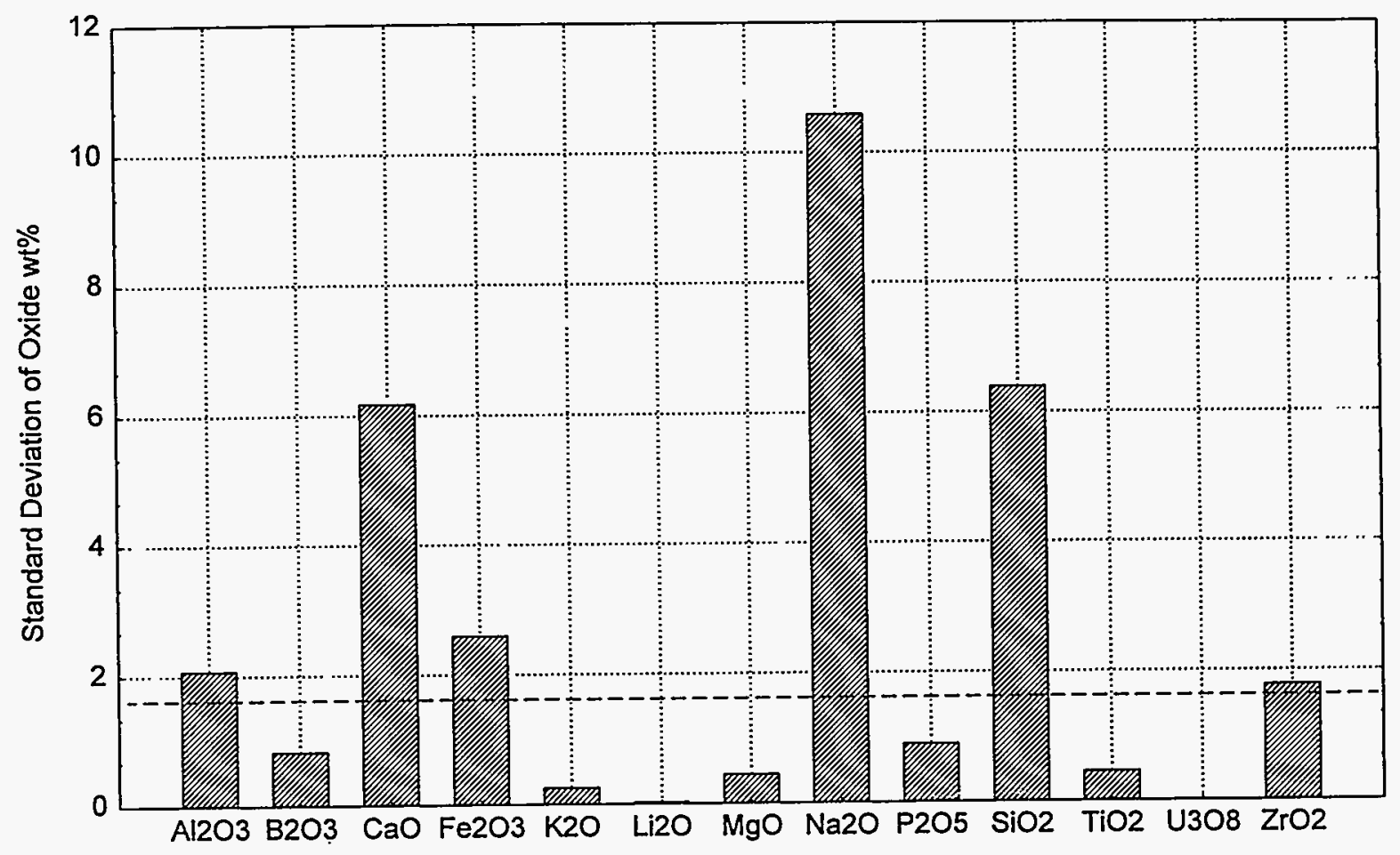

Figure 5.23

Goodness of fit of conductivity model of Hanford glasses.

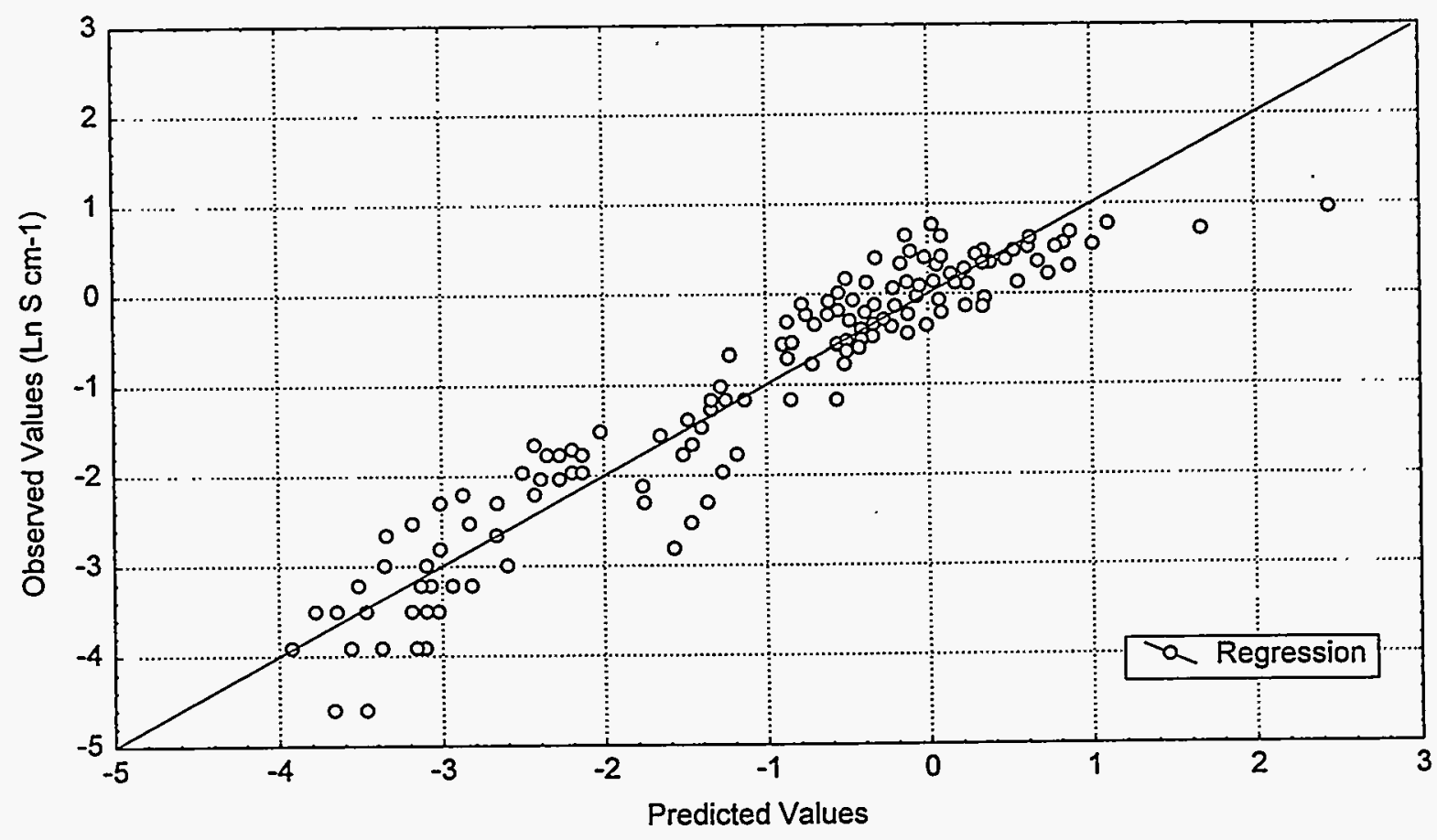




\section{Figure 5.24}

Predicted conductivity is a function of incremental changes of glass components (HAN7 glass as reference). Isothermal conductivities are calculated at $1200^{\circ} \mathrm{C}$ using model for

Hanford glasses.

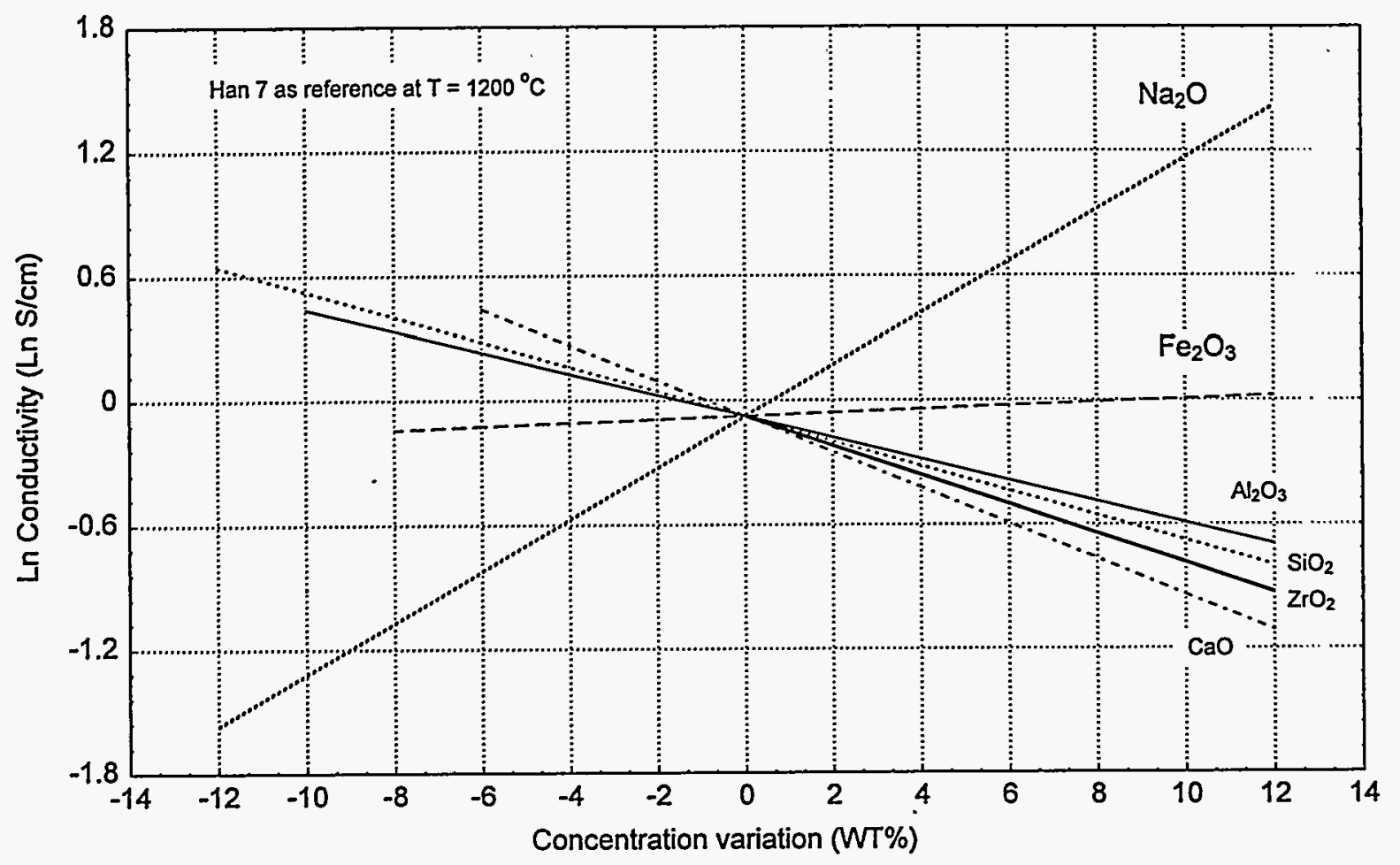

Figure 5.25

Conductivity change with incremental variations in $\mathrm{Na}_{2} \mathrm{O}$ or $\mathrm{CaO}$ wt\% (HAN7 as reference glass). Isothermal conductivity lines $(\mathrm{Ln} \mathrm{S} / \mathrm{cm})$ are calculated at $1200^{\circ} \mathrm{C}$ and $1500^{\circ} \mathrm{C}$ using conductivity model for Hanford glasses.

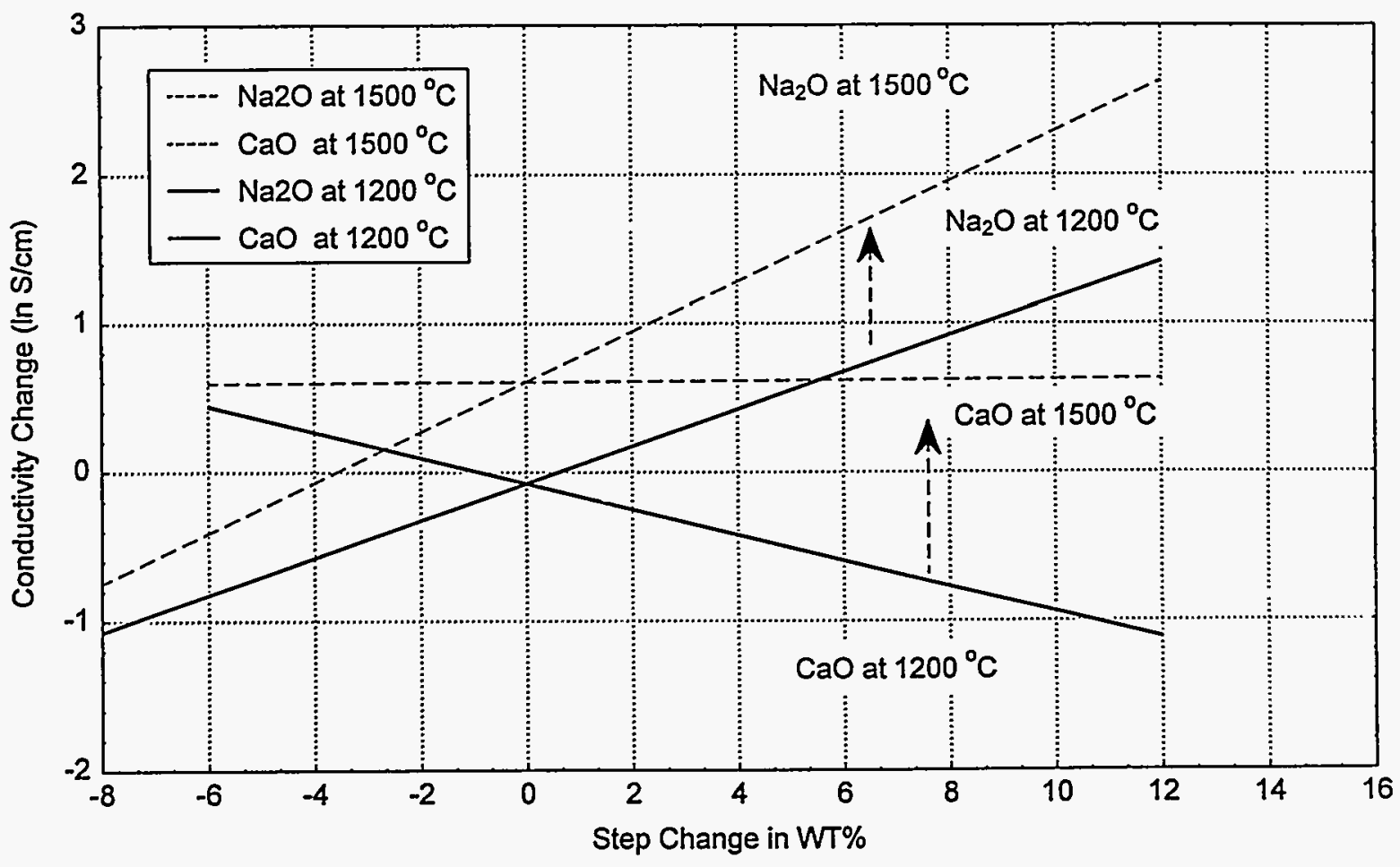


Figure 5.26

Viscosities predicted by models of weighted and unweighted least-squares regression.

Measurement temperature is from $1150^{\circ} \mathrm{C}$ to $1300^{\circ} \mathrm{C}$.

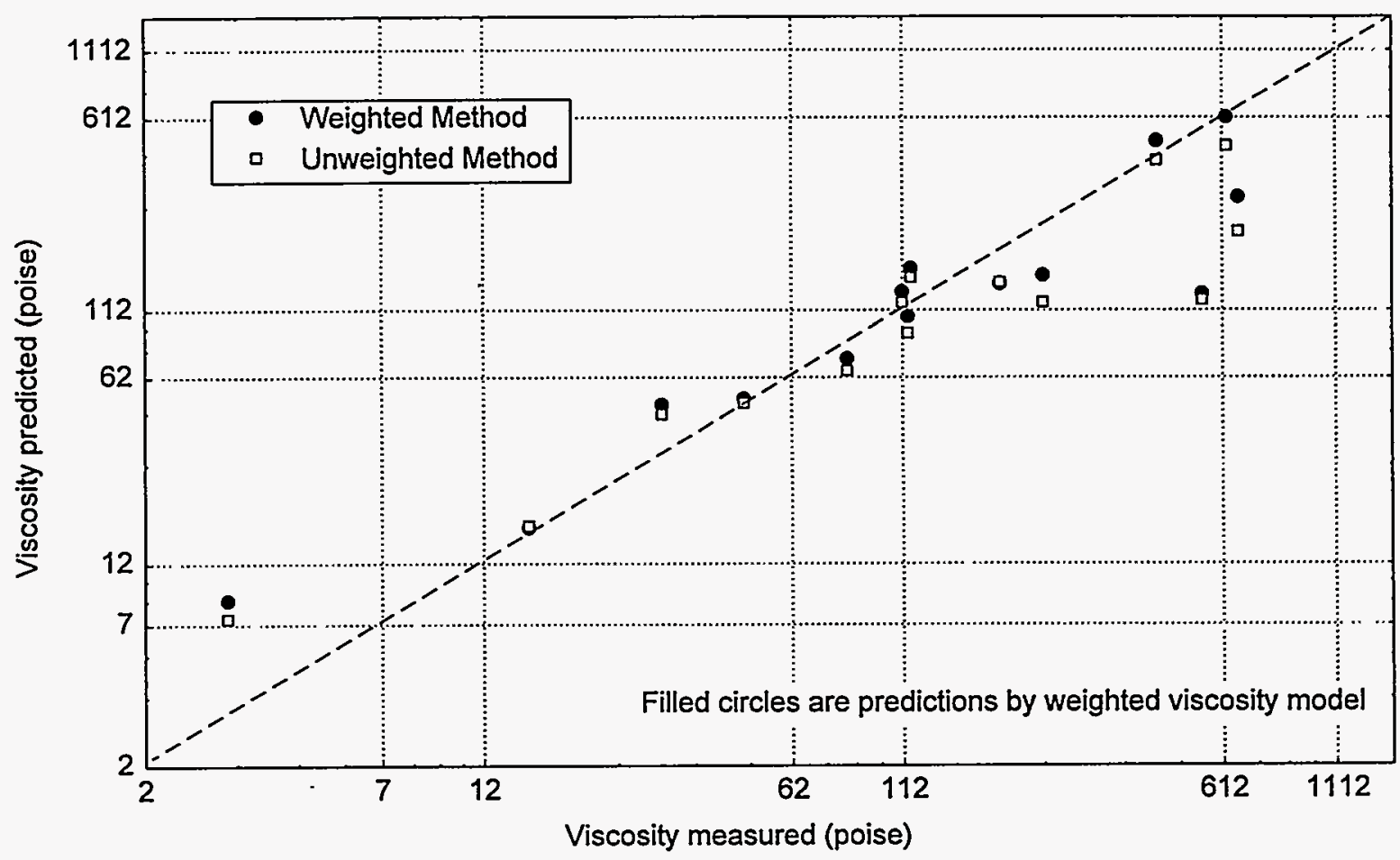

Figure 5.27

Goodness of fit of viscosity model by unweighted least-squares regression. Note that a few points beyond three sigma error were not shown in the plot although they have been included in the regression.

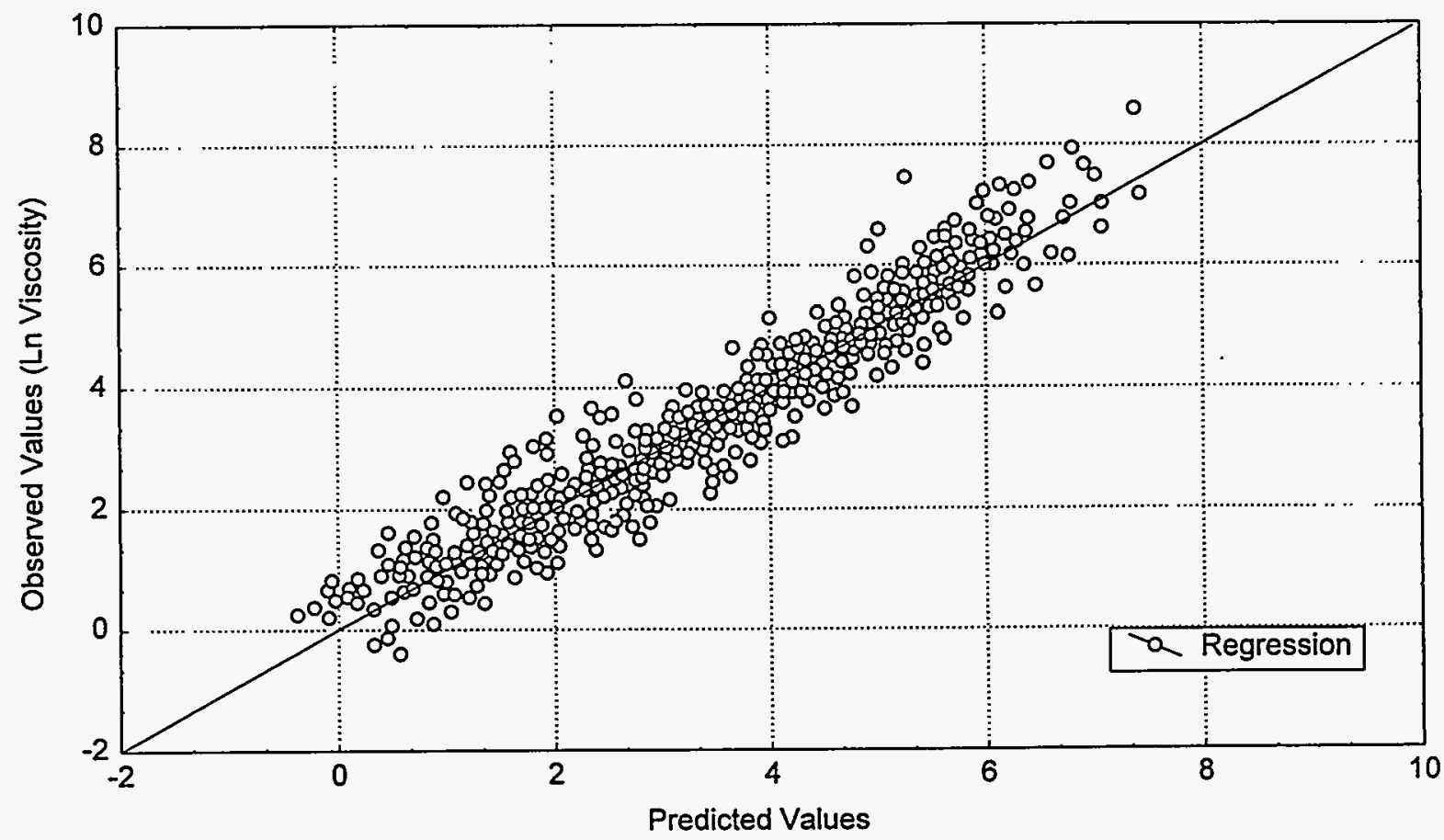


Figure 5.28

Normalized residuals (residual/observed) of viscosity models of weighted and unweighted least-squares regression. Measurement temperature is from $1150^{\circ} \mathrm{C}$ to $1300^{\circ} \mathrm{C}$.

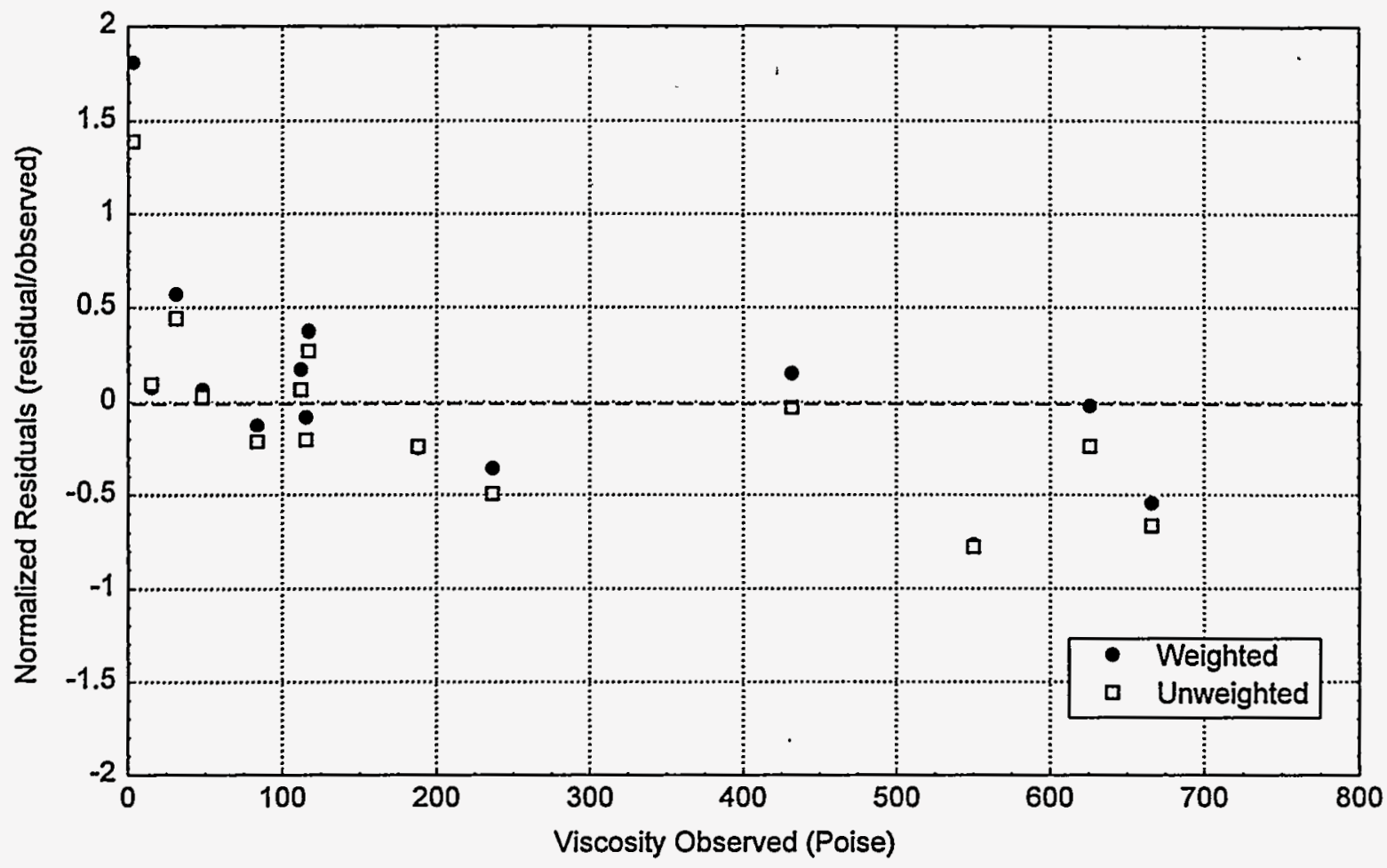

Figure 5.29

Goodness of fit of viscosity model of Hanford glasses by unweighted least-squares regresșion.

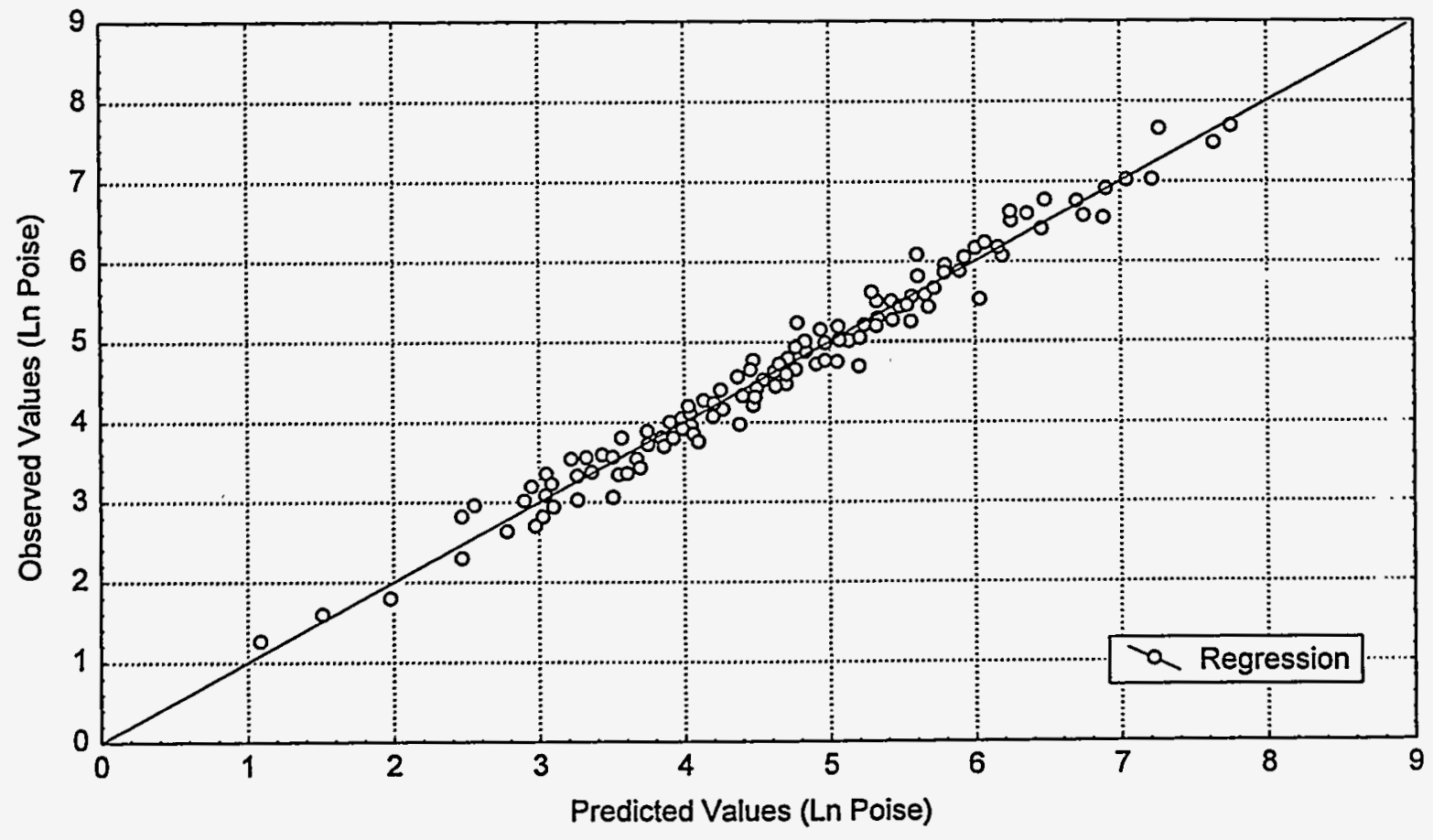


Figure 5.30

Viscosity change with incremental variations in glass composition (adding/removing one glass component in wt\%). Isothermal conductivities are calculated at $1212^{\circ} \mathrm{C}$ by viscosity model for Hanford glasses.

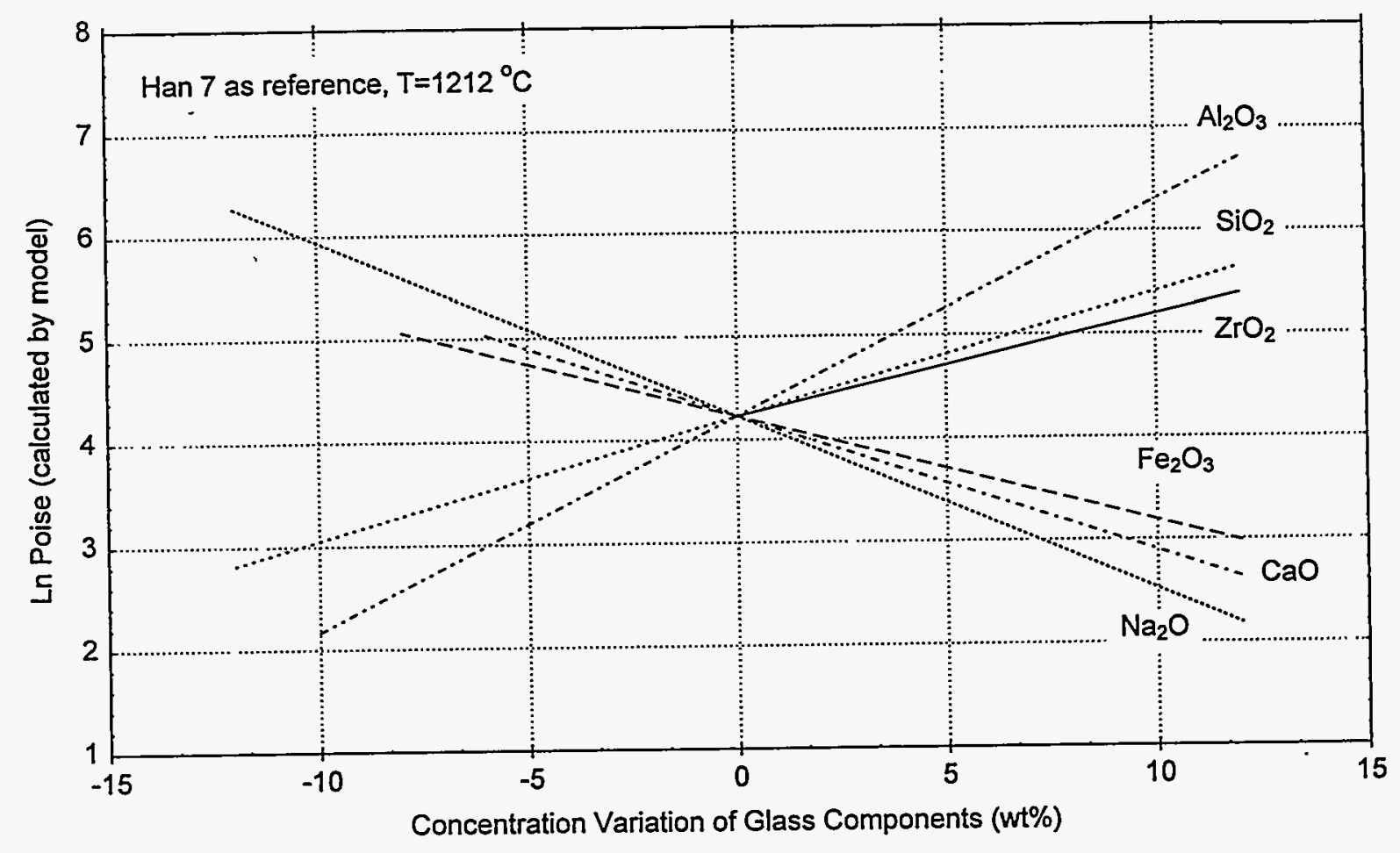

Figure 5.31

Viscosity change with incremental variations in $\mathrm{Na}_{2} \mathrm{O}$ or $\mathrm{CaO}$ wt\% (HAN7 as reference glass). Isothermal viscosity lines ( $\mathrm{Ln}$ Poise) are calculated at $1212^{\circ} \mathrm{C}$ and $1400^{\circ} \mathrm{C}$ by viscosity model for Hanford glasses.

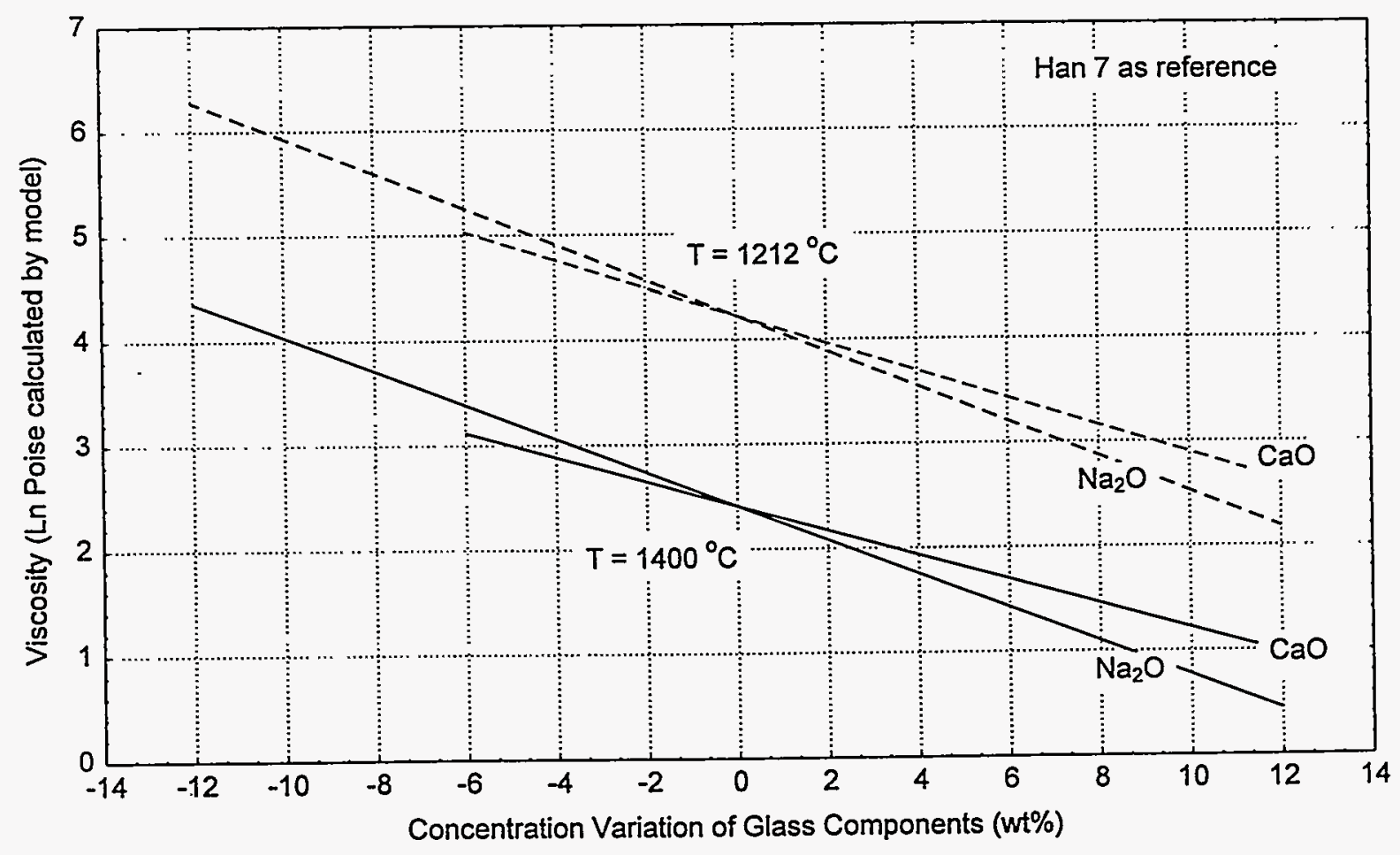


Figure 5.32a

Goodness of fits for 7-Day PCT models for Si.

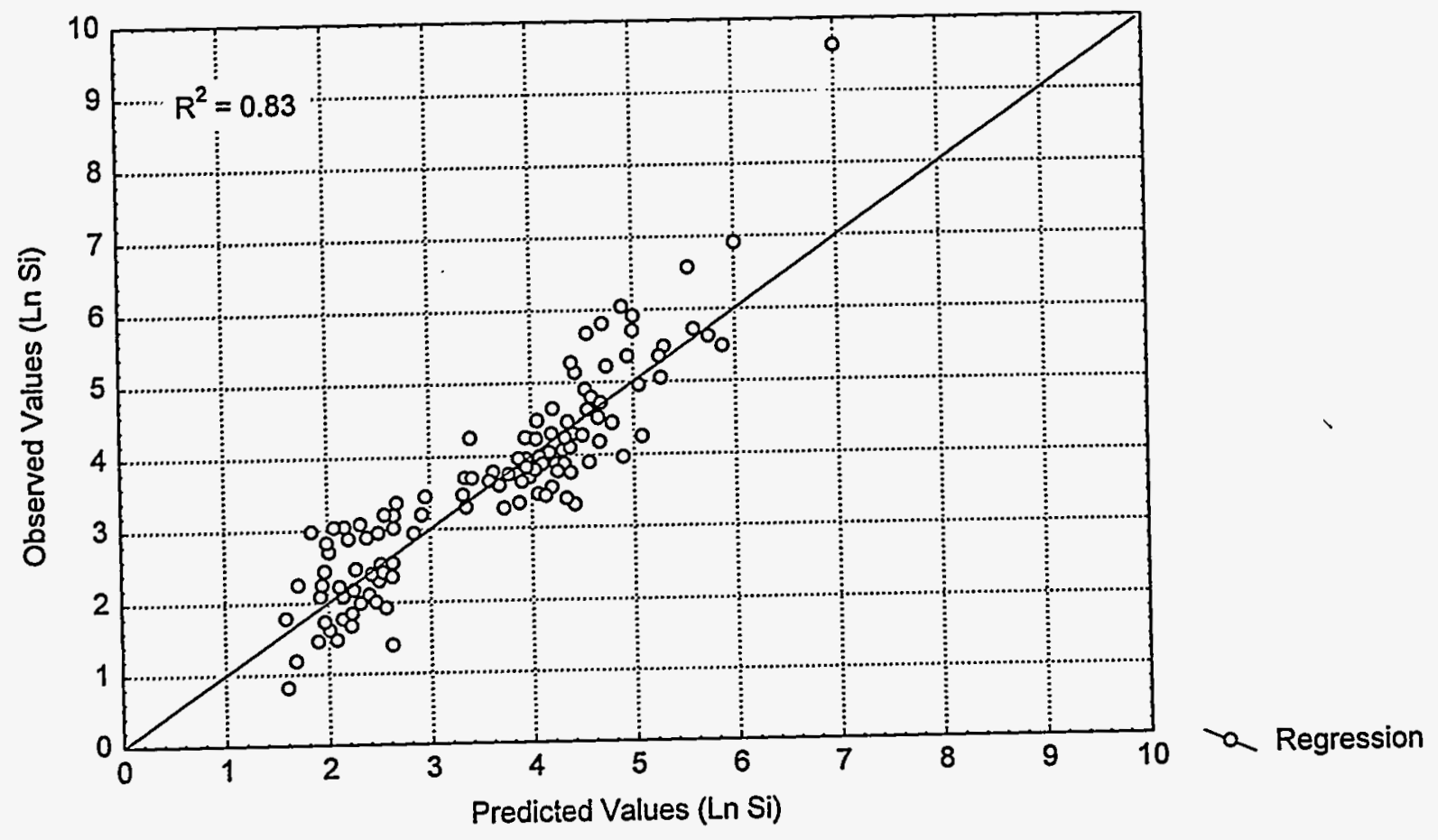

Figure 5.32b

Goodness of fits for 7-Day PCT models for B.

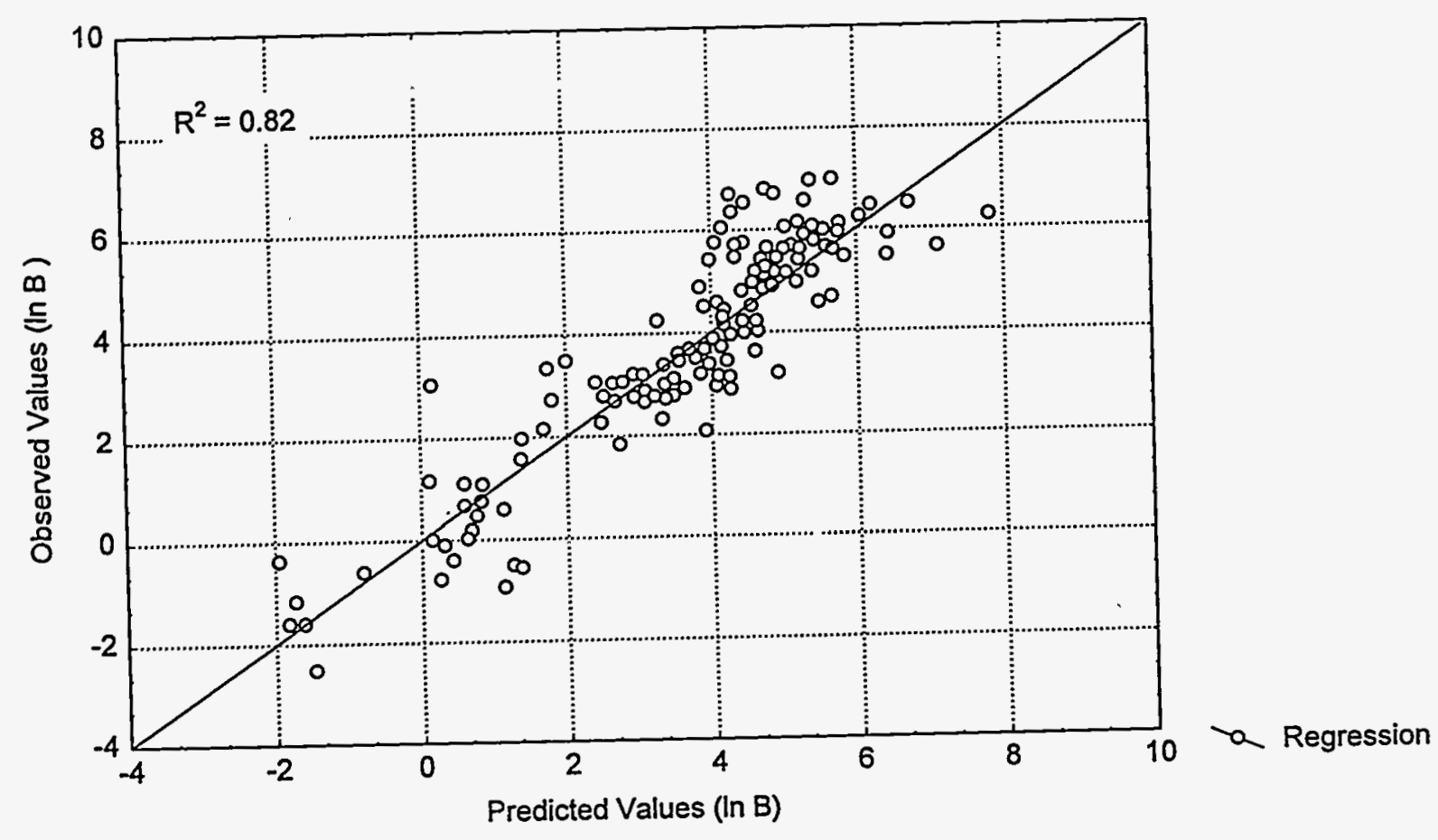


Figure 5.32c

Goodness of fits for 7-Day PCT models for Na.

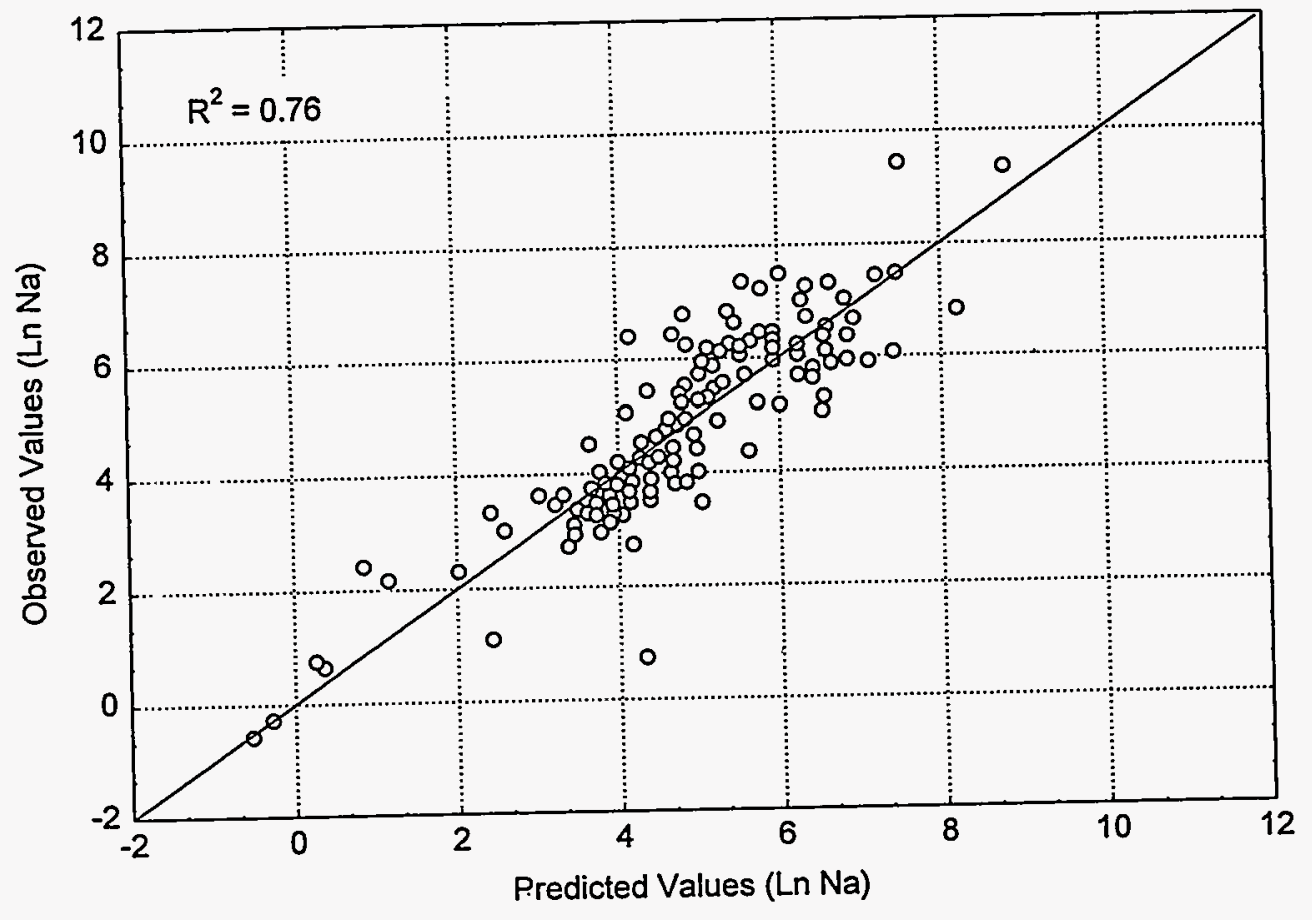

-a Regression

Figure 5.32d

Goodness of fits for 7-Day PCT models for $\mathbf{L}$.

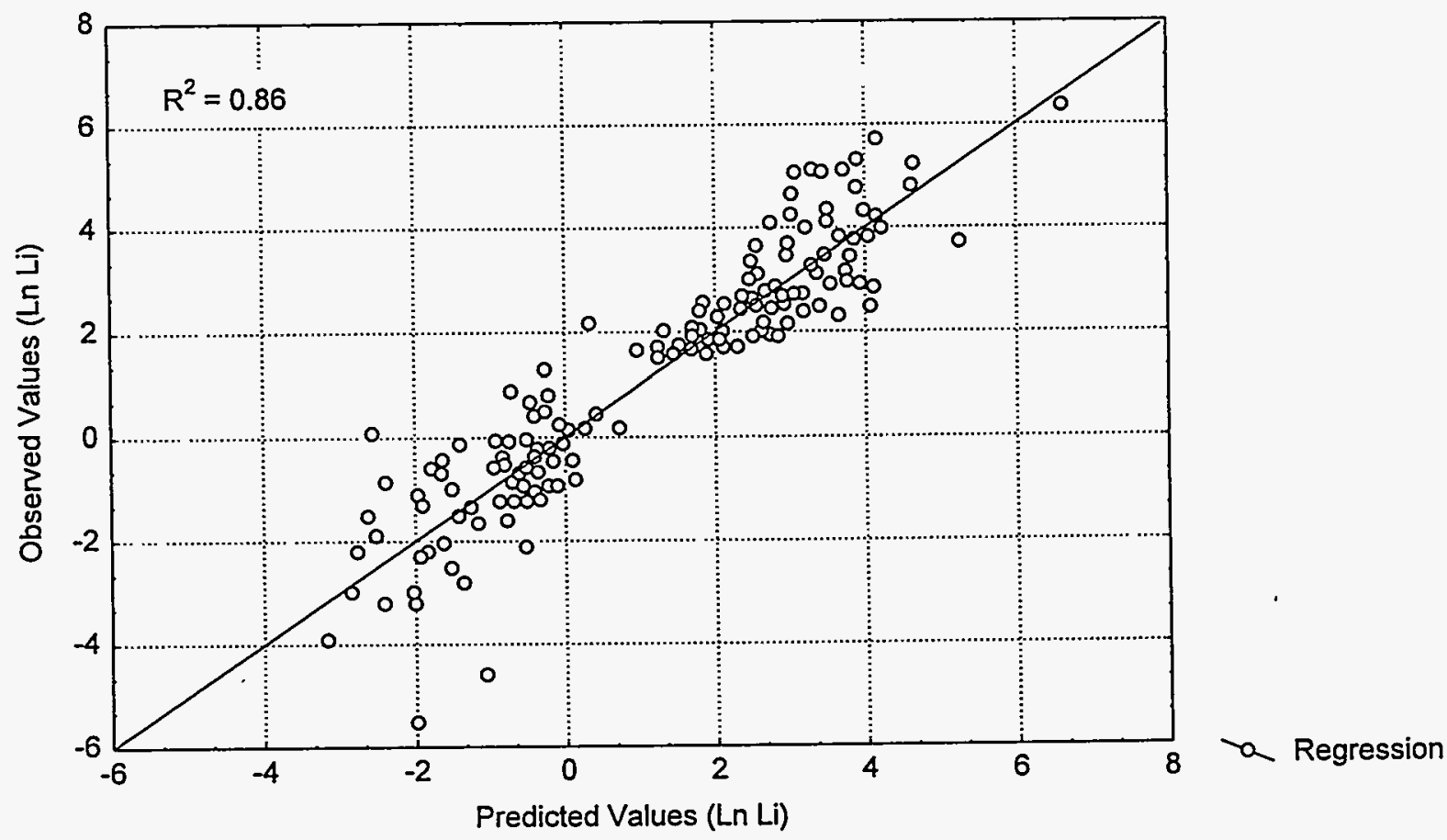




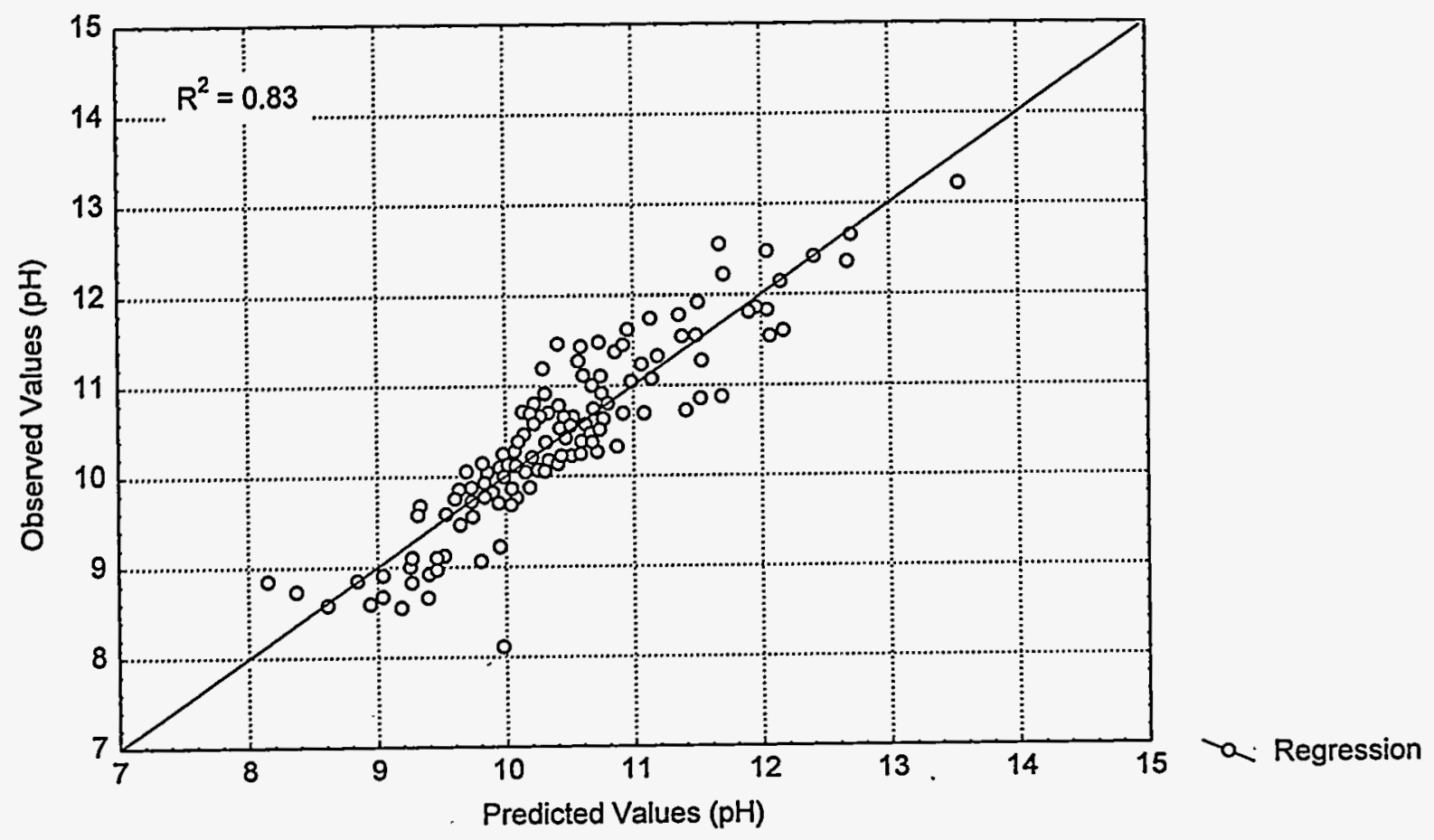


Figure 5.33a

Glass component effects on 7-Day PCT values. Predicted 7-Day PCT values of Si plotted versus incremental changes in glass components (Oxide wt\%).

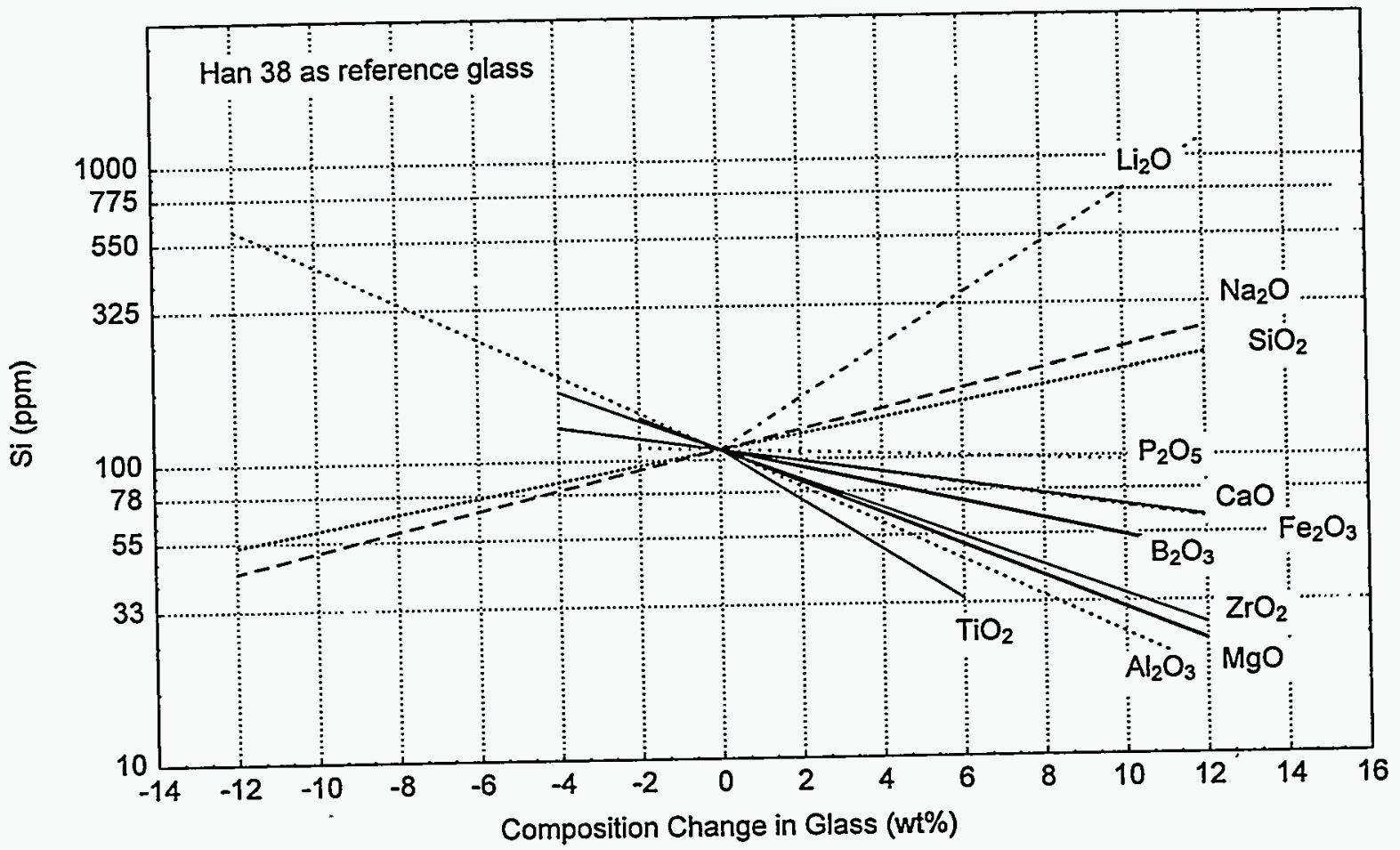

Figure 5.33b

Glass component effects on 7-Day PCT values. Predicted 7-Day PCT values of B plotted versus incremental changes in glass components (Oxide wt\%).

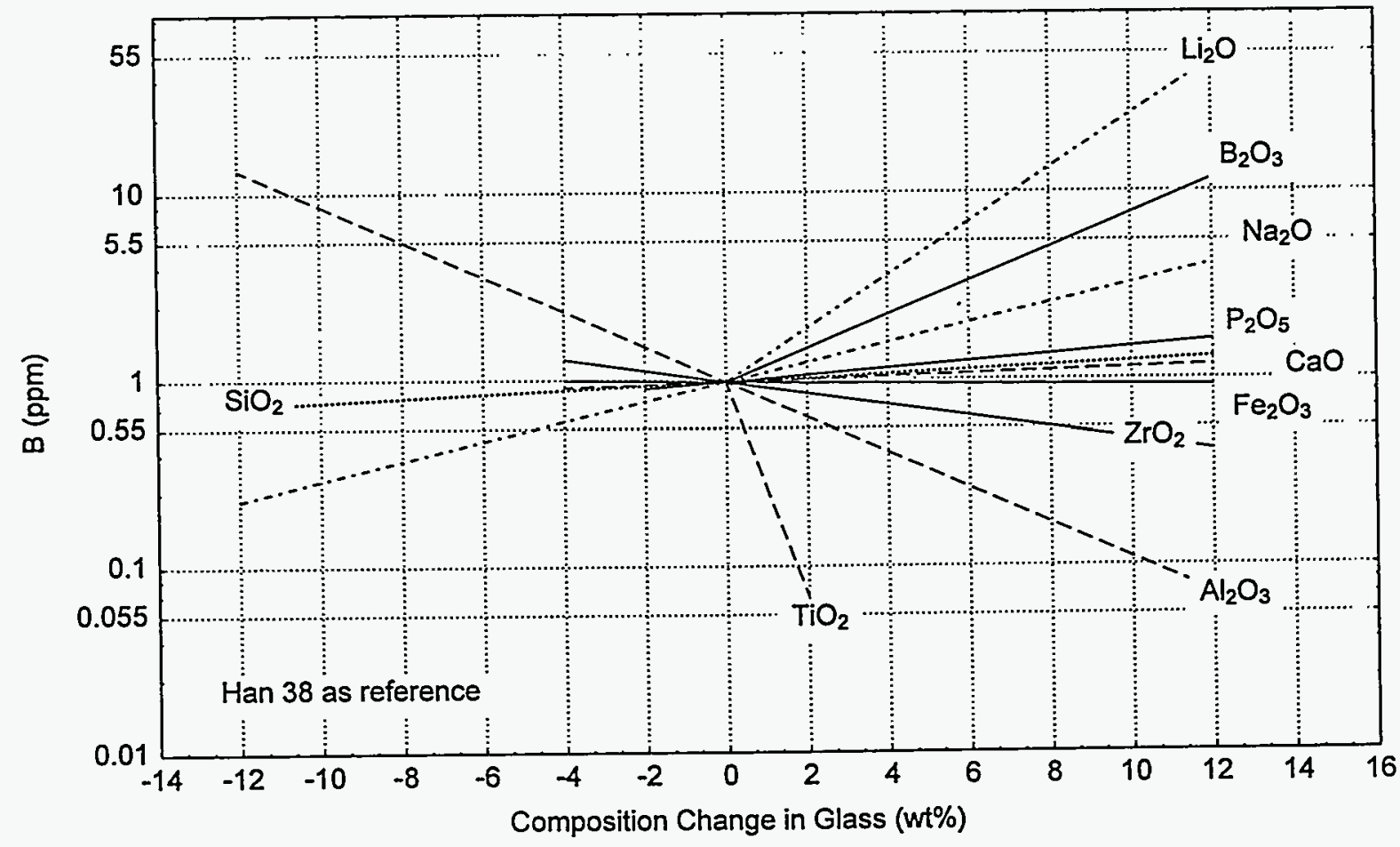


Figure 5.33c

Glass component effects on 7-Day PCT values. Predicted 7-Day PCT values of Na plotted versus incremental changes in glass components (Oxide wt\%).

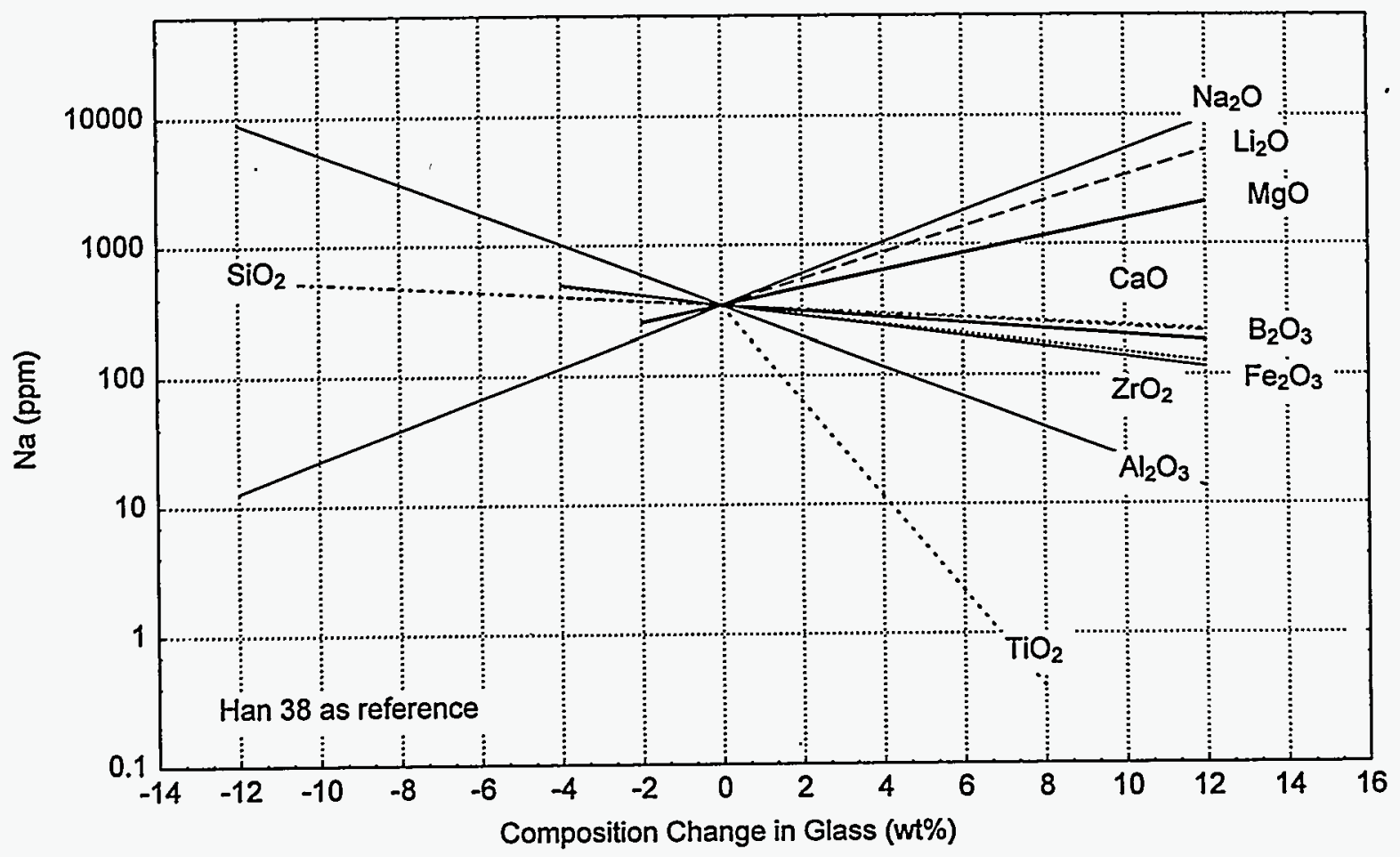

Figure 5.33d

Glass component effects on 7-Day PCT values. Predicted 7-Day PCT values of pH plotted versus incremental changes in glass components (Oxide wt\%).

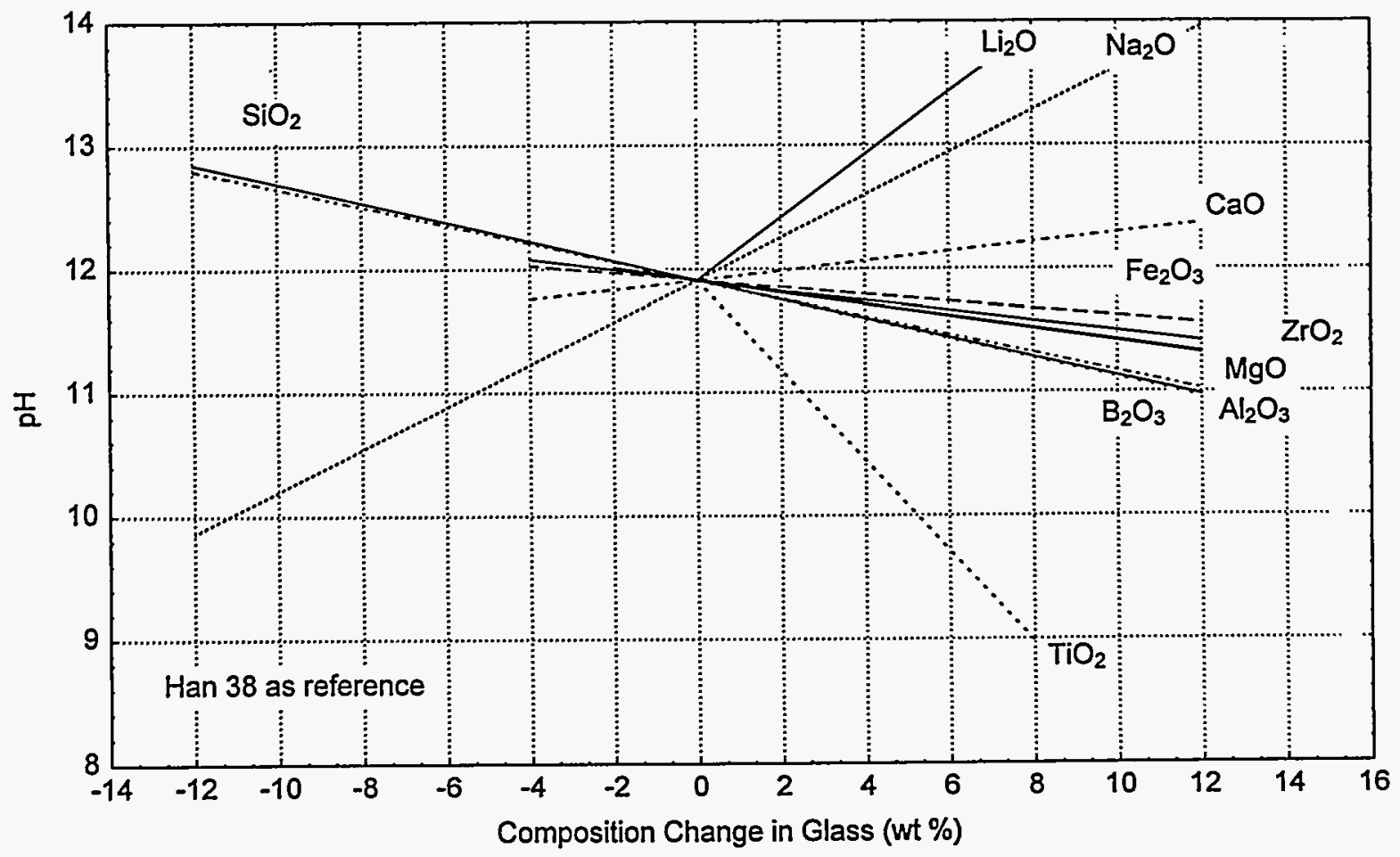


Figure 5.34

Normalized residuals (residual/observed) of 7-Day PCT models for $\mathrm{Si}, \mathrm{B}, \mathrm{Na}, \mathrm{Li}$, and $\mathrm{pH}$.

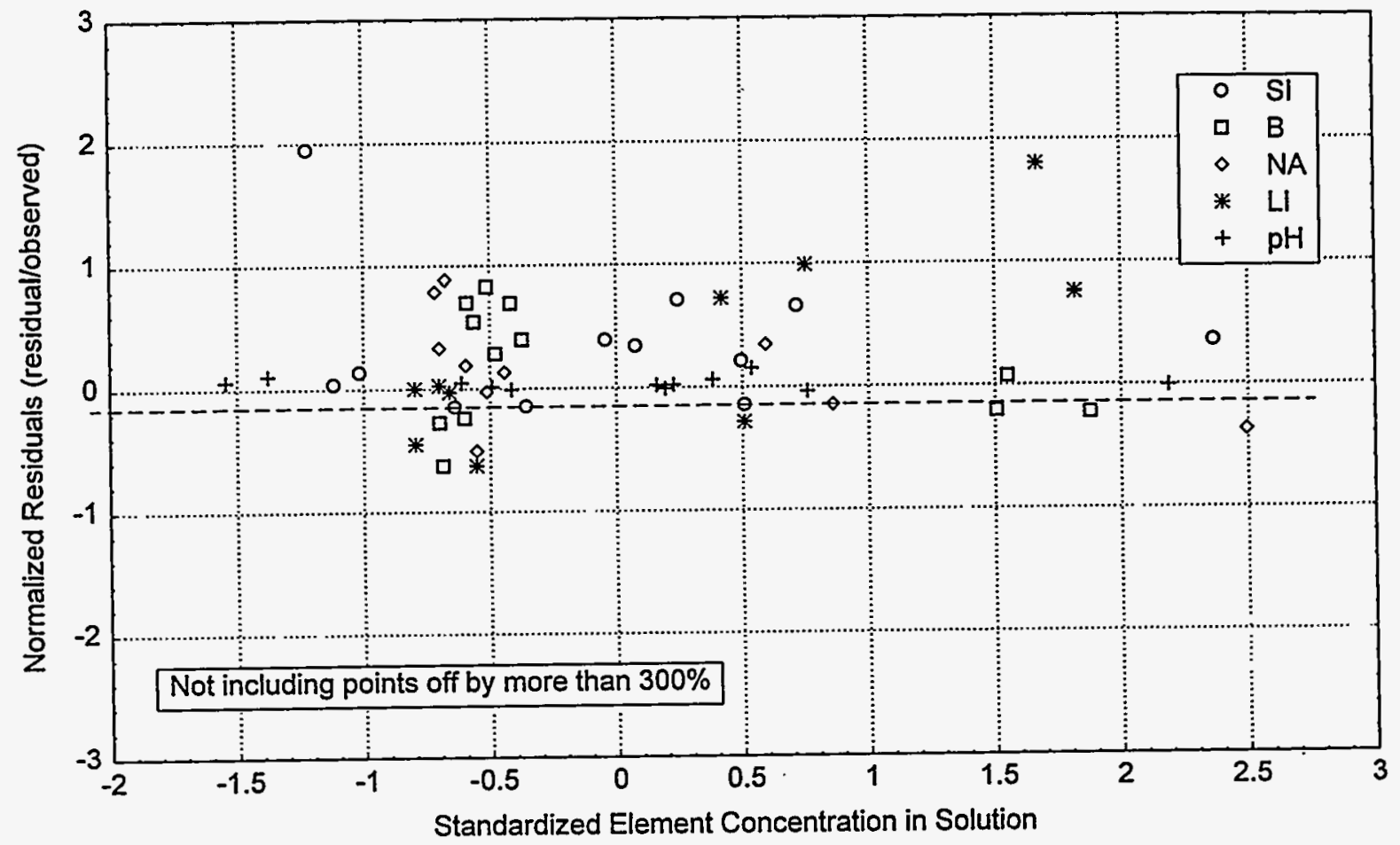


Figure 5.35a

Correlation between Gibbs free energy of hydration and the normalized release of Si from 7-day PCT.

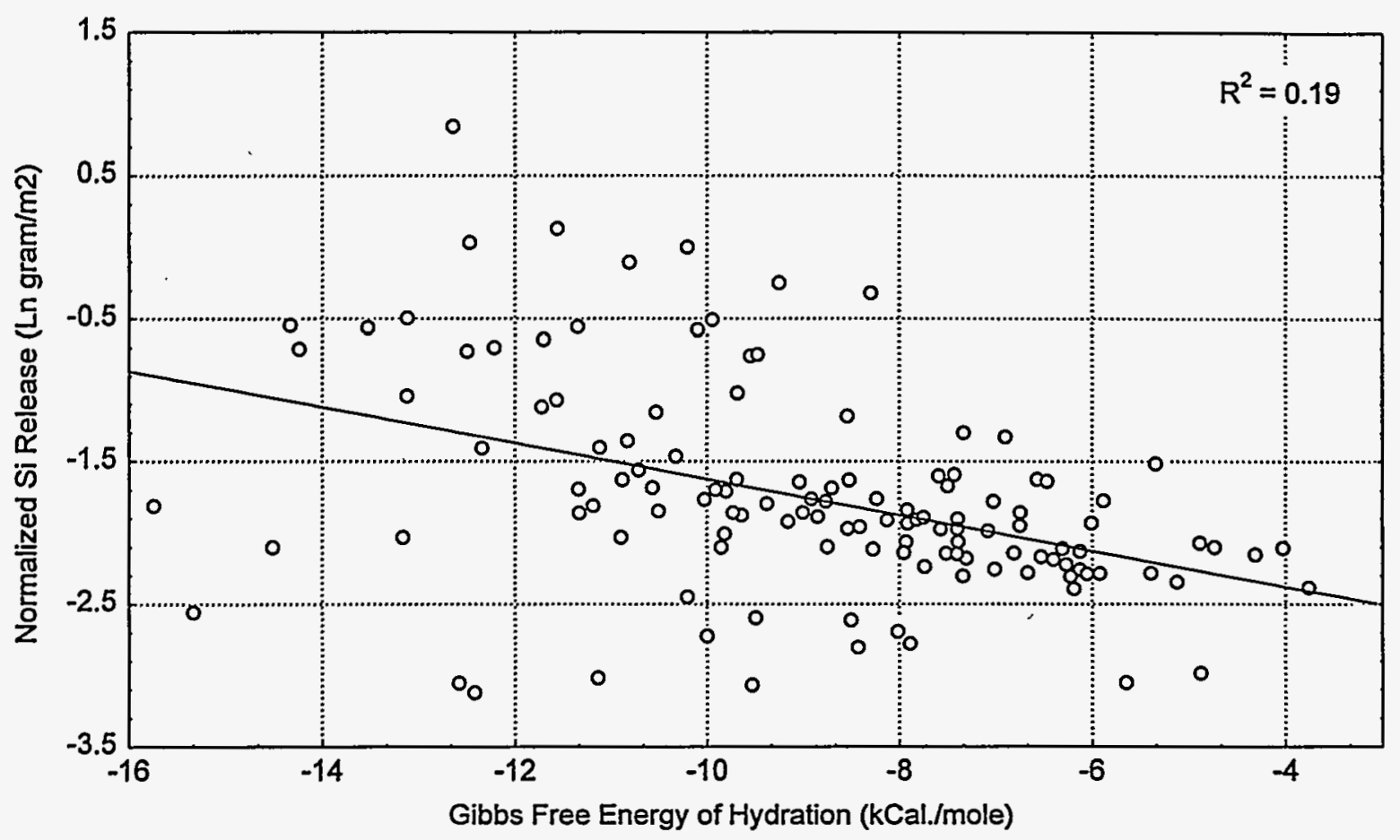

Figure 5.35b

Correlation between Gibbs free energy of hydration and the normalized release of B from 7-day PCT.

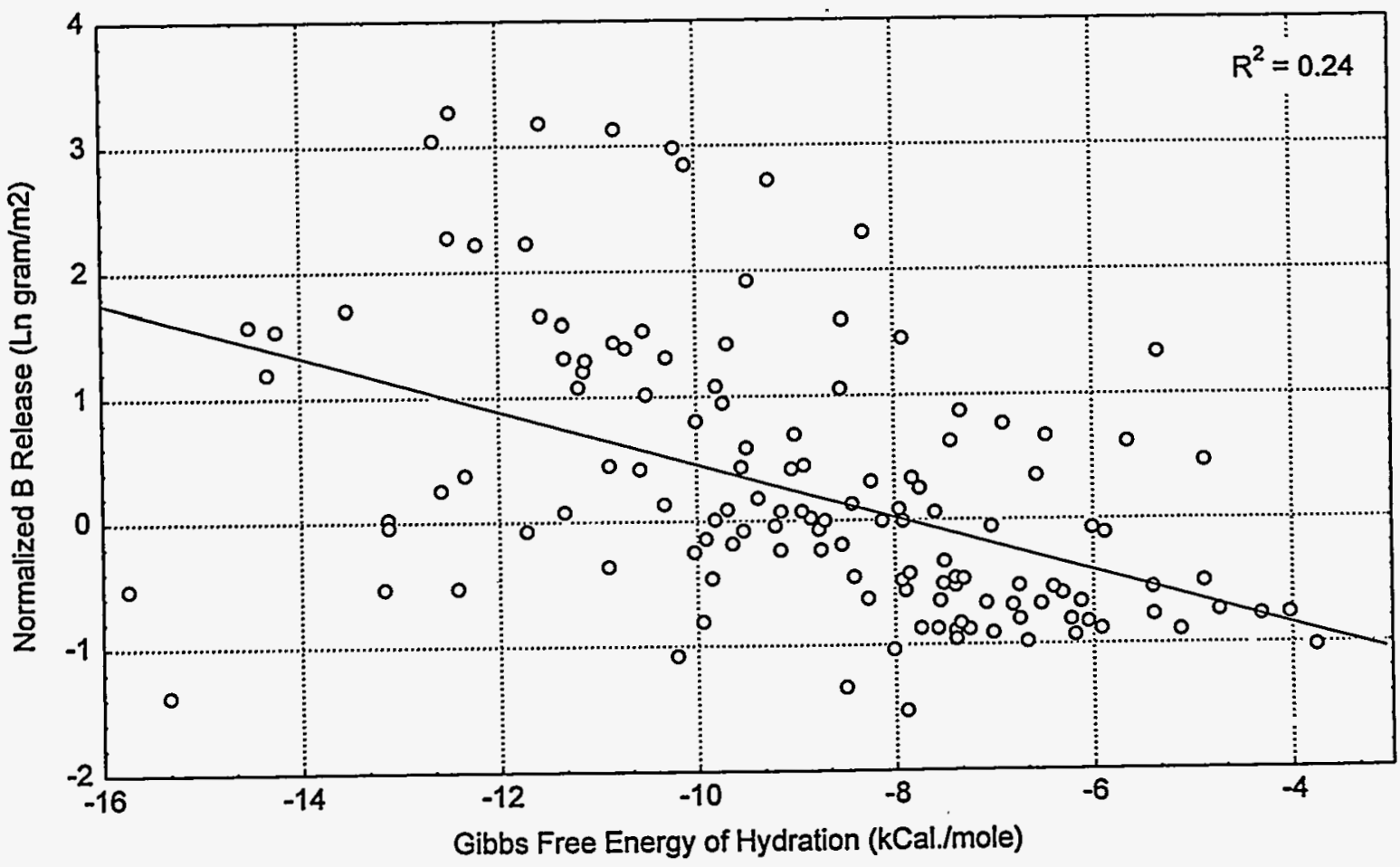


Figure 5.35c

Correlation between Gibbs free energy of hydration and the normalized release of Na from 7-day PCT.

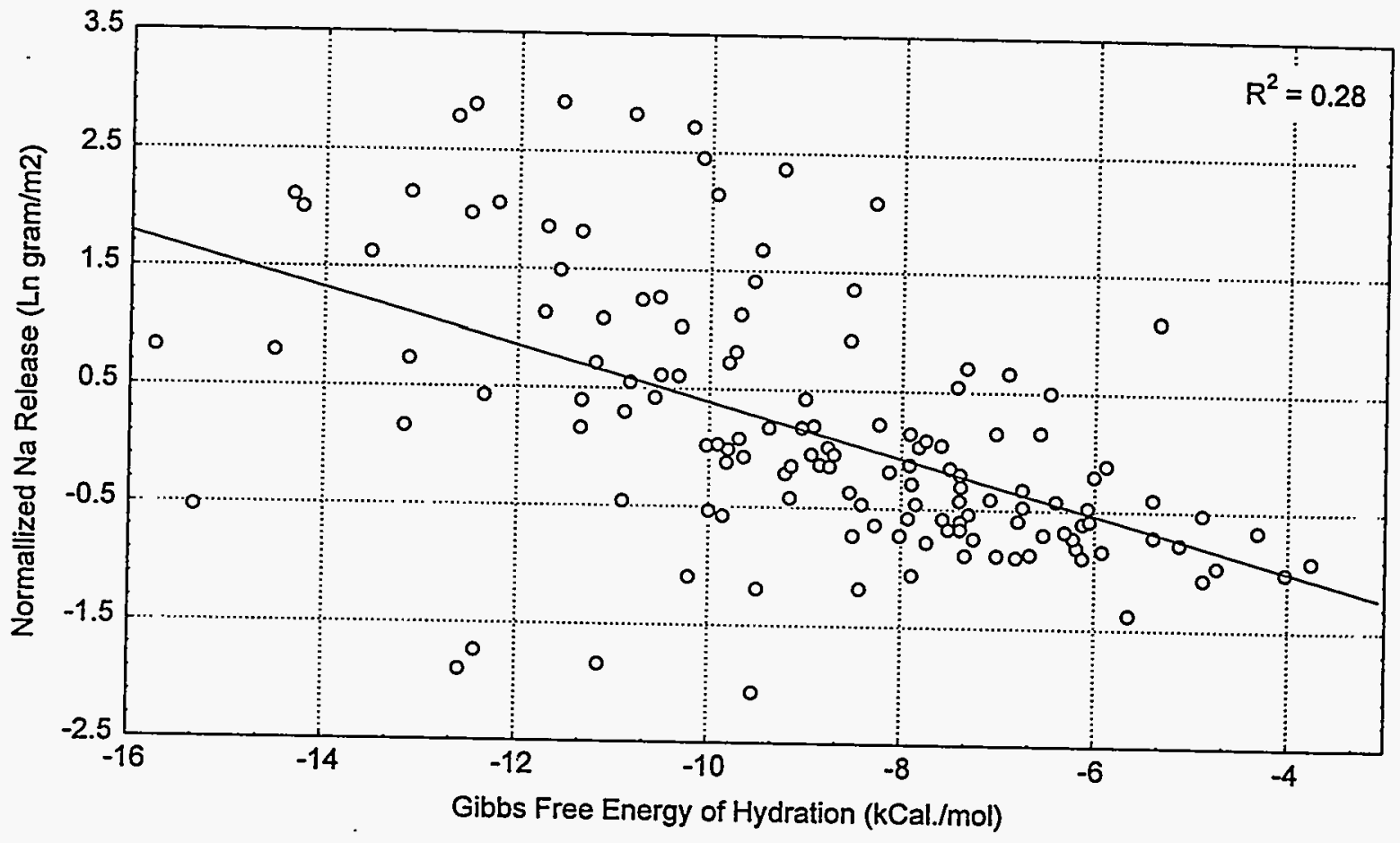

Figure 5.35d

Correlation between Gibbs free energy of hydration and the normalized release of Li from 7-day PCT.

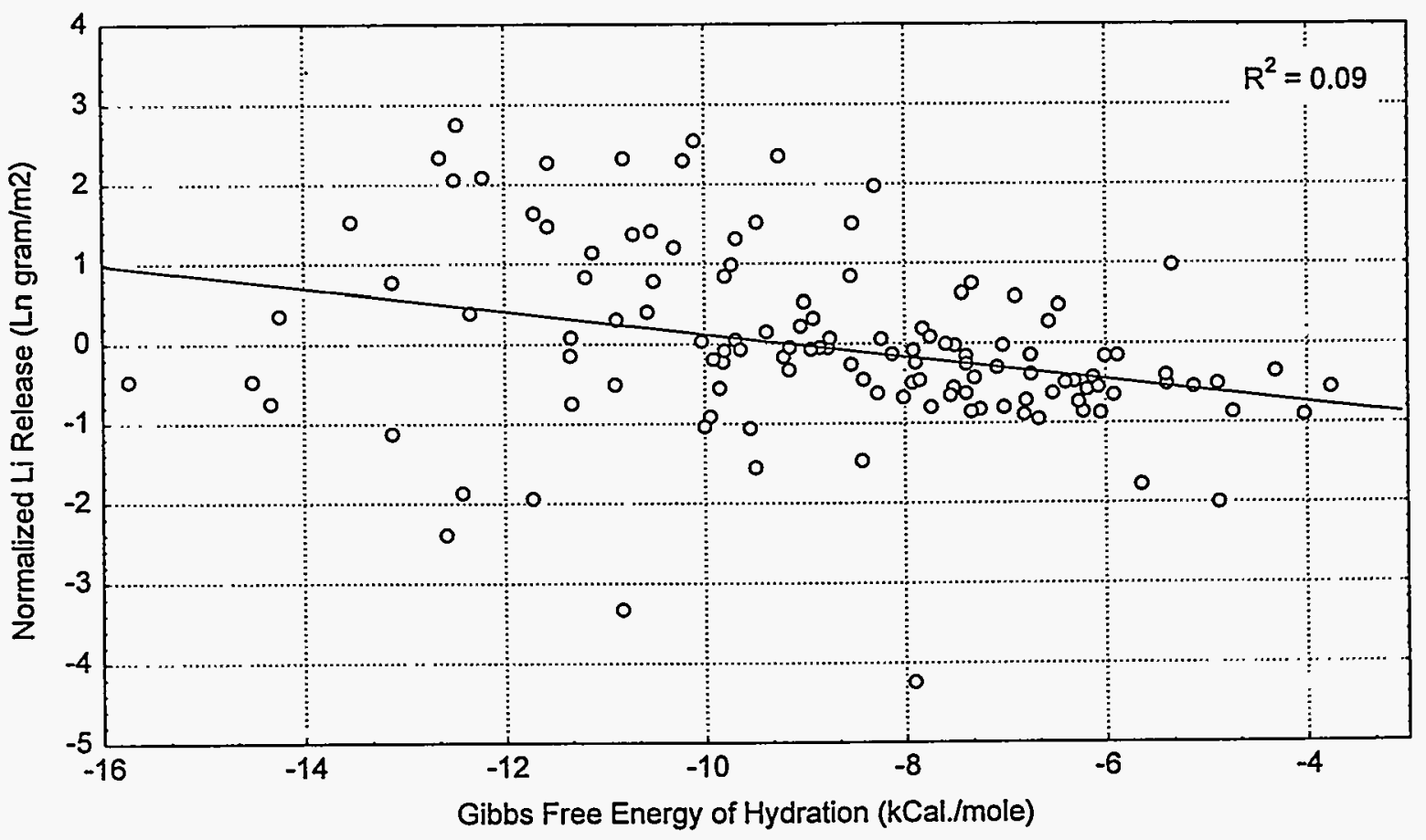


Figure 5.36a

Predicted versus measured viscosity of Group 1 glasses;

these glasses were not included in the database used to generate the model.

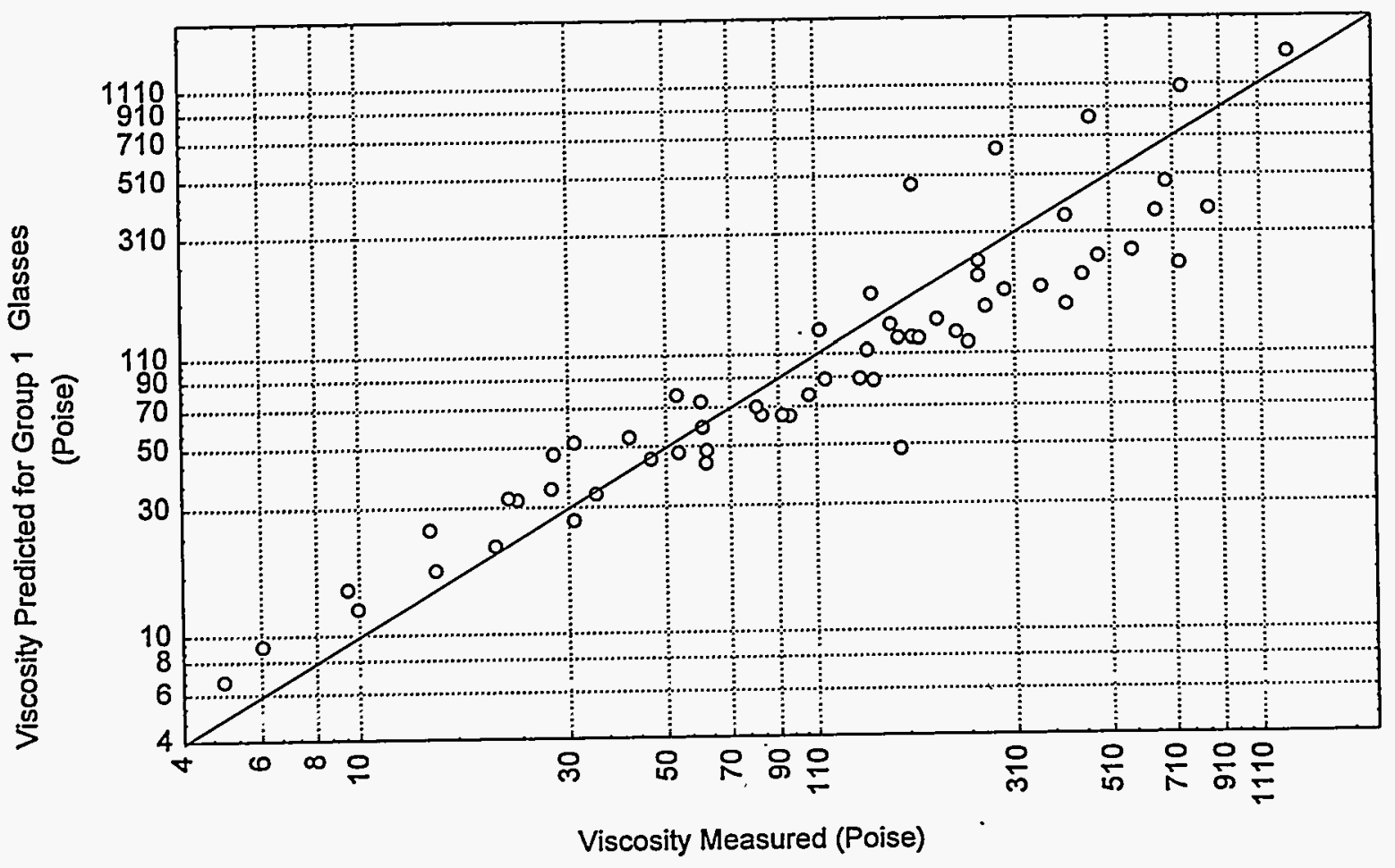

Figure 5.36b

Predicted versus measured viscosity of Group 2 glasses; these glasses were not included in the database used to generate the model.

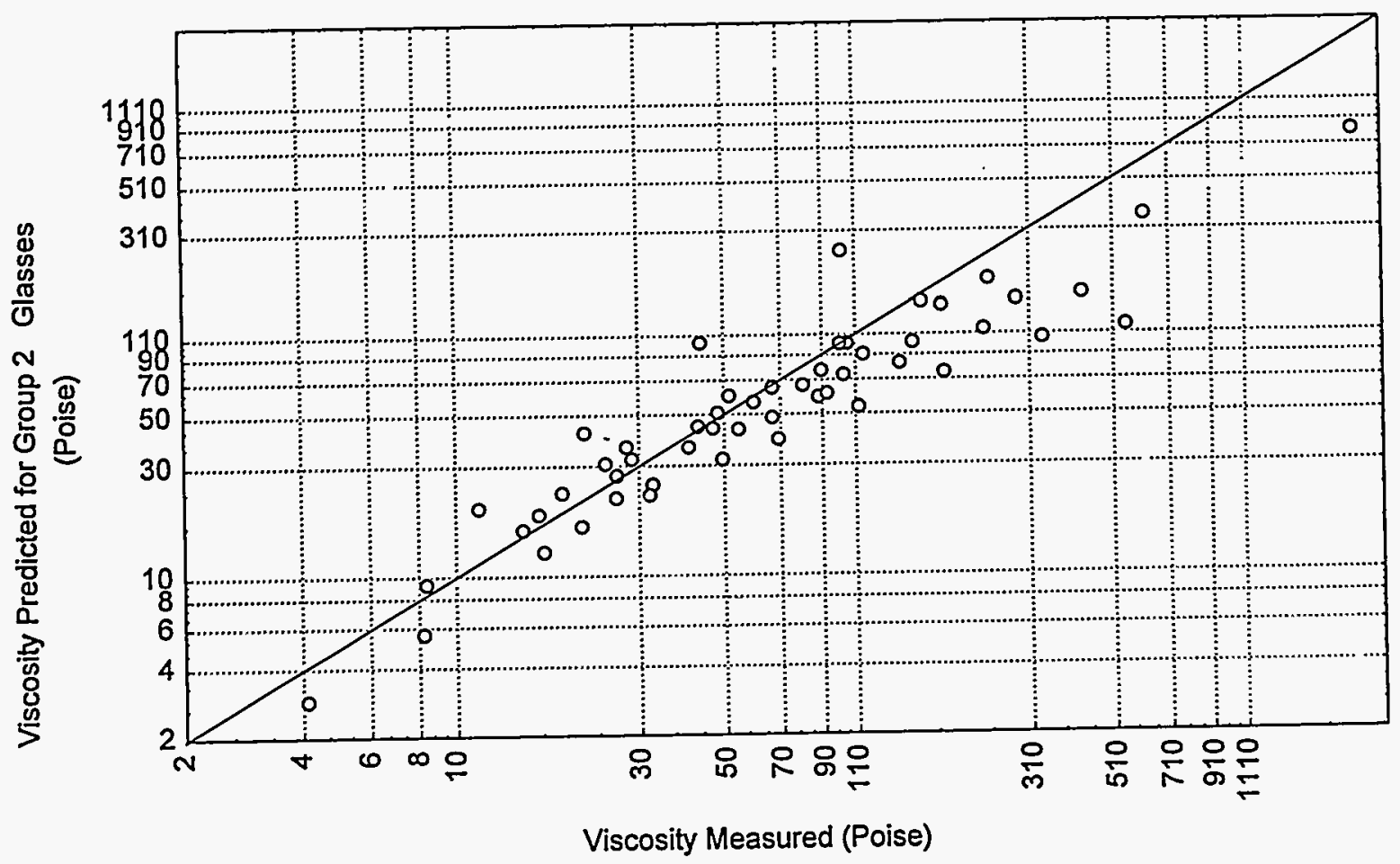

Page 5-71 
Figure 5.36c

Predicted versus measured viscosity of Group 3 glasses; these glasses were not included in the database used to generate the model.

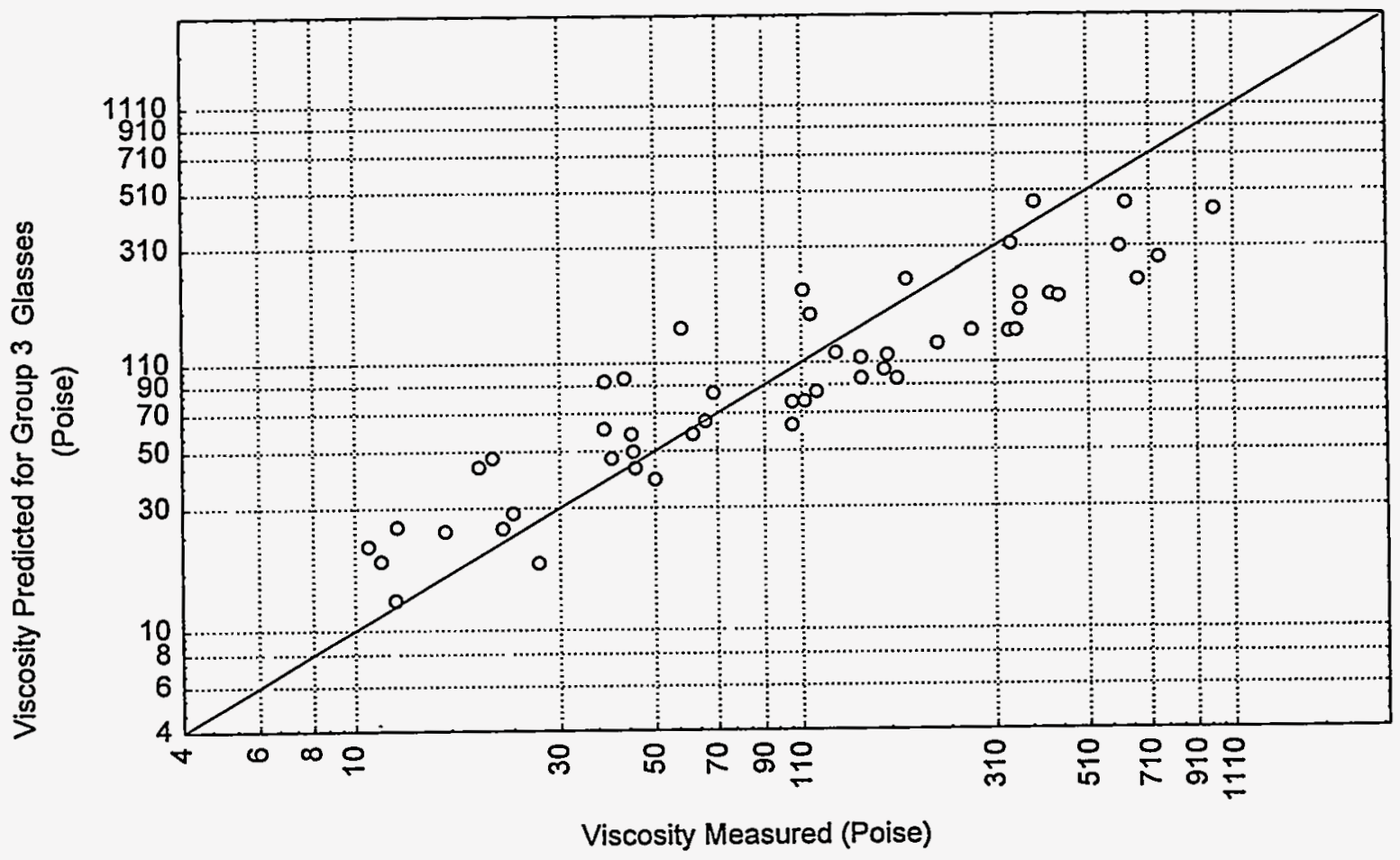


Figure 5.37a

Predicted versus measured sodium concentrations for the 7-day PCT of Group 1 glasses; these glasses were not included in the database used to generate the model.

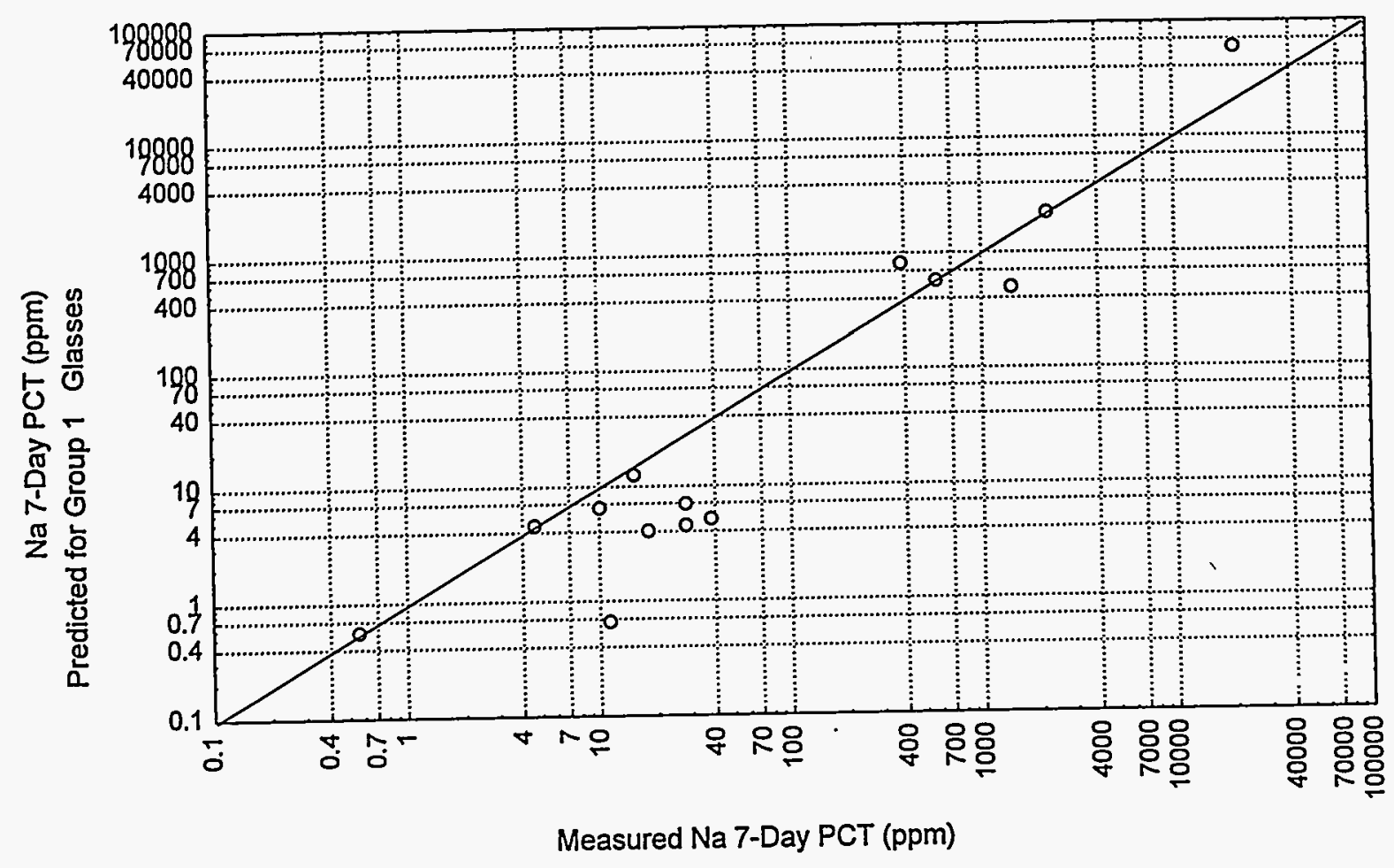

Figure 5.37b

Predicted versus measured sodium concentrations for the 7-day PCT of Group 2 glasses; these glasses were not included in the database used to generate the model.

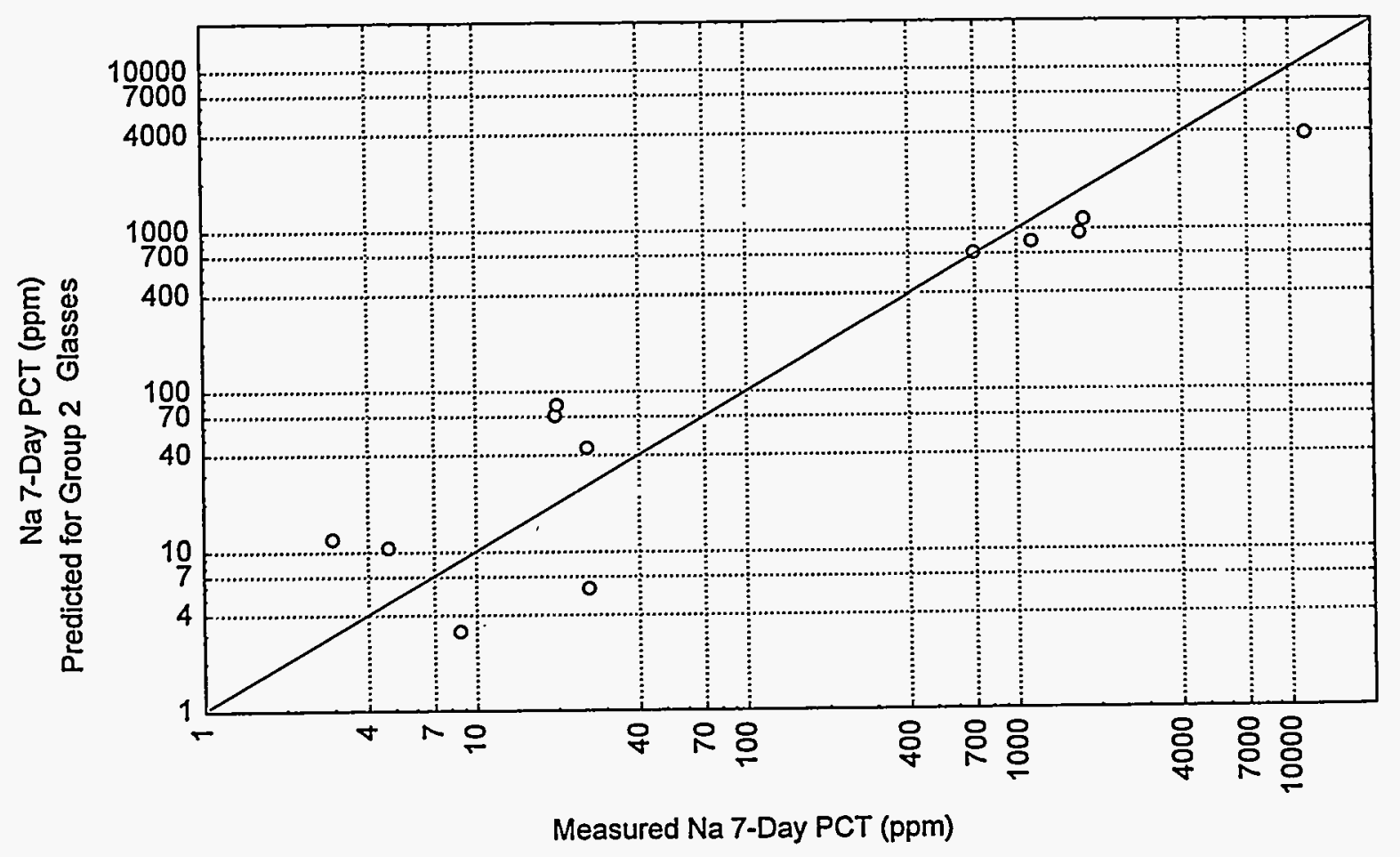


Predicted versus measured sodium concentrations for the 7-day PCT of Group 3 glasses; these glasses were not included in the database used to generate the model.

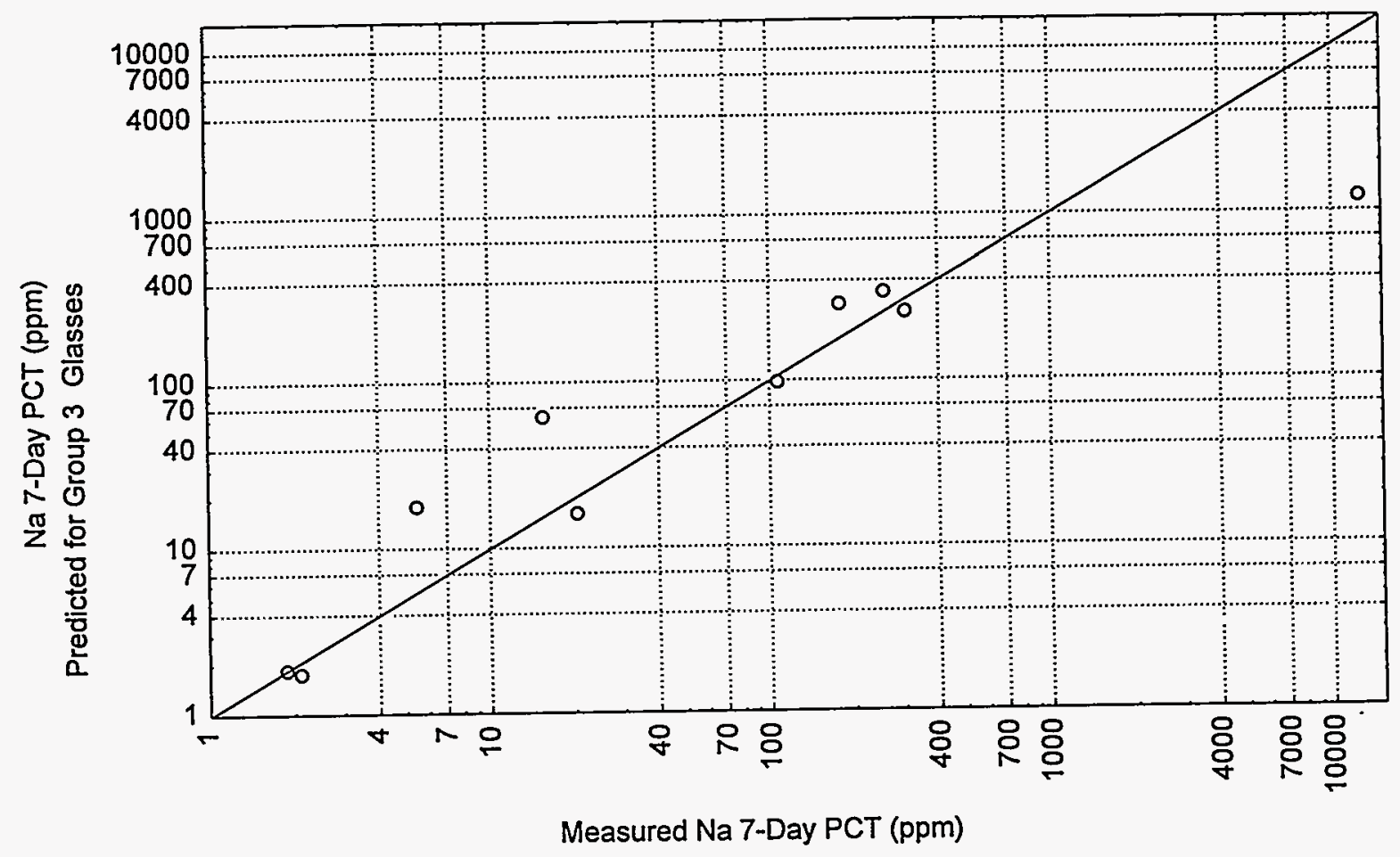




\section{SECTION 6.0 CONCLUSIONS}

The application of the Minimum Additive Waste Stabilization (MAWS) approach using vitrification as a potential treatment technology to a variety of waste streams across the DOE complex has been investigated. This work has involved both experimental vitrification development work using actual mixed wastes from several DOE sites (Hanford, Idaho, and Oak Ridge) as well as the development of a computer-based, integrated glass property-composition database and predictive modeling facility.

The experimental work conducted in this project has provided detailed characterization data with respect to properties of importance for vitrification for several waste streams. Approximately 87 glasses have been prepared from blends of these waste streams and the glasses have been characterized with respect to properties relating to leachability and processability. The core data set consists of 40 glasses on which viscosity, electrical conductivity, and TCLP and PCT leachabilty measurements have been made; the other glasses were partially characterized with respect to these properties. The 40 glasses in the core data set are listed in Table 6.1 together with representative data for each of these properties.

The range of waste blends investigated is represented in Figures 6.1-6.3 which show the weight percentage of each waste stream in each glass. Uniformly high waste loadings are achievable with a wide range of waste stream blends. The volume reductions factors achieved (with respect to total waste) ranged from about 3-14, as discussed in Section 6.4.

Overall, the results suggest that viable glass formulations can be developed for a wide range of combinations of the waste streams investigated with high waste loadings and, in many cases, no additions of chemical additives (i.e., 100\% waste loading). While the limited number of formulations investigated in this study would be unlikely to lead to fully optimized compositions (given the number of waste streams and possible combinations thereof), the results do serve to show the general ranges of glass and melt properties that are achievable with minimal use of chemical additives. The melt properties span ranges that would make the formulations suitable for processing in a variety of vitrification technologies, including the lower-temperature systems. The waste forms produced showed good leach resistance using both the TCLP and the PCT procedures.

The waste streams selected for this study cover a wide range of chemical compositions; this suggests that other waste streams not included in this study could be beneficially blended in similar fashions. The compositional diversity also makes the data of considerable additional value with respect to the compositional envelope modeling objectives of this project in that a relatively 
wide range of glass composition space is spanned which should improve the range of applicability of the models that have been developed.

The major conclusions from the experimental studies on each of the waste stream combinations investigated (i.e., Hanford, Idaho, or Oak Ridge wastes) and from the database/modeling work are reviewed below.

\subsection{Hanford}

The composition of the Hanford soils is typical of that of most soils; the soils are rich in glass formers (such as $\mathrm{SiO}_{2}$ and $\mathrm{Al}_{2} \mathrm{O}_{3}$ ). Conversely, the tank waste is primarily a solution of sodium salts and is, therefore poor in glass formers but rich in fluxes. Samples of contaminated Hanford soils were characterized and vitrified in combination with a radioactive simulant of the Hanford low-level tank waste. The resulting glasses have been subjected to characterization with respect to melt viscosity and electrical conductivity, microstructure, and TCLP and PCT leach resistance. Two groups of glasses were produced; those targeted toward maximized tank waste loadings and those targeted toward maximized soil loadings. The data collected in this study show that, in both cases, these Hanford waste streams can be treated by vitrification to produce leach resistant waste forms.

Glasses with high tank waste loadings (up to about 31 wt\% tank waste oxides) can be made with good chemical durability by using site soil as the major ingredient. With small amounts (around $5 \mathrm{wt} \%$ ) of additives, such as $\mathrm{ZrO}_{2}$, good leach resistance can be achieved for a waste form that is composed of $95 \%$ waste (on an oxide basis). A direct comparison has been made which shows that replacement of the site soil ingredient by a pure, purchased chemical additive such as $\mathrm{SiO}_{2}$ (sand) results in glasses of poorer durability. Realistically achievable tank waste loadings with the use of soil appear to be as high as $31 \%$ on an oxide basis using the presently existing TCLP and PCT specifications. However, site specific requirements on leach resistance may be more stringent which could lead to a corresponding reduction in the tank waste loading. The data obtained from this study should provide a basis for evaluating that trade-off. It is likely that, in the treatment of the enormous volumes of LLW tank waste, upwards of $65 \%$ (on a wt\% oxide basis) of the glass waste form that is produced will be derived from purchased chemicals; using a MAWS blended waste stream approach the additives could be largely replaced by contaminated site soils which would be beneficially co-treated.

Other data obtained from this study show that treatment of contaminated soils alone would benefit (in terms of waste loading) considerably from higher temperature vitrification processes. Very small amounts $(<10 \mathrm{wt} \%)$ of additives would be required to vitrify the site soils using processes operating in the $1400-1500^{\circ} \mathrm{C}$ range. 


\subsection{Idaho}

The results from the chemical characterization of the Idaho waste streams selected for this study (ACM, salt, flyash, and soil) suggest that their compositions are amenable to blending in a MAWS approach with the benefits of minimizing additive requirements and therefore maximizing volume reductions. Furthermore, from a compositional envelope perspective, these waste streams span a wide range of compositions both in terms of their bulk constituents and the hazardous and radioactive contaminants that are present. For example, the silica contents are $0.4 \%$ for the salt, $5.1 \%$ for the fly ash, $45 \%$ in the ACM and $65 \%$ in the soil; similarly, alumina ranges from $0.1 \%$ to $13 \%$, calcia from $0.1 \%$ to $25 \%$, lithia from $0.1 \%$ to $9 \%$ and soda from $1 \%$ to $35 \%$; uranium is present at $9 \%$ in the salt while the flyash contains six RCRA metals at levels of up to a few percent.

The Idaho waste streams exhibit bulk densities between 0.3 to $1.3 \mathrm{~g} / \mathrm{cm}^{3}$ (though particle densities are as high as $2.9 \mathrm{~g} / \mathrm{cm}^{3}$ ) and weight losses upon heating at $1150^{\circ} \mathrm{C}$ reaching as much as $64 \%$. These data indicate the potential for large volume reductions on vitrification if high waste loading can be achieved. Glasses formulated from blends of these waste streams with $100 \%$ waste loadings have been prepared and characterized.

The results obtained show that the four waste streams studied can be effectively blended and vitrified without additives as either binary or quaternary mixtures; other combinations than those investigated are certainly possible, however. In the binary approach, mixtures of salt and ACM were studied. The glass formulations investigated, containing around $10 \mathrm{wt} \%$ salt and 90 $\mathrm{wt} \% \mathrm{ACM}$, produced viable glass products in replicate melts despite the composition variability found in the ACM waste stream. As a further demonstration of the flexibility of MAWS vitrification with respect to a wider variety of waste types, a series of formulations was investigated using all four waste streams.

The leach resistance of the waste forms produced are uniformly high on the basis of the PCT procedure. Many of the glasses produced met all of the TCLP release limits, generally by a wide margin; the high concentration of lead in the fly ash was the primary factor for glasses that exceeded the TCLP release limits.

The most problematic (from a vitrification perspective) of the waste streams investigated in this study was the fly ash due to the high concentrations of carbon, sulfur, and heavy metals, many of which are relatively redox sensitive. As would be expected, the use of the fly ash waste was found to have significant impacts on the redox state of the glass melts that are produced due to the high carbon content of that material. The redox state variations are in turn coupled to the speciation of the redox sensitive species in the glass (primarily sulfur, carbon, and the heavy 
metals) and therefore lead to variations in both the physical and chemical properties of the product and the melt. As a further complication, there are important kinetic effects that must be considered since the melts are produced in a finite time under an oxidizing atmosphere but with reductants in the batch. In this area, some caution should be exercised in using the crucible melt observations to predict what might be expected to occur in a full-scale process since factors such as residence time and oxygen availability are highly dependent on the specific vitrification process concerned. For example, we have recently demonstrated that quite oxidized glasses could be produced from a commercial radioactive waste stream composed of $97 \%$ organics in a prolonged pilot-scale joule-heated melter demonstration (Brandys-1995) though this would not be possible in typical 1-2 hour crucible melt.

Thus, while selection and control of the redox state range is an important consideration for full scale processing of wastes such as the Idaho fly ash, the specific preferred values must be determined on the basis of the particular process characteristics and limitations. As an example, the production of reduced glasses has the advantage of preventing the formation of an immiscible sulfate phase (at the expense of sending the sulfur to the off-gas system) but promotes the formation of molten metal phases (particularly lead in this case) from the melt. This may be an acceptable trade-off for some processes while the reverse may be preferred for others; for yet other processes, neither may be acceptable, in which case the fraction of the problem waste stream in the batch must be limited, perhaps to impractically low levels. Clearly, these decisions cannot be made on the basis of crucible melts alone and are thus outside the scope of the present study. However, an important component of such a decision is, of course, a knowledge of the characteristics and properties of melts made from various waste stream proportions under various conditions; that is the kind information obtained from this study.

\subsection{Oak Ridge}

Of the waste streams selected for this study one sample of actual waste (WETF sludge) was obtained from the Oak Ridge site and three other waste streams (Pond Waste, TSCA Ash, and soil) were simulated; however, the simulants did include the radioactive and hazardous constituents of concern. The waste streams selected introduced new compositional areas into this study as compared to the waste streams from Hanford and Idaho. The WETF sludge is both a relatively large volume waste stream in its own right as well as an example of a large family of wastes generated by the precipitation of heavy metals from various process wastes (in this case, a biodenitration process) with calcium. The TSCA ash is another example of a high-calcium waste, although otherwise of quite different composition and origin from the WETF sludge. After calcium, the next most abundant component in the WETF sludge is aluminum that, as the oxide, constitutes about $20 \mathrm{wt} \%$. The soil is high in silica and represents an obvious source of silica that would otherwise be lacking; together these observations suggest that high waste loading glasses 
would be of the general silica-lime-alumina type. Both the TSCA ash and the Pond Waste contain significant amounts of phosphorus that can lead to phase separation in silicate melts. The primary contaminants in these wastes are uranium and nickel, which are present in significant amounts in both the Pond Waste and the WETF sludge. Barium, chromium and lead are present at the tenth of a percent level while arsenic, silver, and mercury are present at the tens of ppm level.

A variety of waste blends were investigated to demonstrate the flexibility of the general MAWS formulation approach. It was found that both the WETF sludge and the TSCA ash wastes can be blended with contaminated soil and minimal amounts of additives to produce glasses with very good leach resistance as well as acceptable melt characteristics for processing. The ternary systems investigated were the WETF/Pond Waste/Soil system and the TSCA ash/Pond Waste/Soil system. Both of these combinations can be used to produce glasses and melts with acceptable characteristics for vitrification; in addition, the glasses formed from these blends (which tend to be higher in both iron and phosphorus) show a significant reduction in the measured TCLP leachate concentrations. It was also demonstrated that the Pond Waste alone can be melted (i.e., $100 \%$ waste loading) to form a vitroceramic matrix which exhibits very good leach resistance; these results showed clearly the potential benefits of including specific crystalline phases in the waste form (e.g., nickel-containing spinels in this case) in some.instances. Such an approach raises obvious questions related to processing, however.

The results of this study of the potential of MAWS vitrification applied to Oak Ridge wastes suggest that viable glass formulations can be developed for a wide range of combinations of the waste streams investigated with high waste loadings (and in some cases $100 \%$ ). The leach resistance of the waste forms produced are uniformly high on the basis of the PCT procedure. All but one of the glasses produced leached considerably less than any of the RCRA TCLP release limits.

\subsection{Volume Reductions}

The volume reduction can be defined as the volume of waste (or waste blend) that is consumed in the production of a unit volume of glass. The magnitude of the volume reduction achieved on vitrification is the result of three contributions:

(i) The mass loss on vitrification as a result of decomposition and evaporation of thermally unstable and volatile constituents; examples include water, carbonates, nitrates, and hydroxides;

(ii) The mass increase due to the incorporation of additives into the glass; and

(iii) The density difference between the waste (or waste blend) and the glass. 
The characterization data that we have collected on both the waste streams and resultant glasses provides the necessary data on mass losses, additives usage, and densities which, together with the compositions, can be used to calculate the volume reductions in a systematic way.

For each of the waste stream combinations that were studied (Hanford, Idaho, and Oak Ridge) two or three MAWS glass formulations were selected that were judged to be representative of the range of blends achievable and that showed satisfactory leaching and processability characteristics on the basis of the specific glass properties discussed in Sections $2-4$. The waste stream and glass characterization data were then used to calculate the volume reduction for each of the selected glass formulations; these results are presented in Tables 6.2ac. The information presented in Table 6.2a-c warrants explanation with regard to several points:

(i) Whenever a blend of waste streams is involved, one can define volume reductions with respect to each waste stream separately or with respect to any combination of those waste streams. What is usually of most interest is the volume reduction with respect to total waste. However, in other circumstances waste streams may be prioritized for treatment by some set of criteria and then the volume reduction with respect to some sub-set of the wastes involved may be more relevant. For simplicity, we have elected to use the volume reduction with respect to total waste for this comparison; others can be easily calculated from the data presented in Sections 2-4. The one exception to this is the case of the Hanford formulations; since only two waste streams are involved, the volume reductions with respect to total waste and with respect to tank waste alone are both shown.

(ii) It is of interest to compare the volume reductions obtained using the MAWS approach with those obtained by vitrifying each waste stream separately; however, data with respect to the latter were not obtained in this work, particularly with regard to the waste loadings that might be achievable. We have therefore fixed the waste loading arbitrarily at $25 \%$ for each waste stream when it is treated alone in order to calculate a baseline value for the volume reduction; clearly realistic values may be larger or smaller depending on the particular waste stream. The volume reduction values can then be easily scaled up or down to any desired waste loading from the $25 \%$ baseline value that is presented (the value of $25 \%$ is probably reasonable for the Hanford tank waste and the Idaho salt waste, somewhat high for the Idaho flyash, and somewhat low for the other wastes). Since it is reasonably clear that waste loadings of around $90 \%$ should be achievable for soils, that value has been used for those wastes.

The results shown in Table 6.2a-c show that volume reductions for the MAWS glasses range from 1.5-3.7 for the Hanford wastes, 8.7-11.9 for the Idaho wastes, and 6.2-13.7 for the Oak Ridge wastes. It is worth noting that in addition to the volume reduction improvements (and therefore potential disposal cost savings) that are possible by minimizing additives, there are also 
further potential cost savings due to the direct cost of additives and the cost of the operating time required to process the additives.

\subsection{Database and Glass Property Modeling}

In addition to the experimental work, an integrated glass property composition database and modeling facility has been designed, a substantial portion of the development work has been completed, and evaluation tests of the system have been conducted. The major objectives of this facility are to support the design and formulation of glass compositions using the MAWS approach to maximize waste loading and, therefore, improve the overall economics. The longer term objective is that this modeling capability will allow glasses to be formulated from single waste streams or combinations of waste streams subject to a variety of user-imposed constraints including waste stream usage priorities, process related constraints (e.g., melt viscosity, electrical conductivity, etc.), and performance related constraints (e.g., TCLP and PCT leaching characteristics). This capability will be useful in guiding and expediting future glass formulation studies to support any future applications of vitrification technologies in general and those adopting the MAWS approach in particular.

All of the data from the experimental studies outlined above are included in the system, together with data from other sources. We have focused on three properties in this development phase of the program: Melt viscosity, electrical conductivity, and PCT leaching. The database spans a wide range of glass compositions and the property models provide both a good representation of those data as well as reasonable predictions for the properties of glasses that were not included in the data set used to generate the models. The system should be a valuable tool in the design and optimization of glass compositions for waste vitrification activities in general, and for those using the MAWS approach in particular.

While other glass composition databases exist, particularly those developed by the commercial glass industry and some efforts in support of high-level nuclear waste vitrification, these generally relate to rather narrow composition ranges in comparison to those of importance for the present work. The diversity of mixed and low-level waste streams in the DOE complex necessitates a facility of the type developed here which is based firmly on data from vitrification studies conducted predominantly on a similarly diverse set of actual waste streams. The present integrated database-modeling facility incorporates useful models, well explored algorithms to rapidly build new models under different conditions, and a carefully organized glasscomposition-property database.

Additional efforts in this direction that would be of value involve the incorporation of data on other glasses and on other properties of interest, such as longer-term PCT leaching, data 
on TCLP leaching, and liquidus temperature information, as well as the development of models for these properties. It would also be useful to include a database table on DOE waste stream compositional information relevant to vitrification that, together with the present glass property prediction capability, would permit the identification and evaluation of optimized MAWS waste stream blends subject to user imposed constraints characterizing any particular treatment problem. 
Table 6.1a

List of Hanford Glasses with Complete Viscosity, Conductivity, TCLP, and PCT Data (i.e., Glasses in the Core Data Set of $\mathbf{4 0}$ Glasses) and Representative Data for Those

Properties.

\begin{tabular}{|c|c|c|c|c|c|}
\hline \multirow{2}{*}{$\begin{array}{c}\text { Glass } \\
\text { Number }\end{array}$} & \multirow{2}{*}{$\begin{array}{l}\text { Glass } \\
\text { Name }\end{array}$} & \multirow{2}{*}{$\begin{array}{l}\text { Viscosity } \\
@ 1250^{\circ} \mathrm{C}\end{array}$} & \multirow{2}{*}{$\begin{array}{c}\text { Conductivity } \\
@ 1250^{\circ} \mathrm{C}\end{array}$} & TCLP & PCT-7 day \\
\hline & & & & $\mathrm{U}(\mathrm{ppb})$ & $\mathrm{Na}(\mathrm{ppm})$ \\
\hline 1 & HAN22B & 129 & 1.029 & 5 & 1433 \\
\hline 2 & HAN24 & 158 & 0.926 & 10 & 1134 \\
\hline 3 & HAN25 & 307 & 0.953 & 4 & 305 \\
\hline 4 & HAN26 & 74 & 0.995 & 6 & 383 \\
\hline 5 & HAN27 & 220 & 1.075 & 2 & 695 \\
\hline 6 & HAN28 & 333 & 0.752 & 1 & 178 \\
\hline 7 & HAN28A & 319 & 0.894 & 25 & 585 \\
\hline 8 & HAN29A & 101 & 1.319 & 26 & 1765 \\
\hline 9 & HAN29B1 & 61 & 1.065 & 79 & 2201 \\
\hline 10 & HAN29E & 5 & 2.377 & 65 & 20701 \\
\hline 11 & HAN29F & 24 & 1.555 & 65 & 11441 \\
\hline 12 & HAN30A & 353 & 0.418 & 31 & 107 \\
\hline 13 & HAN33 & 165 & 0.022 & 48 & 26 \\
\hline 14 & HAN34 & 1018 & 0.073 & 9 & 20 \\
\hline 15 & HAN35 & 1652 & 0.025 & 35 & 18 \\
\hline 16 & HAN36 & 157 & 0.845 & 12 & 1507 \\
\hline 17 & HAN37 & 88 & 0.779 & 54 & 1702 \\
\hline 18 & HAN38 & 197 & 0.598 & 18 & 257 \\
\hline 19 & HAN39 & 297 & 0.645 & 21 & 12507 \\
\hline
\end{tabular}


Table 6.1b

List of Idaho Glasses with Complete Viscosity, Conductivity, TCLP, and PCT Data (i.e., Glasses in the Core Data Set of $\mathbf{4 0}$ Glasses)

And Representative Data for Those Properties.

\begin{tabular}{|c|c|c|c|c|c|}
\hline \multirow{2}{*}{$\begin{array}{l}\text { Glass } \\
\text { Number }\end{array}$} & \multirow{2}{*}{$\begin{array}{l}\text { Glass } \\
\text { Name }\end{array}$} & \multirow{2}{*}{$\begin{array}{l}\text { Viscosity } \\
@ 1350^{\circ} \mathrm{C}\end{array}$} & \multirow{2}{*}{$\begin{array}{l}\text { Conductivity } \\
@ 1350^{\circ} \mathrm{C}\end{array}$} & TCLP & \multirow{2}{*}{$\frac{\text { PCT-7 day }}{\mathrm{Na}(\mathrm{ppm})}$} \\
\hline & & & & $\mathbf{U}(\mathrm{ppb})$ & \\
\hline 1 & IDG7A & 36 & 0.088 & 400 & 5 \\
\hline 2 & IDG7B & 31 & 0.129 & 452 & 28 \\
\hline 3 & IDG7C & 15 & 0.102 & 506 & 5 \\
\hline 4 & IDG $\dot{8} A$ & 7 & 0.146 & 1879 & 5 \\
\hline 5 & IDG8C & 13 & 0.189 & 612 & 3 \\
\hline 6 & IDG12C & 15 & 0.121 & 664 & 5 \\
\hline 7 & IDG13A & 107 & 0.166 & 244 & 15 \\
\hline 8 & IDG13B & 34 & 0.225 & 1117 & 20 \\
\hline 9 & IDG13C & 66 & 0.227 & 707 & 20 \\
\hline 10 & IDG14A & 427 & $>0.3$ & 474 & 11 \\
\hline 11 & IDG14B & 18 & 0.308 & 2339 & 16 \\
\hline 12 & IDG15A & 65 & 0.191 & 341 & 16 \\
\hline
\end{tabular}


Table 6.1c

List of Oak Ridge Glasses with Complete Viscosity, Conductivity, TCLP, and PCT Data (i.e., Glasses in the Core Data Set of $\mathbf{4 0}$ Glasses) and Representative Data for Those Properties.

\begin{tabular}{|c|c|c|c|c|c|}
\hline \multirow{2}{*}{$\begin{array}{c}\text { Glass } \\
\text { Number }\end{array}$} & \multirow{2}{*}{$\begin{array}{l}\text { Glass } \\
\text { Name }\end{array}$} & \multirow{2}{*}{$\begin{array}{l}\text { Viscosity } \\
@ 1250^{\circ} \mathrm{C}\end{array}$} & \multirow{2}{*}{$\begin{array}{c}\text { Conductivity } \\
\text { @ } 1250^{\circ} \mathrm{C}\end{array}$} & TCLP & PCT-7 day \\
\hline & & & & $\mathrm{U}(\mathrm{ppb})$ & $\mathrm{Na}$ (ppm) \\
\hline 1 & ORNL3 & 490 & 0.150 & 84 & 36 \\
\hline 2 & ORNL9A & 36 & 0.090 & 110 & 9 \\
\hline 3 & ORNL10 & 40 & 0.173 & 39 & 26 \\
\hline 4 & ORNL11 & 62 & 0.042 & 1864 & 2 \\
\hline 5 & ORNL12 & 57 & 0.036 & 576 & 2 \\
\hline 6 & ORNL13 & 16 & 0.113 & 1454 & 10 \\
\hline 7 & ORNL14 & 109 & 0.068 & 272 & 1 \\
\hline 8 & ORNL15 & 81 & 0.044 & 279 & 1 \\
\hline 9 & ORNL16 & 18 & 0.100 & 2252 & 9 \\
\hline
\end{tabular}


Table 6.2a

Volume Reductions Calculated for Hanford Waste Streams

\begin{tabular}{|c|c|c|c|c|}
\hline & Tank Waste & $\begin{array}{c}\text { Contaminated } \\
\text { Soils }\end{array}$ & $\begin{array}{c}\text { Glass } \\
\text { HAN29B1 } \\
\end{array}$ & $\begin{array}{l}\text { Glass } \\
\text { HAN38 } \\
\end{array}$ \\
\hline $\begin{array}{l}\text { Weight Loss at } \\
1150^{\circ} \mathrm{C}\end{array}$ & $71 \%$ & $8 \%$ & N.A. & N.A. \\
\hline $\begin{array}{l}\text { Bulk Density, } \\
\mathrm{g} / \mathrm{cm}^{3}\end{array}$ & 1.42 & $1.2^{\text {(a) }}$ & N.A. & N.A. \\
\hline $\begin{array}{l}\text { Glass Density }{ }^{(\mathrm{d})} \text {, } \\
\mathrm{g} / \mathrm{cm}^{3}\end{array}$ & 2.5 & 2.5 & 2.5 & 2.5 \\
\hline $\begin{array}{l}\text { Total Waste } \\
\text { Loadding, wt\% }\end{array}$ & $25 \%$ & $\therefore \because$ & $95 \%$ & $90 \%$ \\
\hline $\begin{array}{l}\text { Tank Waste } \\
\text { Loading, wt.\% }\end{array}$ & (la & . & $40 \%$ & $\because 25 \%$ \\
\hline $\begin{array}{l}\text { Contaminated } \\
\text { Soil Loading, } \\
\text { wt: } \%\end{array}$ & 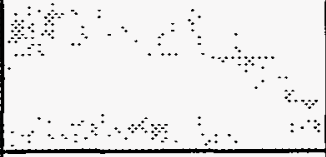 & $\therefore=\ldots$ & 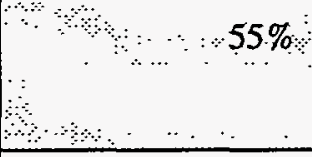 & $\begin{array}{rrr} & & 65 \% \\
& & \\
& \ddots\end{array}$ \\
\hline $\begin{array}{l}\text { Volume Reduction } \\
\text { Factor }\end{array}$ & 1.5 & 2.0 & $\begin{array}{l}3.7^{(\mathrm{b})} \\
2.4^{(\mathrm{c})}\end{array}$ & $\begin{array}{l}3.0^{(\mathrm{b})} \\
1.5^{(\mathrm{c})}\end{array}$ \\
\hline
\end{tabular}

N.A. Not Applicable

(a) Bulk density not measured; average of values for Idaho and Oak Ridge soil used here.

(b) With respect to total waste $=$ tank waste + soil

(c) With respect to tank waste only

(d) Estimated to within $\pm 20 \%$ 
Table 6.2b

Volume Reduction Calculated for Idaho Waste Streams

\begin{tabular}{|c|c|c|c|c|c|c|c|}
\hline & $\mathrm{ACM}$ & Salt & Fly Ash & Soil & $\begin{array}{l}\text { Glass } \\
\text { IDG7 }\end{array}$ & $\begin{array}{c}\text { Glass } \\
\text { mG12C }\end{array}$ & $\begin{array}{c}\text { Glass } \\
\text { IDG15A }\end{array}$ \\
\hline $\begin{array}{l}\text { Weight Loss at } \\
1150^{\circ} \mathrm{C}\end{array}$ & $30 \%$ & $46 \%$ & $64 \%$ & $12 \%$ & N.A. & N.A. & N.A. \\
\hline $\begin{array}{l}\text { Bulk Density, } \\
\mathrm{g} / \mathrm{cm}^{3}\end{array}$ & 0.28 & 1.19 & 0.66 & 1.3 & N.A. & N.A. & N.A. \\
\hline $\begin{array}{l}\text { Glass Density }{ }^{(a)}, \\
\mathrm{g} / \mathrm{cm}^{3}\end{array}$ & 2.5 & 2.5 & 2.5 & 2.5 & 2.5 & 2.5 & 2.5 \\
\hline $\begin{array}{l}\text { Total Waste } \\
\text { Loading, wt } \%\end{array}$ & $25 \%$ & $\begin{array}{l}25 \% \\
\ddots \ldots . \\
\end{array}$ & $25 \%$ & $90 \%$ & $\begin{array}{r}100 \% \\
\quad+1 \\
\end{array}$ & $\begin{array}{c}100 \% \\
: \\
\end{array}$ & $100 \%$ \\
\hline $\begin{array}{l}\mathrm{ACM}, \\
\mathrm{wt} \%\end{array}$ & $25 \%$ & : & & & $90 \%$ & $70 \%$ & $\begin{array}{l}50 \% \\
\therefore \quad \\
\end{array}$ \\
\hline $\begin{array}{l}\text { Salt, } \\
\text { wt\% }\end{array}$ & 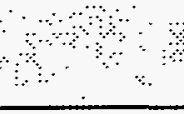 & $\begin{array}{r}25 \% \\
\quad \quad \% \\
\end{array}$ & & : & $\begin{array}{r}10 \% \\
18 \\
\end{array}$ & $\begin{array}{r}10 \% \\
\end{array}$ & $\begin{array}{r}20 \ddot{\%} \\
\therefore\end{array}$ \\
\hline $\begin{array}{l}\text { Fly Ash, } \\
\text { wt\% } \ldots\end{array}$ & $\therefore \quad$ & $\begin{array}{lll} & \ddots \\
\ddots & \ddots \\
\end{array}$ & $\begin{array}{c}25 \% \\
\quad \quad 0 \\
\end{array}$ & & $\begin{array}{r}0 \% \\
\quad 0 \\
\end{array}$ & $10 \%$ & $\begin{array}{l}10 \% \\
\therefore \quad \therefore\end{array}$ \\
\hline $\begin{array}{l}\text { Soil, } \\
\text { wt\% }\end{array}$ & $\therefore$ & 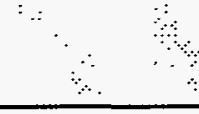 & 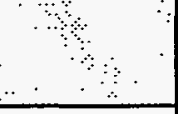 & $90 \%$ & $0 \%$ & $10 \%$ & $20 \%$ \\
\hline $\begin{array}{l}\text { Volume } \\
\text { Reduction } \\
\text { Factor }\end{array}$ & 3.2 & 1.0 & 2.7 & 2.0 & 11.9 & 10.6 & 8.7 \\
\hline
\end{tabular}

N.A. Not Applicable

(a) Estimated to within $\pm 20 \%$ 
Table 6.2c

Volume Reduction Calculated for Oak Ridge WETF Sludge, Pond Waste, TSCA Ash and Soil

\begin{tabular}{|c|c|c|c|c|c|c|c|}
\hline & $\begin{array}{l}\text { WETF } \\
\text { Sludge }\end{array}$ & $\begin{array}{c}\text { Pond } \\
\text { Waste }^{(a)}\end{array}$ & $\begin{array}{r}\text { TSCA } \\
\text { Ash }^{(\mathbf{b})}\end{array}$ & Soil & $\begin{array}{c}\text { Glass } \\
\text { ORNL12 }\end{array}$ & $\begin{array}{c}\text { Glass } \\
\text { ORNL14 }\end{array}$ & $\begin{array}{c}\text { Glass } \\
\text { ORNL15 }\end{array}$ \\
\hline $\begin{array}{l}\text { Weight Loss at } \\
1150^{\circ} \mathrm{C}\end{array}$ & $82 \%$ & $56 \%$ & $90 \%$ & $10 \%$ & N.A. & N.A. & N.A. \\
\hline $\begin{array}{l}\text { Bulk Density, } \\
\mathrm{g} / \mathrm{cm}^{3}\end{array}$ & 1.2 & 1.1 & 1 & 1.13 & N.A. & N.A. & N.A. \\
\hline $\begin{array}{l}\text { Glass Density }{ }^{(\mathrm{c})} \\
\mathrm{g} / \mathrm{cm}^{3}\end{array}$ & 2.5 & 2.5 & 2.5 & 2.5 & 2.5 & 2.5 & 2.5 \\
\hline $\begin{array}{l}\text { Total Waste } \\
\text { Loading }: \cdots\end{array}$ & $25 \%$ & $25 \%$ & $25 \%$ & $90 \%$ & $\begin{array}{r}100 \% \\
\vdots\end{array}$ & $100 \%$ & $90 \%$ \\
\hline $\begin{array}{l}\text { WETF Siudge } \\
\text { wt\% }\end{array}$ & $25 \%$ & & ॥: & $\because$ & $\begin{array}{r}35 \% \\
\because \\
\end{array}$ & $0 \%$ & $0 \%$ \\
\hline $\begin{array}{l}\text { Pond Waste } \\
\text { wt\% }\end{array}$ & & $25 \%$ & & & 20\% & $\quad 0 \%$ & $10 \%$ \\
\hline TSCA Ash wit\% & & & $25 \%$ & & $\therefore \quad 0 \%$ & $50 \%$ & $50 \%$ \\
\hline $\begin{array}{l}\text { Soil Loading } \\
\text { wt\% }\end{array}$ & & & & $90 \%$ & $45 \%$ & $50 \%$ & $30 \%$ \\
\hline $\begin{array}{l}\text { Volume } \\
\text { Reduction } \\
\text { Factor }\end{array}$ & 2.9 & 1.3 & 6.3 & 2.2 & 6.2 & 13.7 & 12.9 \\
\hline
\end{tabular}

(a) (Bostick-1993, Kirkpatrick -1992).

(b) SAIC, File No.RB92004, (1992) and privale communication from Oak Ridge site.

(c) Estimated to within $\pm 20 \%$. 
Figure 6.1.

Waste percentages used in the Hanford glasses.

(Additives used are shown at the top of each bar.)

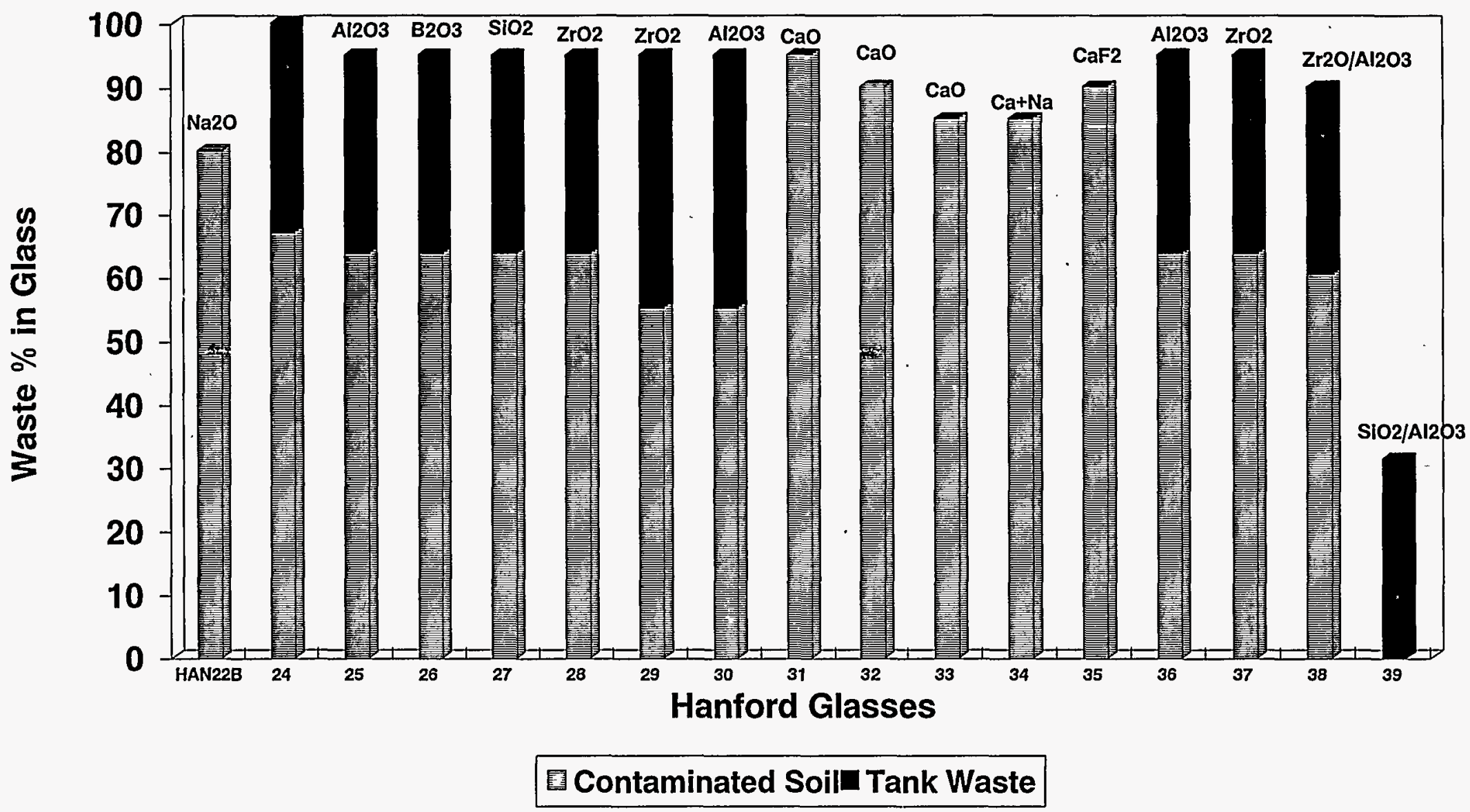

Page 6-15 


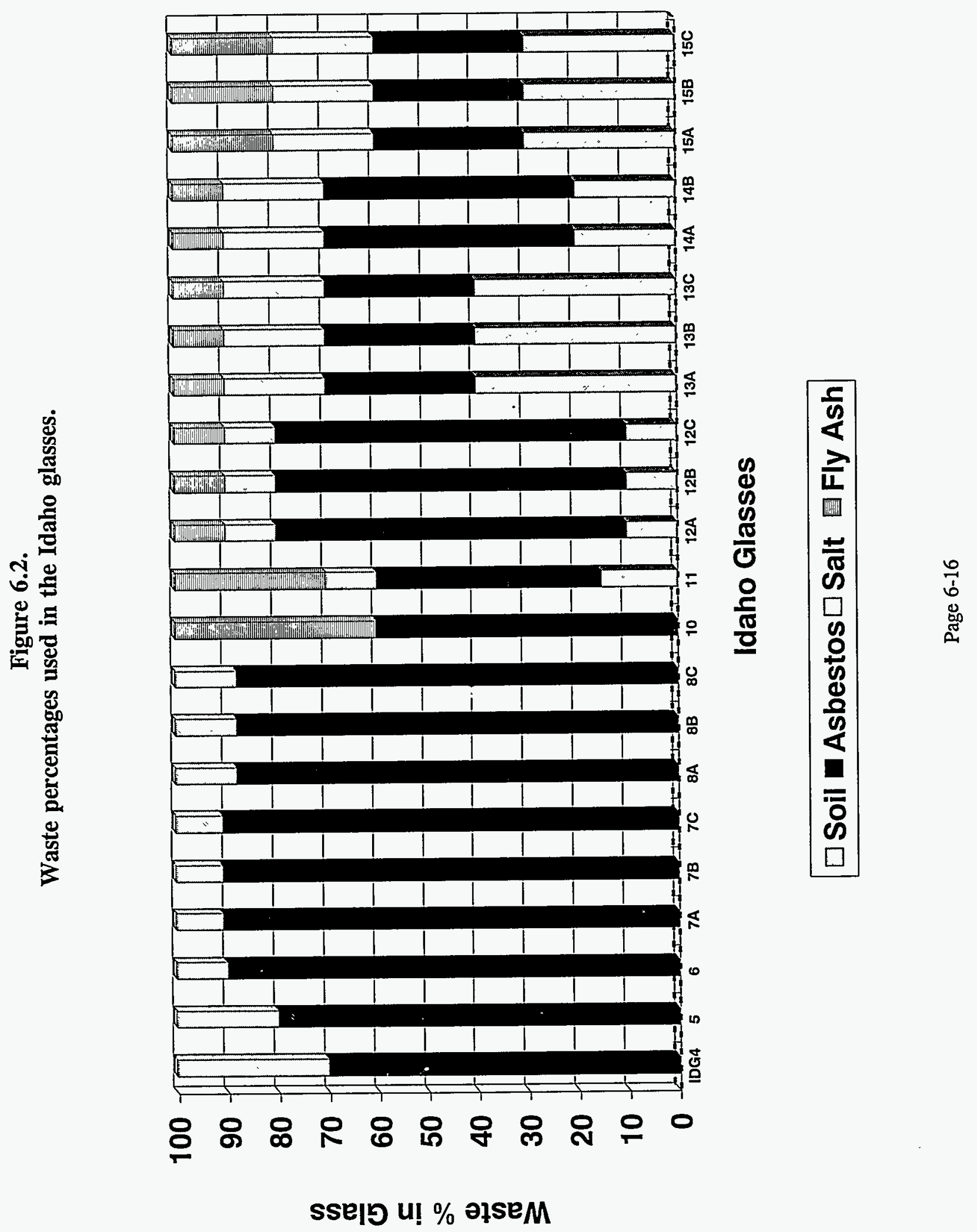


Figure 6.3.

Waste percentages used in the Oak Ridge glasses.

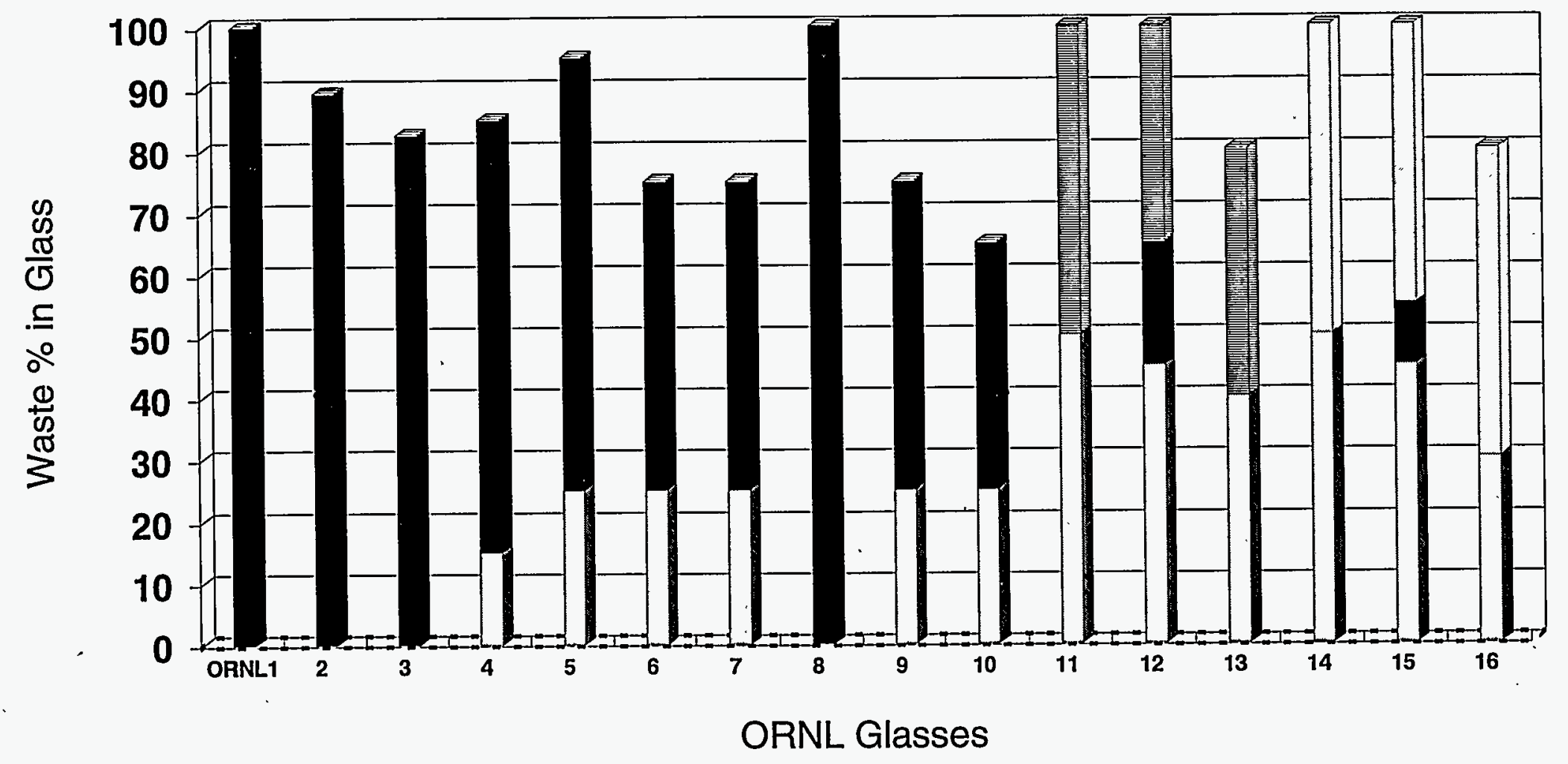

$\square$ Soil $\square$ Pond Waste $\square$ TSCA Ash 国WETF Waste 


\section{SECTION 7.0 \\ REFERENCES}

Adam-1965

G. Adam and J.H. Gibbs, "On the Temperature Dependence of Cooperative Relaxation Properties in Glass-forming Liquids," Journal of Chemical Physics, 43, 139-146 (1965).

\section{Adel-Hadadi-1988}

M. Adel-Hadadi, R. Adiga, Aa. Barkatt, Al. Barkatt, X. Feng, S. Finger, W.P. Freeborn, P. B. Macedo, R. Mohr, C. Montrose, I.L. Pegg, E. Saad, and W. Sousanpour, "Preliminary Results of Durability Testing with Borosilicate Glass Compositions," DOE/NE/44139-34, Barkatt-1991

West Valley Nuclear Services, Inc., West Valley, NY (1988).

Aa. Barkatt, S.A. Olszowska, W. Sousanpor, M.A. Adel-Hadadi, R. Adiga, Al. Barkatt, G. S. Marbury, and S. Li, "Leach Rate Excursions in Borosilicate Glasses: Effcts of Glass and Leachant Composition," in Scientific Basis for Nuclear Waste Management XIV, Eds.

Bockris-1970

T. A. Abrajano and L. H. Johnson, Materials Research Society, Pittsburgh, PA, p. 65 (1991).

J.O'M. Bockris and A.K.N. Reddy, Modern Electrochemistry, 1, Plenum Press, New York (1970).

\section{Bockris-1955}

J.O'M. Bockris, J.D. MacKenzie, and J.A. Kitchener, "Viscous Flow in Silica and Binary Silicates," Trans. Farad. Soc., 51, 1734-1748 (1955).

\section{Bockris-1954}

J.O'M. Bockris and D.C. Lowe, "Viscosity and the Structure of Molten Silicates," Proc.

\section{Boomer-1993}

Roy. Soc. Lond., A226, 423-435 (1954).

K. D. Boomer et al., "Tank Waste Technical Options Report," WHC-EP-0616, Westinghouse

Bostick-1994

Hanford Company, Richland, WA (1993).

W. D. Bostick, D. P. Hoffmann, R. J. Stevenson, A. A. Richmond, and D. F. Bickford, "Surrogate Formulations for Low-Level Mixed Waste, Part IV: Wastewater Treatement Sludges," DOE/MWIP-18, Martin Marietta Energy Systems, Inc., Oak Ridge, TN (1994).

\section{Bourcier-1990}

W. L. Bourcier, D.W. Peiffer, K. G. Knauss, K. D. McKeegan, and D. K. Smith, "A Kinetic Model for Borosilicate Glass Dissolution Based on the Dissolution Affinity of a Surface Alteration Layer," in Scientific Basis for Nuclear Waste Management XIII, Eds. V. M. Oversby and P. W. Brown, Materials Research Society, Pittsburgh, PA, p. 209 (1990). 


\section{Bowan-1994}

B.W. Bowan, M.M. Brandys, R.K. Mohr, I.L. Pegg, and P.B. Macedo, "Design of a Vitrification Process for the Stabilization of Mixed Wastes at the Fernald Environmental Management Project," Ceramic Transactions, Environmental and Waste Management Issues in the Ceramic Industry, Ed. G.B. Mellinger, ACerS, 39, p.23 (1994).

\section{Brandys-1995}

M. Brandys, R. Mohr, H. Hojaji, S. Fu, P. B. Macedo, K. E. Hain, and W. Greenman, "Vitrification of Wet Waste Resin," Waste Management '95, Eds. R. G. Post and M. E. Wacks, WM Symposia, Inc. (1995), in press.

\section{Brown-1988}

G.E. Brown, G. Calas, G.A. Waychunas, and J. Petiau, "X-ray Absorption Spectroscopy and its Applications in Mineralogy and Geochemistry," In Hawthorne FC (ed) Reviews in Mineralogy 18: Spectroscopic Methods in Mineralogy and Geology, 431-512 (1988).

\section{CUA VSL-1992}

"The Catholic University of America, Vitreous State Laboratory Quality Assurance Manual and Implementing Technical Procedures," VSLQAM.MAN, Rev. 11 (1992).

\section{DeJong-1981}

B.H.W.S. DeJong, K.D. Keefer, G.E. Brown, Jr., and C.M. Taylor, "Polymerization of Silicate and Aluminate Tetrahedra in Glasses, Melts and Aqueous Solutions -- III. Local Silicon Environments and Internal Nucleation in Silicate Glasses," Geochimica et Cosmochimica Acta, 45, 1291-1308 (1981).

\section{Dell-1983}

W.J. Dell, P.J. Bray, and X.Z. Xiao, " ${ }^{11} \mathrm{~B}$ NMR Studies and Structural Modeling of $\mathrm{Na}_{2} \mathrm{O}-$ $\mathrm{B}_{2} \mathrm{O}_{3}-\mathrm{SiO}_{2}$ Glasses of High Soda Content," Journal of Non-Crystalline Solids, 58, 1-16 (1983).

\section{Dickenson-1981}

M.P. Dickenson and P.C. Hess, "Redox Equilibria and the Structural Role of Iron in Alumino-silicate Melts," Contribution to Mineralogy and Petrology, 78, 353-357 (1981).

\section{Domine-1986}

F. Domine and B. Piriou, "Raman Spectroscopic Study of the $\mathrm{SiO}_{2}-\mathrm{Al}_{2} \mathrm{O}_{3}-\mathrm{K}_{2} \mathrm{O}$ Vitreous System: Distribution of Silicon and Second Neighbors," American Mineralogist, 71, 38-50 (1986).

\section{Dupree-1986}

R. Dupree, D. Holland, and D.S. William, "The Structure of Binary Alkali Silicate Glasses,"

\section{Dupree-1984}

J. Non-Cryst. Solids, 81, 185-200 (1986).

R. Dupree, D. Holland, P.W. McMillan and R.F. Pettifer, "The Structure of Soda-Silicate

Glasses: A NAS NMR study," J. Non-Cryst. Solids, 68, 399-410 (1984). 


\section{Dyar-1985}

M. D. Dyar, "A Review of Mossbauer Data on Inorganic Glasses: The Effects of Composition on Iron Valency and Coordination," American Mineralogist, 70, 304-316 (1985).

\section{Engelhardt-1985}

G. Engelhardt, M. Nofz, K. Forkel, F.G. Wihsmann, M. Mägi, A. Samoson, and E. Lippmaa, "Structural Studies of Calcium Aluminosilicate Glasses by High Resolution Solid State ${ }^{29} \mathrm{Si}$ and ${ }^{27} \mathrm{Al}$ Magic Angle Spinning Nuclear Magnetic Resonance," Physics and Chemistry of Glasses, 26, 157-165 (1985).

\section{Farnan-1985}

I. Farnan and J.F. Stebbins, "Observation of Slow Atomic Motions Close to Glass Transition Feng-1989 Using 2-D 29Si NMR,” J. Non-Cryst. Solids, 124, 207-215 (1990).

X. Feng, I.L. Pegg, Aa. Barkatt, P.B. Macedo, S. Cucinell, and S. Lai, "Correlation Between Composition Effects on Glass Durability and the Structural Role of the Constituent Oxides," Nuclear Technology, 85, 334 (1989).

Feng-1990

X. Feng, I.L. Pegg, Y. Guo, Aa. Barkatt, and P.B. Macedo, "Effects of Surface-Area-toSolution Volume Ratio on Chemical Durability of Nuclear Waste Glasses," in Scientific Basis for Nuclear Waste Management XIII, p. 383, Ed. V. M. Oversby and P. W. Brown, MRS, Pittsburgh, PA (1990).

\section{Feng-1994}

X. Feng and I. L. Pegg, "A Glass Dissolution Model for the Effects of S/V on Leachate pH," Fu-1993

J. Noncryst. Solids, 175, 281 (1994).

S.S. Fu, J. Ruller, C. Paul, and I.L. Pegg, "Vitrification Development Studies for OU1

Wastes," Final Report to Fernald Environmental Management Corporation, Cincinnati, OH Fu-1994a (1993).

S.S. Fu, K.S. Matlack, R.K. Mohr, M. Brandys, H. Hojaji, S. Bennett, J. Ruller, and I.L. Pegg, "Development and Testing of the Minimum Additive Waste Stabilization (MAWS) Fu-1994b

System for Fernald Wastes," Phase I Final Report, Contract \# 21402401 (1994).

S.S. Fu, K. S. Matlack, and I.L. Pegg, "Vitrification Studies for CRU1 Wastes," Final Report to Fernald Environmental Management Corporation, Cincinnati, OH (1994).

\section{Gan-1994a}

H. Gan, et al., "Phosphorus and Boron Speciation in $\mathrm{K}_{2} \mathrm{O}-\mathrm{B}_{2} \mathrm{O}_{3}-\mathrm{SiO}_{2}-\mathrm{P}_{2} \mathrm{O}_{5}$ Glasses," Geochimica et Cosmochimica Acta, 58, 4633-4647 (1994). 


\section{Gan-1994b}

H. Gan, P.C. Hess and R.J. Kirkpatrick, "Phosphorus and Boron Speciation in $\mathrm{K}_{2} \mathrm{O}-\mathrm{B}_{2} \mathrm{O}_{3}$ $\mathrm{SiO}_{2}-\mathrm{P}_{2} \mathrm{O}_{5}$ Glasses," Geochimica et Cosmochimica Acta, 58, $4633-4647$ (1994).

\section{Geisinger-1988}

K.L. Geisinger, R. Oestrike, A. Navrotsky, G. L. Turner, and R. J. Kirkpatrick, "Thermochemistry and Structure of Glasses along the Join $\mathrm{NaAlSi}_{3} \mathrm{O}_{8}-\mathrm{KBSi}_{3} \mathrm{O}_{8}$," Geochimica et Cosmochimica Acta, 52, 405-2414 (1988).

\section{Goldman-1985}

D. S. Goldman, "Redox and Solubility in Glass Melts," in Gas Bubbles in Glass Melts, prepared by Technical Committee 14 of the International Commission on Glass, Charleroi, Institut National du Verre, p. 74 (1985).

GTS Duratek-1995

"Phase 1 Hanford LLW Melter Tests," GTS Duratek and The Catholic University of America Final Report, Westinghouse Hanford Company, Richland, WA (1995), in press.

\section{Hojaji-1995}

H. Hojaji, M. Brandys, R. K. Mohr, H. Gan, I. L. Pegg, and P. B. Macedo, Unpublished work.

\section{Hrma-1994}

P.R. Hrma et al., "Property/Composition Relationship for Hanford High-Level Waste Glasses Melting at $1150^{\circ} \mathrm{C}$," Report for the US DOE under contract of DE-AC06-76RLO 1830 (1994).

\section{Ingram-1987}

M.D. Ingram, Ionic Conductivity in Glass, Physics and Chemistry of Glasses, 28, 215(1987).

\section{Jantzen-1984}

C.M. Jantzen and M.J. Plodinec, "Thermodynamic Model of Natural Medieval and Nuclear Waste Glass Durability," J. Non-Cryst. Solids, 67, 207-223 (1984).

\section{Jantzen-1991}

C.M. Jantzen, "First Principles Process-Product Models for Vitrification of Nuclear Waste: Relationship of Glass Composition to Glass Viscosity, Resistivity, Liquidus Temperature, and Durability," Ceramic Transactions, Nuclear Waste Management IV, Eds. G.C. Wicks, D.F. Bickford, and L.R. Bunnell, ACerS, 23, 37 (1991).

\section{Kirkpatrick-1986}

R.J. Kirkpatrick, R. Oestrike, C.A. Weiss, Jr., K.A. Smith, and E. Oldfield, "High-resolution

${ }^{27} \mathrm{Al}$ and ${ }^{29} \mathrm{Si}$ NMR Spectroscopy of Glasses and Crystals along the Join $\mathrm{CaMgSi}_{2} \mathrm{O}_{6}-$ $\mathrm{CaAl}_{2} \mathrm{SiO}_{6}, "$ American Mineralogist, 71, 705-711 (1986).

\section{Ludowise-1993}

J. Ludowise, Westinghouse Hanford Company, Personal Communication, (1993). 


\section{MacKenzie-1960}

J.D. MacKenzie, "Structure of Some Inorganic Glasses from High Temperature Studies," In Modern Aspects of the Vitreous State (J.D. MacKenzie, ed.), Butterworths, Washington, D.C. $(1960)$.

Maekawa-1991

H. Maekawa, T. Maekawa, K. Kawamura, and T. Tokokawa, "The Structural Groups of Alkali Silicate Glasses Determined from ${ }^{29}$ Si MAS-NMR," J. Non-Cryst. Solids, 127, 53-64 (1991).

\section{McMenamin-1992}

T. McMenamin, "In Situ Testing of Radioactive WasteForms and Engineered Barriers,"

Proc. of CEC, SCK/CEN and US DOE workshop, Corsendonk, Belgium (1992).

McMillan-1984

P. McMillan, "Structural Studies of Silicate Glasses and Melts: Applications and Limitations of Raman Spectroscopy," American Mineralogist, 69, 622-644 (1984).

\section{Merrill-1994}

R. A. Merrill and K. A. Whittington, "Final Report of Vitrification Development Studies for Fernald CRU-4 Silo Wastes," Battelle Pacific Northwest Laboratory, Richland, WA April 1994.

\section{Muller-1992}

I.S. Muller, I.L. Pegg, and P.B. Macedo, "Non-linear Effects of Glass Composition on Chemical Durability: Physical Stability of Surface Layers," Scientific Basis for Nuclear Waste Management, XV, Mat. Res. Soc. Symp. Proc., Strasbourg, 257, $91-98$ (1992).

\section{Muller-1993a}

I.S. Muller, G. del Rosario, and P.B. Macedo, "Vitrification of Hazardous Waste Incinerator Ashes," unpublished work (1993).

Muller-1993b

I.S. Muller, S.S. Fu, K.S. Matlack, H. Hojaji, I.L. Pegg, and P.B. Macedo, "Vitrification Development and Melter Testing with Actual Savannah River M-Area Wastes," Reports to GTS Duratek, Inc., Columbia, MD (1993-1995).

\section{Mysen-1989}

B.O. Mysen and D. Virgo, "Redox Equilibria, Structure, and Properties of Fe-bearing Aluminosilicate Melts: Relationships among Temperature, Composition and Oxygen Fugacity in the System $\mathrm{Na}_{2} \mathrm{O}-\mathrm{Al}_{2} \mathrm{O}_{3}-\mathrm{SiO}_{2}-\mathrm{Fe}-\mathrm{O}$," American Mineralogist, 74, 58-76 (1989). Mysen-1985

B.O. Mysen, D. Virgo and F.A. Seifert, "Relationships Between Properties and Structure of Aluminosilicate Melts," American Mineralogist, 69, 834-847 (1985). 


\section{Mysen-1981}

B.O. Mysen, D. Virgo and I. Kushiro, "The Structural Role of Aluminum in Silicate Melts a Raman Spectroscopic Study at 1 Atmosphere," American Mineralogist, 66, 678-701 (1981).

\section{Navrotsky-1985}

A. Navrotsky, K.L. Geisinger, P. McMillan, and G. V. Gibbs, "The Tetrahedral Framework in Glasses and Melts--References from Molecular Orbital Calculations and Implications for Structure, Thermodynamics and Physical Properties," Physics and Chemistry of Minerals, 11, 284-298 (1985).

Napolitano-1965

\section{Newton-1980}

A. Napolitano, P. B. Macedo, and E. G. Hawkins, "A wide Range (up to $10^{10} \mathrm{P}$ ) Rotating Cylinder Viscometer," J. Res. Nat. Bur. Standards, 69A, 449 (1965).

R.G. Newton and A. Paul, "A New Approach to Predicting the Durability of Glasses from Their Chemical Compositions," Glass Technology, 21, 307-309 (1980).

\section{Osborn-1960}

E.F. Osborn and A. Muan, "Phase Equilibrium Diagrams of Oxide System," Plate 4, American Ceramic Society and The Edward Orton, Jr., Ceramic Foundation (1960).

\section{Papadopoulos-1973}

\section{Park-1979}

K. Papadopoulos, Phys. Chem. Glasses, 14, 60 (1973).

M.J. Park, K.S. Kim, and P.J. Bray, "The Determination of the Structures of Compounds and Glasses in the System MgO- $\mathrm{B}_{2} \mathrm{O}_{3}$ using "B NMR," Physics and Chemistry of Glasses, 20, 31-34 (1979).

\section{Patyn-1990}

J. Patyn, P. Van Iseghem, W. Timmermans, "The Long-Term Corrosion and Modelling of Two Simulated Belgian Reference High-Level Waste Glasses - Part II," in Scientific Basis for Nuclear Waste Management XIII, Eds. V. M. Oversby and P. W. Brown, Materials Paul-1990

Research Society, Pittsburgh, PA, p. 299 (1990).

\section{Pegg-1991}

A. Paul, The Chemistry of Glasses, Chapman and Hall, 2nd Ed. (1990).

I.L. Pegg, Aa. Barkatt, A.C Buechele, Y. Guo, S.T. Lai, I.S. Muller, C. Sivaprakash, R.E. Sassoon, and P.B. Macedo, Activities at VSL in Support of the West Valley Demonstration Project - Annual Report, West Valley Nuclear Services, Inc., West Valley, NY (1991).

Pegg-1993a

I.L. Pegg, D.C. Grant, Y. Guo, E.J. Lahoda, S. Lai, I.S. Muller, and J. Ruller, "Development of a Combined Soil-Wash/In-Furnace Vitrification System for Soil Remediation at DOE Sites," DOE/CH9204 (1993). 


\section{Pegg-1993b}

I.L. Pegg, S.T. Lai, I.S. Muller, A.C Buechele, C. Sivaprakash, and P.B. Macedo, "Activities at VSL in Support of the West Valley Demonstration Project - Annual Report," West Valley Nuclear Services, Inc., West Valley, NY (1993).

Pegg-1994a

I.L. Pegg, "Development of the Minimum Additive Waste Stabilization (MAWS) Program for Fernald," Ceramic Transactions, Environmental and Waste Management Issues in the Ceramic Industry, Ed. G.B. Mellinger, ACerS, 39, p.13 (1994).

Pegg-1994b

I.L. Pegg, "The Minimum Additive Waste Stabilization (MAWS) Demonstration Program at the Fernald Site," Waste Management '94, Ed. R.G. Post, 3, p.1533 (1994).

Pegg-1994c

I.L. Pegg, I.S. Muller, C. Sivaprakash, S. T. Lai, A.C Buechele, Aa. Barkatt, M. AdelHadadi, N. Balitactac, Y. Guo, C. Paul, and P. B. Macedo, "Activities at VSL in Support of the West Valley Demonstration Project - Annual Report," West Valley Nuclear Services, Inc., West Valley, NY (1994).

Ritchet-1984

P. Ritchet, "Viscosity and Configurational Entropy of Silicate Melts," Geochimica et Cosmochimica Acta, 48, 470-485 (1984).

Schramm-1984

C.M. Schramm, B.H.W.S. DeJong, and V.E. Parziale, ${ }^{" 29}$ Si Magic Angle Spinning NMR Study on Local Silicon Environments in Amorphous and Crystalline Lithium Silicates," Journal of American Chemical Society, 106, 4396-4402 (1984).

Schreiber-1987

H.D. Schreiber, S.J. Kozak, P.G. Leonhard, and K.K. McManus, "Sulfur Chemistry in a Borosilicate Melt, Part 1: Redox Equilibria and Solubility," Glastech. Ber., 60, No. 12, pp. 389-398 (1987).

\section{Shade-1994}

J. W. Shade, "Waste Simulant Development for Evaluation of LLW Melter System Technology," WHC-SD-WM-TI-624, Westinghouse Hanford Company, Richland, WA (1994).

Stebbins-1988

J. Stebbins, "Effects of Temperature and Composition on Silicate Glass Structure and Dynamics: 29Si NMR Result," J. Non-Cryst. Solids, 106, 359-369 (1988).

Taylor-1979

M. Taylor and G.E. Brown, Jr., "Structure of Mineral Glasses -- I. The Feldspar Glasses $\mathrm{NaAlSi}_{3} \mathrm{O}_{8}, \mathrm{KalSi}_{3} \mathrm{O}_{8}$ and $\mathrm{CaAl}_{2} \mathrm{Si}_{2} \mathrm{O}_{8}, "$ Geochimica et Cosmochimica Acta, 43, 61-75 (1979). 
Tickle-1967

R.E. Tickle, "The Electrical Conductance of Molten Alkali Silicates, Part I. Experiments and US EPA-1990

Results," Physics and Chemistry of Glasses, 8, 101-112 (1967).

U.S. Environmental Protection Agency, (1990), "Method 1311 Toxicity Characteristic Leaching Procedure," Federal Register, 55, No. 60 (1990).

\section{US EPA-1986}

U.S. Environmental Protection Agency, "Test Methods for Evaluating Solid Waste," Office of Solid Waste and Emergency Response, SW-846, 3rd Ed., (1986); incorporating updates through Update IIB, March 1995.

\section{Voogd-1993}

J. Voogd, Westinghouse Hanford Company, Personal Communication (1993).

\section{Whittington-1994}

K. Whittington, R. Peters, M. Cooper, D. Bonner, D. Phares, and S. Perez, "Vitrification Development for Sludge and Ash Mixed Wastes," Waste Management '94, Eds. R. G. Post and M. E. Wacks, WM Symposia, Inc., p. 1565 (1994).

\section{Xing-1994a}

S.-B. Xing, I.S. Muller, and I.L. Pegg, "Non-Linearity in Glass Composition Dependence of Dissolution Rates: Effect of Solution pH," Mat. Res. Soc. Symp. Proc., 333, 549-556 (1994).

\section{Xing-1994b}

S.-B. Xing, A.C. Buechele, S.S. Fu, Z.B. Djordjevic, and I.L. Pegg, "Non-Linear Effect of Glass Composition on Glass Dissolution in Both Borosilicate and Fluorosilicate Waste Glasses," in Environmental and Waste Management Issues in the Ceramic Industry II, Ceramic Transactions, Eds. D. Bickford, S. Bates, V. Jain, and G. Smith, ACerS, 45,177 (1994).

\section{Yan-1994}

Q. Yan, Z.B. Djordjevic, F. Perez-Cardenas, I.S. Muller, P.B. Macedo, and G. del Rosario, Vitrification of Incinerator Ashes," in Environmental and Waste Management Issues in the Ceramics Industry, Ceramic Transactions Eds. D. Bickford, S. Bates, V. Jain, and G. Smith, Ceramic Transactions, 45, 13 (1994). 


\section{Distribution for DOE/CH-9601}

Internal:

J. M. Andrew

J. K. Bates

D. J. Chaiko

J. C. Cunnane (50)

W. L. Ebert

External:
G. Edgell

J. E. Harmon

J. J. Laidler

R. Martello
A. D. Pflug

D. M. Strachan

D. J. Wronkiewicz

TIS Files

DOE-OSTI (2)

ANL-E Library

ANL-W Library

Manager, Chicago Operations Office, DOE

R. C. Baker, DOE-CH

A. Bindokas, DOE-CH

J. C. Haugen, DOE-CH

S. L. Webster, DOE-CH

J. Allison, Westinghouse Savannah River Company, Aiken, SC

T. D. Anderson, USDOE, Office of Science and Technology, Germantown, MD

A. Alm, USDOE, Office of Environmental Management, Washington, DC

M. Arndt, Lockheed Environmental Systems \& Technology, Pocatello, ID (5)

D. H. Bandy, USDOE, Albuquerque Operations Office, Albuquerque, NM

M. J. Barainca, USDOE, Office of Science and Technology, Washington, DC

J. E. Baublitz, USDOE, Office of Environmental Restoration, Germantown, MD

J. Bauer, USDOE, Office of Environmental Management, Germantown, MD

R. C. Bedick, USDOE, Morgantown Energy Technology Center, Morgantown, WV

M. Berger, Los Alamos National Laboratory, Los Alamos, NM

N. J. Beskid, Naperville, $\mathbb{L}$

D. Biancosino, USDOE, Office of Science and Technology, Washington, DC

J. Bickel, USDOE, Albuquerque Operations Office, Albuquerque, NM

D. Bottrell, USDOE, Office of Science and Technology, Washington, DC

G. Boyd, USDOE, Office of Science and Technology, Germantown, MD

M. H. Carter, USDOE, Office of Environmental Management, Germantown, MD

K. A. Chacey, USDOE, Office of Environmental Management, Germantown, MD

P. Colombo, Brookhaven National Laboratories, Upton, NY

J. Corones, Ames Laboratory, Iowa State University, Ames, IA

S. P. Cowan, USDOE, Office of Waste Management, Germantown, MD

M. Elliott, Battelle-PNNL, Richland, WA

J. J. Fiore, USDOE, Office of Environmental Management, Germantown, MD

C. W. Frank, USDOE, Office of Science and Technology, Washington, DC

W. Greenman, GTS/Duratek Corporation, Columbia, MD (5)

J. Hall, USDOE, Nevada Field Office, Las Vegas, NV. 
L. H. Harmon, USDOE, Office of Environmental Management, Germantown, MD

E. L. Helminski, Weapons Complex Monitor, Washington, DC

R. D. Hill, U.S. Environmental Protection Agency, Cincinnati, $\mathrm{OH}$

W. Holman, USDOE, San Francisco Operations Office, Oakland, CA

P. Hrma, Battelle-PNNL, Richland, WA

J. Hyde, USDOE, Office of Science and Technology, Washington, DC

S. James, U.S. Environmental Protection Agency, Cincinnati, OH

E. Koglin, U.S. Environmental Protection Agency, Las Vegas, NV

P. Krumrine, Westinghouse Savannah River Company, Aiken, SC

J. M. Lankford, USDOE, Office of Science and Technology, Germantown, MD

J. C. Lehr, USDOE, Office of Environmental Restoration, Germantown, MD

R. G. Lightner, USDOE, Office of Environmental Management, Germantown, MD

P. Lurk, USDOE, Office of Science and Technology, Washington, DC

A. P. Malinauskas, Oak Ridge National Laboratory, Oak Ridge, TN

S. A. Mann, USDOE, Office of Environmental Restoration, Germantown, MD

J. Marra, Savannah River Technology Center, Aiken, GA

J. Mazer, Downers Grove, IL

C. P. McGinnis, Oak Ridge National Laboratory, Oak Ridge, TN

J. Moore, USDOE, Oak Ridge Operations Office, Oak Ridge, TN

K. Morehouse, U.S. Environmental Protection Agency, Washington, DC

J. Paladino, USDOE, Office of Science and Technology, Germantown, MD

G. S. Patton, USDOE, Office of Science and Technology, Washington, DC

I. L. Pegg, GTS Duratek, Columbia, MD

D. Peeler, Savannah River Technology Center, Aiken, GA

J. Perez, Battelle-PNNL, Richland, WA

M. Peterson, Pacific Northwest National Laboratory, Richland, WA

J. Poppiti, USDOE, Office of Science and Technology, Washington, DC

R. E. Prince, Duratek Corporation, Columbia, MD

P. A. Saxman, USDOE, Albuquerque Operations Office, Albuquerque, NM

J. Simpson, USDOE, Office of Science and Technology, Washington, DC

C. Sink, USDOE, Office of Science and Technology, Washington, DC

S. C. Slate, Pacific Northwest National Laboratory, Richland, WA

R. Snipes, Hazardous Waste Remedial Actions Program, Oak Ridge, TN

J. L. Steele, Westinghouse Savannah River Company, Aiken, SC

S. L. Stein, Battelle Seattle Research Center, Seattle, WA

L. H. Taylor, USDOE, Office of Environmental Restoration, Germantown, MD

J. A. Turi, USDOE, Office of Environmental Management, Germantown, MD

J. W. Wagoner, USDOE, Office of Environmental Restoration, Germantown, MD

J. Walker, USDOE, Office of Science and Technology, Washington, DC

R. D. Warner, USDOE, Fernald Field Office, Cincinnati, OH (4)

P. Wichlacz, EG\&G Idaho, Inc., Idaho Falls, ID

W. Wisenbaker, USDOE, Office of Environmental Management, Germantown, MD

J. L. Yow, Livermore, CA 